Journal of Energy and Power Technology (ISSN 2690-1692)
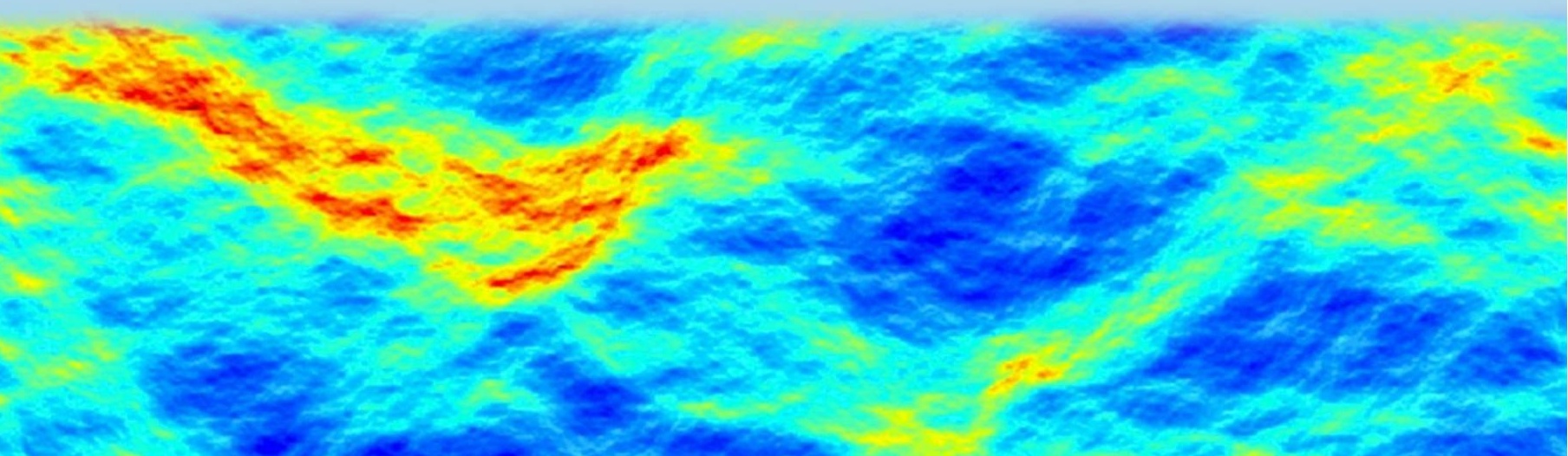

Crustal reservoir fluid flow is

channelised by the empirical relation

$K(x, y, z) \sim \exp (\alpha \varphi(x, y, z))$ linking

permeability $\mathrm{K}$ to porosity $\varphi$

throughout the ambient crust.

Volume 3, Issue 1 | March 2021

SILIDSEN 
Journal of Energy and Power Technology (ISSN 2690-1692)

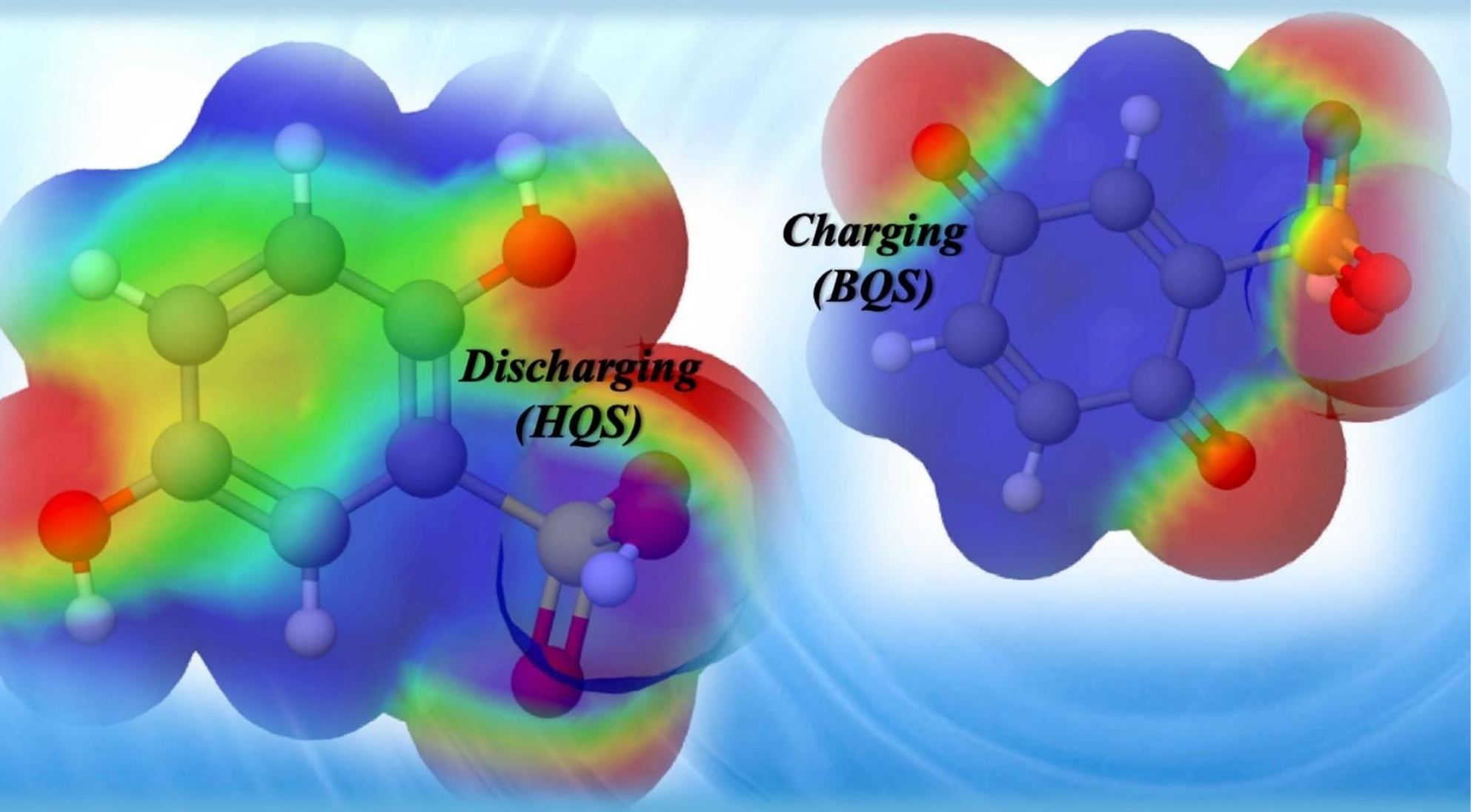

A novel high energy density lithium battery with an aqueous

hydroquinone sulfonic acid/

benzoquinone sulfonic acid redox

couple cathode

Volume 3, Issue 1 | February 2021

$\checkmark$ LIDSEN 
Journal of Energy and Power Technology

(ISSN 2690-1692)

Crustal reservoir fluid flow is

channelised by the empirical relation

$K(X, Y, Z) \sim \exp (\alpha \varphi(x, y, z))$ linking

permeability K to porosity $\varphi$

throughout the ambient crust.

Volume 3 Issue 1 | March 2021 
Journal of Energy and Power Technology

(ISSN 2690-1692)

\section{A novel high energy density lithium battery with an aqueous hydroquinone sulfonic acid/ benzoquinone sulfonic acid redox couple cathode}

Volume 3 Issue 1 | February 2021 
Editorial Office

Journal of Energy and Power Technology Editorial Office, 73 Hongkong Middle Road, Qingdao, China

Tel./Fax: +86-532-8979-9572

LIDSEN Publishing Inc.

2000 Auburn Drive, One Chagrin

Highlands, Suite 200, Beachwood, OH 44122, USA

Tel.: +1-216-370-7293

Fax: +1-216-378-7505

http://www.lidsen.com/

This is a reprint of articles from the Volume 3 Issue 1 published online in the open access journal Journal of Energy and Power Technology (ISSN 2690-1692) from January 1, 2021 to March 31, 2021 (available at: http://www.lidsen.com/journals/jept/jept-issue-03-01).

For citation purposes, cite each article independently as indicated on the article page online and as indicated below:

LastName, AA, LastName, BB, LastName, CC. Article Title. Journal Name Year; Volume(Issue):Article Number; doi.

(C) 2021 by the author. This is an open access article distributed under the conditions of the Creative Commons by Attribution License, which permits unrestricted use, distribution, and reproduction in any medium or format, provided the original work is correctly cited. 


\section{Contents}

Assessment of $\mathrm{Cu}(\mathrm{In}, \mathrm{Ga}) \mathrm{Se}_{2}$ Solar Cells Degradation due to Water Ingress Effect on the CdS Buffer Layer

Deewakar Poudel, Benjamin Belfore, Shankar Karki, Grace Rajan, Sina Soltanmohammad, Angus Rockett, Sylvain Marsillac

Reprinted from: Journal of Energy and Power Technology 2021;3(1):001;

doi:10.21926/jept.2101001 1

Online State of Charge Prediction in Next Generation Vehicle Batteries Using Deep Recurrent Neural Networks and Continuous Model Size Control

Steven Hespeler, Donovan Fuqua

Reprinted from: Journal of Energy and Power Technology 2021;3(1):003;

doi:10.21926/jept.2101003 10

SOFC Stack and System Modeling, Fault Diagnosis and Control

Khaliq Ahmed, Periasamy Vijay, Moses Tadé, Amirpiran Amiri, Zongping Shao, Karl Föger

Reprinted from: Journal of Energy and Power Technology 2021;3(1):004;

doi:10.21926/jept.2101004 .34

Spatio-Temporal Metocean Measurements for Offshore Wind Power

Lucy Wyatt

Reprinted from: Journal of Energy and Power Technology 2021;3(1):005;

doi:10.21926/jept.2101005 66

Quaternary Hydrides $\mathrm{Pd}_{1-y-z} \mathrm{Ag}_{y} \mathrm{Cu}_{z} \mathrm{H}_{x}$ Embedded Atom Method Potentials for Hydrogen Energy Applications

Chaonan Zhang, Robert Fuller, Iyad Hijazi

Reprinted from: Journal of Energy and Power Technology 2021;3(1):006;

doi:10.21926/jept.2101006 81

Ni-Silica-based Catalysts for $\mathrm{CH}_{4}$ Reforming by $\mathrm{CO}_{2}$ with Enhanced Stability: Recent Designs and the Impacts of Ni Confinement, Promoters, and Core-Shell Structures

Oscar Daoura, Maya Boutros, Franck Launay

Reprinted from: Journal of Energy and Power Technology 2021;3(1):007;

doi:10.21926/jept.2101007 106

Modeling of Ceria Reduction in a Solar Thermochemical Reactor via DEM Method

Han Zhang, Joseph D. Smith

Reprinted from: Journal of Energy and Power Technology 2021;3(1):008;

doi:10.21926/jept.2101008

Melting/Solidification Processes of PEG 1500 in Vertical and Horizontal Annular Enclosure

F. A. Hamad, E. Egelle, Abbas Mohammed, S. Gooneratne, P. Russell

Reprinted from: Journal of Energy and Power Technology 2021;3(1):009;

doi:10.21926/jept.2101009 
High Energy Density Rechargeable Aqueous Lithium Batteries with an Aqueous Hydroquinone Sulfonic Acid and Benzoquinone Sulfonic Acid Redox Couple Cathode

Hironori Takagi, Koichi Kakimoto, Daisuke Mori, Sou Taminato, Yasuo Takeda, Osamu Yamamoto, Nobuyuki Imanishi

Reprinted from: Journal of Energy and Power Technology 2021;3(1):010;

doi:10.21926/jept.2101010

Accelerated Controller Tuning for Wind Turbines Under Multiple Hazards

Aly Mousaad Aly, Milad Rezaee

Reprinted from: Journal of Energy and Power Technology 2021;3(1):011;

doi:10.21926/jept.2101011 204

Multi-Disciplinary and Multi-Scale Assessment of Marine Renewable Energy Structure in a Tidal System

Aurore Raoux, Ilan Robin, Jean-Philippe Pezy, Anne-Claire Bennis, Jean-Claude Dauvin

Reprinted from: Journal of Energy and Power Technology 2021;3(1):012;

doi:10.21926/jept.2101012 234

Crustal Reservoir Flow Simulation for Long-Range Spatially-Correlated Random Poroperm Media Peter Leary, Peter Malin

Reprinted from: Journal of Energy and Power Technology 2021;3(1):013;

doi:10.21926/jept.2101013

Nowcasting Wind, Wave, Current and Turbulence Intensity for Offshore Wind Power Operation and Maintenance

Shih-Ang Hsu

Reprinted from: Journal of Energy and Power Technology 2021;3(1):014;

doi:10.21926/jept.2101014 .268 
Original Research

\section{Assessment of $\mathrm{Cu}(\mathrm{In}, \mathrm{Ga}) \mathrm{Se}_{2}$ Solar Cells Degradation due to Water Ingress Effect on the CdS Buffer Layer}

Deewakar Poudel ${ }^{1}$, Benjamin Belfore ${ }^{1}$, Shankar Karki ${ }^{1}$, Grace Rajan ${ }^{1}$, Sina Soltanmohammad ${ }^{2}$, Angus Rockett ${ }^{2}$, Sylvain Marsillac ${ }^{1, *}$

1. Virginia Institute of Photovoltaics, Old Dominion University, Norfolk, VA 23529, USA; E-Mails: dpoud001@odu.edu; bbelf001@odu.edu; skark002@odu.edu; gracecrajan88@gmail.com; smarsill@odu.edu

2. Department of Metallurgical and Materials Engineering, Colorado School of Mines, Golden, CO 80401, USA; E-Mails: sinasm@udel.edu; arockett@mines.edu

* Correspondence: Sylvain Marsillac; E-Mail: smarsill@odu.edu

Academic Editor: Joaquin Alonso-Montesinos

Special Issue: Photovoltaic Solar Systems and Solar Thermal Plants

Journal of Energy and Power Technology

2021, volume 3 , issue 1

doi:10.21926/jept.2101001
Received: October 28, 2020

Accepted: December 28, 2020

Published: January 6, 2021

\section{Abstract}

The effect of water ingress on the surface of the buffer layer of a $\mathrm{Cu}(\mathrm{In}, \mathrm{Ga}) \mathrm{Se}_{2}$ (CIGS) solar cell was studied. Such degradation can occur either during the fabrication process, if it involves a chemical bath as is often the case for $\mathrm{CdS}$, or while the modules are in the field and encapsulants degrade. To simulate the impact of this moisture ingress, devices with a structure sodalime glass/Mo/CIGS/CdS were immersed in deionized water. The thin films were then analyzed both pre and post water soaking. Dynamic secondary ion mass spectroscopy (SIMS) was performed on completed devices to analyze impurity diffusion (predominantly sodium and potassium) and to assess potential degradation mechanisms. The results were compared to device measurements, which indicate a degradation of all device parameters due to an increase in the total and peak trap densities, as shown by simulation. This is potentially due to a modification of the sodium profile in the bulk CIGS, with a decrease

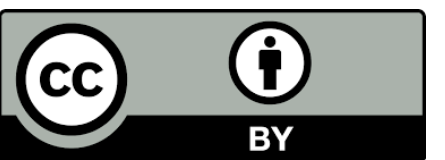

(C) 2021 by the author. This is an open access article distributed under the conditions of the Creative Commons by Attribution License, which permits unrestricted use, distribution, and reproduction in any medium or format, provided the original work is correctly cited. 
content after water soaking or because the oxygen profile increased in the bulk CIGS after water soaking.

\section{Graphical abstract}

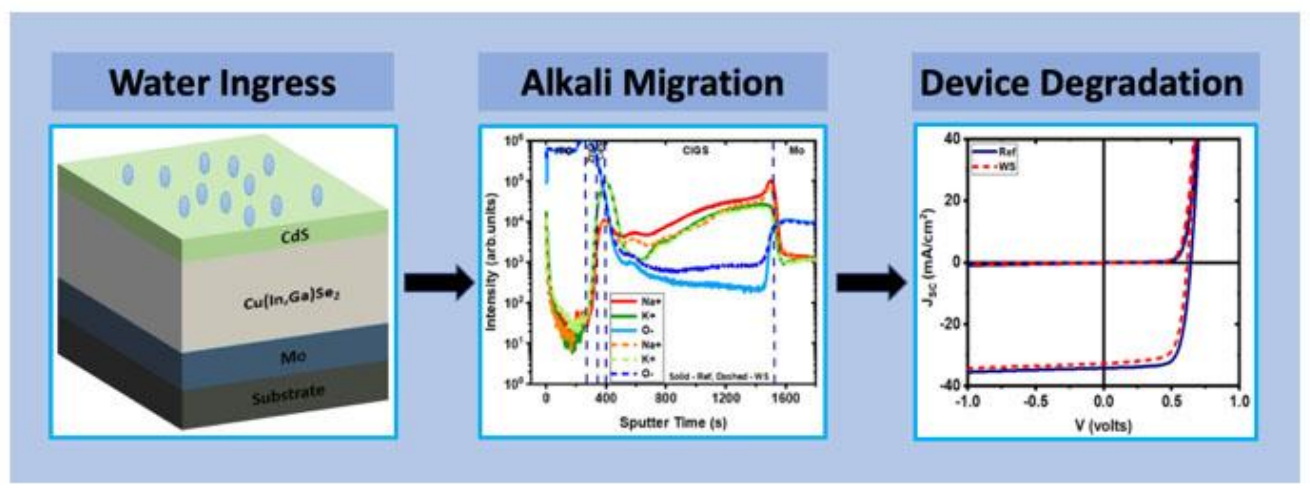

\section{Keywords}

Solar cell; corrosion; CdS; CIGS; alkali

\section{Introduction}

Of all of the degradation mechanisms that can affect a photovoltaic module, water remains one of the most potent ones [1-3]. Degradation can occur all the way from the connection to the electrical system to the degradation of individual layers within the module via corrosion-like processes [4,5]. Most of the studies performed on CIGS solar cells, with regard to the effect of water, have been performed on the solar cell as a whole not on the individual layers $[6,7]$. We have previously reported on the impact of water on Molybdenum, CIGS and TCO (i-ZnO/ITO) components of the CIGS devices [8-10]. We will be focusing on the buffer layer, specifically $\mathrm{CdS}$, here. Among the various choices for buffer layers for CIGS solar cells, cadmium sulfide (CdS) is still the main choice due to its wide bandgap and suitable band alignment with CIGS and TCO [11, 12]. Previous studies into the degradation mechanism due to the CdS layer have mostly focused on the effect on the CIGS layer due to damp heat treatments. Several studies compared the CdS buffer layers to other buffer layers under damp heat treatments and concluded that the CdS layer was often the most stable one. These studies indicate that when degradation occurred, it was often due to a decrease in open circuit voltage, and sometimes due to a decrease in fill factor [13].

In this paper, we focus our study on the effect of water ingress after the CdS deposition on device performance. We assess the potential degradation of the devices using various device characterization and simulation methods.

\section{Materials and Methods}

CIGS solar cells were fabricated using a three-stage co-evaporation process on soda-lime glass (SLG) substrates with the following structure: SLG/Mo/CIGS/CdS/i-ZnO/ITO. The molybdenum layer was deposited by DC magnetron sputtering using a two-step process, with the first step at high 
argon pressure $(5 \mathrm{mTorr})$ and the second at low pressure $(1 \mathrm{mTorr})$ resulting in a tensile/compressive stress dipole. The CIGS films were deposited using a three-stage co-evaporation process [14]. The junction was formed by chemical bath deposition (CBD) of cadmium sulfide (CdS). The initial solution includes a mixture of $\mathrm{H}_{2} \mathrm{O}, \mathrm{Cd}\left(\mathrm{CH}_{3} \mathrm{COO}\right)_{2}$ and $\mathrm{NH}_{4} \mathrm{OH}$ and is kept in a hot bath at $70{ }^{\circ} \mathrm{C}$ for $1 \mathrm{~min}$. Then, thiourea $\left(\mathrm{H}_{2} \mathrm{NCSNH}_{2}\right)$, is added to the solution. Finally, the samples are placed for $16 \mathrm{~min}$ in the heated bath, resulting in an approximate CdS thickness of $\sim 120 \mathrm{~nm}$. This slightly greater thickness compared to standard device structures was intentional to ensure that potential effects due to moisture damage to CdS could be more easily identified. After the CdS deposition, half of the samples were soaked in deionized water $\left(18.2 \mathrm{M} \Omega\right.$ ) at $50^{\circ} \mathrm{C}$ for 24 hours (referred to as water-soaked (WS) samples), while the other half was stored in a dry box until window layer deposition (referred to as reference). Therefore, 24 hours after CdS deposition, all the samples (reference and water soaked) were put together in the sputtering system for window layer deposition. The window layer, consisting of $50 \mathrm{~nm}$ of $\mathrm{i}-\mathrm{ZnO}$ and $150 \mathrm{~nm}$ of ITO was deposited at 5 mTorr of argon by RF sputtering. Finally, a metallic grid of Ni/Al/Ni was used as the front contact and was deposited by e-beam evaporation through a shadow mask. Solar cells were then defined by mechanical scribing with an active area of $0.5 \mathrm{~cm}^{2}$.

Energy dispersive $x$-ray spectroscopy (EDS) and x-ray diffraction (XRD) measurements were performed on the reference films and on water soaked films to measure any overall change in composition or crystalline properties. The elemental depth composition was measured by time of flight secondary ion mass spectrometry (ToF SIMS). The solar cell characteristics were measured by current density-voltage $(\mathrm{J}-\mathrm{V})$ measurements and by external quantum efficiency measurements under simulated $A M 1.5 \mathrm{G}$ with a light intensity of $100 \mathrm{~mW} / \mathrm{cm}^{2}$ at $25^{\circ} \mathrm{C}$.

\section{Results and Discussion}

After CdS deposition and water soaking of one set of samples, the overall composition and crystalline properties of both types of structures was assessed via XRD and EDS measurements. Each experiment was repeated a minimum of 10 times. The experiments lead to reproductive results, consistent with each other. As expected, no change was observed by either of these measurements before and after water soaking. Also, no obvious microstructural changes were observed by STEM [9].

Box plots of the device parameters for reference and water soaked samples are shown in Figure 1 , while representative J-V and QE curves are shown in Figure 2 for the same type of devices. Note that a total of 75 cells have been tested for Figure 1. The devices after water soaking are systematically less efficient, with a decrease in all three major parameters: open circuit voltage (Voc), short-circuit current density (Jsc) and fill factor (FF) (Figure 1), which is consistent with previous reports $[15,16]$. One can see from Figure 2 that there does not seem to be much shunt in the device even after water soaking, while there is an increase in voltage dependent current collection. No dark-to-light crossover can be observed in either case. The QE curves indicate that the loss in current density is due to an overall decrease in current collection from $500 \mathrm{~nm}$ to $1200 \mathrm{~nm}$. To extract diode parameters, a single diode model was used (Table 1). There is an increase in the reverse saturation current density $\left(\mathrm{J}_{0}\right)$ and diode ideality factor $(\mathrm{A})$ after water soaking, indicating a deterioration of the diode quality. At the same time, the series $\left(R_{S}\right)$ and the shunt resistance $\left(R_{S H}\right)$ do not change significantly. 

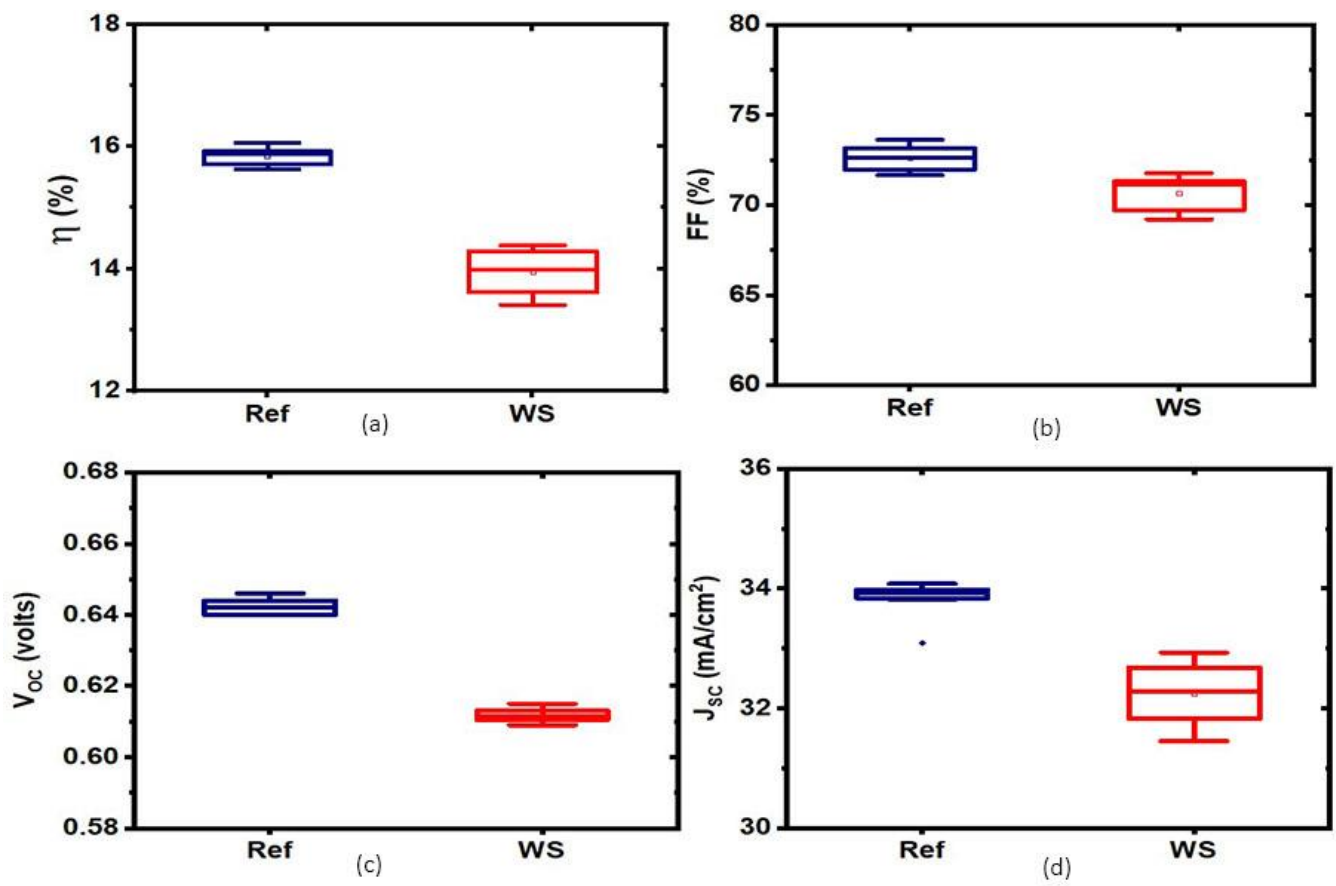

Figure 1 Box plots of device parameters comparing the device characteristics of reference devices (blue) and 24 hour water-soaked devices (red): (a) efficiency; (b): fill factor; (c): open circuit voltage; and (d): short circuit current density.
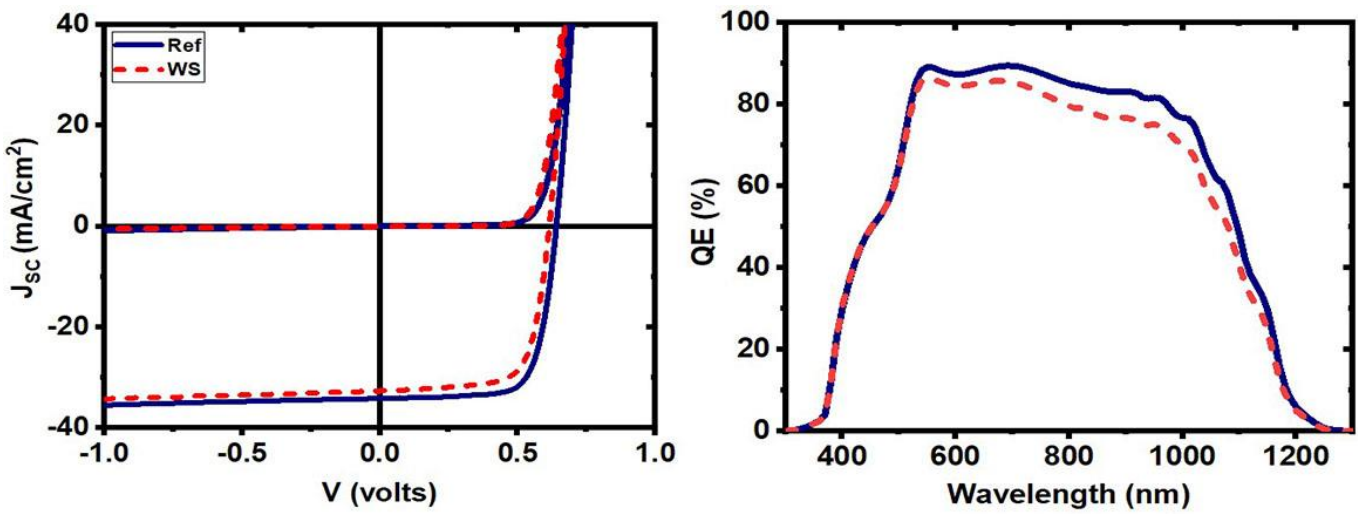

Figure 2 Representative current density-voltage (J-V) and quantum efficiency (QE) curves for reference (solid blue) and water soaked (dashed red) devices.

Table 1 Photovoltaic characteristics and diode parameters (dark j-v) of the representative cells shown in figure 1 and figure 2 .

\begin{tabular}{lllllllll}
\hline Sample & $\operatorname{Voc}(\mathrm{V})$ & $\mathrm{Jsc}\left(\mathrm{mA} / \mathrm{cm}^{2}\right)$ & $\mathrm{FF}(\%)$ & $\eta(\%)$ & $J_{0}\left(\mathrm{~mA} / \mathrm{cm}^{2}\right)$ & $\mathrm{A}$ & $\mathrm{R}_{\mathrm{S}}\left(\Omega . \mathrm{cm}^{2}\right)$ & $\mathrm{R}_{S H}\left(\mathrm{k} \Omega . \mathrm{cm}^{2}\right)$ \\
\hline Reference & 0.64 & 34.0 & 73.2 & 15.9 & $1.0 \mathrm{E}-9$ & 1.6 & 0.7 & 1.4 \\
Water Soaked & 0.61 & 32.7 & 71.3 & 14.3 & $8.0 \mathrm{E}-9$ & 1.8 & 0.6 & 1.8 \\
\hline
\end{tabular}

In order to better understand what could possibly be the underlying mechanism that causes degradation of the devices, simulations of the devices were performed using SCAPS. The main 
parameters used for the simulations are similar to the ones we used previously [9, 17]. The CIGS baseline parameters, such as electron affinity, dielectric permittivity, density of states, thermal velocity, mobilities, acceptor/donor density, were not changed. Parameters like thickness, transmission of the front contact and trap density were slightly modified to replicate the experimental curves.

One change was in the CdS thickness layer, which was set to $120 \mathrm{~nm}$. The other main changes were in the transmission coefficient, which was changed from $89 \%$ for the reference sample to $86 \%$ after water soaking, and in the trap density properties. The change in transmission is likely due to a modification of the sample surface after water soaking, leading to an increase reflection.

Both the total trap density and the trap density peak were changed in the SCAPS simulation to fit the experimental data, from $1.2 \mathrm{E}+15 \mathrm{~cm}^{-3}$ and $6.7 \mathrm{E}+15 \mathrm{~cm}^{-3}$ for the reference sample to $3.0 \mathrm{E}$ $+15 \mathrm{~cm}^{-3}$ and $1.6 \mathrm{E}+16 \mathrm{~cm}^{-3}$ for the water soaked sample. Figure 3 shows the comparison of the J-V and $Q E$ simulated data versus the measured data for both types of devices, indicating a good fit between the two.
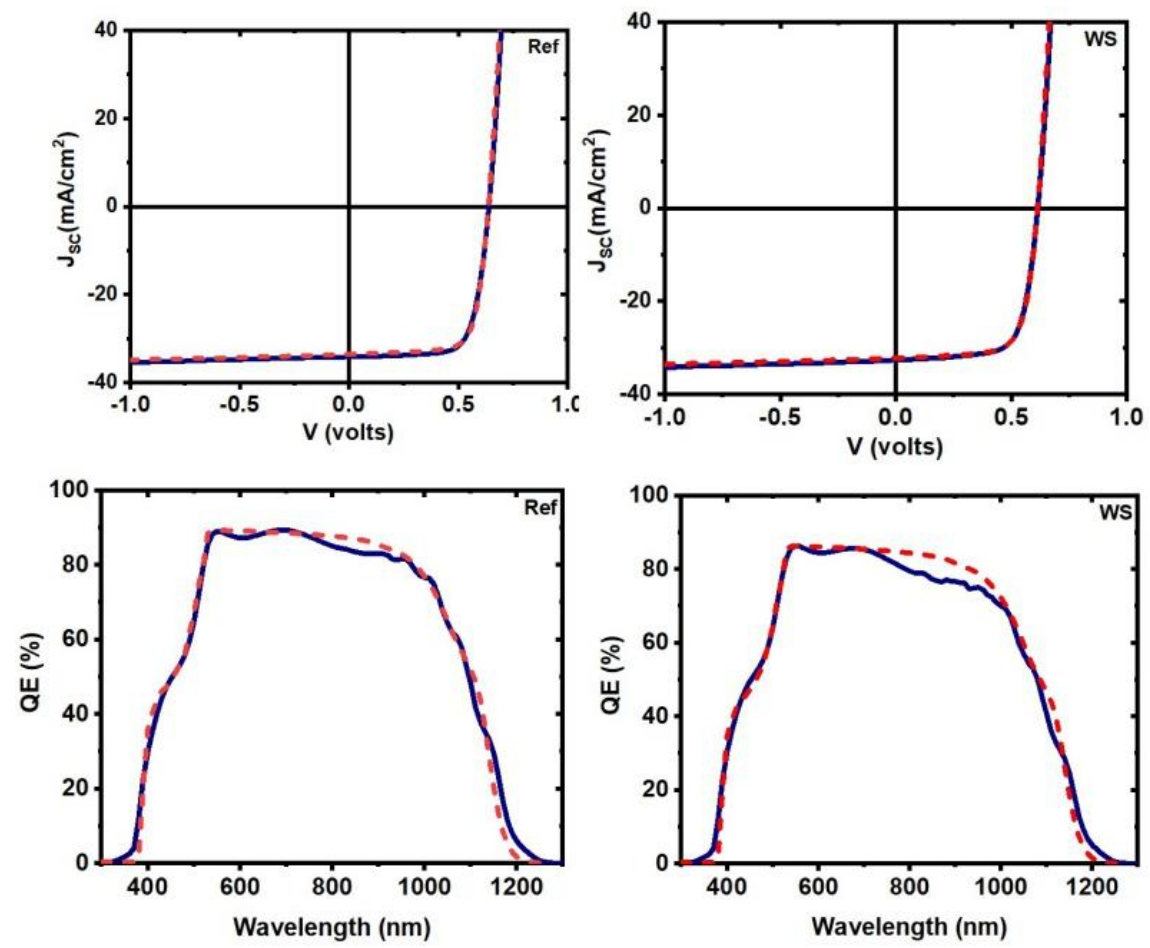

Figure 3 Simulated (dashed red) and measured (solid blue) current density-voltage and external quantum efficiency curves for the reference and water soaked device.

To try to further elucidate where this change in device efficiency could come from, SIMS depth profiles were measured on the samples with and without water soaking. Because we did not have an accurate standard to compare our sample to, no quantitative assessment can be done through the SIMS, but a comparative study of the elemental depth profiles is still possible. Figure 4 indicates clearly that no change in the main elements involved in the CIGS solar cells ( $\mathrm{Cu}, \mathrm{In}, \mathrm{Ga}$ and $\mathrm{Se}$ ) is occurring due to the water soaking, as one would expect, matching the results obtained by EDS and XRD. The gallium profile is also the one expected via the 3-stage deposition process. The next key elements in a CIGS solar cell are the alkali elements, both $\mathrm{Na}$ and $\mathrm{K}$ (since we did not use any postdeposition treatment by RbF or CsF here), as can be seen in Figure 5. One can see that no change 
can be observed for $\mathrm{K}$, while the Na signal decreases in the water soaked sample in comparison to the reference sample. It is known that the alkali metals diffuse from the SLG, through the molybdenum and into the CIGS. Afterwards, notably because the processes are done at much lower temperature, there is less diffusion of the alkali into the other layers. This can be seen for both $\mathrm{Na}$ and $\mathrm{K}$ from their profiles in the reference sample. Interestingly though, the Na profile is lower in the bulk of the CIGS after water soaking, indicating an out-diffusion of the Na through the CdS into the water during water soaking. The effect of alkali migration has been observed before and was correlated with losses in $\mathrm{V}_{\mathrm{OC}}, \mathrm{FF}$ and consequently in efficiency [18]. Interestingly, one can see that the oxygen content in the water-soaked sample is higher than for the reference sample, in the same location where the sodium is lower. One could therefore assume that both ions diffuse under a similar process at grain boundaries, while leaving $\mathrm{K}$ unaffected. The difference of behavior between $\mathrm{Na}$ and $\mathrm{K}$ might be partially explained by the smaller ionic radius of $\mathrm{Na}$ compared to $\mathrm{K}$, or a difference in chemical affinity [19].

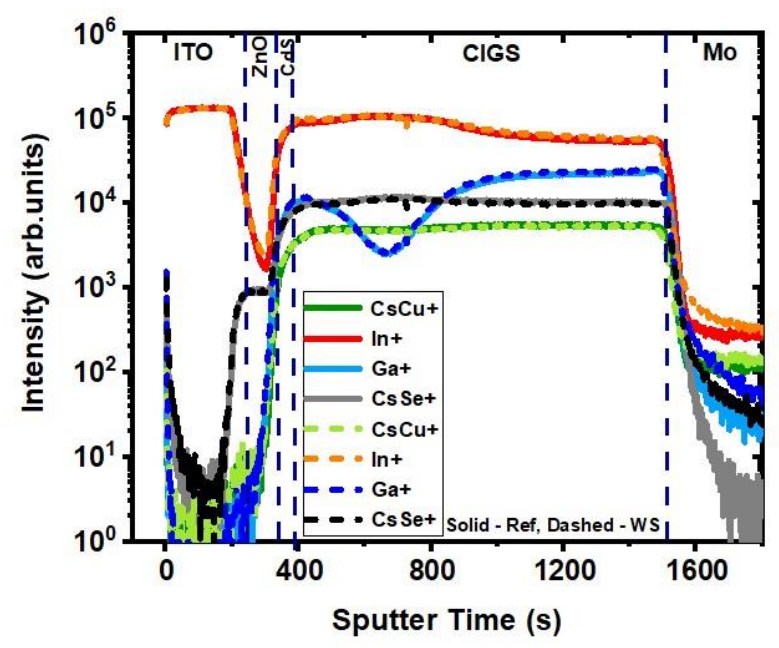

Figure 4 Secondary ion mass spectroscopy (SIMS) depth profiles for the main element of reference (solid lines) and water soaked (dashed lines) device.

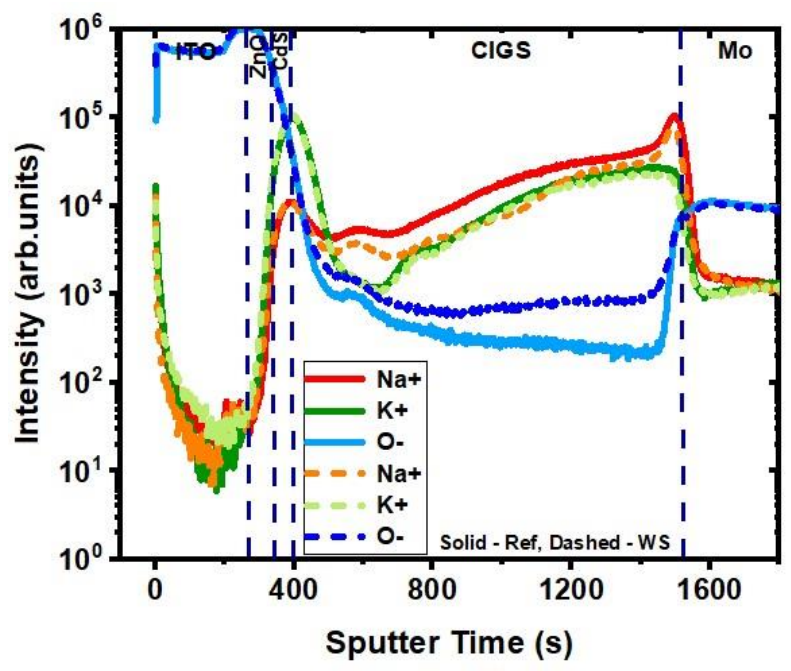

Figure 5 SIMS depth profiles of $\mathrm{Na}^{+}, \mathrm{K}^{+}$and $\mathrm{O}^{-}$in the reference (solid) and water soaked (dashed) device. 


\section{Conclusions}

Because of the nature of the deposition process, often used for CdS buffer layers in CIGS solar cells, which is an aqueous chemical bath method, one would not assume that the resulting layer would be sensitive to water. However, given enough time, water soaking of a SLG/Mo/CIGS/CdS structure can also degrade the future device performance of completed devices. All device parameters are affected by this degradation, which involves primarily a change in the diode quality factor and reverse saturation current density, leading to an overall efficiency dropping from 16\% down to $\sim 14 \%$. Simulation of the devices via SCAPS indicate that a slight modification of the transmission (decreasing by $3 \%$ ) and a slight increase in trap density properties (by a factor of 2) can yield such a change. The chemical origin of these changes seem to be in part due to the out migration of $\mathrm{Na}$ from the bulk of the CIGS and in-migration of $\mathrm{O}$.

\section{Author Contributions}

Conceptualization, Sylvain Marsillac and Angus Rockett; validation, Deewakar Poudel, Shankar Karki, Benjamin Belfore, Grace Rajanand, Sina Soltanmohammad; formal analysis, Sylvain Marsillac, Angus Rockett, Deewakar Poudel, Shankar Karki, Benjamin Belfore, Grace Rajanand and Sina Soltanmohammad; writing - original draft preparation, Sylvain Marsillac, Angus Rockett, Deewakar Poudel, Shankar Karki, Benjamin Belfore, Grace Rajanand and Sina Soltanmohammad; writingreview and editing, Sylvain Marsillac, Angus Rockett, Deewakar Poudel, Shankar Karki, Benjamin Belfore, Grace Rajanand and Sina Soltanmohammad; supervision, Sylvain Marsillac, Angus Rockett.

\section{Competing Interests}

The authors have declared that no competing interests exist.

\section{References}

1. McMahon TJ. Accelerated testing and failure of thin-film PV modules. Prog Photovolt Res Appl. 2004; 12: 235-248.

2. Lee DW, Cho WJ, Song JK, Kwon OY, Lee WH, Park CH, et al. Failure analysis of Cu(In, Ga) $\mathrm{Se}_{2}$ photovoltaic modules: Degradation mechanism of $\mathrm{Cu}(\mathrm{In}, \mathrm{Ga}) \mathrm{Se}_{2}$ solar cells under harsh environmental conditions. Prog Photovolt Res Appl. 2015; 23: 829-837.

3. Nishinaga J, Kamikawa Y, Koida T, Shibata H, Niki S. Degradation mechanism of Cu(In, Ga)Se solar cells induced by exposure to air. Jpn J Appl Phys. 2016; 55: 072301.

4. Wennerberg J, Kessler J, Stolt L. Cu(In, Ga)Se 2 -based thin-film photovoltaic modules optimized for long-term performance. Sol Energy Mater Sol Cells. 2003; 75: 47-55.

5. Pern FJ, Noufi R. Characterization of damp heat degradation of Culn GaSe 2 solar cell components and devices by (electrochemical) impedance spectroscopy. Proceedings of SPIE 8112, Reliability of Photovoltaic Cells, Modules, Components, and Systems IV, 81120S; 2011 August 21-25th; San Diego, California, USA. Bellingham: International Society for Optics and Photonics.

6. Igalson $\mathrm{M}$, Wimbor $\mathrm{M}$, Wennerberg J. The change of the electronic properties of CIGS devices induced by the 'damp heat' treatment. Thin Solid Films. 2002; 403: 320-324.

7. Schmidt M, Braunger D, Schäffler R, Schock HW, Rau U. Influence of damp heat on the electrical properties of $\mathrm{Cu}(\mathrm{In}, \mathrm{Ga}) \mathrm{Se}_{2}$ solar cells. Thin Solid Films. 2000; 361: 283-287. 
8. Karki S, Deitz JI, Rajan G, Soltanmohammad S, Poudel D, Belfore B, et al. Impact of water ingress on molybdenum thin films and its effect on $\mathrm{Cu}(\mathrm{In}, \mathrm{Ga}) \mathrm{Se}_{2}$ solar cells. IEEE J Photovolt. 2019; 10: 696-702.

9. Karki S, Paul P, Deitz JI, Poudel D, Rajan G, Belfore B, et al. Degradation mechanism in Cu(In, $\mathrm{Ga}) \mathrm{Se}_{2}$ material and solar cells due to moisture and heat treatment of the absorber layer. IEEE J Photovolt. 2019; 9: 1138-1143.

10. Poudel D, Karki S, Belfore B, Rajan G, Atluri SS, Soltanmohammad S, et al. Degradation mechanism due to water ingress effect on the top contact of $\mathrm{Cu}(\mathrm{In}, \mathrm{Ga}) \mathrm{Se}_{2}$ solar cells. Energies. 2020; 13: 4545.

11. Naghavi N, Abou-Ras D, Allsop N, Barreau N, Bücheler S, Ennaoui A, et al. Buffer layers and transparent conducting oxides for chalcopyrite $\mathrm{Cu}(\mathrm{In}, \mathrm{Ga})(\mathrm{S}, \mathrm{Se})_{2}$ based thin film photovoltaics: Present status and current developments. Prog Photovolt Res Appl. 2010; 18: 411-433.

12. Witte W, Spiering S, Hariskos D. Substitution of the CdS buffer layer in CIGS thin-film solar cells: Status of current research and record cell efficiencies. Vak Forsch Prax. 2014; 26: 23-27.

13. Theelen M, Daume F. Stability of $\mathrm{Cu}(\mathrm{In}, \mathrm{Ga}) \mathrm{Se}_{2}$ solar cells: A literature review. Sol Energy. 2016; 133: 586-627.

14. Contreras MA, Tuttle J, Gabor A, Tennant A, Ramanathan K, Asher S, et al. High efficiency Cu(In, $\mathrm{Ga}$ )Se/sub 2/-based solar cells: Processing of novel absorber structures. Proceedings of 1994 IEEE 1st World Conference on Photovoltaic Energy Conversion-WCPEC (A Joint Conference of PVSC, PVSEC and PSEC); 1994 December 5-9th; Waikoloa, Hawaii, USA. Piscataway Township: Institute of Electrical and Electronics Engineers.

15. Feist R, Rozeveld S, Mushrush M, Haley R, Lemon B, Gerbi J, et al. Examination of lifetimelimiting failure mechanisms in CIGSS-based PV minimodules under environmental stress. 2008 33rd IEEE Photovoltaic Specialists Conference; 2008 May 11-16th; San Diego, California, USA. Piscataway Township: Institute of Electrical and Electronics Engineers.

16. Tosun BS, Feist RK, Gunawan A, Mkhoyan KA, Campbell SA, Aydil ES. Improving the damp-heat stability of copper indium gallium diselenide solar cells with a semicrystalline tin dioxide overlayer. Sol Energy Mater Sol Cells. 2012; 101: 270-276.

17. Friedlmeier TM, Jackson $P$, Bauer A, Hariskos D, Kiowski $O$, Wuerz $R$, et al. Improved photocurrent in $\mathrm{Cu}(\mathrm{In}, \mathrm{Ga}) \mathrm{Se}_{2}$ solar cells: From $20.8 \%$ to $21.7 \%$ efficiency with CdS buffer and 21.0\% Cd-free. IEEE J Photovolt. 2015; 5: 1487-1491.

18. Theelen M, Hans V, Barreau N, Steijvers H, Vroon Z, Zeman M. The impact of alkali elements on the degradation of CIGS solar cells. Prog Photovolt Res Appl. 2015; 23: 537-545.

19. Contreras MA, Egaas B, Dippo P, Webb J, Granata J, Ramanathan K, et al. On the role of $\mathrm{Na}$ and modifications to $\mathrm{Cu}(\mathrm{In}, \mathrm{Ga}) \mathrm{Se}$ /sub 2/absorber materials using thin-MF ( $\mathrm{M}=\mathrm{Na}, \mathrm{K}, \mathrm{Cs}$ ) precursor layers [solar cells]. Conference Record of the Twenty Sixth IEEE Photovoltaic Specialists Conference-1997; 1997 September 29-October 03; Anaheim, California, USA. Piscataway Township: Institute of Electrical and Electronics Engineers. 
JEPT 2021; 3(1), doi:10.21926/jept.2101001

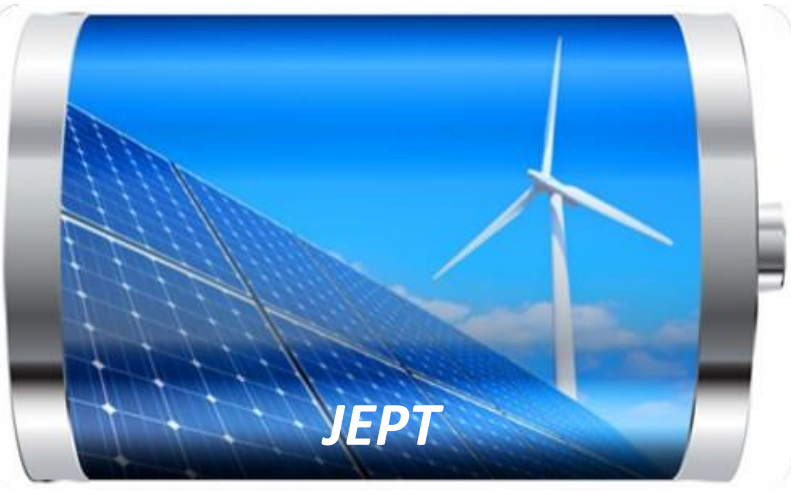

Enjoy JEPT by:

1. Submitting a manuscript

2. Joining in volunteer reviewer bank

3. Joining Editorial Board

4. Guest editing a special issue

For more details, please visit:

http://www.lidsen.com/journal/jept 
Original Research

\title{
Online State of Charge Prediction in Next Generation Vehicle Batteries Using Deep Recurrent Neural Networks and Continuous Model Size Control
}

Steven Hespeler ${ }^{1,}{ }^{+}$, Donovan Fuqua ${ }^{2, *}$

1. College of Engineering, New Mexico State University, Las Cruces, NM, USA; E-Mail: schesp@nmsu.edu

2. College of Business, New Mexico State University, Las Cruces, NM, USA; E-Mail: dfuqua@nmsu.edu

$\dagger$ These authors contributed equally to this work.

* Correspondence: Donovan Fuqua; E-Mail: dfuqua@nmsu.edu

Academic Editor: Zhao Yang Dong

Special Issue: Modeling and Control of Fuel Cell Systems

Journal of Energy and Power Technology

2021, volume 3 , issue 1

doi:10.21926/jept.2101003
Received: July 31, 2020

Accepted: December 29, 2020

Published: January 12, 2021

\begin{abstract}
This investigation presents a data-driven Long-short Term Memory battery model for predicting State of Charge for lithium-ion batteries $\mathrm{LiFePO}_{4}$ for next-generation vehicle operations. Our modified algorithm builds and updates a model using multivariate inputs that include physical properties, voltage, current, and ambient temperature during operations. The primary research goal is to improve prediction performance on future values from multiple training examples using an online learning scheme. Initial results demonstrate excellent predictions that outperform results from literature and other neural network algorithms. Due to computing constraints in on-board vehicle systems, the authors develop online training with autonomous control of lag (window width). The control algorithm embeds in the model with rules that govern and adjust lag during training. This method ensures the
\end{abstract}

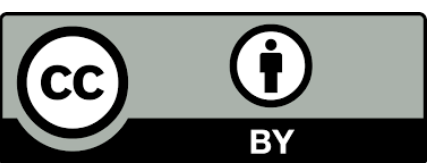

(C) 2021 by the author. This is an open access article distributed under the conditions of the Creative Commons by Attribution License, which permits unrestricted use, distribution, and reproduction in any medium or format, provided the original work is correctly cited. 
minimization of computational cost and prediction errors with the use of standard computing equipment during driving conditions.

\section{Keywords}

Long-short term memory; continuous model size control; battery management system

\section{Introduction}

With the increase of devices and applications that require sophisticated, next-generation batteries, management and control have become critical to maintaining the safety and reliability of these systems. Battery Management Systems (BMS) are used to monitor the overall health of batteries and are customizable to fit a variety of different battery types and various applications. Critical BMS parameters and functions established by Xing, Y, et al. include data acquisition, safety protection, ability to determine and predict state evaluation, ability to control battery charging and discharging, cell balancing, thermal management, delivery of battery status updates to a user interface, communication with all battery components, and prolonged battery life [1].

While battery operational conditions are different for different applications, this research focuses on next-generation automobiles [2]. In the case of Fuel Cell Vehicle (FCV) and Electric Vehicle (EV) operation, the BMS controls many parameters and functions - none being more important than state determination. Researchers consider state of charge (SOC) to be the most influential of the state determination parameters [3]. SOC provides information about the battery's current and remaining life, which is useful for protecting the battery from over-charging and overdischarging [3]. Furthermore, an accurate estimation of the battery state assures reliable and safe operating conditions for the user. In the case of battery operation, SOC is the equivalent of a fuel gauge and indicates to the user how much energy is available for usage. Therefore, accurate SOC prediction remains one of the main challenges in the successful operation of FCVs [2].

SOC estimation methods are typically complex, with scarce literature found to provide a detailed explanation of algorithmic approaches to the problem. Most of the methods have significant issues that become more apparent during the aging of the battery, temperature fluctuation, and change in discharge cycles [4]. Also, many of the methods produce inaccurate estimations of SOC because of the high sensitivity that lithium-ion batteries have to internal/external factors and complex electrochemical reactions. This problem results in a model attempting to evaluate complex calculations with high computation cost and negligence on the effects of time. A small number of Machine Learning (ML) algorithms have been utilized in an attempt to accurately predict SOC due to the ability to adapt and self-learn on a complex nonlinear dataset [5].

He et al. developed one such architecture using a Back Propagation Neural Network (BPNN) along with an Unscented Kalman Filter (UKF). This method estimates SOC during different driving conditions and directly tests recurrent neural network algorithms with algorithmic adjustments against BPNN and BPNN+UKF methods [6].

In a BMS, SOC is widely considered the most influential and essential parameters for batteryoperated applications. SOC is defined as the percentage of residual capacity and the total capacity of the battery cell $[3,7-9]$. When a battery discharges, the formula to calculate SOC is the ratio of 
releasable capacity, $Q_{\text {releasable, }}$ relative to the rated capacity, $Q_{\text {rated }}$, this percentage can be expressed in the following formula: $S O C=Q_{\text {releasable }} * Q_{\text {rated }} * 100$. It is desirable to maintain SOC percentage within certain limits, typically between 20 to 95 percent capacity [10].

While current literature demonstrates that there is no way to measure SOC of the chemical energy directly and precisely, Chang recommends four categories that distribute the many different methods of addressing the issue of SOC estimation [11]. SOC is estimated through direct measurement through physical battery properties such as terminal voltage, book-keeping evaluation, and indirect methods utilizing discharging current as one of the inputs. Adaptive systems automatically adjust the SOC when subjected under various discharging conditions. Finally, hybrid methods employ the advantageous parts of each SOC estimation method to provide estimates. The majority of estimation issues occur at full to partial SOC because the change in impedance is small resulting in a significant prediction error [12].

The voltage measurement method (or terminal voltage method) is based on evaluating terminal voltage drops due to internal impedances as the battery discharges [11]. Sato et al. proposed the following SOC estimation equation that literature accepts as the current standard: $S O C=\alpha V+$ $\beta R+\gamma \sqrt{V}+\partial \sqrt{R}+C[13]$

Typically, AC Impedance measurements are used as a method to indirectly determine battery capacity by inputting a sinusoidal, controlled current or voltage test signal of a single frequency or combination of signals with different frequencies [14]. Huet states that impedance is defined by: $|Z|=\frac{V_{\max }}{I_{\max } e^{j / \varphi}}[15]$. Therefore, the electrochemical impedance of a battery is a frequencydependent complex number characterized either by its real and imaginary parts. Through the mid to late seventies, bridges and lock-in amplifiers were utilized to measure impedance. Later on, impedance measurements were performed by frequency response analyzers based on harmonic analysis [15]. Early literature (before 1977) had conducted experimentation with the procedure involving the battery reaching equilibrium, which results in precise measurement. However, more recent research argues against this procedure and proposes the method of impedance measurement carried out while the battery is charging or discharging $[16,17]$. However, this alteration creates additional errors and increases the measurement period.

Newer literature suggests a model-based approach to predict a state of charge, health, and life (SOC, SOH, SOL). Kozlowski used three models (neural networks, fuzzy logic, and auto-regressive moving average (ARMA) to identify electrochemical parameters in four battery types [18]. These models were offline, where there was a cutoff between training, test, and validation. Offline training, however, is less helpful for real-time SOC measurement. Hung et al. state that the motivation for online estimation is 1 ) the need for initial values or historical data, 2) the inability to perform real-time detection, and 3) the failure to determine the actual SOC from calculations in the event of fluctuations in SOH [19]. Bundy et al. present a multivariate method for predicting SOC using electrochemical data of a nickel-metal hydride ( $\mathrm{NiMH}$ ) battery [20]. This method generates predictions through partial least squares (PLS) regressions. These predictions then evaluate the electrochemical impedance spectra and estimate SOC.

For this research, artificial intelligence methods are utilized to predict SOC in an online training environment. Literature includes techniques involving backward propagation neural networks (BPNN), artificial neural networks (ANN), fuzzy logic, radial basis function neural networks (RBFNN), support vector machines (SVM), Kalman filters (KF) and particle filters (PF) $[5,21-23]$. In the early 
2000s, literature saw several ANN models utilized to estimate SOC in NiMH batteries [24]. Shen et al. created an input layer consisted of temperature, discharge, and regenerative capacity distribution through a total of six input neurons. The hidden layer consists of ten neurons, suggesting that increasing the number of neurons past ten has "no significant improvement in the estimation accuracy." Cai et al. estimated SOC of a high powered NiMH battery with an ANN [25]. In the study, researchers used a three-layered, feed-forward, back-propagation ANN. Since battery behavior is complex and nonlinear, input selection was accomplished by initially testing several different battery parameters (terminal voltage, discharge current, time-average voltage, etc.). Then the correlation coefficient was calculated based on variables and SOC. They rank results in order of the highest correlation to the least correlated. The researchers selected five variables as input neurons to the model: battery discharge current (I), accumulated ampere-hours (Ah), battery terminal voltage (V), time-average terminal voltage (TAV), and twice time-average terminal voltage (TTAV). It is worth noting that the linear correlation coefficients of the variables were very high for several of the selected variables (>0.942). Yanqing presented a novel approach for online SOC determination by using $\mathrm{n}$ neural network-based model and neuro-controller. First, Yanqing erects a nonlinear dynamic system cell model, that can be represented by discrete state-space form calculations [5]. Linda et al. published research on predicting SOC with a feed-forward neural network model using voltage, current, and ambient temperature [26].

Support vector machine (SVM) is a minimization-maximization algorithm that produces hyperplanes within data. Researchers have used SVM to estimate SOC of a variety of batteries [2731]. The fuzzy logic (FL) method is a rule-based option for nonlinear data. FL models include four parts: a relationship in rule-based input-output, the membership function for both input and output, reasoning, and defuzzification of outputs. Singh et al. use an FL based SOC meter developed for use in a Lithium-ion battery in a portable defibrillator [32]. In a study conducted by Hu et al., fuzzy adaptive federated filtering is utilized for SOC estimations for series-connected battery packs to combat inconsistencies in battery cell state [33]. The investigation found that online and offline parameters experience less than $0.4 \%$ and $1 \%$, respectively.

Current literature based on electrochemical battery modeling indicates a keen interest in SOC estimation methods with an attempt to create an efficient BMS [34-39]. Much of the research conducted focuses on external battery influences that do not consider internal dynamics nor energy losses of the battery [40-42]. Other studies estimate SOC without online parameters, which become inaccurate as the battery ages [43]. Some research has investigated internal dynamics and online SOC determination of batteries but does not take into account temperature effects on battery performance [44]. Data-driven methods present a new possibility of explaining internal battery dynamics from an applied systems approach. An intelligent/online BMS that considers internal dynamics and temperature effects work to ensure the Lithium-ion batteries operate efficiently for vehicle operation throughout the life of the battery. Unlike the approaches used in literature, this investigation treats the battery performance data as a time series to ensure the time-dependent relationships of the parameters are accurately monitored.

The issue of computational time during SOC prediction was addressed in the work completed by Kim in [45], which demonstrates a battery model capable of limiting computational time while introducing a robust slider mode observer to compensate for model error. The model restricts SOC error to less than $3 \%$ in most cases. In another study, Skrylnyk et al. investigate slider mode along 
with fuzzy logic modeling for SOC estimation during autonomous solar application [46]. In the study, authors report excellent estimation capabilities with the tradeoff of increased training time.

On an application level, methods like ANN, FL, and KF have their own set of issues. ANN algorithms require a substantial amount of data before they can increase accuracy, which prevents these models from being rapid. FL method requires definitions of its membership functions, making this method undesirable for large models. KF is suited more for linear systems, of which battery models are not. Other versions of the KF like EKF and UKF methods can be challenging to tune and will provide inaccurate estimations if nonlinearities in the battery model are severe [6].

Based on the literature review, the following knowledge gaps are directly addressed in this research:

1) There is limited research on state of charge prediction using state of the art RNN architectures, notably long short-term memory neural networks.

2) Feature selection using statistical learning can determine variable importance in the state of charge prediction that has previously been assumed or reported through observational evidence.

3) There is limited research on comparison of multiple methods for SOC prediction.

4) On-boarding an online algorithm will require controlling data size that can be accomplished through continuously controlling lag as a hyperparameter during learning.

In this study, data used was collected by the CALCE lab at the University of Maryland on $\mathrm{LiFePO}_{4}$ batteries under dynamical stress testing (DST), USO6 highway driving schedule, and the federal driving schedule. We thank CALCE for the use of their data and for their willingness to answer questions about testing procedures [43].

\section{Materials and Methods}

Time computations are performed on a personal computer platform to mimic the capabilities of a next-generation vehicle platform. This setup used an Intel Core i3 $7100 \mathrm{U}$ running up to $2.4 \mathrm{GHz} / \mathrm{s}$ and $8 \mathrm{~GB}$ of RAM. For hyperparameter search and model development, the New Mexico State University Discovery Computing Cluster was utilized. All computations were executed on Python 3 with the Tensor Flow 2.1.0 package.

\subsection{Feature Selection}

An essential aspect of this investigation was to identify the most influential physical battery features to input into the predictive battery model. This dataset is highly dynamical with high dimensionality; therefore, it was essential to decide which features were necessary for high prediction accuracy and which weren't. Therefore, non-informative variables were removed from the dataset through analysis using the Random Forest technique.

\subsubsection{Random Forest Definition}

Random Forest (RF) is a method focused on reducing variance by utilizing the bagging (bootstrap aggregation) technique to comprise a mean of noisy but unbiased models [47]. RF works through a tree-growing process in which random selection of input variables bootstraps on a dataset. The idea is to de-correlate the trees without increasing variance [48]. RF introduces randomness to the treegrowing process. RF selects $m$ variables by randomly pulling from $p$ variables so that $m=\sqrt{p}$. The 
driving force behind this process is the regression predictor, mathematically represented as (for regression):

$$
\hat{f}_{r f}^{B}(x)=\frac{1}{B} \sum_{b=1}^{B} T_{b}(x)
$$

Where $B$ is the total number of trees that we are predicting on a new point $x$.

\subsubsection{Feature Importance}

Random Forests techniques are capable of producing feature importance charts, as seen in Section 3.1. In this method, the importance measure determines the split point when growing the tree. Therefore, highly correlated features show little to no importance measure. The process uses Out of $\mathrm{Bag}(\mathrm{OOB})$ samples at the $b$ th tree to pass down the tree to record prediction accuracy [47]. Then, values at the $j$ th variable are arbitrarily permuted from the OOB samples and again accuracy is computed and recorded. The computed accuracy is then averaged across all trees and used a metric for determining importance at the $j$ th feature with the Random Forest.

\subsection{LSTM}

\subsubsection{Definition}

Long-Short Term Memory (LSTM) is a specific type of recurrent neural network (RNN) architecture developed to solve the vanishing gradient problem $[49,50]$. The LSTM does not experience long term dependency issues seen in other RNN architectures. In practice, the LSTM has demonstrated a superior ability to learn long-range dependencies as compared to simplified RNNs [51]. As seen in Figure 1, this model introduces a memory cell. RNNs utilize long-term memory in the form of weights, which change slowly during training and short-term memory in the form of temporary activations which pass from one node to another [51]. The memory cell in a LSTM network has intermediate storage in the form of gates within each hidden layer. Figure 1 depicts an LSTM architecture that contains four parts within the memory block: an input gate (i), a forget gate (f), an output gate (o), and cell state $\left(C_{t-1}\right)$. The forget gate is responsible for deciding which information is retained or discarded from the cell state. The input gate determines which values will be updated to a vector of new candidate values $\left(C_{t}\right)$. Finally, the output gate decides what information will be passed to the cell state in the next time step. 


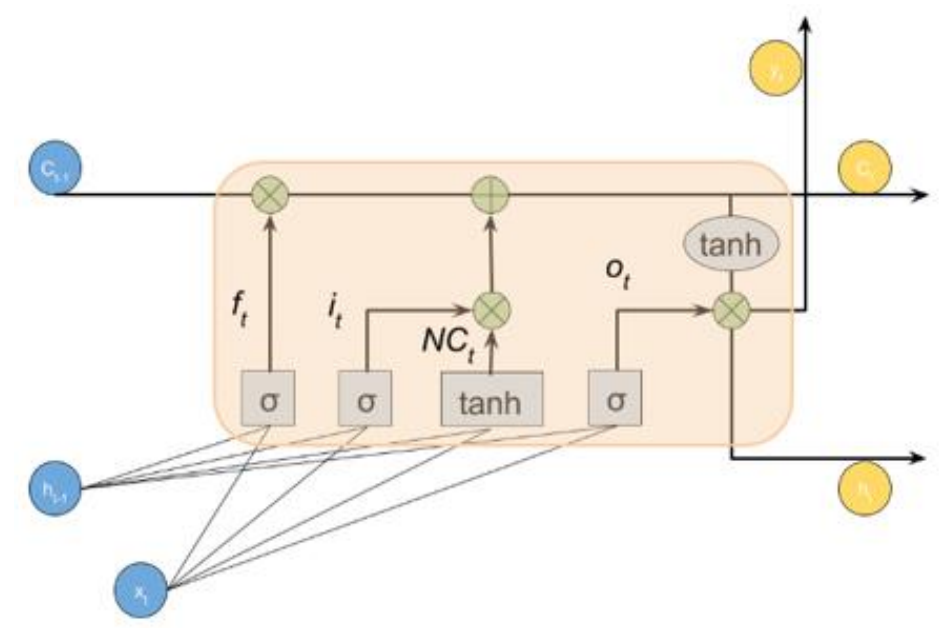

Figure 1 LSTM memory cell.

LSTM connections are represented through the following equations:

$$
\begin{gathered}
f_{t}=\sigma\left(W_{f h} h_{t-1}+W_{f x} x_{t}+b_{f}\right) \\
i_{t}=\sigma\left(W_{i h} h_{t-1}+W_{i x} x_{t}+b_{i}\right) \\
C_{t}=f_{t} \cdot c_{t-1}+i_{t} \cdot N C_{t} \\
N C_{t}=\tanh \left(W_{N C_{t} h} h_{t-1}+W_{N C_{t} x} x_{t}+b_{N C_{t}}\right) \\
o_{t}=\sigma\left(W_{o h} h_{t-1}+W_{o x} x_{t}+b_{o}\right) \\
h_{t}=o_{t} \cdot \tanh \left(c_{t}\right)
\end{gathered}
$$

In these equations, $\sigma$ is a random sigmoid activation, $\left(i_{t}, f_{t}, h_{t}, c_{t}\right.$, and $\left.o_{t}\right)$ are vectors from the input, forget gate, hidden gate, cell gate, and output, $b_{t}$ is the bias vector, and $\mathrm{W}$ represents weight vectors at various portions of the LSTM.

\subsection{Data}

\subsubsection{Collection of Data}

CALCE collected and reported all data used in this experiment [43]. The battery tested was lithium-ion $\left(\mathrm{LiFePO}_{4}\right)$ and was subjected to three battery testing load profiles; dynamical stress testing (DST), US06 highway driving schedule, and federal urban driving schedule (FUDS). DST data is a basic loading profile built for battery testing. US06 and FUDS are complex, highly nonlinear data that simulates real life driving cycles. Figure 2 depicts the load profiles of the DST (top), USO6 (middle), and FUDS (bottom) datasets. 

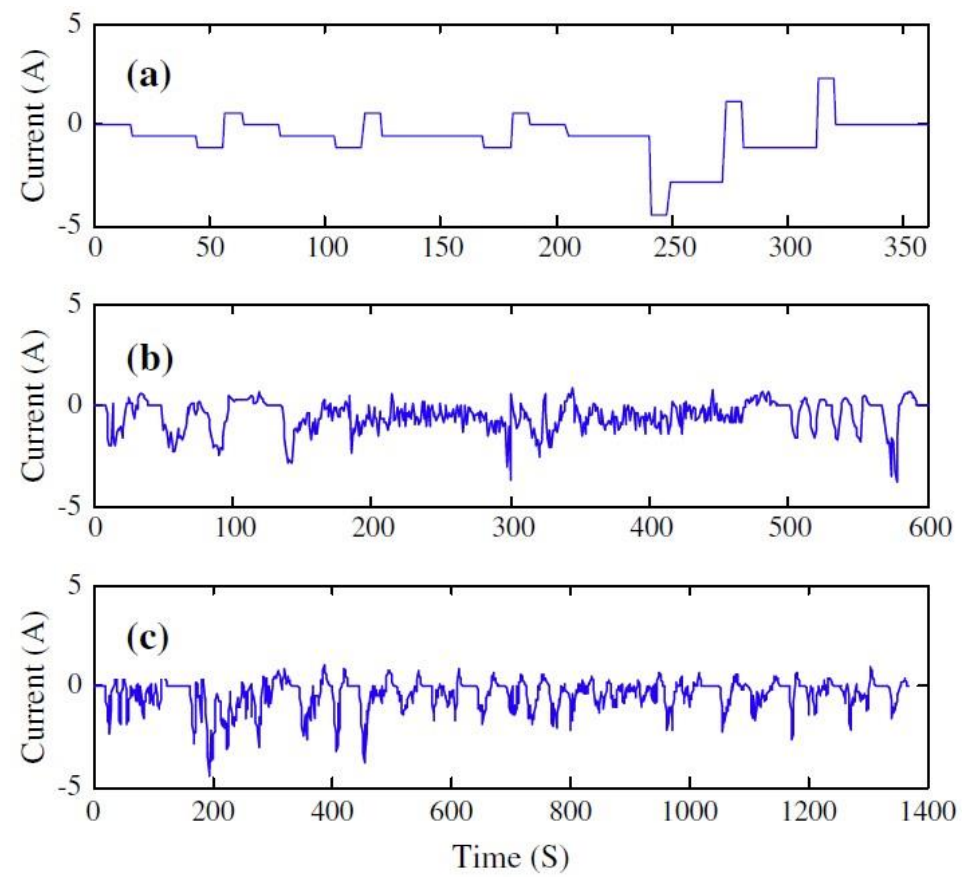

Figure 2 Loading profiles(current) of DST (top), US06 (middle), and FUDS (bottom).

Load Profiles. The DST test is a testing procedure established by the US Department of Energy Vehicle Technologies Program used throughout literature to evaluate the performance of EVs [52]. The test validates models and algorithmic accuracy [25]. During the trial, a battery experiences different DST cycles that alter SOC from $90 \%$ to $20 \%$.

US06 is a high acceleration aggressive driving schedule identified as the "Supplemental FTP" driving schedule EPAUS06. Figure 3 (a) shows the aggressive driving schedule. Figure 3 (b) shows the noticeably more complex FUDS. Researchers subjected all the loading profiles to varying ambient temperatures; $0,10,20,30,40$, and 50 Celsius. This investigation focused on the 0 Celsius datasets to test our algorithm.

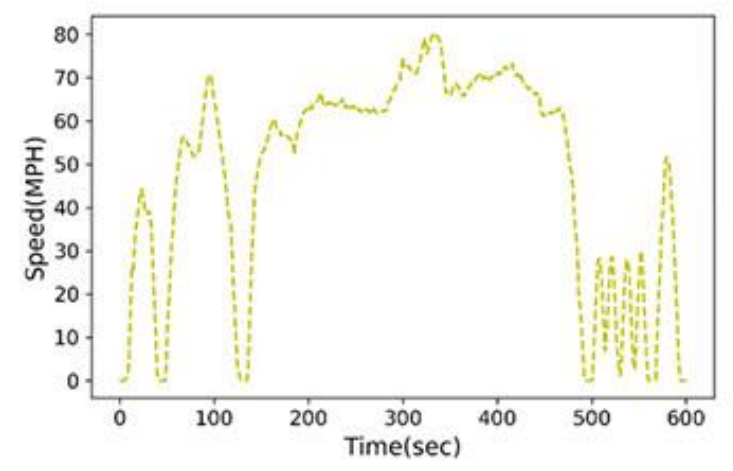

(a)

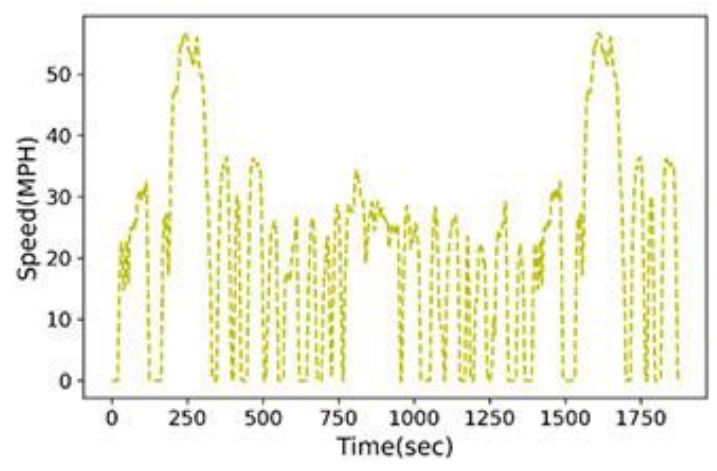

(b)

Figure 3 (a) US06 highway driving schedule (b) FUDS highway driving schedule.

\subsubsection{Data Formatting and Reshape}

Raw data was imported to the predictive battery model as individual load profiles in the commaseparated value (CSV) format. Then, each dataset is cleaned and prepared. The small number of rows containing empty $(\mathrm{NaN})$ values were eliminated and included the current, voltage, 
temperature, and SOC features. Once the cleaning was complete, the time series data was normalized to a range of $(0,1)$.

Next, the data was reshaped and prepared for training on a time-series cross-validation split. Experiments were run for all the data according to the following combinations of lag capacity, horizon, and load profile, with 36 experiments completed in total.

In our construct, a walk-forward validation model was created where the train and test index splits into feature train and test, along with predictor train and test tuples. Since this is a time-series and the data is auto-correlated, our function trains on the feature and predictor training set then uses the $X$ th test sample to generate a $\hat{y}_{+1}$ prediction. Walk-forward validation iterates through the train and test sets. Since this is a time-series dataset, we predict a value $x$ at time $T$ from $T_{-H}$, where $H$ is the pre-set horizon value.

Using the TensorFlow package in Python, the model is formatted to a sequential model with LSTM of 50 input layers (determined through hyperparameter grid search along with batch size and epochs). The raw data (after cleaning and data prep) has 6000 to 8000 -time steps (n) with four features (p). Raw data was reshaped into a 3D tensor with the shape of [batch, time steps, feature]. A reshape() function is executed that accepts a tuple argument. The data now takes the form of:

$$
\text { [X train, }(X \text { train [0], X train[1]), 1] }
$$

Where $X$ train [0] has the shape [batch, time steps, 1] and $X$ train [1] has the shape [batch, time steps, $+1,1]$.

Each experiment runs for 1,000 iterations. Once the input is reshaped, the model is fit to an epoch cycle of 100 and a batch size of 50 . The model trains by slicing inputs into batch sizes and iterating over the specified number of epoch cycles. At the end of each epoch, the model iterates over the validation dataset and computes the validation loss.

\subsection{LSTM-CLC}

\subsubsection{Network Architecture}

Figure 4 depicts how our algorithm predicts SOC on the battery model. The model is compiled with a loss calculation of MAE, using the Adam optimizer. Using a walk-forward validation technique, the model predicts a pre-set horizon value $(1,3$, or 5$)$, indicating 10,30 , and 50 seconds in the future as the time-series is in a 10 -second format. 


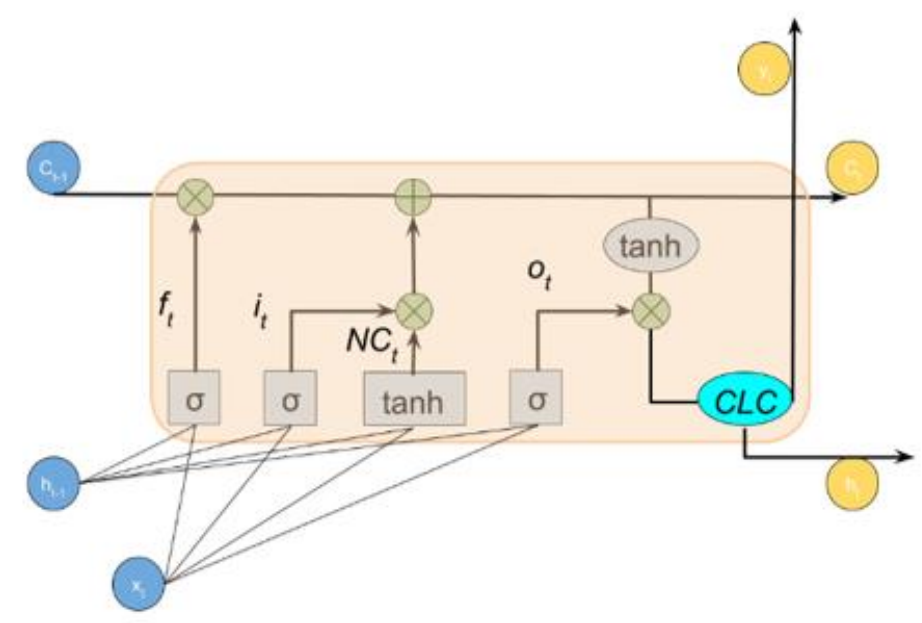

Figure 4 Illustration of LSTM-CLC memory block.

Here, the forget, input, and output gates all function as a vanilla LSTM. However, once the LSTM initiates the backward pass, the lag capacity adjusts (or remains the same from the previous time step), and the next forward pass begins. This next pass uses the lag capacity to train on the allowable data size set by the lag capacity rules. The algorithm runs until all iterations are complete, in this case, 1,000 iterations.

During each grid search, testing parameters are recorded. RMSE, computation time, loss, validation loss, predicted value, and expected value are all appended to respective variables. Once the experiment has completed, the variables are saved and stored in a CSV file for further analysis, discussed in Section 3 and 4.

Figure 5 depicts how the lag capacity could expand during any experiment. However, restriction of the data size up to the lag capacity during experimentation mimics onboard computational capacities. An important note is that during each iteration, an observation has only one time to be in the test set. This type of cross validation ensures low bias in each model.

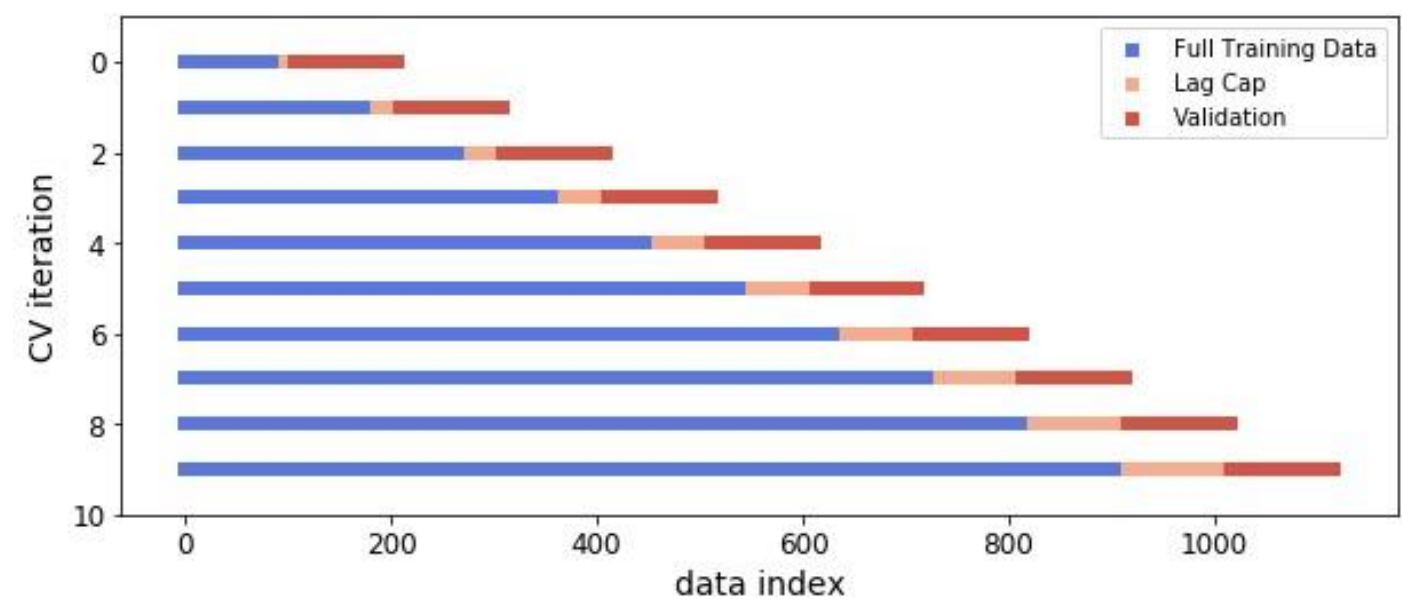

Figure $\mathbf{5}$ Visualization of train set modification with walk forward validation.

\subsubsection{Pseudo Algorithm}

The algorithm is explained in pseudo code below. 


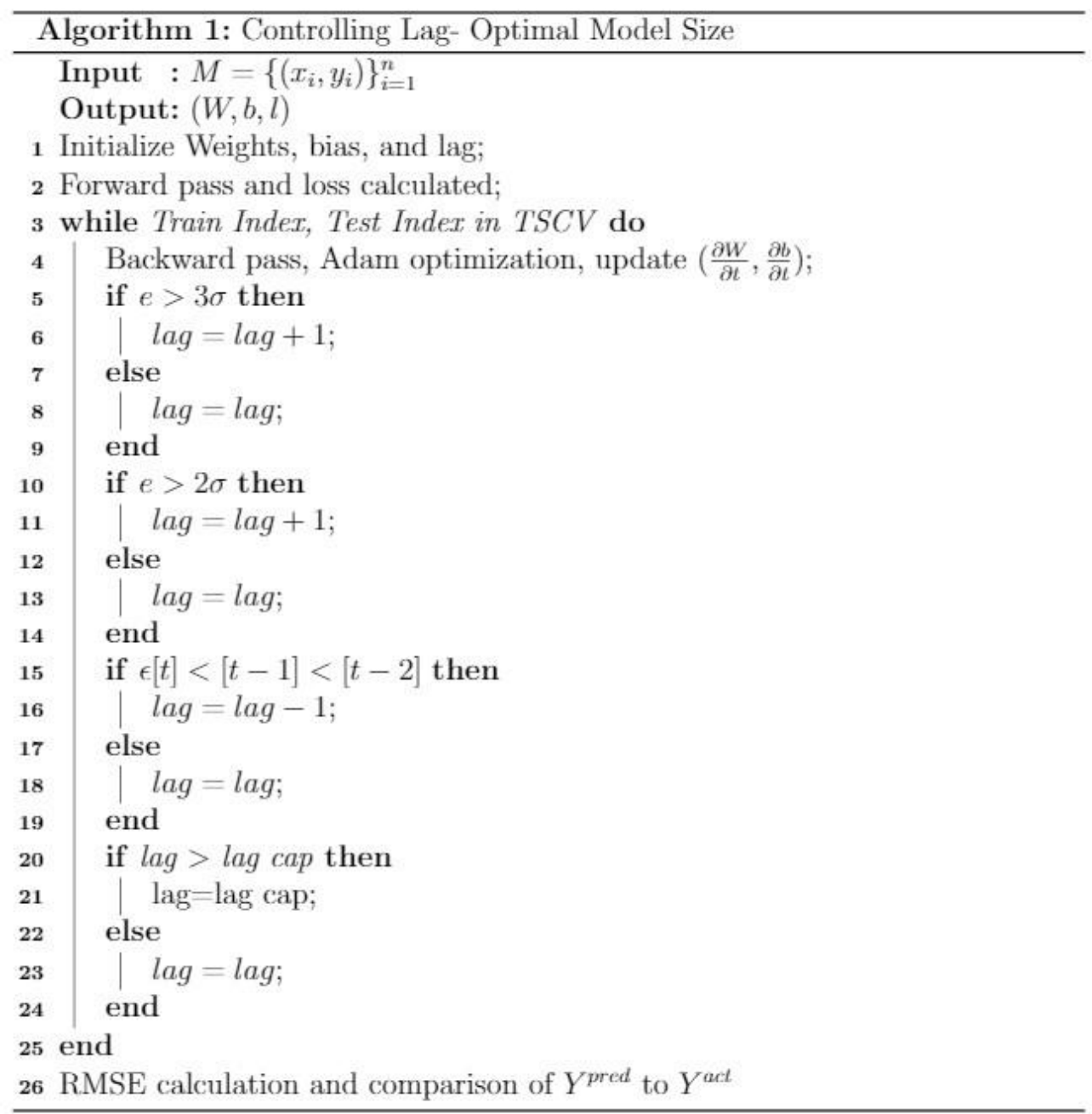

Figure 6 Pseudocode for LSTM-CLC algorithm.

\subsubsection{Rules Governing Lag Control}

Rule 1-Data Capacity. Walk-forward validation is utilized for sequential predictive models to split data into training and test sets. Since the data in sequential models are time dependant, the order of arrival is important. Features must remain in order as they arrived in the dataset. Applying the walk-forward method to an application like HEV proposes difficulty due to ever-increasing memory tax as the model learns. Although with our other rules, lag is capped to a maximum value in the model, which allows the training index to reach back only as far as the lag cap will allow.

This cap minimizes the maximum allowed size of the training index at time $t$ within the planning horizon $[0, T]$. The control algorithm implements the cap by disallowing the training set to be larger than that of the lag cap. If the size of the training set extends beyond the lag cap, the model is adjusted to include the previous training set within the lag cap only. Four different lag caps are run in the experiments: $10,20,30$, and 40 . The lag caps are somewhat arbitrary and can be increased when there is additional on-board computational power.

Rule 2- Sampling Distribution. The lag control algorithm monitors and adjusts to diminishing standard error values. If three consecutive error values are decreasing, that is if $\epsilon[0]<$ 
$\epsilon[-1]$ and $\epsilon[-1]<\epsilon[-2]$, the lag value will pull back from lag to lag -1 . This cap continuously monitors and adjusts the window width to ensure lag controls sampling distribution (standard error).

Rule 3 Outlier Detection. There are three specific cases that the control algorithm adjusts lag based on outlier detection. If the standard error of the residuals is outside two standard deviations from the mean on a particular iteration, lag adjusts from lag to lag +1 .

\section{Results}

\subsection{Feature Analysis}

Figure 7 shows the relative variable importance in each of the driving loading profiles. Although there are differences in the importance levels, we see that current is the most significant predictive feature for SOC prediction, followed by voltage, and then ambient temperature. Test time (degradation of the battery), discharge capacity, charge energy, and charge capacity have a measurable but less significant effect on SOC.

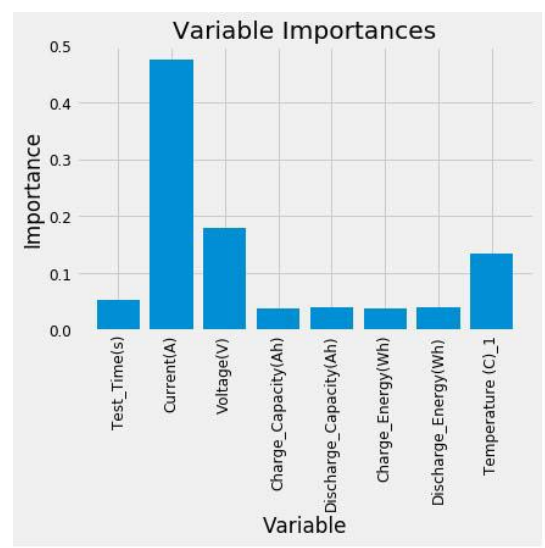

(a)

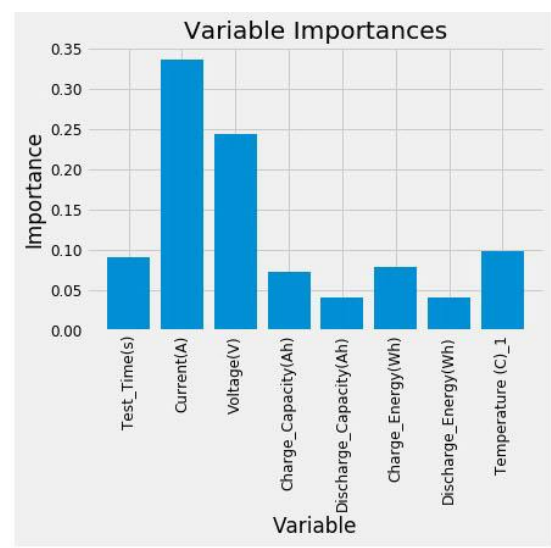

(b)

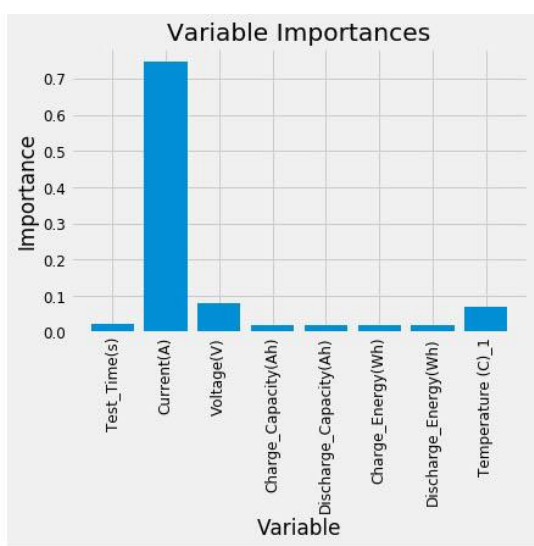

(c)

Figure 7 Feature importance comparison (random forest) for a) DST, b) FUDS, c) US06.

We can see that importance for variable influence changes based on driving cycle, indicating that variable importance is not collinear with the increase of dynamical data.

\subsection{Residual Analysis}

During online training, residuals are plotted for the loading profiles in Figure 8. In Figure 9, we perform QQ plots to check the normality of the residuals, given the assumption that our lag control model identifies an outlier that is three standard deviations from the mean. Figures 8 and 9 show that FUDS has higher residuals than DST or US06, but has a more normal distribution. While there is a lack of residual normality in DST, it is also the simplest of the loading profiles and has the most autocorrelation. There is confidence that while the outlier rule might not help with the DST model, it also does not impede learning. 


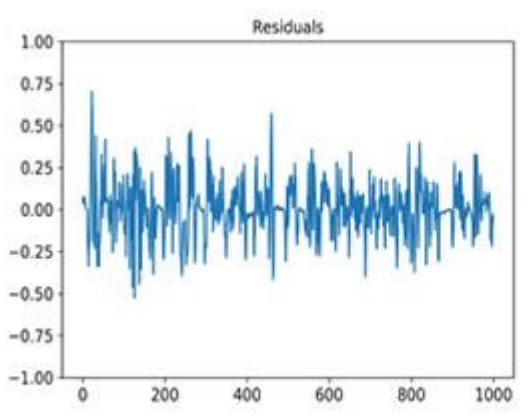

(a)

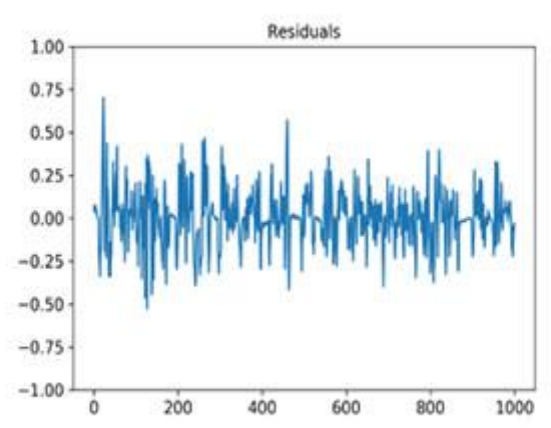

(b)

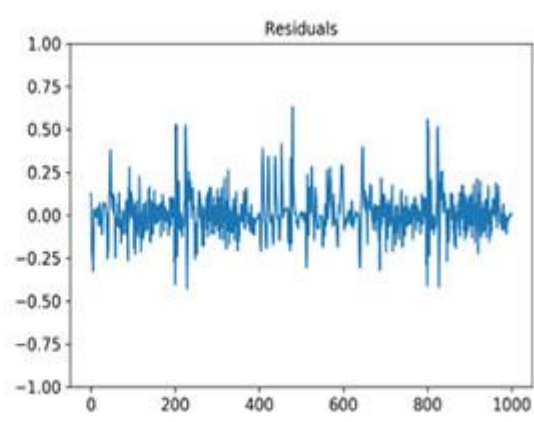

(c)

Figure 8 Residual time series plots for validation results for a) DST, b) FUDS, and c) US06.

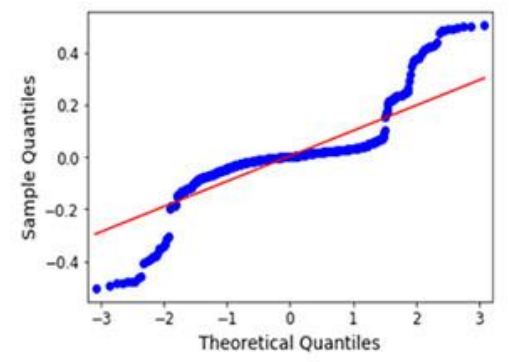

(a)

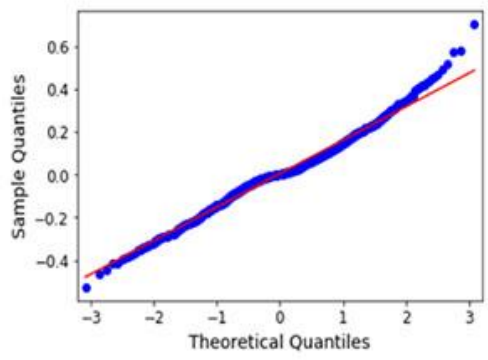

(b)

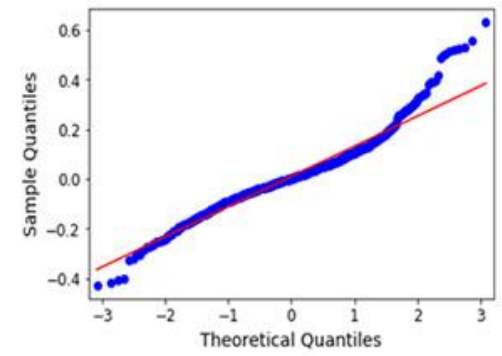

(c)

Figure 9 QQ Plots of residuals for a) DST, b) FUDS, c) US06.

Residual analysis demonstrates a lack of cyclic nature and patterns, which indicates that the errors are random and are a result from the model.

\subsection{Model Performance}

In Table 1, the algorithm is compared with results from literature and computations made on CPU. The first two rows include the NN (neural network, an artificial neural network) and the NN+UKF (neural network with unscented Kalman Filter), results were taken from He (2014) [42]. These two rows do not include results from the DST load profile because the authors from the study trained on that dataset. Results from the vanilla LSTM demonstrate the power of this algorithm on time-series data which set the desire to perform online LSTM and on-board the algorithm to HEV application. The models were trained and tested on each load profile which is likely the reason that the model performs at such a high level. Literature involving SOC prediction using SVM does not perform tests on the DST, US06, and FUDS datasets, therefore tests were conducted with the three most common kernel types using the skLearn toolset in Python and included in the comparison. Finally, results were averaged over ten runs and employ the CLC modification to show that the LSTM+CLC has the best predictive performance.

Table 1 Comparison of different predictive models.

\begin{tabular}{llll}
\hline & DST & US06 & FUDS \\
\hline NN (42) & N/A & 4.100 & 4.200
\end{tabular}


JEPT 2021; 3(1), doi:10.21926/jept.2101003

\begin{tabular}{llll} 
NN+UKF (42) & N/A & 2.400 & 2.200 \\
Vanilla LSTM & 0.372 & 0.464 & 0.493 \\
SVM (RBF) +CLC & 0.126 & 0.155 & 0.210 \\
SVM (Poly) + CLC & 0.119 & 0.124 & 0.201 \\
SVM (Linear) +CLC & 0.099 & 0.128 & 0.193 \\
LSTM + CLC & $\mathbf{0 . 0 7 6}$ & $\mathbf{0 . 1 0 2}$ & $\mathbf{0 . 1 6 6}$ \\
\hline
\end{tabular}

In Table 2, average RMSE values are displayed (over ten runs) with a ten-second $(H=1)$, thirtysecond $(\mathrm{H}=3)$, and fifty-second $(\mathrm{H}=5)$ future prediction. It was anticipated that predictions would degrade given longer horizon time however, the results demonstrate robust future prediction capabilities. Longer predictive horizons benefit from a larger lag cap. Table 3 shows the standard deviations in each of the average runs. This table illustrates relatively low variability between runs and demonstrates that the CLC is effective at controlling outliers.

Table 2 Average (Mean) of RMSE results.

\begin{tabular}{|c|c|c|c|c|c|c|c|c|c|}
\hline & \multicolumn{3}{|c|}{$\mathrm{H}=1$} & \multicolumn{3}{|c|}{$\mathrm{H}=3$} & \multicolumn{3}{|c|}{$\mathrm{H}=5$} \\
\hline & DST & US06 & FUDS & DST & US06 & FUDS & DST & US06 & FUDS \\
\hline Lag Cap 10 & 0.064 & 0.091 & 0.140 & 0.078 & 0.111 & 0.202 & 0.084 & 0.120 & 0.212 \\
\hline Lag Cap 20 & 0.070 & 0.088 & 0.132 & 0.076 & 0.104 & 0.180 & 0.083 & 0.113 & 0.184 \\
\hline Lag Cap 30 & 0.069 & 0.087 & 0.128 & 0.076 & 0.104 & 0.172 & 0.082 & 0.112 & 0.180 \\
\hline Lag Cap 40 & 0.070 & 0.086 & 0.125 & 0.078 & 0.102 & 0.170 & 0.084 & 0.112 & 0.178 \\
\hline
\end{tabular}

Table 3 Standard deviation of RMSE results.

\begin{tabular}{lcccccccccc}
\hline & \multicolumn{3}{c}{$\mathrm{H}=1$} & & \multicolumn{3}{c}{$\mathrm{H}=3$} & & & $\mathrm{H}=5$ \\
& DST & US06 & FUDS & DST & US06 & FUDS & DST & US06 & FUDS \\
\hline Lag Cap 10 & 0.011 & 0.008 & 0.024 & 0.012 & 0.013 & 0.029 & 0.012 & 0.013 & 0.027 \\
Lag Cap 20 & 0.011 & 0.006 & 0.020 & 0.010 & 0.014 & 0.023 & 0.011 & 0.012 & 0.022 \\
Lag Cap 30 & 0.012 & 0.006 & 0.018 & 0.011 & 0.011 & 0.022 & 0.011 & 0.011 & 0.024 \\
Lag Cap 40 & 0.012 & 0.006 & 0.018 & 0.012 & 0.013 & 0.023 & 0.012 & 0.011 & 0.024 \\
\hline
\end{tabular}

Table 4 illustrates ten-second $(\mathrm{H}=1)$ predictions for each of the loading profiles. A significant result is that with the simulated on-board system (Core i3 processor), average computational time was 2.05 seconds per online prediction. Therefore, despite using an advanced deep learning 
JEPT 2021; 3(1), doi:10.21926/jept.2101003

technique, this BMS would receive a forecast for an event 8 seconds in the future with excellent prediction performance.

Table 4 Results of ten-second horizon.

\begin{tabular}{lllllll}
\hline Lag Cap & Load Profile & Ave SDE & Max SDE & Ave Time & Max Time & Total Time \\
\hline 10 & DST & 0.064 & 0.080 & 2.065 & 3.577 & 2061.141 \\
& USO6 & 0.091 & 0.127 & 2.053 & 3.351 & 2048.867 \\
& FUDS & 0.140 & 0.208 & 2.100 & 3.150 & 2095.649 \\
\hline \multirow{2}{*}{20} & DST & 0.069 & 0.087 & 2.051 & 2.755 & 2051.213 \\
& US06 & 0.087 & 0.123 & 2.035 & 3.053 & 2034.995 \\
& FUDS & 0.131 & 0.169 & 2.058 & 3.225 & 2057.557 \\
\hline \multirow{2}{*}{30} & DST & 0.069 & 0.087 & 2.108 & 3.948 & 2108.107 \\
& USO6 & 0.086 & 0.112 & 2.128 & 5.079 & 2127.751 \\
& FUDS & 0.127 & 0.152 & 2.164 & 4.512 & 2164.085 \\
\hline \multirow{2}{*}{40} & DST & 0.070 & 0.088 & 2.031 & 2.440 & 2030.791 \\
& USO6 & 0.085 & 0.123 & 2.039 & 2.851 & 2039.138 \\
& FUDS & 0.124 & 0.155 & 2.037 & 2.854 & 2037.492 \\
\hline
\end{tabular}

In Table 5 and Table 6 , the predictive horizon is pushed to thirty seconds $(\mathrm{H}=3$ ) and fifty seconds $(\mathrm{H}=5)$ and have roughly the same computational time per iteration. It can be stated that horizon window has minimal effect on computational time in the model.

Table 5 Results of thirty-second horizon.

\begin{tabular}{lllllll}
\hline Lag Cap & Load Profile & Ave SDE & Max SDE & Ave Time & Max Time & Total Time \\
\hline \multirow{2}{*}{10} & DST & 0.096 & 0.129 & 2.037 & 3.125 & 2037.435 \\
& USO6 & 0.126 & 0.171 & 2.145 & 4.049 & 2144.721 \\
& FUDS & 0.200 & 0.268 & 2.171 & 5.119 & 2171.391 \\
\hline \multirow{2}{*}{30} & DST & 0.098 & 0.129 & 2.062 & 3.610 & 2062.110 \\
& US06 & 0.121 & 0.179 & 2.067 & 2.649 & 2066.734 \\
& FUDS & 0.179 & 0.223 & 2.071 & 2.939 & 2071.450 \\
\hline \multirow{2}{*}{40} & DST & 0.097 & 0.128 & 2.173 & 3.643 & 2173.444 \\
& USO6 & 0.119 & 0.172 & 2.145 & 3.370 & 2144.582 \\
& FUDS & 0.171 & 0.219 & 2.110 & 3.347 & 2110.102 \\
\hline & DST & 0.095 & 0.128 & 2.152 & 2.591 & 2152.111 \\
& USO6 & 0.119 & 0.177 & 2.154 & 4.550 & 2153.868
\end{tabular}


Table 6 Results of fifty-second horizon.

\begin{tabular}{lllllll}
\hline Lag Cap & Load Profile & Ave SDE & Max SDE & Ave Time & Max Time & Total Time \\
\hline 10 & DST & 0.117 & 0.145 & 2.230 & 4.335 & 2229.806 \\
& USO6 & 0.089 & 0.131 & 2.034 & 3.051 & 2030.082 \\
& FUDS & 0.211 & 0.287 & 2.060 & 2.876 & 2060.138 \\
\hline \multirow{2}{*}{30} & DST & 0.115 & 0.143 & 2.131 & 3.769 & 2131.025 \\
& USO6 & 0.127 & 0.146 & 2.092 & 3.221 & 2092.484 \\
& FUDS & 0.183 & 0.251 & 2.154 & 4.162 & 2154.042 \\
\hline \multirow{2}{*}{40} & DST & 0.110 & 0.144 & 2.171 & 5.362 & 2171.285 \\
& USO6 & 0.125 & 0.156 & 2.166 & 4.142 & 2165.733 \\
& FUDS & 0.180 & 0.251 & 2.203 & 3.992 & 2202.841 \\
\hline
\end{tabular}

Figure 10 displays RMSE plots through 1000 iterations for all experiments including DST, US06, and FUDS load profiles. Datasets with less variability (DST) train similarly, while there is more training variability in complex driving profiles (FUDS). These plots demonstrate that training takes roughly 200-300 iterations before the model reaches a constant prediction error. Training error increases as the datasets become more dynamical, which is expected.

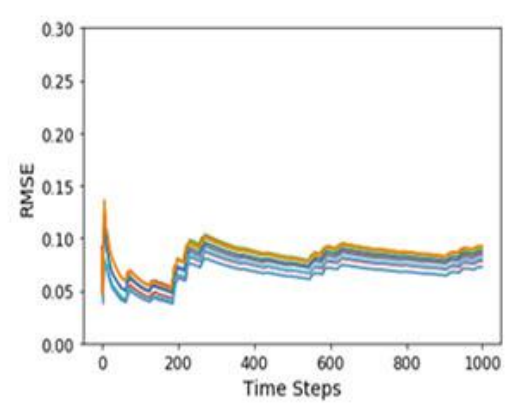

(a)

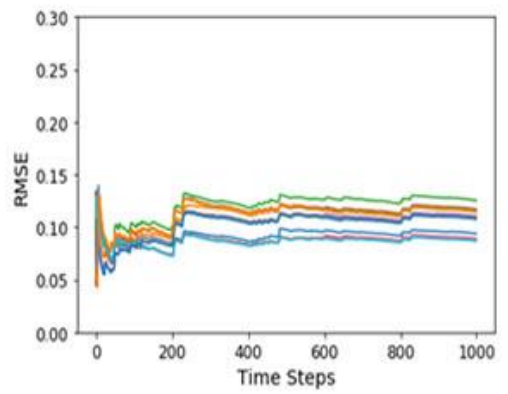

(b)

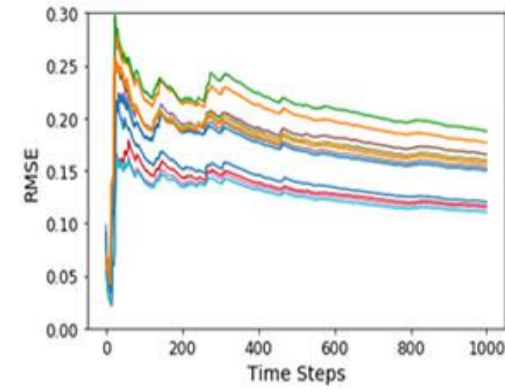

(c)

Figure 10 RMSE Values for runs of a) DST, b) US06, and c) FUDS.

In Figure 11, the effect of controlling training data size is shown and how valuable the CLC is for a possible on-board application. Figure 11a shows the necessity of adding a lag control as training exponentially grows and becomes computationally too complex after 500 iterations. This additional time and computational complexity does not equate to better training or a lower RMSE as seen in 
Figure $11 \mathrm{~b}$. It can be seen that model performance remains excellent with minimal computation time in 11b.

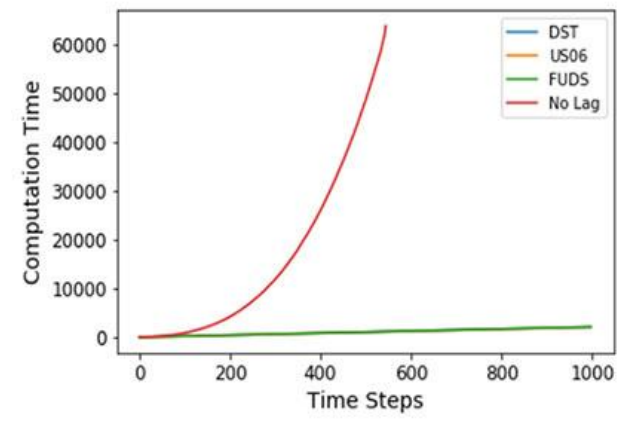

(a)

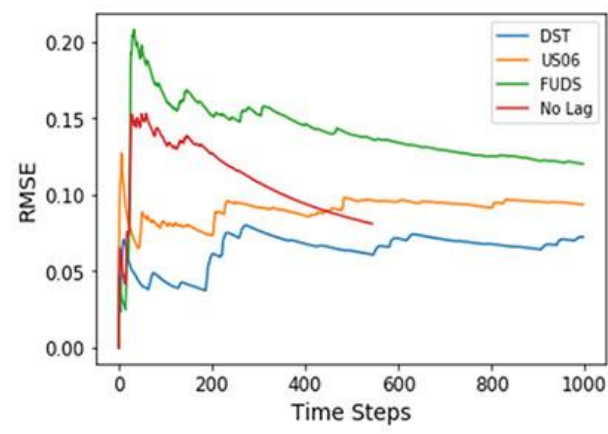

(b)

Figure 11 a) Comparison of computation of load profiles with a lag cap and one profile with a lag cap and b) Standard error plots of the same load profiles.

In Figure 12, Figure 13, Figure 14, Figure 15, Figure 16 and Figure 17, actual values versus predictive values are plotted with varying values of lag capacity limits and validation horizon. As stated in Section 2, it was ensured that training and validation sets were separate during online training. Therefore, an apparent decrease of predictive power can be seen with broader training horizons and a smaller increase of power with larger lag capacity limits.

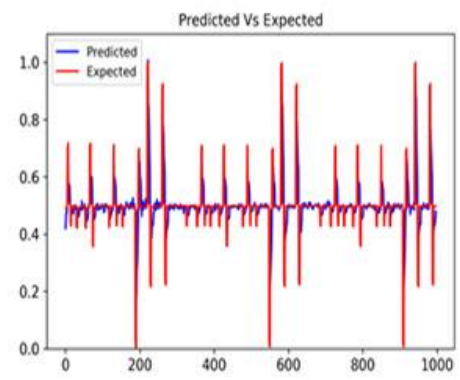

(a)

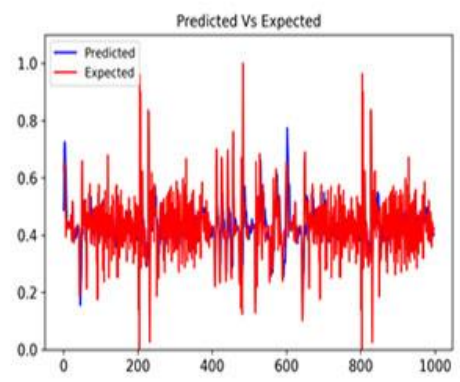

(b)

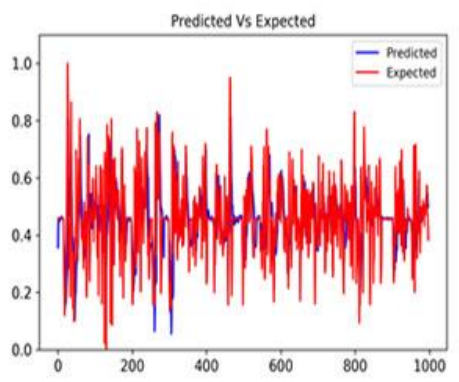

(c)

Figure 12 Expected vs. predicted plots with horizon set at one and lag cap set to 10 for a) DST, b) USO6, c) FUDS.

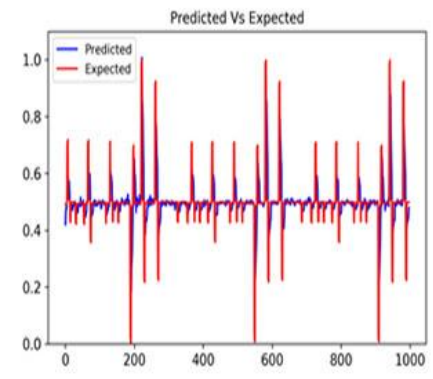

(a)

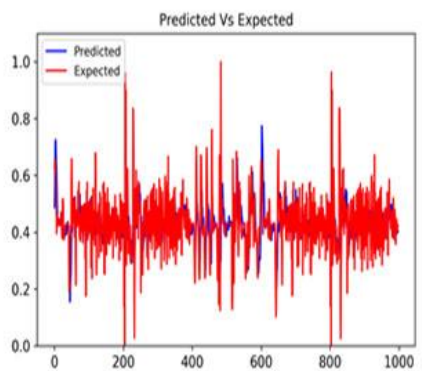

(b)

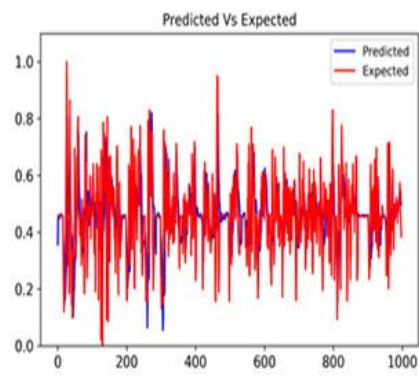

(c)

Figure 13 Expected vs. predicted plots with horizon set at three and lag cap set to 10 for a) DST, b) USO6, c) FUDS. 


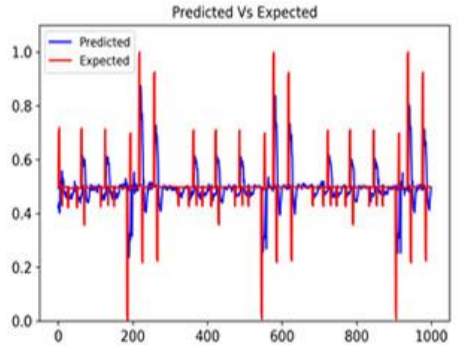

(a)

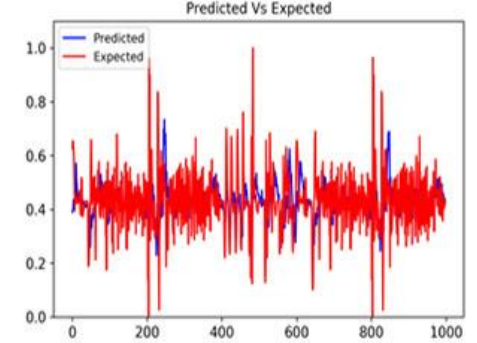

(b)

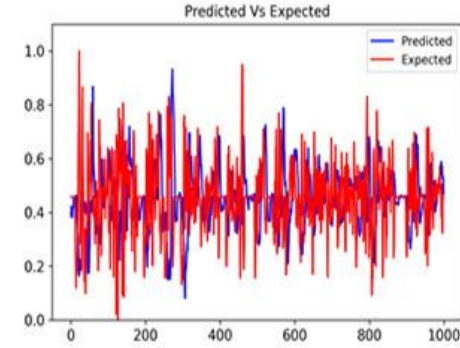

(c)

Figure 14 Expected vs. predicted plots with horizon set at five and lag cap set to 10 for a) DST, b) USO6, c) FUDS.

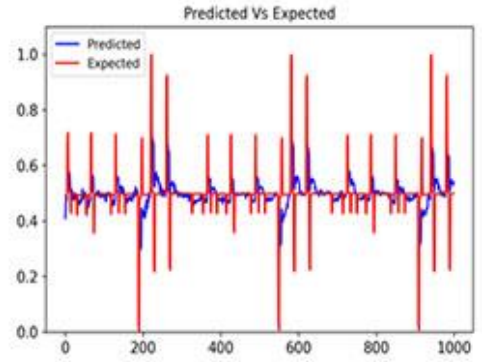

(a)

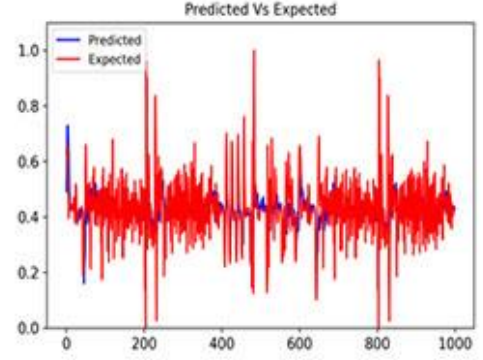

(b)

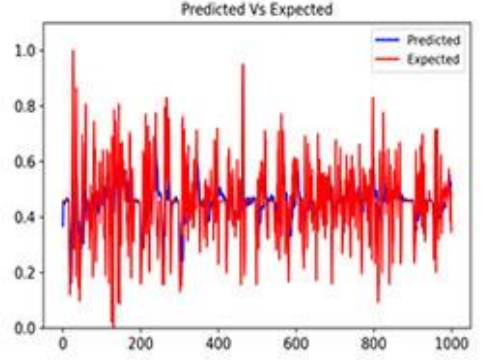

(c)

Figure 15 Expected vs. predicted plots with horizon set at one and lag cap set to 40 for a) DST, b) USO6, c) FUDS.

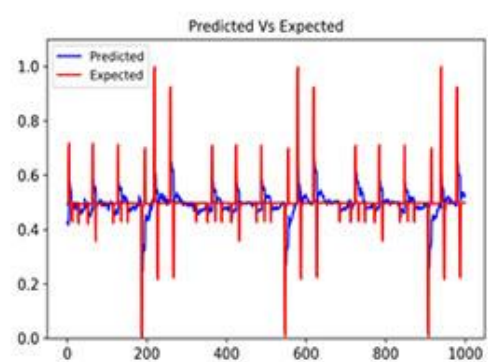

(a)

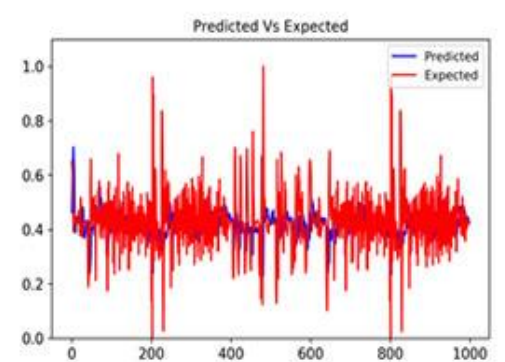

(b)

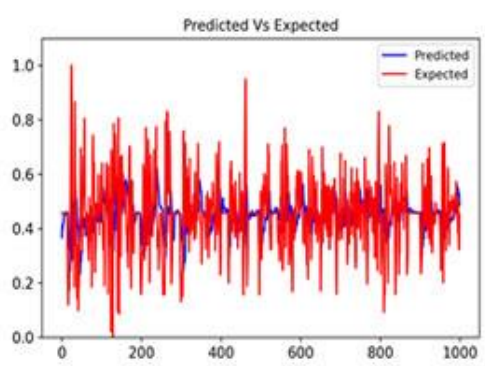

(c)

Figure 16 Expected vs. predicted plots with horizon set at three and lag cap set to 40 for a) DST, b) USO6, c) FUDS.

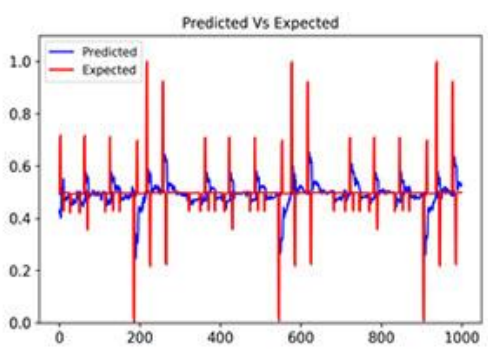

(a)

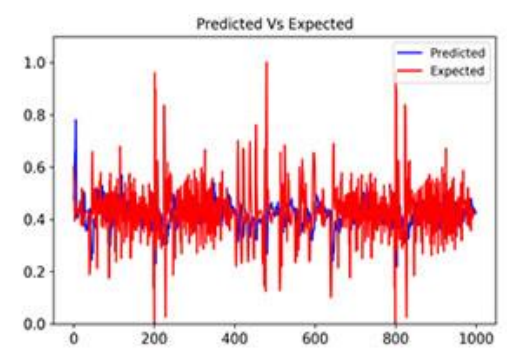

(b)

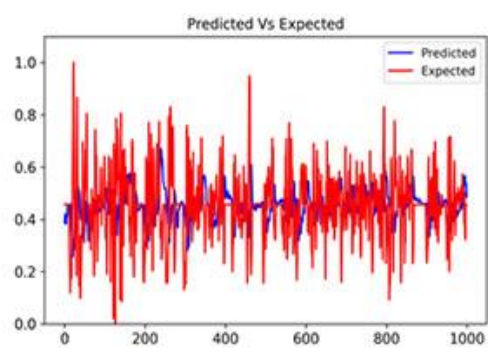

(c)

Figure 17 Expected vs. predicted plots with horizon set at five and lag cap set to 40 for a) DST, b) US06, c) FUDS. 
False positive predictions (Type II error) increase slightly as the prediction horizon increases from 10 to 30 seconds. Although an error of this type would be hazardous during operation, we don't see false positive predictions to be a significant issue with this battery model.

Finally, extension of the prediction window out to 50 seconds demonstrates excellent prediction between actual and predicted plots. Compared to the 30 second horizon plot there is a slight increase in type II error however, even at the extended prediction window there doesn't appear to be enough error to cause the model to fail to meet expectations.

Plots 15 through 17 show the prediction performance while extending the training window to 40 . When comparing these plots to corresponding plots above (same horizon windows), it is determined the expanding the training size from 10 to 40 doesn't have a drastic effect on performance.

Using offline training (where one section of the data is trained and validate once over another portion of the data), plots are generated (Figure 18) to ensure that bias is not introduced to the model. Although the following plot is only a snapshot, training and validation losses are consistent during training. It can be seen visually that 20 epochs are where training levels stabilize during offline training.

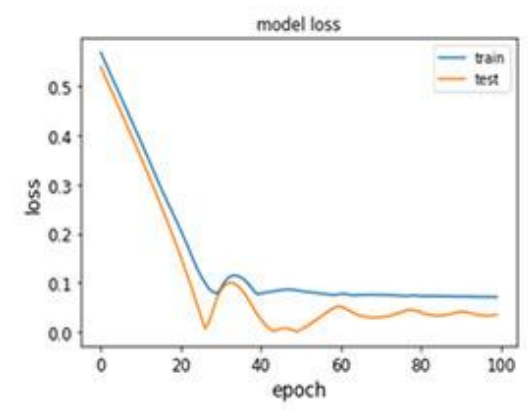

(a)

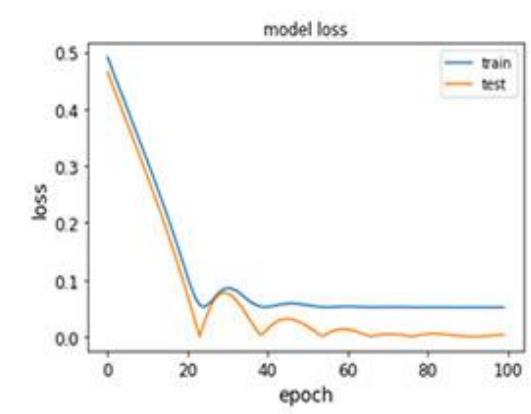

(b)

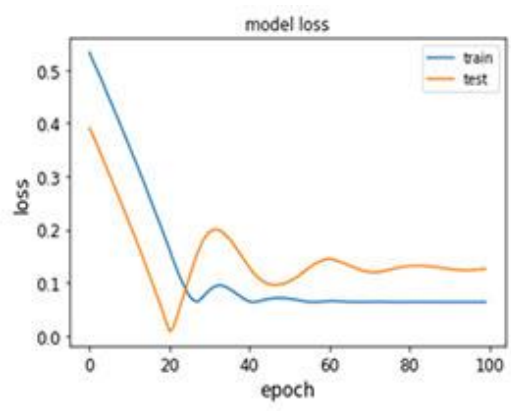

(c)

Figure 18 Training versus validation loss for offline profiles a) DST, b) US06, c) FUDS.

\section{Conclusions}

Although many consider deep learning to be a "black box" method, this study provides illumination into a methodology for using advanced recurrent neural networks within a vehicle battery management system. By examining feature importance, hyperparameter impact, contrasting model performance, and developing an algorithm for vehicle on-boarding, it is shown that recurrent neural networks combined with online training are a viable and impactful solution for next-generation vehicles.

In this research, feature analysis using a random forest algorithm to determine the impact of various parameters on the state of charge prediction was conducted. Although the literature assumes the impact of variables, this research quantifies the impact of temperature, voltage, and current on the state of charge. Next, hyperparameter search and comparison between an LSTM algorithm and models used in literature for the state of change prediction was performed. Tests were conducted on a high-performance cluster to perform gradient search to optimize the number of epochs, batch size, and gradient methods.

Although the first two objectives to this research are new applications for state of charge prediction, the methods are within deep learning literature. However, to on-board a recurrent 
neural network into a vehicle system required moving away from high-performance cluster computing. By considering computational performance and the notion that a late predictor is worthless, a rules-based model was created and a continuous training data size control mechanism embedded into a recurrent neural network architecture. Through this mechanism, the feasibility of online predictions from 10 seconds up to 50 seconds in the future is demonstrated.

\section{Conclusions and Future Studies}

In this study, the feasibility of using advanced deep learning for online battery management in a vehicle platform is examined. Limiting training time $(\sim 2 \mathrm{sec})$ and computational complexity allows for this battery predictive model to be on-boarded to a HEV application without hindering model performance. While these results indicate that continuous data size control allows for the mechanism of optimizing energy pulls from fuel cells to batteries, we plan to improve our model for eventual vehicle tests.

One limitation of this study is the use of only three driving profiles. Actual driving consists of a combination of many iterations of stop-and-go traffic, open freeway, urban driving, and city driving. To improve the BMS, the plan is to use a classifier in coordination with a recurrent neural network to enhance regression. More data would allow the use of a Conv-LSTM (Convolutional Neural Network classifier embedded in a Long Short-Term neural network regression algorithm) that optimizes data size and improves predictions during online training.

The authors are interested in studying capacity fade over long term use and implementing these effects into our model. This is an open area of research that could benefit from deep learning. Capacity fade is not considered in this study and is a limitation of this model since a battery performs differently as it ages. Future experiments are planned to account for how aging batteries in a HEV application effect state of charge, state of health, and overall effects on a BMS.

\section{Acknowledgments}

The authors wish to thank the Center for Advanced Life Cycle Engineering (CALCE) at the University of Maryland for the use of their battery data. The authors also want to thank the Graduate School, College of Engineering, and College of Business at New Mexico State University for their support.

\section{Author Contributions}

Both authors designed the model, organized the computational framework, and analyzed the data. Steven Hespeler carried out the implementation and performed the calculations. Donovan Fuqua provided academic guidance and planning for the research. Both authors conceived the study and wrote the manuscript.

\section{Funding}

The authors thank the Graduate School and College of Business at New Mexico State University for partial funding of this research.

\section{Competing Interests}


The authors have declared that no competing interests exist.

\section{References}

1. Xing Y, Ma EW, Tsui KL, Pecht M. Battery management systems in electric and hybrid vehicles. Energies. 2011; 4: 1840-1857.

2. Piller $S$, Perrin $M$, Jossen $A$. Methods for state-of-charge determination and their applications. J Power Sources. 2001; 96: 113-120.

3. Hannan MA, Lipu MS, Hussain A, Mohamed A. A review of lithium-ion battery state of charge estimation and management system in electric vehicle applications: Challenges and recommendations. Renew Sustain Energy Rev. 2017; 78: 834-854.

4. Zenati A, Desprez P, Razik H. Estimation of the SOC and the SOH of Li-ion batteries, by combining impedance measurements with the fuzzy logic inference. Proceedings of the IECON 2010-36th Annual Conference on IEEE Industrial Electronics Society; 2010 November 7-10th; Glendale, California, USA. Piscataway Township: Institute of Electrical and Electronics Engineers.

5. Shen Y. Adaptive online state-of-charge determination based on neuro-controller and neural network. Energy Convers Manag. 2010; 51: 1093-1098.

6. He Y, Liu X, Zhang C, Chen Z. A new model for State-of-charge (SOC) estimation for high-power Li-ion batteries. Appl Energy. 2013; 101: 808-814.

7. Waag W, Fleischer C, Sauer DU. Critical review of the methods for monitoring of lithium-ion batteries in electric and hybrid vehicles. J Power Sources. 2014; 258: 321-339.

8. Cuma MU, Koroglu T. A comprehensive review on estimation strategies used in hybrid and battery electric vehicles. Renew Sustain Energy Rev. 2015; 42: 517-531.

9. Sauer DU, Bopp G, Jossen A, Garche J, Rothert M, Wollny M. State of charge-What do we really speak about? Proceedings of the 21st international telecommunications energy conference; 1999 June 9th; Copenhagen, Denmark. Piscataway Township: Institute of Electrical and Electronics Engineers.

10. Chiasson J, Vairamohan B. Estimating the state of charge of a battery. IEEE Trans Control Syst Technol. 2005; 13: 465-470.

11. Chang WY. The state of charge estimating methods for battery: A review. ISRN Appl Math. 2013; 2013: 953792.

12. Coleman M, Lee CK, Zhu C, Hurley WG. State-of-charge determination from EMF voltage estimation: Using impedance, terminal voltage, and current for lead-acid and lithium-ion batteries. IEEE Trans Ind Electron. 2007; 54: 2550-2557.

13. Sato S, Kawamura A. A new estimation method of state of charge using terminal voltage and internal resistance for lead acid battery. Proceedings of the Power Conversion ConferenceOsaka 2002 (Cat No 02TH8579); 2002 April 2-5th; Osaka, Japan. Piscataway Township: Institute of Electrical and Electronics Engineers.

14. Robinson RS. System noise as a signal source for impedance measurements on batteries connected to operating equipment. J Power Sources. 1993; 42: 381-388.

15. Huet F. A review of impedance measurements for determination of the state-of-charge or stateof-health of secondary batteries. J Power Sources. 1998; 70: 59-69.

16. Stoynov Z, Savova-Stoynov B, Kossev T. Non-stationary impedance analysis of lead/acid batteries. J Power Sources. 1990; 30: 275-285. 
17. Blanchard P. Electrochemical impedance spectroscopy of small $\mathrm{Ni}-\mathrm{Cd}$ sealed batteries: Application to state of charge determinations. J Appl Electrochem. 1992; 22: 1121-1128.

18. Kozlowski JD. Electrochemical cell prognostics using online impedance measurements and model-based data fusion techniques. 2003 IEEE Aerospace Conference Proceedings (Cat No 03TH8652); 2003 March 8-15th; Big Sky, Montana, USA. Piscataway Township: Institute of Electrical and Electronics Engineers.

19. Hung $\mathrm{MH}$, Lin $\mathrm{CH}$, Lee LC, Wang CM. State-of-charge and state-of-health estimation for lithiumion batteries based on dynamic impedance technique. J Power Sources. 2014; 268: 861-873.

20. Bundy K, Karlsson M, Lindbergh G, Lundqvist A. An electrochemical impedance spectroscopy method for prediction of the state of charge of a nickel-metal hydride battery at open circuit and during discharge. J Power Sources. 1998; 72: 118-125.

21. Liu J, Saxena A, Goebel K, Saha B, Wang W. An adaptive recurrent neural network for remaining useful life prediction of lithium-ion batteries. Proceedings of the annual conference of the prognostics and health management society 2010; 2010 October 10-16th; Portland, Oregon, USA. New York: The Prognostics and Health Management Society.

22. Charkhgard M, Farrokhi M. State-of-charge estimation for lithium-ion batteries using neural networks and EKF. IEEE Trans Ind Electron. 2010; 57: 4178-4187.

23. Xu L, Wang J, Chen Q. Kalman filtering state of charge estimation for battery management system based on a stochastic fuzzy neural network battery model. Energy Convers Manag. 2012; 53: 33-39.

24. Shen WX, Chau KT, Chan CC, Lo EW. Neural network-based residual capacity indicator for nickelmetal hydride batteries in electric vehicles. IEEE Trans Veh Technol. 2005; 54: 1705-1712.

25. Cai C, Du D, Liu Z, Ge J. State-of-charge (SOC) estimation of high power Ni-MH rechargeable battery with artificial neural network. Proceedings of the 9th International Conference on Neural Information Processing, 2002. ICONIP '02; 2002 November 18-22; Singapore. Piscataway Township: Institute of Electrical and Electronics Engineers.

26. Linda O, William EJ, Huff M, Manic M, Gupta V, Nance J, et al. Intelligent neural network implementation for SOCl development of Li/CFx batteries. Proceedings of the 2009 2nd International Symposium on Resilient Control Systems; 2009 August 11-13th; Idaho Falls, Idaho, USA. Piscataway Township: Institute of Electrical and Electronics Engineers.

27. Zhang Y, Song W, Lin S, Feng Z. A novel model of the initial state of charge estimation for $\mathrm{LiFePO}_{4}$ batteries. J Power Sources. 2014; 248: 1028-1033.

28. Antón JC, Nieto PJ, de Cos Juez FJ, Lasheras FS, Vega MG, Gutiérrez MN. Battery state-of-charge estimator using the SVM technique. Appl Math Model. 2013; 37: 6244-6253.

29. Wu X, Mi L, Tan W, Qin JL, Zhao MN. State of charge (SOC) estimation of Ni-MH battery based on least square support vector machines. Adv Mat Res. 2011; 211-212: 1204-1209.

30. Chen $Y$, Long $B$, Lei $X$. The battery state of charge estimation based weighted least squares support vector machine. Proceedings of 2011 Asia-Pacific Power and Energy Engineering Conference; 2011 March 25-28; Wu Han, China. Piscataway Township: Institute of Electrical and Electronics Engineers.

31. Shi QS, Zhang CH, Cui NX. Estimation of battery state-of-charge using v-support vector regression algorithm. Int J Automot Technol. 2008; 9: 759-764.

32. Singh $P$, Fennie Jr C, Reisner D. Fuzzy logic modelling of state-of-charge and available capacity of nickel/metal hydride batteries. J Power Sources. 2004; 136: 322-333. 
33. Hu L, Hu X, Che Y, Feng F, Lin X, Zhang Z. Reliable state of charge estimation of battery packs using fuzzy adaptive federated filtering. Appl Energy. 2020; 262: 114569.

34. Zhong $\mathrm{F}$, Li H, Zhong S, Zhong $\mathrm{Q}$, Yin C. An SOC estimation approach based on adaptive sliding mode observer and fractional order equivalent circuit model for lithium-ion batteries. Commun Nonlinear Sci Numer Simul. 2015; 24: 127-144.

35. Zhang Z, Cheng X, Lu Z, Gu D. SOC estimation of lithium-ion batteries with AEKF and wavelet transform matrix. IEEE Trans Power Electron. 2017; 32: 7626-7634.

36. Lin X. Theoretical analysis of battery SOC estimation errors under sensor bias and variance. IEEE Trans Ind Electron. 2018; 65: 7138-7148.

37. Ouyang $Q$, Chen J, Zheng J, Hong Y. SOC estimation-based quasi-sliding mode control for cell balancing in lithium-ion battery packs. IEEE Trans Ind Electron. 2018; 65: 3427-3436.

38. Guo Y, Zhao Z, Huang L. SoC estimation of lithium battery based on improved BP neural network. Energy Procedia. 2017; 105: 4153-4158.

39. He T, Li D, Wu Z, Xue Y, Yang Y. A modified luenberger observer for SoC estimation of lithiumion battery. Proceedings of the 2017 36th Chinese Control Conference (CCC 2017); 2017 July 26-28; Dalian, China. Piscataway Township: Institute of Electrical and Electronics Engineers.

40. Purvins A, Sumner M. Optimal management of stationary lithium-ion battery system in electricity distribution grids. J Power Sources. 2013; 242: 742-755.

41. Zong Y, Mihet-Popa L, Kullmann D, Thavlov A, Gehrke O, Bindner HW. Model predictive controller for active demand side management with pv self-consumption in an intelligent building. Proceedings of the 3rd IEEE PES Innovative Smart Grid Technologies (ISGT) Europe Conference; 2012 October 14-17th; Berlin, Germany. Piscataway Township: Institute of Electrical and Electronics Engineers.

42. Castillo-Cagigal M, Gutiérrez A, Monasterio-Huelin F, Caamaño-Martín E, Masa D, JiménezLeube J. A semi-distributed electric demand-side management system with PV generation for self-consumption enhancement. Energy Convers Manag. 2011; 52: 2659-1666.

43. He W, Williard N, Chen C, Pecht M. State of charge estimation for Li-ion batteries using neural network modeling and unscented Kalman filter-based error cancellation. Int J Electr Power Energy Syst. 2014; 62: 783-791.

44. Li J, Danzer MA. Optimal charge control strategies for stationary photovoltaic battery systems. J Power Sources. 2014; 258: 365-373.

45. Kim I. The novel state of charge estimation method for lithium battery using sliding mode observer. J Power Sources. 2006; 163: 584-590.

46. Skrylnyk $O$, Lepore R, loakimidis CS, Remy M, Frère M. State-of-charge observers for lead-acid storage units used in autonomous solar applications. J Energy Storage. 2017; 14: 1-7.

47. Hastie T, Tibshirani R, Friedman J. Random forests. In: The elements of statistical learning. New York: Springer; 2009. pp. 587-604.

48. Kuhn M, Johnson K. Regression Trees and Rule-Based Models. In: Applied Predictive Modeling. New York: Springer; 2013. pp. 173-220.

49. Sak H, Senior A, Beaufays F. Long short-term memory recurrent neural network architectures for large scale acoustic modeling. Proceedings of the Interspeech 2014: 15th Annual Conference of the International Speech Communication Association; 2014 September 14-18th; Singapore. Baixas: International Speech Communication Association.

50. Hochreiter S, Schmidhuber J. Long short-term memory. Neural Comput. 1997; 9: 1735-1780. 
51. Lipton ZC, Berkowitz J, Elkan C. A critical review of recurrent neural networks for sequence learning. Available from: https://arxiv.org/abs/1506.00019.

52. USABC electric vehicle battery test procedures manual. Revision 2. Washington, DC, USA. United States Department of Energy. 1996.

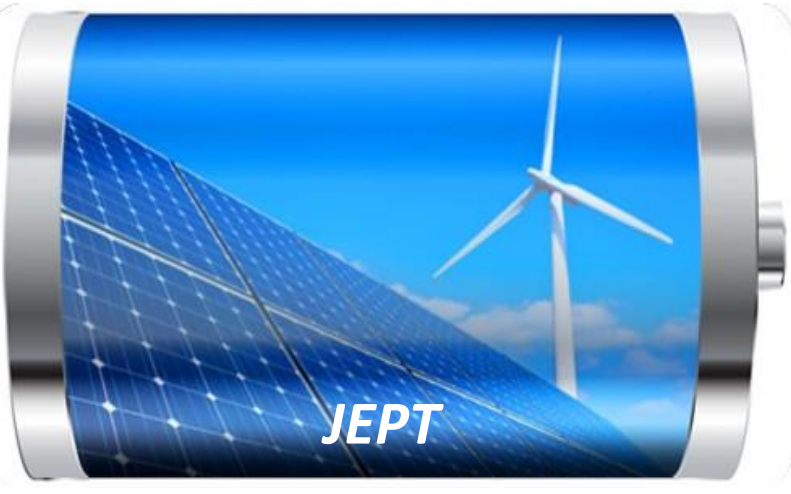

Enjoy JEPT by:

1. Submitting a manuscript

2. Joining in volunteer reviewer bank

3. Joining Editorial Board

4. Guest editing a special issue

For more details, please visit:

http://www.lidsen.com/journal/jept 
Project Report

\title{
SOFC Stack and System Modeling, Fault Diagnosis and Control
}

Khaliq Ahmed ${ }^{1}$, Periasamy Vijay ${ }^{1}$, Moses Tadé ${ }^{1,}{ }^{*}$, Amirpiran Amiri ${ }^{2}$, Zongping Shao ${ }^{1}$, Karl Föger ${ }^{3}$

1. Chemical Engineering Department, Curtin University, Kent St, Perth, WA 6102, Australia; E-Mails: khaliq.ahmed83@alumni.ic.ac.uk; V.Periasamy@curtin.edu.au; M.O.Tade@curtin.edu.au; Zongping.shao@curtin.edu.au

2. Energy and Bioproducts Research Institute, School of Engineering \& Applied Science, Aston University, Birmingham B4 7ET, United Kingdom; E-Mail: a.p.amiri@aston.ac.uk

3. Xinnotec Pty Ltd. Kew, Victoria 3101, Australia; E-Mail: kf@xinnotec.com.au

* Correspondence: Moses Tadé; E-Mail: M.O.Tade@curtin.edu.au

Academic Editor: Ramon Costa-Castello

Special Issue: Modeling and Control of Fuel Cell Systems

Journal of Energy and Power Technology

2021, volume3,issue 1

doi:10.21926/jept.2101004
Received: July 31, 2020

Accepted: December 15, 2020

Published: January 18, 2021

\begin{abstract}
This report is an account of research and development activities undertaken by the Centre for Process Systems Computations, Department of Chemical Engineering at Curtin University, Western Australia in the area of solid oxide fuel cells. The focus of work of the group included 1)effect of cell macrostructure and microstructure on electrochemical performance with a view to optimise both macro- and micro-structure 2 ) electrochemistry modeling for simulating electrochemical performance 3) internal reforming aspects impacting performance at cell/stack and system levels 4) system level modeling investigating cell internal profiles (temperature, gas composition), homogeneity improvement, thermal management, anode recycle, fuel diversity, oxygen quality, and 5) monitoring for diagnostics, optimisation and control. The report summarizes work done over a period of 15 years and highlights areas of research gaps and future directions for research in the path to mass-scale commercialisation of the solid oxide fuel cell technology.
\end{abstract}

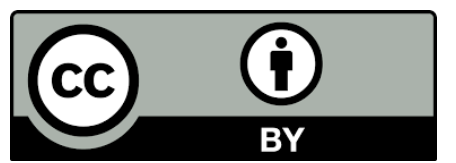

(C)2021 by the author. This is an open access article distributed under the conditions of the Creative Commons by Attribution License, which permits unrestricted use, distribution, and reproduction in any mediumor format, provided the original work is correctly cited. 


\section{Keywords}

SOFC stack; process synthesis and analysis; optimisation; simulation studies; control; process diagnostics; electrochemistry; thermal management

\section{Introduction}

Population growth and human activities - in particular, increased digitalization, transport electrification, consumption behaviour and rapid growth in the building (residential and commercial) sector are driving an increasing need for electricity. In this regard, fuel cells are attractive as they are highly efficient, practically noiseless and produce substantially lower emissions than conventional power plants. Amongst the different types of fuel cells, the solid oxide fuel cell (SOFC) technology, despite its challenges relating to durability and manufacturing cost, continues to draw attraction and attention by researchers and developers of commercial products for power generation. The most compelling reason for this, is the fact that the SOFC can contribute to a significant reduction in global emissions even when operating on a fossil fuel like the abundantly available reticulated pipeline natural gas, on account of the high efficiency of conversion of the chemical energy in the fuel to electrical energy and its ability to modulate power without significant efficiency loss. Net electrical efficiencies of 50-60\% have been reported, even for small generators $[1,2]$, which is significantly higher than any of the conventional technologies, particularly for smallscale distributed power generation; the SOFC is a highly efficient and fully controllable power source. Thus, SOFC is the ideal technology to ensure a stable grid when combined with intermittent renewable sources, as well as enable the transition from fossil-fuel dependency to a sustainable world utilizing renewable energy sources for electricity generation. SOFC technology not only provides a solution in the transition period, it can be a part of the renewable solution as it is amenable to operating on renewable fuels like bio-ethanol, landfill gas and bio-gas and green hydrogen [3-8].

Research and development in the SOFC field are currently focussed mainly in three areas - I) manufacturability (volume production and cost reduction) ii) reliability (reduced maintenance cycle and component failure i.e. reduced downtime) and iii) life (reduced material degradation and degradation of performance). Modeling is an important tool in each of these areas. At the cell/electrode level, modeling can provide valuable insight into the physics and chemistry of the internal processes occurring within the cell. Such insight can lead to designing components with improved performance and reduced degradation. Flow field and thermal and structural stability of the stack can be designed and improved by modeling without the need for expensive and timeconsuming experiments. System level modeling enables simulation for performance prediction and the development of diagnostic tools as well as the formulation of control algorithms, both for steady state and dynamic operating conditions.

In this technical report we bring together the work carried out by our research team including collaborative work with our previous industrial partner Ceramic Fuel Cells Ltd., some of which were funded by the Australian Research Council. The focus of the work of our group has been in the area of modeling and control - at the cell, stack and system levels, both steady state and dynamic. 


\section{Modeling of SOFC}

\subsection{Cell and Stack Level Modeling}

In our early work [9] a 3D CFD model of an internal reforming cell was developed where the anode was treated as two distinct layers - a diffusion layer and a catalyst layer. As shown in Figure 1 , the model was validated with experimental data in the range of $700-750^{\circ} \mathrm{C}$ from a single layer stack with cells and interconnects of the same geometry. The complex interaction between macroand micro-structural parameters of the anode was found to significantly influence the distribution of the key parameters - temperature, reaction rates and species concentration profiles along different spatial domains. An optimal set of the anode diffusion layer (ADL) microstructure, which promotes the reforming reaction rate, did not necessarily result in improved SOFC performance. For example, for the chosen reaction kinetics scheme [10], a porosity of 0.4 was found to be optimal for the internal reforming reaction in terms of methane conversion and temperature drop caused by the endothermic steam reforming reaction. However, a porosity of 0.2 was found to be more effective from the consideration of heat released by the exothermic electrochemical reactions. Different kinetic models will lead to different concentration and temperature profiles, which, in turn, will result in differences in the rates of physical processes within the pores of the anode and in the external supply and distribution channels in the anode chamber.

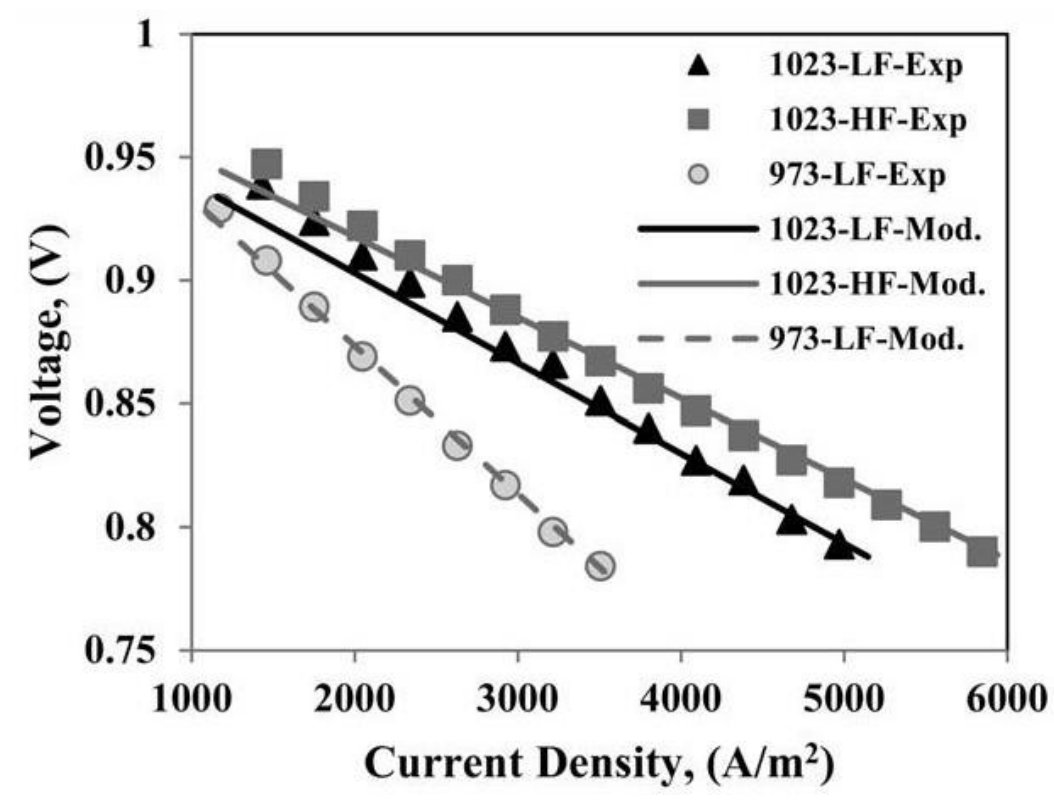

Figure 1 Validation of 3D CFD model with experimental data of the same geometry. LF: $0.067 \mathrm{SLM}$ (dry gas) + 0.073SLM (steam); HF: 0.127SLM (dry gas) + 0.139SLM (steam) [3].

A study of the effects of pore size [9] showed that pore size affects reaction rates, and consequently, species molar fractions and electrochemical performance, in addition to temperature profiles. While larger pore sizes reduce diffusion resistance (as evidenced by smaller differences of the species concentrations between gas-phase and the ADL), smaller pores result in higher reaction rates. An optimal pore-size is therefore found in the mid-range.

An increase in the macrostructural parameter of ADL thickness affects the SOFC performance predominantly within the initial length of the cell; hydrogen residence time was increased and the 
reforming reaction rate was reduced due to dilution of the reactants with hydrogen. The developed model is a powerful tool for studying the complex interactions of the chemical reactions involved and the accompanying physical processes.

\subsubsection{Tank in Series SOFC Modeling}

SOFC modeling at the cell and stack levels are useful for analysis of design considerations for flow fields and manifolds. CFD models are very effective, but with the disadvantage of high computational costs. In our work [11] the lumped parameter modeling concept of tank in series reactor (TSR) commonly used for modeling chemical reactors was introduced for SOFC cell level modeling to capture the spatial variation in the variables. The developed model for planar SOFC accounted for the charge balances in the electrodes and electrolyte and the component and energy balances in the cell and simulates the potentiostatic operation of the cell.

A two-dimensional model of the cell area was constructed through this modeling approach with lumping in the direction perpendicular to the direction of flow. Mass and energy balance equations were formulated for each lump along with the constitutive relations governing the electrochemical phenomena. The model generated polarisation curves were then validated using literature data [12]. The model was utilised to study the effect of the flow configurations on the spatial distributions of the variables. Three flow configurations were studied, namely, co-flow, counter-flow and cross-flow configurations. The transient behaviour of the key variables like current density, temperature and concentrations were also studied. The study found that the co flow configuration is most suited for SOFC as it provided uniform transient behaviour throughout the cell along with a good efficiency. We applied the same TSR methodology to analyse the spatial distributions in a tubular SOFC in [13]. One of the model simulation results for the temperature distribution along the tube is shown in Figure 2. The results show significant gradients within the tubular cell with a maximum difference between the inlet and in-cell temperature of $500 \mathrm{~K}$, which is comparable to trends reported in the literature $[14,15]$. This demonstrates the ability of the TSR methodology to simulate SOFCs with tubular configurations.
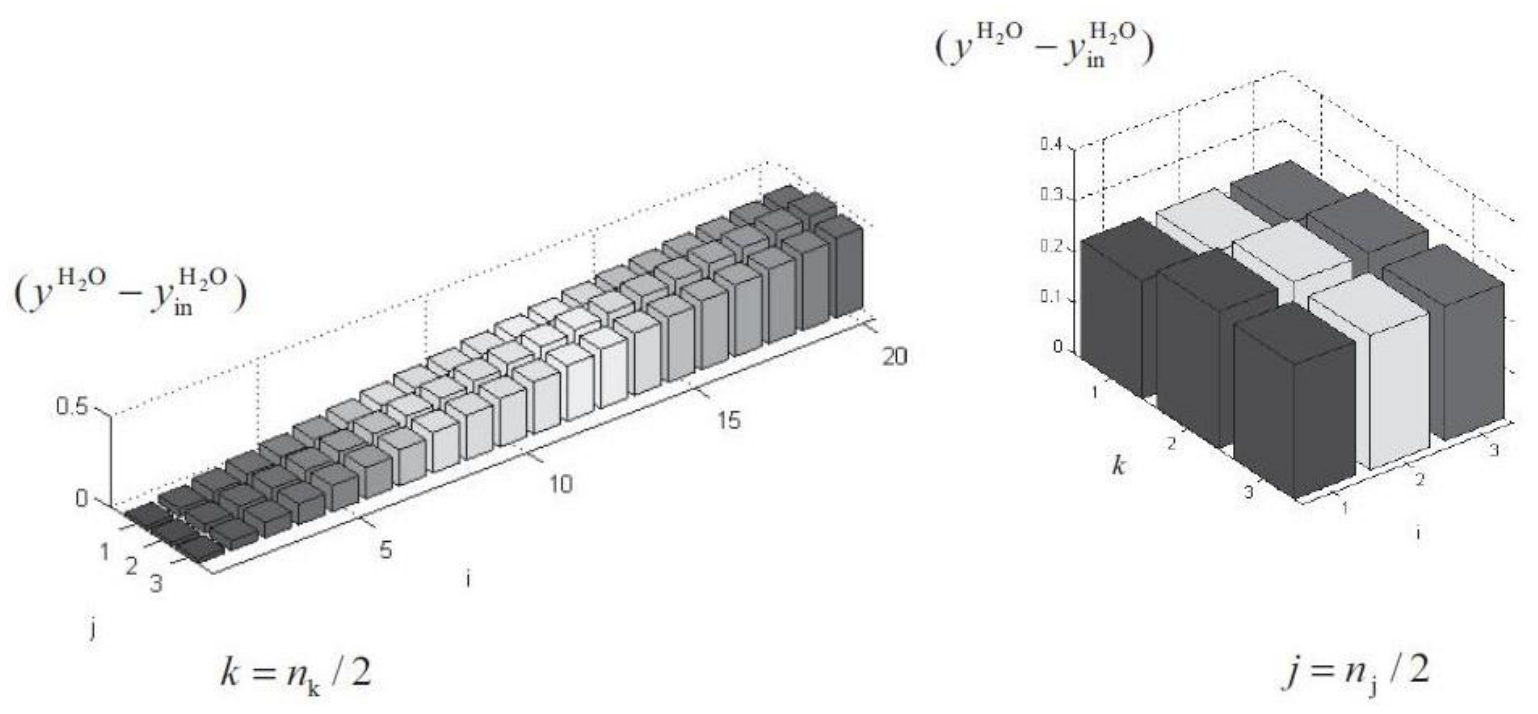

Figure 2 Local current density predicted by TSR model for the tubular SOFC stack. $i_{\text {avg }}^{A}=$ $1360 \mathrm{~A} / \mathrm{m}^{2} ; T_{\text {avg }}^{C}=1266 \mathrm{~K} ; \mathrm{T}_{\text {avg }}^{A}=1283 \mathrm{~K} ; \mathrm{T}_{\text {avg }}^{S}=1283 \mathrm{~K}[13]$. 


\subsubsection{Planar SOFC Thermal Management}

Excessive thermal gradients inside the cell during operation can result in many kinds of failures by inducing thermal stresses. It is well documented that air flow can be used to reduce the thermal gradients in planar SOFC $[16,17]$. However, this involves the trade-offs of reduced cell average temperature and reduced fuel utilisation, which are not desirable. A novel design for the planar SOFC interconnect was proposed by us in [18] to reduce the excessive thermal gradients. This design tackles the problem by introducing a secondary air stream in the counter direction and orifice based localised cooling to even out temperature differences and reduce hot spots. This way the thermal distribution can be evened out without drastic change in overall air inlet flow. Furthermore, the presence of orifices allows mass transfer between the two air streams and thus aids localised cooling. A schematic of the proposed design is shown in Figure 3. The proposed design involved minor changes to the interconnect and the inlet manifold design, which can be fabricated using standard methods while retaining all the advantages of the planar cell configuration. The effectiveness of this design was evaluated through model simulations.A TSR model of thehydrogen fuelled planar SOFC including the secondary air stream is developed and is utilised to compare the performance of the normal and the new designs in terms of the temperature uniformity in the cell. Using simulations, the primary air channel inlet flow rate and the secondary air channel inlet temperature are identified as suitable variables for manipulation so as to achieve the goal of temperature uniformity, while maintaining a desirable fuel utilisation (FU) and cell operating temperature. It is concluded from the simulations that the new design can restrict the temperature difference across the cell to less than $2 \mathrm{~K}$ over the range of operating voltages while achieving a FU of above 75\% [18-20].
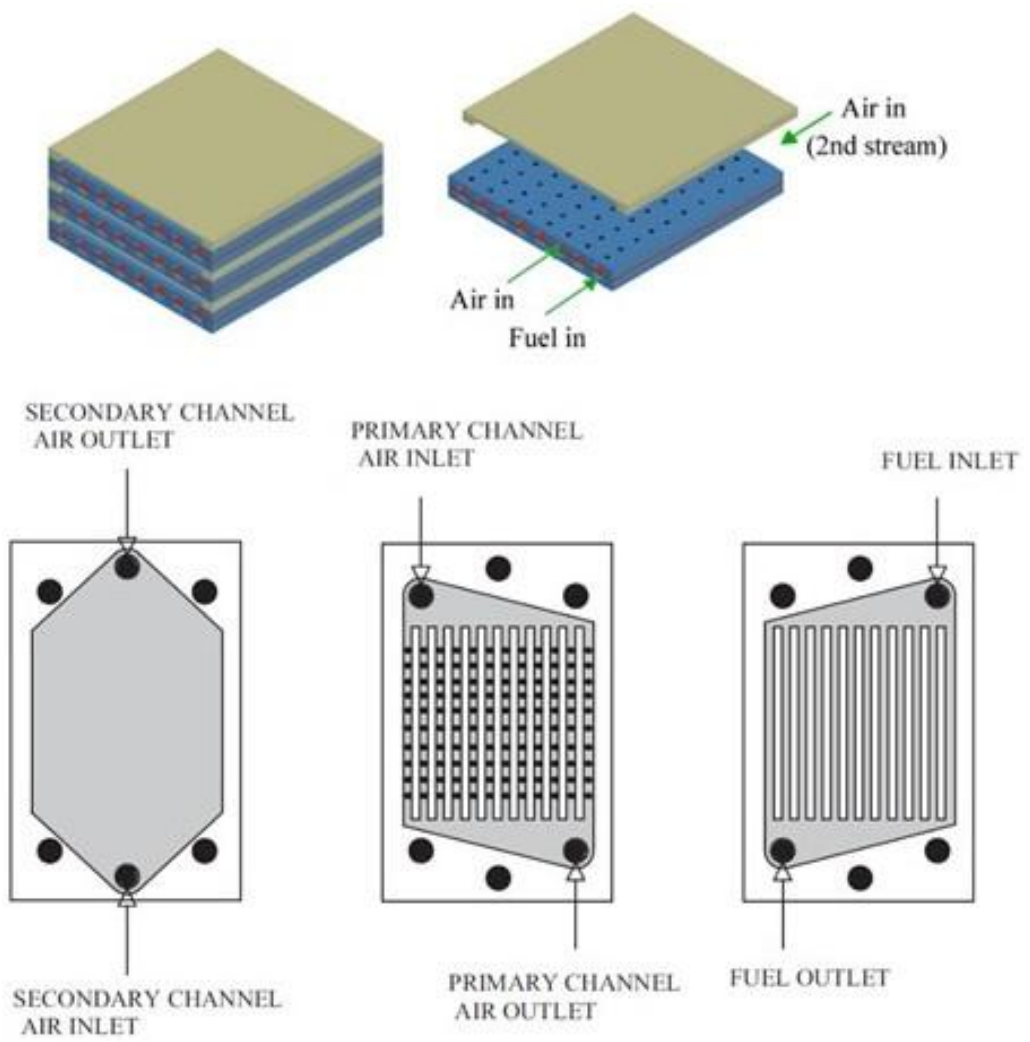

Figure 3 Schematic of the proposed interconnect and manifold designs for thermal management [19]. 
Understanding the thermal behaviour of the SOFC is essential for formulating good thermal management plans. Effective thermal management leads to minimising thermal gradients and hot spots in the cell during various operating modes resulting in improved durability of the cell. For the purpose of gaining an enhanced understanding of SOFC thermal behaviour, we conducted studies of the SOFC in a heated furnace environment [20]. The TSR modeling method is scaled for cell level modeling in creating a quasi-3D model with 4 layers accounting for various modes of heat transfer such as conduction, convection and radiation. The heat transfer characteristics of the cell in a heated environment were studied under adiabatic and non-adiabatic conditions. The experimental set-up for model validation contains the SOFC within a temperature-controlled furnace. Three models were formulated for understanding the cell thermal behaviour. A simple model that represents a perfectly insulated cell, an improved model that also accounts for the convective and conductive heat transfers between cell layers but is still insulated from atmosphere and a comprehensive model that includes the radiation heat transfer to the surrounding environment in addition to the internal heat transfer mechanisms.

Continuing with this development of the TSR model, a short stack consisting of three cells was modelled with the same approach for the purpose of evaluating the thermal behaviour and devising thermal management strategies [21]. Three different strategies for improving thermal behaviour of the stack were studied, viz. using excess air, adjustment of gas inlet temperature difference and usage of air enriched with oxygen. It was found that that temperature gradient minimisation can be achieved only at the cost of efficiency. All the three strategies investigated result in reduced energy conversion efficiency, therefore requiring trade-off optimisation. To reveal the trade-offs for design purposes, a multi-objective optimisation was performed with the two objectives of reducing thermal gradients in the stack and increasing stack efficiency. The trade-off optimisation is presented in the form of Pareto chart in Figure 4. This will serve to guide the design decision process using acceptable thresholds for the thermal gradients and efficiency. If the thermal gradient minimisation is dominant for a particular application, then a region near point III can be chosen at the cost of efficiency. If obtaining high efficiency is the primary objective then strategies can be devised to achieve operation near point I.

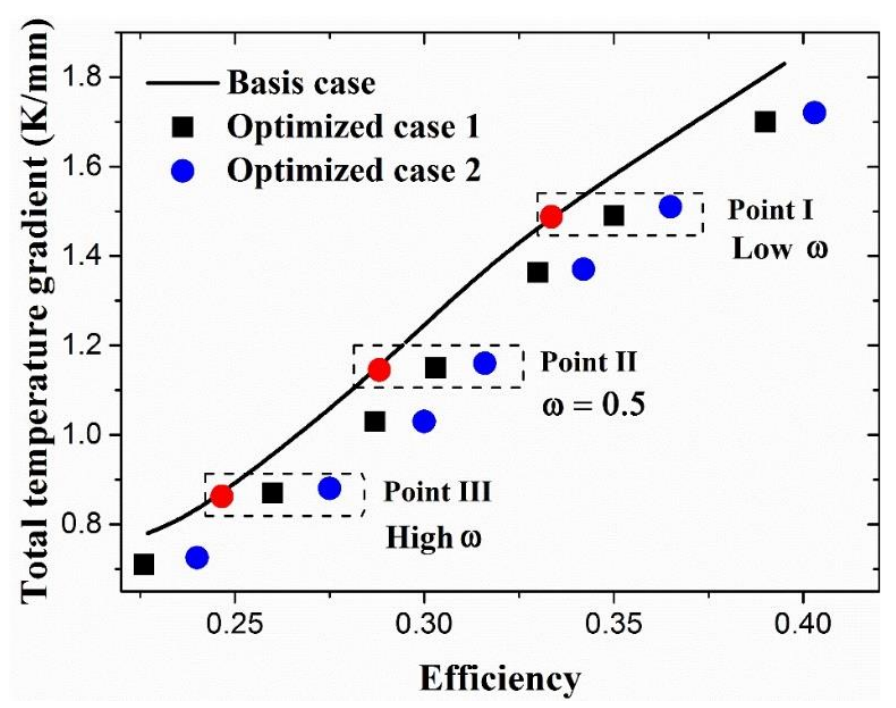

Figure 4 Trade-off between efficiency and temperature gradient; Pareto points for basis case and optimization trials $\left(F_{\mathrm{H}_{2}}=900 \mathrm{ml} / \mathrm{min}\right)$ [21]. 
The methane reforming reaction is a key element of SOFCs operating on methane-based fuels such as natural gas, biogas, pre-reformed higher hydrocarbons (eg. propane or butane). Both, the extent of reforming and the rate at which it occurs directly contribute to the fuel cell performance. Furthermore, the extent of reforming indirectly contributes to fuel cell efficiency owing to heat abstraction by the endothermic steam reforming reaction which increases fuel value and impacts on the cooling requirements by external air. The rate of reforming i.e. the kinetics of the steam reforming reactions plays a significant role in the profiles of voltage, gas composition, temperature and current density along the cell and stack - all of which contribute to cell and stack degradation and/or thermal stresses. In general, the reactions considered to be involved are the methane steam reforming reaction, MSR (1) and the water-gas shift reaction, WGS (2):

$$
\begin{gathered}
\mathrm{CH}_{4}+\mathrm{H}_{2} \mathrm{O} \rightleftharpoons \mathrm{CO}+3 \mathrm{H}_{2} \\
\mathrm{CO}+\mathrm{H}_{2} \mathrm{O} \rightleftharpoons \mathrm{CO}_{2}+\mathrm{H}_{2}
\end{gathered}
$$

There are two approaches to modeling the reforming reactions $-i$ ) assuming the reactions are at thermodynamic equilibrium and ii) using kinetic expressions derived from out-of-cell or in-cell experiments. More commonly reaction (2) is assumed to be at equilibrium and reaction (1) is considered to be kinetically controlled. In a comprehensive analysis [22] our work in this area showed how different modeling schemes - thermodynamic, kinetic and combinations of kinetic and thermodynamic schemes result in vastly different distribution of temperature, current, voltage and compositions on the SOFC anode. The results also showed that a third reaction - the reverse methanation reaction, RMTN (3), when considered to be occurring directly on the anode surface leads to significant differences in the profiles.

$$
\mathrm{CH}_{4}+2 \mathrm{H}_{2} \mathrm{O} \rightleftharpoons \mathrm{CO}_{2}+4 \mathrm{H}_{2}
$$

From their study of intrinsic steam reforming, Xu and Froment [23] concluded that it is unlikely that $\mathrm{CO}_{2}$ would be formed solely out of $\mathrm{CO}$ and therefore the reaction scheme needs to account for the direct parallel formation of $\mathrm{CO}_{2}$ via reaction (3).

Different modeling schemes led to significant differences in some of the performance parameters like maximum current density, cell temperature gradient, average cell voltage and cell efficiency. There are significant differences for different kinetic expressions, see Ahmed \& Föger [10] and Achenbach\& Riensche [24]. Furthermore, there are significant differences when the reverse methanation reaction is included in the model and when the WGS reaction is kinetically controlled (Tingey [25]).

Steepest profiles of current density, $\mathrm{H}_{2}$ mole fraction and Nernst voltage are obtained with a thermodynamic model for both MSR and WGS and the inclusion of RMTN makes little or no difference. The latter is because under equilibrium conditions RMTN is a combination of the MSR and WGS reactions. The flattest profiles are obtained with a kinetic model for MSR combined with a kinetic or thermodynamic model for WGS, while not including RMTN. The fact that there is little difference between models with kinetic expressions for WGS and those assuming thermodynamic equilibrium indicates that the WGS reaction is at or near equilibrium under SOFC operating conditions, where the reaction progress is shifted to the right due to consumption of $\mathrm{H}_{2}$ by the fuel cell reaction i.e. the hydrogen electrochemical oxidation. This work highlighted the importance of 
developing internal reforming models which are representative of anodes in real systems with particular attention to MSR kinetics and the inclusion of RMTN. It was shown that both of these can significantly influence the profiles of gas composition, temperature, current density and voltage. Furthermore, the associated mass and heat transport limitations can also influence these profiles. These are important considerations when developing simulation models and control algorithms for the entire system including the balance of plant (BoP).

A HYSYS (Aspen) model for an ethane fed internal reforming SOFC was developed using in-built unit operation modules of HYSYS and without the need for linking with any programming code [26]. The model includes electrochemistry and diffusion and reforming kinetics. Three methods for representing the SOFC were investigated, out of which the recycled reforming model gave reasonable results over a wide range of operating conditions. The electrochemical model that gives good agreement with experimental data was also identified. From the simulations, it was concluded that the developed model is reasonably accurate over a wide operating range and can be used for steady state analysis. Two main drawbacks of this work are the assumptions of isothermal conditions for internal reforming and the use of a GIBBS reactor for the fuel cell reactions. In a real system the SOFC stack does not operate in an isothermal mode. Our subsequent work [27] addresses these issues by using a non-isothermal plug-flow reactor for the internal reforming MSR, an equilibrium reactor for WGS and a conversion reactor for the fuel cell oxidation reactions to represent the fuel utilisation level. The main findings from this work are presented in the following section as this was a system level study compared to [26], which was a cell-level study.

\subsection{Modeling and Simulation at the System Scale}

In the area of multi-scale modeling of SOFC systems, the application of suitable commercial flowsheet software was adopted for a number of reasons. Firstly, it obviates the need for writing codes for modeling of process units like heat-exchangers, chemical reactors and burners or process operations like fluid flow and separation. Most commercial process simulators include model libraries for the various units and operations in process and chemical engineering and these are available as in-built modules. The SOFC system units (except detailed electrochemical reactors including fuel cells) can be selected and used from these established high-fidelity libraries. Detailed modeling and simulation of the SOFC is possible through the interfacing of these process simulators with modeling environments such as Aspen Custom Modeler (ACM), numerical routines written in an external platform like FORTRAN, C+ or in embedded Spreadsheets as in ASPEN HYSYS and Pro-II. The externally coded module will be integrated to the main flowsheet by using efficient tools such as ACM, CAPE-OPEN, and integrated Spreadsheet. Secondly, the estimation of the thermo-physical properties of chemical species/mixtures, reaction kinetics, and equilibrium, etc., that are involved in SOFC system operation can be conducted using well established databases and thermodynamicsbased property methods available in the commercial simulators. Thirdly, they offer efficient process analysis facilities such as sensitivity analysis, optimisation, and specification design, etc., that are essential tools for SOFC process synthesis and analysis.

Using this approach, a simulation platform was developed [28] in Aspen. This multiscale simulation included models at the element (OD), channel (1D) and cell (2D) scales. A SOFC model was initially developed in ACM to be deployed as an imported model in Aspen Plus. In order to demonstrate the capabilities and the preciseness of the model prediction at various scales, several 
case studies were conducted including dynamic and steady-state performances modeling, sensitivity analysis and optimisation strategies. The model allows for modification of the governing equations which is a vital feature for internal and external reformation processes and also fuel quality or fuel type variation. Moreover, simulation of the fuel and air flow patterns, including coflow, counter-flow, and cross-flow patterns, were applied at the flowsheet level. The model has been used for studies at various scales. Initially, the dynamic behaviour of microscale element of a cell was examined by using the platform capabilities for lumped modeling. Even though a dynamic and distributed model is promising for SOFC system dynamics evaluation, OD dynamic models are still widely reported in the literature. Our study [28] demonstrated the effectiveness of such a model for investigation of the SOFC dynamics. This element was later used as the building block for more complex SOFC configurations. Secondly, at the channel (1D) scale, a sensitivity analysis was performed to evaluate the effects of different flow patterns in air and fuel sides. Thirdly, the effect of fuel and air flow rates on the cell internal profiles was tested on a 2D assembly. Finally, internal thermal gradients study was conducted by using 2D model. This led to an optimal air distribution profile which minimises the internal temperature gradients (Figure 5) and avoids hot spots in the cell. The most important outcome was the established numerical platform, in Aspen Plus, that could be readily employed for a wide spectrum of SOFC system technical challenges without further programming tasks. Accordingly, several further publications were presented based on this platform, each, dealing with a state-of-the-art challenge relevant to SOFC Research and Development. A brief summary of these is presented below.

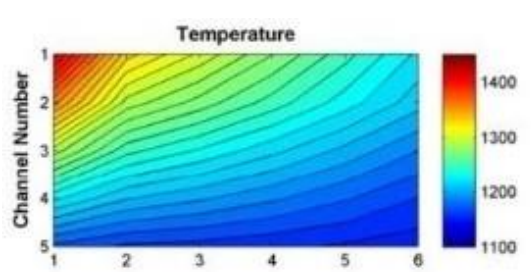

Current Density, $\mathrm{A} / \mathrm{m}^{2}$
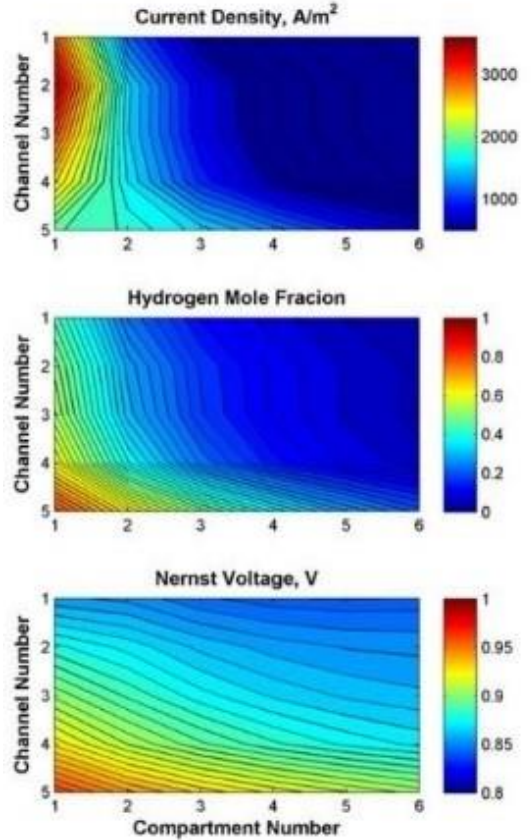

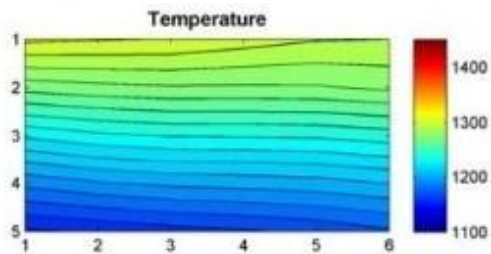

Current Density, A/m²
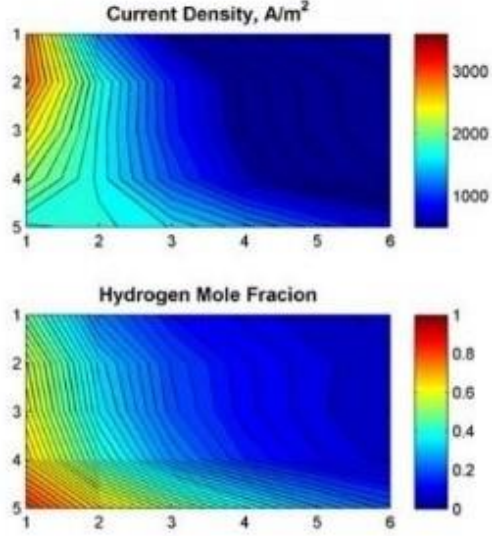

Nernst Voltage, $\mathrm{v}$

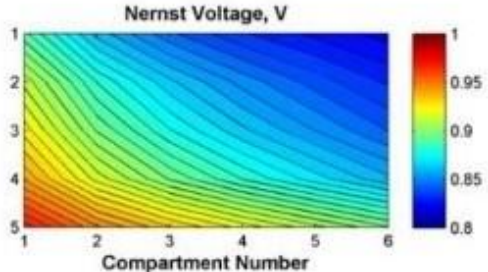

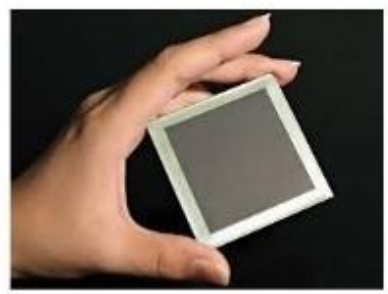

Figure 5 2D visualisation of internal variables at cell scale using flowsheet data, (left) basis case, (right) optimised case [28]. 
The impact of the BoP units on the SOFC thermal behaviour could be investigated through a system scale model that captures SOFC internal thermal homogeneity. The leading feature of the modeling strategy was the capability to evaluate the internal distribution profiles of characteristic variables such as hydrogen concentration, current, over-potentials and temperature in the flowsheeting environment. This becomes possible not only at the cell scale but also at stack scale. For instance, Figure 6 (left) depicts the effect of anode tale gas recycling rate on the current distribution in a cell embedded in a short stack. Figure 6 (right) shows the effects of the same operating variable on the stack (4 cells) current and temperature counters presented in 2D including the fuel flow (stack width) direction and stacking (stack height) directions.
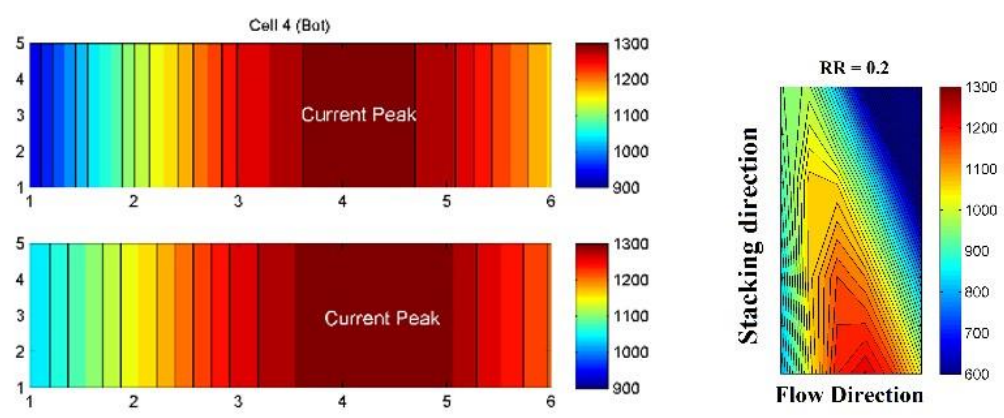

Current Density (A/Cm2)
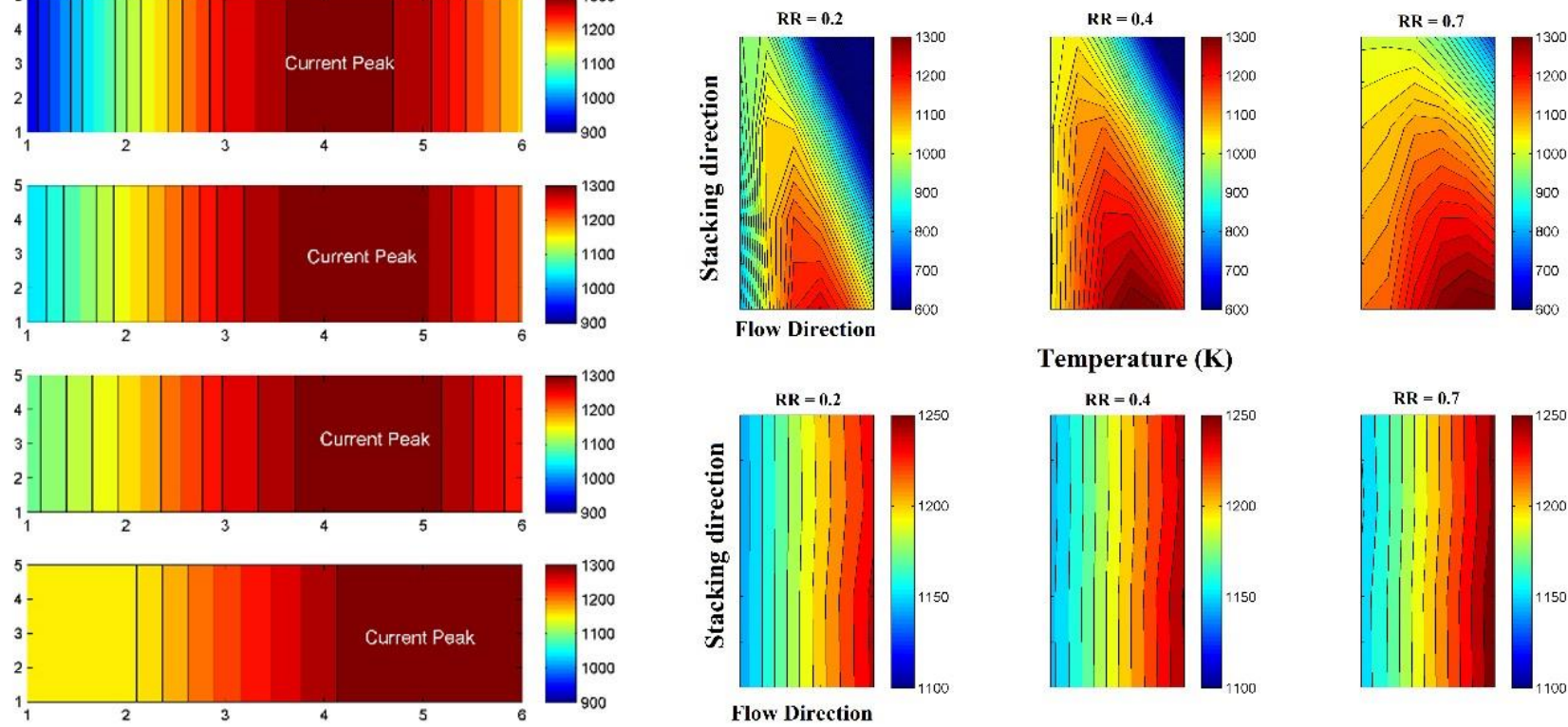

Temperature (K)
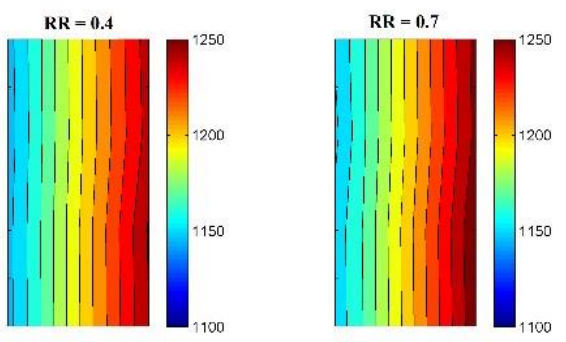

Figure 6 (left) Current distribution in single cell embedded in a short stack. (right) Effects of the same operating variable on the stack (4 cells) current and temperature counters presented in 2D including the fuel flow (stack width) direction (x) and stacking (stack height) direction (y).

Our subsequent work [29] extends the approach to stack and system scales in which we evaluated SOFC (as a 3D geometry) embedded in a system including main BoP units such as the external steam reformer. We and our industrial partner CFCL Ltd. aimed to use the model for the SOFC system thermal management. This becomes feasible owing to the stack features included in the flowsheet for the first time. The model also effectively integrated BoP units demonstrating the flowsheeting environment advantages mentioned earlier. Two case studies were studied for a short stack operating with BoP in system, i.e., the impacts of flow maldistribution in the stack manifold and the depleted fuel recycling on stack thermal inhomogeneity. It was shown that anode gas recycling could be used strategically for managing the gradients within the stack, while also enhancing fuel utilisation and water management. The thermal management challenges and opportunities were further investigated in subsequent work $[20,30]$ in which we sought further operation relevant strategies to improve the SOFC thermal homogeneity. This work resulted in novel opportunities such as enriched air utilisation and also combination of various approaches to achieve optimum results. Note that these opportunities identified using a system level modelingshow that 
the devolving macro scale models and micro scale ones must proceed interactively and in parallel to each other.

Fuel flexibility is an attractive feature of SOFC technology. Due to its high temperature (600$700{ }^{\circ} \mathrm{C}$ ) operation, most of hydrogen carrier fuels, including renewable fuels, can be reformed internally through various chemical pathways such as steam reforming, partial oxidation, etc. In a designed system, however, fuel change is not practical. It may cause several system performance problems, making dynamic fluctuations, control difficulties and undesirable measures that do not meet the design load/voltage requirements. In order to demonstrate the system fuel diversity capabilities, these consequences must be assessed through quantifying the operating measures for a range of fuel and also of potential combinations of fuel. From this viewpoint, the numerical predictive models play a critical role given the experiments cost and limitations of such as large number of possible fuels. This obviously needs a system level model with internal and external fuel reformation capabilities.

In [31], fuel diversity in SOFC systems was evaluated using the system level simulation platform. This paper examined the technical and environmental challenges and opportunities relevant to the potential SOFC fuels with specific focus on promising renewable fuels. The research results were validated using an industrial scale SOFC system published by Pacific Northwest National Laboratory (USA) [32]. The modeling results were based on steady-state operation. The control relevant issues caused by fuel change must be assessed in dynamic mode. The next challenge is to develop the modeling platform as a predictive tool for time-dependent evaluation of internal gradients resulting from the fuel change. Moreover, the impact of this scenario on the SOFC longevity is a crucial aspect to be modelled.

A distributed variable 1D-model of the SOFC was developed [27] in Aspen HYSYS with representation of internal reforming on the anode within the plug-flow reactor (PFR) module of the flowsheet software. The reforming reaction calculations were carried out in the PFR module and the electrochemical details were calculated in the spreadsheet module. By interlinking the two modules, temperature, composition and current density profiles could be readily calculated using empirical correlations and without the necessity of using an external coding platform, such as MATLAB or FORTRAN. Results for different reforming activity were discussed with respect to temperature, current density and concentration profiles, anode oxidation risk and carbon formation potential, all of which impact on performance and/or durability and life. While reduced reforming activity was shown to result in more gradual profiles for current density and temperature compared to fast reforming, such gradual reforming comes at the expense of reduced Nernst voltage and consequently results in a loss of fuel cell efficiency. Figure 7shows that for constant voltage operation gradual or distributed reforming results in a lower average current density compared to fast reforming and therefore leads to a loss in efficiency. 


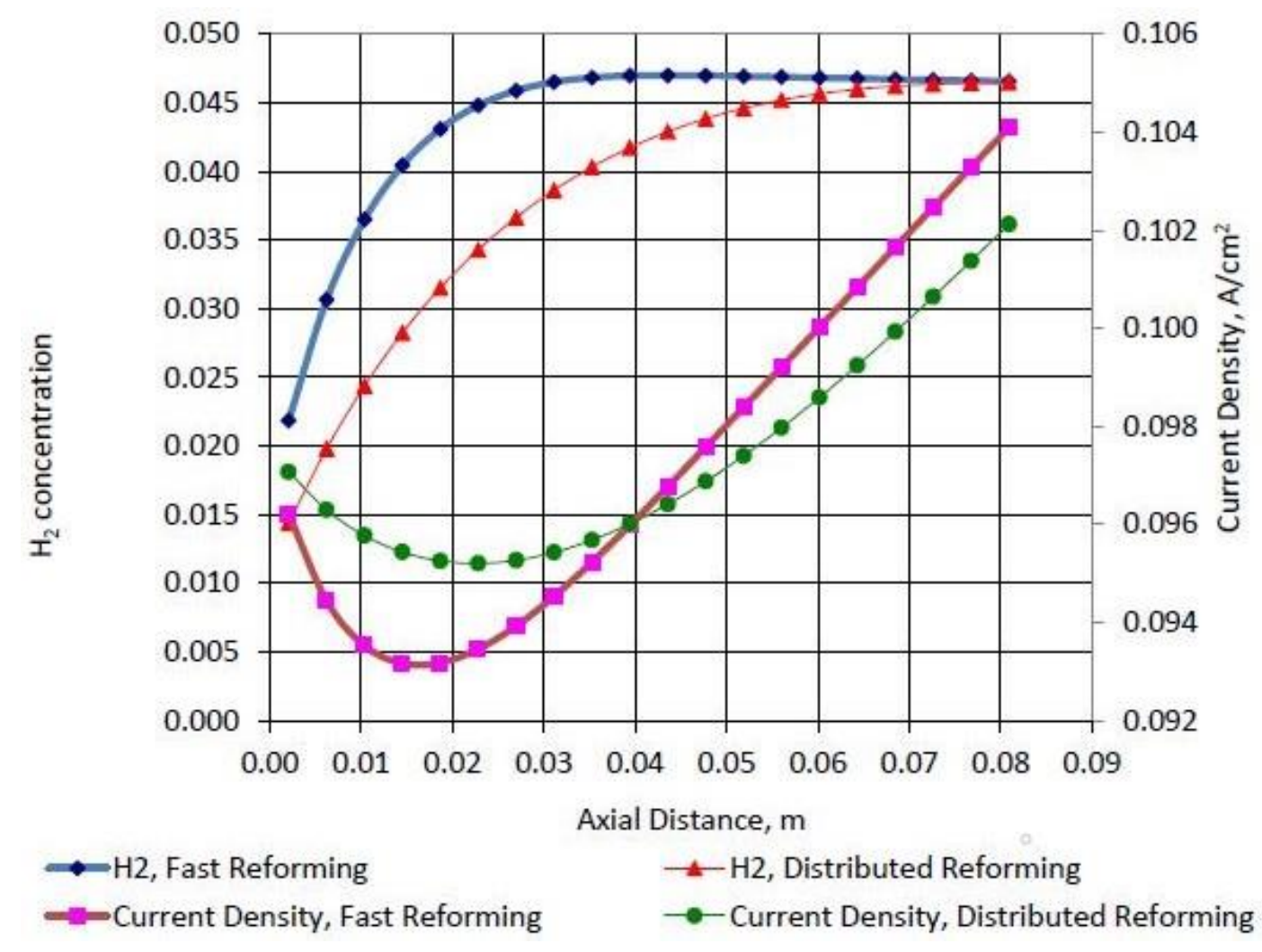

Figure $7 \mathrm{H}_{2}$ and current density profiles for fast and distributed reforming.

At cell scale, the temperature differences between cell elements were simulated in [20] using a pseudo 3D model. The model consists of a four-layer structure including stand, two interconnects, and PEN (Positive electrode-Electrolyte-Negative electrode). A OD model approximated each individual layer. The heating process through the surrounding furnace was considered. The model results show a good agreement with practical data, offering a significant improvement compared to reduced models in which the impact of external heat loss is neglected. Temperatures of the layers were estimated for adiabatic and non-adiabatic operations. This allows capturing the detailed thermal behaviour of a single cell followed by a polarisation loss assessment. Finally, model-based design of experiment was demonstrated. The model was tuned and validated with experimental data from an industrial scale SOFC system manufactured by Ceramic Fuel Cells Ltd.

In an extension to the above work at system level, a modular simulator of dynamic behaviour of the Solid Oxide Fuel Cell (SOFC) system, suitable for use as a training tool was developed [33], as an alternative to expensive practical tests. This simulator, developed in ACM, captures all of the processes in an SOFC module: mass, energy, and charge balances, overpotential losses, ohmic losses and electrochemical reaction kinetics. The developed simulator effectively minimises the requirements of mathematical and programming skills on the part of the user. The ease of application of the simulator was demonstrated through i) steady-state simulation and estimation of the stack's distributed internal variables followed by 1D optimisation and ii) illustration and interpretation of the transient behaviour of the SOFC system. The simulator not only serves as a useful tool for practical applications and as a virtual laboratory, it also opens up opportunities for developing an in-built SOFC simulator module in flowsheet simulation software such as Aspen Plus and Aspen HYSYS. 


\subsection{Electrochemistry, Microstructure and Reaction Kinetics}

\subsubsection{SOFC Electrochemistry}

At a fundamental electrochemistry level, our work [34] demonstrated that accounting for the variation in the reversible potential under closed circuit, along the cell length, as a separate "Nernst loss" allows prediction of the effects of operating parameters. The Nernst losses are attributed to the change in composition of the fuel along the length of the cell. The extent of Nernst voltage loss can be seen in Figure 8 -almost one-third and one-fourth of the total voltage loss at lower (1000$3000 \mathrm{~A} / \mathrm{m}^{2}$ ) and higher $\left(4000-7000 \mathrm{~A} / \mathrm{m}^{2}\right)$ current densities, respectively. The Nernst losses increase with the utilization of oxidant and fuel with respect to all the constituting parameters except the fuel composition, in which case it depends on the inlet concentration of $\mathrm{H}_{2}$. Since the Nernst losses are shown to contribute significantly towards voltage losses, particularly for high temperature fuel cells, it was suggested that they be treated separately from ohmic, activation, and concentration losses in any analysis of voltage losses [22]. This is an important consideration for optimizing cell composition and microstructure in order to reduce ohmic, activation and overpotential losses.

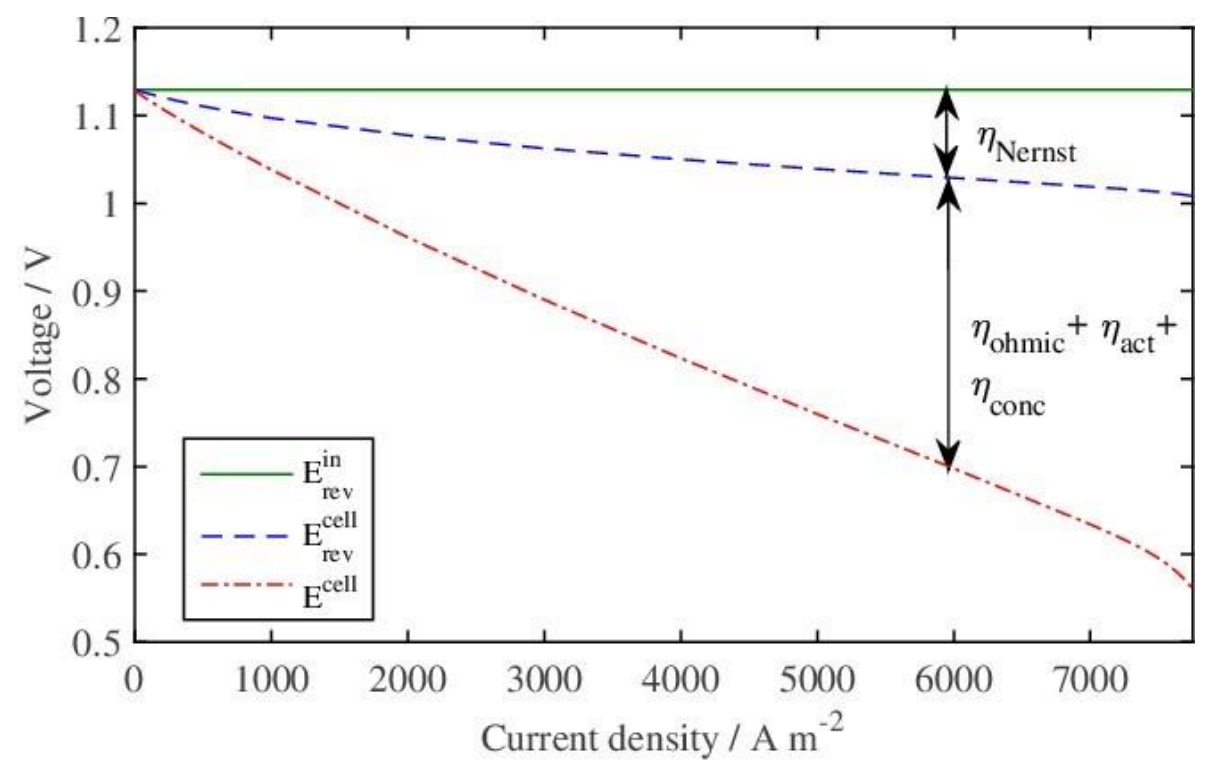

Figure 8 Voltage breakdown for a SOFC at $800{ }^{\circ} \mathrm{C}$ with $97 \% \mathrm{H}_{2}, 3 \% \mathrm{H}_{2} \mathrm{O}$. Breakdown of cell voltage losses accounting for Nernst voltage loss; other voltage losses comprise activation, ohmic and concentration overpotential losses [34].

Fundamental electrochemical modeling provides insights into the electrochemical reaction phenomena that form the core of the fuel cell operation. Primarily, three different types of polarisation affect the cell voltage. The activation polarisation represents the potential lost in overcoming the activation barrier for charge transfer reactions. The most widely used mathematical model for the activation phenomena is the Butler-Volmer (BV) equation.

In general, incorporation of the Butler-Volmer (BV) equation into multi-scale modeling frameworks requires iterative solution of the equation, which is in an implicit form. However, in applications where an iterative solution is not feasible, approximations for the BV equation are generally used to convert it into an explicit form. One particular example will be dynamic models used for controller or observer design. For this purpose, several approximations for the BV equation 
are used in literature like the popular Tafel type equation [35], the hyperbolic sine approximation and the high field approximation [36]. While these approximations are good in limited scenarios [37, 38], a better approximation that can provide wider applicability and lower computational cost is desirable. In our work [39], we presented an improved approximation scheme for the BV equation based on the form $x=f(x)$.

Approximations Proposed Based on Modified BV Equation. The BV equation was modified to the form $x=f(x)$ for deriving approximations. Three forms are discussed as given in the following.

$$
\begin{gathered}
\eta=\frac{R T}{\frac{\alpha_{t o t}}{2} F} \sin ^{-1}\left(\frac{i}{2 i_{0}} \exp \left(\frac{-x F \eta}{R T}\right)\right) \\
\eta=\frac{R T}{\alpha_{1} F} \ln \left[\frac{i}{i_{0}}+\exp \left(\frac{-\alpha_{2} F \eta}{R T}\right)\right] \\
\eta=\frac{R T}{\alpha_{1} F} \ln \left[\frac{i}{i_{0}\left(1-\exp \left(\frac{-\alpha_{t o t} F \eta}{R T}\right)\right)}\right]
\end{gathered}
$$

In these expressions, $x=\alpha_{1}-\frac{\alpha_{t o t}}{2}=\frac{\alpha_{t o t}}{2}-\alpha_{2}$, where $\alpha_{t o t}$ is the sum of the two transfer coefficients. These forms can be used to formulate iterative solution schemes that are shown to perform better compared to the standard form of the BV equation. Alternatively, explicit form approximations for the BV equation can also be constructed by approximating the over-potentials on the right-hand sides of the expressions with standard approximations like the high field or hyperbolic sine approximation.

Performance Comparison. Comparisons of the performance of the proposed forms of the BV equation with traditional approximations and the actual implicit form solution are performed to ascertain the effectiveness of the proposed forms. It was found that the proposed second form of the BV equation performs best with the hyperbolic sine approximation for the right-hand side of the expression.

Some of the results of the comparison of the proposed forms with standard assumptions are presented in Figure 9. Literature studies indicate that generally for $\alpha_{1}>\alpha_{2}$, the more important region is $x>0$. These results along with other results [39] confirm that the second form of the BV equation is the best approximation for the region $x>0$ throughout the $\frac{i}{i_{0}}$ range and for $\frac{i}{i_{0}}>1$ in the $x<1$ region, although it has some small error in the $x<1$ region with $\frac{i}{i_{0}}<1$. 


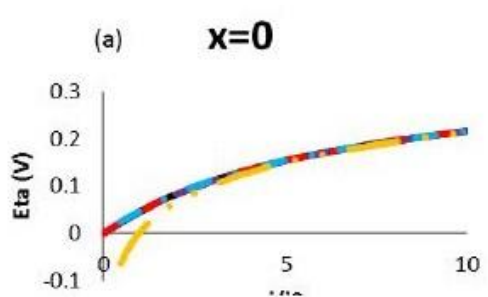

(c) $\quad x=0.8$
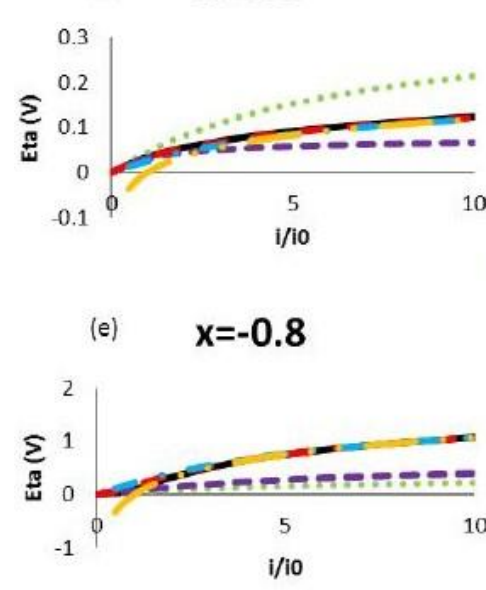

(b) $\quad x=0.4$

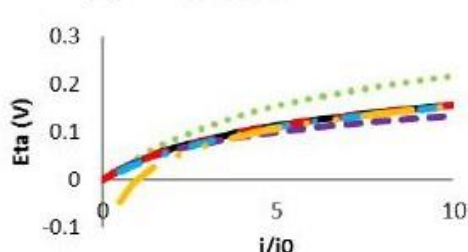

(d) $\quad x=-0.4$
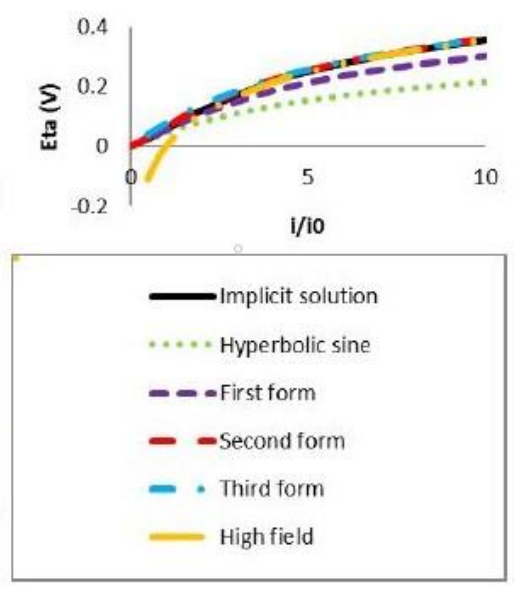

Figure 9 Performance comparison of proposed BV approximation with other common approximations [39].

Convergence Properties with Fixed-Point Iterative Calculations. In addition to enabling better explicit approximations for the BV equation, the proposed forms also enable efficient iterative schemes using the fixed-point theorem. The convergence results for such iterative schemes are reported in detail in [38]. It was found that the second form proposed earlier converges with less than one iteration (for a specified accuracy of 1e-3) throughout the range studied, especially in the range $x>0$ with guaranteed convergence properties.

In effect, the proposed approximation schemes for the BV equation not only provides an explicit expression, but is also useful in formulating better iterative schemes in its implicit form. The proposed iterative algorithm enables ease of implementation, faster computations and elimination of the need for Jacobian information.

\subsubsection{SOFC Electrochemical Reaction Kinetics Modeling}

Our work in this area was focussed on the electrochemical oxidation of hydrogen. Generally, CO electro-oxidation is considered less facile and for modeling it is assumed that CO consumption occurs via the WGS reaction only. Because the stoichiometry of CO conversion is the same for both routes, this assumption has no effect on mass balance.

Thermodynamic Consistency. Modeling the electrode electrochemical reaction kinetics in the SOFC can be useful for increasing the rate of the overall reaction. The parameters of the reaction kinetic models often need to be estimated from experimental data. Methods for monitoring and generating data include electrochemical impedance spectroscopy, temperature programmed desorption, near-infrared spectroscopy, short-wavelength near-infrared spectroscopy and ultraviolet visible spectroscopy. The fundamental requirement while estimating the parameters is 
the satisfaction of thermodynamic consistency conditions. This was achieved in [37] using constrains that relate the reaction enthalpies and the entropies to the activation energies and the preexponential constants respectively. To achieve this, thermodynamic data for many intermediate species participating in the mechanism are required and these are seldom available. Further, this approach is an overkill if our aim is the estimation of the reaction rate coefficients (excluding preexponential constants and activation energies).

In our work [40], we proposed a thermodynamically consistent formulation of the SOFC Ni/YSZ anode reaction kinetics for the estimating the parameters. The rate determining steps in the mechanism were also identified using the concept of 'degree of rate control'.

We demonstrated that the reaction mechanisms could form loops thereby introducing constraints on the reaction rate constants through the anode reaction mechanism proposed in [37]. The reaction mechanism contains a loop as shown in Figure 10.

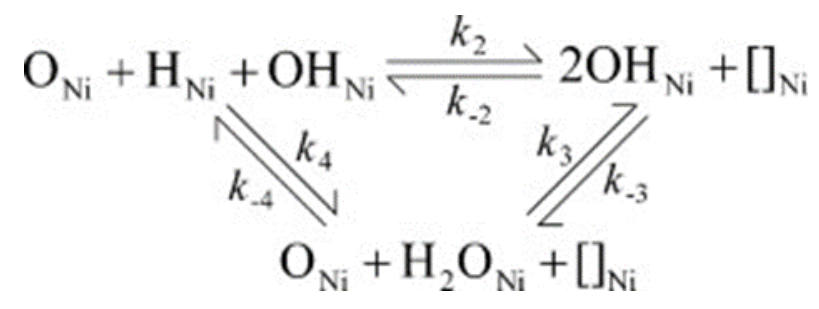

Figure 10 The loop in the SOFC anodic reaction mechanism [41].

Applying the equilibrium conditions, we can obtain the following condition for the rate constants known as the Wegscheider condition [41].

$$
W r=\frac{k_{-2} k_{+3} k_{+4}}{k_{+2} k_{-3} k_{-4}}=1
$$

The reaction rate parameters must satisfy the condition failing which the model will represent a physically impossible system since the equilibrium flux becomes non-zero representing a permanent deviation of concentrations from their equilibrium ratios [41]. This condition was found to be violated using the parameter values estimated in [37].

New Formulation of the Reaction Kinetics Equations. The traditional formulation of the reaction kinetics using law of mass action results in differential equations with the surface coverage as the dependent variables. This formulation does not consider the driving forces of the reactions explicitly. Chemical reactions are driven by the chemical potential difference, the Gibbs free energies being the flow variables. Due to the difficulty in using these quantities, a formulation for the reaction kinetics is developed using the new thermo-kinetic force proposed in [41]. Expressions for the species' capacities are required in the new formulation, which can be calculated from their standard chemical potentials [40]. If this information is not available, any set of equilibrium concentrations can be used as capacities as proven in our paper [40]. It is also shown that this formulation satisfies the De Donder conditions given in the following equation.

$$
\frac{J_{+i}}{J_{-i}}=\exp \left(-\frac{(\Delta G)_{i}}{R T}\right)
$$


Where $J_{+i}$ and $J_{-i}$ are the forward and reverse reaction fluxes and $\Delta G$ is the Gibbs free energy.

Using this new formulation can avoid the detailed thermodynamic consistency tests needed in parameter estimation requiring extensive thermodynamic data. Parameter estimation using this new formulation will also reduce the search space to consistent values thus speed up the searching time. The number of parameters to be estimated is also reduced to half that of the traditional formulation. An iterative algorithm was developed to exploit the new formulation in estimating the reaction rate constants without violating the thermodynamic constraints. More details of the algorithm can be found in our work [40].

The parameter estimation results using the new formulation are presented in the form of frequency response plots [40]. They compare favourably with other estimates [37] and result in a lower least squares error. The difference with experimentally generated data is attributed to the non-inclusion of low frequency processes like diffusion into the model.

Graph based reaction kinetics modelling. Graphs form an intuitive and convenient basis to represent the reaction mechanism networks that allows elegant visualisation of reaction routes leading to the overall reaction. This representation further allows the use of several well-established network analysis methods from other domains like electrical, thermal, mechanical etc. to chemical reaction networks. In our collaborative work with Worcester Polytechnic Institute, we explored the application of reaction route graphs for SOFC reaction mechanism analysis. Theoretical basis for the application of the graph-based method to linear and non-linear chemical reaction mechanisms were established in [42]. An algorithm for utilising the graph theory-based approach to elucidate unknown reaction mechanism was presented in [43].

This graphical approach was applied to analyse the hydrogen oxidation mechanism on the standard Nickel/Yttria-stabilised Zirconia (Ni/YSZ) SOFC anode in our work [44]. This mechanism consisted of surface and charge transfer reactions occurring on the Ni and YSZ surfaces and their interfaces in SOFC anode. The mechanism consisted of 13 reaction steps, 8 intermediate and 5 terminal species as shown in Figure 11.

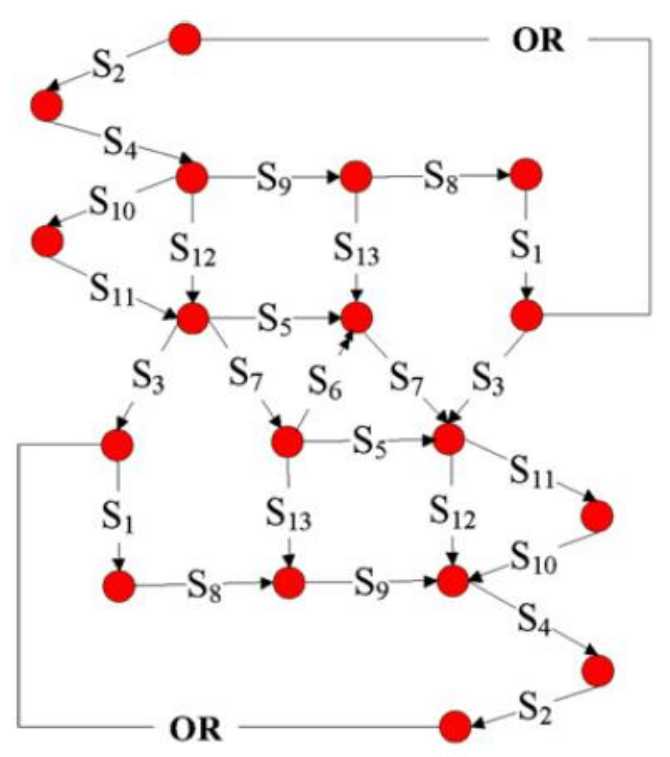

Figure 11 Graphical approach to analyse the hydrogen oxidation mechanism [45]. 
In the reaction route graph for the mechanism, the nodes represent the intermediate states during the reaction progression through a particular path and the directed edges represent the different reaction steps. The OR represents the overall reaction. The graph illustrates the different paths possible to achieve the overall reaction.

$$
H_{2(\text { gas })}+\mathrm{O}_{\mathrm{O} Y S Z}^{\mathrm{x}} \leftrightarrow \mathrm{H}_{2} \mathrm{O}_{\text {(gas) }}+V_{o Y S Z}^{*}+2 e^{-}
$$

The graph is exploited using electrical analogy to identify critical paths and rate limiting steps. Further, expression for the overall reaction rate was derived using quasi steady state assumptions and the graph connections. The rate expression was validated through comparison of polarisation curve with experimental data. This study revealed the dominant pathway to be the one containing the two-hydrogen spill-over reactions. The hydrogen spill-over to oxide ion was identified as the slowest reaction step with the highest resistance governing the rate of the over-all reaction.

\subsubsection{SOFC Electrode Microstructure Modeling}

The SOFC electrodes and electrolyte are the key components where the chemical and electrochemical reactions take place. The microstructure of the electrodes plays a vital role in determining the fuel cell performance. There is ample scope for improving fuel cell performance through optimisation of the cell microstructure. Efforts are underway to devise methods for specific microstructure tailoring [46-49]. Best cell performance can be obtained through a trade-off between electrochemical reactions and species transport processes. Mathematical modeling of the electrode microstructure can offer many benefits for understanding and optimising the microstructure. Our work [50-52] in this area focussed on mathematical modeling of SOFC microstructures.

The analytical models of the electrodes were developed using analytical methods. Principle of coordination numbers and percolation theory formed the basis for these models. In [53], we presented analytical models for the $\mathrm{H}^{+} / \mathrm{e}^{-} / \mathrm{O}^{2-}$ mixed conducting electrodes. The microstructural properties like the triple phase boundaries and the conductivities were presented in nondimensional forms for generalisation and ease of application to different geometries and scales. A recently introduced method for improving fuel cell performance is the preparation of the electrodes through infiltration method [50]. This produces a unique microstructure for the electrodes where the infiltrated element deposits as man-sized particles while the other element forms the backbone (Figure 12). This arrangement of the electrode particles can result in increasing the triple phase areas and thereby resulting in improved performance. In our work [52], we proposed an analytical model for infiltrated electrodes based on percolation theory and coordination principles. The triple phase boundary density of the electrodes is defined in terms of the percolation probabilities, the particle sizes, their volume fractions and porosity. The percolation probabilities were defined based on empirical correlations and were validated with literature data. Several parametric analyses were performed through simulations to facilitate a good understanding of the microstructure. The results were also validated comparing them to the predictions of numerically simulated electrode structures. 


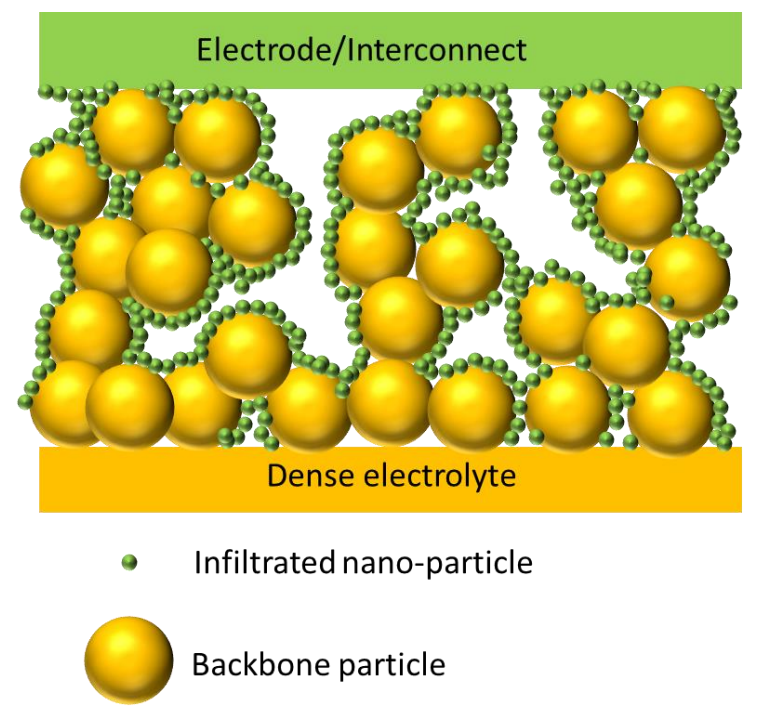

Figure 12 Schematic of an infiltrated electrode with micro-meter sized backbone particles and nanometer sized infiltrated particles [51].

One of the practical uses of microstructural modeling is that it provides an opportunity to study different configurations through simulations with little investment in time and cost. These models can also be incorporated into optimisation routines to search for microstructures that can provide best performance. Since several kinds of microstructures have been introduced for the SOFC electrodes, it is useful to perform optimisation studies on these to perform a comparative analysis. This was the subject of our publication [53], where different microstructure models including the conventional two particle electrodes, two particle infiltrated electrodes, one and two particles based mixed ionic and electronic conducting electrodes, three particle based electrodes and electrodes that change their structure dynamically during operation were all validated using data from literature and comparative optimisation studies were presented. This study resulted in offering insights into the influence of microstructure parameters on microstructural properties. The plots of TPB density surfaces as functions of particle sizes and volume fractions revealed that infiltration modifies the symmetrical behaviour of the triple phase boundary density (exhibited in conventional electrodes) from which we can understand the importance of proper selection of microstructure parameters to achieve best cell performance.

\subsection{Control, Monitoring, Diagnostics and Optimisation}

\subsubsection{SOFC Model Validation}

With wide availability of computing power, model-based design and analysis methods are gaining more and more ground in science and engineering. Mathematical models can be very useful for enhancing understanding and exploring the design space at reduced cost and time. However, the models will have to be rigorously validated with data for providing sufficient confidence in their predictions. This important step of model validation is often neglected resulting in lesser model usability.

SOFC modeling involves representation of several processes that have significant uncertainties as the underlying mechanisms of electrochemical reactions are not fully understood. Model validation is approached from several viewpoints in the literature. Methods applied for model 
validation include classical statistics, Bayesian methods and artificial intelligence methods. In our work [54], we enumerated a classical statistics-based model calibration and validation strategy for a cell level, lumped parameter SOFC model. Our work was concerned with the steady state validation, but the concepts can be applied to other model types. For example, dynamic validation can be approached as a special case with space and time dependent variables. However, data generation at faster sampling times are required to validate dynamic models.

For the model validation strategy presented in our work [54], several quantitative methods like statistical hypothesis testing, sensitivity analysis and design of experiments are employed along with the expert knowledge of the physical and chemical fuel cell process. Two specific types of model errors are identified, viz., model structural errors and model parametric errors. The validation strategy deploys two stages. In the first stage, the model structural errors are identified employing knowledge-based interpretation of model response to change in operating inputs. This stage involves application of quantitative methods like the design of experiments but also depends on qualitative knowledge-based interpretation of results. In the second stage, model parameter calibration is carried out using various quantitative methods to address the parametric errors. Figure 13 illustrates various stages in the proposed validation strategy, where the blue region represents the first stage of validation.

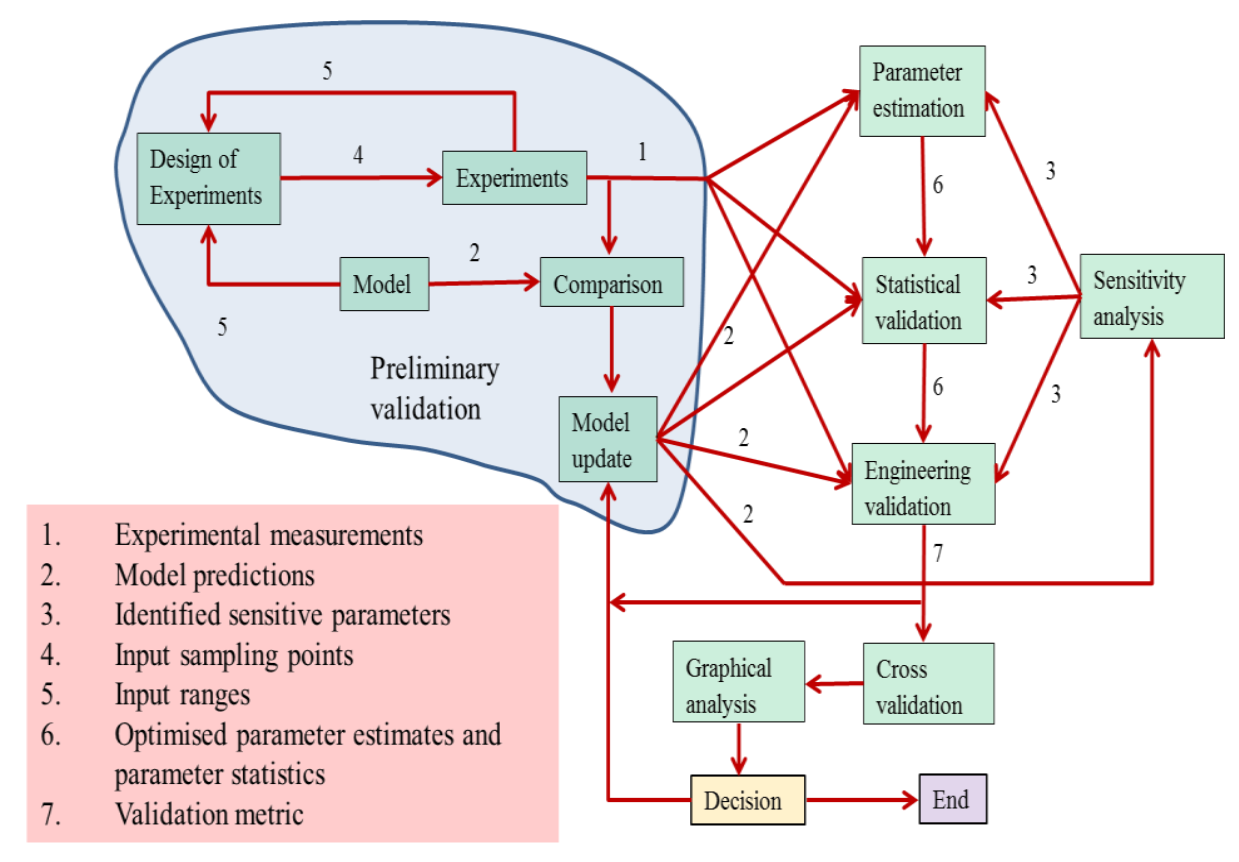

Figure 13 Flowchart for the two-stage model validation [54].

The data for model validation were generated through custom designed experiments on an SOFC test rig in collaboration with Ceramic Fuel Cells Limited. The experiments were aimed at generating data to cover the design space consisting of the SOFC operating range. In order to limit the number of runs, the experiments are designed using Latin hypercube sampling plan with the additional criteria of maximising the minimum distance between the points. This experimental design methodology will help to cover the design space and obtain random samples. The source of voltage error between the measured and the model predicted data is rigorously analysed considering all possible causes as shown in the fish bone diagram in Figure 14. Once the responsible model structural error is identified and amended, the second phase of the validation is performed. 


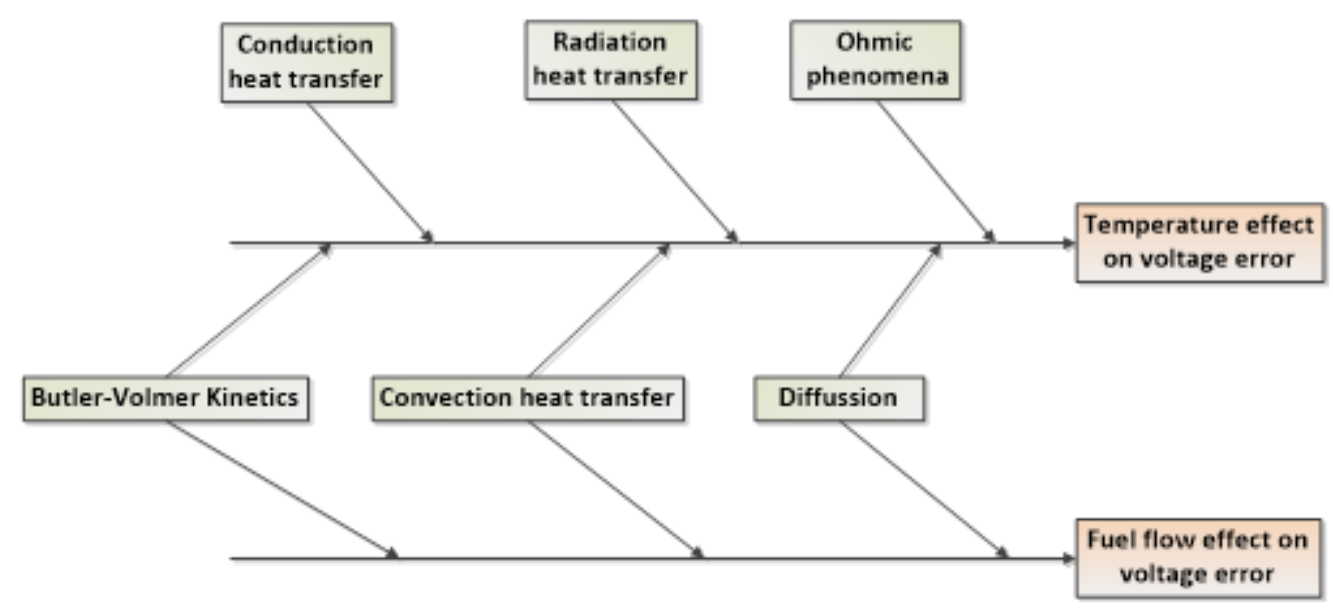

Figure 14 Fishbone diagram for root cause analysis of voltage error [54].

The data for model validation were generated through custom designed experiments on an SOFC test rig in collaboration with Ceramic Fuel Cells Limited. The experiments were aimed at generating data to cover the design space consisting of the SOFC operating range. In order to limit the number of runs, the experiments are designed using Latin hypercube sampling plan with the additional criteria of maximising the minimum distance between the points. This experimental design methodology will help to cover the design space and obtain random samples. The source of voltage error between the measured and the model predicted data is rigorously analysed considering all possible causes as shown in the fish bone diagram in Figure 14. Once the responsible model structural error is identified and amended, the second phase of the validation is performed.

For the second stage of the validation, data on SOFC model parameters are collected and statistically analysed for uncertainties. The electrochemical parameters were found to have the most uncertainty as expected. The elementary effects-based screening of the parameters helped in identifying the most sensitive ones. Model parameter statistics were derived from the data using least squares estimation. The model is then calibrated and validated using hypothesis testing resulting in model acceptance with less $8 \%$ maximum error in predicting the cell current.

\subsubsection{Control Structure Design}

Even the basic operation of the SOFC requires a control system to regulate the flows, fuel utilisation, temperature and voltage. Although sophisticated control schemes are possible, simple PID controllers in separate loops are the simplest, easily implemented and most commonly employed control structures in SOFCs. In such a linear multi-loop control structure design, careful attention is needed in their design due to the possible interaction s between the loops. In addition, SOFCs can be operated in several operating modes depending on their applications.

Depending on the application, three different operating modes with respect to control are identified for the SOFC. They are the a) constant fuel utilisation and temperature operation, b) constant voltage and temperature operation and c) constant temperature and power tracking operation modes. The input variables that can be manipulated include the fuel and air flows and the cell current. With these set of manipulable variables, controlled variables and operation modes, 18 different multi-loop control structures are possible. All these control structures were evaluated through a process interaction analysis for effective control structure design in our work [55]. The 
choice of analysis method was the frequency dependent relative gain array (RGA) to account for the process dynamics. The best control structures were then used to design PID controllers and their control performance was evaluated. Due to the presence of minimum interactions, a model predictive controller designed for these control structures also provided superior performance compared to control structures with large loop interactions. The results of this study provide guidelines for the selection of manipulated and control variables and designing effective control structures for plant-wide control of the SOFC system.

\subsubsection{Monitoring}

SOFC technology has advanced significantly during the last decade. However, it has yet to meet all durability and lifetime requirements that will enable it to be competitive against existing and other competing technologies. There is a lot of scope for improving SOFC durability by solving materials related issues. Yet another avenue for improving the cell durability is through effective operational management by continuous and online monitoring of critical variables so that incipient failures can be detected and rectified in a timely manner.

One of the important quantities that can indicate the health of the SOFC are the thermal profiles inside the cell. Excessive thermal gradients can indicate failures or abnormalities in the cell or other components connected to the cell (for example fuel and air supply, electrical load etc.). Due to several practical difficulties, the cell's internal thermal gradients are not available for in-situ measurement. In this context, online estimation of the internal thermal profiles in the SOFC can be very useful in the overall monitoring and diagnostic scheme.

We had presented a non-linear model based adaptive observer for estimating the internal thermal profiles of the SOFC in [56]. The dynamic model is formulated based on the concepts of tank in series reactor with six lumps to capture the dynamic spatial temperature profiles. Simulations establish good tracking ability of the adaptive observer. Comparisons with a linear Luenberger observer also establish its superior tracking performance. This non-linear observer offers advantages of requiring fewer measured variables, validity over a wide range of operating conditions and robustness to fluctuations in inlet flows.

In a later work [57], the adaptive observer was extended to estimate the inputs in addition to the states as an unknown input observer that simultaneously estimates the states and the inputs of the SOFC. In this case, the cell current and fuel and air inlet temperatures were considered as slowly varying parameters that need to be estimated in addition to the anodic and cathodic inlet flows.

Details of the observer stability proof using non-linear theory can be found in [56]. Simulations established the input and state tracking abilities of the observer. Figure 15 shows the response of the adaptive parameters that are estimated online during a step change in current density. Besides offering the same advantages as the previous adaptive observer, this unknown input observer can robustly handle disturbances to be SOFC input variables effectively and provide accurate estimates of the cell internal thermal profiles. 


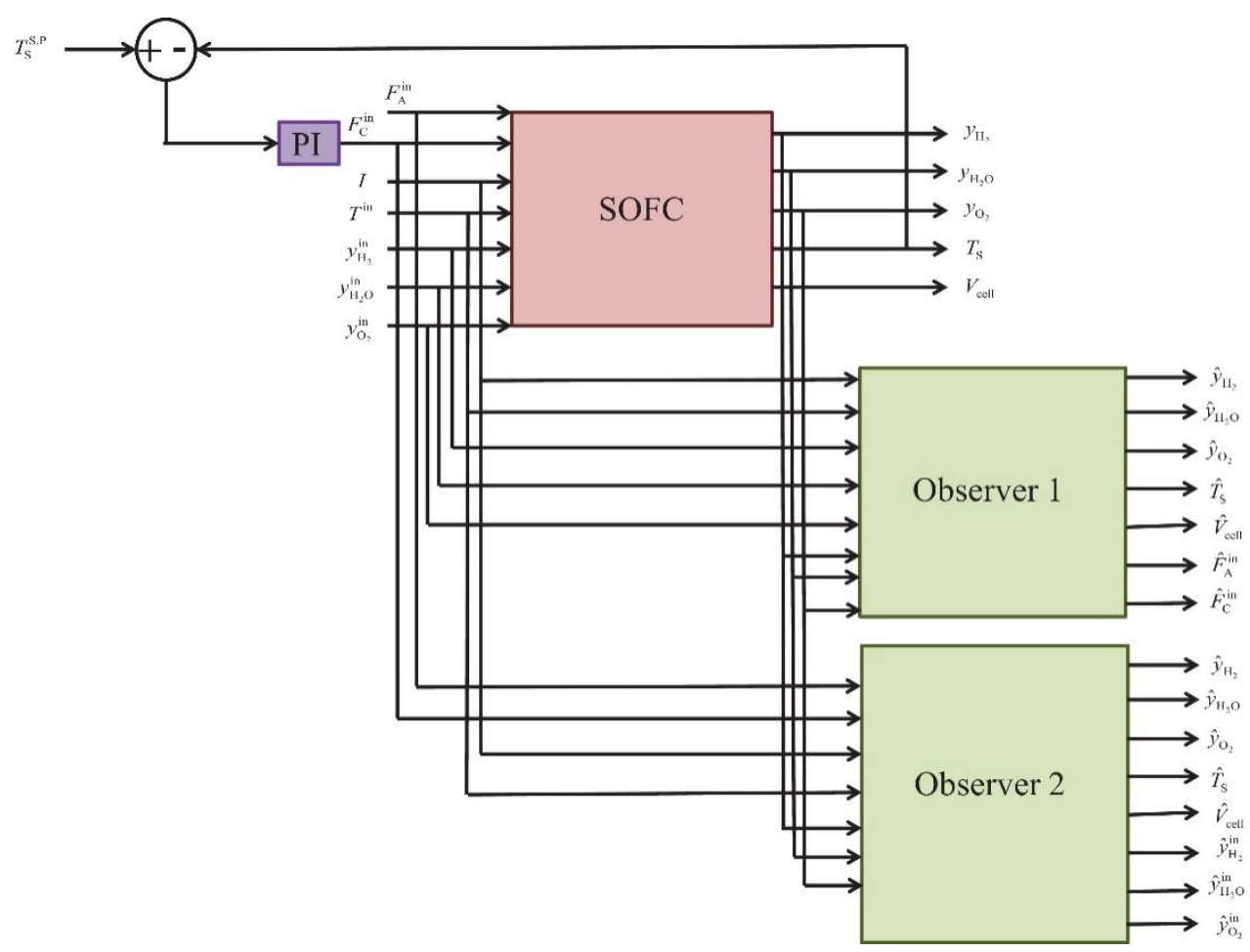

Figure 15 Schematic depiction of the multiple adaptive observer-based fault identification approach [36].

\subsubsection{Fault Diagnosis}

The SOFC is susceptible to a number of faults in the cell that lead to reducing its general reliability. A monitoring system can indicate some abnormal situation arising in the cell. Due to the complex interactions in the fuel cell, identification of the specific root cause of a fault requires a diagnostic system. The diagnostic system should be capable of isolating different causes for the observed symptoms and give an indication of the root cause for action. Due to the complexity of the fuel cell system with many interactions, a model-based approach is well suited. We proposed a multiple observer-based approach to the problem of SOFC diagnosis in [36]. Five common faults affecting the SOFC (given in Table 3) were simulated in this work. In the Table, $\alpha$ is the loss factor, which is a multiplicative variable imposed on the overall irreversible voltage to simulate the fault. Two different adaptive non-linear observers were designed targeting specific faults.

Table 1 Details of the SOFC fault simulation [36].

\begin{tabular}{llll}
\hline Fault name & Description & $\begin{array}{l}\text { Corresponding } \\
\text { variable }\end{array}$ & Fault simulation steps \\
\hline Fault 1 & Fuel leak & $Q_{A}^{\text {in }}\left(\mathrm{mL} \mathrm{min}^{-1}\right)$ & $-50 \mathrm{~mL} \mathrm{~min}^{-1}$ \\
Fault 2 & Air leak & $Q_{C}^{\text {in }}\left(\mathrm{mL} \mathrm{min}^{-1}\right)$ & $-500 \mathrm{~mL} \mathrm{~min}^{-1}$ \\
Fault 3 & Anode inlet composition & $y_{H_{2}}^{\text {in }}$ & -0.1 \\
Fault 4 & Cathode inlet composition & $y_{O_{2}}^{\text {in }}$ & +0.1 \\
Fault 5 & Cell fault & $\alpha$ & +0.2 \\
\hline
\end{tabular}


Detailed stability proofs for these observers can be found in [36]. Following the successful evaluation of the observer performances, they were tested for fault diagnostic application. The schematic of the proposed fault diagnostic approach is shown in Figure15.

A fault diagnosis strategy is formulated based on the simultaneous application of the two adaptive observers. The observer performance, both for designed and out of the design conditions is presented in Table 4. The symbol 'ü' in the table indicates that the observer estimation is same as the measured value resulting in zero residual and the symbol ' $\hat{u}$ ' indicates non-aero residual. The symbol ' $x$ ' means simulation failing due to unrealistic values. Fault diagnosis is based on the ability of the two observers to track the changes in operating conditions which is monitored through the residual. In general, if an observer reports at least one non-zero residual with the other observer correctly estimates all variables; the fault indicated by the second observer is the root cause of the problem. The cell fault (Fault number 5 ) is the root cause if both observers provide at least on nonzero residuals. More details about these and the detailed simulation studies can be found in [36].

Table 2 Observer tracking performance under different fault scenarios[36].

\begin{tabular}{|c|c|c|c|c|c|c|c|c|c|c|c|c|c|}
\hline Faults & Obse & ver 1 & & & & & Obse & ver 2 & & & & & \\
\hline & $y_{H_{2}}$ & $y_{O_{2}}$ & $V_{\text {cell }}$ & $T_{S}$ & $F_{A}^{\text {in }}$ & $F_{C}^{\text {in }}$ & $y_{H_{2}}$ & $y_{O_{2}}$ & $V_{\text {cell }}$ & $T_{S}$ & $y_{H_{2} O}^{\text {in }}$ & $y_{H_{2}}^{\mathrm{in}}$ & $y_{O_{2}}^{\mathrm{in}}$ \\
\hline 1 & ü & ü & ü & $\ddot{u}$ & ü & ü & ü & ü & û & û & $\hat{u}$ & $\hat{u}$ & ü \\
\hline 2 & ü & ü & ü & ü & ü & ü & ü & ü & û & $\ddot{u}$ & ü & ü & û \\
\hline 3 & ü & ü & û & $\hat{u}$ & ü & û & ü & ü & ü & $\ddot{u}$ & $\ddot{u}$ & $\ddot{u}$ & ü \\
\hline 4 & $x$ & $x$ & $x$ & $x$ & $x$ & $x$ & ü & ü & ü & $\ddot{u}$ & ü & $\ddot{u}$ & ü \\
\hline 5 & ü & ü & $\hat{u}$ & $\hat{u}$ & ü & ü & ü & $\ddot{u}$ & û & $\hat{u}$ & ü & ü & ü \\
\hline
\end{tabular}

No human, animal or plant subjects were involved in the any of the studies that comprise this technical report.

\section{Discussion}

SOFC modeling is of fundamental importance in the on-goingdevelopment/improvement and successful mass-scale deployment of SOFC-based combined heat and power systems, auxiliary power units and other SOFC-based systems. Optimisation of system operating conditions requires reliable models at the system scale. This can be done at $0 \mathrm{D}$ or $1 \mathrm{D}$ levels by simulating for different stack performance parameters which will vary over the useful life of the stack.

Understanding stack failure modes and their causes require thermo-mechanical-electrical models of stacks in 3D configuration. Simulation of ageing and degradation caused by impurity poisoning of electrodes and catalysts e.g. for internal reforming, requires detailed kinetic and mechanistic models at the cell level. Modeling for monitoring and control is another area which is of vital importance but is fraught with the challenges of integrating performance variables and their time-based changes in system i.e. BoP-components with those of the myriad of stack components 
- electrodes, electrolyte, contact layers, compliant layers, poisoning guards, interconnects, current collectors and interfaces with BoP components. Incorporating the effects of thermal cycling is an additional challenge.

At the cell and stack level, detailed models identifying the dominant modes of degradation at different stages of life followed by development of appropriate mitigation strategies is a key requirement for the commercial success of the SOFC.

High efficiency SOFC are invariably of the internal reforming type with conversion of methane on the anode and anode chamber via the steam reforming reaction through upgrading the fuel with the heat produced by the electrochemical oxidation of hydrogen [58]. The reforming reaction plays a key role in the performance of the SOFC at all levels - cell performance; stack thermal gradients and stress; and system level steady-state and dynamic performance. Any changes occurring during the operational life of the SOFC system that can have a significant impact on the internal reforming reaction, will consequently not only affect the electrical efficiency, it will also affect stack durability. Therefore, it is important to determine the effect of anode degradation on the kinetics of internal reforming which consequently reduces the efficiency of the system. Ageing and degradation occur from a number of processes which include coarsening of Ni particles due to sintering, particularly at high fuel utilization, and poisoning by contaminants present in the stack materials (e.g. seals) or carried through by the fuel stream over a period of time. Both of which can significantly reduce the kinetic rate of the steam reforming reaction, as known from the petrochemical industry.

The internal reforming kinetics plays a significant role in the cell and stack's temperature, concentration, current density, and voltage profiles during steady and transient operation and consequently, influences the durability of the SOFC stack. Reforming kinetics on an aged anode can be significantly different than on a fresh anode or an early-life anode. There are several SOFC models in the literature for internal reforming SOFCs, which include kinetics of methane reforming derived from empirical correlations, based on data from fresh catalysts. However, there is no reported work in the open literature on reforming on an aged or degraded anode. With changes occurring on the anode during its operating lifetime, and the potential impact on internal reforming kinetics, there is little value in developing detailed models for cell and stack performance. This is also the case for thermal stress modeling of stacks or for developing control algorithms for steady-state and dynamic operation of systems, based on internal reforming kinetics which may not be applicable for anodes that have aged and degraded from prolonged operation under varying operating conditions including thermal cycling.

Degradation also is caused by an increase in ohmic resistance due to formation of dielectric layers between cell and stack components. Cross-leaks and external leaks resulting from seal failure also contribute to degradation. None of these can be avoided completely and can influence the kinetics of the internal reforming reaction.

Mathematical modeling is used as a tool for detecting undesirable gradients in temperature and current density profiles within the cell and for predicting hot-spot locations and high current density points. The fidelity of mathematical modeling of the SOFC system relies largely on the adequate representation of the internal reforming chemistry and kinetics. It is desirable to develop a macromicro kinetic model of the SOFC anode accounting for transport processes and the changing reforming kinetics. The main objective of such modeling work is to create a platform for stack and system level modeling accounting for the influence of anode degradation and ageing on internal 
reforming kinetics with the purpose of creating more reliable models for predicting the performance of real stacks and systems.

The reliability and thus commercial viability of the SOFC technology must be demonstrated at system scale in order to achieve commercial success. Therefore, during the past decade SOFC system level modeling has been increasingly receiving attention by researchers and industries due to its importance in solving the commercialisation relevant challenges. However, in order to make future models more efficient, computational tools for achieving fundamental insights and technical applications need to be target-oriented focusing on the state-of-the-art research gaps as follows:

1) Advances have been made to reduce time-based degradation to a level that has the potential of making the technology commercially viable [59], predominantly through materials science improved protective coatings and scavenger compounds for poisons. However, fuel cell lifetime and reliability is still a barrier to its commercialisation. Practical degradation tests, in particular, at system scale, are complicated, costly and time-consuming. Thus, multiscale models capable of capturing the fuel cell degradation features against dominant factors such as fuel impurities and thermal gradients are in high demand. It is of particular interest to estimate the degradation rate imposed by the balance-of-the-plant components such as the heat exchanger and burner. Due to the lack of knowledge in the interacting mechanisms involved in the SOFC degradation, a comprehensive physics-based model is currently not possible. Utilisation of Artificial Intelligence methods is a prospective solution for this problem given the availability of large data sets that have been generated in large demonstration projects worldwide.

2) Most of the models developed for process control purposes are $O D$ and mostly for the singlevariable-single-objective control design. Smart control design is possible through distributed and dynamic models developed for multi-variable-multi-objective control design projects. This is important as several dominant variables and interacting mechanisms are involved in a SOFC system and its operation that must not be compromised in an optimised control system.

3) The stack design challenges can be addressed by models that capture the stack hydrodynamics as well as the inhomogeneous performance in different layers of the stack. The reduction in efficiency and longevity due to stacking is an important issue in SOFC scale up as it demands multidisciplinary modeling in which chemical, mechanical, and electrical design aspects are taken into account.

It is clear that significant knowledge gaps still exist in understanding the interactions between stack and BoP that lead to substantial performance loss and/or catastrophic stack failures. Detailed analysis of large data sets from field test units are required for developing a thorough understanding of these issues and their impact on stack performance and stack durability [60]. This understanding will be critical in defining optimum control strategies for the system, that ensure reliable and longterm operation of the system. Validated dynamic models are required to address this knowledge gap.

In addition, degradation and sudden stack failures are key areas where more focused investigations are required to arrive at a commercial SOFC product. However, apart from lifetime and system reliability, cost reductions and improved manufacturability are further key requirements for a commercial success of the technology.

However, apart from lifetime and system reliability, further cost reductions, improved manufacturability and reduced installation complexity are key requirements for a commercial success of the technology [61]. 
Materials R\&D is one critical approach to push fuel cells into commercialisation. Material selection holds the key to lifetime and costs. The focus needs to be on materials for improved performance yielding to smaller, more compact fuel cells and more robust SOFC stacks, and replacement of expensive materials for cheaper ones wherever possible. For example, recent progress in lifetime and performance of stainless-steel supported cells operating below $650^{\circ} \mathrm{C}[62$ 63] could represent a step change towards simpler and smaller stack designs and therefore substantial cost reductions. Combinatorial methods combined with fast materials screening techniques are increasingly used in the difficult task to find new materials and improve existing ones. Materials used in fuel cells commonly have to satisfy multiple functionalities and the mathematical methods are ideally suited for defining the path to performance improvements.

Numerous detailed studies on cost reduction drivers of SOFC systems have been carried out, for example the US Department of Energy Study [64].

Reduced complexity of the integrated products (part count reduction) and volume manufacturing with high yield and uniform high quality are key in the strategy to arrive at commercially viable fuel cells. Like with any other commercial technology, product manufacturing volume with high yield significantly influences product costs, and uniform high-quality components are necessary for reliability and life. This is of critical importance for fuel cells, which are largely serial devices, where one low performing component determines the performance of the fuel cell stack. Fuel cells designed for manufacture, automated streamlined manufacturing processes, efficient supply chains, and use of common components will be key goals for achieving commercial success. Extensive experience with Technology Learning Curves for other technology products gives us excellent guidelines for extrapolation of installed costs of SOFC systems with increased volume, and according to the US Department of Energy study, SOFC systems have an excellent change to achieve commercial targets.

\section{Conclusions}

Over the past 15 years our team has engaged in research and development activities in many of the areas discussed above, a summary of which is provided in this technical report. Our work also highlighted the areas where more focussed work is required.

The authors hope that this technical report will be a useful complimentary source for initiating further work in SOFC research as well as provide an exposé of the contributions of our group to this area over the past 15 years.

Notations

\begin{tabular}{cl}
$\begin{array}{c}e^{-} \\
\mathrm{E} \text { and } \mathrm{V}\end{array}$ & Electron \\
& Voltage $(\mathrm{V})$ \\
$F$ & Mole flow rate $\left(\mathrm{mol} \mathrm{s}^{-1}\right)$ (appears in table 2, but it is the same symbol as for Faraday's \\
& constant) \\
\hline $\mathrm{G}$ & Faraday's constant $\left(96485 \mathrm{C} \mathrm{mol}^{-1}\right)$ \\
$i$ & Gibb's free energy $\left(\mathrm{J} \mathrm{mol}^{-1}\right)$ \\
$i_{0}$ & Current density $\left(\mathrm{A} \mathrm{m}^{-2}\right)$ \\
& Exchange current density $\left(\mathrm{A} \mathrm{m}^{-2}\right)$
\end{tabular}


JEPT 2021; 3(1), doi:10.21926/jept.2101004

$\begin{array}{ll}\mathrm{J} & \text { Reaction flux }\left(\mathrm{mol} \mathrm{min}^{-1}\right) \\ k & \text { Reaction rate constant } \\ Q & \text { Volume flow rate }\left(\mathrm{mL} \mathrm{min}^{-1}\right) \\ R & \text { Ideal gas constant }\left(\mathrm{J} \mathrm{mol}^{-1} \mathrm{~K}^{-1}\right) \\ T & \text { Temperature }(\mathrm{K}) \\ y & \text { Species mole fraction }\end{array}$

Greek letters
$\alpha \quad$ Charge transfer coefficient
$\eta \quad$ Overpotential (V)
$\omega \quad$ Weight factor

Abbreviations

$\begin{array}{ll}\text { ACM } & \text { Aspen Custom Modeler } \\ \text { ADL } & \text { Anode Diffusion layer } \\ \text { BOP } & \text { Balance of Plant } \\ \text { BV } & \text { Butler-Volmer } \\ \text { FU } & \text { Fuel Utilisation } \\ \text { MSR } & \text { Methane Steam Reforming } \\ \text { PEN } & \text { Positive electrode-Electrolyte-Negative electrode } \\ \text { RMTN } & \text { Reverse Methanation } \\ \text { SOFC } & \text { Solid Oxide Fuel Cells } \\ \text { TSR } & \text { Tank in Series Reactor } \\ \text { WGS } & \text { Water-gas Shift }\end{array}$

\section{Acknowledgments}

The authors acknowledge the contribution of staff at Curtin University, Ceramic Fuel Cells Ltd and Aston University that have technically supported this work.

\section{Author Contributions}

The contribution of each author is in the order of author names on page 1.

\section{Competing Interests}

The authors have declared that no competing interests exist.

\section{References}

1. Payne R, Love J, Kah M. Generating electricity at $60 \%$ electrical efficiency from 1-2 kWe SOFC products. ECS Trans. 2009; 25: 231. 
2. Powell M, Meinhardt K, Sprenkle V, Chick L, McVay G. Demonstration of a highly efficient solid oxide fuel cell power system using adiabatic steam reforming and anode gas recirculation. J Power Sources. 2012; 205: 377-384.

3. Langnickel H, Rautanen M, Gandiglio M, Santarelli M, Hakala T, Acri M, et al. Efficiency analysis of 50 kWe SOFC systems fueled with biogas from waste-water. J Power Sources Adv. 2020; 2: 100009.

4. Lanzini A, Ferrero D, Papurello D, Santarelli M. Reporting degradation from different fuel contaminants in Ni-anode SOFCs. Fuel Cells. 2017; 17: 423-433.

5. Papurello $D$, Lanzini $A$, Leone $P$, Santarelli $M$, Silvestri S. Biogas from the organic fraction of municipal solid waste: Dealing with contaminants for a solid oxide fuel cell energy generator. Waste Manag. 2014; 34: 2047-2056.

6. Papurello D, Chiodo V, Maisano S, Lanzini A, Santarelli M. Catalytic stability of a Ni-Catalyst towards biogas reforming in the presence of deactivating trace compounds. Renew Energ. 2018; 127: 481-494.

7. Inac S, Unverdi SO, Midilli A. A parametric study on thermodynamic performance of a SOFC oriented hybrid energy system. Int J Hydrog Energy. 2019; 44: 10043-10058.

8. Barrett S. Wärtsilä SOFC using landfill gas passes first phase field trial. Fuel Cells Bull. 2010; 2010: 5-6.

9. Hosseini S, Ahmed K, Tadé MO. CFD model of a methane-fuelled single cell SOFC stack for analysing the combined effects of macro/micro structural parameters. J Power Sources. 2013; 234: 180-196.

10. Ahmed K, Foger K. Kinetics of internal steam reforming of methane on Ni/YSZ-based anodes for solid oxide fuel cells. Catal Today. 2000; 63: 479-487.

11. Hosseini S, Danilov VA, Vijay P, Tadé MO. Improved tank in series model for the planar solid oxide fuel cell. Ind Eng Chem Res. 2011; 50: 1056-1069.

12. Ravussin F, Van herle J, Autissier N, Molinelli M, Larrain D, Favrat D. Local current measurement in a solid oxide fuel cell repeat element. J Eur Ceram Soc. 2007; 27: 1035-1040.

13. Danilov VA, Vijay P, Tadé MO. Improved tank in series reactor model for tubular solid oxide fuel cell stacks. Chem Eng Technol. 2011; 34: 737-745.

14. Sundén B, Faghri M. Transport Phenomena in fuel cells (Development in heat transfer). Boston: WIT Press; 2005.

15. Bove R, Ubertini S. Modeling solid oxide fuel cells. Berlin: Springer; 2008. p.207-p.239.

16. Inui $\mathrm{Y}$, Ito $\mathrm{N}$, Nakajima $\mathrm{T}$, Urata A. Analytical investigation on cell temperature control method of planar solid oxide fuel cell. Energy Convers Manag. 2006; 47: 2319-2328.

17. Fardadi M, Mueller F, Jabbari F. Feedback control of solid oxide fuel cell spatial temperature variation. J Power Sources. 2010; 195: 4222-4233.

18. Hosseini S, Vijay P, Ahmed K, Tadé MO, Pareek V, Utikar R. Dynamic tank in series modeling of direct internal reforming SOFC. Int J Energy Res. 2017; 41: 1563-1578.

19. Vijay $P$, Hosseini $S$, Tadé MO. A novel concept for improved thermal management of the planar SOFC. Chem Eng Res Des. 2013; 91: 560-572.

20. Tang S, Amiri A, Vijay $P$, Tadé MO. Development and validation of a computationally efficient pseudo 3D model for planar SOFC integrated with a heating furnace. Chem Eng J. 2016; 290: 252-262. 
21. Amiri A, Tang S, Vijay $P$, Tadé MO. Planar solid oxide fuel cell modeling and optimization targeting the stack's temperature gradient minimization. Ind Eng Chem Res. 2016; 55: 74467455.

22. Ahmed K, Föger K. Analysis of equilibrium and kinetic models of internal reforming on solid oxide fuel cell anodes: Effect on voltage, current and temperature distribution. J Power Sources. 2017; 343: 83-93.

23. Xu J, Froment GF. Methane steam reforming, methanation and water-gas shift: I. Intrinsic kinetics. AIChE J. 1989; 35: 88-96.

24. Achenbach E, Riensche E. Methane/steam reforming kinetics for solid oxide fuel cells. J Power Sources. 1994; 52: 283-288.

25. Tingey GL. Kinetics of the water-Gas equilibrium reaction. I. The reaction of carbon dioxide with hydrogen. J Phys Chem. 1966; 70: 1406-1412.

26. Anderson T, Vijay P, Tade MO. An adaptable steady state Aspen Hysys model for the methane fuelled solid oxide fuel cell. Chem Eng Res Des. 2014; 92: 295-307.

27. Ahmed K, Amiri AO. Tadé M. Simulation of solid oxide fuel cell anode in aspen HYSYS-A study on the effect of reforming activity on distributed performance profiles, carbon formation, and anode oxidation risk. Processes. 2020; 8: 268.

28. Amiri A, Vijay $P$, Tadé MO, Ahmed $K$, Ingram GD, Pareek V, et al. Solid oxide fuel cell reactor analysis and optimisation through a novel multi-scale modeling strategy. Comput Chem Eng. 2015; 78: 10-23.

29. Amiri A, Vijay P, Tadé MO, Ahmed K, Ingram GD, Pareek V, et al. Planar SOFC system modeling and simulation including a 3D stack module. Int J Hydrog Energy. 2016; 41: 2919-2930.

30. Tang, S, Amiri A, Tadé MO. System level exergy assessment of strategies deployed for solid oxide fuel cell stack temperature regulation and thermal gradient reduction. Ind Eng Chem Res. 2019; 58: 2258-226

31. Amiri A., Tang S, Steinberger-Wilckens R, Tadé MO. Evaluation of fuel diversity in solid oxide fuel cell system. Int J Hydrog Energy. 2018; 43: 23475-87.

32. Powell M, Meinhardt K, Sprenkle V, Chick L, Mcvay G. Demonstration of a highly efficient solid oxide fuel cell power system using adiabatic steam reforming and anode gas recirculation. J Power Sources. 2012; 205: 377-384.

33. Amiri A, Ahmed K, Tadé MO. A steady-state and dynamic simulation tool for solid oxide fuel cell operation applications. Comput Aided Chem Eng. 2019; 46: 595-600.

34. Sharma AK, Ahmed K, Birgersson E. Nernst voltage losses in planar fuel cells caused by changes in chemical composition: Effects of operating parameters. Ionics 2018; 24: 2047-2054.

35. Huang B, Qi Y, Murshed M. Solid oxide fuel cell: Perspective of dynamic modeling and control. J Proc Control. 2011; 21: 1426-1437.

36. Vijay $P$, Tadé MO, Shao Z. Adaptive observer based approach for the fault diagnosis in solid oxide fuel cells. J. Process Control. 2019; 84: 101-114.

37. Noren DA, Hoffman MA. Clarifying the Butler-Volmer equation and related approximations for calculating activation losses in solid oxide fuel cell models. J Power Sources. 2005; 152: 175-181.

38. Mann RF, Amphlett JC, Peppley BA, Thurgood CP. Application of Butler-Volmer equations in the modeling of activation polarization for PEM fuel cells. J Power Sources. 2006; 161: 775-781.

39. Vijay P, Tadé MO. Improved approximation for the Butler-Volmer equation in fuel cell modeling. Comput Chem Eng. 2017; 102: 2-10. 
40. Bessler WG. A new computational approach for SOFC impedance from detailed electrochemical reaction-diffusion models. Solid State Ion. 2005; 176: 997-1011.

41. Vijay $P$, Samantaray AK, Mukherjee A. Development of a thermodynamically consistent kinetic model for reactions in the solid oxide fuel cell. Comput Chem Eng. 2010; 34: 866-877.

42. Ederer M, Gilles ED. Thermodynamically feasible kinetic models of reaction networks. Biophys J. 2007; 92: 1846-1857.

43. Vijay $P$, Tadé MO, Datta R. Insights into the applicability of the R dot approach for reaction mechanism kinetics studies. Chem Eng Sci. 2012; 69: 616-627.

44. Vijay P, Tadé MO, Fishtik I, Datta R. A graph theoretical approach to the elucidation of reaction mechanisms: Analysis of the chlorine electrode reaction. Comput Chem Eng. 2013; 49: 85-94.

45. Dar YR, Vijay $P$, Tadé MO, Datta R. Topological analysis of hydrogen oxidation reaction kinetics at $\mathrm{Ni} / \mathrm{YSZ}$ anode of the solid oxide fuel cell. J Electroanal Chem. 2012; 677-680: 15-23.

46. Connor PA, Yue X, Savaniu CD, Price R, Triantafyllou G, Cassidy M, et al. Tailoring SOFC electrode microstructures for improved performance. Adv Energy Mater. 2018; 8: 1800120.

47. Zhang Y, Sun Q, Xia C, Ni M. Geometric properties of nanostructured solid oxide fuel cell electrodes. J Electrochem Soc. 2013; 160: F278.

48. Ruiz-Morales JC, Canales-Vázquez J, Peña-Martínez J, Marrero-López D, Irvine JT, Núñez P. Microstructural optimisation of materials for SOFC applications using PMMA microspheres. J Mater Chem. 2006; 16: 540-542.

49. Hanifi AR, Laguna-Bercero MA, Sandhu NK, Etsell TH, Sarkar P. Tailoring the microstructure of a solid oxide fuel cell anode support by calcination and milling of YSZ. Sci Rep. 2016; 6: 27359.

50. Jiang SP. Nanoscale and nano-structured electrodes of solid oxide fuel cells by infiltration: Advances and challenges. Int J Hydrog Energy. 2012; 37: 449-470.

51. Vijay $P$, Tadé MO, Shao Z, Ni M. Modeling the triple phase boundary length in infiltrated SOFC electrodes. Int J Hydrog Energy. 2017; 42: 28836-28851.

52. Vijay $P$, Tadé $M O$, Shao Z. Model based evaluation of the electrochemical reaction sites in solid oxide fuel cell electrodes. Int J Hydrog Energy. 2019; 44: 8439-8459.

53. Chen D, Zhang Q, Lu L, Periasamy V, Tade MO, Shao Z. Multi scale and physics models for intermediate and low temperatures $\mathrm{H}^{+}$-solid oxide fuel cells with $\mathrm{H}^{+} / \mathrm{e}^{-} / \mathrm{O}^{2-}$ mixed conducting properties: Part A, generalized percolation theory for LSCF-SDC-BZCY 3-component cathodes. J Power Sources. 2016; 303: 305-316.

54. Vijay P, Tadé MO, Shao Z. Statistical method-based calibration and validation of a solid oxide fuel cell model. Int J Energy Res. 2019; 43: 2478-2500.

55. Vijay $P$, Tadé MO, Datta R. Effect of the operating strategy of a solid oxide fuel cell on the effectiveness of decentralized linear controllers. Ind Eng Chem Res. 2011; 50: 1439-1452.

56. Vijay $P$, Tadé MO. An adaptive non-linear observer for the estimation of temperature distribution in the planar solid oxide fuel cell. J Process Control. 2013; 23: 429-443.

57. Vijay P, Tadé MO, Ahmed K, Utikar R, Pareek V. Simultaneous estimation of states and inputs in a planar solid oxide fuel cell using nonlinear adaptive observer design. J Power Sources. 2014; 248: 1218-1233.

58. Ahmed K, Föger K. Fuel processing for high -temperature high-efficiency fuel cells. Ind Eng Chem Res. 2010; 49: 7239-7256. 
59. Ceramic fuel cells limited technology update: Substantial reduction of stack degradation rates achieved. Melbourne: Ceramic Fuel Cells Ltd; 2014. Available from: http://www.asx.com.au/asxpdf/20140630/pdf/42qi2zq90rbhxt.pdf.

60. Ahmed K, Föger K. Perspectives in solid oxide fuel cell based micro-combined heat and power systems. J Electrochem Energy Convers Storage. 2017; 14: 031005.

61. Föger K. Materials Basics for Fuel Cells. New York: Woodhead; 2009.p.6-p.64.

62. Udomsilp D, Rechberger J, Neubauer R, Bischof C, Thaler F, Schafbauer W, et al. MetalSupported solid oxide fuel cells with exceptionally high power density for range extender systems. Cell Rep Phys Sci. 2020; 1: 100072.

63. Leah R, Bone A, Lankin M, Selcuk A, Pierce R, Rees L, et al. Low cost, REDOX-stable, lowtemperature SOFC developed by ceres power for multiple applications: Latest development update. ECS Trans.2013; 57: 461.

64. James BD, DeSantis DA. Manufacturing costs and installed price analysis of stationary fuel cell systems.Arlington,VA: Strategic Analysis Inc; 2015.

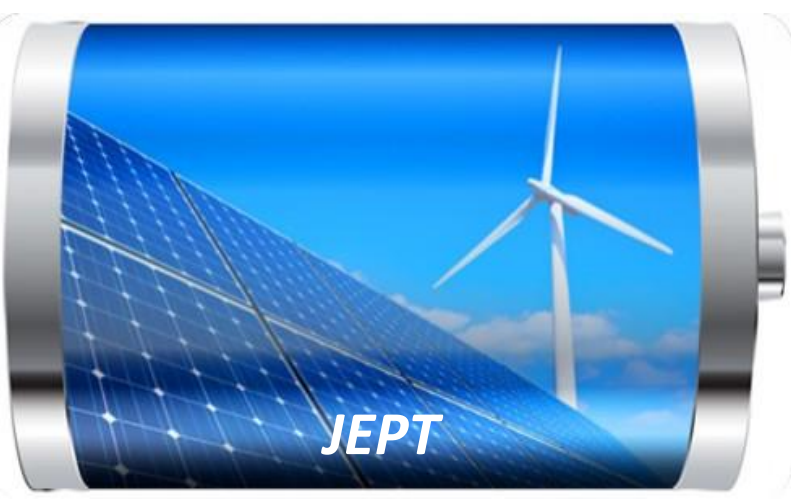

Enjoy JEPT by:

1. Submitting a manuscript

2. Joining in volunteer reviewer bank

3. Joining Editorial Board

4. Guest editing a special issue

For more details, please visit:

http://www.lidsen.com/journal/jept 
Technical Note

\title{
Spatio-Temporal Metocean Measurements for Offshore Wind Power
}

Lucy Wyatt *

School of Mathematics and Statistics, University of Sheffield, Sheffield, UK and Seaview Sensing Ltd;

E-Mail: l.wyatt@sheffield.ac.uk

* Correspondence: Lucy Wyatt; E-Mail: I.wyatt@sheffield.ac.uk

Academic Editor: Andrés Elías Feijóo Lorenzo

Special Issue: Offshore Wind Farms

Journal of Energy and Power Technology

2021, volume 3 , issue 1

doi:10.21926/jept.2101005
Received: November 24, 2020

Accepted: January 19, 2021

Published: January 22, 2021

\begin{abstract}
This paper presents the case for the use of HF radar to provide wave, current and wind measurements and maps during the surveying, resource and impact assessment, construction, maintenance and performance assessment phases of an offshore fixed or floating wind farm or other marine renewable project. The data presented are from the Celtic Sea which is currently the focus of a lot of offshore wind interest. The data are from a dual radar system deployed at the Wave Hub site off the Cornwall coast since 2011, a single radar system in North Devon reactivated in 2017, and data from 2005 when the North Devon radar was operated as a dual radar with a second site in South Wales. The accuracy of the wave and wind data is discussed and examples of the sort of data that could be available are presented.
\end{abstract}

\section{Keywords}

HF radar; metocean measurements; offshore wind; resource assessment; monitoring

\section{Introduction}

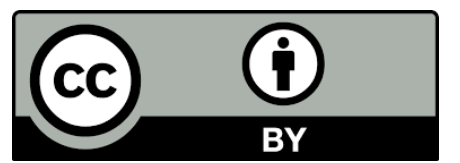

(C) 2021 by the author. This is an open access article distributed under the conditions of the Creative Commons by Attribution License, which permits unrestricted use, distribution, and reproduction in any medium or format, provided the original work is correctly cited. 
The UK is the world's largest generator of electricity from offshore wind. Surveying, resource and impact assessment, construction, maintenance and performance assessment all require information about winds, waves and currents in the region of interest. For example:

- $\quad$ Resource and metocean assessment is carried out to provide atmospheric and oceanographic datasets to inform the engineering design of a wind farm, the potential future energy production, and to fully describe the likely operating conditions at the proposed wind farm location" [1].

- $\quad$ "better weather monitoring together with the availability of vessels with lower metocean limitations can help to reduce weather downtime" [2].

- The cost benefit will also differ between concepts, largely due to the met-ocean limitations and conditions in which the structures can be towed back to shore and then back to site"[2].

Short-term forecasting is needed as discussed in [3] from which the following are extracted.

- $\quad$ "An accurate short-term prediction (few hours - one day) of the power output is needed both to bid at energy auctions (where energy companies sell power) and to plan maintenance of the energy harvesters. The power output is due to a combination of the weather forecast and the control system of the farm. It requires advanced integrated modelling of the whole weather-farm system."

- $\quad$ "Accurate forecasting of site met conditions and consequent plans and decisions about work at site are typically based on a small number of in site devices coupled with algorithms to extrapolate across the site. With a reduction in cost, many more sensors could be deployed leading to much better information about the conditions at site (e.g. wind speed, wind direction, wave height, wave period etc.)."

Accurate forecasts require accurate measurements and, as emphasised in the above quote, accurate forecasts in coastal seas require measurements that reflect the spatial and temporal variability of these seas due to local topography, orography and bathymetry. The spatial picture is important for optimal siting of devices, for assessing safe passage from coast to site and also for impact assessment which isn't necessarily limited to the immediate vicinity of wind farms. This can be achieved using multiple in situ devices or using land- or satellite- based remote sensing. This paper is aimed at presenting the advantages of land-based remote sensing using HF radar systems, see section 3, but first in section 2 the other options are briefly reviewed.

\section{Technology Options}

\subsection{Wind}

The wind is of course the primary resource for offshore wind power and accurate measurements are required to assess the resource and monitor performance. Wind measurement for offshore wind applications have traditionally been made using tower based anemometers. In recent years these are being replaced by lidars on platforms or metocean buoys which have been shown to provide measurements of similar accuracy and are much cheaper [1]. These devices can make measurements at the height of a proposed turbine and therefore provide reliable estimates of resource at that location. The Carbon Trust [4] requires a linear regression slope of 0.98-1.02 and $\mathrm{R}^{2}>$ 0:98 for wind speed measured with LiDAR compared with mast data.

Satellite scatterometer measurements are available to provide global and regional maps of wind climate and estimated wind power resource [5-7]. The accuracy requirements (compared with buoy 
measurements) for operational satellite scatterometer wind speeds are bias $<0.5 \mathrm{~m} / \mathrm{s}$ and standard deviation $<2 \mathrm{~m} / \mathrm{s}$ on a monthly basis [8]. The latest results [9] show biases of $0.2-0.3 \mathrm{~m} / \mathrm{s}$ and standard deviations of 1.2-2.2 m/s. These measurements are not at mast height, modelling of the vertical profile is required to extrapolate; are not as accurate as LIDAR; are not available continuously at a specific location but do provide a spatial picture of wind fields when the satellite is crossing the region of interest.

\subsection{Wave}

In the context of offshore wind, wave measurements are needed to inform the location and design of structures, both platform-based and floating, and cables and to ensure safe conditions for installation, maintenance and decommissioning operations. Wave buoys, in particular the Datawell directional waverider, are considered to be the industry standard against which all other measurements are assessed. While individual buoys are relatively cheap [1], they require suitable weather windows and ship costs to deploy and maintain and can lose their moorings especially in busy shipping and fishing areas. Multiple buoys to provide more detailed information soon increases the cost of course.

Microwave radar systems, of navigation radar [10] or altimeter [11] type, mounted on platforms are also an option for wave measurement. These can measure wave parameters such as significant waveheight, peak period and direction, as well as the directional spectrum. Like buoys these are point measurements and multiple systems would be required to get a more complete spatial picture.

Waves can be measured from satellite using altimeters [12] and synthetic aperture radars (SAR) [13] (note that these papers also refer to wind speed measurements). Altimeters only measure limited non-directional wave parameters, significant waveheight and period; SARs measure the directional spectrum of long waves. Coverage from both are of course limited by their orbits.

\subsection{Current}

Current measurements need to be accounted for similar reasons to those referred to in section 2.2 and also because wind farms may have an impact on the local dynamics which can have environmental impacts in the immediate vicinity and downstream. Standard systems for current measurement are current meters mounted below buoys and ADCPs (acoustic doppler current meters) either mounted on buoys or at the sea bed. Microwave radars can also provide local surface current measurements [10].

Satellites can measure large scale geostrophic currents using altimeters [14]. More detailed measurements of currents in coastal areas are being developed using interferometric SAR techniques [15] but these are not yet operational products.

\subsection{The Alternative}

$\mathrm{HF}$ radar located on the coast is the only technology that can provide continuous monitoring of waves, winds and currents in coastal waters on a spatial scale (from the coast to over $120 \mathrm{~km}$ offshore) suitable for offshore wind. HF radars are now routinely used for surface current measurement in many parts of the globe with networks around most of the coast of the USA and 
other countries aiming to achieve this [16-19]. In the next section this technology is reviewed and examples of available data are presented.

\section{HF radar}

\subsection{Theory and Methods}

Metocean measurements are obtained from the radar signal scattered from ocean waves. The motion of the waves, i.e their intrinsic phase speed and any underlying current, impose a Doppler shift on the received signal and the amplitude of the signal is related to the energy in the wave field which in turn is linked to the local wind plus any swell systems. The Doppler shift can be measured very accurately from what is usually referred to as the first order return in the power spectrum of the received signal which is due to waves with half the radio wavelength propagating towards (producing a positive Doppler shift) and away from (negative) the radar. The current measurement is obtained from the frequency asymmetry of these two signals since, for example, a current propagating towards the radar will impose a positive Doppler shift in both cases [20]. The two waves generating the first order signals are normally driven by the local wind and their relative amplitudes, together with a wind wave directional model, allow wind direction to be measured [21-23].

Wave measurements are obtained from the so-called second-order part of the power spectrum following the work of Barrick [24, 25]. A number of methods have been developed to solve the Barrick equations. In this paper the method of Wyatt and colleagues [21, 26, 27], is used. This method obtains the ocean wave directional spectrum up to a maximum frequency which depends on radar operating frequency and includes both swell and wind waves unless the wind speed is very low. Wave parameters such as significant wave height, peak and mean period and direction are determined from the spectrum using standard methods. The method has been tested on hundreds of hours of data from many different sites in the UK, Europe, USA, Australia, Canada, Taiwan and on different radar systems establishing that where radar data quality is good, good quality wave measurements are obtained [28].

Wind speed is a more difficult parameter to extract from the HF radar signal. The first order peaks are generated by the local wind but the wind wave spectrum saturates as wind speed increases. The full directional spectrum is contributing to the signal amplitude so could include waves that are not generated by the local wind. A number of potential methods have been suggested which look for regions of the radar power spectrum that are more likely to be locally wind related [29-31], relate wind to the detided current [32] or which assume the impact of swell on the measured wave parameter (or the features from which they are derived) is small so relationships can be found using wave model [33] or neural network [34] methods. The Dexter and Theodorides method [33] is currently implemented in the Seaview Sensing Ltd (SV) software package from which wave, current and wind measurements are presented in Section 3.2. The wind speed results are not sufficiently robust and this aspect of the package is not normally promoted. Research into the use of machine learning methods is underway with encouraging early results (see Section 3.2.3). Another approach currently being investigated involves partitioning the directional spectrum into swell and wind sea fields [35] and applying the modeling approach to the wind-sea partition alone.

Note that at least two radar systems are needed to make all of these measurements, unless other information from models or other measurements is available, in order to resolve direction and amplitude ambiguities inherent in the scattering process [28]. 


\subsection{Data and Validations}

\subsubsection{The Celtic Sea}

The Celtic Sea between Ireland and the south-west of Great Britain is one area around the UK being considered for offshore wind exploitation and in particular floating wind [36, 37]. The exploitation of wave and current power is also being considered in this region [37]. It is also an area where the original UK work on ground-based HF radar was carried out [38] and is currently the only site in the UK where there are HF radar installations: a Pisces radar [39] is located in North Devon and operated by Neptune Radar Ltd and two WERAs [40] are located in North Cornwall operated by the University of Plymouth (UoP) (see Figure 1). The Seaview Sensing software metocean measurement package is used with both these installations. The wave and wind direction measurements have been validated in a number of different deployments [22, 23, 41-45], as well as at these sites $[46,47]$. This paper will show results from the Celtic Sea.

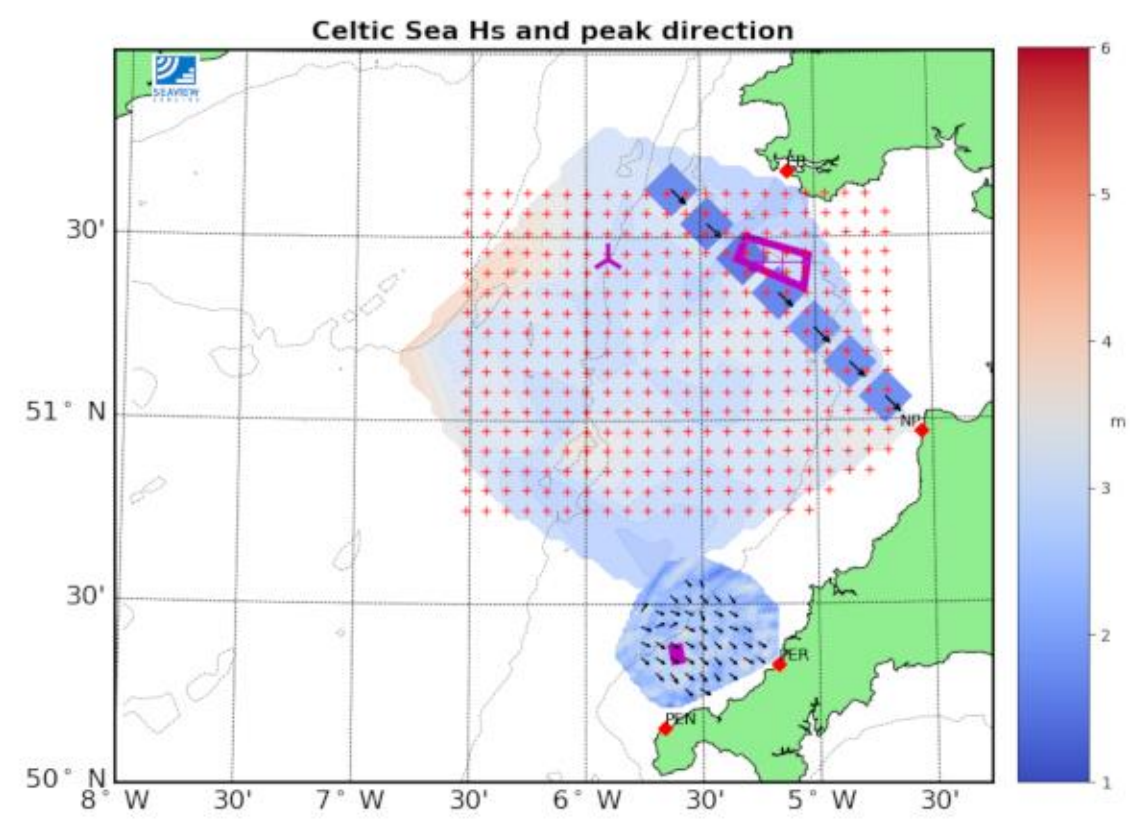

Figure 1 Map of the Celtic Sea showing radar coverage and offshore wind and wave power sites. Radar sites are shown with red diamonds. Sample measured wave maps are shown with the colour-coding scale on the right. + mark positions of a potential measurement grid for future work. Sites earmarked for offshore wind and/or wave power exploitation are shown with magenta outlines (Pembrokeshire and Cornwall Wave Hub) and the symbol $入$ (Erebus). See text for more details.

The measurements in Wyatt et al [46] were made using the North Devon Pisces together with a second Pisces which was located on the South Wales coast. The second site was removed after the operational trial was completed in 2005. From 2017, the Devon site was put back into operation to test a new version of Pisces capable of operating simultaneously on two different frequencies which would provide more robust measurements over a wider range of wave conditions and, it was hoped, would provide the additional information needed to resolve the ambiguities in single radar wave measurement. The results showed promise [39] but more work was needed before a full trial of the 
system and this work is ongoing. The aim is to install a second site for a thorough test of the new system. Such a test would also provide operational data using already proven methods.

The UoP radars were installed in 2011 with the aim of providing wave measurements to support the Wave Hub site being developed for wave power device testing [37]. This system has been operating continuously since that time, has been used for wave validations [47] over extensive periods, for testing new methods [48], and has recently begun supplying data to the EU HFR Node for inclusion in CMEMS-INSTAC.

A map of the radar sites in the Celtic Sea is shown in Figure 1. The UoP radar sites are at Pendeen (PEN) and Perranporth (PER). Pisces is located at Nabor Point (NP) and the S. Wales site used for the operational trial is shown with EB. A sample UoP radar waveheight/peak direction measurement is shown with directions sub-sampled for clarity. The wind was blowing towards the radar but there was also some swell from the west. The shaded region under the red grid shows a waveheight map from the Pisces operational trial using single radar data from both sites to show maximum coverage. A sample of the more recent single radar dual-frequency measurements for a case when winds were blowing towards the NP radar is shown. Measurements were made along a single radar beam. These are not at the same time as the UoP measurements. The red + mark positions of a potential grid for future testing of this system with just one radar at NP and possibly with a second radar in South Wales. Sites that have been earmarked for initial offshore wind and/or wave power exploitation are marked with magenta outlines and the symbol $入$.

\subsubsection{Validations}

Figure 2 shows a significant waveheight comparison for three months of the Pisces deployment. Unfortunately the buoy had lost its mooring in Dec 2004 and was not recovered and replaced until after the stormy period in early January. The radar storm waveheights are consistent with wave model data for this period. Figure 3 shows significant waveheight, energy period and peak period and direction measured with the UoP WERA system during a one month period in 2012.

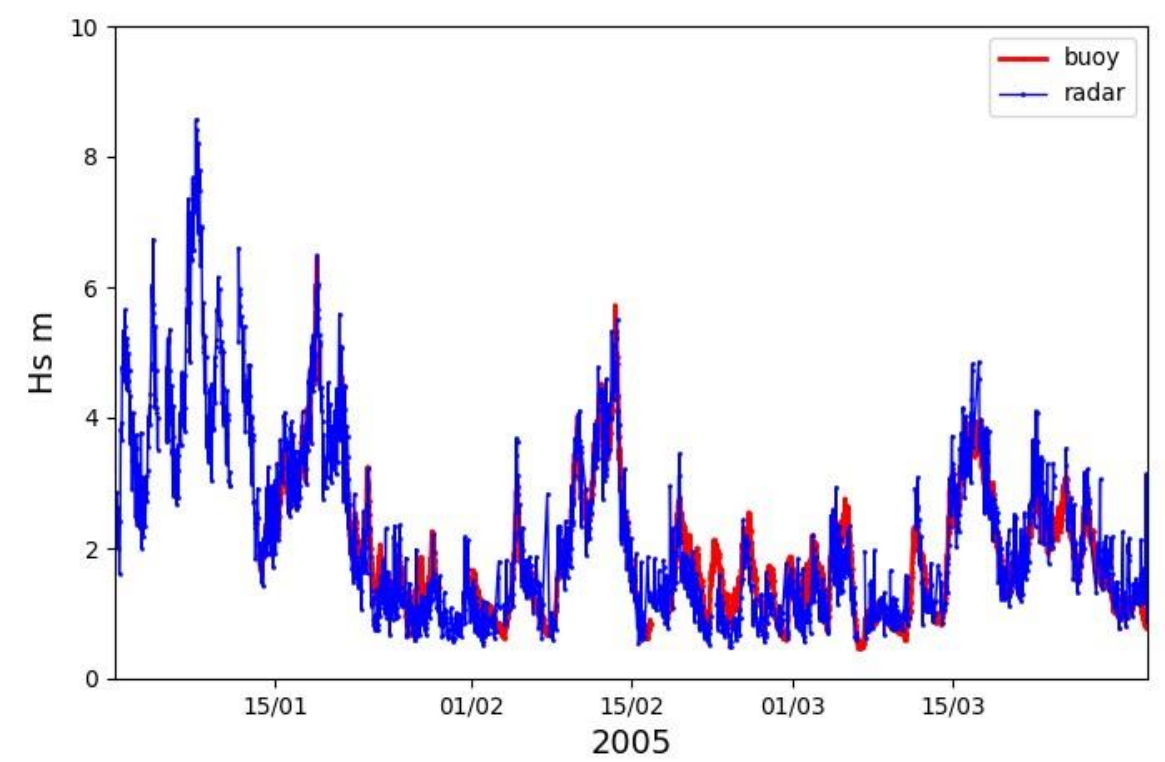

Figure 2 Pisces significant waveheight comparison between buoy (red) and radar (blue). 

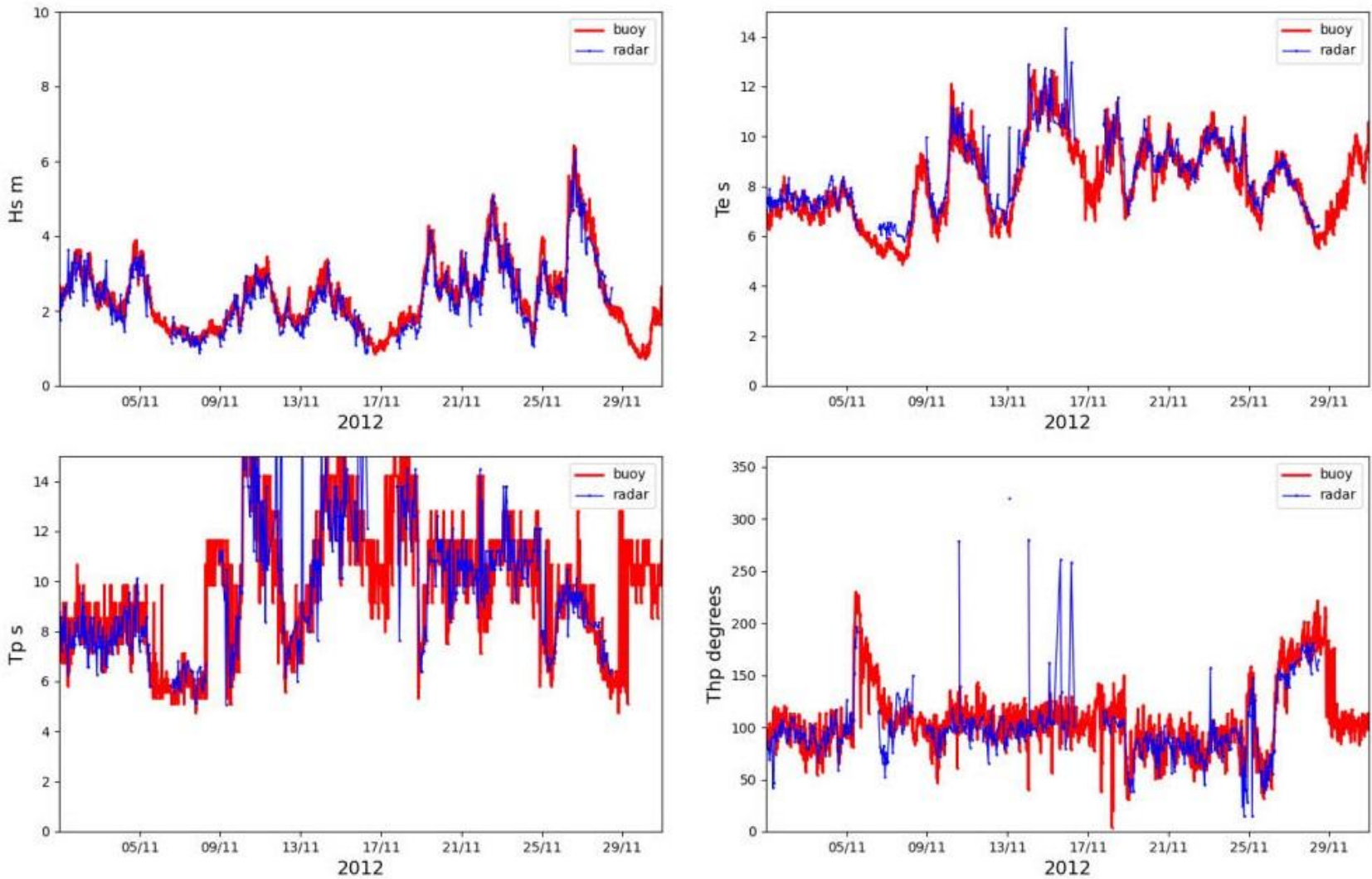

Figure 3 WERA wave parameter comparisons between buoy (red) and radar (blue).

Both data sets show good agreement. There are differences, usually in periods of low waveheights when other signals such as ships or sidelobe impacts [49] corrupt the sea signal. Peak period and direction have more variability with any measurement system (as can be seen in both buoy and radar data in Figure 3) because they do not involve any integration over frequency. The statistics of the UoP radar wave comparisons are presented in Table 1. These are similar to those presented in Lopez et al [47] where a longer data set of 8 months was used and validations were carried out at more than one location.

Table 1 Statistics of the UoP wave parameters. rms - root mean square difference; SI scatter index; cc- correlation coefficient, vc - vector correlation; diff - mean difference; $95 \% \mathrm{ci}$ is the $95 \%$ confidence interval for the mean difference; conc - is an inverse measure of the spread of the directional difference.

\begin{tabular}{llllllll}
\hline parameter & buoy mean/std & radar mean/std & bias & rms & SI & cc & N \\
\hline$H s, \mathrm{~m}$ & $2.59 / 0.87$ & $2.43 / 0.87$ & 0.15 & 0.37 & 0.14 & 0.93 & 488 \\
$T e, \mathrm{~s}$ & $8.25 / 1.55$ & $8.54 / 1.45$ & -0.29 & 0.73 & 0.09 & 0.9 & 477 \\
$T p, \mathrm{~s}$ & $9.8 / 2.56$ & $9.69 / 2.68$ & 0.1 & 1.79 & 0.18 & 0.77 & 477 \\
\hline & & & $\mathrm{Vc}$ & Diff & $95 \% \mathrm{ci}$ & $\mathrm{conc}$ & \\
\hline Thm & $95.38 / 28.74$ & $93.61 / 29.78$ & 0.93 & 3.03 & 21.29 & 7.96 & 477 \\
$T h p$ & $99.98 / 29.84$ & $94.7 / 31.82$ & 0.92 & 6.19 & 25.26 & 6.83 & 477 \\
\hline
\end{tabular}


Note that there are more $\mathrm{Hs}(\mathrm{N})$ cases for comparison. The spectral parameters of period and direction are flagged and not included in the statistics if $\mathrm{kOHs}<0.32$ where $\mathrm{kO}$ is the radar wavenumber [50]. A lower limit of 0.12 is used for significant waveheight. Very low values of kOHs are cases where the spectral peak is close to the first order Bragg peak frequency where the theoretical underpinning of the inversion process fails. There are more low waveheight cases in the Pisces measurements shown in Figure 2, many are close to the $\mathrm{kOHs}$ limits consequently the statistics are not quite as good for the spectral parameters (they are reported in [46, 50]). Pisces can operate at a number of different frequencies and during that trial these were optimised to minimise external interference: low frequencies in the day, higher at night. They can also be optimised to wave conditions, i.e. to keep $\mathrm{kOHs}$ high enough. The availability now of simultaneous operation on two frequencies will also help in this respect.

\subsubsection{Wind Speed and Direction}

It was noted in Section 3.1 that wind speed is not yet an operational parameter although an estimate is provided in the SV software package. SV has begun to investigate machine learning methods. The work is in its early stages and details of the methods being tested are not included here although we do show some preliminary and encouraging results. To develop an effective algorithm for wind speed we need wind measurements at the position of the radar measurements. To date this has not been available in the Celtic Sea although we have obtained wind data from a coastal station at Perranporth through the Channel Coastal Observatory [51] and this is being used for algorithm development.

We are currently working with only one month of UoP radar data and have established that this is not really enough to provide a sufficiently reliable model using the machine learning methods we have tested. However, we do obtain results that are much better than our wave model based approach. Figure 4 shows scatter plots of wind speed for the model generated by one of the methods we are testing using $90 \%$ of the data on the left and the prediction using the remaining $10 \%$ on the right. The correlation coefficients of over 0.9 in both cases, over wind speed ranges from 1 to over $20 \mathrm{~m} / \mathrm{s}$, are huge improvements on 0.46 which is obtained with the currently SVimplemented wave model method at this location due to the ubiquitous presence of swell often dominating the wave spectrum. It is worth noting that we have obtained an $\mathrm{rms}$ figure of $<1.5 \mathrm{~m} / \mathrm{s}$ (compared to about $2 \mathrm{~m} / \mathrm{s}$ here) for a different data set (not in the Celtic Sea) for which, although radar data quality was not as good, we do have some local wind speed data. We expect an $r m s$ of $<$ $1 \mathrm{~m} / \mathrm{s}$ to be achievable when we have completed this work. This will not reach the accuracy expected for lidar measurements, nor is the measurement location nominally at turbine height although a model could be used to scale it, but the data will provide a large-scale complementary spatial map of wind speed and direction. Figure 5 shows a scatter plot of wind direction for all the UoP data set colour-coded with radar-measured wind speed. Bearing in mind that the radar measurements are not at the same location as the anemometer which is at the coast, the agreement is good with largest direction differences at low wind speeds. The statistics for the wind vector comparisons are presented separately for the $90 \%$ model development data and the $10 \%$ test data. These are very encouraging results. 

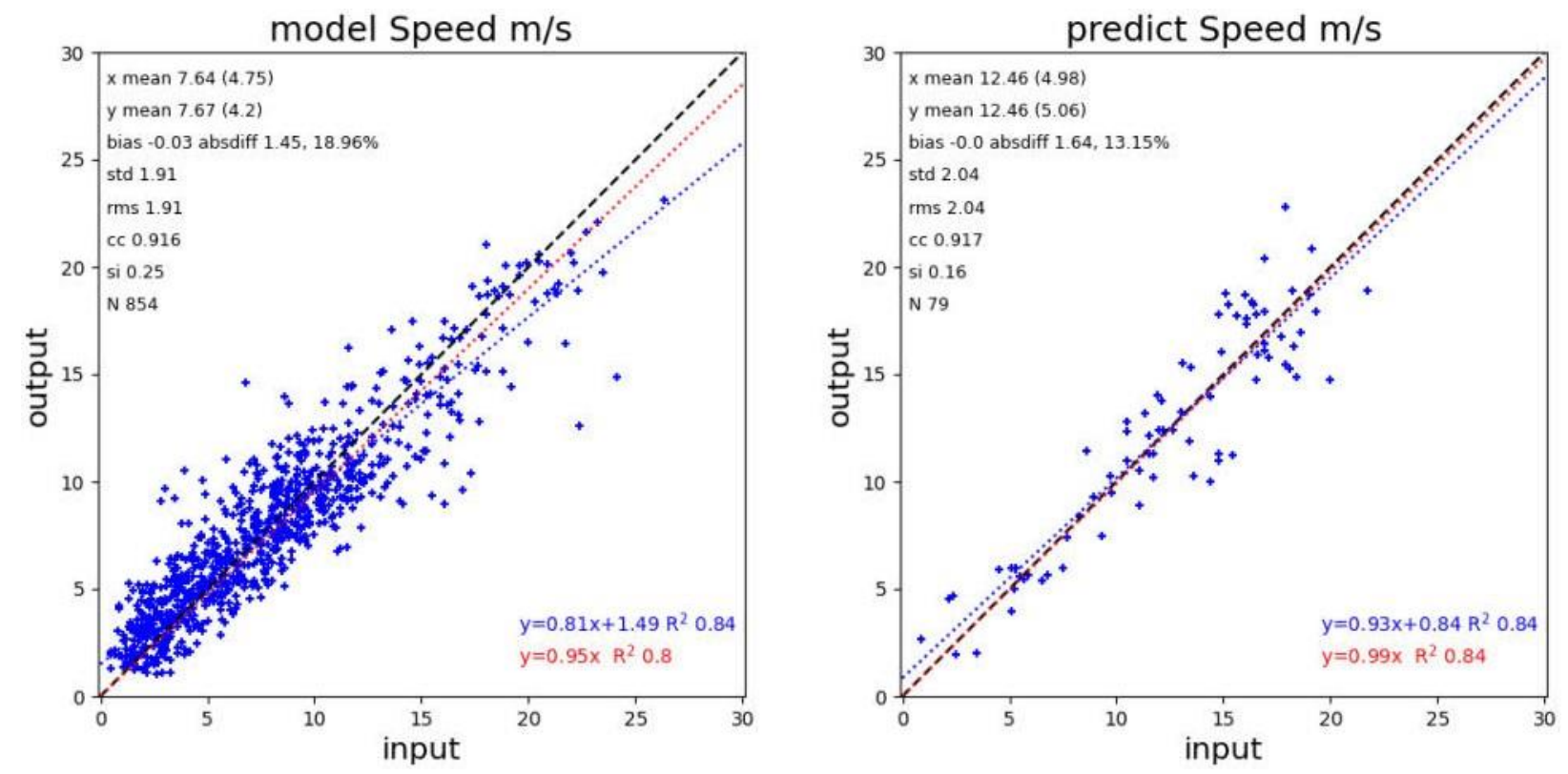

Figure 4 Wind speed scatter plots and statistics.

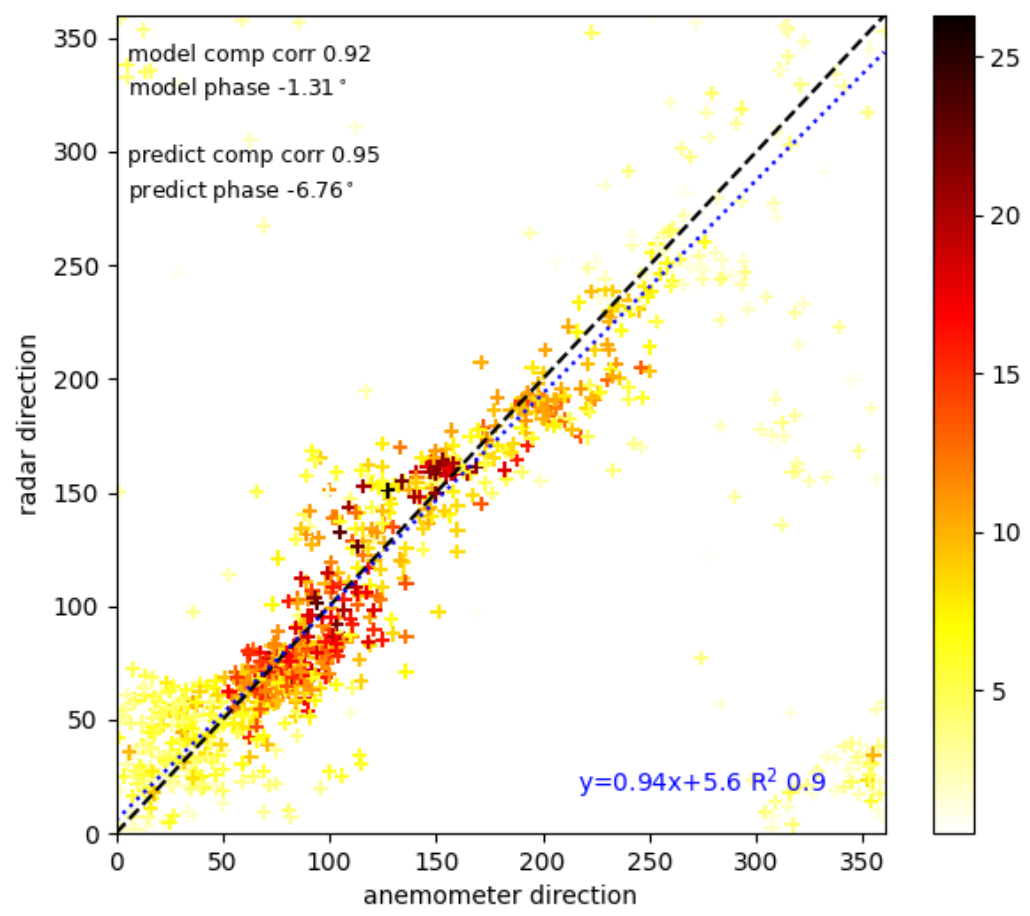

Figure 5 Direction scatter plot colour-coded by radar wind speed and vector statistics.

\subsubsection{Metocean Maps}

Having established the validity of the measurements the maps in Figure 6 show the spatial coverage potential of the radar systems in the Celtic Sea. The UoP measurements cover the Wave Hub site in the southern Celtic Sea; Pisces (with a second site in S Wales) could cover offshore wind sites in the northern Celtic Sea. The Pisces measurements are from the operational trial on 8/4/2005 @ 00:00 and the UoP measurements are from 24/11/2012 @22:05 so at different times, different 
metocean conditions and different phases of the tide although wind directions are roughly the same. The arrows are scaled according to the spatial resolution of the measurements and thus the scaling is different for the two radar systems. The details are not important. They have been combined here to illustrate coverage and spatial variability not to describe a particular event. The UoP wave, current and wind vectors and spectral data have been sub-sampled for clarity. For the same reason not all Pisces wave spectra are shown. Note that the wind speeds shown here are obtained with the standard SV method. The new method has not yet been extended to all locations. In the wave plot, the lighter shading, beyond the area containing arrows, shows the extent of single radar measurements.

(a)
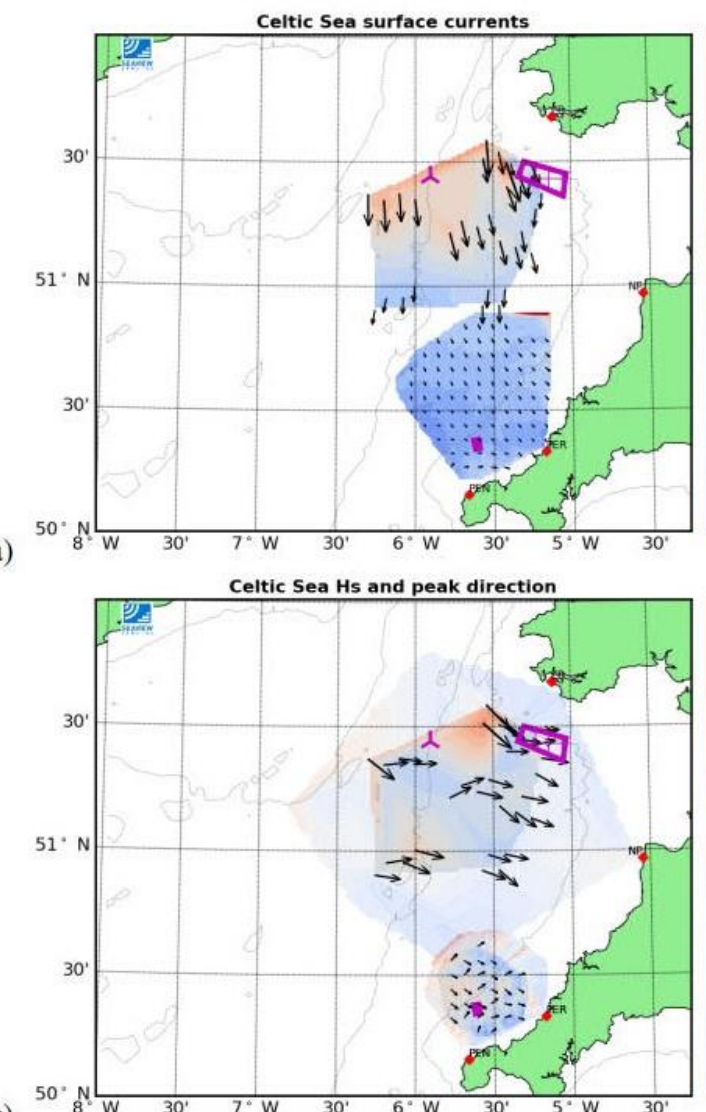

(b)
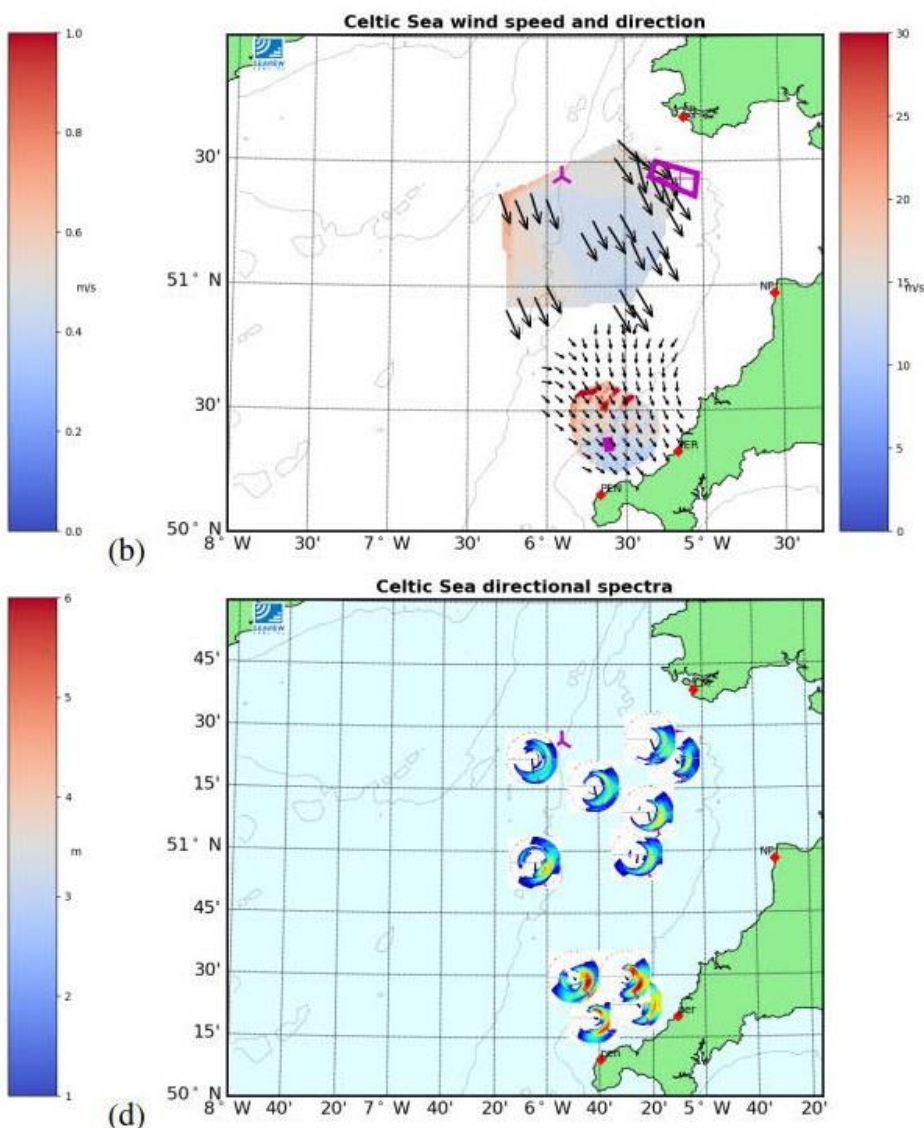

Figure 6 Maps of metocean measurements in the Celtic Sea: (a) surface current, (b) wind speed and direction, (c) waveheight and peak direction, (d) directional wave spectra, to illustrate potential coverage. Note that the measurements from the UoP and Pisces radars are at different times, different metocean conditions, different phases of the tide and with different spatial resolutions.

The Pisces operational trial was focussed on validating wave measurements at one location, approximately $60 \mathrm{~km}$ from both radar sites, and high spatial resolution was not required. Pisces is a more powerful radar than WERA and can measure waves, currents and winds to over $100 \mathrm{~km}$ as seen here. The UoP radar provides measurements over a more limited range but with high spatial resolution. It is normally that case that wave and wind speed measurements have a more limited range than current and wind direction, because signal to noise requirements are more strict, and this can be seen in the UoP plots. 


\section{Concluding Remarks}

We have demonstrated that wave measurements can be obtained with good accuracy and mapped over wide areas of the coastal ocean. New wind speed algorithms are being developed that show great promise. These will never be as accurate as lidar or mast data but they have the advantage of providing a spatial picture which is particularly important in coastal regions such as the Celtic Sea. Surface current measurement with HF radar is now well accepted and used in operational networks. We expect that the accuracy and coverage will be of value to the offshore wind sector.

HF radars will therefore provide very useful data for resource assessment, and the planning, operation and maintenance stages for wind farms. Once the wind farms are in operation the turbine motion produces identifiable signals in the radar return that do impact on wave and wind measurement accuracy in the immediate vicinity of the farms; less so on current measurement accuracy [52]. Removing these signals is an area of active and promising research. In the USA this is coordinated by the Wind Turbine Radar Interference Mitigation Working Group, a consortium of federal agencies together with interested research groups and radar operators both in the US and elsewhere including the UK where the issue was first identified. Success in this research effort will make it possible to measure metocean parameters accurately within wind farms.

At present there are just three radar systems on the Celtic Sea coast all on the north coast of the SW of England (PEN, PER and NP in Figure 6). The vision is to re-install a radar on the Welsh coast to provide coverage of the whole of the Eastern sector of the Celtic Sea combining information from all 4 radars. To obtain full coverage of the region, to meet the longer term aspirations of floating offshore wind developers radars will also be needed on the South-East coast of Ireland. Such a network of radars could also provide valuable data for many other applications such as shipping, fisheries, meteorological services and ground-truthing of satellite missions. The lifetime cost of a radar is equivalent to a small number of in-situ systems without the need for ship-time to deploy, maintain and recover. The cost of a radar will reduce over time if the offshore wind sector takes up this technology in the UK and the rest of the world.

\section{Acknowledgments}

The Pisces Celtic Sea data were provided by Neptune Radar and collected during a project funded by DEFRA and the MetOffice. The buoy data for this experiment have been provided by CEFAS. The UoP radar and buoy data were provided by Daniel Conley with financial support from the Natural Environment Research Council (Grant NE/J004219/1). The wind data were obtained from the Channel Coastal Observatory. Daniel Conley and Mike Moorhead (Neptune Radar Ltd) also provide helpful comments to improve the manuscript.

\section{Author Contributions}

The author did all work.

\section{Competing Interests}

The author has declared that no competing interests exist. 


\section{References}

1. BVG associates. Guide to an offshore wind farm. Glasgow: Offshore Renewable Energy Catapult; 2019. [cited date 2020 October 29]. Available from: https://www.thecrownestate.co.uk/media/2861/guide-to-offshore-wind-farm-2019.pdf.

2. Carbon Trust. Floating offshore wind market technology review. London: Carbon Trust; 2015. [cited date 2020 October 29]. Available from: https://www.carbontrust.com/resources/floating-offshore-wind-market-technology-review.

3. Offshore Wind Innovation Hub. O\&M and windfarm lifecycle. [cited date 2020 October 29]. Available from: https://offshorewindinnovationhub.com/category/operations-maintenance.

4. DNV GL, Frazer-Nash Consultancy, Multiversum Consulting, and Fraunhofer IWES. Carbon Trust Offshore Wind Accelerator Roadmap for the Commercial Acceptance of Floating LiDAR Technology. Version 2.0. 2018. [cited date 2020 October 29]. Available from: https://www.carbontrust.com/resources/roadmap-for-commercial-acceptance-of-floatinglidar.

5. Verhoef A, Vogelzang J, Verspeek J, Stoffelen A. Long-Term scatterometer wind climate data records. IEEE J Sel Top Appl Earth Obs Remote Sens. 2017; 10: 2186-2194.

6. Ahsbahs TT, Badger M, Volker P, Hansen KS, Hasager CB. Applications of satellite winds for the offshore wind farm site anholt. Wind Energy Sci. 2018; 3: 573-588.

7. Remmers T, Cawkwell F, Desmond C, Murphy J, Politi E. The potential of advanced scatterometer (ASCAT) $12.5 \mathrm{~km}$ coastal observations for offshore wind farm site selection in Irish Waters. Energies. 2019; 12: 206.

8. Meteo-France, Ifremer, MET Norway, DMI, KNMI. OSI SAF CDOP3 Product Requirement Document Version 1.4. 2018. [cited date 2020 October 29]. Available from: http://www.osisaf.org/sites/default/files/dynamic/public doc/osisaf cdop3 gen prd 1.4.pdf.

9. Meteo-France, Ifremer, MET Norway, DMI, KNMI. OSI SAF Half-Yearly Operations Report Version 1.4. 2020. [cited date 2020 October 29]. Available from: http://www.osisaf.org/sites/default/files/dynamic/public doc/osisaf cdop3 rp hyr20 h1 1 0.pdf.

10. Hessner KG, Nieto-Borge JC, Bell PS. Nautical radar measurements in Europe: Applications of WAMOS II as a sensor for sea state, current and bathymetry. In Remote Sensing of the European Seas. Berlin: Springer; 2008. pp. 435-446.

11. van der Vlugt $\mathrm{T}$, van der Vlugt R. Comparison of directional WaveGuide Radar with Directional WaveRider buoy. Madrid: Environmental XPRT; 2014. [cite date 2020 October 30]. Available from: https://www.environmental-expert.com/articles/comparison-of-directional-waveguideradar-with-directionalwaverider-buoy-503676/full-article.

12. Ribal A, Young IR. 33 years of globally calibrated wave height and wind speed data based on altimeter observations. Sci Data. 2019; 6: 77.

13. Zhang B, Li X, Perrie W, He Y. Synergistic measurements of ocean winds and waves from SAR. J Geophys Res Oceans. 2015; 120: 6164-6184.

14. Dohan K, Maximenko N. Monitoring ocean currents with satellite sensors. Oceanography. 2010; 23: 94-103.

15. Martin AC, Gommenginger C. Towards wide-swath high-resolution mapping of total ocean surface current vectors from space: Airborne proof-of-concept and validation. Remote Sens Environ. 2017; 197: 58-71. 
16. Fujii S, Heron ML, Kim K, Lai JW, Lee SH, Wu X, et al. An overview of developments and applications of oceanographic radar networks in Asia and Oceania countries. Ocean Sci J. 2013; 48: 69-97.

17. Wyatt L. The IMOS ocean radar facility, ACORN. In Coastal Ocean Observing Systems. Cambridge, MA: Academic Press; 2015. pp.143-158.

18. Rubio A, Mader J, Corgnati L, Mantovani C, Griffa A, Novellino A, et al. HF radar activity in European coastal seas: Next steps toward a pan-European HF radar network. Front Mar Sci. 2017; 4: 8.

19. Roarty H, Cook T, Hazard L, George D, Harlan J, Cosoli S, et al. The global high frequency radar network. Front Mar Sci. 2019; 6: 164.

20. Wyatt LR, Mantovanelli A, Heron ML, Roughan M and Steinberg CR. Assessment of surface currents measured with High-Frequency Phased-Array Radars in two regions of complex circulation. IEEE J Ocean Eng. 2018; 43: 484-505.

21. Wyatt LR, Ledgard L, Anderson C. Maximum-likelihood estimation of the directional distribution of 0.53-Hz ocean waves. J Atmos Ocean Technol. 1997; 14: 591-603.

22. Wyatt LR. Shortwave direction and spreading measured with HF radar. J Atmos Ocean Technol. 2012; 29: 286-299.

23. Wyatt LR. A comparison of scatterometer and HF radar wind direction measurements. J Oper Oceanogr. 2018; 11: 54-63.

24. Barrick DE. The ocean waveheight nondirectional spectrum from inversion of the HF sea-echo Doppler spectrum. Remote Sens Environ. 1977; 6: 201-227.

25. Barrick D. Extraction of wave parameters from measured HF radar sea-echo Doppler spectra. Radio Sci. 1977; 12: 415-424.

26. Wyatt LR. Limits to the inversion of HF radar backscatter for ocean wave measurement. J Atmos Ocean Technol. 2000; 17: 1651-1666.

27. Green JJ, Wyatt LR. Row-action inversion of the Barrick-Weber equations. J Atmos Ocean Technol. 2006; 23: 501-510.

28. Wyatt L. Ocean wave measurement. In Ocean Remote Sensing Technologies: High Frequency, Marine and GNSS-Based Radar. To be published in 2021.

29. Gaffard C, Parent J. Remote sensing of wind speed at sea surface level using HF skywave echoes from decametric waves. Geophys Res Lett. 1990; 17: 615-618.

30. Shen W, Gurgel KW, Voulgaris G, Schlick T, Stammer D. Wind-speed inversion from HF radar first-order backscatter signal. Ocean Dyn. 2012; 62: 105-121.

31. Kirincich A. Remote sensing of the surface wind field over the coastal ocean via direct calibration of HF radar backscatter power. J Atmos Ocean Technol. 2016; 33: 1377-1392.

32. Seroka G, Kohut J, Palamara L, Glenn S, Roarty H, Bowers L, et al. Spatial evaluation of highresolution modeled offshore winds using estimated winds derived from a network of HF radars. 2013 OCEANS - San Diego. San Diego, CA: IEEE; 2013. pp. 1-5.

33. Dexter PE, Theodorides S. Surface wind speed extraction from HF sky-wave radar doppler spectra. Radio Sci. 1982; 17: 643-652.

34. Zeng $\mathrm{Y}$, Zhou $\mathrm{H}$, Roarty $\mathrm{H}$, Wen $\mathrm{B}$. Wind speed inversion in high frequency radar based on neural network. Int J Antennas Propa. 2016; 2016: 2706521.

35. Waters J, Wyatt L, Wolf J, Hines A. Data assimilation of partitioned HF radar wave data into wavewatch III. Ocean Model. 2013; 72: 17-31. 
36. Erebus. Wales: Blue Gem Wind Ltd. [cited date 2020 November 04]. Available from: https://www.bluegemwind.com/our-projects/erebus/.

37. Wave Hub Limited. Cornwall: Wave Hub. [cited date 2020 November 04]. Available from: https://www.wavehub.co.uk.

38. Wyatt LR, Venn J, Burrows G, Ponsford A, van Heteren J, Moorhead M. HF radar measurements of ocean wave parameters during NURWEC. IEEE J Ocean Eng. 1986; 11: 219-234.

39. Wyatt LR, Moorhead M, Fairley IA. Developments in metocean HF radar technology, applications and accuracy. Proceedings of the ASME 2019 38th International Conference on Ocean, Offshore and Arctic Engineering; 2019 June 9-14; Glasgow, Scotland, UK. New York: American Society of Mechanical Engineers.

40. Gurgel KW, Antonischki G, Essen HH, Schlick T. Wellen radar (WERA): A new ground-wave HF radar for ocean remote sensing. Coast Eng. 1999; 37: 219-234.

41. Wyatt LR, Thompson SP, Burton RR. Evaluation of high frequency radar wave measurement. Coast Eng. 1999; 37: 259-282.

42. Wyatt LR, Green JJ, Gurgel KW, Borge JN, Reichert K, Hessner K, et al. Validation and intercomparisons of wave measurements and models during the EuroROSE experiments. Coast Eng. 2003; 48: 1-28.

43. Wyatt LR, Graber H, Haus B. Factors affecting the accuracy of SHOWEX HF radar wave measurements. J Atmos Ocean Technol. 2005; 22: 847-859.

44. Wyatt L. Measuring the ocean wave directional spectrum 'First Five' with HF radar. Ocean Dyn. 2018; 69: 123-144.

45. James C, Collopy M, Wyatt L, Middleditch A. Suitability of the southern Australia integrated marine observing system's (SA-IMOS) HF-radar for operational forecasting. J Oper Oceanogr. 2019; 12: 73-85.

46. Wyatt LR, Green JJ, Middleditch A, Moorhead MD, Howarth J, Holt M, et al. Operational wave, current and wind measurements with the Pisces HF radar. IEEE J Ocean Eng. 2006; 31: 819-834.

47. Lopez G, Conley DC. Comparison of HF radar fields of directional wave spectra against in situ measurements at multiple locations. J Mar Sci Eng. 2019; 7: 271.

48. Hardman R, Wyatt L. Inversion of HF radar Doppler spectra using a neural network. J Mar Sci Eng. 2019; 7: 255.

49. Wyatt LR, Liakhovetski G, Graber H, Haus B. Factors affecting the accuracy of SHOWEX HF radar wave measurements. J Atmos Ocean Technol. 2005; 22: 844-856.

50. Wyatt LR, Green JJ, Middleditch A. HF radar data quality requirements for wave measurement. Coast Eng. 2011; 58: 327-336.

51. Regional Coastal Monitoring Programmes. Perraporth. Southampton: National Network of Regional Coastal Monitoring Programmes of England. [cited date 2020 November 06]. Available from: https://www.channelcoast.org/realtimedata/?chart=76\&tab=qc\&disp option=.

52. Robinson A, Wyatt L, Howarth M. HF Radar data availability and measurement accuracy in Liverpool Bay before and after the construction of Rhyl-Flats wind farm. J Oper Oceanogr. 2013; 6: 1-12. 
JEPT 2021; 3(1), doi:10.21926/jept.2101005

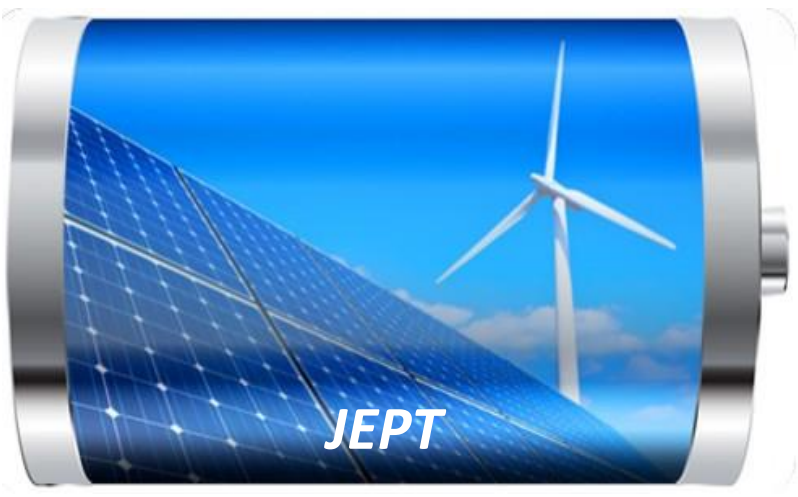

Enjoy JEPT by:

1. Submitting a manuscript

2. Joining in volunteer reviewer bank

3. Joining Editorial Board

4. Guest editing a special issue

For more details, please visit:

http://www.lidsen.com/journal/jept 
Original Research

\title{
Quaternary Hydrides $\mathrm{Pd}_{1-y-z} \mathrm{Ag}_{y} \mathrm{Cu}_{z} \mathrm{H}_{x}$ Embedded Atom Method Potentials for Hydrogen Energy Applications
}

\author{
Chaonan Zhang ${ }^{1,{ }^{\dagger}}$, Robert Fuller ${ }^{1,{ }^{\dagger}}$, Iyad Hijazi ${ }^{1,{ }^{+}, *}$
}

Weisberg Department of Mechanical Engineering, Marshall University, Huntington, United States; E-Mails: zhang127@marshall.edu; fuller94@marshall.edu; hijazii@marshall.edu

$\dagger$ These authors contributed equally to this work.

* Correspondence: Iyad Hijazi; E-Mail: hijazii@marshall.edu

Academic Editor: Alfonso Chinnici

Special Issue: $\underline{\text { Hydrogen Energy: Sustainable Production, Storage and Utilisation }}$

Journal of Energy and Power Technology

2021, volume 3, issue 1

doi:10.21926/jept.2101006
Received: November 10, 2020

Accepted: January 12, 2021

Published: January 25, 2021

\begin{abstract}
The $\mathrm{Pd}-\mathrm{H}$ system has attracted extensive attention. Pd can absorb considerable amount of $\mathrm{H}$ at room temperature, this ability is reversible, so it is suitable for multiple energy applications. $\mathrm{Pd}$-Ag alloys possess higher $\mathrm{H}$ permeability, solubility and narrower miscibility gap with better mechanical properties than pure $\mathrm{Pd}$, but sulfur poisoning remains an issue. $\mathrm{Pd}-\mathrm{Cu}$ alloys have excellent resistance to sulfur and carbon monoxide poisoning and hydrogen embrittlement, good mechanical properties, and broader temperature working environments over pure Pd, but relatively lower hydrogen permeability and solubility than pure $\mathrm{Pd}$ and $\mathrm{Pd}-\mathrm{Ag}$ alloys. This suggests that alloying $\mathrm{Pd}$ with $\mathrm{Ag}$ and $\mathrm{Cu}$ to create $\mathrm{Pd}-\mathrm{Ag}$ - $\mathrm{Cu}$ ternary alloys can optimize the overall performance and substantially lowers the cost. Thus, in this paper, we provide the first embedded atom method potentials for the quaternary hydrides $\mathrm{Pd}_{1-y-z} \mathrm{Ag}_{y} \mathrm{Cu}_{z} \mathrm{H}_{x}$. The fully analytical potentials are fitted utilizing the central atom method without performing timeconsuming molecular dynamics simulations.
\end{abstract}

\section{Keywords}

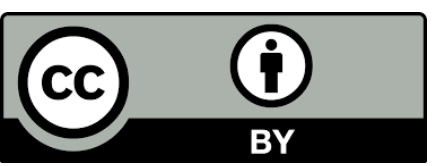

(C) 2021 by the author. This is an open access article distributed under the conditions of the Creative Commons by Attribution License, which permits unrestricted use, distribution, and reproduction in any medium or format, provided the original work is correctly cited. 
EAM; DFT; metal hydrides; hydrogen storage; miscibility Gap; Pd-Ag-Cu-H

\section{Introduction}

The palladium hydride $(\mathrm{Pd}-\mathrm{H})$ system has attracted various studies [1-8]. Palladium ( $\mathrm{Pd})$ possesses the capacity to absorb a considerable amount of hydrogen $(\mathrm{H})$ at room temperature and atmospheric pressure. It has excellent hydrogen selectivity, permeability and $\mathrm{H}$ diffusivity with high mobility [9-12]. Therefore, it has diverse applications such as fuel cells, hydrogen storage, refrigeration, hydrogen separation and purification, nuclear radiation adsorption and catalytic converter [9-15].

However, the coexistence of low $\mathrm{H}$ alpha $(\alpha)$ and high $\mathrm{H}$ beta $(\beta)$ phases in Pd hydride causes a miscibility gap. The strain caused by the lattice mismatch between the two phases increases the possibility of mechanical failure [16-18]. In addition, $\mathrm{Pd}$ is vulnerable to hydrogen embrittlement after some cycles of $\alpha$ phase and $\beta$ phase transformations in $\mathrm{Pd}$ membranes or absorption/desorption cycles in hydrogen storage $[18,19]$. $\mathrm{Pd}$ membranes are also prone to $\mathrm{H}_{2} \mathrm{~S}$ and CO poisoning [20-22]. Finally, palladium is an expensive noble metal, with a price currently higher than gold.

$\mathrm{Pd}$ can be alloyed with silver $(\mathrm{Ag})$ and copper $(\mathrm{Cu})$ to solve the above listed problems and reduce costs [23-25]. Although $\mathrm{H}$ is practically insoluble in $\mathrm{Ag}, \mathrm{Pd}-\mathrm{Ag}$ alloys possess higher $\mathrm{H}$ solubility than $\mathrm{Pd}$ [24]. As with Pd, Pd-Ag alloys are $100 \%$ selective when absorbing $\mathrm{H}$, and therefore, are also ideal for separating $\mathrm{H}$ from mixture of gases [18]. Pd-Ag alloys also possess excellent $\mathrm{H}$ permeability, with the greatest permeability observed in the $\mathrm{Pd}_{77} \mathrm{Ag}_{23}$ structure $[23,26]$. Depending on $\mathrm{Ag}$ concentration, $\mathrm{H}$ diffusion rate can be also increased in $\mathrm{Pd}-\mathrm{Ag}$ alloys as compared with pure $\mathrm{Pd}$ [5]. $\mathrm{Pd}$-Ag alloys are reported to possess more steady mechanical properties than pure $\mathrm{Pd}$, and better resistance to $\mathrm{H}$ embrittlement [18], however, strong poisoning by $\mathrm{H}_{2} \mathrm{~S}$ remains an issue [27].

$\mathrm{Pd}-\mathrm{Cu}$ alloys are also known as one of the most effective materials in hydrogen separation field, as $\mathrm{Cu}$ is a much cheaper metal than $\mathrm{Pd}$. $\mathrm{Pd}-\mathrm{Cu}$ alloys also have better mechanical properties, high thermal stability [28], avoidance of hydrogen embrittlement at room temperature, improved sulfur poisoning resistance than $\mathrm{Pd}$ and $\mathrm{Pd}-\mathrm{Ag}$ alloys [29], and more resistance to carbon monoxide poisoning than pure $\mathrm{Pd}$ [30]. In addition, $\mathrm{Pd}-\mathrm{Cu}$ alloys are also completely selective for hydrogen [31], and $\mathrm{H}$ diffusion rate in body-centered-cubic (bcc) Pd-Cu alloys is faster than those in pure $\mathrm{Pd}$ and face-centered-cubic ( $\mathrm{fcc}$ ) Pd-Cu alloys [32]. However, $\mathrm{H}$ permeation rate in bcc $\mathrm{Pd}$-Cu has been found to be slower than in pure $\mathrm{Pd}$, but faster than in $\mathrm{fcc} \mathrm{Pd}-\mathrm{Cu}$ [33], and $\mathrm{H}$ solubility in $\mathrm{Pd}-\mathrm{Cu}$ alloy is lower than pure Pd, and decreases strongly as Cu content increases [33].

Since Pd-Ag alloys have excellent $\mathrm{H}$ selectivity, permeability and solubility, and Pd-Cu alloys have excellent resistance to sulfur and carbon monoxide poisoning and hydrogen embrittlement, good mechanical properties, broader working conditions than pure $\mathrm{Pd}$ and relatively low cost, one way to optimize the overall performance and substantially lower the cost is alloying Pd with $\mathrm{Ag}$ and $\mathrm{Cu}$ [34-38]. To accelerate the search for Pd-Ag-Cu alloys with optimal hydrogen selectivity, permeability, diffusivity, absorption, thermal stability and resistance to sulfur and $\mathrm{CO}$ poisoning, an accurate and efficient modeling of the Pd-Ag-Cu-H system is needed. The most reliable simulation technique utilizes first principle (ab initio) calculations, but its high processing costs make it infeasible for 
simulations containing a large number of atoms. Molecular dynamics (MD) simulations using the embedded atom method (EAM) offers an efficient way to investigate alloys with larger atomic structures. EAM is well suitable to model binary and ternary hydrides with metallic crystal structures and interstitial $\mathrm{H}$ atoms. An EAM Pd-H potential which can predict the miscibility gap was formulated by Zhou et al. [4]. The Pd-H EAM potential was then expanded into Pd-Ag-H ternary potential by Hale et al. [5]. Their Pd-Ag-H potential predicted smooth changes in structures parameters, elastic properties and energy with increasing $H$ concentrations, $H$ sites occupation shift and the disappearance of the miscibility gap by the addition of $\mathrm{Ag}$ at $300 \mathrm{~K}$. However, their model was built on Foiles et al.'s Pd potential, which is available in a tabular form but does not include a full explanation of the analytical form and parameters [39], and therefore impeding further improvement of the Pd-Ag-H ternary system.

In previous work, Pd-H EAM potentials with fewer fitting parameters than Zhou et al. [4] were fitted by Hijazi et al. [40] that can predict many of the properties of the $\mathrm{Pd}-\mathrm{H}$ structures accurately. In this paper, the previously developed $\mathrm{Pd}-\mathrm{H}$ potentials are expanded into the $\mathrm{Pd}-\mathrm{Ag}-\mathrm{Cu}-\mathrm{H}$ quaternary potentials. First principles simulations using SIESTA were carried out to obtain the fitting parameters. The fully analytical potentials are fitted utilizing the central atom method without performing time-consuming MD simulations. The Pd-Ag-Cu-H EAM potentials can capture the miscibility gap behavior; preferred $\mathrm{H}$ occupancy sites; and predicts the trends for the lattice constants, cohesive energies, and elastic properties during MD simulations.

\section{The Atomic Potentials}

A total of 18 functions are needed to create the $\mathrm{Pd}-\mathrm{Ag}-\mathrm{Cu}-\mathrm{H}$ quaternary atomic EAM potentials. Which include 4 embedding energy functions, $F_{P d}, F_{A g}, F_{C u}$ and $F_{H}, 4$ electron density functions, $\rho_{P d}$ $\rho_{A g}, \rho_{C u}$ and $\rho_{H}$, and 10 pair functions, $\varphi_{P d-P d}, \varphi_{A g-A g}, \varphi_{C u-C u}, \varphi_{H-H}, \varphi_{P d-A g}, \varphi_{P d-C u}, \varphi_{A g-C u}, \varphi_{P d-H}, \varphi_{A g-H}$ and $\varphi_{C u-H}$. In EAM, each atom is embedded into a lattice that include all host atoms. The pair potential between atoms, and the energy related to embedding an atom inside the host lattice is modeled. The total energy $E_{\text {tot }}$ of an EAM system is given by [41].

$$
\begin{gathered}
E_{\text {tot }}=\sum_{i=1}^{N} F_{i}\left(\rho_{i}\right)+\frac{1}{2} \sum_{\substack { i=1 \\
\begin{subarray}{c}{j=1 \\
j \neq i{ i = 1 \\
\begin{subarray} { c } { j = 1 \\
j \neq i } }\end{subarray}}^{N} \varphi_{i j}\left(r_{i j}\right) \\
\rho_{i}=\sum_{j \neq i}^{N} f_{j}\left(r_{i j}\right)
\end{gathered}
$$

where $\rho_{i}$ is the electron density for atom $i, F_{i}$ is the embedding energy, $\phi_{i j}$ is the pair potential between atom $i$ and atom $j, r_{i j}$ represents the distance from atom $i$ and to atom $j, f_{j}$ is the electron density function of distance from the center of atom $j$. The EAM model by Hijazi et al. was used in fitting the $\mathrm{Pd}-\mathrm{Pd}, \mathrm{Ag}-\mathrm{Ag}, \mathrm{Cu}-\mathrm{Cu}, \mathrm{Pd}-\mathrm{Ag}, \mathrm{Pd}-\mathrm{Cu}$ and $\mathrm{Ag}-\mathrm{Cu}$ potentials [42-46]. The embedding function is represented by:

$$
F(\rho)=F\left(\rho_{e}\right)\left[1-\eta \ln \left(\frac{\rho}{\rho_{e}}\right)\right]\left(\frac{\rho}{\rho_{e}}\right)^{\eta}
$$


The host electron density is given by:

$$
f=f_{e} e^{-\chi\left(r-r_{e}\right)}
$$

where $f_{e}$ is a scaling factor that can be obtained from $f_{e}=E_{c} / \Omega, \Omega$ is the atomic volume and $E_{c}$ is the cohesive energy, $r_{e}$ is the equilibrium closest distance, and $\chi$ is a fitting parameter. The pair potential function is the modified potential created by Rose et al. [47] and has the form:

$$
\varphi=-\varphi_{e}\left[1+\delta\left(\frac{r}{r_{e}}-1\right)\right] e^{-\beta\left(\frac{r}{r_{e}}-1\right)}
$$

where $\phi_{e}, \delta$, and $B$ are the 3 adjustable parameters. Therefore, for an fcc metal, there are 6 fitting parameters $\chi, \phi_{e}, \delta, 6, \eta$, and $\rho_{e}$.

The analytical expressions proposed by Zhou et al. [4, 5] was used in fitting the $\mathrm{H}-\mathrm{H}, \mathrm{Pd}-\mathrm{H}, \mathrm{Ag}-\mathrm{H}$ and $\mathrm{Cu}-\mathrm{H}$ potentials. The pair potentials have the form of the generalized Morse potential and is given by:

$$
\varphi(r)=D\left(\beta e^{-\alpha\left(r-r_{0}\right)}-\alpha e^{\beta\left(r-r_{0}\right)}\right)
$$

where $D, \alpha, b$, and $r_{0}$ are fitting parameters, $r_{0}$ defines the interatomic spacing between two atoms, and $D(B-\alpha)$ is the binding energy. The $\mathrm{H}$ electron density function is given by:

$$
\rho_{H}(r)=C_{H} e^{-\delta_{H} \cdot r}
$$

which has 2 fitting parameters $C_{H}$ and $\delta_{H}$, while the embedding function for $\mathrm{H}$ has the form:

$$
F_{H, u}(\rho)=-C_{H} \cdot\left(\begin{array}{l}
\frac{1}{2+d_{H}} \cdot\left(\rho+\varepsilon_{H}\right)^{2+d_{H}}-\frac{a_{H}+b_{H}}{1+d_{H}} \cdot\left(\rho+\varepsilon_{H}\right)^{1+d H} \\
+\frac{a_{H} \cdot b_{H}}{d_{H}} \cdot\left(\rho+\varepsilon_{H}\right)^{d_{H}}
\end{array}\right)
$$

where $a_{H}, b_{H}, c_{H}, d_{H}$ are fitting parameters, and $\varepsilon_{H}=0.0540638$.

The EAM total energy is a linear summation of the embedding energy and the pair potentials. $A$ unique feature of the elemental EAM potential is that it will not change due to the transformation of the embedded energy functions. Thus, the embedding and pair potentials for $\mathrm{Pd}-\mathrm{H}$ can be transformed utilizing the two equations below:

$$
\begin{gathered}
F_{i}^{\text {Final }}\left(\rho_{i}\right)=F_{i}^{\text {initial }}\left(\rho_{i}\right)+k \rho_{i} \\
\varphi_{i j}^{\text {Final }}\left(r_{i j}\right)=\varphi_{i j}^{\text {initial }}\left(r_{i j}\right)+2 k \rho_{i}\left(r_{i j}\right)
\end{gathered}
$$

$k$ represents an arbitrary constant. The embedding and pair potentials for $\mathrm{Pd}-\mathrm{H}$ were thus converted in this pattern according to the method of Zhou et al. [4].

\subsection{Palladium Silver Copper Alloys}

2.1.1 Ag and Cu Fitting Parameters 
For the pure metals $\mathrm{Ag}$ and $\mathrm{Cu}$, the EAM potentials were fitted the same way as previously done for Pd [40]. For each metal, the six fitting parameters included $a_{0}, E_{c}, C_{11}, C_{12}, C_{44}$, and $E_{v f}$. Where $a_{0}$ is the lattice constant, $E_{c}$ is the cohesive energy, $C_{11}, C_{12}$, and $C_{44}$ are three elastic constants, and $E_{v f}$ is the vacancy formation energy. Table 1 below lists the $\mathrm{Pd}, \mathrm{Ag}$ and $\mathrm{Cu}$ fitting parameters.

Table 1 Fitting parameters for $\mathrm{Pd}, \mathrm{Ag}$ and $\mathrm{Cu}$.

\begin{tabular}{ccccccc}
\hline & $\boldsymbol{\chi}$ & $\boldsymbol{\varphi} \boldsymbol{e}$ & $\boldsymbol{\delta}$ & $\boldsymbol{B}$ & $\boldsymbol{\eta}$ & $\boldsymbol{\rho} \boldsymbol{e}$ \\
\hline $\mathrm{Pd}$ & 2.054085 & 0.216817 & 8.414105 & 7.221224 & 0.999999 & 3.316887 \\
$\mathrm{Ag}$ & 1.584768 & 0.154164 & 8.491335 & 7.183185 & 1.022270 & 2.213230 \\
$\mathrm{Cu}$ & 2.504500 & 0.175425 & 8.713725 & 6.906629 & 0.560027 & 3.648665 \\
\hline
\end{tabular}

As can be seen from Table 2, the calculated fitting results were almost identical to the experimental values [42] and those obtained by Foiles et al. [48]. In addition, as with Pd, the plots of cohesive energy vs. lattice constant for $\mathrm{Ag}$ and $\mathrm{Cu}$ were also in very good consistency with the equation of state obtained from Rose et al. [47], as shown in Figure 1.

Table 2 Fitting results for $\mathrm{Ag}$ and $\mathrm{Cu}$.

\begin{tabular}{|c|c|c|c|c|c|c|c|}
\hline $\mathrm{Ag}$ & $\begin{array}{l}a_{0} \\
(\mathrm{~nm})\end{array}$ & $\begin{array}{l}E_{c} \\
(\mathrm{eV})\end{array}$ & $\begin{array}{l}C_{11} \\
(\mathrm{GPa})\end{array}$ & $\begin{array}{l}C_{12} \\
(\mathrm{GPa})\end{array}$ & $\begin{array}{l}C_{44} \\
(\mathrm{GPa})\end{array}$ & $\begin{array}{l}B \\
\text { (GPa) }\end{array}$ & $\begin{array}{l}E_{v f} \\
(\mathrm{eV})\end{array}$ \\
\hline Calculations & 0.409 & 2.85 & 123.1 & 94.4 & 46.9 & 104 & 1.08 \\
\hline Simulation & 0.409 & 2.85 & 123.1 & 94.4 & 46.9 & 104 & 1.08 \\
\hline Foiles et al. & 0.409 & 2.85 & 124.0 & 91.0 & 57.0 & 102 & 0.97 \\
\hline Experimental & 0.409 & 2.85 & 124.0 & 93.4 & 46.1 & 104 & 1.10 \\
\hline $\mathrm{Cu}$ & $\begin{array}{l}a_{o} \\
(\mathrm{~nm})\end{array}$ & $\begin{array}{l}E_{c} \\
(\mathrm{eV})\end{array}$ & $\begin{array}{l}C_{11} \\
(\mathrm{GPa})\end{array}$ & $\begin{array}{l}C_{12} \\
(\mathrm{GPa})\end{array}$ & $\begin{array}{l}C_{44} \\
(\mathrm{GPa})\end{array}$ & $\begin{array}{l}\text { B } \\
\text { (GPa) }\end{array}$ & $\begin{array}{l}E_{v f} \\
(\mathrm{eV})\end{array}$ \\
\hline Calculations & 0.3615 & 3.54 & 168.4 & 122.8 & 76.7 & 138 & 1.30 \\
\hline Simulation & 0.3615 & 3.54 & 168.4 & 122.8 & 76.7 & 138 & 1.30 \\
\hline Foiles et al. & 0.3615 & 3.54 & 167.0 & 124.0 & 76.0 & 138 & 1.28 \\
\hline Experimental & 0.3615 & 3.54 & 170.0 & 122.5 & 75.8 & 138 & 1.30 \\
\hline
\end{tabular}




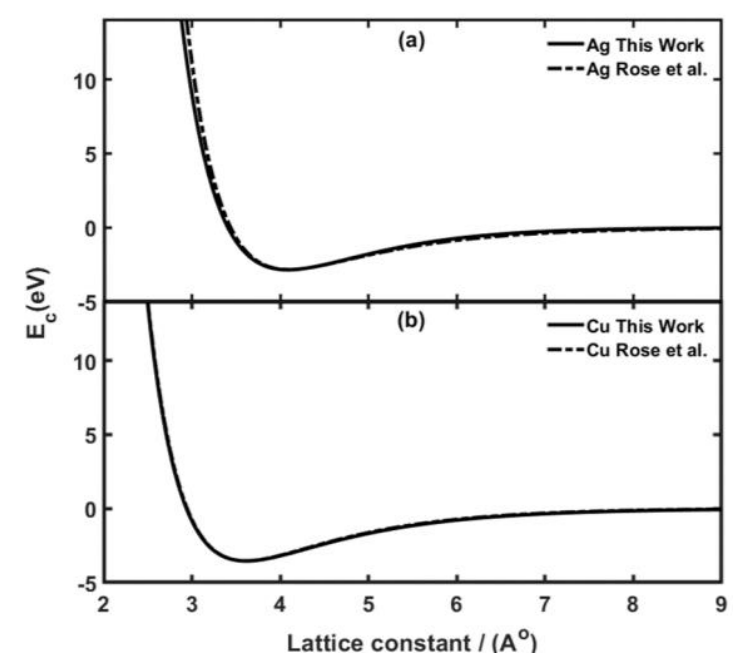

Figure $1 \mathrm{Ag}$ and $\mathrm{Cu}$ Cohesive Energy and Rose et al. Equation of State [47].

To account for the pair potential interactions for $\mathrm{Pd}-\mathrm{Ag}, \mathrm{Pd}-\mathrm{Cu}$ and $\mathrm{Ag}-\mathrm{Cu}$ alloys, the mixing rule between a type-a and a type-b atom interaction introduced by Johnson [49] was applied, and is given by the equation:

$$
\phi_{a b}(r)=\frac{1}{2}\left[\frac{f_{b}(r)}{f_{a}(r)} \phi_{a a}(r)+\frac{f_{a}(r)}{f_{b}(r)} \phi_{b b}(r)\right]
$$

For each type in the alloy, the electron density parameter can be calculated from the equation $f_{\mathrm{e}}=S\left(E_{\mathrm{c}} / \Omega\right)$, where $\Omega$ is the atomic volume and $S$ is a scaling factor, with $S=1$ for pure metals. For type-a atom as a host (solvent) and type-b as impurity (solute), the unrelaxed dilute limit heat of solution can be determined by the five steps given below:

(a) Host removal: $F^{b}\left(\bar{\rho}^{a}\right)+\sum_{i \neq 1} \phi^{a b}\left(r_{l i}^{a}\right)$

(b) Add impurity: $-F^{a}\left(\bar{\rho}^{a}\right)-\sum_{i \neq 1} \phi^{a a}\left(r_{l i}^{a}\right)$

(c) Adjust neighbors: $-\sum_{i \neq 1} F^{a}\left(\bar{\rho}^{a}\right)-\sum_{i \neq 1} F_{i}^{a}\left(\bar{\rho}^{a}-f^{a}\left(r_{l i}^{a}\right)+f^{b}\left(r_{l i}^{a}\right)\right)$

(d) Adjust cohesive energy: $-E_{c}^{a}+E_{c}^{b}$

(e) Relaxation energy: $E_{r}=\left[1.167\left(\Omega_{b} / \Omega_{a}-1\right)\right]^{2}$

where $\bar{\rho}^{a}$ is the expression of electron density for type-a atom, $\mathrm{r}^{\mathrm{a}}$ is the distance to its closest neighbor and $E_{r}$ is the drop in total energy caused by relaxation and is predominantly dependent on the unit cell volume mismatch.

The electron density scaling factors for type-a and type-b atoms, $S_{a}$ and $S_{b}$, for the Pd-Ag, Pd-Cu and $\mathrm{Ag}$-Cu pair potentials, obtained from fitting the experimental heat of solutions, are listed in Table 3 along with the calculated heat of solutions values for each metal. After applying the relaxation energy $E_{r}$, the values for the relaxed heat of solution are very consistent with experimental obtained data and overall better than those obtained by Foiles et al. [48] and Hijazi et al. [42]. 
Table $3 \mathrm{Pd}-\mathrm{Ag}, \mathrm{Pd}-\mathrm{Cu}$ and $\mathrm{Ag}-\mathrm{Cu}$ heat of solution and scaling factors from fitting.

\begin{tabular}{llllll}
\hline $\begin{array}{l}\Delta \mathbf{H}_{\text {solution }} \\
(\mathbf{e V})\end{array}$ & $\begin{array}{l}\text { This work } \\
\text { (relaxed) }\end{array}$ & $\begin{array}{l}\text { Foiles et al. } \\
\text { (relaxed) }\end{array}$ & $\begin{array}{l}\text { Hijazi \& Park } \\
\text { (relaxed) }\end{array}$ & $\begin{array}{l}\text { Exp. } \\
\text { (relaxed) }\end{array}$ & $\begin{array}{l}\text { Scaling } \\
\text { Factor } \\
\text { (S) }\end{array}$ \\
\hline $\mathrm{Pd}$ in $\mathrm{Ag}$ & -0.186 & -0.36 & -0.23 & -0.11 & 1.1063 \\
$\mathrm{Ag}$ in $\mathrm{Pd}$ & -0.207 & -0.24 & -0.17 & -0.29 & 1.8319 \\
$\mathrm{Pd}$ in $\mathrm{Cu}$ & -0.364 & -0.33 & -0.36 & -0.39 & 1.7097 \\
$\mathrm{Cu}$ in $\mathrm{Pd}$ & -0.461 & -0.34 & -0.46 & -0.44 & 1.3419 \\
$\mathrm{Ag}$ in $\mathrm{Cu}$ & 0.257 & 0.18 & 0.19 & 0.25 & 1.4626 \\
$\mathrm{Cu}$ in $\mathrm{Ag}$ & 0.389 & 0.11 & 0.41 & 0.39 & 1.6275 \\
\hline
\end{tabular}

\subsubsection{Validation}

The fitted parameters for $\mathrm{Pd}-\mathrm{Pd}, \mathrm{Ag}-\mathrm{Ag}, \mathrm{Cu}-\mathrm{Cu}, \mathrm{Pd}-\mathrm{Ag}, \mathrm{Pd}-\mathrm{Cu}$ and $\mathrm{Ag}-\mathrm{Cu}$ have been applied to create a tabulated EAM potential file in DYNAMO setfl format for the ternary Pd-Ag-Cu system. Utilizing the tabulated EAM potential file, MD annealing simulations with a Nose-Hoover NPT ensemble from $500 \mathrm{~K}$ to $1 \mathrm{~K}$ in 100 ns were performed for $\mathrm{Ag}-\mathrm{Ag}, \mathrm{Cu}-\mathrm{Cu}, \mathrm{Pd}-\mathrm{Ag}, \mathrm{Pd}-\mathrm{Cu}$ and $\mathrm{Ag}-\mathrm{Cu}$ structures using a LAMMPS script code [50]. A molecular statics (MS) simulation utilizing the conjugate gradient $(\mathrm{cg})$ minimization method was then applied after each MD simulation. The MD simulation results for $\mathrm{Ag}-\mathrm{Ag}, \mathrm{Cu}-\mathrm{Cu}$ were in excellent agreement with the calculated fitting results, as can be seen from Table 2, and the MD results proved the reliability of the $\mathrm{Pd}-\mathrm{Ag}, \mathrm{Pd}-\mathrm{Cu}$ and $\mathrm{Ag}$ $\mathrm{Cu}$ EAM potentials as illustrated in Figure 2, Figure 3 and Figure 4.

Figure 2(a) and (b) show our lattice constant and cohesive energy results for the $\operatorname{Pd}_{x} A g_{1-x}$ and $\mathrm{Pd}_{x} \mathrm{Cu}_{1-x}(0 \leq x \leq 1)$ structures are almost identical with the experimental data [51]. For the $\mathrm{Pd}_{x} \mathrm{Ag}_{1-x}$ structures, the lattice constant results are closer to the experimental values than the results calculated using the Hale et al.'s EAM potential with Morse pair function [5]. On the other hand, the density functional theory (DFT) data collected from Løvvik et al. [52] reveal a similar trend but tend to overestimate the values for all compositions. Figure 2(b) shows that our cohesive energy values for $\mathrm{Pd}-\mathrm{Ag}$ are very much in line with the values that are derived using the Hale et al. with Morse function [5]. The Hale et al. EAM potentials were obtained from the Interatomic Potential Repository [53]. However, their EAM potential with the hybrid model produced erratic results and was not included with the Figures.

For the $\mathrm{Pd}_{x} \mathrm{Cu}_{1-x}$ structures, the lattice constant values from MD simulations are almost identical with the experimental values and those obtained by Kart et al. [28] as shown in Figure 2(a). For the cohesive energies for $\mathrm{Pd}_{x} \mathrm{Cu}_{1-x}$, our MD values have an increasing trend similar to the data obtained by Kart et al. [28], as can be seen in Figure 2(b). 


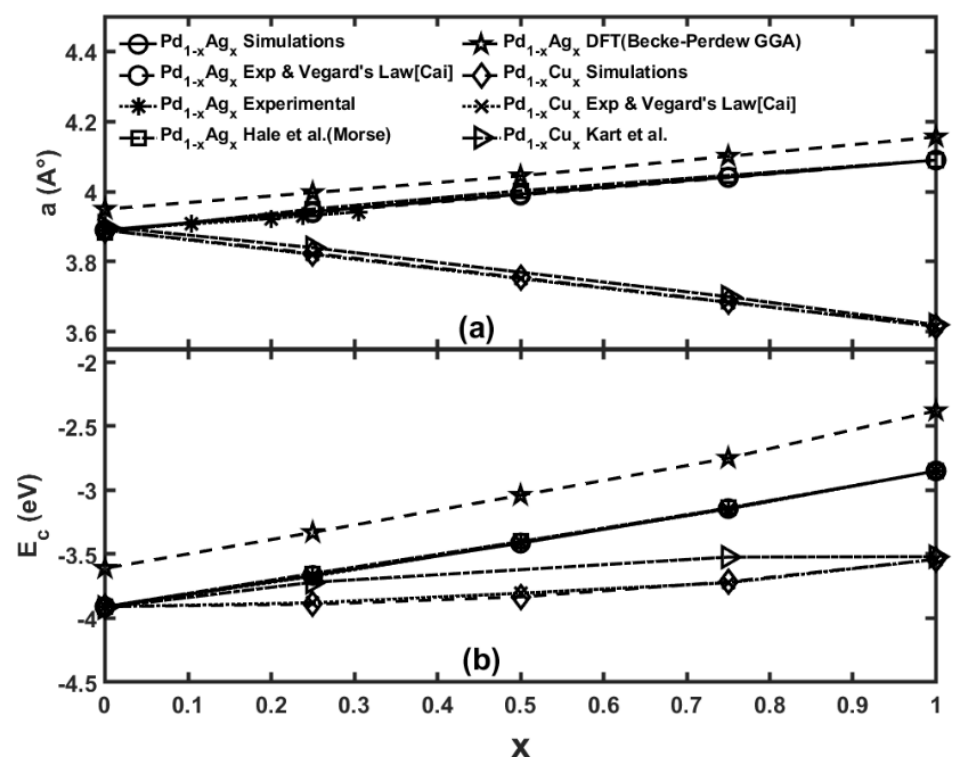

Figure $2 \mathrm{Pd}_{1-x} \mathrm{Ag}_{x}$ and $\mathrm{Pd}_{x} \mathrm{Cu}_{1-x}$ structures $a_{o}$ and $E_{c}$ results obtained from MD simulations, experimental and DFT calculations $[28,55,52,51]$.

In Figure 3(a) and (b) the values for the elastic constants $C_{11}, C_{12}$ from MD simulations for $\mathrm{Pd}_{1-x} \mathrm{Ag}_{x}$ and $\mathrm{Pd}_{1-x} \mathrm{Cu}_{x}$ structures show consistent trend with the DFT calculations and the results from Hale et al. and Kart et al. $[54,5,28]$. The bulk modulus for $\mathrm{Pd}_{1-x} \mathrm{Ag}_{x}$ and $\mathrm{Pd}_{1-x} \mathrm{Cu}_{x}$ structures obtained from MD simulations match the softening trends predicted by the DFT calculations as well $[54,52,28]$ and match the given experimental data at the edge of the composition range quite closely, as shown in Figure 3(c). It's worth noticing that the Hale et al. [5] EAM potential overestimates $C_{11}, C_{12}$ and bulk modulus for the pure Pd metal, as can be seen at the left edges of Figure 3(a), (b) and (c).

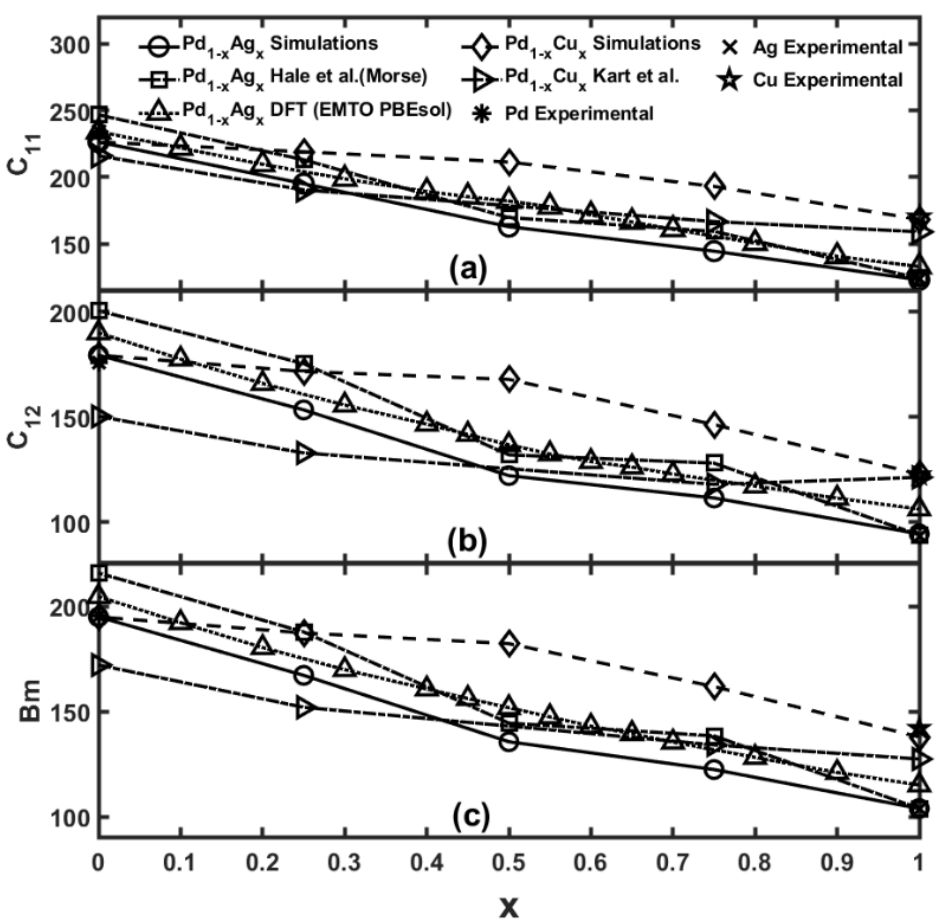

Figure $3 \mathrm{Pd}_{1-x} \mathrm{Ag}_{x}$ and $\mathrm{Pd}_{x} \mathrm{Cu}_{1-x}$ alloys $C_{11}, C_{12}$ elastic constant and $\mathrm{B}_{\mathrm{m}}$ results obtained from MD simulations, experimental and DFT calculations $[28,56]$. 
In Figure 4, the values for the elastic constants $C_{44}$ and $C^{\prime}$ from MD simulations for $\mathrm{Pd}_{1-x} A g_{x}$ show that our results are closer to the experimental data at the edge of the composition range than those of Hale et al. [5]. As with the Hale et al. EAM Morse model [5], our $\mathrm{Pd}_{1-x} \mathrm{Ag}_{x}$ potential underestimates $C_{44}$ relative to the DFT results and have an overall decreasing trend. For $\mathrm{Pd}_{1-x} \mathrm{Cu}_{x}$, our $C_{44}$ and $C^{\prime}$ values have a slightly increasing trend, with $C^{\prime}$ for $P d$ being underestimated, but still more consistent with the experimental data than the results from Kart et al. [28].

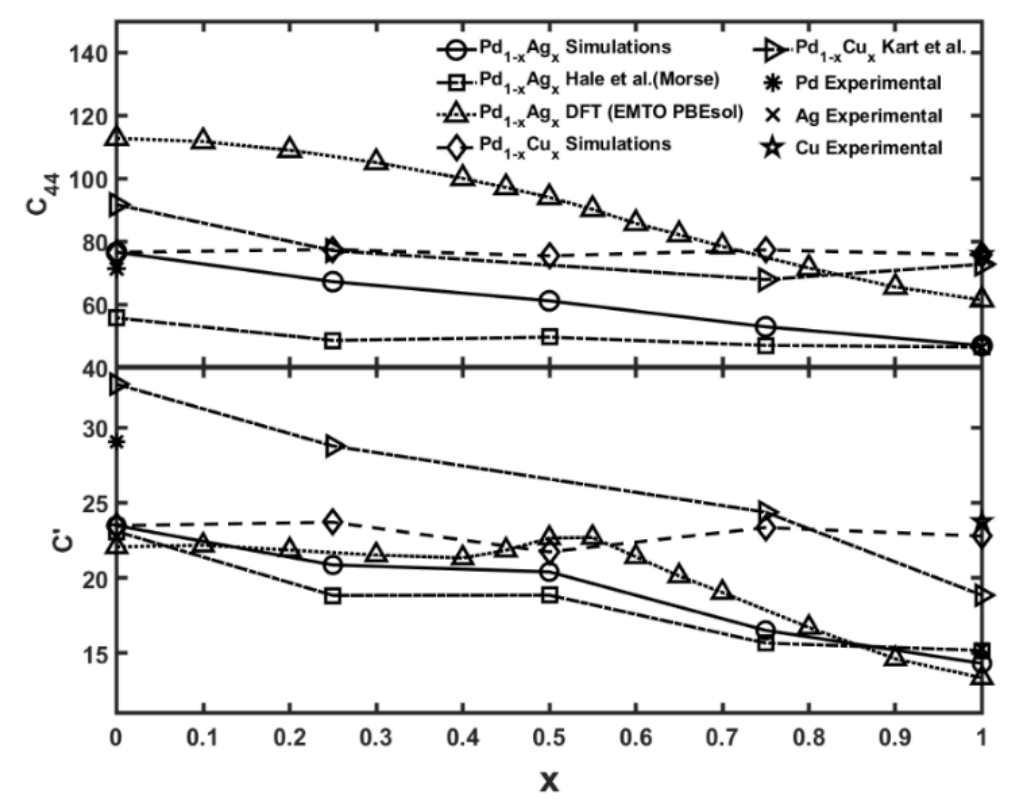

Figure $4 \mathrm{Pd}_{1-x} \mathrm{Ag}_{x}$ and $\mathrm{Pd}_{x} \mathrm{Cu}_{1-x}$ alloys $C_{44}$ and $C^{\prime}$ elastic constant results obtained from MD simulations, experimental and DFT calculations $[28,56]$.

\section{Palladium Silver and Palladium Copper Hydrides}

\subsection{DFT Calculations}

Since $\mathrm{H}$ is almost insoluble in $\mathrm{Ag}[24,57]$, and no experimental fitting data was found for $\mathrm{Ag}-\mathrm{H}$ and $\mathrm{Cu}-\mathrm{H}$ systems [40], therefore, the $\mathrm{Pd}-\mathrm{Ag}-\mathrm{H}$ and $\mathrm{Pd}-\mathrm{Cu}-\mathrm{H}$ properties were used as fitting data to fit the $\varphi_{A g-H}$ and $\varphi_{C u-H}$ pair functions. However, only a limited experimental and ab initio data were available to utilize a full $\mathrm{H}$ concentration in the fitting procedure. Hale et al. obtained their fitting data by utilizing DFT calculations for the Pd-Ag-H system [5], but failed to provide the lattice constant values, and only the cohesive energy values were given. Wei et al. performed DFT calculations on $\mathrm{Pd}-\mathrm{Cu}-\mathrm{H}$ phase stability, heat of formations and elastic property based on generalized gradient approximations (GGA) for the range of hydrogen concentration $0 \leq x \leq 0.5$, but they failed to report the exact values for the lattice constants and the cohesive energies [58]. In this paper, the open source SIESTA software was used to perform ab initio simulations to get full fitting data for the Pd-Ag-H and Pd-Cu-H structures. The SIESTA pseudopotentials were obtained from the Abinit's Fritz-Haber-Institute (FHI) pseudo database [59]. The local density approximation (LDA) method with Ceperley-Alder exchange and correlation form using the norm-conserving TroullierMartins scheme was utilized in the pseudopotentials. Valence states were described using double zeta-polarized (DZP) basis sets with split-valence scheme for multiple-zeta. The ab initio simulations were conducted with a dense $18 \times 18 \times 18$ Monkhorst-Pack grid, a cutoff energy of $100 \mathrm{Ry}$, a $25 \mathrm{~K}$ 
electronic temperature, and electron spin polarization during the DFT calculations. For our Pd-Ag-H and $\mathrm{Pd}-\mathrm{Cu}-\mathrm{H}$ structures, the calculations utilized periodic boundary conditions with a unit cell of 3 $\mathrm{Pd}$ atoms, $1 \mathrm{Ag}$ or $\mathrm{Cu}$ atom, and 1 to $4 \mathrm{H}$ atoms at different locations.

During the DFT simulations, the $\mathrm{Pd}_{0.75} \mathrm{Ag}_{0.25} \mathrm{H}_{x}$ and $\mathrm{Pd}_{0.75} \mathrm{Cu}_{0.25} \mathrm{H}_{x}$ structures were constructed with five different $\mathrm{H}$ concentrations: $x=0,0.25,0.50,0.75$, and 1.00. In the Pd-Ag and Pd-Cu fcc lattice, $\mathrm{H}$ atoms were located in three different interstitial positions. As shown in Figure 5 , these positions included the octahedral $(\mathrm{O})$ positions, in which $\mathrm{O} 1$ represent a body center position and $\mathrm{O} 2$ an edge center position, and the tetrahedral (TE) positions.
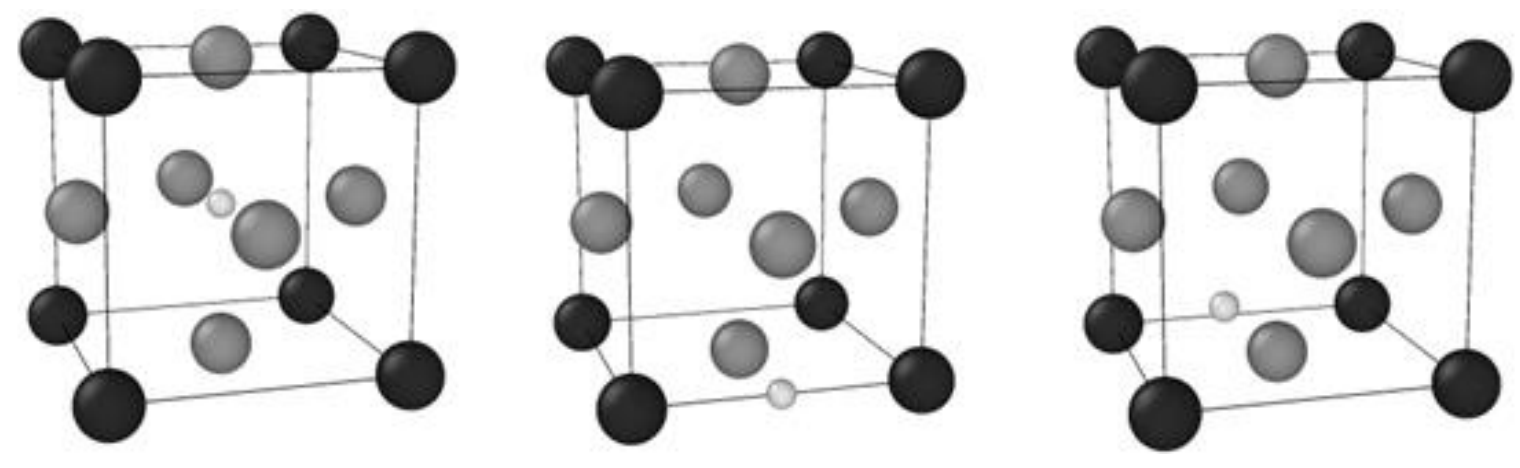

Figure $5 \mathrm{H}$ atoms (white) interstitial different positions in the $\mathrm{Pd}$ atoms (gray) and $\mathrm{Ag}$ or $\mathrm{Cu}$ atoms (black) lattice.

As with Løvvik et al. $\mathrm{Pd}_{1-x} \mathrm{Ag}_{x}$ DFT results from Figure 2(a) and (b) [52], the lattice constant and cohesive energy results from our DFT simulations for the $\mathrm{Pd}-\mathrm{Ag}-\mathrm{H}$ and $\mathrm{Pd}-\mathrm{Cu}-\mathrm{H}$ structures were also overestimated in comparison to the available experimentally obtained data for the $\mathrm{Pd}, \mathrm{Ag}, \mathrm{Cu}$, $\mathrm{PdH}_{0.50}, \mathrm{PdH}_{1.00}, \mathrm{Pd}_{0.75} \mathrm{Ag}_{0.25}$ and $\mathrm{Pd}_{0.75} \mathrm{Cu}_{0.25}$ structures. The calculated DFT values can be shifted, if multiplied with a selected factor, to make it consistent with the experimental data [60]. Equations 12 to 15 describe the shifting procedure for the cohesive energies for $\mathrm{Pd}_{0.75} \mathrm{Ag}_{0.25} \mathrm{H}_{x}$ structures, which have been applied in a similar manner to the $\mathrm{Pd}_{0.75} \mathrm{Cu}_{0.25} \mathrm{H}_{x}$ case by replacing $\mathrm{Ag}$ atoms with $\mathrm{Cu}$. The shifting procedure was also applied in a similar fashion to the lattice constants case. The shifting data for $\mathrm{Pd}_{0.75} \mathrm{Ag}_{0.25} \mathrm{H}_{x}$ and $\mathrm{Pd}_{0.75} \mathrm{Cu}_{0.25} \mathrm{H}_{x}$ structures are given in Table 4 and the shifting factors in Table 5.

$$
\begin{aligned}
& \left(\text { Cohesive Energy }{ }_{\mathrm{Pd}_{0.75} \mathrm{Ag}_{0.25} \mathrm{H}_{\mathrm{X}}}\right)_{\text {SIESTA shifted }}=\left(\mathrm{C}_{\mathrm{Pd}_{0.75} \mathrm{Ag}_{0.25}}+\mathrm{x} \cdot \mathrm{C}_{\mathrm{H}}\right) \\
& \text { · }\left(\text { Cohesive Energy }{ }_{\mathrm{Pd}_{0.75}} \mathrm{Ag}_{0.25} \mathrm{H}_{\mathrm{X}}\right)_{\text {SIESTA }} \\
& \mathrm{C}_{\mathrm{Pd}_{0.75} \mathrm{Ag}_{0.25}}=\frac{\left(\text { Cohesive Energy } \mathrm{Pd}_{0.75} \mathrm{Ag}_{0.25}\right)_{\text {Experimental }}}{\left(\text { Cohesive Energy } \mathrm{Pd}_{0.75} \mathrm{Ag}_{0.25}\right)_{\text {SIESTA }}} \\
& \mathrm{C}_{H}=2 \cdot\left\{\frac{\left(\text { Cohesive Energy }_{\mathrm{PdH}_{0.50}}\right)_{\text {Experimental }}}{\left(\text { Cohesive Energy }{ }_{\mathrm{PdH}_{0.50}}\right)_{\text {SIESTA }}}-\mathrm{C}_{P d}\right\}
\end{aligned}
$$

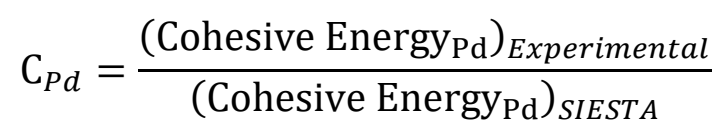


Table 4 Experimental values used in shifting ab initio data.

\begin{tabular}{lcl}
\hline Structure & Lattice Constant $\left(\mathrm{A}^{\mathbf{0}}\right)$ & Cohesive Energy $(\mathrm{eV})$ \\
\hline $\mathrm{Pd}$ & 3.89 & -3.91 \\
$\mathrm{PdH} \mathrm{H}_{0.50}$ & - & -3.4877 \\
$\mathrm{PdH}_{1.00}$ & 4.12 & - \\
$\mathrm{Pd}_{0.75} \mathrm{Ag}_{0.25}$ & 3.94 & -3.65 \\
$\mathrm{Pd}_{0.75} \mathrm{Cu}_{0.25}$ & 3.821 & -3.8775 \\
\hline
\end{tabular}

Table 5 Shifting factors for Pd-Ag-H and Pd-Cu-H ab initio data.

\begin{tabular}{cccc}
\hline \multicolumn{2}{c}{ Lattice Constant } & \multicolumn{2}{c}{ Cohesive Energy } \\
\hline Structure & Factor & Structure & Factor \\
$\mathrm{C}_{\mathrm{Pd}_{0.75} \mathrm{Ag}_{0.25}}$ & 0.98546 & $\mathrm{C}_{\mathrm{Pd}_{0.75} \mathrm{Ag}_{0.25}}$ & 0.78677 \\
$\mathrm{C}_{\mathrm{Pd}_{0.75} \mathrm{Cu}_{0.25}}$ & 0.98255 & $\mathrm{C}_{\mathrm{Pd}_{0.75} \mathrm{Cu}_{0.25}}$ & 0.79091 \\
$\mathrm{C}_{H}$ & 0.01768 & $\mathrm{C}_{H}$ & 0.02107 \\
$\mathrm{C}_{P d}$ & 0.98232 & $\mathrm{C}_{P d}$ & 0.79165 \\
\hline
\end{tabular}

The shifted cohesive energy values for the $\mathrm{Pd}_{0.75} \mathrm{Ag}_{0.25} \mathrm{H}_{x}$ structures are close to those obtained by Hale et al. DFT calculations [5] and have similar trend, as can be seen in Figure 6 . Figure 6 also shows that the $\mathrm{Pd}_{0.75} \mathrm{Cu}_{0.25} \mathrm{H}_{x}$ shifted data have a similar trend to the $\mathrm{Pd}_{0.75} \mathrm{Ag}_{0.25} \mathrm{H}_{x}$ shifted data, but with lower cohesive energy values.

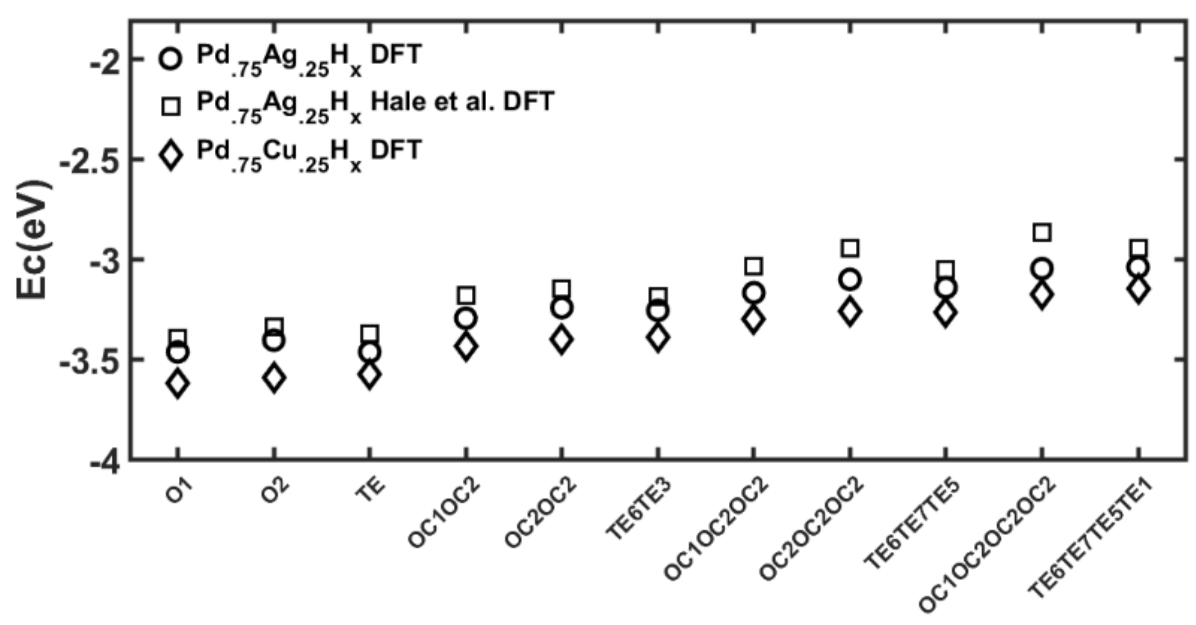

Figure $6 \mathrm{Pd}_{0.75} \mathrm{Ag}_{0.25} \mathrm{H}_{x}$ and $\mathrm{Pd}_{0.75} \mathrm{Cu}_{0.25} \mathrm{H}_{x}$ shifted cohesive energies compared with Hale et al. DFT results.

The shifted values for the cohesive energy and lattice constant obtained from the DFT simulations are listed in Table S1 in the supplementary data. The shifted cohesive energies for 7 OC structures and 7 TE structures with 4 different $\mathrm{H}$ concentrations, were used in fitting the $\varphi_{\mathrm{Ag}-\mathrm{H}}$ and $\varphi_{\mathrm{C} U-H}$ pair potential functions during the fitting process. The $\varphi_{\mathrm{Ag}-\mathrm{H}}$ and $\varphi_{\mathrm{Cu}-\mathrm{H}}$ pair functions take the same generalized Morse potential mathematical form, as used previously in the $\mathrm{Pd}-\mathrm{H}$ interaction 
[46]. Since a third atom type was added to the binary Pd-H structures to create the ternary Pd-Ag$\mathrm{H}$ and $\mathrm{Pd}-\mathrm{Cu}-\mathrm{H}$ structures, all $\mathrm{Pd}-\mathrm{H}$ potentials and property equations utilized in the fitting procedure were expanded by adding a central atom expression as a third type [40]. For the ternary system, the cohesive energy equation has an additional host term and is given by:

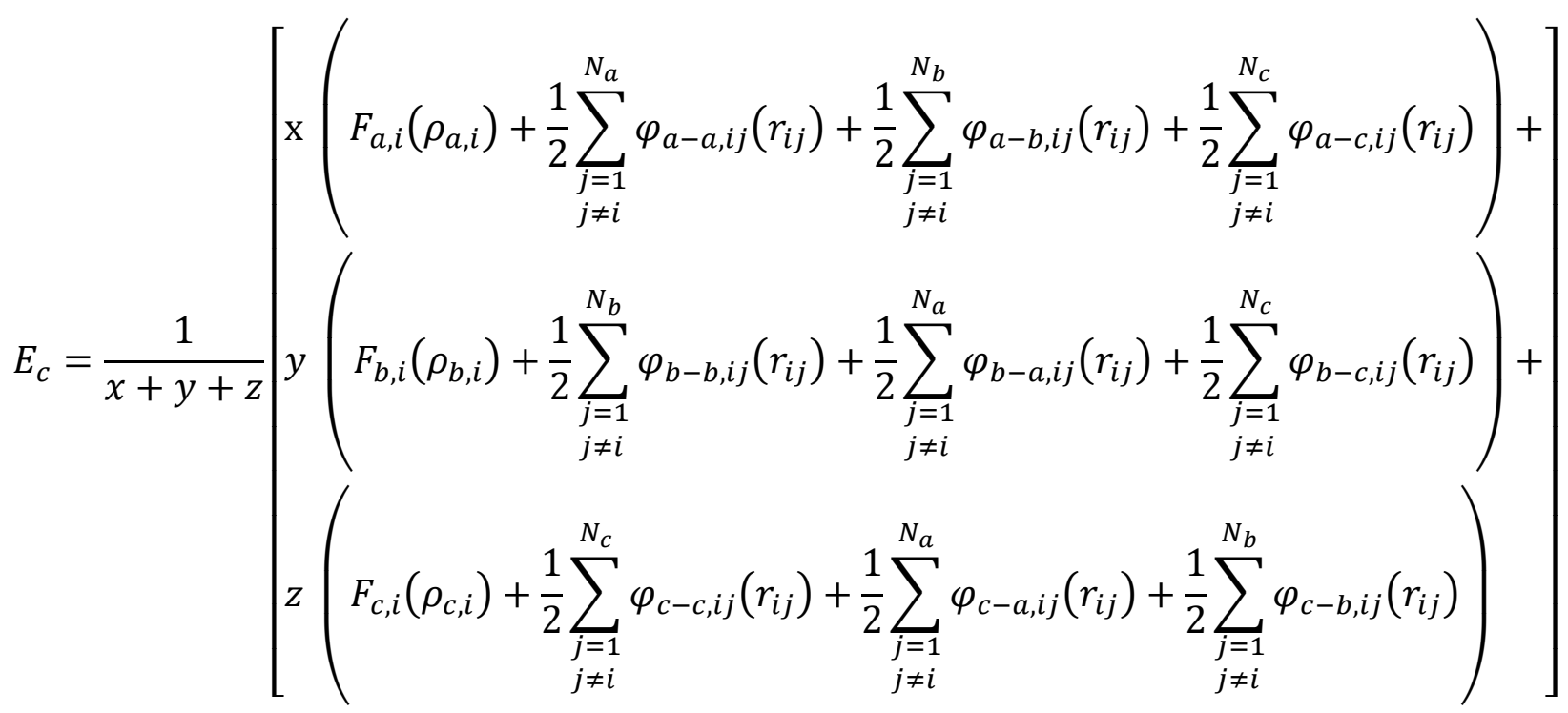

$$
\begin{aligned}
& \rho_{a, i}=\rho_{a-a, i}+\rho_{a-b, i}+\rho_{a-c, i}=\sum_{\substack{j=1 \\
j \neq i}}^{N_{a}} f_{a}\left(r_{i j}\right)+\sum_{\substack{j=1 \\
j \neq i}}^{N_{b}} f_{b}\left(r_{i j}\right)+\sum_{\substack{j=1 \\
j \neq i}}^{N_{c}} f_{c}\left(r_{i j}\right) \\
& \rho_{b, i}=\rho_{b-a, i}+\rho_{b-b, i}+\rho_{b-c, i}=\sum_{\substack{j=1 \\
j \neq i}}^{N_{a}} f_{a}\left(r_{i j}\right)+\sum_{\substack{j=1 \\
j \neq i}}^{N_{b}} f_{b}\left(r_{i j}\right)+\sum_{\substack{j=1 \\
j \neq i}}^{N_{c}} f_{c}\left(r_{i j}\right) \\
& \rho_{c, i}=\rho_{c-a, i}+\rho_{c-b, i}+\rho_{c-c, i}=\sum_{\substack{j=1 \\
j \neq i}}^{N_{a}} f_{a}\left(r_{i j}\right)+\sum_{\substack{j=1 \\
j \neq i}}^{N_{b}} f_{b}\left(r_{i j}\right)+\sum_{\substack{j=1 \\
j \neq i}}^{N_{c}} f_{c}\left(r_{i j}\right)
\end{aligned}
$$

where $a, b$, and $c$ are three different type of atoms, and $x, y$, and $z$ are the concentrations for each type of atom in the structure respectively. A constrained nonlinear optimization MATLAB code was used during the fitting procedure to obtain the fitting parameters. Table 6 lists the parameters for the $\mathrm{Ag}-\mathrm{H}$ and $\mathrm{Cu}-\mathrm{H}$ from fitting, and the $\mathrm{Pd}-\mathrm{H}$ parameters from our previous work [40].

Table 6 Fitting data used for $\mathrm{Pd}-\mathrm{H}, \mathrm{Cu}-\mathrm{H}$ and $\mathrm{Ag}-\mathrm{H}$.

\begin{tabular}{lllll}
\hline System & $\mathbf{D}$ & $\boldsymbol{\alpha}$ & $\boldsymbol{\beta}$ & $\mathbf{r}_{\mathbf{0}}$ \\
\hline $\mathrm{Pd}-\mathrm{H}$ & 0.740938 & 2.373944 & 1.702142 & 1.300000 \\
$\mathrm{Ag}-\mathrm{H}$ & 1.476745 & 1.967649 & 1.741865 & 1.850017 \\
$\mathrm{Cu}-\mathrm{H}$ & 0.799995 & 1.730082 & 1.400001 & 1.500002 \\
\hline
\end{tabular}


The previously obtained fitting data for the $\mathrm{H}-\mathrm{H}$ potential are also included in Table 7 [40]. The two body potential functions used in our Pd-Ag-Cu- $\mathrm{H}$ model are plotted in Figure 7 and Figure 8 . The calculated cohesive energies for $\mathrm{Pd}_{0.75} \mathrm{Ag}_{0.25} \mathrm{H}_{x}$ and $\mathrm{Pd}_{0.75} \mathrm{Cu}_{0.25} \mathrm{H}_{x}$ structures from fitting are consistent with the fitting data for most of the $\mathrm{H}$ concentrations, but the results start to diverge from the fitting data at high $\mathrm{H}$ concentrations as can be seen in Figure 9(b).

Table 7 Fitting data for $\mathrm{H}-\mathrm{H}$.

\begin{tabular}{lllll}
\hline $\boldsymbol{D}_{\boldsymbol{H}}$ & $\boldsymbol{\alpha}_{\boldsymbol{H}}$ & $\boldsymbol{B}_{\boldsymbol{H}}$ & $\boldsymbol{r}_{\mathrm{o}, H H}$ & $\boldsymbol{C}_{\boldsymbol{H}}$ \\
\hline 0.589510 & 1.104827 & 0.942490 & 3.474173 & 2.145808 \\
$\delta_{H}$ & $a_{H}$ & $b_{H}$ & $C_{H}$ & $d_{H}$ \\
0.942201 & 8.370790 & 62.343273 & 0.000100 & 1.187000 \\
\hline
\end{tabular}

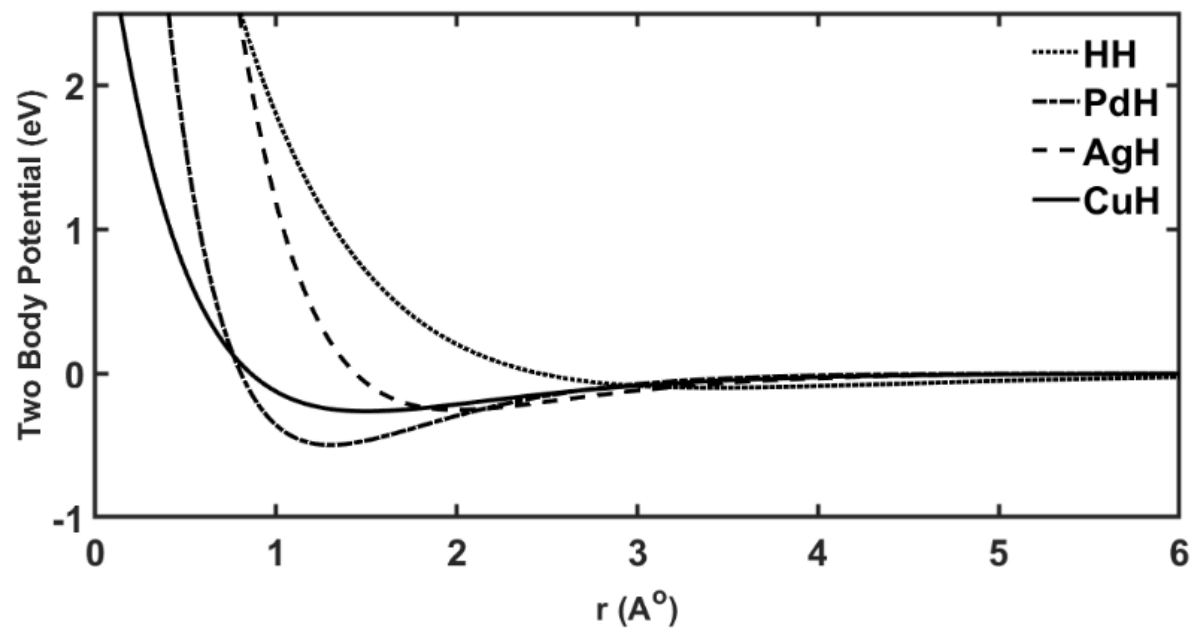

Figure $7 \mathrm{H}-\mathrm{H}, \mathrm{Pd}-\mathrm{H}, \mathrm{Ag}-\mathrm{H}$ and $\mathrm{Cu}-\mathrm{H}$ two body potential functions.

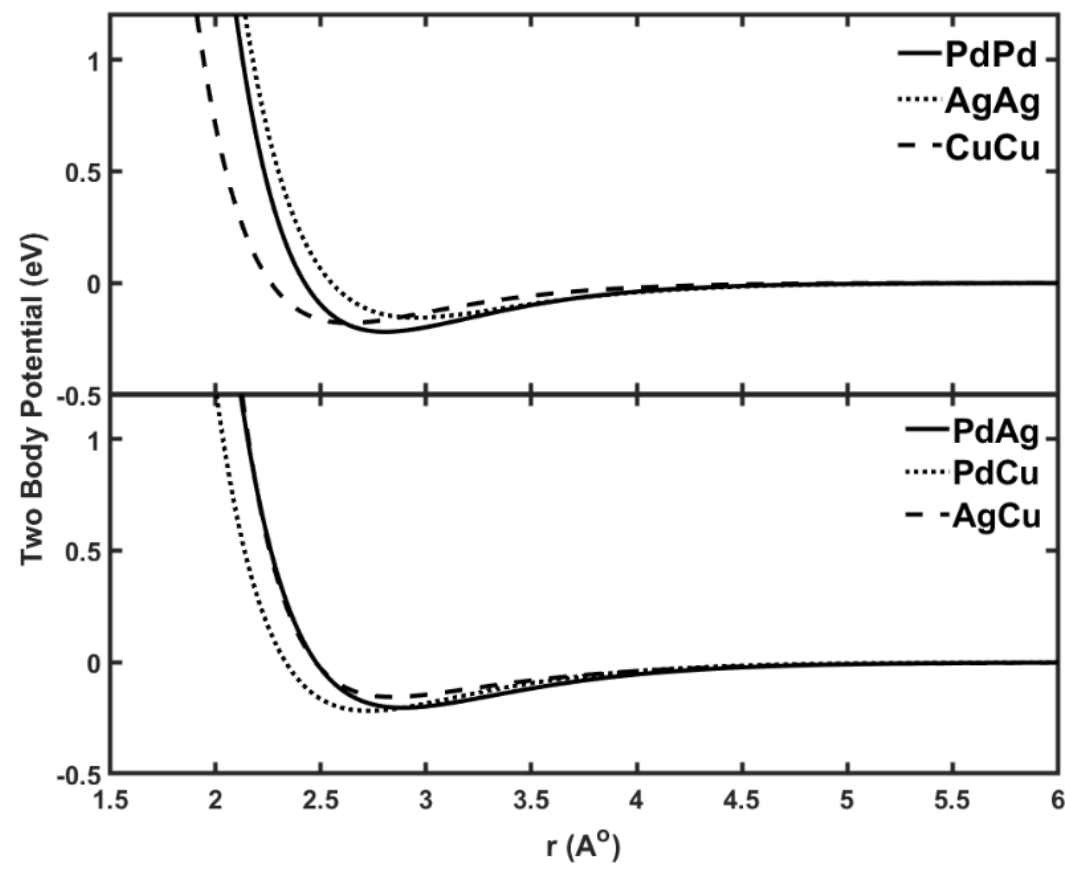

Figure $8 \mathrm{Pd}-\mathrm{Pd}, \mathrm{Ag}-\mathrm{Ag}, \mathrm{Cu}-\mathrm{Cu}, \mathrm{Pd}-\mathrm{Ag}, \mathrm{Pd}-\mathrm{Cu}$ and $\mathrm{Ag}-\mathrm{Cu}$ two body potential functions. 


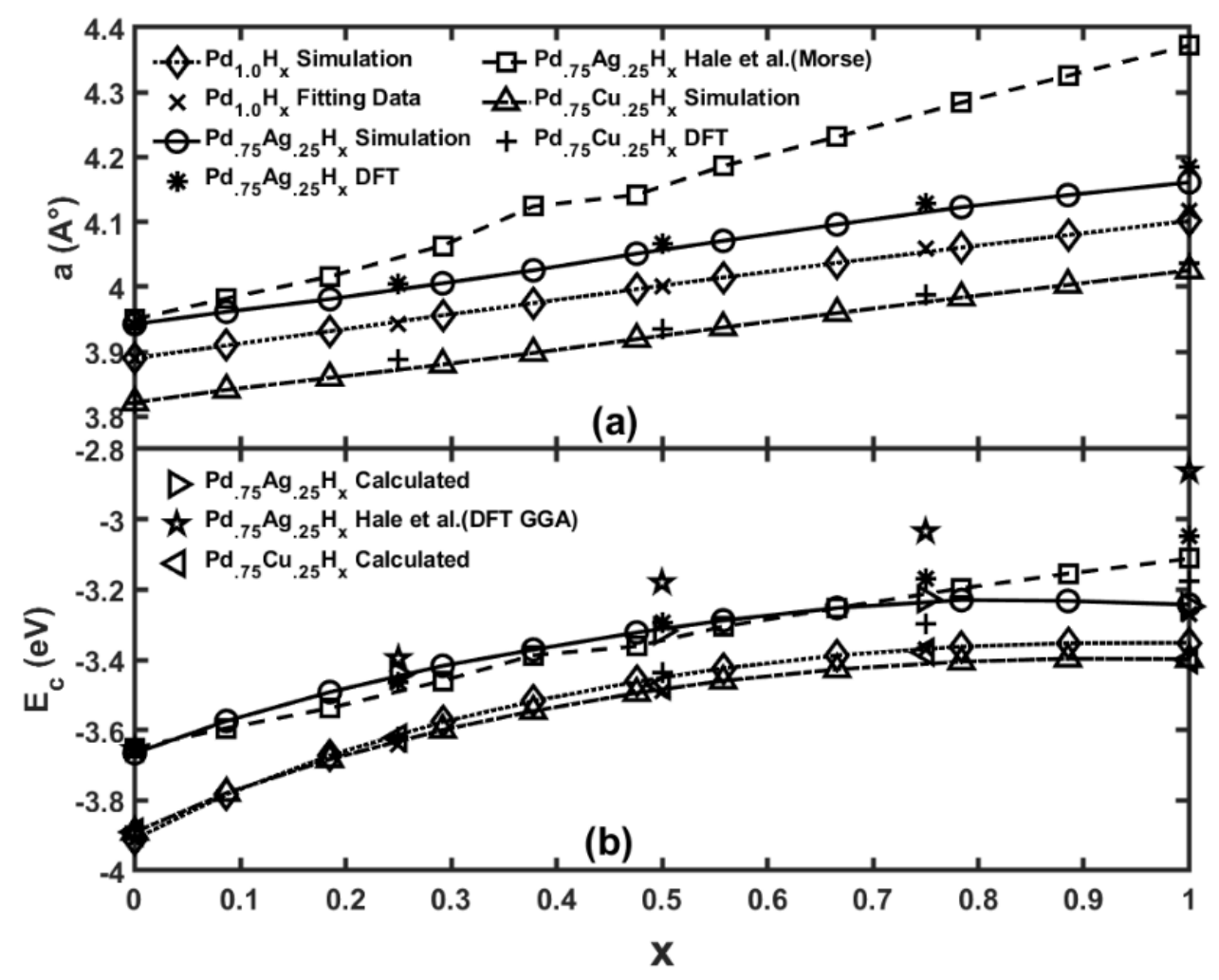

Figure $9 \mathrm{Pd}_{1.00} \mathrm{H}_{x}, \mathrm{Pd}_{0.75} \mathrm{Ag}_{0.25} \mathrm{H}_{x}$ and $\mathrm{Pd}_{0.75} \mathrm{Cu}_{0.25} \mathrm{H}_{x} a_{o}$ and $E_{c}$ results obtained from $\mathrm{MD}$ simulations and fitting.

\subsection{Results}

To test the reliability of the $\mathrm{Pd}-\mathrm{Ag}-\mathrm{Cu}-\mathrm{H}$ potentials, a tabulated potential file in DYNAMO setfl format was generated utilizing the final fitting parameters. Utilizing LAMMPS and the tabulated potential file, MD annealing simulations with a Nose-Hoover NPT ensemble from $500 \mathrm{~K}$ to $1 \mathrm{~K}$ in 100 ns with random hydrogen atom positions were performed for the $\mathrm{Pd}_{0.75} \mathrm{Ag}_{0.25} \mathrm{H}_{x}$ and $\mathrm{Pd}_{0.75} \mathrm{Cu}_{0.25} \mathrm{H}_{x}$ structures. A molecular statics (MS) simulation utilizing the conjugate gradient (cg) minimization method was then applied after each MD simulation. Ten sets of data were generated for each $\mathrm{H}$ composition and their average values were taken to ensure accuracy.

\subsection{Lattice Constant and Cohesive Energy Results}

The stress triggered by variation in the lattice constants in regions with different $\mathrm{H}$ concentrations at equilibrium is of important consideration [4]. Therefore, the influence of $\mathrm{H}$ concentration on the equilibrium lattice constant was investigated. As can be seen in Figure 9(a), the lattice constant values obtained from MD simulations for $\mathrm{Pd}_{0.75} \mathrm{Ag}_{0.25} \mathrm{H}_{x}$ and $\mathrm{Pd}_{0.75} \mathrm{Cu}_{0.25} \mathrm{H}_{x}$ structures are almost identical with the DFT results used in the fitting process. The lattice constant plots show an increasing trend with increasing $\mathrm{H}$ concentrations, similar with the DFT calculated data and the $\mathrm{Pd}_{1.00} \mathrm{H}_{x}$ results in our previous work [40]. The increasing trend from our plots is also consistent with the results using the Hale et al. EAM potential with the Morse function for $\mathrm{Pd}_{0.75} \mathrm{Ag}_{0.25} \mathrm{H}_{x}$ [5], and the DFT simulation results for the fcc Pd-Cu-H (O1) from Wei et al. [58].

For the cohesive energy, the results for $\mathrm{Pd}_{0.75} \mathrm{Ag}_{0.25} \mathrm{H}_{x}$ and $\mathrm{Pd}_{0.75} \mathrm{Cu}_{0.25} \mathrm{H}_{x}$ structures shown in Figure $9(\mathrm{~b})$, were in good agreement with our fitting results and DFT data, and also have a similar 
trend to the $\mathrm{Pd}_{1.00} \mathrm{H}_{x}$ plot [40]. For the $\mathrm{Pd}_{0.75} \mathrm{Ag}_{0.25} \mathrm{H}_{x}$ structures, our MD results are closer to our DFT data than the Hale et al. EAM Morse potential MD results with respect to their own DFT fitting data [5].

\subsection{Elastic Constants and Bulk Modulus}

Using the relaxed $\mathrm{Pd}_{0.75} \mathrm{Ag}_{0.25} \mathrm{H}_{x}$ and $\mathrm{Pd}_{0.75} \mathrm{Cu}_{0.25} \mathrm{H}_{x}$ structures obtained from our $\mathrm{MD}+\mathrm{MS}$ simulations and utilizing an adaptation of the general-purpose LAMMPS script written by Aiden P. Thompson at Sandia National Laboratories [50], the elastic constants and bulk modulus values were estimated. Figure 10 shows that the elastic constants $C_{11}$ and $C_{12}$ and bulk modulus for $P_{0.75} A_{0.25} H_{x}$, $\mathrm{Pd}_{0.75} \mathrm{Cu}_{0.25} \mathrm{H}_{x}$ and $\mathrm{Pd}_{1.00} \mathrm{H}_{x}$ display smooth curves with similar trends. Although, as stated previously, that the Hale et al. EAM potential overestimates the bulk modulus for pure Pd, our overall decreasing trend matches well with the results obtained by Hale et al. EAM Morse potential for the $\mathrm{Pd}_{0.75} \mathrm{Ag}_{0.25} \mathrm{H}_{x}$ structures and in a good agreement with our previously obtained results for $\mathrm{Pd}_{1.00} \mathrm{H}_{x}$ $[5,40]$. The bulk modulus values from our MD simulations for $\mathrm{Pd}_{0.75} \mathrm{Cu}_{0.25} \mathrm{H}_{x}$ also have a similar decreasing trend with those obtained by Wei et al. DFT simulations for fcc Pd-Cu-H (01) [58]. Other researchers have also documented this softening behavior with increasing $\mathrm{H}$ concentrations [61-63]. The results for $\mathrm{Pd}_{0.75} \mathrm{Ag}_{0.25} \mathrm{H}_{x}$ structures obtained from Hale et al. Hybrid potential yielded an unstable trend but still had an overall similar softening trend [5,53]. As for the $\mathrm{Pd}_{0.75} \mathrm{Cu}_{0.25} \mathrm{H}_{x}$ compositions, our simulation results for $C_{11}$ and $C_{12}$ also have a similar smooth overall decreasing trend to those obtained by Wei et al. [58].

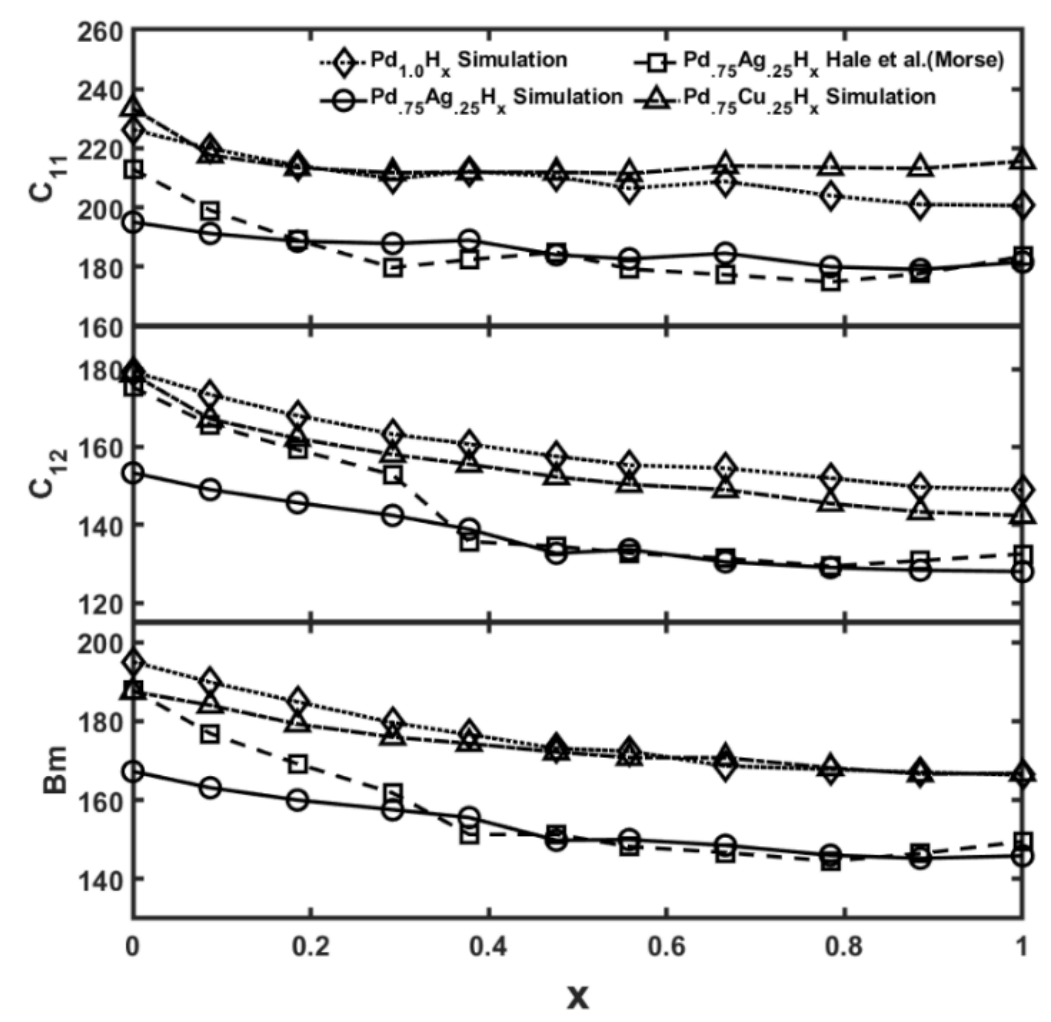

Figure $10 \mathrm{Pd}_{1.00} \mathrm{H}_{\mathrm{x}}, \mathrm{Pd}_{0.75} \mathrm{Ag}_{0.25} \mathrm{H}_{\mathrm{x}}$ and $\mathrm{Pd}_{0.75} \mathrm{Cu}_{0.25} \mathrm{H}_{x} C_{11}, \mathrm{C}_{12}$ elastic constant and $\mathrm{B}_{\mathrm{m}}$ results from MD simulations. 
Figure 11 shows the elastic constants $C_{44}$ and $C^{\prime}$ for $\mathrm{Pd}_{1.00} \mathrm{H}_{x}, \mathrm{Pd}_{0.75} \mathrm{Ag}_{0.25} \mathrm{H}_{x}$ and $\mathrm{Pd}_{0.75} \mathrm{Cu}_{0.25} \mathrm{H}_{x}$ alloys. As previously obtained for $\mathrm{Pd}_{1.00} \mathrm{H}_{x}$ [40], the plot values for $C_{44}$ for $\mathrm{Pd}_{0.75} \mathrm{Ag}_{0.25} \mathrm{H}_{x}$ and $\mathrm{Pd}_{0.75} \mathrm{Cu}_{0.25} \mathrm{H}_{x}$ decrease while the shear elastic constants $C^{\prime}$ increase with increasing $\mathrm{H}$ composition.

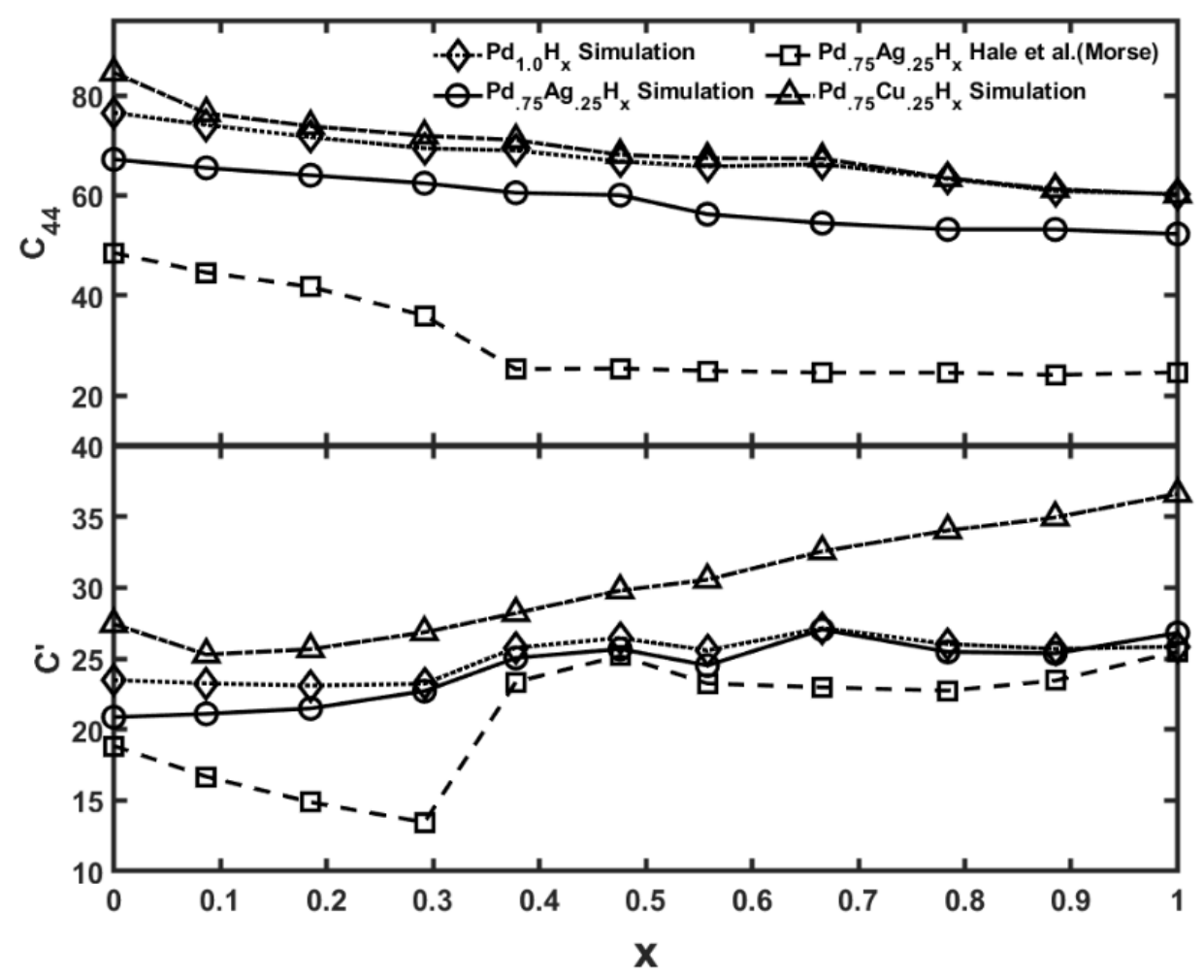

Figure $11 \mathrm{Pd}_{1.00} \mathrm{H}_{x}, \mathrm{Pd}_{0.75} \mathrm{Ag}_{0.25} \mathrm{H}_{x}$ and $\mathrm{Pd}_{0.75} \mathrm{Cu}_{0.25} \mathrm{H}_{x} \mathrm{C}_{44}$ elastic constant and $C^{\prime}$ shear elastic constant results from MD simulations.

In addition, our elastic constants values for the various $\mathrm{Pd}_{1.00} \mathrm{H}_{x}, \mathrm{Pd}_{0.75} \mathrm{Ag}_{0.25} \mathrm{H}_{x}$ and $\mathrm{Pd}_{0.75} \mathrm{Cu}_{0.25} \mathrm{H}_{x}$ alloys shown in Figure 10 and Figure 11 satisfy the theory of strain energy for cubic structures [64]. According to strain energy theory, the following formulas can be applied to a mechanically stable cubic: $C_{11}-C_{12}>0, C_{11}+2 C_{12}>0$ and $C_{44}>0$. From Figure 10 and Figure 11 , it can also be seen that the $\mathrm{Pd}_{0.75} \mathrm{Ag}_{0.25} \mathrm{H}_{x}$ and $\mathrm{Pd}_{0.75} \mathrm{Cu}_{0.25} \mathrm{H}_{x}$ structures have smaller $C_{12}$ and bigger $C_{44}$ than the $\mathrm{Pd}_{1.00} \mathrm{H}_{x}$ structures, implying that alloying $\mathrm{Pd}$ with $\mathrm{Cu}$ or $\mathrm{Ag}$ has a significant impact on the elastic constant properties for $\mathrm{Pd}-\mathrm{Cu}-\mathrm{H}$ and $\mathrm{Pd}-\mathrm{Ag}-\mathrm{H}$ phases [58].

\subsection{Additional Compositions}

To demonstrate the validity of our EAM potentials beyond the $\mathrm{Pd}, \mathrm{Ag}$ and $\mathrm{Cu}$ concentrations utilized during the fitting process, Figure 12 shows the lattice constants and cohesive energies for the $\mathrm{Pd}_{0.50} \mathrm{Ag}_{0.50} \mathrm{H}_{x}, \mathrm{Pd}_{0.50} \mathrm{Cu}_{0.50} \mathrm{H}_{x}$ and $\mathrm{Pd}_{0.50} \mathrm{Ag}_{0.25} \mathrm{Cu}_{0.25} \mathrm{H}_{x}$ hydrides. The $\mathrm{Pd}_{50} \mathrm{Cu}_{50}$ structure was chosen on purpose because of its relative similarity to the $\mathrm{Pd}_{52.5} \mathrm{Cu}_{47.5}$ structure which proved to have the highest $\mathrm{H}$ permeability by experimental findings [58]. As can be seen from Figure 12, the lattice constant and cohesive energy results for these additional compositions display a similar trend consistent with our previously obtained results for $\mathrm{Pd}_{1.00} \mathrm{H}_{x}, \mathrm{Pd}_{0.75} \mathrm{Ag}_{0.25} \mathrm{H}_{x}$ and $\mathrm{Pd}_{0.75} \mathrm{Cu}_{0.25} \mathrm{H}_{x}$ hydrides. 


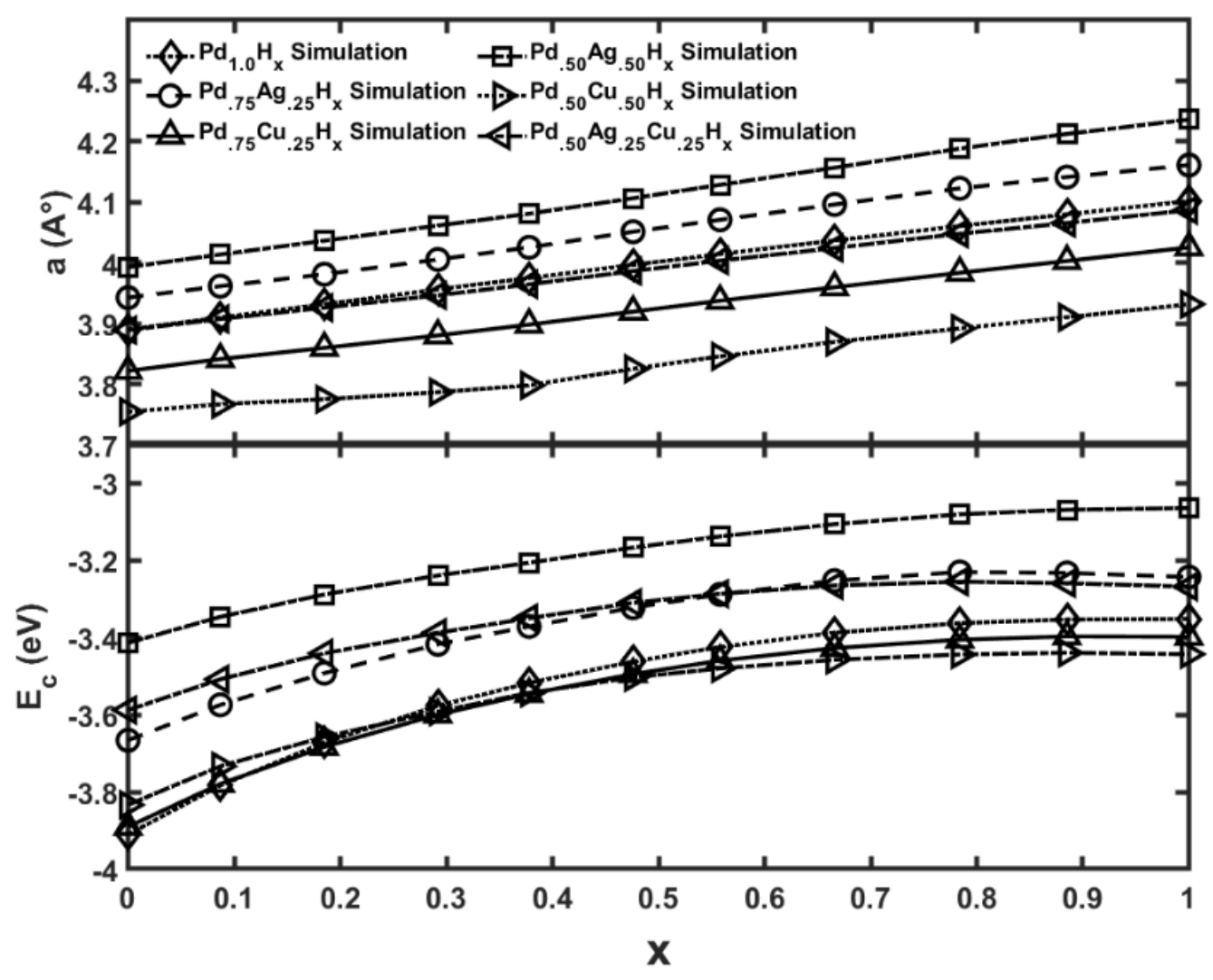

Figure $12 \mathrm{Pd}_{1.00} \mathrm{H}_{x}, \mathrm{Pd}_{0.75} \mathrm{Ag}_{0.25} \mathrm{H}_{x}, \mathrm{Pd}_{0.50} \mathrm{Ag}_{0.50} \mathrm{H}_{x}, \mathrm{Pd}_{0.75} \mathrm{Cu}_{0.25} \mathrm{H}_{x}, \mathrm{Pd}_{0.50} \mathrm{Cu}_{0.50} \mathrm{H}_{x}$ and $\mathrm{Pd}_{0.50} \mathrm{Ag}_{0.25} \mathrm{Cu}_{0.25} \mathrm{H}_{x} a_{o}$ and $E_{c}$ results from MD simulations.

In Figure 13(a), (b) and (c) the MD simulation results for the elastic constants and bulk modulus for $\mathrm{Pd}_{0.50} \mathrm{Ag}_{0.50} \mathrm{H}_{x}, \mathrm{Pd}_{0.50} \mathrm{Cu}_{0.50} \mathrm{H}_{x}$ and $\mathrm{Pd}_{0.50} \mathrm{Ag}_{0.25} \mathrm{Cu}_{0.25} \mathrm{H}_{x}$ are plotted. It can be seen that they also have a consistent trend with our previous results for the $\mathrm{Pd}_{1.00} \mathrm{H}_{x}, \mathrm{Pd}_{0.75} \mathrm{Ag}_{0.25} \mathrm{H}_{x}$ and $\mathrm{Pd}_{0.75} \mathrm{Cu}_{0.25} \mathrm{H}_{x}$ hydrides. The plots for bulk modulus for $\mathrm{Pd}_{0.50} \mathrm{Ag}_{0.50} \mathrm{H}_{x}, \mathrm{Pd}_{0.50} \mathrm{Cu}_{0.50} \mathrm{H}_{x}$ and $\mathrm{Pd}_{0.50} \mathrm{Ag}_{0.25} \mathrm{Cu}_{0.25} \mathrm{H}_{x}$ have a similar softening trend to our previous results for $\mathrm{Pd}_{1.00} \mathrm{H}_{x}, \mathrm{Pd}_{0.75} \mathrm{Ag}_{0.25} \mathrm{H}_{x}$ and $\mathrm{Pd}_{0.75} \mathrm{Cu}_{0.25} \mathrm{H}_{x}$. For $\mathrm{Pd}_{0.50} \mathrm{Ag}_{0.25} \mathrm{Cu}_{0.25} \mathrm{H}_{x}$, the bulk modulus values are higher than those for $\mathrm{Pd}_{0.50} \mathrm{Ag}_{0.50} \mathrm{H}_{x}$ and closer to $\mathrm{Pd}_{0.50} \mathrm{Cu}_{0.50} \mathrm{H}_{x}$, indicating that adding $\mathrm{Cu}$ has a strengthening impact on the solid solution while adding $\mathrm{Ag}$ has a softening effect.

The elastic constant values for $C_{11}$ and $C_{12}$ for the various $\mathrm{Pd}_{0.50} \mathrm{Ag}_{0.50} \mathrm{H}_{x}, \mathrm{Pd}_{0.50} \mathrm{Cu}_{0.50} \mathrm{H}_{x}$ and $\mathrm{Pd}_{0.50} \mathrm{Ag}_{0.25} \mathrm{Cu}_{0.25} \mathrm{H}_{x}$ structures shown in Figure 13 also satisfy the strain energy theory for cubic structures [58], implying these structures also possess mechanical stability. 


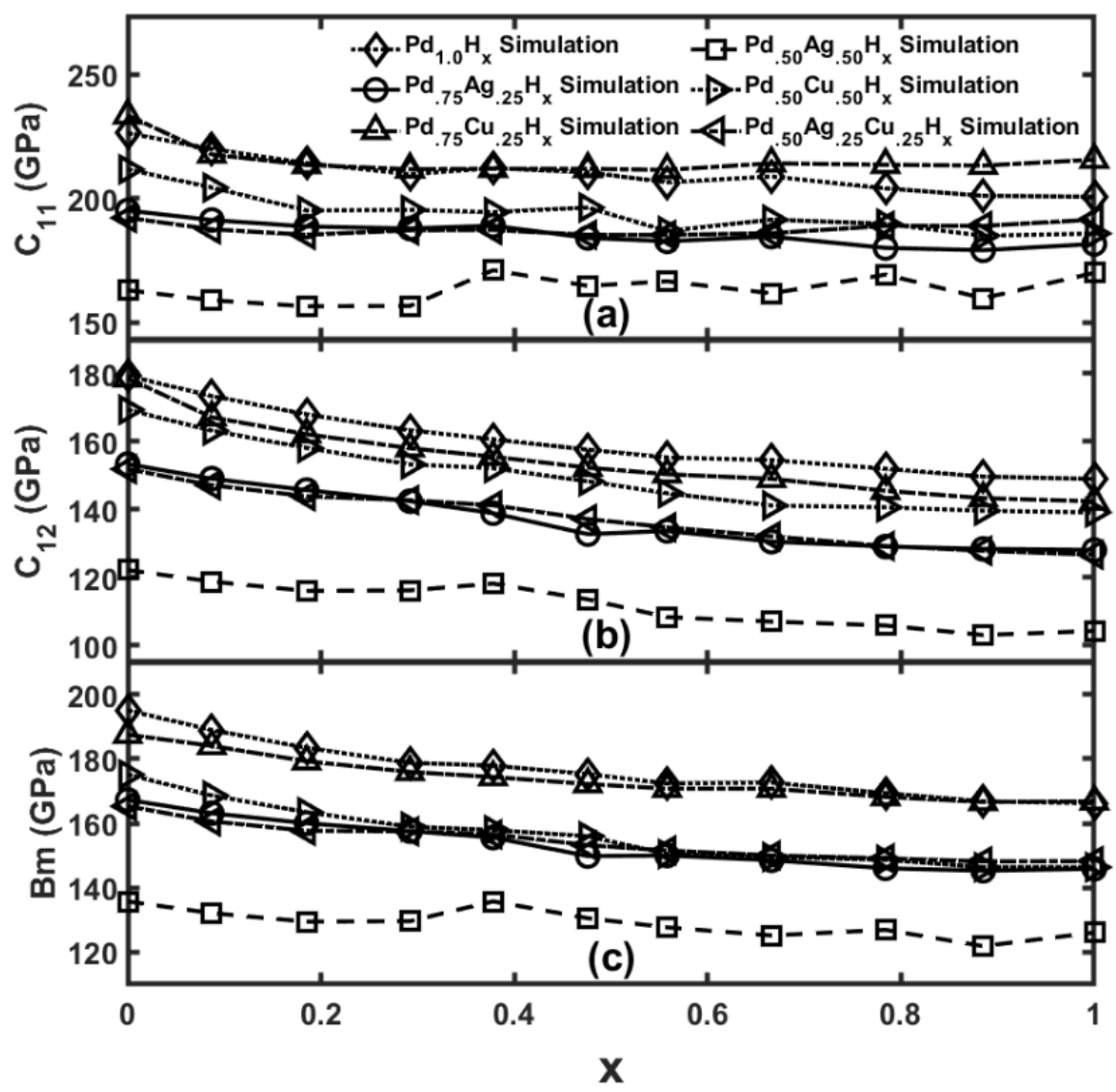

Figure $13 \mathrm{Pd}_{1.00} \mathrm{H}_{x}, \mathrm{Pd}_{0.75} \mathrm{Ag}_{0.25} \mathrm{H}_{x}, \mathrm{Pd}_{0.50} \mathrm{Ag}_{0.50} \mathrm{H}_{x}, \mathrm{Pd}_{0.75} \mathrm{Cu}_{0.25} \mathrm{H}_{x}, \mathrm{Pd}_{0.50} \mathrm{Cu}_{0.50} \mathrm{H}_{x}$ and $\mathrm{Pd}_{0.50} \mathrm{Ag}_{0.25} \mathrm{Cu}_{0.25} \mathrm{H}_{x} C_{11}, C_{12}$ elastic constant and $\mathrm{B}_{\mathrm{m}}$ results from MD simulations.

\subsection{Dynamic Stability}

In the $\mathrm{Pd}_{1.00} \mathrm{H}_{x}$ hydride, $\mathrm{H}$ atoms tend to take the $\mathrm{OC}$ sites in the $\mathrm{Pd}$ fcc lattice [65]. The DFT calculation results show that the $\mathrm{OC}$ sites in $\mathrm{Pd}_{0.75} \mathrm{Ag}_{0.25} \mathrm{H}_{x}$ and $\mathrm{Pd}_{0.75} \mathrm{Cu}_{0.25} \mathrm{H}_{x}$ structures are highly energetically favorable to $\mathrm{H}$ atoms; this behavior was also observed and reported by other researchers $[5,52]$. In order to verify the stability for the $\mathrm{Pd}_{1-y} \mathrm{Ag}_{y} \mathrm{H}_{x}, \mathrm{Pd}_{1-y} \mathrm{Cu}_{y} \mathrm{H}_{x}$ and $\mathrm{Pd}_{1-y-z} \mathrm{Ag}_{y} \mathrm{Cu}_{z} \mathrm{H}_{x}$ structures using our EAM potentials, structures with TE sites occupied by $\mathrm{H}$ atoms were created using LAMMPS, as shown in Figure 14(a). MD simulations were carried out with an NPT ensemble, each TE structure was annealed from $500 \mathrm{~K}$ to $1 \mathrm{~K}$ for $100 \mathrm{~ns}$, and then followed by cg energy minimization. After each MD simulation, the $\mathrm{H}$ atoms moved to lower energy $\mathrm{OC}$ sites, as was reported. The resulting structure for a $\mathrm{Pd}_{0.50} \mathrm{Ag}_{0.25} \mathrm{Cu}_{0.25} \mathrm{H}_{x}$ is shown in Figure 14 (b). 


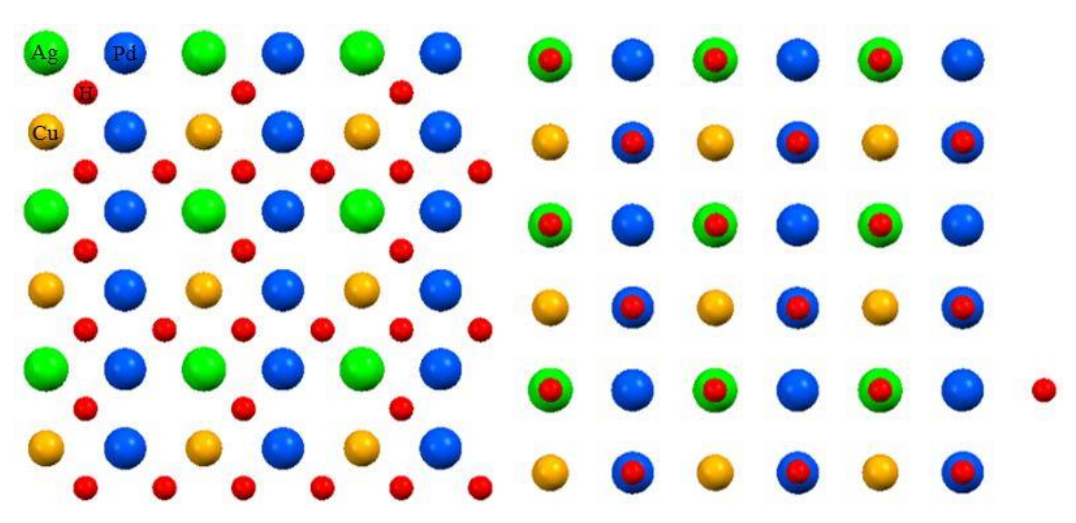

(a)

(b)

Figure 14 (a) $\mathrm{H}$ atoms (red) in TE sites before simulation. (b) $\mathrm{H}$ atoms moved to OC sites after simulation.

\subsection{Miscibility Gap and Gibbs Free Energy of Mixing}

For the previously studied $\mathrm{Pd}_{1.00} \mathrm{H}_{x}$ hydrides, the miscibility gap was predicted based on the Gibbs free energy of mixing as a function of $\mathrm{H}$ concentration [40]. Following the method of Hale et al. [5], the mixing enthalpy term was modified to obtain the Gibbs free energy functions for $P d_{1-y} A g_{y} H_{x}, P d_{1-}$ ${ }_{y} \mathrm{Cu}_{y} \mathrm{H}_{x}$ and $\mathrm{Pd}_{1-y-z} \mathrm{Ag}_{y} \mathrm{Cu}_{z} \mathrm{H}_{x}$ hydrides as follows:

$$
\begin{gathered}
\Delta G^{\mathrm{mix}}=\Delta H^{\mathrm{mix}}-\Delta S^{\mathrm{mix}} \cdot T \\
\Delta \mathrm{H}^{\mathrm{mix}}=E_{\mathrm{Pd}_{1-y} A g_{y} H_{x}}-2 X \cdot E_{\mathrm{Pd}_{1-y} A g_{y} H_{1.0}}-(1-2 X) \cdot E_{\mathrm{Pd}_{1-y} A g_{y}}
\end{gathered}
$$

where the cohesive energies $E_{\mathrm{Pd}_{1-y} A g_{y} H_{x}}, E_{\mathrm{Pd}_{1-y} A g_{y} H_{1.0}}$, and $E_{\mathrm{Pd}_{1-y} A g_{y}}$ were applied, and $X$ is the mole fraction which $X=x /(1+x)$.

$$
\Delta \mathrm{S}_{t}=-k_{B} \cdot\left[\begin{array}{l}
X \cdot \ln \left[\frac{X}{(1-X)}\right]+ \\
(1-2 \cdot X) \cdot \ln \left[\frac{1-2 X}{(1-X)}\right]
\end{array}\right]
$$

where $k_{B}$ is Boltzmann's constant.

At $0 \mathrm{~K}$, the Gibbs free energy values in Figure 15, as expected, are all above zero for all structures and various $\mathrm{H}$ concentrations, indicating that the average attractive interactions between different atom types are weaker than those between the same atom types. At $300 \mathrm{~K}$, Figure 16 shows that the Gibbs free energy plot for $\mathrm{Pd}_{1.00} \mathrm{H}_{x}$ has two minima at $x=0.034$ and 0.95 , corresponding to the mole fraction of $X=0.033$ and 0.49 and represent the $\alpha$ and $B$ phases, respectively. They describe a miscibility gap region in an alloy, where two phases are more stable than a single one. The Hale et al. EAM Morse model predicts the $\alpha$ and $B$ phases to be $X=0.0$ and 0.47 [5]. Experimentally obtained phase boundaries for $\mathrm{Pd}_{1.00} \mathrm{H}_{x}$ at $300 \mathrm{~K}$ are $x=0.03$ and 0.6 , corresponding to mole fraction of $X=$ 0.029 and 0.375 . Therefore, our model is in better agreement in predicting the $\alpha$ phase but the $\beta$ phase is slightly overestimates compared to Hale et al. EAM Morse potential. In Figure 16, our MD results for $\mathrm{Pd}_{1-y} \mathrm{Ag}_{y} \mathrm{H}_{x}$ at $300 \mathrm{~K}$ show that when $\mathrm{Ag}$ concentration increases, the values become more negative relative to the $\mathrm{Pd}_{1.00} \mathrm{H}_{x}$ system, indicating more favorable mixing, and the miscibility gap 
become narrower. No miscibility gap observed when $y=0.5$. At $300 \mathrm{~K}$, the experimental values indicate that the $\alpha$ phase and $B$ phase cease to be distinct at $y=0.25-0.30$ for $\operatorname{Pd}_{1-y} A_{y}$ [56]. This shows that our EAM potentials are able to detect the miscibility gap, and are consistent with the experimental results regarding the miscibility gap overall behavior as $\mathrm{Ag}$ concentration increases. For the $\mathrm{Pd}_{1-y} \mathrm{Cu}_{y} \mathrm{H}_{x}$ compositions, experimental data at $303 \mathrm{~K}$ indicated that by increasing $\mathrm{Cu}$ concentration, the $\alpha$ phase and 6 phase shift to the right and to the left respectively, the miscibility gap narrows, and finally disappears at $y=0.29[66,67]$. Our values from MD simulations at $300 \mathrm{~K}$ in Figure 16 also indicate that adding $\mathrm{Cu}$ causes the Gibbs free energy to increase for all $\mathrm{H}$ concentrations in comparison to the $\mathrm{Pd}_{1.00} \mathrm{H}_{x}$ structures. At $y=0.25$, all calculated energies are positive indicating unfavorable mixing, no miscibility gap observed at $y=0.5$. This shows that our model predicts the miscibility gap to disappear at high $\mathrm{Cu}$ concentration. For $\mathrm{Pd}_{0.50} \mathrm{Ag}_{0.25} \mathrm{Cu}_{0.25} \mathrm{H}_{x}$ compositions, the Gibbs free energy plot has a similar trend with those obtained from the $\mathrm{Pd}_{1.00} \mathrm{H}_{x}$ structures, indicating that the addition of copper and silver with equal concentration seems to have an opposite effect on the Gibbs free energy and tend to offset each other.

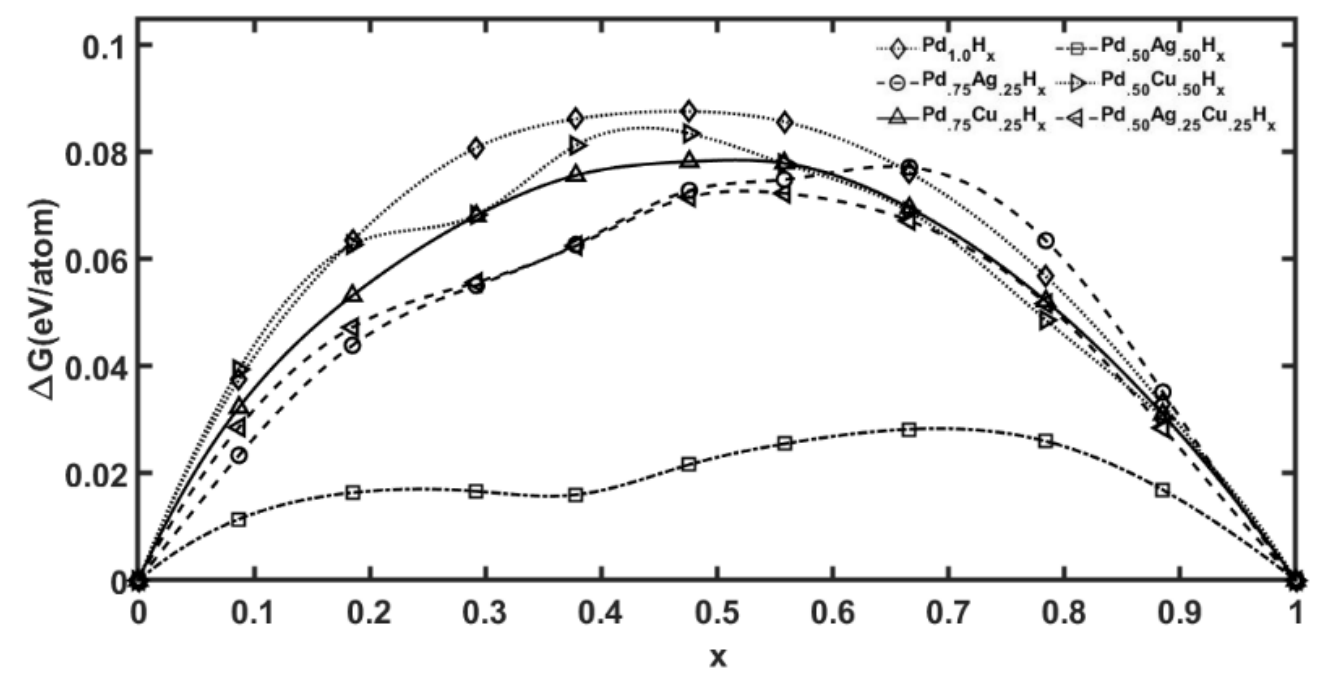

Figure 15 Gibbs free energy plot for different structures at $0 \mathrm{~K}$.

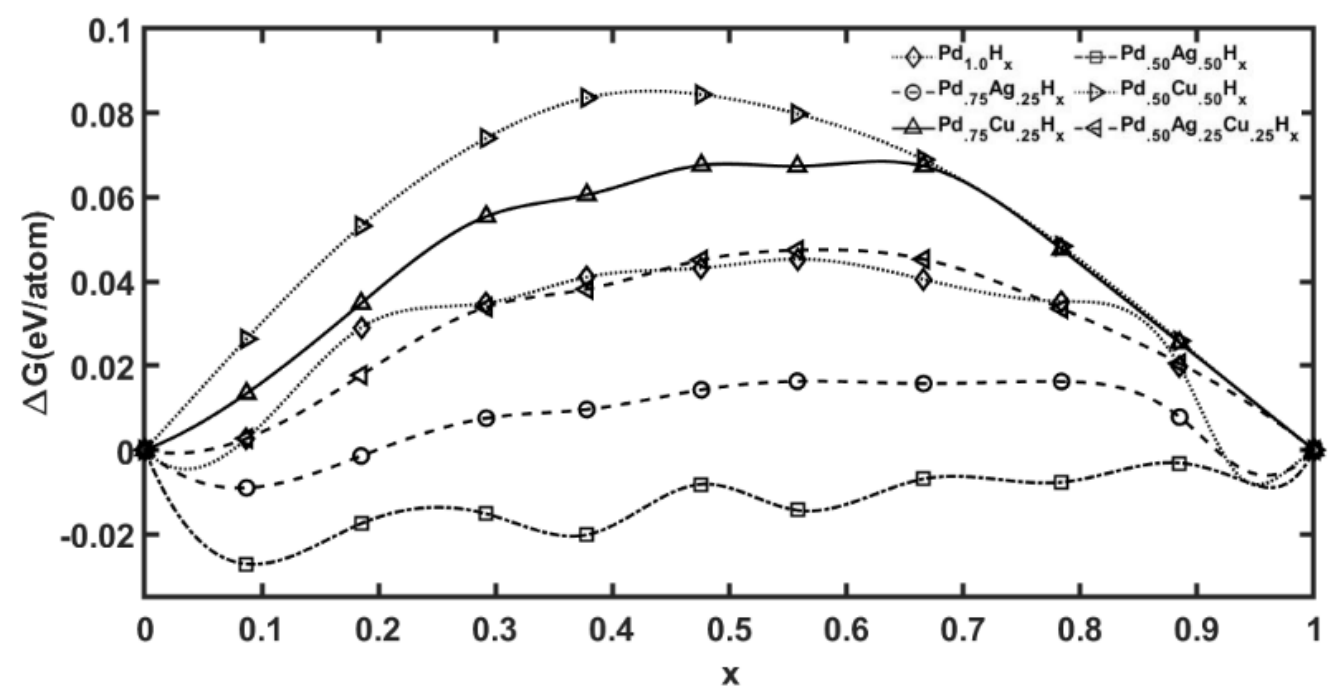

Figure 16 Gibbs free energy plot for different structures at 300K. 


\section{Conclusion}

In this paper, the central atom method was used to fit fully analytical Pd-Ag-Cu-H EAM potentials without utilizing the time-intensive MD simulations during the fitting process. The potentials were efficient in minimizing the objective functions during the fitting calculations, and the number of fitting parameters were reduced compared to previously developed EAM potentials. There were 6 fitting parameters for each $\mathrm{Pd}-\mathrm{Pd}, \mathrm{Ag}-\mathrm{Ag}$ and $\mathrm{Cu}-\mathrm{Cu}$ EAM potential, 2 scaling factors calculated by a mixing rule for each $\mathrm{Pd}-\mathrm{Ag}, \mathrm{Pd}-\mathrm{Cu}$ and $\mathrm{Ag}-\mathrm{Cu}$ pair interaction, 10 fitting parameters for $\mathrm{H}-\mathrm{H}$, and 4 for each $\mathrm{Pd}-\mathrm{H}, \mathrm{Ag}-\mathrm{H}$ and $\mathrm{Cu}-\mathrm{H}$ EAM potential. Our MD simulation results validated that these EAM potentials can be applied accurately in further simulations.

The experimentally obtained heat of solutions values were used in fitting the $\mathrm{Pd}-\mathrm{Ag}, \mathrm{Pd}-\mathrm{Cu}$ and $\mathrm{Ag}$ - $\mathrm{Cu}$ pair potentials. The Ag-H and $\mathrm{Cu}-\mathrm{H}$ EAM potentials were fitted to the cohesive energies for $14 \mathrm{Pd}_{0.75} \mathrm{Ag}_{0.25} \mathrm{H}_{x}$ and $14 \mathrm{Pd}_{0.75} \mathrm{Cu}_{0.25} \mathrm{H}_{x}$ structures, obtained from ab initio SIESTA simulations. The MD simulations utilizing LAMMPS demonstrated that our lattice constant and cohesive energy results for $\mathrm{Pd}_{0.75} \mathrm{Ag}_{0.25} \mathrm{H}_{x}$ and $\mathrm{Pd}_{0.75} \mathrm{Cu}_{0.25} \mathrm{H}_{x}$ structures were consistent with the ab initio fitting data for most of the $\mathrm{H}$ concentrations. The MD results for the $\mathrm{Pd}_{1-y} \mathrm{Ag}_{y} \mathrm{H}_{x}, \mathrm{Pd}_{1-y} \mathrm{Cu}_{y} \mathrm{H}_{x}$ and $\mathrm{Pd}_{1-y-z} \mathrm{Ag}_{y} \mathrm{Cu}_{z} \mathrm{H}_{x}$ structures also demonstrated a consistent trend with our previously obtained values for the $\mathrm{Pd}_{1.00} \mathrm{H}_{x}$ hydride. The elastic constants trend was as expected, with the bulk modulus decreasing with increasing $\mathrm{H}$ concentration. As with $\mathrm{Pd}_{1.00} \mathrm{H}_{x}$, dynamic stability testing for the $\mathrm{Pd}_{1-y-z} \mathrm{Ag}_{y} \mathrm{Cu}_{z} \mathrm{H}_{x}$ quaternary structures also predicted $\mathrm{H}$ atoms transferring from higher energy TE sites to lower energy OC sites. Our EAM potentials also captured the existence of a miscibility gap for the $\mathrm{Pd}_{1-y-}$ ${ }_{z} \mathrm{Ag}_{y} \mathrm{Cu}_{z} \mathrm{H}_{x}$ and predicted it to narrow and disappear when $\mathrm{Ag}$ and $\mathrm{Cu}$ concentration increases as was predicted by the experimental findings.

\section{Additional Materials}

The following additional materials are uploaded at the page of this paper:

1. Table S1: PdAgH and PdCuH ab initio data, fitting results, and MD results.

\section{Author Contributions}

Robert Fuller and Iyad Hijazi contributed to the work on the PdAgH EAM potentials and related calculations. Chaonan Zhang and lyad Hijazi contributed to the work on the PdCuH and PdAgCuH EAM potentials and related calculations.

\section{Funding}

West Virginia Space Grant Consortium (Grant No. 217164, Funder ID. 10.13039/100005768).

\section{Competing Interests}

The authors have declared that no competing interests exist.

\section{References}

1. Nelin G. A neutron diffraction study of palladium hydride. Phys Status Solidi B. 1971; 45: 527536. 
2. Caputo R, Alavi A. Where do the $\mathrm{H}$ atoms reside in $\mathrm{PdH}_{\mathrm{x}}$ systems? Mol Phys. 2003; 101: 17811787.

3. Manchester FD, San-Martin A, Pitre JM. The H-Pd (hydrogen-palladium) system. J Phase Equilib. 1994; 15: 62-83.

4. Zhou XW, Zimmerman JA, Wong BM, Hoyt JJ. An embedded-atom method interatomic potential for Pd-H alloys. J Mater Res. 2008; 23: 704-718.

5. Hale LM, Wong BM, Zimmerman JA, Zhou XW. Atomistic potentials for palladium-silver hydrides. Modell Simul Mater Sci Eng. 2013; 21: 045005.

6. Hsu DK, Leisure RG. Elastic constants of palladium and $\beta$-phase palladium hydride between 4 and 300 K. Phys Rev B. 1979; 20: 1339.

7. Dong $W$, Ledentu V, Sautet $P$, Eichler A, Hafner J. Hydrogen adsorption on palladium: A comparative theoretical study of different surfaces. Surf Sci. 1998; 411: 123-136.

8. Goltsova MV, Artemenko YA, Zaitsev VI. Kinetics and morphology of the reverse $\beta \rightarrow \alpha$ hydride transformation in thermodynamically open Pd-H system. J Alloys Compd. 1999; 293-295: 379384.

9. Alefeld G, Völkl J. Hydrogen in Metals II: Application-Oriented Properties. Berlin Heidelberg: Springer-Verlag; 1978.

10. Muetterties EL. Transition metal--hydrogen interaction. Transition Met Hydrides. 1971; 4: 1113.

11. Siegel B, Libowitz GG, Mueller WM, Blackledge JP. Metal Hydrides. New York: Academic Press; 1968.

12. Fukai Y. The Metal-Hydrogen System: Basic Bulk Properties. Berlin Heidelberg: Springer-Verlag; 1993.

13. Lässer R. Tritium and Helium-3 in Metals. Berlin Heidelberg: Springer-Verlag; 1989.

14. Alefeld G, Völkl J. Hydrogen in Metals I: Basic Properties. Berlin Heidelberg: Springer-Verlag; 1978.

15. Povel R, Feucht K, Gelse W, Withalm G. Hydrogen fuel for motorcars. Interdiscip Sci Rev. 1989; 14: 365-373.

16. Knapton AG. Palladium alloys for hydrogen diffusion membranes. Platinum Met Rev. 1977; 21: 44-50.

17. Adams BD, Chen A. The role of palladium in a hydrogen economy. Mater Today. 2011; $14: 282-$ 289.

18. Jimenez G, Dillon E, Dahlmeyer J, Garrison T, Garrison T, Darkey S, et al. A comparative assessment of hydrogen embrittlement: Palladium and palladium-silver (25 weight\% silver) subjected to hydrogen absorption/desorption cycling. Adv Chem Eng Sci. 2016; 6: 246.

19. Foletto EL, Wirbitzki Da Silveira JV, Jahn SL. Preparation of palladium-silver alloy membranes for hydrogen permeation. Lat Am Appl Res. 2008; 38: 79-84.

20. Chen $\mathrm{CH}, \mathrm{Ma} \mathrm{YH}$. The effect of $\mathrm{H}_{2} \mathrm{~S}$ on the performance of $\mathrm{Pd}$ and $\mathrm{Pd} / \mathrm{Au}$ composite membrane. J Membr Sci. 2010; 362: 535-544.

21. O'Brien CP, Gellman AJ, Morreale BD, Miller JB. The hydrogen permeability of $\mathrm{Pd}_{4} \mathrm{~S}$. J Membr Sci. 2011; 371: 263-267.

22. Gabitto J, Tsouris C. Modeling sulfur poisoning of palladium membranes used for hydrogen separation. Int J Chem Eng. 2019; 2019: 9825280. 
23. Amandusson $\mathrm{H}$, Ekedahl LG, Dannetun $\mathrm{H}$. Hydrogen permeation through surface modified Pd and PdAg membranes. J Membr Sci. 2001; 193: 35-47.

24. Semidey-Flecha L, Sholl DS. Combining density functional theory and cluster expansion methods to predict $\mathrm{H}_{2}$ permeance through Pd-based binary alloy membranes. J Chem Phys. 2008; 128: 144701.

25. Qin L, Jiang C. First-principles based modeling of hydrogen permeation through $\mathrm{Pd}-\mathrm{Cu}$ alloys. Int J Hydrog Energy. 2012; 37: 12760-12764.

26. Adhikari S, Fernando S. Hydrogen membrane separation techniques. Ind Eng Chem Res. 2006; 45:875-881.

27. Martin $\mathrm{MH}$, Galipaud J, Tranchot A, Roué L, Guay D. Measurements of hydrogen solubility in $\mathrm{Cu}_{x} \mathrm{Pd}_{100-\mathrm{x}}$ thin films. Electrochim Acta. 2013; 90: 615-622.

28. Kart SÖ, Erbay A, Kılıç H, Cagin T, Tomak M. Molecular dynamics study of Cu-Pd ordered alloys. J Achiev Mater Manuf Eng. 2008; 31: 41-46.

29. McKinley DL. Method for hydrogen separation and purification. Washington, DC: U.S. Patent and Trademark Office; 1969; 3,439,474.

30. Illas F, López N, Ricart JM, Clotet A, Conesa JC, Fernández-García M. Interaction of CO and NO with PdCu (111) surfaces. J Phys Chem B. 1998; 102: 8017-8023.

31. O'Brien CP, Lee IC. The interaction of $\mathrm{CO}$ with $\mathrm{PdCu}$ hydrogen separation membranes: An operando infrared spectroscopy study. Catal Today. 2019; 336: 216-222.

32. Guerreiro BH, Martin MH, Roué L, Guay D. Hydrogen permeability of PdCuAu membranes prepared from mechanically-alloyed powders. J Membr Sci. 2016; 509: 68-82.

33. Liu LC, Wang JW, He YH, Gong HR. Solubility, diffusivity, and permeability of hydrogen at PdCu phases. J Membr Sci. 2017; 542: 24-30.

34. Tarditi AM, Braun F, Cornaglia LM. Novel PdAgCu ternary alloy: Hydrogen permeation and surface properties. Appl Surf Sci. 2011; 257: 6626-6635.

35. Semidey-Flecha L, Ling C, Sholl DS. Detailed first-principles models of hydrogen permeation through PdCu-based ternary alloys. J Membr Sci. 2010; 362: 384-392.

36. Ling C, Semidey-Flecha L, Sholl DS. First-principles screening of PdCuAg ternary alloys as $\mathrm{H}_{2}$ purification membranes. J Membr Sci. 2011; 371: 189-196.

37. Yuan L, Goldbach A, Xu H. Real-time monitoring of metal deposition and segregation phenomena during preparation of PdCu membranes. J Membr Sci. 2008; 322: 39-45.

38. Zhao L, Goldbach A, Xu H. Tailoring palladium alloy membranes for hydrogen separation from sulfur contaminated gas streams. J Membr Sci. 2016; 507: 55-62.

39. Foiles SM, Hoyt JJ. Computer simulation of bubble growth in metals due to He. Sandia Natl Lab. 2001; SAND2001-0661.

40. Hijazi I, Zhang Y, Fuller R. A simple embedded atom potential for Pd-H alloys. Mol Simul. 2018; 44: 1371-1379.

41. Daw MS, Baskes MI. Semiempirical, quantum mechanical calculation of hydrogen embrittlement in metals. Phys Rev Lett. 1983; 50: 1285.

42. Hijazi IA, Park YH. Consistent analytic embedded atom potential for face-centered cubic metals and alloys. J Mater Sci Technol. 2009; 25: 835-846.

43. Park YH, Hijazi IA. Simple analytic embedded atom potential for FCC materials. Int J Microstruct. Mater Prop. 2011; 6: 378-396. 
44. Hijazi IA, Park YH. Structure of pure metallic nanoclusters: Monte carlo simulation and ab initio study. Eur Phys J D. 2010; 59: 215-221.

45. Park YH, Hijazi IA. Critical size of transitional copper clusters for ground state structure determination: Empirical and ab initio study. Mol Simul. 2012; 38:241-247.

46. Hijazi IA. Structural, electronic and magnetic properties of pure metallic and bimetallic nanoclusters: Empirical and density functional studies. Ann Arbor: ProQuest Dissertations; 2010.

47. Rose JH, Smith JR, Guinea F, Ferrante J. Universal features of the equation of state of metals. Phys Rev B. 1984; 29: 2963-2969.

48. Foiles SM, Baskes MI, Daw MS. Embedded-atom-method functions for the fcc metals $\mathrm{Cu}, \mathrm{Ag}$, $\mathrm{Au}, \mathrm{Ni}, \mathrm{Pd}, \mathrm{Pt}$, and their alloys. Phys Rev B. 1986; 33: 7983.

49. Johnson RA. Alloy models with the embedded-atom method. Phys Rev B. 1989; 39:12554-12559.

50. Thompson A. In.elastic. Sandia National Laboratory. Available from: https://github.com/lammps/lammps/tree/master/examples/ELASTIC.

51. Cai J, Ye YY. Simple analytical embedded-atom-potential model including a long-range force for fcc metals and their alloys. Phys Rev B. 1996; 54:8398-8410.

52. Løvvik OM, Olsen RA. Density functional calculations on hydrogen in palladium-silver alloys. J Alloys Compd. 2002; 330-332: 332-337.

53. Hale LM, Wong BM, Zimmerman JA, Zhou XW. Atomistic potentials for palladium-silver hydrides. Model Simul Mat Sci Eng. 2013; 21: 045005.

54. Delczeg-Czirjak EK, Delczeg L, Ropo M, Kokko K, Punkkinen MP, Johansson B, et al. Ab initio study of the elastic anomalies in Pd-Ag alloys. Phys Rev B. 2009; 79: 085107.

55. Uemiya S, Matsuda T, Kikuchi E. Hydrogen permeable palladium-silver alloy membrane supported on porous ceramics. J Membr Sci. 1991; 56: 315-325.

56. Burch R. On the role of silver atoms in the absorption of hydrogen by palladium-silver alloys. Solid State Commun. 1969; 7: 1313-1317.

57. Karakaya I, Thompson WT. The Ag- Pd (silver-palladium) system. Bull Alloy Phase Diagrams. 1988; 9:237-243.

58. Wei C, Kong FT, Gong HR. Phase stability and elastic property of PdH and PdCuH phases. Int J Hydrog Energy. 2013; 38: 16485-16494.

59. ABINIT. Accessed Dec. 31, 2020. [Online]. Available from: https://www.abinit.org/downloads/psp-links/pseudopotentials.

60. Fuller R. Improved embedded atom method potentials for metal hydride systems. Huntington: Marshall University; 2018. Available from: https://mds.marshall.edu/etd/1124.

61. Ilawe NV, Zimmerman JA, Wong BM. Breaking badly: DFT-D2 gives sizeable errors for tensile strengths in palladium-hydride solids. J Chem Theory Comput. 2015; 11: 5426-5435.

62. Schwarz RB, Bach HT, Harms U, Tuggle D. Elastic properties of Pd-hydrogen, Pd-deuterium, and Pd-tritium single crystals. Acta Mater. 2005; 53: 569-580.

63. Zhong W, Cai Y, Tomanek D. Computer simulation of hydrogen embrittlement in metals. Nature. 1993; 362: 435-437.

64. Mouhat F, Coudert FX. Necessary and sufficient elastic stability conditions in various crystal systems. Phys Rev B. 2014; 90: 224104.

65. Antonov VE. Phase transformations, crystal and magnetic structures of high-pressure hydrides of d-metals. J Alloys Compd. 2002; 330: 110-116. 
66. Flanagan TB, Luo S, Clewley JD. Calorimetric enthalpies for the reaction of $\mathrm{H}_{2}$ with $\mathrm{Pd}-\mathrm{Cu}$ alloys at 303 K. J Alloys Compd. 2003; 356: 13-16.

67. Huang W, Opalka SM, Wang D, Flanagan TB. Thermodynamic modelling of the Cu-Pd-H system. Calphad. 2007; 31: 315-329.

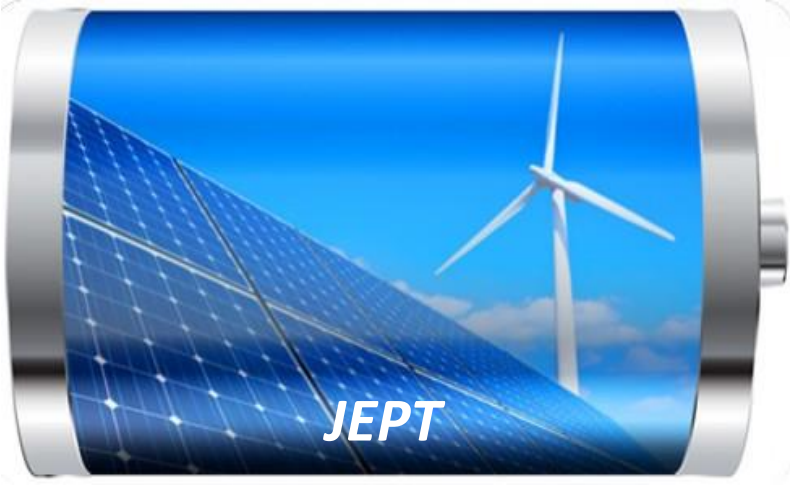

Enjoy JEPT by:

1. Submitting a manuscript

2. Joining in volunteer reviewer bank

3. Joining Editorial Board

4. Guest editing a special issue

For more details, please visit:

http://www.lidsen.com/journal/jept 


\title{
Ni-Silica-based Catalysts for $\mathrm{CH}_{4}$ Reforming by $\mathrm{CO}_{2}$ with Enhanced Stability: Recent Designs and the Impacts of Ni Confinement, Promoters, and Core-Shell Structures
}

Oscar Daoura ${ }^{1,2,}{ }^{*}$, Maya Boutros ${ }^{1}$, Franck Launay ${ }^{2}$

1. Lebanese University, Laboratoire de Chimie Physique des Matériaux (LCPM/PR2N), Faculté des Sciences II, Campus Fanar, BP 90696 Jdeideh, Lebanon; E-Mails: oscardawra@hotmail.com; boutrosmaya@hotmail.com

2. Sorbonne Université, CNRS, Laboratoire de Réactivité de Surface, LRS, Campus Pierre et Marie Curie, F-75005 Paris, France; E-Mail: franck.launay@sorbonne-universite.fr

* Correspondence: Oscar Daoura; E-Mail: oscardawra@hotmail.com

Academic Editor: Zhao Yang Dong

Special Issue: $\underline{\text { Hydrogen Energy: Sustainable Production, Storage and Utilisation }}$

Journal of Energy and Power Technology

2021, volume 3 , issue 1

doi:10.21926/jept.2101007
Received: September 01, 2020

Accepted: December 31, 2020

Published: January 26, 2021

\begin{abstract}
$\mathrm{CO}_{2}$ reforming of $\mathrm{CH}_{4}$, also referred to as the Dry Reforming of Methane (DRM), is considered an excellent method to produce $\mathrm{H}_{2}$ and $\mathrm{CO}$ (syngas), which are known to be used for the production of higher alkanes and oxygenates. Despite nickel's moderate toxicity, Ni-based heterogeneous catalysts are considered excellent candidates for use in DRM due to their reasonable performances and economic advantages. However, these materials also present severe drawbacks, such as sintering of the active phase and coke (carbon) deposition, which may, in certain cases, lead to severe catalyst deactivation. Several synthesis strategies, mostly based on the stabilization of nickel through oxide support, have been developed to overcome these issues. Silica-based materials are investigated widely due to their availability, high surface area, and the confinement capacity conferred by their controlled porosity. The present review summarizes the progress in the design of $\mathrm{Ni} /$ silica-based catalysts for the dry
\end{abstract}

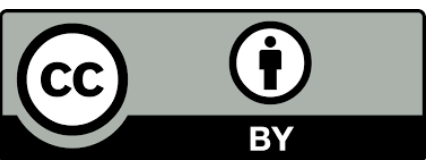

(c) 2021 by the author. This is an open access article distributed under the conditions of the Creative Commons by Attribution License, which permits unrestricted use, distribution, and reproduction in any medium or format, provided the original work is correctly cited. 
reforming of methane between the years 2015 and 2018. The different strategies implemented are discussed to assist future research works in designing the anti-coking and anti-sintering nickel-silica-based catalysts.

\section{Keywords}

$\mathrm{Ni}$-silica; $\mathrm{CH}_{4}$ reforming; $\mathrm{CO}_{2} ; \mathrm{Ni}$

\section{Introduction}

Given the abundance of methane $\left(\mathrm{CH}_{4}\right)$ and the diversity of its sources [1-3], along with its greenhouse gas character (second after carbon dioxide [4] but 25 times more specific [5]), the development of efficient strategies to take advantage of its energetic and chemical properties appears to be essential. Methane conversion is achieved through direct or indirect pathways. The direct pathway of combustion is the main approach followed to generate heat and electricity. However, the conversion of $\mathrm{CH}_{4}$ through the indirect pathway of reforming processes, which produces syngas, which is further converted to valuable liquid and chemical intermediates, is also one of the possibilities considered [6]. Indeed, methane can react with the oxidants, such as $\mathrm{H}_{2} \mathrm{O}$, $\mathrm{CO}_{2}$, and $\mathrm{O}_{2}$, to produce syngas that is composed of dihydrogen and carbon monoxide in different molar ratios (Equation 1).

$$
\mathrm{CH}_{4}+\left(\mathrm{H}_{2} \mathrm{O} \text { or } \mathrm{CO}_{2} \text { or } \mathrm{O}_{2}\right) \rightarrow \mathrm{x} \mathrm{H}_{2}+\mathrm{y} \mathrm{CO}
$$

Among the most important applications of syngas, the Fischer-Tropsch synthesis (FTS) reaction, which involves the conversion of $\mathrm{CO}$ and $\mathrm{H}_{2}$ into valuable products, particularly alkanes, is of particular interest. However, FTS requires an $\mathrm{H}_{2} / \mathrm{CO}$ reactant ratio of less than 1 , and such a composition can neither be achieved directly through the partial oxidation of methane $\left(\mathrm{H}_{2}: \mathrm{CO}=2\right)$ nor through steam reforming (most often $\mathrm{H}_{2}: \mathrm{CO}=3$ ); it can be achieved only after additional treatments, such as the use of selective membranes, which lead to additional costs and greater time consumption. Therefore, Dry Reforming of Methane (Equation 2, with $\mathrm{CO}_{2}$ ), which produces the lowest $\mathrm{H}_{2}$ :CO ratio, appears to be a more convenient and direct approach. Besides the economic and energy benefits, DRM provides an important added advantage of environmental protection as it consumes another greenhouse gas, i.e., carbon dioxide [7-18].

$$
\mathrm{CH}_{4}+\mathrm{CO}_{2} \rightarrow 2 \mathrm{H}_{2}+2 \mathrm{CO}
$$

Unfortunately, DRM is a highly endothermic reaction requiring high temperatures to reach the desired conversion levels. According to thermodynamic simulations, the maximal $\mathrm{CH}_{4}$ conversion possible at $650{ }^{\circ} \mathrm{C}$ is approximately $80 \%$, and a total conversion can be achieved only when the reaction temperature exceeds $800{ }^{\circ} \mathrm{C}[12,16]$. A major objective of the research works conducted on dry methane reforming is the development of stable catalysts that enable achieving high conversion rates at the mildest possible operating conditions and allow, under the stream, a constant selectivity in $\mathrm{CO}$ and $\mathrm{H}_{2}$. However, syngas production is accompanied by side reactions, such as methane decomposition, the Boudouard reaction, and the Reverse Water Gas shift reaction. 
The supporting noble metals, such as $\mathrm{Rh}, \mathrm{Ru}$, and $\mathrm{Ir}$, are considered highly active, selective, and stable catalysts for DRM $[19,20]$. However, due to economic concerns, the substitution of these noble metals with the less expensive and more abundant metals such as Ni that can demonstrate comparable catalytic activities is considered advantageous [21], even though $\mathrm{Ni}$-based catalysts result in higher sintering and carbon deposition and also exhibit a certain level of toxicity [22].

The size of the metal particles, the physicochemical properties of the support, and the metal/support interaction are crucial parameters to be considered to optimize the stability of the catalysts. A wide number of oxides are used as supports, including $\mathrm{SiO}_{2}, \mathrm{La}_{2} \mathrm{O}_{3}, \mathrm{ZrO}_{2}, \mathrm{TiO}_{2}, \mathrm{CeO}_{2}$, $\mathrm{Al}_{2} \mathrm{O}_{3}$, and $\mathrm{MgO}$ [22]. Among these, $\mathrm{SiO}_{2}$ has been investigated widely in terms of application in the preparation of nickel-based catalysts for the DRM reaction. Indeed, it is possible to prepare $\mathrm{SiO}_{2}$ supports with high surface areas, tunable nanoscale pore structures/sizes, and variable accessible morphologies with the potential benefits of $\mathrm{Ni}$ dispersion and confinement. Moreover, it is supposed that the mechanism of the DRM reaction in the presence of $\mathrm{SiO}_{2}$ support follows a monofunctional pathway, where both the reactants are activated by nickel alone [22]. This fact is crucial when studying the intrinsic activity of nickel. Although there are several published reviews on DRM [22-27], there is none that considers Ni-based-siliceous support catalysts specifically. In this context, the present bibliographic survey examines the current diversity of the $\mathrm{Ni} / \mathrm{SiO}_{2}$ catalysts. First, the advances in the $\mathrm{Ni}$ size control and confinement methods are discussed, following which different $\mathrm{Ni}$-promoter combinations are explored. Next, the recent pathways used for the design of $\mathrm{Ni} / \mathrm{SiO}_{2}$ core-shell are highlighted while emphasizing their advantages and inconveniences. Particular focus is placed on the catalytic performance of these different systems evaluated according to the gas hourly space velocity (GHSV) used during their catalytic evaluation.

\section{Ni Size Control/Confinement Strategies with Silica}

In DRM, the use of small Ni nanoparticles is often preferred due to their known resistance toward coke deposition (Table 1, entries 1 and 2) [28, 29]. However, optimizing the size of these particles and keeping them relatively unchanged during the whole reaction is difficult. 
JEPT 2021; 3(1), doi:10.21926/jept.2101007

Table $1 \mathrm{~A}$ selection of recent papers dealing with nickel size control and confinement within silica [28-48].

\begin{tabular}{|c|c|c|c|c|c|c|c|c|c|c|c|c|c|c|}
\hline$E$ & Year & $\begin{array}{l}\mathrm{Ni} \\
\text { (wt.\%) }\end{array}$ & $\begin{array}{l}\mathrm{NiO} \text { or } \\
\left(\mathrm{Ni}^{0}\right) \\
(\mathrm{nm})\end{array}$ & $\begin{array}{l}\text { Siliceous } \\
\text { support }\end{array}$ & $\begin{array}{l}\text { Preparation } \\
\text { Method }\end{array}$ & $\begin{array}{l}\text { Total } \\
\text { flow } \\
(\mathrm{mL} \\
\left.\mathrm{min}^{-1}\right)\end{array}$ & $\begin{array}{l}\text { GHSV } \\
\mathrm{Lg}^{-1} \mathrm{~h}^{-1}\end{array}$ & $\begin{array}{l}\text { Weight } \\
\text { (mg) }\end{array}$ & $\begin{array}{l}\mathrm{T} \\
\left({ }^{\circ} \mathrm{C}\right)\end{array}$ & $\begin{array}{l}\text { Time } \\
\text { (h) }\end{array}$ & $\mathrm{CH}_{4} \%$ & $\begin{array}{l}\mathrm{CO}_{2} \\
\%\end{array}$ & Objectives & Ref \\
\hline 1 & 2017 & 7.5 & (4.1) & SBA-15 & IWI & 30 & 22.5 & 80 & 700 & 50 & 79 & 85 & $\begin{array}{l}\text { Use of a chelating agent } \\
\text { to incorporate smaller } \\
\text { nanoparticle in the } \\
\text { porosity }\end{array}$ & [28] \\
\hline 2 & 2017 & 5 & (3.2) & $\begin{array}{l}\text { SBA-15, } \\
\text { MCM-41, } \\
\text { KIT-6 }\end{array}$ & WI, Griding & 30 & 22.5 & 80 & 700 & 100 & 77 & 86 & $\begin{array}{l}\text { Preparation through solid } \\
\text { grinding for better } \\
\text { dispersion and smaller } \\
\text { particles }\end{array}$ & [29] \\
\hline 3 & 2017 & 11 & (11) & $\begin{array}{l}\text { SBA-15, } \\
\text { Al-MCM- } \\
41\end{array}$ & WI with ethanol & 100 & 150 & 40 & 750 & 23 & 91 & 94 & $\begin{array}{l}\text { Comparative study of } \\
\text { micro and mesoporous } \\
\text { materials }\end{array}$ & [30] \\
\hline 4 & 2017 & 10 & 27 & SBA-15 & IWI & 60 & 24 & 150 & 750 & 4 & 90 & 92 & $\begin{array}{l}\text { Influence of operation } \\
\text { conditions such as } \\
\text { reaction temperature and } \\
\text { gas feed }\end{array}$ & [31] \\
\hline 5 & 2015 & $4,5,10$ & 3.8 & M. $\mathrm{SiO}_{2}$ & IWI & 60 & $\begin{array}{l}72,36 \\
16 \\
14.4\end{array}$ & 50 & 800 & 20 & 70 & 78 & $\begin{array}{l}\text { Effect of carbon chain } \\
\text { number and amine } \\
\text { concentration of } \mathrm{Ni}- \\
\text { aliphatic amine complex }\end{array}$ & [32] \\
\hline
\end{tabular}


JEPT 2021; 3(1), doi:10.21926/jept.2101007

$6 \quad 2015 \quad 10$

(8.2) $\quad \mathrm{Mi} . \mathrm{SiO}_{2} \quad$ Electrospinning

OP

$\mathrm{Ni}$ Deposition Coating

$2017 \begin{aligned} & 0.16 \\ & 0.32\end{aligned}$

(2.6) $\quad \mathrm{np} \mathrm{SiO}_{2}$

$9 \quad 2017 \quad 10$

(5-9) $\quad \mathrm{np} \mathrm{SiO} 2$

Ultrasonic assisted I

Glucose

40

12

200

$\begin{array}{lll}700 & 40 \quad 72\end{array}$

$10 \quad 2017 \quad 5$

3.3 (5) SBA-15

TS

$60 \quad 180$

20

$\begin{array}{lll}650 & 12 \quad 63\end{array}$

1120165,10

4

$\mathrm{np} \mathrm{SiO} 2 \quad$ WI

60

$12 \quad 2016 \quad 5$

(2.5) M. $\mathrm{SiO}_{2}$

IWI

60

0

6.6

$13 \quad 2016 \quad 10$

Confinement of nickel inside nanofiber through electrospinning preparation

Development of active catalysts via simple route

Particles size effect on the catalytic performances

Promotion of the catalysts using pretreatments by $\mathrm{CO}_{2}, \mathrm{~N}_{2}$ and $\mathrm{H}_{2}$ before test Influence of the calcination (rate and [37] atmosphere)

Influence of the pretreatment (reduction- [38] oxidation-reduction)

Hydrogen treatment before calcination $\mathrm{Ni}-$

Hexamethylenetetramine as precursor for better [40] confinement 
JEPT 2021; 3(1), doi:10.21926/jept.2101007

$14 \quad 2015 \quad 0.5-2 \quad$ n.d. SBA-15 IW

$152015 \quad 5$

$16 \quad 2017 \quad 7$

$172016 \quad 5$

\section{3}

18

$2016 \quad 2.5-7.5$
$5 \quad \mathrm{SBA}-15$,
M. $\mathrm{SiO}_{2}$

TS

5.7 SBA-15,

(3.7) M. $\mathrm{SiO}_{2}$

$I W I+$ ultrasound DP

80

240

20

$750 \quad 35$

60

$20 \quad 2015 \quad 10$

5

SBA-15

IWI and DP and RM

100
$\mathrm{Ni}_{4} \mathrm{O}_{4}$ cluster as $\mathrm{Ni}$ precursor for better incorporation and confinement

Use of OAm/OAc organic pair toward anti-coking [42] $\mathrm{Ni} / \mathrm{SiO}_{2}$

Confinement of $\mathrm{Ni}$ 7 particles inside different [43] pores sizes

PVP-assisted

impregnation of nickel for better dispersion and confinement

Confinement of nickel using SBA-15

Confinement of nickel inside the porosity of [46] SBA-15 or $\mathrm{SiO}_{2}$

Non-conventional impregnation method for better confinement and dispersion 
E: Entry; M.: Mesoporous; Mi.: Microporous; np: Non-Porous; TS: Two solvents; I.: Impregnation; IWI: Incipient wetness impregnation; WI: wet impregnation; DP: deposition-precipitation; OP: one-pot; RM: precipitation in presence of ascorbic acid; PVP: Polyvinylpyrrolidone; KIT: Korean Institute of Technology mesoporous siliceous support; HMS: Hollow mesoporous silica; n.d.: Not determined. 
Examples of the studies concerning nickel and silica alone are presented in Table 1 . The size control and stabilization of the nanoparticles are achieved mainly by confining the metal into the channels of porous silica supports (Figure 1). Various preparation methods are listed in Table 1 (entries 3-6) [30-33]. This strategy is quite promising as it protects the nanoparticles, particularly against the growth of carbon filaments $[49,50]$, without any decrease in their activity that might otherwise occur due to mass transfer limitation or due to coverage of the active sites as the nickel particles remain accessible to the reactants in the pores of the sample (Table 1, entry 7) [34].

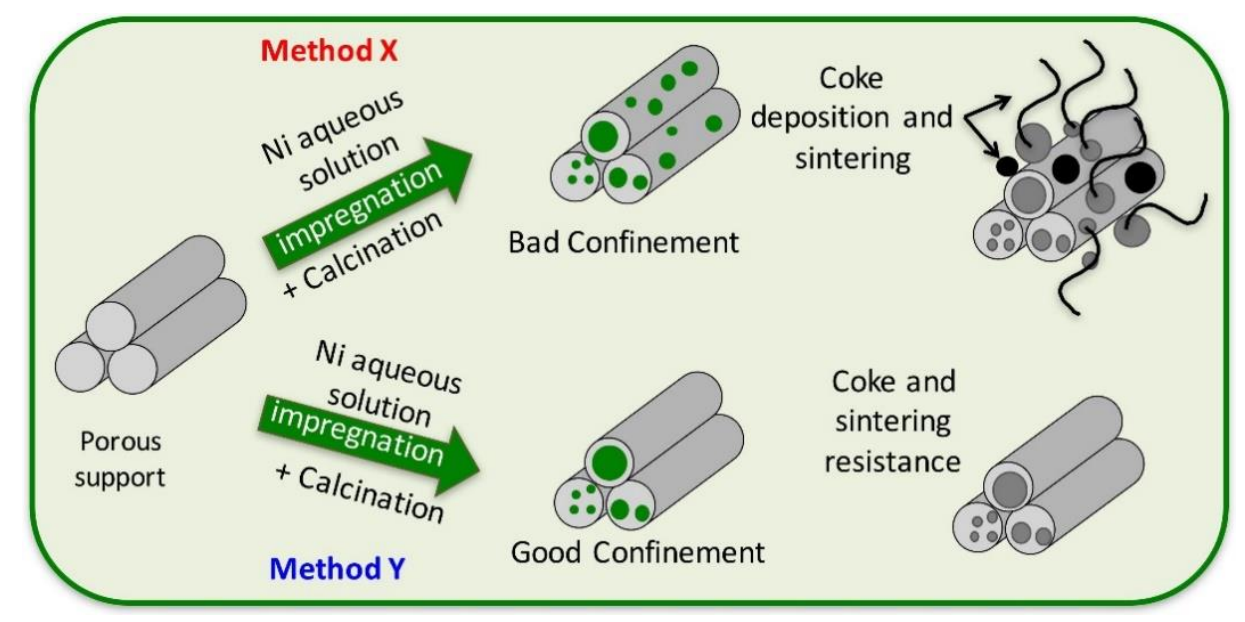

Figure 1 Illustration of the effect of nickel confinement in porous siliceous supports on nickel particle dispersion and DRM side reactions.

A few examples of efficient control of the particle properties using non-porous silica involve the dispersion of preformed Ni NPs (colloids) in such support (Table 1, entry 8) [35]. It is noteworthy that the modifications in the calcination step (heating rate, under air or inert gases, etc.) and certain pretreatments after the original preparation procedure (with $\mathrm{H}_{2}, \mathrm{~N}_{2}, \mathrm{CO}_{2}$, etc.) may also impact the nickel particle size and dispersion (Table 1, entries 9 to 12) [36-39].

\subsection{Examples with Non-Porous Supports}

Woo Han et al. (Table 1, entry 8) [35] prepared dispersions of Ni nanoparticles of highly uniform sizes centered on 2.6, 5.2, 9.0, and $17.3 \mathrm{~nm}$, using a procedure that involved nickel acetylacetonate, trioctylphosphine, and oleylamine, and demonstrated that these colloids could be homogeneously deposited on non-porous silica spheres $(400 \mathrm{~nm})$ using ultrasonic-assisted wet impregnation. The obtained materials with $\mathrm{Ni}$ wt.\% ranging between $0.16 \%$ and $0.32 \%$ were used in $\mathrm{DRM}$ at $800{ }^{\circ} \mathrm{C}$ to study the effect of the Ni NP size on the catalytic performances. The results revealed that the Ni nanoparticles of size $2.6 \mathrm{~nm}$ led to a 4-times higher turnover frequency for methane conversion compared to the NPs of size $17.3 \mathrm{~nm}\left(61.7 \mathrm{~s}^{-1} \mathrm{vs} .15 .1 \mathrm{~s}^{-1}\right)$. The small Ni nanoparticles were definitely more advantageous in ensuring high activity and selectivity (39\% and $65 \%$ of $\mathrm{CH}_{4}$ and $\mathrm{CO}_{2}$ conversion, respectively, with $\mathrm{H}_{2} / \mathrm{CO}=0.7$ for $2.6 \mathrm{~nm}$ colloids vs. $15 \%$ and $22 \%$ and $\mathrm{H}_{2} / \mathrm{CO}=0.5$ for the $17.3 \mathrm{~nm}$ ones) as long as their size was preserved. However, it is noteworthy that the $\mathrm{Ni} / \mathrm{silica}$ catalysts reported by Woo Han et al. were evaluated under a rather low GHSV $\left(12 \mathrm{~L} \mathrm{~g}^{-1} \mathrm{~h}^{-1}\right)$.

Non-porous siliceous supports were also utilized by Li et al. (Table 1, entry 9) [36], who demonstrated that $\mathrm{Ni} / \mathrm{C}-\mathrm{SiO}_{2}(10 \mathrm{wt} . \%)$ catalysts prepared using an in-situ reduction impregnation 
method involving glucose solution were characterized (using XRD analysis) as having smaller and greatly dispersed NPs compared to those obtained using a traditional incipient impregnation method without glucose. However, the preparation procedure involving glucose initially led to poor catalytic performances due to carbon deposition associated with the use of an organic additive. Therefore, the authors had to use several pretreatments (during the preparation) to overcome such limitations. After optimization, the $\mathrm{CO}_{2}$ pretreated catalyst was observed to exhibit higher coke deposition resistance due to the ability of $\mathrm{CO}_{2}$ to eliminate carbon deposition under high temperature $\left(\mathrm{CO}_{2}+\mathrm{C} \rightarrow 2 \mathrm{CO}\right)$. However, the catalyst performances $\left(70 \%\right.$ conversion of $\mathrm{CH}_{4}$ and $\mathrm{CO}_{2}$ at $700{ }^{\circ} \mathrm{C}$ ) were evaluated under quite low GHSV $\left(12 \mathrm{~L} \mathrm{~g}^{-1} \mathrm{~h}^{-1}\right)$. In another study, Lovell et al. (Table 1 , entry 11) [38] demonstrated that a reduction-oxidation-reduction (ROR) pretreatment (reduction followed by oxidation, and then re-reduction again) of calcined Ni-based solid might exert a significant impact on $\mathrm{Ni}$-non-porous $\mathrm{SiO}_{2}\left(\mathrm{Ni}_{-} \mathrm{SiO}_{2}\right)$ catalysts prepared using an impregnation method involving Ni wt.\% of 5 and 10 . This pretreatment has been applied to different supported metals to generate small particles with high metal-support interaction. For instance, ROR method was used for the activation of cobalt catalysts used in the Fischer-Tropsch reaction [51]. After the ROR pretreatment, the Co species were re-dispersed, resulting in the formation of smaller Co deposits with stronger metal-support interaction. In other examples, the ROR pretreatment resulted in a reduction in the Ni deposit size for $\mathrm{Ni}_{-} \mathrm{SiO}_{2}$ [52] or an increase in the dispersion of $\mathrm{Ru}$ in $\mathrm{Ru}-\mathrm{Co} / \mathrm{Al}_{2} \mathrm{O}_{3}$, which ultimately increased the CO conversion in the Fischer-Tropsch reaction [53]. In comparison to the samples that were just reduced without any pretreatment ( $\mathrm{R}$ samples), Lovel et al (Table 1, entry 11) showed that the ROR pretreated samples were characterized by smaller and greatly dispersed $\mathrm{Ni}$ (12.8 $\mathrm{nm}$ for calcined vs. $8.7 \mathrm{~nm}$ for ROR pretreated $10 \mathrm{wt}$.\% Ni-SiO 2 ). Using a rather important GHSV of $144 \mathrm{~L} \mathrm{~g}^{-1} \mathrm{~h}^{-1}$, the authors revealed that after the ROR pretreatment, an enhancement occurred in the $\mathrm{CH}_{4}$ conversion (from $57 \%$ to $68 \%$ at $800{ }^{\circ} \mathrm{C}$ ), particularly for the solid containing $10 \mathrm{wt} . \%$ of $\mathrm{Ni}$. Such improvement was attributed to the increased active metallic surface area caused by this type of pretreatment. However, the pretreatment did not lead to better stability

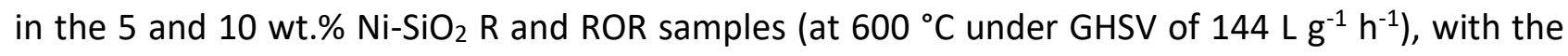
particles exhibiting a dramatic decrease $(\approx 30 \%)$ in activity after approximately $3-4 \mathrm{~h}$ of run time.

\subsection{Examples with Porous Supports}

Mesoporous supports provide a huge specific surface area, which facilitates metal dispersion. However, most of the available surface area has to be accessible to the metal precursors, which implies that dispersion strategies have to be implemented to take full advantage of these supports. Significant efforts have been put into utilizing original nickel precursors or the assistance of ligands or organic molecules. Certain authors also investigated the impact of support properties. The effects of the impregnation conditions have also been studied. Most of the works discussed in this section concern the materials obtained through the impregnation of preformed supports, although certain examples concerning one-pot strategies for $\mathrm{Ni}$ incorporation are presented as well.

\subsubsection{Use of Original Nickel Precursors or the Assistance of Organic Molecules}

One approach for controlling the size of the targeted $\mathrm{Ni}^{0}$ NPs prepared using impregnation techniques is the use of specific nickel precursors. Li et al. prepared and utilized a hexamethylenetetramine ( $\mathrm{HMA}$ ) $\mathrm{Ni}(\mathrm{II})$ complex (referred to as $\left(\mathrm{NO}_{3}\right)_{2} \mathrm{Ni}\left(\mathrm{H}_{2} \mathrm{O}\right)_{6}(\mathrm{HMA})_{2} \cdot 4 \mathrm{H}_{2} \mathrm{O}$ by the 
authors), the formation of which could be attested using FTIR spectroscopy (Figure 2), to improve the insertion of Ni into mesoporous support of type SBA-15 (Table 1, entry 13) [40].
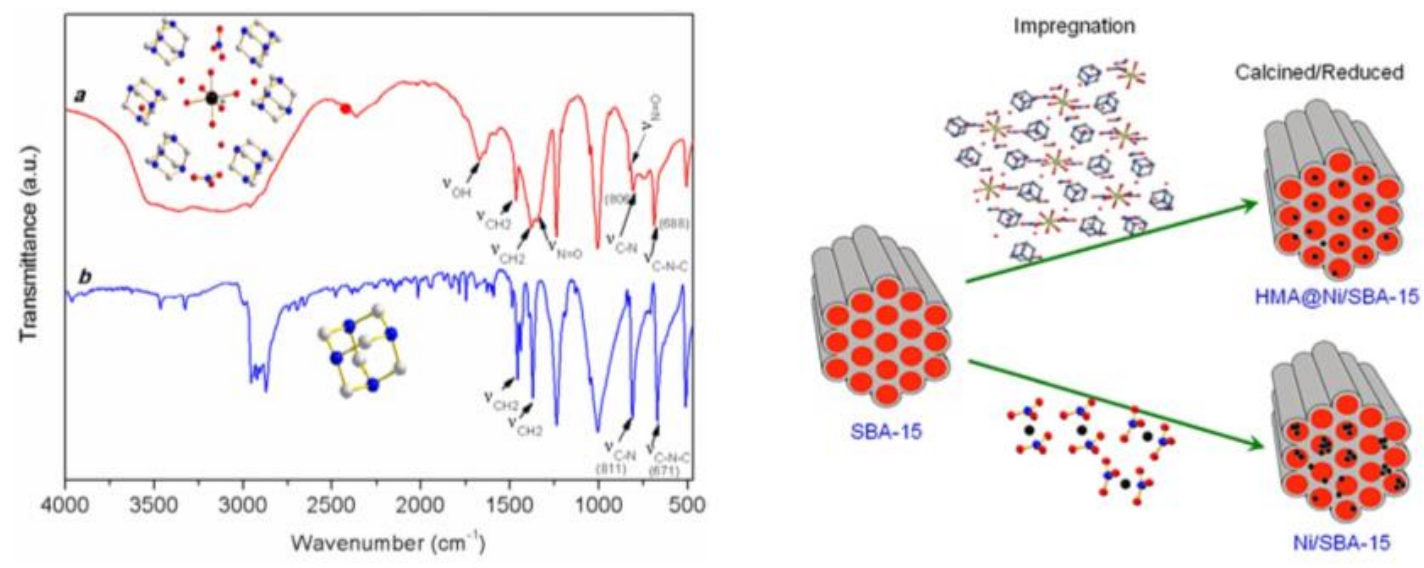

Figure 2 Left: The FTIR spectra of a: the as-synthesized $\left(\mathrm{NO}_{3}\right)_{2} \mathrm{Ni}\left(\mathrm{H}_{2} \mathrm{O}\right)_{6}(\mathrm{HMA})_{2} \cdot 4 \mathrm{H}_{2} \mathrm{O}$ complex and b: HMA; Right: The schematic illustration of the improved Ni dispersion for HMA@Ni/SBA-15 compared to Ni/SBA-15 [40].

HMA@Ni/SBA-15 (10.5 wt.\% of $\mathrm{Ni}$ ) was prepared through the incipient wetness impregnation of SBA-15 with the minimum amount of $\left(\mathrm{NO}_{3}\right)_{2} \mathrm{Ni}\left(\mathrm{H}_{2} \mathrm{O}\right)_{6}(\mathrm{HMA})_{2} \cdot 4 \mathrm{H}_{2} \mathrm{O}$ solution. For comparison, another material, Ni/SBA-15 (12.7 wt.\% of $\mathrm{Ni}$ ), was synthesized in a similar manner using a nickel nitrate aqueous solution. Smaller Ni NPs were observed for HMA@Ni/SBA-15 (4.9 nm) compared to $\mathrm{Ni} / \mathrm{SBA}-15(7.5 \mathrm{~nm})$. Better incorporation of $\mathrm{Ni}$, less porosity plugging, and higher dispersion were also deduced from the results of TEM analysis, $\mathrm{N}_{2}$ physisorption analysis, and XRD measurements for HMA@Ni/SBA-15. Therefore,HMA@Ni/SBA-15 was a promising catalyst in good agreement with the characteristics of the materials used. At $750{ }^{\circ} \mathrm{C}$, under a relatively high GHSV of $100 \mathrm{~L} \mathrm{~g}^{-1} \mathrm{~h}^{-1}$, HMA@Ni/SBA-15 exhibited a considerably good stability during $30 \mathrm{~h}$, with the $\mathrm{CH}_{4}$ and $\mathrm{CO}_{2}$ conversion rates of $76 \%$ and $85 \%$, respectively. On the contrary, the $\mathrm{CH}_{4}$ and $\mathrm{CO}_{2}$ conversion rates achieved with Ni/SBA-15 derived from the nickel nitrate aqueous solution decreased from $71 \%$ and $77 \%$ to $65 \%$ and $68 \%$, respectively.

In another study, the controlled formation of nickel oxide nanoparticles on SBA-15 was achieved through incipient wetness impregnation of the support using $\mathrm{Ni}_{4} \mathrm{O}_{4}$ cluster as the precursor $(0.5<$ $\mathrm{Ni}$ wt.\% < 5) (Table 1, entry 14) [41] generating $\mathrm{Ni}_{4} / \mathrm{SBA}-15$. The use of such a ligand-stabilized cluster enabled the deposition of four $\mathrm{Ni}$ ions in close proximity on the silica support, which in turn, according to the authors, led to the formation of small and highly-dispersed NiO NPs after heat treatment. These clusters would exert a significant influence on the generation of NiO NPs compared to a conventional nickel(II) salt such as $\mathrm{Ni}(\mathrm{OAc})_{2}$. The utilization of the cubane $\mathrm{Ni}_{4} \mathrm{O}_{4}$ precursor would result in, as the author hypothesized, a faster formation of small and highlydispersed NiO nanoparticles, although the mean size and dispersion of the particles were not reported. Undoubtedly, the resulting materials demonstrated enhanced stability toward DRM. Further precisely, at $550{ }^{\circ} \mathrm{C}, \mathrm{Ni}_{4} / \mathrm{SBA}-15$ (1 wt.\%) led to $23 \% \mathrm{CH}_{4}$ conversion even after $20 \mathrm{~h}$, while a reference material Ni/SBA-15 containing 1 wt.\% of $\mathrm{Ni}$ (1-Ni/SBA-15) was completely deactivated under similar conditions [41]. However, these results must be considered with a critical mindset as the DRM tests in both the evaluations were performed under a rather modest GHSV $\left(36 \mathrm{~L} \mathrm{~g}^{-1} \mathrm{~h}^{-1}\right)$ 
compared to that used in work reported by Li et al. (Table 1, entry 13).

Organic additives may also be useful in this regard. Gao et al. (Table 1, entry 15) [42] prepared $5 \% \mathrm{Ni} / \mathrm{SiO}_{2}$ catalysts promoted with oleylamine (OAm) and/or oleic acid (OAc) using an incipient wetness impregnation method. In this approach, OAm and/or OAc were used as surfactants, ligands, and/or reducing agents added on purpose to the solution of nickel nitrate prior to the impregnation in order to improve the dispersion of $\mathrm{Ni}$ on silica due to the strong interaction of the Ni precursors with OAm and OAc. Using a mixture of OAm and OAc with the spheres of mesoporous silica (particles size $=40-60 \mathrm{~mm}$, specific surface area $=753 \mathrm{~m}^{2} \mathrm{~g}^{-1}$, mean pore size $=7.5 \mathrm{~nm}$ ) produced, after calcination, small $\mathrm{NiO}$ particles in the $\mathrm{Ni} / \mathrm{SiO}_{2}-\mathrm{OAmc}$ material, and therefore, after reduction by $\mathrm{H}_{2}$, to small $\mathrm{Ni}^{0} \mathrm{NPs}$ (Table 1 , entries 5,15$)[32,42]$. Using this approach, the authors managed to achieve $5 \% \mathrm{Ni} /$ silica with a nickel dispersion of c.a. $22 \%$ (mean diameter of the particles: $4.6 \mathrm{~nm}$ ). The $\mathrm{Ni}$ dispersion was only $11.5 \%$ ( $8.8 \mathrm{~nm}$ particle diameter size) with $\mathrm{OAc}$ only $\left(\mathrm{Ni} / \mathrm{SiO}_{2}-\mathrm{OAc}\right.$ ). Accordingly, $\mathrm{Ni} / \mathrm{SiO}_{2}-\mathrm{OAmc}$ presented the highest conversion rate values in the series $\left(\mathrm{CH}_{4}: 73 \%\right.$, $\mathrm{CO}_{2}: 75 \%$, with a rather good GHSV of $72 \mathrm{~L} \mathrm{~g}^{-1} \mathrm{~h}^{-1}$ ) and the best stability (a decrease of $3 \%-5 \%$ after $17 \mathrm{~h})$.

Zhang et al. evaluated certain other ligands, such as ethylenediamine (en) and citric acid (cit), which are known to be strong chelating agents, with aqueous $\mathrm{Ni}\left(\mathrm{NO}_{3}\right)_{2} \cdot 6 \mathrm{H}_{2} \mathrm{O}$, to generate metal chelates prior to the impregnation of SBA-15 (7.5 wt.\% of Ni) (Table 1, entry 1) [28]. The DRM catalysis tests with the corresponding materials revealed that ethylenediamine (en) and citric acid (cit) play a positive role in promoting the dry reforming of $\mathrm{CH}_{4}$. Basically, the strong coordination ability of these ligands would inhibit the aggregation of the nickel species in the early stage of catalyst preparation, thereby contributing to the formation of well-dispersed Ni particles with low diameter size [below $6 \mathrm{~nm}$ for $7.5 \mathrm{wt} . \% \mathrm{Ni} / \mathrm{SBA}-15(\mathrm{en})$ ] compared to the $18.9 \mathrm{~nm}$ diameter size of a reference $\mathrm{Ni} / \mathrm{SBA}-15$ prepared using a more conventional impregnation method [7.5 wt.\% Ni/SBA15(IWI)]. Again, the better dispersion obtained in the presence of chelating agents led to improved catalysis results [ $45 \%$ vs. $12 \%$ for $\mathrm{CH}_{4}$ and $55 \%$ vs. $25 \%$ for $\mathrm{CO}_{2}$ conversions at $600{ }^{\circ} \mathrm{C}$ for $\mathrm{Ni} / \mathrm{SBA}$ 15(en) and Ni/SBA-15(IWI), respectively]. It is noteworthy that the GHSV used was more modest ( $22.5 \mathrm{~L} \mathrm{~g}^{-1} \mathrm{~h}^{-1}$ ) compared to the one in the study by Gao et al. $\left(72 \mathrm{~L} \mathrm{~g}^{-1} \mathrm{~h}^{-1}\right)$, who used oleylamine and oleic acid. However, Zhang et al. performed the stability test for a longer duration (50 h) without observing any significant decreases in the $\mathrm{CH}_{4}$ and $\mathrm{CO}_{2}$ conversion rates.

Furthermore, functionalized polymers are employed for improving the incorporation of $\mathrm{Ni}$ precursor without affecting the dispersion of the resulting $\mathrm{Ni}(0)$ particles. Polyethyleneimine (PEI), which is used as a stabilizer, reducing agent, and capping agent for the synthesis of nanoparticles in aqueous solutions [54, 55], was evaluated by Kang et al. (Table 1, entry 16) [43] for the introduction of nickel(II) within hexagonally-ordered mesoporous materials such as SBA-15 and the mesocellular foams such as SBA-15-TMB. PEI was first dissolved in deionized water and then contacted with $\mathrm{Ni}\left(\mathrm{NO}_{3}\right)_{2} .6 \mathrm{H}_{2} \mathrm{O}$ to obtain PEI-Ni complexes prior to the impregnation of the supports up to 7 wt.\% (inorganic weight percentage), producing PEI-Ni/SBA-15 and PEI-Ni/SBA-15-TMB, respectively. In the impregnation step, PEI-Ni complexes were expected to develop strong interactions with the mesoporous silica. The best nickel incorporation was achieved for SBA-15-TMB, probably due to a better fit between the mean pore diameter and the PEI-Ni complexes. Both PEI-Ni/SBA-15 and PEI$\mathrm{Ni} / \mathrm{SBA}-15-\mathrm{TMB}$ exhibited reasonable performances [c.a. $85 \%$ for $\mathrm{CH}_{4}$ and $\mathrm{CO}_{2}$ conversions] and acceptable stability. A decrease of only $5 \%$ to $8 \%$ was observed in the $\mathrm{CH}_{4}$ and $\mathrm{CO}_{2}$ conversion rates within $40 \mathrm{~h}$. However, it is noteworthy that the authors used a rather low GHSV $\left(20 \mathrm{~L} \mathrm{~g}^{-1} \mathrm{~h}^{-1}\right)$. In a 
parallel investigation, Yang et al. (Table 1, entry 17) [44] investigated the influence of poly(N-vinyl2-pyrrolidone) (PVP), another nonionic polymer [56], on the Ni dispersion of the materials upon the wet impregnation of $\mathrm{SBA}-15$ using $\mathrm{Ni}\left(\mathrm{NO}_{3}\right)_{2} \cdot 6 \mathrm{H}_{2} \mathrm{O}$ as the nickel source. Here, similar to the previous examples, which involved PEI, PVP was used as a ligand of $\mathrm{Ni}^{2+}$ during its incorporation onto silica. In order to better understand the potential role of PVP, the authors varied the amounts used, for which they prepared a series of Ni/SBA15-PVP materials targeting $5 \mathrm{wt} . \%$ of Ni (PVP not included) with different $n P V P / n N i$ molar ratios $(1 / X=1 / 7500,1 / 5000,1 / 2000,1 / 1000,1 / 100$, etc.) and one reference sample ( $\mathrm{Ni} / \mathrm{SBA} 15$, no PVP). The TEM, $\mathrm{H}_{2}$ sorption, and XRD measurement results demonstrated that the $1 / \mathrm{X}$ ratio influenced the nickel confinement and dispersion. Therefore, the $\mathrm{Ni} / \mathrm{SBA} 15-\mathrm{PVP}$ solids with $1 / \mathrm{X}=1 / 5000,1 / 2000$, and $1 / 1000$ presented the dispersion values of $12.5 \%, 11.8 \%$, and $12.2 \%$, respectively and small $\mathrm{Ni}^{0}$ particles $(8,8.5$, and $8.2 \mathrm{~nm}$, respectively), while $\mathrm{Ni} / \mathrm{SBA} 15, \mathrm{Ni} / \mathrm{SBA} 15-\mathrm{PVP}$ (1/7500), and Ni/SBA15-PVP(1/100) presented larger aggregates and bigger particles on the external surface of the support. These characteristics were in agreement with the catalysis performances exhibited by the materials measured under a reasonable GHSV ( $75 \mathrm{~L} \mathrm{~g}^{-1}$ $\left.\mathrm{h}^{-1}\right)$. Under these conditions, the Ni/SBA15-PVP solids with $1 / 5000,1 / 2000$, and $1 / 1000$ ratios exhibited rather good stability at $750{ }^{\circ} \mathrm{C}$ after $25 \mathrm{~h}$ on stream, with almost no decrease in the $\mathrm{CH}_{4}$ and the $\mathrm{CO}_{2}$ conversions (stable at $80 \%$ for both), while with $\mathrm{Ni} / \mathrm{SBA} 15, \mathrm{Ni} / \mathrm{SBA} 15-\mathrm{PVP}(1 / 7500)$, and $\mathrm{Ni} /$ SBA15-PVP(1/100), the activity decreased by $15 \%$ after $25 \mathrm{~h}$. A detailed study of the solids recovered at each step of catalyst preparation, i.e., at impregnation, drying, and calcination, generated results that were compatible with the formation of specific complexes of $\mathrm{Ni}$ by its coordination to the $\mathrm{N}$ and $\mathrm{O}$ atoms of PVP, which would reduce the redistribution of the metal, resulting in better dispersion and stabilization of the metal.

\subsubsection{Impact of Supports and Impregnation Conditions}

The silica support may itself play an important role in the control and confinement of nickel NPs. Certain works concerning the influence of the porosity of the supports (either micropores vs. mesopores or among mesopores with different sizes) on the nickel size and dispersion are discussed ahead.

In 2017, Drobna et al. (Table 1, entry 3) [30] reported a complete study that involved the use of zeolites such as $\mathrm{MFI}$ and $\mathrm{FAU}, \mathrm{Al}_{2} \mathrm{O}_{3}$, mesoporous aluminosilica such as $\mathrm{Al}-\mathrm{MCM}-41$, or mesoporous silica such as SBA-15, coupled with $\mathrm{Ni}$, to investigate the relationship between the catalysis performances and the textural properties of the supports. The Ni-based catalysts with $11 \mathrm{wt} . \%$ of $\mathrm{Ni}$ were prepared through wet impregnation in ethanol, although such a loading did not allow all the Ni NPs to be confined within the porosity even in the cases of Ni/SBA-15 and Ni/Al-MCM-41. Nevertheless, both Ni/SBA-15 and Ni/AI-MCM-41, with Ni particle sizes of $11 \mathrm{~nm}$, exhibited the best performances in DRM (approximately $80 \% \mathrm{CH}_{4}$ conversion) after $20 \mathrm{~h}$ on stream under a GHSV of $150 \mathrm{~L} \mathrm{~g}^{-1} \mathrm{~h}^{-1}$. In the case of $\mathrm{Ni} / \mathrm{MFI}$ with the nickel particle size of $29 \mathrm{~nm}$, the conversion value was $55 \%$, which appeared to be rather unusual with such size and under such harsh test conditions.

Similarly, Zhang et al. (Table 1, entry 2) [29] reported a study focusing on ordered mesoporous silica materials with either different pore diameters (Ni-SBA-15 vs. Ni-MCM-41) or different pore structures (hexagonal vs. cubic such as KIT-6) and used an original Ni incorporation method based on solid-state grinding. The materials with $5 \mathrm{wt} . \%$ of Ni were prepared from the mixtures of the support and the $\mathrm{Ni}$ source $\left(\mathrm{Ni}\left(\mathrm{NO}_{3}\right)_{2} \cdot 6 \mathrm{H}_{2} \mathrm{O}\right)$ without any significant degradation in the textural 
properties of the support compared to the reference ones obtained using conventional impregnation. The Ni particles on the Ni-SBA-15 samples prepared using this solid-state grinding method were among the smallest ones obtained in the study (compared to MCM-41 and KIT), implying that the nickel species were much more dispersed and in apparently greater interaction with the SBA-15 support, as a consequence of which, the Ni-SBA-15 sample exhibited the lowest decrease in activity (approximately 3\% to 4\% in general) in the DRM reaction after $100 \mathrm{~h}$ at $700{ }^{\circ} \mathrm{C}$ compared to $14 \%-15 \%$ decrease obtained for the Ni/SBA-15 sample prepared using an impregnation method. The catalytic activity and stability were observed to decline in the following sequence: $\mathrm{Ni}$ SBA-15 > Ni-KIT-6 > Ni-MCM-41 > conventional Ni/SBA-15. However, the GHSV used in this work was relatively low $\left(22.5 \mathrm{~L} \mathrm{~g}^{-1} \mathrm{~h}^{-1}\right)$. Therefore, Zhang et al. suggested that the solid-state grinding method improves the dispersion of the active component on carriers, although they did not report measuring the Ni dispersion.

Structured mesoporous materials provide high specific surface areas and high pore volumes that should facilitate high metal dispersions. However, the impregnation method used might be quite determining, as demonstrated in various previous studies. In the study reported by Kaydouh et al. (Table 1, entry 18) [45], who claimed to have prepared a series of Ni/SBA-15 catalysts with $2.5<\mathrm{Ni}$ wt.\% $<7.5 \%$ with improved metal insertion into the pore structure, better confinement of the metal in comparison to that obtained using the conventional incipient wetness impregnation was achieved by using, for the first time, "Two-Solvents" (TS) impregnation. The innovation resided in the protocol, which involved: i) suspending the siliceous support in an apolar organic solvent (cyclohexane) and ii) adding a volume of an aqueous solution containing the adequate quantity of Ni precursor that was precisely equal to the pore volume of the support. Owing to the extremely low miscibility of water with the organic solvent, the aqueous solution containing the Ni precursor was supposed to penetrate the porosity more easily compared to its penetration in the absence of the organic cosolvent [57]. The success of the procedure was confirmed by the TEM and SEM results, particularly for the materials with 2.5 and $5 \mathrm{wt} . \%$ of $\mathrm{Ni}$. The resulting catalysts reduced under $\mathrm{H}_{2}$ at $650{ }^{\circ} \mathrm{C}$ and tested in DRM exhibited rather high activity and considerably good stability for $12 \mathrm{~h}$ (GHSV $=52.8 \mathrm{~L}$ $\left.\mathrm{g}^{-1} \mathrm{~h}^{-1}\right)$.

Other approaches that have been implemented include the deposition-precipitation (DP) method, originally reported by Geus et al. [58]. Practically, the preparation of $\mathrm{Ni} / \mathrm{SiO}_{2}$ catalysts using the DP method involves the precipitation of the nickel species onto the silica surface via an increase in the $\mathrm{pH}$ of the $\mathrm{Ni}$ salt solution used for impregnation. Subsequently, $\mathrm{Ni}$-anchoring occurred on the basis of the kinetic competition between two types of reactions, the $\mathrm{Ni}-\mathrm{O}-\mathrm{Si}$ heterocondensation/polymerization and the $\mathrm{Ni}-\mathrm{OH}-\mathrm{Ni}$ olation/polymerization, which lead to the formation and growth of nickel phyllosilicate and nickel hydroxide, respectively [59]. Rodriguez et al. (Table 1, entry 19) [46] prepared four materials with up to $10 \mathrm{wt} . \%$ of Ni impregnated onto SBA15 , and another mesoporous $\mathrm{SiO}_{2}$ support using either the DP method (in the presence of urea) or the incipient wetness impregnation (IWI) combined with ultrasound. The TEM images (Figure 3) depicted fibrous structures for SBA-15-DP and $\mathrm{SiO}_{2}$-DP calcined samples, while no such observations were made when the IWI impregnation was used. 

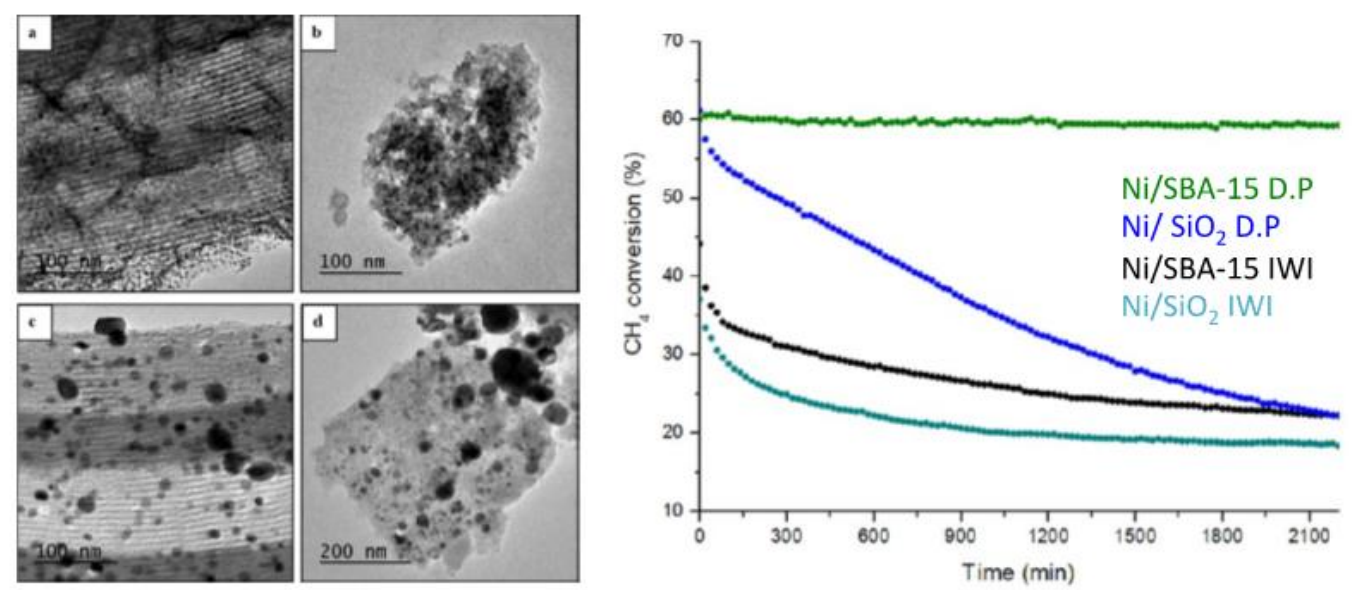

Figure 3 Left - TEM images of reduced: a: Ni/SBA-15 DP, b: Ni/SiO 2 DP, c: Ni/SBA-15 IWI, and $\mathrm{d}$ : $\mathrm{Ni} / \mathrm{SiO}_{2} \mathrm{IWI}$; Right $-\mathrm{CH}_{4}$ conversion in the reaction of DRM at $750{ }^{\circ} \mathrm{C}$ under one of the best GHSV $\left(240 \mathrm{~L} \mathrm{~g}^{-1} \mathrm{~h}^{-1}\right)$ reported [46].

According to the XRD, XANES, and EXAFS results, these observed structures corresponded to $\mathrm{Ni}$ phyllosilicates that are formed upon calcination of dried impregnated solid and, which, after reduction, produce small and well-dispersed Ni NPs (mean size of approximately $4.4 \mathrm{~nm}$ as confirmed by the particles size distribution measurement using TEM). The authors stated that the use of DP impregnation led to the confinement of the nickel particles at the inner surface of Ni/SBA15 , rendering them much more active ( $60 \%$ for $\mathrm{CH}_{4}$ conversion) compared to those prepared using a conventional approach (35\%-40\%) and also more stable [no deactivation in the case of Ni/SBA-15DP vs. a 20\% deactivation in the other case (IWI)] (Figure 3). These results demonstrated that the confinement of nickel prevents sintering and coke deposition. Moreover, the GHSV value used in that work is one of the highest ones $\left(240 \mathrm{~L} \mathrm{~g}^{-1} \mathrm{~h}^{-1}\right)$ reported in the literature.

Galvez et al. (Table 1, entry 20) [47] also attempted to evaluate the influence of the preparation method on the synthesis of $10 \mathrm{wt} . \% \mathrm{Ni} / \mathrm{SBA}-15$. Three different synthesis methods were evaluated, i.e., the incipient wetness impregnation (IWI), the deposition-precipitation (DP), and precipitation in the presence of ascorbic acid as a reducing agent (RM). In the last method, the catalyst was prepared following a procedure quite similar to the conventional DP method, except for the addition of ascorbic acid to the support suspended in distilled water after the introduction of urea. The authors of this work demonstrated that the physicochemical features of the obtained materials were influenced strongly by the preparation procedure used. The DP and RM methods led to the $\mathrm{Ni}$ particles being confined in the mesopores of SBA-15 (verified by TEM), while the simple IWI method led to the Ni particles being mostly deposited on the outer surface of the silica grains. The dispersion values of the $\mathrm{Ni}(0)$ phase obtained from the $\mathrm{H}_{2}$ chemisorption experiments were observed to be enhanced for the DP (8.5\%) and RM (8.7\%) catalysts in comparison to the IWI catalyst (2.7\%). Such differences were also reflected in the activity, selectivity, and stability exhibited in the DRM. After $24 \mathrm{~h}$ on stream, under a relatively modest GHSV $\left(20 \mathrm{~L} \mathrm{~g}^{-1} \mathrm{~h}^{-1}\right)$, the Ni/SBA-15-RM prepared using ascorbic acid produced a $\mathrm{CH}_{4}$ conversion rate $(65 \%)$ that was quite close to the conversion rate obtained with $\mathrm{Ni} / \mathrm{SBA}-15-\mathrm{DP}(67 \%)$ and significantly higher than the rate obtained in the presence of the solid prepared using the IWI method (40\%) that underwent rapid deactivation. Therefore, the authors concluded that the addition of a reducing agent during the precipitation synthesis method 
led to the catalyst exhibiting improved stability, relatively higher activity, and enhanced selectivity toward DRM. It is, however, noteworthy that the dispersions of nickel on the solids prepared using the DP and RM methods were quite similar, although the TPR results revealed higher reduction temperatures in the case of the DP catalyst, which was attributed to better metal-support interactions. The authors concluded that the decrease in the textural properties upon $\mathrm{Ni}$ addition was due to the incorporation of $\mathrm{Ni}$ inside the pores. However, such decrease (approximately 65\% and $50 \%$ of the specific surface area and the pore volume, respectively) can rather be explained by a certain level of degradation of SBA-15 due to the presence of urea, which when thermally decomposed, generates $\mathrm{NH}_{3}$ that might be responsible for the alkaline attack of silica [59].

\subsubsection{One-Pot Strategies for Ni Incorporation}

One-pot (OP) incorporation of $\mathrm{Ni}$ is an alternative strategy that involves incorporating the nickel source directly into the silica's synthesis gel. This strategy could be promising in terms of simplicity and nickel dispersion throughout the resulting material. However, the one-pot preparation method is limited by the partial incorporation of the metal cation, particularly during the synthesis reactions performed under acidic conditions. Another strong limitation of this method when applied to mesoporous materials is associated with the decrease in the textural properties of the resulting samples. Indeed, when the metal salt is added directly into the synthesis gel, the structuration of the pores could be modified in comparison to the use of the silica precursor alone. For instance, while a decrease of approximately $20 \%$ could be achieved in the $S_{B E T}$ with the use of post-synthesis methods such as TS, IWI, etc. [45-47] for metal introduction, a worse deterioration (approximately $80 \%$ ) is achieved with OP pathways $[12,17]$. In this section, a few examples of the works concerning the successful preparation of $\mathrm{Ni}$-based silica using the one-pot strategy are discussed.

Wang et al. (Table 1, entry 7) [34] evaluated the OP procedure with HMS mesoporous silica obtained using an $\mathrm{S}^{0} \mathrm{I}^{0}$ assembly pathway [60]. Four HMS samples (denoted as $\mathrm{xNi}$-HMS) incorporating different $\mathrm{Ni}$ loadings $(x=2.5 \%, 5 \%, 7.5 \%, 10 \%$, and $15 \%)$ were synthesized using dodecylamine as the structure-directing agent, followed by a comparison of these samples with $\mathrm{Ni} / \mathrm{HMS}$ (7.5 wt.\% of $\mathrm{Ni}$ ) prepared through incipient wetness impregnation (IWI). The authors indicated different advantages of using the one-pot strategy with HMS. First, such a synthesis was considered time-saving and convenient in operation. Second, the xNi-HMS materials were prepared at room temperature without hydrothermal crystallization, which minimized energy consumption. Third, the template used for HMS was a primary amine, which is much less expensive compared to block polymers such as Pluronic 123 (used for SBA-15) and can be recovered through solvent extraction. Wang et al. reported that highly dispersed/confined small and homogeneous particles of $\mathrm{Ni}$ were observed in the XNi-HMS samples. However, the $\mathrm{CO}$ chemisorption results revealed that the dispersion range obtained for these solids was between $6.9 \%$ and $5.2 \%$, which are not adequately high values. Moreover, the dispersions appeared to be close to the one obtained with impregnated $\mathrm{Ni} / \mathrm{HMS}$ (4.3\%). The 2.5 $\mathrm{Ni}$-HMS exhibited the highest $\mathrm{Ni}$ dispersion (6.9\%) and led to higher catalysis performances in DRM compared to its analogs with $\mathrm{x}=5,7.5,10$, and $15 \mathrm{wt} . \%$ of $\mathrm{Ni}$ as well as to the reference solid 7.5Ni/HMS. According to Wang et al., the solids obtained using this one-pot approach exhibited excellent stability, with approximately $90 \%$ of $\mathrm{CH}_{4}$ and $\mathrm{CO}_{2}$ conversion rates, respectively, at $800^{\circ} \mathrm{C}$, due to the strong anchoring effect of the silica wall that improved the resistance of the particles toward coking and sintering. However, it is noteworthy that these 
conversions were obtained under a rather low GHSV $\left(22.5 \mathrm{~L} \mathrm{~g}^{-1} \mathrm{~h}^{-1}\right)$.

Tian and co-workers (Table 1, entry 21) [48] also used a one-pot synthesis to obtain $\mathrm{Ni@SiO}_{2}$ catalyst for DRM, beginning with $\mathrm{Ni}$ nanoparticles encapsulated in dendritic silica structures. $\mathrm{Ni@SiO} 2$ with 4.9 wt.\% of $\mathrm{Ni}$ thus prepared exhibited a better dispersion of $\mathrm{Ni}$ with smaller nanoparticles $(2-3 \mathrm{~nm}$ ) compared to the reference material (4.8 Ni wt.\% with $16 \mathrm{~nm}$ particles) obtained using a conventional impregnation method. In the latter, the Ni NPs were observed at both inner and outer surfaces, and the resulting solid underwent a total deactivation at $700{ }^{\circ} \mathrm{C}$ after $50 \mathrm{~h}$ on stream, while the material prepared using the one-pot synthesis exhibited high stability (81\% and $72 \%$ for $\mathrm{CO}_{2}$ and $\mathrm{CH}_{4}$ conversions, respectively, after $50 \mathrm{~h}$ ) although at a rather low GHSV value $\left(40 \mathrm{~L} \mathrm{~g}^{-1} \mathrm{~h}^{-1}\right)$. Using theoretical calculation, the authors determined the formation energy of the different $\mathrm{C}$ clusters on the Ni particles, revealing that the growth of carbon nanotubes was difficult on the surface of small and confined nanoparticles obtained using the one-pot strategy.

\section{Promoters and Bimetallic Ni-silica based Catalysts}

Combining nickel with other metals or oxides may lead to synergistic effects as it would change the surface properties of $\mathrm{Ni}$, thereby conferring better catalytic performances than exhibited when using $\mathrm{Ni}$ alone [26]. In this section, the contribution of the various additives that were used to promote nickel-silica based catalysts in the DRM reaction is presented (Table 2). 
Table 2 A selection of papers dealing with promoters and bimetallic catalysts published between 2015-2018 [45, 61-92].

\begin{tabular}{|c|c|c|c|c|c|c|c|c|c|c|c|c|c|c|c|}
\hline $\mathbf{E}$ & Year & $\begin{array}{l}\mathrm{Ni} \\
\text { (wt.\%) }\end{array}$ & $\begin{array}{l}\mathrm{NiO} \\
\text { or } \\
\left(\mathrm{Ni}{ }^{0}\right) \\
(\mathrm{nm})\end{array}$ & $\begin{array}{l}\text { Promoters } \\
\text { element }\end{array}$ & $\begin{array}{l}\text { Siliceous } \\
\text { support }\end{array}$ & $\begin{array}{l}\text { Preparation } \\
\text { method }\end{array}$ & $\begin{array}{l}\text { Total } \\
\text { flow } \\
(\mathrm{mL} \\
\mathrm{min}^{-} \\
\left.{ }^{1}\right)\end{array}$ & $\begin{array}{l}\text { GHSV } \\
L g^{-1} \\
h^{-1}\end{array}$ & $\begin{array}{l}\text { Weight } \\
\text { (mg) }\end{array}$ & $\begin{array}{l}\mathrm{T} \\
\left({ }^{\circ} \mathrm{C}\right)\end{array}$ & $\begin{array}{l}\text { Time } \\
\text { (h) }\end{array}$ & $\begin{array}{l}\mathrm{CH}_{4} \\
\%\end{array}$ & $\begin{array}{l}\mathrm{CO}_{2} \\
\%\end{array}$ & Objectives & Ref \\
\hline 1 & 2017 & $0-10$ & (5.7) & $\begin{array}{l}\text { Co } \quad(0-10 \\
\text { wt.\%) }\end{array}$ & $\begin{array}{l}\mathrm{SiO}_{2} \quad \text { from } \\
\text { phyllosilicates }\end{array}$ & HM & 30 & 60 & 30 & 750 & 100 & 86 & 89 & $\begin{array}{l}\text { Ni-Co alloy derived from } \\
\text { phyllosilicates }\end{array}$ & [61] \\
\hline 2 & 2018 & $2.5-10$ & n.d. & $\begin{array}{l}\text { Co } \\
(0-10 \\
\text { wt.\%) }\end{array}$ & SBA-15 & Co-P & 60 & 72 & 50 & 700 & 50 & 70 & 60 & $\begin{array}{l}\text { Bimetallic } \mathrm{Ni}-\mathrm{Co} \text { for } \\
\text { higher activity and } \\
\text { stability }\end{array}$ & [62] \\
\hline 3 & 2017 & $4-5$ & n.d. & Co (5 wt.\%) & $\begin{array}{l}\text { Spherical } \\
{\mathrm{M} . \mathrm{SiO}_{2}}\end{array}$ & IWI & 60 & 72 & 50 & 700 & 30 & 79 & 82 & $\begin{array}{l}\text { OAm/OAc organic pair } \\
\text { for the improvement of } \\
\text { performances of } \mathrm{Ni}-\mathrm{Co} \\
\text { bimetallic catalysts }\end{array}$ & [63] \\
\hline 4 & 2018 & $2.5-5$ & 16.9 & $\begin{array}{l}\text { Co ( } \\
1-5 w t . \%)\end{array}$ & SBA-15 & WI & 60 & 36 & 100 & 750 & 4 & 80 & 90 & $\begin{array}{l}\mathrm{Ni} / \mathrm{SBA}-15 \text { promoted by } \\
\text { Co }\end{array}$ & [64] \\
\hline 5 & 2015 & 20.9 & (8) & $\begin{array}{l}\mathrm{Cu} \\
(0-16 \\
\text { wt. } \%)\end{array}$ & 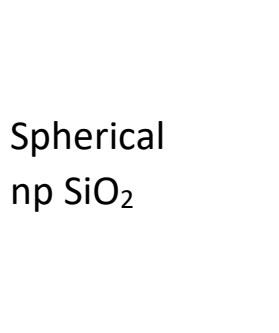 & HM & 20 & 13.3 & 90 & 700 & 30 & 71 & n.d. & $\begin{array}{l}\text { Hierarchical } \mathrm{Ni} \text { and } \mathrm{Ni}- \\
\mathrm{Cu} / \mathrm{SiO}_{2} \text { derived from } \\
\text { phyllosilicates } \\
\text { (nanoparticles } \\
\text { embedded } \\
\text { nanosheets) }\end{array}$ & [65] \\
\hline 6 & 2016 & $\begin{array}{l}2.5- \\
7.5\end{array}$ & 5 & $\begin{array}{l}\mathrm{Ce} \\
(6 \text { wt.\%) }\end{array}$ & $\begin{array}{l}\text { SBA-15, } \\
\text { M. } \mathrm{SiO}_{2}\end{array}$ & TS & 88 & $\begin{array}{l}52.8 \\
264\end{array}$ & 20,100 & 500 & 12 & 69 & 63 & $\begin{array}{l}\text { Confinement of nickel } \\
\text { using SBA-15 }\end{array}$ & [45] \\
\hline 7 & 2017 & 10 & 5.3 & $\mathrm{Ce}, \mathrm{Zr}$ & $\mathrm{np} \mathrm{SiO} 2$ & WI & 60 & 144 & 25 & 700 & 24 & 50 & n.d. & $\begin{array}{l}\text { Examination of the } \\
\text { intricate relationship }\end{array}$ & [66] \\
\hline
\end{tabular}


JEPT 2021; 3(1), doi:10.21926/jept.2101007

(CeZr both

57-84

wt.\%)

$8 \quad 2017 \quad 5-7$

$9 \quad 2016 \quad 10$

$10 \quad 2016 \quad 10$

(16.3)

(6.2)

$\begin{array}{ll}\mathrm{Ce} & (2.5 \\ \text { wt.\%) }\end{array}$

In situ particles

preparation

30

12

150

n.d.

30

12

150

Illite clay

$112015 \quad 10$

9

Ce, Z

(both CeZr SBA-15

10 wt.\%)

$12 \quad 2015 \quad 10$

Ce

(3 wt.\%)

$\mathrm{M} . \quad \mathrm{SiO}_{2}$
nanospheres

(mainly

containing

Co-I

$\mathrm{SiO}_{2}$ )

$12.2015 \quad 10$

$13 \quad 2015 \quad 5$
IWI

SBA-15
TS and Co-I

\begin{abstract}
Ce
(6 wt.\%)
\end{abstract}
between

support

structure and catalytic

performances

In-situ preparation of $\mathrm{Ni}$

nanoparticles

nanochannels of cerium-

modified silica aerogels

Design of NiCe@m-SiO

core-shell structure for [68]

better confinement

Illite clay as support for promoted $\mathrm{Ni}$ by $\mathrm{Ce}$

Non conventional impregnation method for better confinement and dispersion

Immobilizing $\mathrm{Ni}$ in the porosity of mesoporous $\mathrm{SiO}_{2}$ and preparation of modified $\mathrm{Ce}-\mathrm{SiO}_{2}$ support

Order of $\mathrm{Ni}$ and $\mathrm{Ce}$ addition on the catalytic [72] performances 


\begin{tabular}{|c|c|c|c|c|c|c|c|c|c|c|c|c|c|c|c|}
\hline & & & & & & & & & & & & & & Facile & \\
\hline 14 & 2107 & 17.5 & $\begin{array}{l}9.7 \\
(8.4)\end{array}$ & $\begin{array}{l}\text { La } \\
\text { (1-7 wt.\%) }\end{array}$ & M. $\mathrm{SiO}_{2}$ & $\mathrm{OP}$ & n.d. & 72 & 50 & 700 & 40 & 72 & 74 & $\begin{array}{l}\text { preparation of } \mathrm{Ni}- \\
\mathrm{La}_{2} \mathrm{O}_{3} / \mathrm{SiO}_{2}\end{array}$ & [73] \\
\hline 15 & 2017 & 1 & $2-3$ & $\begin{array}{l}\text { La }(0.2-34 \\
\text { wt.\%) }\end{array}$ & $\mathrm{np} \mathrm{SiO} 2$ & IWI & n.d. & n.d. & n.d. & 500 & 43 & n.d. & n.d. & $\begin{array}{l}\text { Study the improvement } \\
\text { of La doping }\end{array}$ & [74] \\
\hline 16 & 2016 & 5 & 2.5 & $\begin{array}{l}\text { La (0-5 } \\
\text { wt.\%) }\end{array}$ & SBA-15 & Modified IWI & 60 & 72 & 50 & 700 & 12 & 83 & 87 & Promotional effect of La & [75] \\
\hline 17 & 2016 & 10 & 19 & La (3 wt.\%) & SBA-15 & IWI and Co-I & 60 & 24 & 150 & 750 & 24 & 92 & 95 & $\begin{array}{l}\text { Influence of Lanthanide } \\
\text { promoters on Ni/SBA-15 }\end{array}$ & [76] \\
\hline 18 & 2018 & 12 & n.d. & $\begin{array}{l}\text { La } \\
\text { (n.d.) }\end{array}$ & M. $\mathrm{SiO}_{2}$ & Stöber & 30 & 18 & 100 & 800 & 8 & 81 & 84 & $\begin{array}{l}\mathrm{LaNiO}_{3} \text { nanocube used as } \\
\mathrm{Ni} \text { precursor embedded } \\
\text { in mesoporous } \mathrm{SiO}_{2}\end{array}$ & [77] \\
\hline 19 & 2017 & 10 & 10 & $\begin{array}{l}\text { Sm, Y, Zr } \\
\text { (3 wt.\%) }\end{array}$ & SBA-15 & TS & 40 & 12 & 200 & 700 & 5 & 71 & 75 & $\begin{array}{l}\text { Comparative study of } \\
\text { samaria, yttria and } \\
\text { zirconia as promoters for } \\
\mathrm{Ni} / \mathrm{SBA}-15\end{array}$ & [78] \\
\hline 20 & 2017 & 10 & 7.2 & $\begin{array}{l}\text { Sm (0.5-1.5 } \\
\text { wt.\%) }\end{array}$ & SBA-15 & TS & 40 & 12 & 200 & 700 & 5 & 54 & 64 & $\begin{array}{l}\text { Sm promotional effect on } \\
\mathrm{Ni} \text { and Co based catalysts }\end{array}$ & [79] \\
\hline 21 & 2017 & 10 & 7 & $\begin{array}{l}\text { Sm }(0.5-3 \\
w t . \%)\end{array}$ & SBA-15 & TS & 40 & 12 & 200 & 700 & 11.5 & 73 & 75 & $\begin{array}{l}\text { Promotional effect of } \\
\text { samarium for better } \\
\text { dispersion }\end{array}$ & [80] \\
\hline 22 & 2018 & $2.5-10$ & n.d. & $\begin{array}{l}\mathrm{Ti} \\
(5-18 \\
\text { wt.\%) }\end{array}$ & SBA-15 & WI & n.d. & 1.5 & 50 & 700 & 12 & 66 & 71 & $\begin{array}{l}\text { Promotional effect of } \\
\text { titanium nitride (due to } \\
\text { its thermal stability) }\end{array}$ & [81] \\
\hline
\end{tabular}




\begin{tabular}{|c|c|c|c|c|c|c|c|c|c|c|c|c|c|c|c|}
\hline 23 & 2016 & $\begin{array}{l}3,5, \\
10\end{array}$ & n.d. & $\begin{array}{l}\text { Gd } \\
\text { (0.2 wt.\%) }\end{array}$ & M. ZSM-5 & WI & $\begin{array}{l}33, \\
166\end{array}$ & 10,50 & 200 & 750 & 100 & 93 & n.d. & $\begin{array}{l}\text { Effect of Gadolinium on } \\
\text { the stabilization of } \\
\text { Nickel/ZSM-5 }\end{array}$ & [82] \\
\hline 24 & 2015 & 10 & n.d. & $\begin{array}{l}\mathrm{Fe}_{2} \mathrm{O}_{3} \\
\text { (n.d.) }\end{array}$ & Mi. $\mathrm{SiO}_{2}$ & OP & 90 & 54 & 100 & 800 & 1 & 66 & 93 & $\begin{array}{l}\text { Nickel ferrite supported } \\
\text { catalysts (effect of } \\
\text { dispersion) }\end{array}$ & [83] \\
\hline 25 & 2018 & 5 & n.d. & $\begin{array}{l}\text { Sc } \\
\text { (0-3 wt.\%) }\end{array}$ & MCM-41 & Co-I & 65 & $39 / 78$ & $100 / 50$ & 800 & 7 & 85 & 90 & $\begin{array}{l}\text { Promotional effect of } \\
\text { scandium }\end{array}$ & [84] \\
\hline 26 & 2017 & 5 & (4) & $\begin{array}{l}\text { Boron } \\
\text { nitride } \\
\text { (n.d.) }\end{array}$ & M. $\mathrm{SiO}_{2}$ & $\begin{array}{l}\text { I in sonic bath } \\
\text { followed by } \\
\text { rotary } \\
\text { evaporation }\end{array}$ & 30 & 15 & 120 & n.d. & 100 & 89 & n.d. & 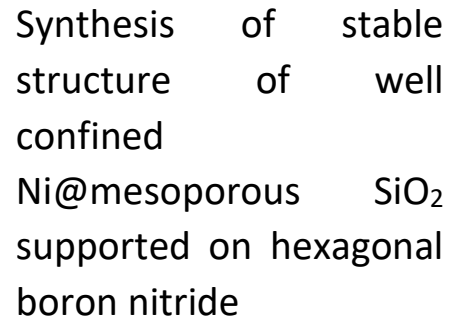 & [85] \\
\hline & & & & & & & & & & & & & & Low temperature & \\
\hline 27 & 2016 & n.d. & n.d. & $\operatorname{Zr}$ (n.d.) & $\mathrm{SiO}_{2}$ & WI & 60 & 14.4 & 250 & n.d. & n.d. & n.d. & n.d. & $\begin{array}{l}\text { reforming using } \mathrm{Zr} \text { - } \\
\text { promoted } \mathrm{Ni} / \mathrm{SiO}_{2}\end{array}$ & [86] \\
\hline 28 & 2018 & 3 & (8.6) & $\begin{array}{l}\text { In } \\
\text { (2 wt.\%) }\end{array}$ & $\mathrm{np} \mathrm{SiO}{ }_{2}$ & DP & 20 & 40 & 30 & 675 & 24 & 31 & 69 & $\begin{array}{l}\text { Reducing the coke } \\
\text { formation by adding } \\
\left.\text { Indium ( } \mathrm{Ni}-\ln / \mathrm{SiO}_{2}\right)\end{array}$ & [87] \\
\hline 29 & 2017 & n.d. & (4) & $\begin{array}{l}\mathrm{Zr}, \mathrm{Mn} \\
\text { (n.d.) }\end{array}$ & $\mathrm{np} \mathrm{SiO} 2$ & Co.I & 60 & 14.4 & 250 & n.d. & n.d. & n.d. & n.d. & $\begin{array}{l}\text { Influence of reduction } \\
\text { temperature on the } \\
\text { performances } \\
\text { catalysts }\end{array}$ & [88] \\
\hline 30 & 2015 & n.d. & n.d. & $\begin{array}{l}\mathrm{Mn}, \mathrm{Zr} \\
\text { (n.d.) }\end{array}$ & $\mathrm{np} \mathrm{SiO} 2$ & WI & 60 & 20 & n.d. & 800 & 60 & 75 & n.d. & $\begin{array}{l}\text { Promotional effect of } \\
\text { both } \mathrm{Mn} \text { and } \mathrm{Zr} \text { in the } \\
\text { same time }\end{array}$ & {$[8$} \\
\hline
\end{tabular}


JEPT 2021; 3(1), doi:10.21926/jept.2101007

\begin{tabular}{|c|c|c|c|c|c|c|c|c|c|c|c|c|c|c|}
\hline 2015 & n.d. & n.d. & Mn (n.d.) & $\mathrm{np} \mathrm{SiO} 2$ & Sol-Gel + P & 30 & 90 & 20 & 750 & 50 & 16 & 30 & Promotional effect of $\mathrm{Mn}$ & {$[90]$} \\
\hline 2017 & 9 & n.d. & $\begin{array}{l}\text { Ca } \\
\text { (0-9 wt.\%) }\end{array}$ & MCF's zeolite & Sol-Gel and WI & 60 & 24 & 150 & 750 & 75 & 91 & 99 & $\begin{array}{l}\text { Effect of } \mathrm{CaO} \text { on the } \\
\text { stabilization towards } \\
\text { coking }\end{array}$ & [91] \\
\hline 2018 & $18-27$ & (6.6) & $\begin{array}{l}\text { Mg } \\
(0-11 \\
\text { wt. \%) }\end{array}$ & $\begin{array}{l}\text { Hollow } \\
\text { Hierarchical } \\
\mathrm{SiO}_{2}\end{array}$ & $\begin{array}{l}\text { Hydrothermal- } \\
\text { P }\end{array}$ & 18 & 30 & 100 & 700 & 50 & 89 & 92 & $\begin{array}{l}\text { Promotional effect of } \\
\text { MgO }\end{array}$ & [92] \\
\hline
\end{tabular}

E: Entry; M.: Mesoporous; np: Non-Porous; TS: Two solvents; I.: Impregnation; IWI: Incipient wetness impregnation; WI: wet impregnation; Co-P: Coprecipitation; DP: deposition-precipitation; OP: one-pot; P: precipitation; HM: hydrothermal method; n.d.: Not determined; OAc: Oleic acid; OAm: Oleylamine 
Metals, such as Pt, Ru, and Ir, have been well-recognized as DRM catalysts for decades, demonstrating higher activity and better resistance to carbon compared to $\mathrm{Ni}$ [93-97]. When associated with $\mathrm{Ni}$, a small amount of noble metal usually assists in promoting the reducibility of nickel due to the higher affinity of the noble metal toward $\mathrm{H}_{2}$ and the increase in the number of active sites [98-107, 108]. For instance, when Pt is added to nickel-based catalysts, a significant decrease in the barrier energy of $\mathrm{CO}_{2}$ dissociation was observed $[109,110]$.

In the literature between 2015 and 2018, not many examples concerning the use of noble metals as promoters for $\mathrm{Ni}$-silica based catalysts are available. Other metals, such as $\mathrm{Co}, \mathrm{Mg}, \mathrm{Cu}$, and In, are preferred for DRM application. The combination of $\mathrm{Ni}$ and $\mathrm{Co}$, for example, has been studied extensively. Bian et al. (Table 2, entry 1) [61] and Xin et al. (Table 2, entry 2) [62] separately demonstrated that the $\mathrm{Ni} / \mathrm{Co}$ ratio, in a $\mathrm{Ni}$-Co alloy, is crucial for the catalytic performances of the resulting $\mathrm{Ni}$-Co-silica catalysts. It was reported that $7 \mathrm{Ni3Co}$ exhibits high and stable activity, while $5 \mathrm{Ni5Co}, 3 \mathrm{Ni7Co}$, and $10 \mathrm{Co}$ exhibit severe deactivation (e.g., $\mathrm{CH}_{4}$ conversion after $30 \mathrm{~h}$ on stream decreased from $60 \%$ to $40 \%$ in the case of $10 \% \mathrm{Ni} / \mathrm{SBA}-15$, while it remained stable $(65 \%)$ for $50 \mathrm{~h}$ in the presence of Co [62]). Gao et al. (Table 2, entry 3) [63] observed small shifts in the binding energy values in the XPS (increase for $\mathrm{Co}$ and decrease for $\mathrm{Ni}$ compared to isolated $\mathrm{Co}$ and $\mathrm{Ni}$, respectively), and emphasized the existence of an electron transfer from Co to $\mathrm{Ni}$ when both are combined, thereby revealing an intimate interaction between the two [111, 112]. According to the authors, the higher electron density in Ni would improve the metal-support interaction and protect $\mathrm{Ni}$ from sintering during the DRM reaction (Table 2, entry 4) [64, 113-115]. In addition, the better affinity of Co toward the oxygen species leads to an excellent coke resistance, resulting in a catalyst with higher stability and reactivity (Table 2, entries 2 and 4) [62,64].

Wu et al. (Table 2, entry 5) [65] proposed Cu-Ni alloy nanoparticles species supported on silica nanosheets using a phyllosilicate intermediate, prepared using nickel and copper nitrate salts. Such a preparation method resulted in much higher metal-support interaction compared to the conventional impregnation methods, thereby producing finely-divided Cu-Ni nanoparticles with an average diameter of $\mathrm{ca} .7 \mathrm{~nm}$. However, the $\mathrm{CuNi}_{3} / \mathrm{SiO}_{2}$ catalyst [containing $5.5 \mathrm{wt} . \%$ and $20.9 \mathrm{wt} . \%$ of $\mathrm{Cu}$ and $\mathrm{Ni}$, respectively] led to a slightly higher $\mathrm{CH}_{4}$ conversion (72\%) compared to that observed for $\mathrm{Ni} / \mathrm{SiO}_{2}$ [20.9 wt.\% of nickel (66\%)]. Indeed, the activation energy for $\mathrm{CH}_{4}$ conversion derived from the slope of Arrhenius plots (logarithms of the conversion rates vs. 1/T at a temperature range of $500-700^{\circ} \mathrm{C}$ ) was similar for $\mathrm{Ni}$ and $\mathrm{CuNi}_{x}$, when $\mathrm{x}$ was larger than 3 . On the other hand, there was a significant impact on the stability and the minimization of carbon whisker formation after $30 \mathrm{~h}$ on stream at $700{ }^{\circ} \mathrm{C}$, although this was achieved using a relatively low GHSV $\left(13.3 \mathrm{~L} \mathrm{~g}^{-1} \mathrm{~h}^{-1}\right)$. The authors explained that $\mathrm{Cu}$ would assist in promoting the sintering resistance of the alloy nanoparticles, although for $\mathrm{Cu}$-overdosed materials, the activation energy for $\mathrm{CH}_{4}$ conversion was apparently higher.

Various other elements or their oxides/nitrides have also been investigated previously as promoters to improve the stability of nickel-based catalysts toward coke deposition, such as Ce (Table 2, entries 6-13) [45, 66-72], La (Table 2, entries 14-18) [73-77], Sm (Table 2, entries 19-21) [78-80], Titanium nitride [81], Gadolinium [82], Ferrite [83], Sc [84], and boron nitride [85], (Table 2, entries 22-26), etc. In a study, Yao et al. [86] (Table 2, entry 27) studied Ni-Zr-silica catalysts prepared through impregnation of the $\mathrm{SiO}_{2}$ support with an aqueous solution containing $\mathrm{Ni}\left(\mathrm{NO}_{3}\right)_{2}$ and $\mathrm{Zr}\left(\mathrm{NO}_{3}\right)_{4}$ and demonstrated that such materials were catalytically more active in the DRM reaction at low temperatures compared to their non-promoted counterpart $\mathrm{Ni} / \mathrm{SiO}_{2}$. Such a behavior could 
be related to the high activation ability of the $\mathrm{Ni}-\mathrm{Zr} / \mathrm{SiO}_{2}$ materials for both $\mathrm{CH}_{4}$ and $\mathrm{CO}_{2}$. Therefore, the activation energy values obtained with $\mathrm{Ni}-\mathrm{Zr} / \mathrm{SiO}_{2}$ were much lower than those obtained with $\mathrm{Ni} / \mathrm{SiO}_{2}\left[30.0\left(\mathrm{CH}_{4}\right)\right.$ and $21.9\left(\mathrm{CO}_{2}\right) \mathrm{kJ} \mathrm{mol}^{-1}$ vs. $473.9\left(\mathrm{CH}_{4}\right)$ and $704.6\left(\mathrm{CO}_{2}\right) \mathrm{kJ} \mathrm{mol}^{-1}$, respectively]. According to Yao et al., $\mathrm{Zr}$-promoted catalysts would facilitate the activation of the $\mathrm{CH}$ bond in $\mathrm{CH}_{4}$ and activate $\mathrm{CO}_{2}$ by forming significant proportions of carbonates (Table 2, entry 27) [86]. In addition, the $\mathrm{Ni}-\mathrm{Zr} / \mathrm{SiO}_{2}$ catalysts exhibited $\mathrm{CH}_{4}$ and $\mathrm{CO}_{2}$ conversions of only $4.5 \%$ and $9.1 \%$, respectively, at $450{ }^{\circ} \mathrm{C}$. Unfortunately, these authors did not work under high GHSV values (only $14.4 \mathrm{~L} \mathrm{~g}^{-1} \mathrm{~h}^{-1}$ ). Károlyi et al. (Table 2, entry 28) [87] combined $\mathrm{Ni}$ with Indium (In) on $\mathrm{SiO}_{2}$ support using a deposition-precipitation method, which involved the mixing of the $\mathrm{Ni}$ and the In precursors. The presence of $2 \mathrm{wt} . \%$ of In on the surface of $3 \mathrm{wt} . \% \mathrm{Ni} / \mathrm{SiO}_{2}$ prevented coke formation in the DRM at $675^{\circ} \mathrm{C}$ for $24 \mathrm{~h}$ under an acceptable GHSV of $40 \mathrm{~L} \mathrm{~g}^{-1} \mathrm{~h}^{-1}$, as evidenced by the TPO curves of the spent catalysts. The improved stability exhibited by $\mathrm{Ni}-\mathrm{In} / \mathrm{SiO}_{2}$ compared to $\mathrm{Ni} / \mathrm{SiO}_{2}$ was also related, as stated by the authors, to a change in the nickel adsorption properties [and its electronic structure] when indium was used as the promoter.

Oxides, such as $\mathrm{MnO}_{x}$ (Table 2, entries 29-31) [88-90], $\mathrm{CaO}, \mathrm{MgO}, \mathrm{Y}_{2} \mathrm{O}_{3}$, and $\mathrm{Sm}_{2} \mathrm{O}_{3}$, have also been evaluated for improving the performances of $\mathrm{Ni}$ catalysts. For instance, Amin et al. (Table 2, entry 32) [91] concluded that the introduction of $\mathrm{CaO}$ to Ni-based mesocellular silica foams using a sol-gel method renders the obtained catalyst more stable toward the DRM limitations. Similarly, MgO-promoted $\mathrm{Ni} / \mathrm{SiO}_{2}$ was evaluated by Zhang et al. (Table 2, entry 33) [92], who demonstrated that the uniformly-dispersed $\mathrm{MgO}$ basic promoter resulted in an increased $\mathrm{CO}_{2}$-adsorbing affinity. Moreover, the formation of $\mathrm{Mg}$-phyllosilicate induced $\mathrm{CO}_{2}$ activation by the support, thereby improving the catalytic activity and coking resistance of the resulting material.

In another study, Taherian et al. (Table 2, entry 19) [78] compared the effect of three oxides (3 wt.\%) on a $10 \mathrm{wt} . \% \mathrm{Ni} / \mathrm{SBA}-15$ catalyst. The authors used zirconia $\left(\mathrm{ZrO}_{2}\right)$, yttria $\left(\mathrm{Y}_{2} \mathrm{O}_{3}\right)$, and samaria $\left(\mathrm{Sm}_{2} \mathrm{O}_{3}\right)$, and the precursors of these were added using the "two-solvent" method. The activity tests, which used a rather low GHSV $\left(12 \mathrm{~L} \mathrm{~g}^{-1} \mathrm{~h}^{-1}\right)$ and the TPO revealed that the incorporation of samaria i) improved the metal-support interaction and the dispersion of $\mathrm{Ni}$, leading to high methane conversion (approximately 75\%) and ii) decreased the coke formation. The addition of yttria increased the $\mathrm{CH}_{4}$ conversion, although it also enhanced the coke formation, unfortunately. Several other oxides of rare earth elements have been used in association with silica. Oemar et al. (Table 2, entry 16) [75] added $\mathrm{La}_{2} \mathrm{O}_{3}$ onto $\mathrm{Ni} / \mathrm{SBA}-15$ and demonstrated that the quantity of oxide has to be adjusted. Only a small amount of La (approximately $1 \mathrm{wt} . \%)$ was necessary to obtain the best catalytic performances [83\% of $\mathrm{CH}_{4}$ conversion at $700{ }^{\circ} \mathrm{C}$ for $10 \mathrm{~h}$ under a reasonable GHSV $\left(72 \mathrm{~L} \mathrm{~g}^{-}\right.$ $\left.{ }^{1} \mathrm{~h}^{-1}\right)$ ]. The $1 \%$ La-promoted Ni/SBA-15 material was much more active and stable compared to the un-promoted one. These authors concluded that in the presence of La, the highly-dispersed $\mathrm{Ni}$ catalyst was very active in the decomposition of $\mathrm{CH}_{4}$, resulting in higher $\mathrm{CH}_{4}$ conversion. In addition, the La oxide also actively adsorbs $\mathrm{CO}_{2}$ and would assist in the removal of the deposited carbon on the catalyst surface, resulting in high catalytic stability. In addition, the presence of La was demonstrated to strongly inhibit the RWGS reaction (Table 2, entry 14) [73].

$\mathrm{Ce}$ has also been often employed as a promoter for the Ni-silica based catalysts. The synthesis of such catalysts is performed, for example, by using natural illite clay, which is a low-cost precursor (Table 2, entry 10) [69], or, more often, by beginning with non-natural silica supports. Irrespective of the structure (mesoporous, core-shell, or aerogels), the incorporation of $\mathrm{Ce}$ in the catalyst formulation improved its performance in the DRM reaction (Table 2, entries 6-13) [45, 66-72]. Ce 
introduction also conferred self-decoking properties and favored the formation of small highlydispersed Ni particles and the strengthening of the interaction between the metal and the support (Table 2, entries 8-13) [67-72]. It is noteworthy that more complex catalyst formulations, such as the combination of $\mathrm{Ce}$ and $\mathrm{Zr}$, were considered to synthesize a silica/ceria-zirconia support for $\mathrm{Ni}$ deposition (Table 2, entry 7) [66]. Another rare-earth element, gadolinium has been tested successfully for the promotion of Ni/ZSM-5 in DRM (Table 2, entry 23) [82]. Sarkar et al. reported the formation of $\mathrm{GdNi5}$ alloy with a particle size of 2-10 $\mathrm{nm}$. In this case, the strong interaction and charge transfer from $\mathrm{Gd}$ to $\mathrm{Ni}$, as evidenced by the XPS results, explained the low carbon deposition and the sintering of $\mathrm{Ni}$.

\section{Ni@silica Core-Shell Nanoparticles}

One possible physical protection to prevent carbon deposition and efficiently inhibit the sintering of the metal (Table 3, entry 1) [116] is to cover the metal particles with at least one microporous oxide material that allows the access of the DRM reactants. This approach was tested with various materials, ranging from core-shell structures involving silica particles to porous materials that incorporate, into their walls, the metal introduced during the synthesis of the silica support (Figure 4). The latter has already been described in Section 2.2.3 and will not be discussed below.

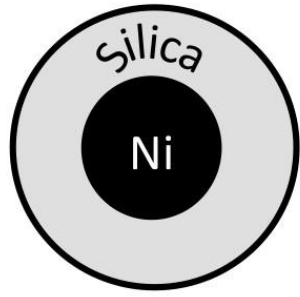

Typical Core-shell

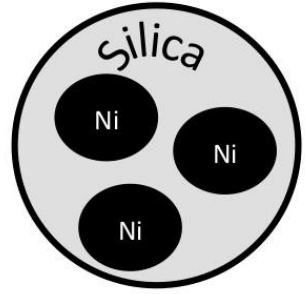

Core-shell or also called Multiple Cores-shell

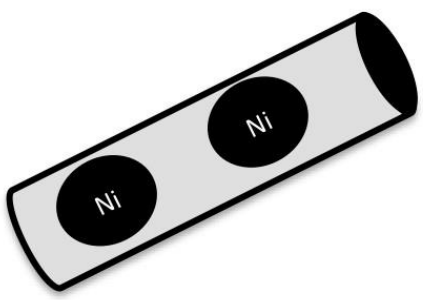

Embedded in siliceous structure

Figure 4 Different approaches leading to the physical protection of nickel by porous silica. 
JEPT 2021; 3(1), doi:10.21926/jept.2101007

Table 3 A selection of papers dealing with core-shell catalysts published between 2015-2018 [66, 68, 71, 116-122].

\begin{tabular}{|c|c|c|c|c|c|c|c|c|c|c|c|c|c|c|c|}
\hline $\mathbf{E}$ & Year & $\begin{array}{l}\mathrm{Ni} \\
\text { (wt.\%) }\end{array}$ & $\begin{array}{l}\mathrm{NiO} \\
\text { or } \\
\left(\mathrm{Ni}{ }^{0}\right) \\
(\mathrm{nm})\end{array}$ & $\begin{array}{l}\text { Promoters } \\
\text { element }\end{array}$ & $\begin{array}{l}\text { Siliceous } \\
\text { support }\end{array}$ & $\begin{array}{l}\text { Preparation } \\
\text { method }\end{array}$ & $\begin{array}{l}\text { Total } \\
\text { flow } \\
(\mathrm{mL} \\
\left.\mathrm{min}^{-1}\right)\end{array}$ & $\begin{array}{l}\text { GHSV } \\
\mathrm{Lg}^{-1} \mathrm{~h}^{-} \\
1\end{array}$ & $\begin{array}{l}\text { Weight } \\
\text { (mg) }\end{array}$ & $\begin{array}{l}\mathrm{T} \\
\left({ }^{\circ} \mathrm{C}\right)\end{array}$ & $\begin{array}{l}\text { Time } \\
\text { (h) }\end{array}$ & $\begin{array}{l}\mathrm{CH}_{4} \\
\%\end{array}$ & $\begin{array}{l}\mathrm{CO}_{2} \\
\%\end{array}$ & Objectives & Ref \\
\hline 1 & 2016 & $\begin{array}{l}11.6 \\
18.8\end{array}$ & (8.5) & $\begin{array}{l}\mathrm{Mg} \quad(5.1, \\
9.2 \mathrm{wt} . \%)\end{array}$ & $\mathrm{M} . \mathrm{SiO}_{2}$ & $\mathrm{HM}$ & 30 & 60 & 30 & 750 & 72 & 85 & 88 & $\begin{array}{l}\mathrm{Ni}-\mathrm{Mg} \quad \text { phyllosilicates } \\
\text { @silica used as catalyst }\end{array}$ & [116] \\
\hline 2 & 2017 & 10 & 5.3 & $\begin{array}{l}\mathrm{Ce}, \mathrm{Zr} \\
\text { (CeZr both } \\
57-84 \\
\text { wt.\%) }\end{array}$ & $\mathrm{np} \mathrm{SiO} 2$ & WI & 60 & 144 & 25 & 700 & 24 & 50 & n.d. & $\begin{array}{l}\text { Examination of the intricate } \\
\text { relationship between } \\
\text { support structure and } \\
\text { catalytic performances }\end{array}$ & {$[66]$} \\
\hline 3 & 2016 & 10 & (18) & $\begin{array}{ll}\mathrm{Ce} & (4.3 \\
\text { wt.\%) } & \end{array}$ & M. $\mathrm{SiO}_{2}$ & n.d. & 30 & 12 & 150 & 750 & 40 & 89 & 97 & $\begin{array}{l}\text { Design of } \mathrm{NiCe@m}-\mathrm{SiO}_{2} \\
\text { core-shell structure for } \\
\text { better confinement }\end{array}$ & [68] \\
\hline 4 & 2015 & 10 & (2.3) & Ce (3 wt.\%) & $\begin{array}{l}\text { M. } \mathrm{SiO}_{2} \\
\text { nanospheres }\end{array}$ & IWI & 30 & 12 & 150 & 750 & 20 & 90 & 95 & $\begin{array}{l}\text { Immobilizing } \mathrm{Ni} \text { in the } \\
\text { porosity of } \mathrm{SiO}_{2} \text { mesopores } \\
\text { and preparation of modified } \\
\text { Ce-SiO } 2 \text { support }\end{array}$ & [71] \\
\hline 5 & 2015 & 29 & 10 & No & $\begin{array}{l}\mathrm{Mi} \text {. and } \mathrm{M} \text {. } \\
\mathrm{SiO}_{2}\end{array}$ & $\begin{array}{l}\mathrm{NiO} \\
\text { suspended } \\
\text { in } \quad \mathrm{CTABr}\end{array}$ & 40 & 48 & 50 & 750 & 25 & 54 & 66 & $\begin{array}{l}\text { Preparation of core-shell } \\
\text { structure for better stability }\end{array}$ & [117] \\
\hline
\end{tabular}




\section{then coated}

by $\mathrm{SiO}_{2}$

\begin{tabular}{|c|c|c|c|c|c|c|c|c|c|c|c|c|c|c|c|}
\hline \multirow[b]{2}{*}{6} & \multirow[b]{2}{*}{2017} & \multirow[b]{2}{*}{5} & \multirow[b]{2}{*}{$(4.3)$} & \multirow[b]{2}{*}{ No } & \multirow[b]{2}{*}{$\mathrm{Mi} \mathrm{SiO}_{2}$} & \multirow[b]{2}{*}{$\mathrm{OP}$} & \multirow[b]{2}{*}{15} & \multirow[b]{2}{*}{18} & \multirow[b]{2}{*}{50} & \multirow[b]{2}{*}{800} & \multirow[b]{2}{*}{100} & \multirow[b]{2}{*}{88} & \multirow[b]{2}{*}{89} & \multirow{2}{*}{\multicolumn{2}{|c|}{$\begin{array}{l}\text { One-pot for } \begin{array}{r}\text { core-shell } \\
\text { structure better } \\
\text { confinement }\end{array}\end{array}$}} \\
\hline & & & & & & & & & & & & & & & \\
\hline 7 & 2018 & 10 & $(5-6)$ & No & $\begin{array}{l}\text { silica nano- } \\
\text { capsule }\end{array}$ & $\begin{array}{l}\text { Reverse } \\
\text { micelle } \\
\text { approach }\end{array}$ & 80 & 48 & 100 & 700 & 325 & 75 & 80 & $\begin{array}{l}\text { Siliceous nanocapsule for } \\
\text { the confinement of nickel }\end{array}$ & [119] \\
\hline 8 & 2018 & $3-7$ & (4) & No & $\mathrm{np} \mathrm{SiO}_{2}$ & $\mathrm{OP}$ & 30 & 60 & 30 & 800 & 23 & 45 & 55 & $\begin{array}{l}\text { Sandwich-Like } \\
\text { Silica@Ni@Silica Multicore- } \\
\text { ShellCatalyst: Confinement } \\
\text { effect against coke } \\
\text { deposition }\end{array}$ & [120] \\
\hline 9 & 2018 & 25 & $(5-8)$ & No & $\begin{array}{l}\mathrm{SiO}_{2} \\
\text { nanotubes }\end{array}$ & HM & n.d. & 36 & n.d. & 700 & 70 & 81 & 78 & $\begin{array}{l}\text { Confinement of } \mathrm{Ni} \text { particles } \\
\text { in silica nanotubes }\end{array}$ & [121] \\
\hline 10 & 2017 & $\begin{array}{l}2.6- \\
2.8\end{array}$ & $(7-9)$ & No & $\mathrm{np} \mathrm{SiO} 2$ & IWI & n.d. & 5 & 800 & 800 & 500 & 90 & 89 & $\begin{array}{l}\text { Core-shell structure using } \\
\text { thin-felt microfibrous- } \\
\text { structured } \\
\mathrm{Ni@SiO} / \mathrm{Al}_{2} \mathrm{O}_{3} / \mathrm{FeCrAl} \\
\text { fabricated by one-step }\end{array}$ & [122] \\
\hline
\end{tabular}

E: Entry; M.: Mesoporous; Ma: Macroporous; np: Non-Porous; Mi: Microporous; IWI: Incipient wetness impregnation; WI: wet impregnation; OP: onepot; HM: hydrothermal method; n.d.: Not determined. 
Core-Shell Nanoparticles (CSNs) are a class of nanostructured materials that have been receiving increasing attention recently due to their important roles in various catalytic processes [123]. As discussed earlier, promoters are often added to the catalyst formulation to improve the performance of $\mathrm{Ni}$ in the DRM reaction. This strategy has also been considered in the approaches involving $\mathrm{Ni} /$ silica core-shell particles. The available examples in the literature deal with Ce (Table 3, entries 2-4) [66, 68, 71], Zr (Table 3, entry 2) [66], and Mg (Table 3, entry 1) [116]. The following paragraph presents a few examples of core-shell systems with varying scales of complexity. In all the cases, the ordered porosity of silica is generated using organic structure-directing agents that are later removed through calcination.

In a quite interesting work reported by Zhang et al. (Table 3, entry 5) [117], core-shell NiO@SiO nanoparticles were prepared from the commercially available NiO NPs (10-20 nm) added to a silica precursor dispersed in a cetyltrimethylammonium bromide solution to construct a mesoporous silica framework around the NiO NPs, thereby preventing the formation of carbon species on their surface. The mesopores were generated after removing the surfactant molecules through calcination. As expected, the shell of silica suppressed the sintering of $\mathrm{Ni}$ and the growth of carbon filaments, while the mesopore channels allowed the reactants to diffuse with no limitation toward the surface of $\mathrm{Ni}$, which could also be encouraging for other reactions such as $\mathrm{CO}_{2}$ methanation. In DRM, coke deposition was demonstrated to be negligible after $39 \mathrm{~h}$ on stream at $850{ }^{\circ} \mathrm{C}$ under an acceptable GHSV $\left(40 \mathrm{~L} \mathrm{~g}^{-1} \mathrm{~h}^{-1}\right)$, while the catalyst was relatively stable for $24.5 \mathrm{~h}$. In addition, it was

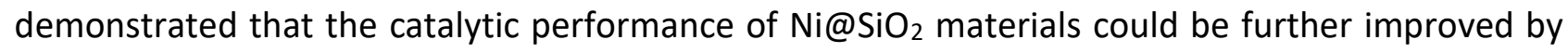
optimizing the microstructure of the core-shell nanoparticles and the surface of the Ni NPs inside the shell. Promotion with cerium was evaluated, for example, with the $\mathrm{NiCe@m-SiO} 2$ catalyst containing 10.6 wt.\% of $\mathrm{Ni}$ and 4.3 wt.\% of Ce (Table 3, entry 3) [68]. The material was prepared through the hydrolysis and condensation of silica precursors added to a reverse microemulsion obtained by dispersing aqueous $\mathrm{Ce}\left(\mathrm{NO}_{3}\right)_{3} \cdot 6 \mathrm{H}_{2} \mathrm{O}$ and $\mathrm{Ni}\left(\mathrm{NO}_{3}\right)_{2}$ in a mixture of cetyltrimethylammonium bromide, 1-butanol, and cyclohexane. NiCe@m-SiO 2 with nickel particle size ranging between 14 and $24 \mathrm{~nm}$ was tested for $40 \mathrm{~h}$ at $750{ }^{\circ} \mathrm{C}$, and it proved to be a better catalyst ( $90 \% \mathrm{CH}_{4}$ conversion) compared to $\mathrm{NiCe} / \mathrm{SiO}_{2}$ prepared through the impregnation of silica (75\% of $\mathrm{CH}_{4}$ conversion). However, the GHSV value $\left(12 \mathrm{~L} \mathrm{~g}^{-1} \mathrm{~h}^{-1}\right)$ used in the study was quite low.

Multiple-cores@shell catalysts comprising multiple Ni nanoparticles as the core and microporous silica as the shell $\left(\mathrm{M}-\mathrm{Ni} @ \mathrm{SiO}_{2}\right)$ may also be synthesized using a one-pot reverse-phase microemulsion strategy. As an example, in the work of Peng et al. (Table 3, entry 6) [118], the M$\mathrm{Ni@SiO} 2$ material containing 5 wt.\% of nickel was prepared by introducing concentrated ammonia and tetraethoxysilane into a reverse microemulsion prepared by dispersing an aqueous $\mathrm{Ni}^{2+}$ solution in a mixture of polyethylene glycolmono-4-nonylphenyl ether (NP-5) and cyclohexane. Following two days of hydrothermal treatment, the particles were destabilized using ethanol and then collected through centrifugation at $10000 \mathrm{rpm}$, followed by calcination at $800^{\circ} \mathrm{C}$ and vacuum drying. In the freshly calcined Multiple-NiO@ $\mathrm{SiO}_{2}$ samples, the particle size of the ultra-fine Ni cores was approximately $4.3 \mathrm{~nm}$, while the total average size of the core-shell particle was $30 \mathrm{~nm}$ and the pore size of the microporous shell was $1.5 \mathrm{~nm}$. Peng et al. demonstrated that after in situ reduction, $\mathrm{M}$ $\mathrm{Ni} @ \mathrm{SiO}_{2}$ exhibits good catalytic activity and stability $\left(90 \%\right.$ and $92 \%$ for $\mathrm{CH}_{4}$ and $\mathrm{CO}_{2}$ conversions, respectively, after $100 \mathrm{~h}$ ). No carbon species could be detected in TGA-DSC after $100 \mathrm{~h}$ of DRM at $800{ }^{\circ} \mathrm{C}$, although the GHSV worked with was quite low (18 $\mathrm{L} \mathrm{g}^{-1} \mathrm{~h}^{-1}$ ) (Figure 5). M-Ni@SiO 2 led to a $\mathrm{CH}_{4}$ conversion quite similar to that of $\mathrm{Ni} / \mathrm{SiO}_{2}$ prepared using a conventional impregnation method 
and that of the commercial $\mathrm{Ni} / \mathrm{Al}_{2} \mathrm{O}_{3}$. However, $\mathrm{Ni} / \mathrm{SiO}_{2}$ and $\mathrm{Ni} / \mathrm{Al}_{2} \mathrm{O}_{3}$ presented more coke deposition, as revealed in the TGA characterization of the spent catalysts. In the case of $\mathrm{M}-\mathrm{Ni} @ \mathrm{SiO}_{2}$, all the Ni particles were confined in the microporous silica shell, which led to a lack of physical space for the growth of carbon species, particularly the filamentous and encapsulating carbon. However, it is noteworthy that the comparison was not really fair as the $\mathrm{Ni}$ particles in $\mathrm{M}-\mathrm{Ni} @ \mathrm{SiO}_{2}$ were smaller than those in the other catalysts, which could also have contributed to the superior carbon resistance ability of the core-shell catalyst.
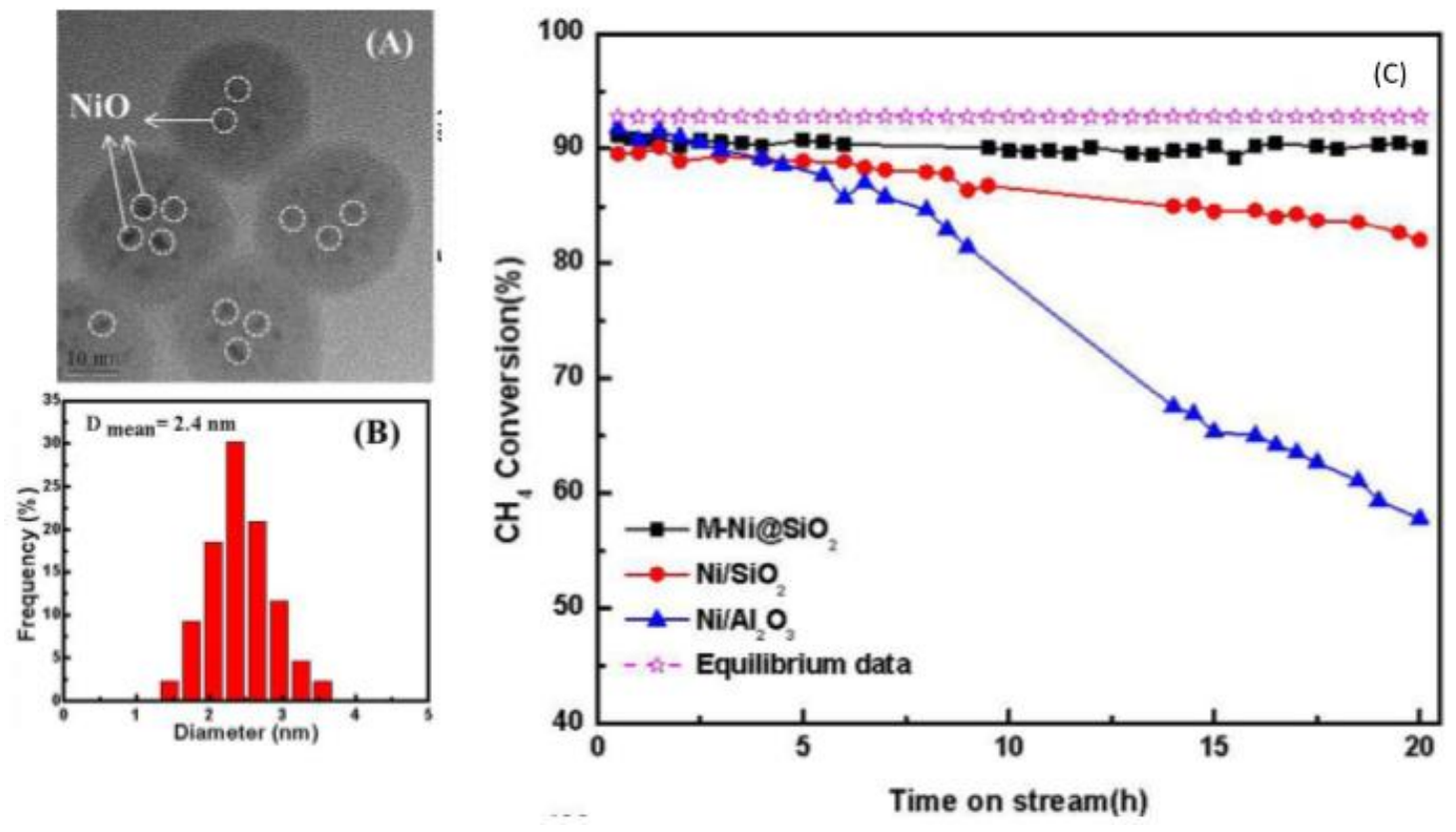

Figure $5 \mathrm{~A}$ and $\mathrm{B}$ : TEM and $\mathrm{Ni}$ particle size distribution of $\mathrm{M}-\mathrm{Ni} @ \mathrm{SiO}_{2}$ and $\mathrm{C}$ : comparison of the stability of different Ni-based silica or alumina catalysts toward the $\mathrm{CH}_{4}$ conversion [118].

Further sophisticated objects have also been designed, such as hollow silica capsules, including Ni nanoparticles (work of Wang et al.) (Table 3, entry 7) [119]) or the sandwich-like Silica@Ni@Silica Multicore-shell systems (Table 3, entry 8) [120]. The latter were synthesized by Bian et al. using a four-step approach, in which $\mathrm{SiO}_{2}$ nanospheres were prepared first, which were then covered by $\mathrm{Ni}$ phyllosilicates (PS) that were further protected by a silica shell and reduced under $\mathrm{H}_{2}$. In a similar approach, Z. Li et al. (Table 3, entry 9) [121] developed an original method that involved using $\mathrm{Ni}$ phyllosilicates nanotubes as precursors to obtain, after $\mathrm{H}_{2}$ treatment, small and well-dispersed nanoparticles $\left(5-8 \mathrm{~nm}\right.$ ) of $\mathrm{Ni}^{0}$ confined in the $\mathrm{SiO}_{2}$ nanotubes with a porous shell having a thickness of $26.1 \mathrm{~nm}$. The strong interaction with the support produced Ni nanoparticles that were resistant to the migration in the silica tubes, and consequently, to sintering. However, the dispersion estimated using $\mathrm{H}_{2}$ chemisorption was quite low (3.7\% for the highest one). The catalysts led to $81 \%$ and $78 \%$ conversions for $\mathrm{CO}_{2}$ and $\mathrm{CH}_{4}$, respectively, after $70 \mathrm{~h}$ at $700{ }^{\circ} \mathrm{C}$ under a relatively modest GHSV of $36 \mathrm{~L} \mathrm{~g}^{-1} \mathrm{~h}^{-1}$.

Bian et al. (Table 3, entry 1) [116] also worked on a multicore-shell material with $\mathrm{Mg}$ as a promoter, which was synthesized successfully through the silica coating (thickness of $10 \mathrm{~nm}$ ) of NiMg phyllosilicate nanotubes (PSNTS). The authors reported that the thermal stability of the catalyst was significantly improved due to the confinement of nickel and good interaction between $\mathrm{Ni}$ and 
the nanotubular support (core@tube confinement).

Despite the promising advances brought by the use of core-shell structures, the limitations of severe intra-bed and intra-particle heat transfer are nonetheless encountered with the Ni@oxide nanostructured catalysts that are implemented for the DRM process due to their poor thermal conductivity that generates cold-spots. According to the thermodynamic studies, cold spots not only decrease the reactor conversion, but they also facilitate carbon deposition, particularly on Ni-based catalysts. In this context, Chai and co-workers [122] (Table 3, entry 10) proposed the $\mathrm{Ni@SiO} 2 / \mathrm{Al}_{2} \mathrm{O}_{3} / \mathrm{FeCrAl}$-fiber catalysts where the FeCrAl-alloy-based microfiber (referred to as FeCrAl-fiber) is an attractive material for the preparation of structured catalysts owing to its high temperature tolerance, mechanical robustness, high permeability, and particularly high heat transfer [124]. $\mathrm{Ni@SiO} / \mathrm{Al}_{2} \mathrm{O}_{3} / \mathrm{FeCrAl}$-fibers were prepared using a one-step, top-down, macromicro-nano organization with the aid of cross-linking molecules, followed by a calcination treatment. The prepared fibers exhibited good activity $\left(95 \%\right.$ and $98 \%$ for $\mathrm{CH}_{4}$ and $\mathrm{CO}_{2}$ conversion, respectively, at $800^{\circ} \mathrm{C}$ ) and remained stable for $500 \mathrm{~h}$ due to their enhanced resistance to coke and Ni sintering resulting from their core-shell-like nanostructure. However, the reaction conditions tested were not so harsh $\left(800{ }^{\circ} \mathrm{C}, \mathrm{GHSV}=5 \mathrm{~L} \mathrm{~g}^{-1} \mathrm{~h}^{-1}, \mathrm{CH}_{4} / \mathrm{CO}_{2}=1.0 / 1.1\right.$ (Table 3, entry 10) [122]).

Different structures have been obtained by following this strategy. However, it should be noted that in certain cases, this method may lead to a loss in the active sites due to the decreased accessibility of nickel. In addition, the synthesis of core-shell catalysts is difficult and the preparation methods are not so easy to implement. Moreover, only $10 \%$ of the reports involved GHSV values higher than $60 \mathrm{~L} \mathrm{~g}^{-1} \mathrm{~h}^{-1}$. Evidently, this approach allows close control of the nature of the catalyst, which is of fundamental interest, although one may wonder regarding the applicability of such a synthesis approach in large-scale catalyst production. Therefore, metal deposition onto silica using conventional approaches, followed by coating with a porous silica layer, is perhaps a more practical solution in this regard.

\section{Conclusion}

Small Ni NPs confined in porous silica are capable of resisting against coke deposition and sintering by increasing the interactions with the support and/or exploiting the presence of physical barriers that reduce the migration of atoms and consequently their growth under harsh conditions. However, the conventional impregnation of porous silica is usually not sufficient to obtain the right materials. The key factor is the control of the interface between silica and nickel, which may rely heavily on the deposition method used. In the present review, several combinations of "nickel sources/support/impregnation methods" were discussed. Certain strategies beginning with original nickel precursors and/or implementing impregnations assisted by ligands or organic molecules appear to be complicated and are not always environment-friendly.

Therefore, it appears better to emphasize the use of the traditional deposition-precipitation method in combination with SBA-15 support [46], which generates a $10 \mathrm{wt} \%$ catalyst that remains stable for $35 \mathrm{~h}$ under quite a high value of GHSV $\left(240 \mathrm{~L} \mathrm{~g}^{-1} \mathrm{~h}^{-1}\right)$. One-pot approaches that incorporate nickel precursors in the synthesis gel of mesoporous supports, which are in the initial stages of development, could also be promising as they confer protection to nickel particles by a porous silica coating. Combining $\mathrm{Ni}$ with promoters generates active and stable Ni-silica catalysts for DRM. The reasons for the improved stability of nickel vary with the nature of the promoter used. However, 
the addition of such species renders the preparation as well as the characterization of the catalysts further difficult. In addition, adding promoters may increase the cost of the catalysts. A simple comparison of Table 1 and Table 2 reveals that both the strategies (confinement and promoters) generate catalysts with good performances. However, just $8 \%$ of the reports concerning the use of promoters involved GHSV values higher than $100 \mathrm{~L} \mathrm{~g}^{-1} \mathrm{~h}^{-1}$, while only $22 \%$ of the reports concerned the strategy of controlling the nickel particle size/confinement. In consideration of these remarks, it could be considered that the control of particle size and confinement might be more interesting provided that it is realized with an approach that is less complex and more feasible with industrial application.

The core-shell structure design is another approach that appears to be efficient for confining nickel nanoparticles in porous silica. The materials resulting from this approach appear to be quite interesting for DRM due to the possibility of nickel stabilization and the possibility of using different combinations with promoters.

\section{Author Contributions}

O. Daoura collected the data, wrote the paper. F. Launay and M. Boutros have initiated the project, helped O.D. for the structuration of the review and did various corrections.

\section{Competing Interests}

The authors have declared that no competing interests exist.

\section{References}

1. Ross JR, van Keulen AN, Hegarty ME, Seshan K. The catalytic conversion of natural gas to useful products. Catal Today. 1996; 30: 193-199.

2. Rani S, Padmanabhan E, Prusty BK. Review of gas adsorption in shales for enhanced methane recovery and $\mathrm{CO}_{2}$ storage. J Pet Sci Eng. 2019; 175: 634-643.

3. Starr K, Gabarrell X, Villalba G, Talens L, Lombardi L. Life cycle assessment of biogas upgrading technologies. Waste Manage. 2012; 32: 991-999.

4. Talyan V, Dahiya RP, Anand S, Sreekrishnan TR. Quantification of methane emission from municipal solid waste disposal in Delhi. Resour Conserv Recy. 2007; 50: 240-259.

5. Todd RW, Cole NA, Casey KD, Hagevoort R, Auvermann BW. Methane emissions from southern High Plains dairy wastewater lagoons in the summer. Anim Feed Sci Technol. 2011; 166: 575580.

6. Kumar N, Shojaee M, Spivey JJ. Catalytic bi-reforming of methane: From greenhouse gases to syngas. Curr Opin Chem Eng. 2015; 9: 8-15.

7. Aresta $\mathrm{M}$, Dibenedetto $\mathrm{A}$. Utilisation of $\mathrm{CO}_{2}$ as a chemical feedstock: Opportunities and challenges. Dalton Trans. 2007; 2975-2992.

8. Arakawa H, Aresta M, Armor JN, Barteau MA, Beckman EJ, Bell AT, et al. Catalysis research of relevance to carbon management: Progress, challenges, and opportunities. Chem Rev. 2001; 101: 953-996.

9. Guo J, Hou Z, Gao J, Zheng X. Syngas production via combined oxy- $\mathrm{CO}_{2}$ reforming of methane over $\mathrm{Gd}_{2} \mathrm{O}_{3}$-modified $\mathrm{Ni} / \mathrm{SiO}_{2}$ catalysts in a fluidized-bed reactor. Fuel. 2008; 87: 1348-1354. 
10. Kawi S, Kathiraser Y, Ni J, Oemar U, Li Z, Saw ET. Progress in synthesis of highly active and stable nickel-based catalysts for carbon dioxide reforming of methane. ChemSusChem. 2015; 8: 35563575 .

11. Sivaiah MV, Petit S, Beaufort MF, Eyidi D, Barrault J, Batiot-Dupeyrat C, Valange S. Nickel based catalysts derived from hydrothermally synthesized 1:1 and 2:1 phyllosilicates as precursors for carbon dioxide reforming of methane. Microporous Mesoporous Mater. 2011; 140: 69-80.

12. Daoura O, Daher S, Kaydouh MN, El Hassan N, Massiani P, Launay F, Boutros M. Influence of the swelling agents of siliceous mesocellular foams on the performances of $\mathrm{Ni}$-based methane dry reforming catalysts. Int J Hydrog Energy. 2018; 43: 17205-17215.

13. Balat $M$, Balat $M$, Kırtay $E$, Balat $H$. Main routes for the thermo-conversion of biomass into fuels and chemicals. Part 1: Pyrolysis systems. Energ Convers Manage. 2009; 50: 3147-3157.

14. Zhang $X$, Zhang $Q$, Tsubaki N, Tan $Y$, Han $Y$. Carbon dioxide reforming of methane over $\mathrm{Ni}$ nanoparticles incorporated into mesoporous amorphous $\mathrm{ZrO}_{2}$ matrix. Fuel. 2015; 147: 243-252.

15. Wang $C$, Zhang $Y$, Wang $Y$, Zhao $Y$. Comparative studies of non-noble metal modified mesoporous $\mathrm{M}-\mathrm{Ni}-\mathrm{CaO}-\mathrm{ZrO}_{2}(\mathrm{M}=\mathrm{Fe}, \mathrm{Co}, \mathrm{Cu})$ catalysts for simulated biogas dry reforming. Chin J Chem. 2017; 35: 113-120.

16. Daoura O, Kaydouh MN, El Hassan N, Massiani P, Launay F, Boutros M. Mesocellular silica foambased $\mathrm{Ni}$ catalysts for dry reforming of $\mathrm{CH}_{4}$ (by $\mathrm{CO}_{2}$ ). J $\mathrm{CO}_{2}$ Util. 2018; 24: 112-119.

17. Karaca H, Safonova OV, Chambrey S, Fongarland P, Roussel P, Griboval-Constant A, et al. Structure and catalytic performance of Pt-promoted alumina-supported cobalt catalysts under realistic conditions of Fischer-Tropsch synthesis. J Catal. 2011; 277: 14-26.

18. Khodakov AY, Chu W, Fongarland P. Advances in the development of novel cobalt Fischer-Tropsch catalysts for synthesis of long-chain hydrocarbons and clean fuels. Chem Rev. 2007; 107: 1692-1744.

19. Portugal UL, Santos AC, Damyanova S, Marques CM, Bueno JM. $\mathrm{CO}_{2}$ reforming of $\mathrm{CH}_{4}$ over Rhcontaining catalysts. J Mol Catal A Chem. 2002; 184: 311-322.

20. Gheno SM, Damyanova S, Riguetto BA, Marques CM, Leite CA, Bueno JM. $\mathrm{CO}_{2}$ reforming of $\mathrm{CH}_{4}$ over Ru/zeolite catalysts modified with Ti. J Mol Catal A Chem. 2003; 198: 263-275.

21. Şener AN, Günay ME, Leba A, Yıldırım R. Statistical review of dry reforming of methane literature using decision tree and artificial neural network analysis. Catal Today. 2018; 299: 289302.

22. Pakhare $\mathrm{D}$, Spivey J. A review of dry $\left(\mathrm{CO}_{2}\right)$ reforming of methane over noble metal catalysts. Chem Soc Rev. 2014; 43: 7813-7837.

23. Aramouni NA, Touma JG, Tarboush BA, Zeaiter J, Ahmad MN. Catalyst design for dry reforming of methane: Analysis review. Renew Sust Energ Rev. 2018; 82: 2570-2585.

24. Arora S, Prasad R. An overview on dry reforming of methane: Strategies to reduce carbonaceous deactivation of catalysts. RSC Adv. 2016; 6: 108668-108688.

25. Nair MM, Kaliaguine S. Structured catalysts for dry reforming of methane. New J Chem. 2016; 40: 4049-4060.

26. Bian Z, Das S, Wai MH, Hongmanorom P, Kawi S. A review on bimetallic nickel-based catalysts for $\mathrm{CO}_{2}$ reforming of methane. ChemPhysChem. 2017; 18: 3117-3134.

27. Lavoie JM. Review on dry reforming of methane, a potentially more environmentally-friendly approach to the increasing natural gas exploitation. Front Chem. 2014; 2: 1-17. 
28. Zhang $\mathrm{Q}$, Long $\mathrm{K}$, Wang J, Zhang $\mathrm{T}$, Song $\mathrm{Z}$, Lin $\mathrm{Q}$. A novel promoting effect of chelating ligand on the dispersion of $\mathrm{Ni}$ species over Ni/SBA-15 catalyst for dry reforming of methane. Int J Hydrog Energy. 2017; 42: 14103-14114.

29. Zhang $Q$, Zhang $T$, Shi $Y$, Zhao B, Wang $M$, Liu $Q$, et al. A sintering and carbon-resistant Ni-SBA-

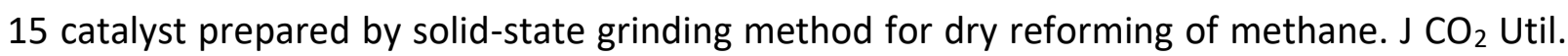
2017; 17: 10-19.

30. Drobná $H$, Kout M, Sołtysek A, González-Delacruz VM, Caballero A, Čapek L. Analysis of Ni species formed on zeolites, mesoporous silica and alumina supports and their catalytic behavior in the dry reforming of methane. Reac Kinet Mech Cat. 2017; 121: 255-274.

31. Omoregbe O, Danh HT, Nguyen-Huy C, Setiabudi HD, Abidin SZ, Truong QD, et al. Syngas production from methane dry reforming over Ni/SBA-15 catalyst: Effect of operating parameters. Int J Hydrog Energy. 2017; 42: 11283-11294.

32. Gao XY, Ashok J, Widjaja S, Hidajat $\mathrm{K}$, Kawi S. Ni/SiO 2 catalyst prepared via Ni-aliphatic amine complexation for dry reforming of methane: Effect of carbon chain number and amine concentration. Appl Catal A Gen. 2015; 503: 34-42.

33. Wen S, Liang M, Zou J, Wang S, Zhu X, Liu L, et al. Synthesis of a $\mathrm{SiO}_{2}$ nanofibre confined $\mathrm{Ni}$ catalyst by electrospinning for the $\mathrm{CO}_{2}$ reforming of methane. J Mater Chem A. 2015; 3: 1329913307.

34. Wang $M$, Zhang $Q$, Zhang $T$, Wang $Y$, Wang J, Long $K$, et al. Facile one-pot synthesis of highly dispersed Ni nanoparticles embedded in HMS for dry reforming of methane. Chem Eng J. 2017; 313: 1370-1381.

35. Han JW, Parka JS, Choib MS, Lee H. Uncoupling the size and support effects of Ni catalysts for dry reforming of methane. Appl Catal B Environ. 2017; 203: 625-632.

36. Li H, He Y, Shen D, Cheng S, Wang J, Liu H, et al. Design an in-situ reduction of $\mathrm{Ni} / \mathrm{C}-\mathrm{SiO}_{2}$ catalyst and new insights into pretreatment effect for $\mathrm{CH}_{4}-\mathrm{CO}_{2}$ reforming reaction. Int J Hydrog Energy. 2017; 42: 10844-10853.

37. Karam L, Casale S, El Zakhem H, El Hassan N. Tuning the properties of nickel nanoparticles inside SBA-15 mesopores for enhanced stability in methane reforming. $\mathrm{J} \mathrm{CO}_{2}$ Util. 2017; 17: 119-124.

38. Lovell EC, Fuller A, Scott J, Amal R. Enhancing $\mathrm{Ni}_{-} \mathrm{SiO}_{2}$ catalysts for the carbon dioxide reforming of methane: Reduction-oxidation-reduction pre-treatment. Appl Catal B Environ. 2016; 199: 155-165.

39. Gao XY, Hidajat $\mathrm{K}$, Kawi S. Facile synthesis of $\mathrm{Ni} / \mathrm{SiO}_{2}$ catalyst by sequential hydrogen/air treatment: A superior anti-coking catalyst for dry reforming of methane. J $\mathrm{CO}_{2}$ Util. 2016; 15: 146-153.

40. Li W, Zhao Z, Guo X, Wang G. Employing a nickel-containing supramolecular framework as Ni precursor for synthesizing robust supported $\mathrm{Ni}$ catalysts for dry reforming of methane. ChemCatChem. 2016; 8: 2939-2952.

41. Baktash E, Littlewood P, Pfrommer J, Schomacker R, Driess M, Thomas A. Controlled formation of nickel oxide nanoparticles on mesoporous silica using molecular $\mathrm{Ni}_{4} \mathrm{O}_{4}$ clusters as precursors: Enhanced catalytic performance for dry reforming of methane. ChemCatChem. 2015; 7: 12801284.

42. Gao XY, Liu H, Hidajat $\mathrm{K}$, Kawi S. Anti-coking $\mathrm{Ni} / \mathrm{SiO}_{2}$ catalyst for dry reforming of methane: Role of oleylamine/oleic acid organic pair. ChemCatChem. 2015; 7: 4188-4196. 
43. Kang D, Lim HS, Lee JW. Enhanced catalytic activity of methane dry reforming by the confinement of Ni nanoparticles into mesoporous silica. Int J Hydrog Energy. 2017; 42: 1127011282.

44. Yang W, He D. Role of poly(N-vinyl-2-pyrrolidone) in Ni dispersion for highly-dispersed Ni/SBA15 catalyst and its catalytic performance in carbon dioxide reforming of methane. Appl Catal A Gen. 2016; 524: 94-104.

45. Kaydouh MN, El Hassan N, Davidson A, Casale S, El Zakhem H, Massiani P. Highly active and stable Ni/SBA-15 catalysts prepared by a "two solvents" method for dry reforming of methane. Microporous Mesoporous Mater. 2016; 220: 99-109.

46. Rodriguez-Gomez A, Pereñiguez R, Caballero A. Nickel particles selectively confined in the mesoporous channels of SBA-15 yielding a very stable catalyst for DRM reaction. J Phys Chem B. $2018 ; 122:$ 500-510.

47. Gálvez ME, Albarazi A, Da Costa P. Enhanced catalytic stability through non-conventional synthesis of Ni/SBA-15 for methane dry reforming at low temperatures. Appl Catal A Gen. 2015; 504: 143-150.

48. Tian J, Ma B, Bu S, Yuan Q, Zhao C. One-pot synthesis of highly sintering- and coking-resistant $\mathrm{Ni}$ nanoparticles encapsulated in dendritic mesoporous $\mathrm{SiO}_{2}$ for methane dry reforming. Chem Commun. 2018; 54: 13993-13996.

49. Xu L, Zhang J, Wang F, Yuan K, Wang L, Wu K, et al. One-step synthesis of ordered mesoporous $\mathrm{CoAl}_{2} \mathrm{O}_{4}$ spinel-based metal oxides for $\mathrm{CO}_{2}$ reforming of $\mathrm{CH}^{+}$. RSC Adv. 2015; 5: 48256-48268.

50. $\mathrm{Xu} \mathrm{L}$, Song $\mathrm{H}$, Chou L. One-pot synthesis of ordered mesoporous $\mathrm{NiO}-\mathrm{CaO}-\mathrm{Al}_{2} \mathrm{O}_{3}$ composite oxides for catalyzing $\mathrm{CO}_{2}$ reforming of $\mathrm{CH}_{4}$. ACS Catal. 2012; 2: 1331-1342.

51. Kobylinski TP, Kibby CL, Pannell RB, Eddy EL. ROR-activated catalyst for synthesis gas conversion. Patent US4729981. 1986.

52. Unmuth EE, Schwartz LH, Butt JB. Iron alloy Fischer-Tropsch catalysts: I: Carburization studies of the Fe Ni system. J Catal. 1980; 63: 404-414.

53. Tang L, Yamaguchi D, Leita B, Sage V, Burke N, Chiang K. The effects of oxidation-reduction treatment on the structure and activity of cobalt-based catalysts. Catal Commun. 2015; 59: 166169.

54. Yu T, Wu Z, Kim WS. Mild temperature synthesis of gold nanoplates using polyethyleneimine and their improved surface enhanced Raman signal. RSC Adv. 2014; 4: 37516-37521.

55. Shahzad A, Kim WS, Yu T. Synthesis, stabilization, growth behavior, and catalytic activity of highly concentrated silver nanoparticles using a multifunctional polymer in an aqueous-phase. RSC Adv. 2015; 5: 28652-28661.

56. Yu T, Kim R, Park H, Yi T, Kim WS. Mechanistic study of synthesis of gold nanoparticles using multi-functional polymer. Chem Phys Lett. 2014; 592: 265-271.

57. Van der meer J, Bardez-Giboire I, Mercier C, Revel B, Davidson A, Denoyel R. Mechanism of metal oxide nanoparticle loading in SBA-15 by the double solvent technique. J Phys Chem C. 2010; 114: 3507-3515.

58. J. W. Geus, Dutch pat. Appl. 6705,259, 1967: 6813,236, 1968.

59. Burattin $\mathrm{P}, \mathrm{Che} \mathrm{M}$, Louis $\mathrm{C}$. Molecular approach to the mechanism of deposition-precipitation of the Ni(II) phase on silica. J Phys Chem B. 1998; 102: 2722-2732.

60. Tanev PT, Pinnavaia TJ. A neutral templating route to mesoporous molecular sieves. Science. 1995; 267: 865-867. 
61. Bian Z, Kawi S. Highly carbon-resistant $\mathrm{Ni}-\mathrm{Co} / \mathrm{SiO}_{2}$ catalysts derived from phyllosilicates for dry reforming of methane. $\mathrm{J} \mathrm{CO}_{2}$ Util. 2017; 18: 345-352.

62. Xin J, Cui H, Cheng Z, Zhou Z. Bimetallic Ni-Co/SBA-15 catalysts prepared by urea coprecipitation for dry reforming of methane. Appl Catal A Gen. 2018; 554: 95-104.

63. Gao X, Tan Z, Hidajat $\mathrm{K}$, Kawi $\mathrm{S}$. Highly reactive $\mathrm{Ni}-\mathrm{Co} / \mathrm{SiO}_{2}$ bimetallic catalyst via complexation with oleylamine/oleic acid organic pair for dry reforming of methane. Catal Today. 2017; 281: 250-258.

64. Erdogan B, Arbag H, Gazi NY. SBA-15 supported mesoporous Ni and Co catalysts with high coke resistance for dry reforming of methane. Int J Hydrog Energy. 2018; 43: 1396-1405.

65. Wu T, Zhang Q, Cai W, Zhang P, Song X, Sun Z, et al. Phyllosilicate evolved hierarchical Ni- and $\mathrm{Cu}-\mathrm{Ni} / \mathrm{SiO}_{2}$ nanocomposites for methane dry reforming catalysis. Appl Catal A Gen. 2015; 503: 94-102.

66. Lovell EC, Horlyck J, Scott J, Amal R. Flame spray pyrolysis-designed silica/ceria-zirconia supports for the carbon dioxide reforming of methane. Appl Catal A Gen. 2017; 546: 47-57.

67. Zhao X, Lu M, Li H, Fang J, Shi L, Zhang D. In situ preparation of Ni nanoparticles in ceriummodified silica aerogels for coking- and sintering-resistant dry reforming of methane. New J Chem. 2017; 41: 4869-4878.

68. Zhao X, Li H, Zhang J, Shi L, Zhang D. Design and synthesis of NiCe@m-SiO 2 yolk-shell framework catalysts with improved coke- and sintering-resistance in dry reforming of methane. Int J Hydrog Energy. 2016; 41: 2447-2456.

69. Akri M, Chafik T, Granger P, Ayrault P, Batiot-Dupeyrat C. Novel nickel promoted illite clay based catalyst for autothermal dry reforming of methane. Fuel. 2016; 178: 139-147.

70. Albarazi A, Gálvez ME, Da Costa P. Synthesis strategies of ceria-zirconia doped Ni/SBA-15 catalysts for methane dry reforming. Catal Commun. 2015; 59: 108-112.

71. Xie T, Zhao X, Zhang J, Shi L, Zhang D. Ni nanoparticles immobilized Ce-modified mesoporous silica via a novel sublimation-deposition strategy for catalytic reforming of methane with carbon dioxide. Int J Hydrog Energy. 2015; 40: 9685-9695.

72. Kaydouh MN, El Hassan N, Davidson A, Casale S, El Zakhem H, Massiani P. Effect of the order of $\mathrm{Ni}$ and Ce addition in SBA-15 on the activity in dry reforming of methane. C R Chim. 2015; 18: 293-301.

73. Chen C, Wang X, Zhang L, Zou X, Ding W, Lu X. Synthesis of mesoporous $\mathrm{Ni}-\mathrm{La}_{2} \mathrm{O}_{3} / \mathrm{SiO}_{2}$ by ploy(ethylene glycol)-assisted sol-gel route as highly efficient catalysts for dry reforming of methane with $\mathrm{a} \mathrm{H}_{2} / \mathrm{CO}$ ratio of unity. Catal Commun. 2017; 94: 38-41.

74. Baudouin D, Margossian T, Rodemerck U, Webb PB, Veyre L, Krumeich F, et al. Origin of the improved performance in lanthanum-doped silica-supported $\mathrm{Ni}$ catalysts. ChemCatChem. 2017; 9: 586-596.

75. Oemar $\mathrm{U}$, Kathiraser $\mathrm{Y}, \mathrm{Mo} \mathrm{L}, \mathrm{Ho} \mathrm{XK}$, Kawi S. $\mathrm{CO}_{2}$ reforming of methane over highly active Lapromoted Ni supported on SBA-15 catalysts: Mechanism and kinetic modelling. Catal Sci Technol. 2016; 6: 1173-1186.

76. Omoregbe O, Danh HT, Abidin SZ, Setiabudi HD, Abdullah B, Vud KB, et al. Influence of lanthanide promoters on $\mathrm{Ni} / \mathrm{SBA}-15$ catalysts for syngas production by methane dry reforming. Procedia Eng. 2016; 148: 1388-1395. 
77. Zhang L, Lian J, Li L, Peng C, Liu W, Xu X, et al. $\mathrm{LaNiO}_{3}$ nanocube embedded in mesoporous silica for dry reforming of methane with enhanced coking resistance. Microporous Mesoporous Mater. 2018; 266: 189-197.

78. Taherian Z, Yousefpour M, Tajally $M$, Khoshandam B. A comparative study of $\mathrm{ZrO}_{2}, \mathrm{Y}_{2} \mathrm{O}_{3}$ and $\mathrm{Sm}_{2} \mathrm{O}_{3}$ promoted Ni/SBA-15 catalysts for evaluation of $\mathrm{CO}_{2}$ /methane reforming performance. Int J Hydrog Energy. 2017; 42: 16408-16420.

79. Taherian Z, Yousefpour M, Tajally M, Khoshandam B. Catalytic performance of Samariapromoted $\mathrm{Ni}$ and Co/SBA-15 catalysts for dry reforming of methane. Int J Hydrog Energy. 2017; 42: 24811-24822.

80. Taherian Z, Yousefpour M, Tajally M, Khoshandam B. Promotional effect of samarium on the activity and stability of Ni-SBA-15 catalysts in dry reforming of methane. Microporous Mesoporous Mater. 2017; 251: 9-18.

81. Chotirach $M$, Tungasmita S, Tungasmita DN, Tantayanon S. Titanium nitride promoted Ni-based SBA-15 catalyst for dry reforming of methane. Int J Hydrog Energy. 2018; 43: 21322-21332.

82. Sarkar B, Goyal R, Pendem C, Sasaki T, Bal R. Highly nanodispersed Gd-doped Ni/ZSM-5 catalyst for enhanced carbon-resistant dry reforming of methane. J Mol Catal A Chem. 2016; 424: 1726.

83. Benrabaa R, Löfberg A, Caballero JG, Bordes-Richard E, Rubbens A, Vannier RN, et al. Sol-gel synthesis and characterization of silica supported nickel ferrite catalysts for dry reforming of methane. Catal Commun. 2015; 58: 127-131.

84. Al-Fatesh AS, Atia H, Abu-Dahrieh JK, Ibrahim AA, Eckelt R, Armbruster $U$, et al. Hydrogen production from $\mathrm{CH}_{4}$ dry reforming over Sc promoted $\mathrm{Ni} / \mathrm{MCM}-41$. Int J Hydrog Energy. 2019; 44: 20770-20781.

85. Cao Y, Lu M, Fang J, Shi L, Zhang D. Hexagonal boron nitride supported mesoSiO ${ }_{2}$-confined $\mathrm{Ni}$ catalysts for dry reforming of methane. Chem Commun. 2017; 53: 7549-7552.

86. Yao L, Shi J, Xu H, Shen W, Hu C. Low-temperature $\mathrm{CO}_{2}$ reforming of methane on Zr-promoted $\mathrm{Ni} / \mathrm{SiO}_{2}$ catalyst. Fuel Process Technol. 2016; 144: 1-7.

87. Károlyi J, Németh $M$, Evangelisti $C$, Sáfrán $G$, Schay Z, Horváth $A$, et al. Carbon dioxide reforming of methane over $\mathrm{Ni}-\mathrm{In} / \mathrm{SiO}_{2}$ catalyst without coke formation. J Ind Eng Chem. 2018; 58: 189201.

88. Yao L, Wang $Y$, Shi J, Xu H, Shen $W, H u$ C. The influence of reduction temperature on the performance of $\mathrm{ZrO}_{x} / \mathrm{Ni}-\mathrm{MnO}_{x} / \mathrm{SiO}_{2}$ catalyst for low-temperature $\mathrm{CO}_{2}$ reforming of methane. Catal Today. 2017; 281: 259-267.

89. Yao L, Shi J, Hu C. The structure, carbon deposition and stability of a $\mathrm{ZrO} \times{ }_{x} / \mathrm{Ni}-\mathrm{MnO}_{x} / \mathrm{SiO}_{2}$ catalyst for the $\mathrm{CO}_{2}$ reforming of methane. RSC Adv. 2015; 5: 90168-90177.

90. Littlewood $\mathrm{P}$, Xie $X$, Bernicke $\mathrm{M}$, Thomas $\mathrm{A}$, Schomäcker R. $\mathrm{Ni}_{0.05} \mathrm{Mn}_{0.95} \mathrm{O}$ catalysts for the dry reforming of methane. Catal Today. 2015; 242: 111-118.

91. Amin R, Liu B, Ullah S, Biao HZ. Study of coking and catalyst stability over CaO promoted Nibased MCF synthesized by different methods for $\mathrm{CH}_{4} / \mathrm{CO}_{2}$ reforming reaction. Int J Hydrog Energy. 2017; 42: 21607-21616.

92. Zhang $Q$, Feng $X$, Liu J, Zhao L, Song $X$, Zhang $P$, et al. Hollow hierarchical $\mathrm{Ni} / \mathrm{MgO}-\mathrm{SiO}_{2}$ catalyst with high activity, thermal stability and coking resistance for catalytic dry reforming of methane. Int J Hydrog Energy. 2018; 43: 11056-11068. 
93. Nematollahi B, Rezaei M, Khajenoori M. Combined dry reforming and partial oxidation of methane to synthesis gas on noble metal catalysts. Int J Hydrog Energy. 2011; 36: 2969-2978.

94. Wang F, Xu L, Zhang J, Zhao Y, Li H, Li HX, et al. Tuning the metal-support interaction in catalysts for highly efficient methane dry reforming reaction. Appl Catal B Environ. 2016; 180: 511-520.

95. Wang HY, Au CT. Carbon dioxide reforming of methane to syngas over $\mathrm{SiO}_{2}$-supported rhodium catalysts. Appl Catal A Gen. 1997; 155: 239-252.

96. Nagaoka K, Seshan K, Aika K, Lercher JA. Carbon deposition during carbon dioxide reforming of methane-comparison between $\mathrm{Pt}_{\mathrm{Al}} \mathrm{O}_{3}$ and Pt/ZrO 2 . J Catal. 2001; 197: 34-42.

97. Claridge JB, Green ML, Tsang SC. Methane conversion to synthesis gas by partial oxidation and dry reforming over rhenium catalysts. Catal Today. 1994; 21: 455-460.

98. Nurunnabi M, Mukainakano Y, Kado S, Miyazawa T, Okumura K, Miyao T, et al. Oxidative steam reforming of methane under atmospheric and pressurized conditions over $\mathrm{Pd} / \mathrm{NiO}-\mathrm{MgO}$ solid solution catalysts. Appl Catal A Gen. 2006; 308: 1-12.

99. Miyata T, Li D, Shiraga M, Shishido T, Oumi Y, Sano T, et al. Promoting effect of Rh, Pd and Pt noble metals to the $\mathrm{Ni} / \mathrm{Mg}(\mathrm{Al}) \mathrm{O}$ catalysts for the DSS-like operation in $\mathrm{CH}_{4}$ steam reforming. Appl Catal A Gen. 2006; 310: 97-104.

100.Li D, Shishido T, Oumi Y, Sano T, Takehira K. Self-activation and self-regenerative activity of trace Rh-doped $\mathrm{Ni} / \mathrm{Mg}(\mathrm{Al}) \mathrm{O}$ catalysts in steam reforming of methane. Appl Catal A Gen. 2007; 332: 98-109.

101.Lucci FR, Marcinkowski MD, Lawton TJ, Sykes EC. $\mathrm{H}_{2}$ activation and spillover on catalytically relevant Pt-Cu single atom alloys. J Phys Chem C. 2015; 119: 24351-24357.

102.Tanksale A, Beltramini JN, Dumesic JA, Lu GQ. Effect of Pt and Pd promoter on Ni supported catalysts-A TPR/TPO/TPD and microcalorimetry study. J Catal. 2008; 258: 366-377.

103.Damyanova S, Pawelec B, Arishtirova K, Fierro JL, Sener C, Dogu T. MCM-41 supported PdNi catalysts for dry reforming of methane. Appl Catal B Environ. 2009; 92: 250-261.

104.Pinheiro AN, Valentini A, Sasaki JM, Oliveira AC. Highly stable dealuminated zeolite support for the production of hydrogen by dry reforming of methane. Appl Catal A Gen. 2009; 355: 156168.

105.Liu D, Cheo WN, Lim YW, Borgna A, Lau R, Yang Y. A comparative study on catalyst deactivation of nickel and cobalt incorporated MCM-41 catalysts modified by platinum in methane reforming with carbon dioxide. Catal Today. 2010; 154: 229-236.

106.de Miguel SR, Vilella IM, Maina SP, San Jose-Alonso D, Román-Martínez MC, Illán-Gómez MJ. Influence of Pt addition to $\mathrm{Ni}$ catalysts on the catalytic performance for long term dry reforming of methane. Appl Catal A Gen. 2012; 435-436: 10-18.

107. Hou T, Lei Y, Zhang S, Zhang J, Cai W. Ethanol dry reforming for syngas production over $\mathrm{Ir} / \mathrm{CeO}_{2}$ catalyst. J Rare Earths. 2015; 33: 42-45.

108.Greeley J, Mavrikakis M. Alloy catalysts designed from first principles. Nat Mater. 2004; 3: 810815.

109.Niu J, Ran J, Ou Z, Du X, Wang R, Qi W, et al. $\mathrm{CO}_{2}$ dissociation over $\mathrm{Pt}_{\mathbf{x}} \mathrm{Ni}_{4-\mathrm{x}}$ bimetallic clusters with and without hydrogen sources: A density functional theory study. J $\mathrm{CO}_{2}$ Util. 2016; 16: 431441.

110.Li L, Zhou L, Ould-Chikh S, Anjum DH, Kanoun MB, Scaranto J, et al. Controlled surface segregation leads to efficient coke-resistant nickel/platinum bimetallic catalysts for the dry reforming of methane. ChemCatChem. 2015; 7: 819-829. 
111.You XJ, Wang X, Ma YH, Liu JJ, Liu WM, Xu XL, et al. Ni-Co/Al $\mathrm{O}_{3}$ bimetallic catalysts for $\mathrm{CH}_{4}$ steam reforming: Elucidating the role of Co for improving coke resistance. ChemCatChem. 2014; 6: 3377-3386.

112.Xu JK, Zhou W, Li ZJ, Wang JH, Ma JX. Biogas reforming for hydrogen production over nickel and cobalt bimetallic catalysts. Int J Hydrog Energy. 2009; 34: 6646-6654.

113.Li LD, Anjum DH, Zhu HB, Saih Y, Laveille PV, D'souza L, et al. Synergetic effects leading to cokeresistant NiCo bimetallic catalysts for dry reforming of methane. ChemCatChem. 2015; 7: 427433.

114.Zhang JG, Wang H, Dalai AK. Development of stable bimetallic catalysts for carbon dioxide reforming of methane. J Catal. 2007; 249: 300-310.

115.Takanabe K, Nagaoka K, Nariai K, Aika K. Titania-supported cobalt and nickel bimetallic catalysts for carbon dioxide reforming of methane. J Catal. 2005; 232: 268-275.

116. Bian Z, Suryawinata IY, Kawi S. Highly carbon resistant multicore-shell catalyst derived from NiMg phyllosilicate nanotubes@silica for dry reforming of methane. Appl Catal B Environ. 2016; 195: 1-8.

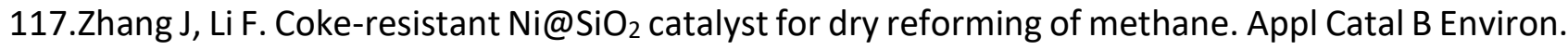
2015; 176-177: 513-521.

118.Peng H, Zhang X, Zhang L, Rao C, Lian J, Liu W, et al. One-pot facile fabrication of multiple nickel nanoparticles confined in microporous silica giving a multiple-cores@shell structure as a highly efficient catalyst for methane dry reforming. ChemCatChem. 2017; 9: 127-136.

119.Wang C, Qiu Y, Zhang X, Zhang Y, Sun N, Zhao Y. Geometric design of a Ni@silica nano-capsule catalyst with superb methane dry reforming stability: Enhanced confinement effect over the nickel site anchoring inside a capsule shell with an appropriate inner cavity. Catal Sci Technol. 2018; 8: 4877-4890.

120.Bian Z, Kawi S. Sandwich-like silica@Ni@silica multicore-shell catalyst for the low-temperature dry reforming of methane: Confinement effect against carbon formation. ChemCatChem. 2018; 10: 320-328.

121.Li Z, Wang Z, Jiang B, Kawi S. Sintering resistant Ni nanoparticles exclusively confined within $\mathrm{SiO}_{2}$ nanotubes for $\mathrm{CH}_{4}$ dry reforming. Catal Sci Technol. 2018; 8: 3363-3371.

122.Chai R, Zhao G, Zhang Z, Chen P, Liu Y, Lu Y. High sintering-/coke-resistance $\mathrm{Ni@SiO} / \mathrm{Al}_{2} \mathrm{O}_{3} / \mathrm{FeCrAl}$-fiber catalyst for dry reforming of methane: One-step, macro-to-nano organization via cross-linking molecules. Catal Sci Technol. 2017; 7: 5500-5504.

123.Gawande MB, Goswami A, Asefa T, Guo H, Biradar AV, Peng DL, et al. Core-shell nanoparticles: Synthesis and applications in catalysis and electrocatalysis. Chem Soc Rev. 2015; 44: 7540-7590.

124.Wu P, Li X, Ji S, Lang B, Habimana F, Li C. Steam reforming of methane to hydrogen over Nibased metal monolith catalysts. Catal Today. 2009; 146: 82-86. 
JEPT 2021; 3(1), doi:10.21926/jept.2101007

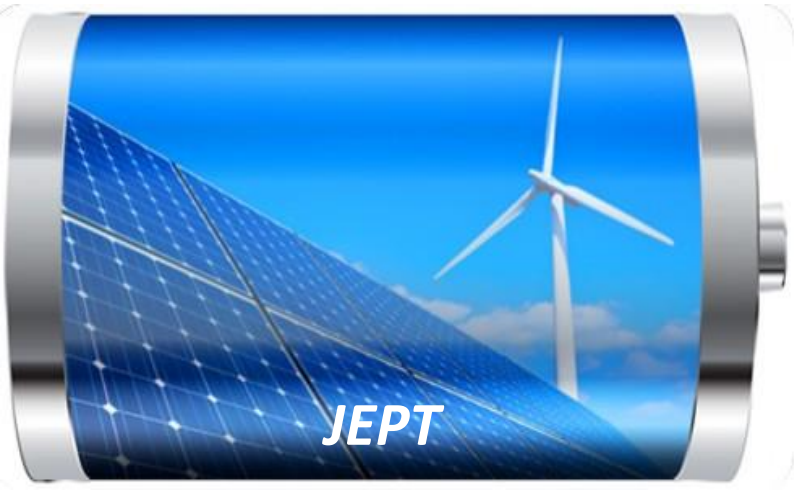

Enjoy JEPT by:

1. Submitting a manuscript

2. Joining in volunteer reviewer bank

3. Joining Editorial Board

4. Guest editing a special issue

For more details, please visit:

http://www.lidsen.com/journal/jept 
Original Research

\title{
Modeling of Ceria Reduction in a Solar Thermochemical Reactor via DEM Method
}

\author{
Han Zhang, Joseph D. Smith * \\ Department of Chemical and Biochemical Engineering, Missouri University of Science and \\ Technology, Rolla, MO 65409, United States; E-Mails: han.zhang@mst.edu, \\ smithjose@umsystem.edu
}

* Correspondence: Joseph D. Smith; E-Mail: smithjose@umsystem.edu

Academic Editor: Joaquin Alonso-Montesinos

Special Issue: Photovoltaic Solar Systems and Solar Thermal Plants

Journal of Energy and Power Technology

2021, volume 3 , issue 1

doi:10.21926/jept.2101008
Received: November 09, 2020

Accepted: January 19, 2021

Published: January 30, 2021

\begin{abstract}
Solar thermochemical reactor provides an attractive approach that utilizes the most common solar radiation as the thermal driving force to motivate the reaction between $\mathrm{CO}_{2}$ and metal oxides, which is also called metal oxide redox pair-based thermochemical cycles. The $\mathrm{CeO}_{2} / \mathrm{CeO}_{2-\delta}$ is widely used in the two-step redox process due to its advantages including fastredox kinetics, high crystallographic stability of a wide range of reacting oxygen nonstoichiometry, and relatively high oxygen solid-state conductivity. In this work, a threedimensional transient numerical analysis has been completed to study the performance of a $\mathrm{CeO}_{2}$ reduction reaction in a $1 / 8^{\text {th }}$ segment region of a novel partition cavity-receiver reactor. The porous $\mathrm{CeO}_{2}$ catalyst was analyzed using the discrete element method (DEM) to capture the heat transfer and reactive performances. The catalyst textural properties (particle size and void fraction) and reaction conditions (gas flow rate and radiative power input) were investigated in the $\mathrm{CeO}_{2}$ reduction reaction. The results indicated that increasing the catalyst specific surface area and the temperature are beneficial to the $\mathrm{O}_{2}$ production and further $\mathrm{CO}_{2}$ conversion.
\end{abstract}

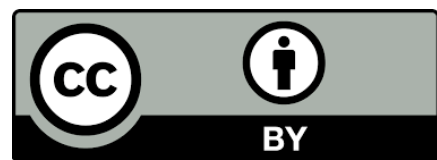

(C) 2021 by the author. This is an open access article distributed under the conditions of the Creative Commons by Attribution License, which permits unrestricted use, distribution, and reproduction in any medium or format, provided the original work is correctly cited. 


\section{Keywords}

Solar thermochemical reactor; ceria reduction; radiation; transient; DEM; chemical energy storage

\section{Introduction}

With the growing energy demands in the world, environmental protection issues have attracted a lot of attention related to energy technology development. Among various energy resources, fossil fuel remains the primary and stable energy source. Along with the use of fossil fuels, carbon dioxide emission rate is a serious factor that affects climate change and the global environment. Figure 1 shows global carbon dioxide emissions from 1990 to 2019 [1].

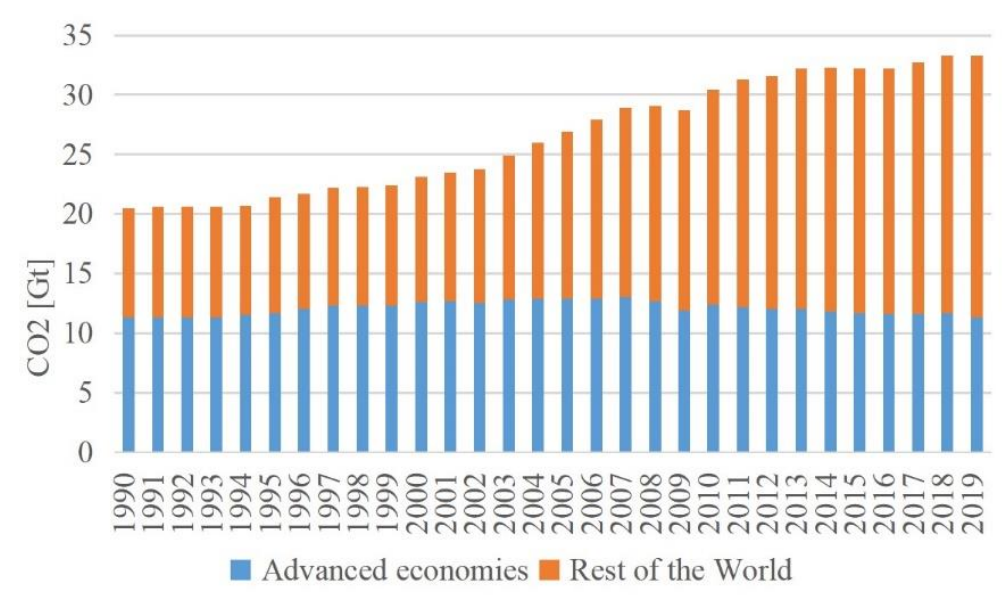

Figure 1 Global carbon dioxide emissions from 1990 to 2019 [1].

From this figure, $\mathrm{CO}_{2}$ emission continues at a high level in the recent three years. However, the emission of 2019 kept flat in 2019 due to the expanding application of renewable energy. Thanks to the numerous studies focusing on using clean energies or novel technologies, they showed important effect on the reduction of $\mathrm{CO}_{2}$ emission [2-6]. Since $\mathrm{CO}_{2}$ is also a carbon source and can be converted into useful and variable materials, work has been focused on finding efficient technology to accomplish this process. Decades ago, people began studying water electrolysis to produce hydrogen, since water is a cheap and available resource. However, hydrogen storage is an issue due to its high volatile property [7]. Compared with water electrolysis, syngas production from $\mathrm{CO}_{2}$ and $\mathrm{H}_{2} \mathrm{O}$ does not only provide an option for environmental protection and transportation safety but also offers an attractive energy fuel for further applications during the chemical process, like Fischer-Tropsch technology [8]. Solar energy is essentially a green technology with inexhaustible power production potential. Under the concentrated energy from the sun, $\mathrm{CO}_{2}$ and $\mathrm{H}_{2} \mathrm{O}$ can be converted to syngas using proper metal oxide redox pairs such as $\mathrm{ZnO} / \mathrm{Zn}$ [9], $\mathrm{Fe}_{3} \mathrm{O}_{4} / \mathrm{FeO}$ [10], and many more advanced couples [11]. The appearance of producing syngas via solar energy provides a brand-new idea for both solar energy application and chemical fuel production. 
The two-step solar thermochemical process is a redox cycle based on metal oxide redox. This chemical process is represented as the high-temperature reduction (see Eq. (1)) and lowtemperature oxidation (see Eq. (2) and (3)).

High-temperature reduction:

$$
M_{x} O_{y} \rightarrow M_{x} O_{y-\delta}+\frac{\delta}{2} O_{2}
$$

Low-temperature oxidation:

$$
\begin{aligned}
& M_{x} O_{y-\delta}+\delta C O_{2} \rightarrow M_{x} O_{y}+\delta C O \\
& M_{x} O_{y-\delta}+\delta H_{2} O \rightarrow M_{x} O_{y}+\delta H_{2}
\end{aligned}
$$

In the first step, the metal oxide is reduced to form pure metal or reductive metal oxide that releases oxygen over $1273 \mathrm{~K}$ [12]. This reaction is endothermic, so excess energy must be supplied using concentrated solar energy. In the second step, the oxidation reactions are exothermic and occur at a lower temperature $(<1000 \mathrm{~K})$. The feed $\mathrm{CO}_{2} / \mathrm{H}_{2} \mathrm{O}$ react with metal or reduced metal oxide and lose oxygen to form $\mathrm{CO} / \mathrm{H}_{2}$. Correspondingly, the metal or reduced metal oxide is oxidized to the former oxidation state. Compared with syngas production cycles, the most remarkable advantage of the two-step solar thermochemical process is that the water splitting and carbon dioxide splitting share the same reaction mechanism, which provides a great convenience for further analysis [13]. The reaction formula are shown in Eq. (2) and Eq. (3).

The metal oxide pairs for the two-step solar thermochemical redox process can be divided into two groups, volatile and non-volatile. The classification is based on the material physical properties and whether phase change is occurred during the metal oxide reduction [14]. Since the temperature requirement for reduction is in the range of $1500 \mathrm{~K}$ to $2000 \mathrm{~K}$ and up to $2300 \mathrm{~K}$, possibly higher than the boiling temperature of the reduced species, the volatile metal oxide may experience a phase change. There are some representative metal oxide pairs, including $\mathrm{ZnO} / \mathrm{Zn}, \mathrm{CdO} / \mathrm{Cd}, \mathrm{SnO}_{2} / \mathrm{SnO}$, $\mathrm{GeO}_{2} / \mathrm{GeO}$, and so forth [15]. Even though the volatile redox pair results in an extra entropy gain for the system due to the phase transition, the recombination of oxygen and reduced metal or metal oxide decreases the available reduced metal or metal oxide in the oxidization process, which has a negative impact on fuel production of volatile pairs [16]. Non-volatile redox pairs (e.g. ferrites, ceria, hercynite, and perovskites) $[17,18]$ can maintain their solid state at high temperatures, which avoid the recombination issue. Other morphology characteristics of non-volatile redox pairs, such as pore size, porosity, and specific surface area, also affect the reaction rate.

Cerium oxide is a representative redox catalyst used to produce carbon monoxide and hydrogen in the early 1980s due to its excellent performance in releasing and recovering oxygen [19]. The application of the non-stoichiometric $\mathrm{CeO}_{2}$ redox pair for solar thermochemical reactions flourished in the last decade. In 2006, Flamant et al. [20] proposed the application of $\mathrm{CeO}_{2} / \mathrm{Ce}_{2} \mathrm{O}_{3}$ redox pair to split water. The reduction of $\mathrm{CeO}_{2}$ was proceeded at $2000{ }^{\circ} \mathrm{C}, 100 \sim 200 \mathrm{mbar}$, followed by the oxidation step in the temperature range of $400-600^{\circ} \mathrm{C}$. Since reduction temperature is high enough to transfer $\mathrm{CeO}_{2}$ to a molten state, which strongly reduces the catalyst activity and quality, the study of $\mathrm{CeO}_{2}$ was focused on how to avoid the phase transfer under a high reduction temperature. Non- 
stoichiometric $\mathrm{CeO}_{2}\left(\mathrm{CeO}_{2-\delta}\right)$ was proposed to solve the issue of melting at high temperature. Based on the two-step thermochemical process, the reactions can be represented by

$$
\begin{gathered}
\mathrm{CeO}_{2} \rightarrow \mathrm{CeO}_{2-\delta}+\frac{\delta}{2} \mathrm{O}_{2} \\
\mathrm{CeO}_{2-\delta}+\delta \mathrm{CO}_{2} \rightarrow \mathrm{CeO}_{2}+\delta \mathrm{CO} \\
\mathrm{CeO}_{2-\delta}+\delta \mathrm{H}_{2} \mathrm{O} \rightarrow \mathrm{CeO}_{2}+\delta \mathrm{H}_{2}
\end{gathered}
$$

The net reaction enthalpies of $\mathrm{CO}_{2}$ splitting $\left(\mathrm{CO}_{2} \rightarrow \mathrm{CO}+\frac{1}{2} \mathrm{O}_{2}\right)$ and $\mathrm{H}_{2} \mathrm{O}$ splitting $\left(\mathrm{H}_{2} \mathrm{O} \rightarrow \mathrm{H}_{2}+\frac{1}{2} \mathrm{O}_{2}\right)$ are $283 \mathrm{~kJ}$ per mole of $\mathrm{CO}_{2}$ and $242 \mathrm{~kJ}$ per mole of $\mathrm{H}_{2} \mathrm{O}$, respectively [21, 22]. At 1500 and $0.1 \mathrm{mbar}$ $\mathrm{O}_{2}$ partial pressure, the enthalpy change of ceria reduction is around $475 \mathrm{~kJ}$ per half mole of $\mathrm{O}_{2}$ [21]. Therefore, the reaction enthalpies to generate one mole of $\mathrm{CO}$ and $\mathrm{H}_{2}$ are estimated $-192 \mathrm{~kJ} / \mathrm{mol}$ and $-233 \mathrm{~kJ} / \mathrm{mol}$.

The solar-to-fuel efficiency (see Eq. (7)) is used to show the reaction performance [22].

$$
\eta_{\text {solar-to-fuel }}=\frac{\eta_{\text {fuel }} H H V}{Q_{\text {solar }}}
$$

Chueh et al. [13] presented the application of porous monolithic ceria on the two-step solar thermochemical $\mathrm{CO}_{2} / \mathrm{H}_{2} \mathrm{O}$ splitting. The results showed that porous monolith ceria can reach a stable fuel generation state after 500 redox cycles with an estimated $0.8 \%$ peak solar-to-fuel efficiency. Furler et al. [23] proposed using porous ceria felt as the catalyst to support solar $\mathrm{CO}_{2} / \mathrm{H}_{2} \mathrm{O}$ splitting. The $\mathrm{O}_{2}$ and fuel production are $2.89 \pm 0.27 \mathrm{~mL} \mathrm{~g}-1 \mathrm{CeO}_{2}$ and $5.88 \pm 0.43 \mathrm{~mL} \mathrm{~g}-1 \mathrm{CeO}_{2}$. Furler et al. [24] also proposed a reticulated porous ceria (RPC) foam structure for redox reactions, which derived a $3.53 \%$ peak solar-to-fuel efficiency. The efficiency improved more than four times compared with former porous monolithic ceria. Venstrom et al. [25] presented a novel morphology of $\mathrm{CeO}_{2}$, three-dimensional-ordered macroporous (3DOM) $\mathrm{CeO}_{2}$, which has both higher specific surface area and effective pore interconnections. The fuel production rate was enhanced by as much as $260 \%$ and $175 \%$ compared with traditional low porosity ceria and non-ordered mesoporous ceria. Oliveira et al. [26] further studied the 3DOM ceria structure for solar thermochemical $\mathrm{CO}_{2}$ splitting in a tubular reactor. The result showed that the maximum fuel production rate is three times higher than the dual-scale porosity ceria reticulated porous foam.

Based on the difference of heat transfer type, solar thermochemical reactors can be classified as indirectly-irradiated reactors and directly-irradiated reactors. For indirectly-irradiated reactors, the reactor chamber material is generally made from opaque metals. Incoming solar radiant flux to the external wall of the reactor is transferred to heat conduction to raise the temperature of the metal oxide. For directly-irradiated reactors, the solar radiation can directly touch the surface of the metal oxide and use the incident radiation to heat up the oxide. Since the radiation term in the energy conservation equation also participates in heat transfer in directly-irradiated reactors, the reactor can achieve a higher temperature. To fully utilize the solar radiation, the cavity-receiver reactor is commonly used for directly-irradiated solar thermochemical processes. In a cavity-receiver reactor, the space provided in the cavity provides or enhances radiation to the catalyst inner surface, which forms an approximate blackbody. Different types of cavity-receiver reactors have been discussed 
and used over the past several years to prove that direct irradiation has a huge advantage on the two-step solar $\mathrm{H}_{2} \mathrm{O} / \mathrm{CO}_{2}$ splitting process [27].

To better predict and validate the $\mathrm{CeO}_{2}$ supported solar thermochemical two-step redox cycle, several chemical kinetics were studied and utilized to model the solar thermochemical process performances in recent years. Thermodynamic equilibrium model is a representative reaction model that widely used in the simulations of $\mathrm{CeO}_{2}$ two-step redox reactions. Calle et al. [28], Groehn et al. [29], Bulfin et al. [30] introduced the relation between non-stoichiometric coefficient with oxygen partial pressure and temperature [31] in their process simulation and reactor modeling, respectively. It was proved that the model has a higher agreement with the model of Zinkevich et al. [32]. Zoller et al. [26] used the thermodynamic equilibrium model to simulate a 50kW solar receiver-reactor, which obtained the solar-to-fuel efficiency larger than $10 \%$ with dual-scale reticulated porous ceramic. The most basic chemical kinetics reaction model is based on the Arrhenius equation proposed by Ishida et al. [33]. This model was applied in different tubular reactor studies [34, 35]. However, the accuracy needs to be improved, due to the limitation of the current reported values of coefficients in the Arrhenius equation [36]. Arifin et al. [37] and McDaniel et al. [38] used the improved solid-state kinetic model, which includes the influences of reaction order of the oxidant mole fraction and the progress of the rate of oxidation [39], With their studies, the solid-state kinetic model is further parametrized for accurately evaluating the reaction rates. Based on the theory of Kröger et al. [40], the crystal site relations of $\mathrm{CeO}_{2}$ reduction was discussed in the work of Scheffe et al. [41] and corresponding reaction mechanism was applied in the work of Bader et al. [42] to study the $\mathrm{CeO}_{2}$ reduction performances in a particle suspension system. Haeussler et al. $[43,44]$ studied the two-step redox cycling of reticulated porous ceria structures in a monolithic cavity-type reactor. By increasing the reduction temperature or decreasing the operating pressure, the fuel production yields can reach up to $341 \mu \mathrm{mol} / \mathrm{g}$ and the peak solar-tofuel efficiency achieves up to $8 \%$. Parthasarathy et al. [45] used CFD to validate a solar thermochemical reactor with ceria RPC structure. In the studied power range (0.8 kW to $3.8 \mathrm{~kW})$, the temperature of the CFD model has an error range between $-0.3 \%$ to $7.15 \%$ compared with experiment results. Kyrimis et al. [46] numerical studied the scale-up solar thermochemical reactor geometry. The results showed that the scale-up geometry has a benefit for the solar-to-fuel efficiency at the beginning of the reduction but limited due to the thickness of $\mathrm{CeO}_{2} \mathrm{RPC}$.

Gas-solid performance plays a vital role in the numerical simulation of solar thermochemical processes. The Eulerian-Lagrangian (CFD-DEM) method, which uses the DEM method to solve the transfer performances of particles individually in the gas continuum phase, is a representative approach to capture the variations of the dispersed phase. It was widely used in the simulations of fluidized bed reactors $[47,48]$, however, the application in the solar thermochemical processes for high-temperature chemical reactions is still in the infantile stage. Bellan et al. did a series of work on the heat transfer performances in different types of fluidized bed reactors for solar gasification and solar thermochemical cycles using the CFD-DEM method and the results showed high agreement between the experimental and simulation results $[49,50]$. Morris et al. used CFD-DEM method to study the heat transfer performances in the novel solar receiver designed by the National Renewable Energy Laboratory and proved that the improved continuum model has a high accuracy to predict the heat transfer coefficient [51]. Although some studies have been reported to focus on the heat transfer performances in the solar fluidized bed reactors using the CFD-DEM method, very few works consider the reactions in the solar thermochemical two-step metal redox cycles. 
Additionally, the transfer performances in the porous catalyst simulated by the DEM methods are worthy of discussing intensively.

In this paper, a three-dimensional (3D) transient multi-physics computational fluid dynamics model is applied to study heat and mass transfer of a high-temperature $\mathrm{CeO}_{2}$ reduction process in a novel partition cavity solar thermochemical reactor. The model uses the discrete element method (DEM) [52] to approximate the structure of a $\mathrm{CeO}_{2}$ layer as a packed bed. Several parameters defining the operating conditions and the catalyst layer formation have been studied to investigate the performance of the $\mathrm{CeO}_{2}$ reduction in the novel partition-cavity thermochemical reactor.

\section{Reactor Design Concept}

The reactor (shown in Figure 2) consists of a cylindrical cavity and a solar radiation receiver. An annular cylindrical catalyst region (60 mm i.d., $100 \mathrm{~mm}$ o.d., and $150 \mathrm{~mm}$ height) is integrated into the cavity section. The reactive region is separated from the cavity center by a transparent quartz partition (145 mm height). A $5 \mathrm{~mm}$ gap between the bottom of the transparent partition and cavity bottom leaves space for fluid to pass through the entire length of the reactive packed bed. Additionally, the transparent quartz allows solar radiation to enter the reactive region. With the partition design, the reactive cavity not only provides a relatively long path for contact of gas and solid but also enhance radiation heat transfer to the reactive region. The reactive region is surrounded by a ceramic insulation material inside the stainless-steel outer shell. Concentrated solar radiation enters the reactor through the $240 \mathrm{~mm}$ receiver window and focuses on the interface of the solar receiver and reactive cavity. Concentrated radiation distributes over the inner cavity surface and enters the reactive region. Eight inlets and outlets, each with a diameter of $8 \mathrm{~mm}$, are evenly distributed around the top edge of the solar receiver and reactive cavity side walls (see Figure 2 and Figure 3).

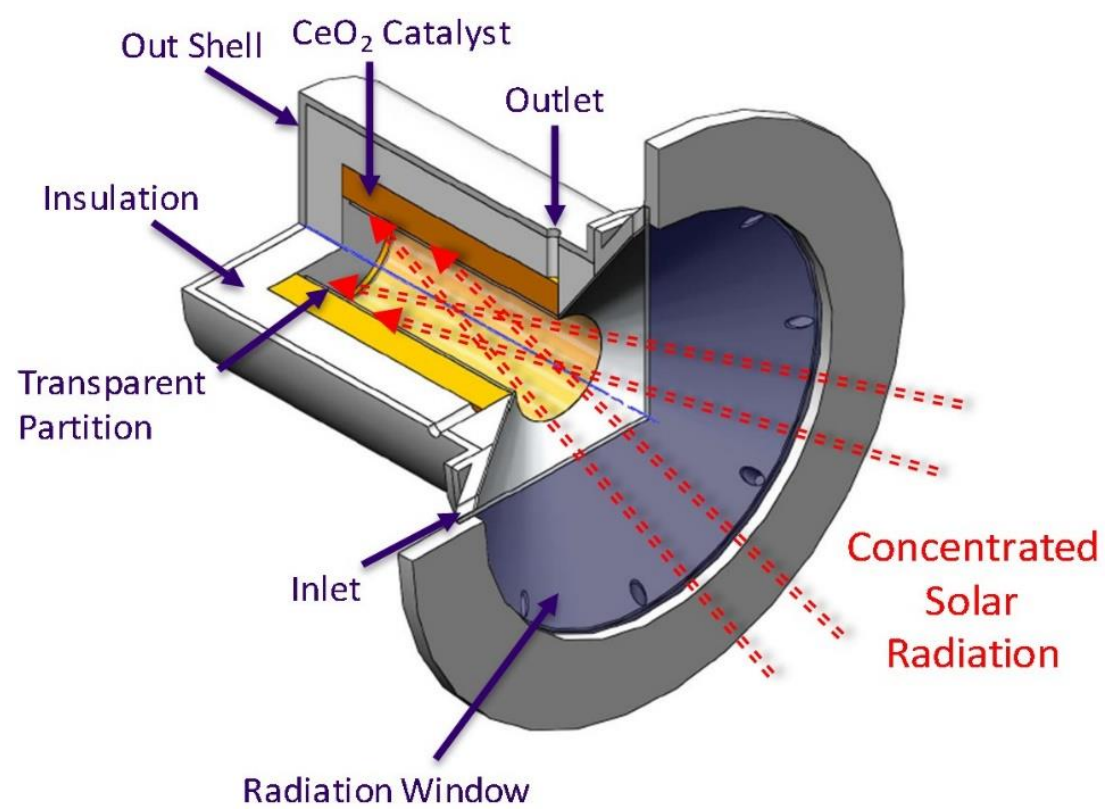

Figure 2 Partition cavity solar reactor schematic diagram. 
(a)

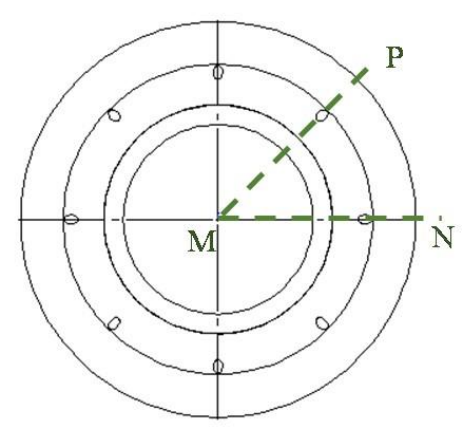

(b)

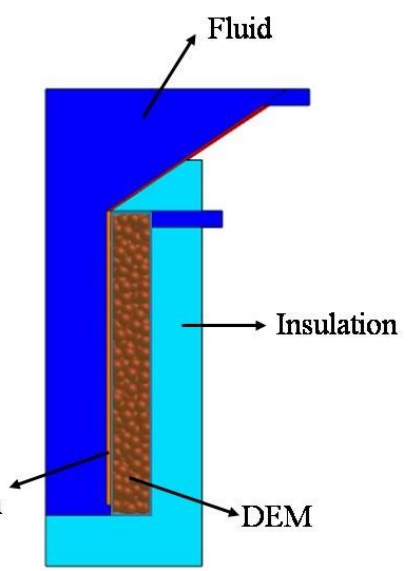

Figure 3 Simulation reactor model. (a) top view; (b) cross-section.

\section{Approach}

\subsection{Reactor Model}

Figure 3 shows the solar reactor modeling geometry in top view and cross-section. Since the reactor has a symmetric structure, only one-eighth of the entire geometry is simulated, with the assumption that radiation distribution and thermal losses are symmetric. Plane MN and MP are periodic boundary conditions with a 450 rotational angle. The stainless-steel housing and receiver are considered insignificant resistances, which are included in the wall boundary conditions to approximate heat loss from the reactor. Cerium dioxide $\left(\mathrm{CeO}_{2}\right)$ is packed in the reactive region to form a porous media structure approximated by the DEM method. By controlling the DEM injecting conditions, the catalyst particles are uniformly distributed through the entire reactive region that creates the appropriate gas flow paths to effectively mimic the structure of a porous media region. The reactive section is housed in the ceramic insulation material $\mathrm{Al}_{2} \mathrm{O}_{3}-\mathrm{SiO}_{2}$ with surfaces to exchange radiation heat transfer. Radiative heat transfer is implemented using the discrete ordinates model (DOM) [52] with gray thermal radiation model, which considers the radiative properties of participated media and the surrounding surfaces are identical for all wavelengths [53]. $\mathrm{CeO}_{2}$ is assumed to be a black body radiator and $\mathrm{Al}_{2} \mathrm{O}_{3}-\mathrm{SiO}_{2}$ layer is assumed to be a good thermal insulator with an emittance of 0.28 [54]. The receiver side wall is highly reflective material with a reflectance of 0.9 [55]. The incident solar power is assumed to be evenly distributed on the partition surface for simplification. Argon (Ar), entering the reactor from radial inlets on the solar receiver, works as an inert purge gas during preheating and reduction processes to improve the heat convection and accelerates the motion of generated oxygen.

\subsection{Numerical Analysis and Validation}

The simulated reactor schematic diagram in Figure 3 (b) represents the computational domains considered in the reactor model. Generally, the domains can be divided into non-reactive regions and reactive regions. Non-reactive domains include the solar receiver, the reactor cavity, and the inlets and outlets, which are simulated as fluid regions with laminar flow and ideal gas mixture. The transparent quartz partition and $\mathrm{Al}_{2} \mathrm{O}_{3}-\mathrm{SiO}_{2}$ insulation are considered as solid walls with corresponding thermal properties (conductivity and emittance). The reactive domain is a 
multiphase region gas mixture flowing through a packed bed of $\mathrm{CeO}_{2}$ particles. To set up the link between the particles and fluid phases, a two-way coupling method is introduced that solves the heat and mass transfer conservation equations in both phases [52].

\subsubsection{Non-reactive Regions}

The continuity, momentum, energy, and mass conservation equations of fluid regions areas follow:

$$
\begin{gathered}
\frac{\partial \rho_{f}}{\partial t}+\nabla \cdot\left(\rho_{f} \boldsymbol{v}\right)=0 \\
\frac{\partial\left(\rho_{f} \boldsymbol{v}\right)}{\partial t}+\nabla \cdot\left(\rho_{f} \boldsymbol{v} \boldsymbol{v}\right)=-\nabla p+\nabla \cdot \mu\left(\nabla \boldsymbol{v}+(\nabla \boldsymbol{v})^{T}-\frac{2}{3} \boldsymbol{I} \nabla \cdot \boldsymbol{v}\right)+\boldsymbol{f}_{\boldsymbol{b}} \\
\frac{\partial\left(\rho_{f} H_{f}\right)}{\partial t}+\nabla \cdot\left(\rho_{f} H_{f} \boldsymbol{v}\right)=\nabla \cdot(k \nabla T) \\
\frac{\partial\left(\rho_{f} Y_{i}\right)}{\partial t}+\nabla \cdot\left(\rho_{f} \boldsymbol{v} Y_{i}\right)=0
\end{gathered}
$$

where $\rho_{\mathrm{f}}$ is fluid density, $v$ is velocity vector, $\mathrm{p}$ is pressure, $\boldsymbol{\mu}$ is fluid dynamic viscosity, $\boldsymbol{I}$ is identity matrix, $\mathrm{f}_{\mathrm{b}}$ is momentum body force (gravity), $\mathrm{H}_{\mathrm{f}}$ is fluid enthalpy, $k$ is fluid thermal conductivity, and $Y_{i}$ is component concentration [52].

Since there is no flow in the solid regions, only the energy conservation equation is considered. Eq. (12) and (13) show the energy conservation of partition and insulation:

$$
\begin{gathered}
\frac{\partial\left(\rho_{\text {part }} H_{\text {part }}\right)}{\partial t}+\nabla \cdot\left(\rho_{\text {part }} H_{\text {part }} \boldsymbol{v}\right)=\nabla \cdot\left(k_{\text {part }} \nabla T\right)+S_{E, \text { rad }} \\
\frac{\partial\left(\rho_{s} H_{s}\right)}{\partial t}+\nabla \cdot\left(\rho_{s} H_{s} v\right)=\nabla \cdot\left(k_{s} \nabla T\right)+\nabla q_{r}
\end{gathered}
$$

where $\rho_{\text {part }}$ is partition density, $\rho_{s}$ is insulation density, $H_{\text {part }}$ is partition enthalpy, $H_{s}$ is insulation enthalpy, $\mathrm{k}_{\text {part }}$ is partition thermal conductivity, $\mathrm{k}_{\mathrm{s}}$ is insulation thermal conductivity, $\mathrm{S}_{E, \text { rad }}$ is source term of radiation, $\nabla q_{r}$ is re-radiation term. The input radiation and the re-radiation are included in $\mathrm{S}_{E, \mathrm{rad}}$, which can be solved from the radiative transfer equation:

$$
\frac{d I}{d s}=-\beta I+\kappa_{a} I_{b}+\frac{\kappa_{s}}{4 \pi} \int_{4 \pi} I(\Omega) d(\Omega)+\kappa_{p a} I_{p b}+\frac{\kappa_{p s}}{4 \pi} \int_{4 \pi} I(\Omega) d(\Omega)
$$

where $I$ is radiative intensity, $\mathrm{K}_{\mathrm{a}}$ is absorption coefficient, $\mathrm{k}_{\mathrm{s}}$ is scattering coefficient, $I_{b}$ is black body intensity, $\Omega$ is solid angle, $K_{p a}$ is particle absorption coefficient, $K_{p s}$ is particle scattering coefficient, $I_{p b}$ is particle black body intensity, $\beta$ is extinction coefficient, and $\mathrm{s}$ is distance in the $\Omega$ direction. As the $\mathrm{Ar}$ and $\mathrm{O}_{2}$ are low radiative materials, the radiative properties $\mathrm{K}_{\mathrm{a}}$ and $\mathrm{K}_{\mathrm{s}}$ are assumed to equal zero [56]. Re-radiation is used to couple the fluid and radiant energy field, which is shown as: 


$$
\nabla q_{r}=\kappa_{p a}\left(4 \pi I_{b}-\int_{4 \pi} I d \Omega\right)
$$

\subsubsection{Reactive Region}

The multiphase domain is the core-reactive region in the reactor consisting of two phases: gas mixture fluid phase and $\mathrm{CeO}_{2}$ particle Lagrangian phase. The fluid continuous phase should consider the interaction between gas mixture and $\mathrm{CeO}_{2}$ particles, which can be conveyed as source terms in governing equations. The conservation equations are as follows:

$$
\begin{gathered}
\frac{\partial\left(\alpha \rho_{f}\right)}{\partial t}+\nabla \cdot\left(\alpha \rho_{f} \boldsymbol{v}\right)=S_{m, O_{2}} \\
\frac{\partial\left(\alpha \rho_{f} \boldsymbol{v}\right)}{\partial t}+\nabla \cdot\left(\alpha \rho_{f} \boldsymbol{v} \boldsymbol{v}\right)=-\nabla \alpha p+\nabla \cdot \alpha \mu\left(\nabla \boldsymbol{v}+(\nabla \boldsymbol{v})^{T}-\frac{2}{3} \boldsymbol{I} \nabla \cdot \boldsymbol{v}\right)+\alpha \boldsymbol{f}_{\boldsymbol{b}}+S_{V} \\
\frac{\partial\left(\alpha \rho_{f} H_{f}\right)}{\partial t}+\nabla \cdot\left(\alpha \rho_{f} H_{f} \boldsymbol{v}\right)=\nabla \cdot(\alpha k \nabla T)+h_{f s} A_{f s}\left(T_{s}-T_{f}\right)+\nabla q_{r}+S_{E} \\
\frac{\partial\left(\alpha \rho_{f} Y_{O_{2}}\right)}{\partial t}+\nabla \cdot\left(\alpha \rho_{f} \boldsymbol{v} Y_{O_{2}}\right)=S_{m, O_{2}}
\end{gathered}
$$

where $\alpha$ is the void fraction of fluid phase, $S_{m, O_{2}}, \mathrm{~S}_{\mathrm{V}}$, and $\mathrm{S}_{\mathrm{E}}$ are the binary phase coupling mass source term, momentum source term, and energy source term, respectively. These terms can be expanded and shown as:

$$
\begin{gathered}
S_{V}=-\frac{1}{\Delta t} \sum_{\pi}\left(\int_{t}^{t+\Delta t} \int_{V_{c}} \delta\left(\boldsymbol{r}-\boldsymbol{r}_{\pi}\right) n_{\pi}\left(\boldsymbol{F}_{\boldsymbol{s}}+\dot{m}_{p} \boldsymbol{v}_{\boldsymbol{p}}\right) d V d t\right) \\
S_{E}=-\frac{1}{\Delta t} \sum_{\pi}\left(\int_{t}^{t+\Delta t} \int_{V_{c}} \delta\left(\boldsymbol{r}-\boldsymbol{r}_{\pi}\right) n_{\pi}\left(Q_{t}+\boldsymbol{F}_{\boldsymbol{s}} \cdot v_{p}+\frac{1}{2} \dot{m}_{p} \boldsymbol{v}_{\boldsymbol{p}}{ }^{2}+\dot{m}_{p} h\right) d V d t\right) \\
S_{m, O_{2}}=-\frac{1}{\Delta t} \sum_{\pi}\left(\int_{t}^{t+\Delta t} \int_{V_{c}} \delta\left(\boldsymbol{r}-\boldsymbol{r}_{\pi}\right) n_{\pi} \dot{m}_{p} d V d t\right)
\end{gathered}
$$

where $F_{s}$ is particle surface force and $m_{p}$ is particle mass transfer rate. The volume integral is based on cells. The Dirac delta function accounts for the parcels not represented in the cell. The concrete formations of $F_{s}$ and $m_{p}$ are discussed with the governing equations for DEM particles. By taking the particle motion, energy, and mass influences into consideration, the fluid governing equations approximate fluid/particle interactions using the DEM packing conditions.

Since $\mathrm{CeO}_{2}$ particles work as discrete elements, the momentum conservation is shown based on surface and body force. Particle mass and heat transfer happen on interphase, which should be considered together. The governing equations are represented below:

$$
m_{p} \frac{d \boldsymbol{v}_{\boldsymbol{p}}}{d t}=\boldsymbol{F}_{\boldsymbol{s}}+\boldsymbol{F}_{\boldsymbol{b}}
$$




$$
\begin{gathered}
m_{p} c_{p} \frac{d T_{p}}{d t}=Q_{c o n v}+Q_{r a d}+Q_{\text {reac }} \\
\frac{d m_{p}}{d t}=\dot{m}_{p}
\end{gathered}
$$

where $F_{s}$ is surface force including drag force and pressure gradient, $F_{b}$ is body force including gravity and contact force, $Q_{\text {conv }}$ is convective heat transfer from the fluid phase to the particle, $Q_{\text {rad }}$ is radiative heat transfer rate, $Q_{\text {reac }}$ is heat of reduction reaction. Eq. (26) shows how $Q_{\text {conv }}$ can be formulated:

$$
Q_{\text {conv }}=h_{f p} A_{s}\left(T-T_{p}\right)
$$

where $h_{f p}$ is heat transfer coefficient, $\mathrm{A}_{s}$ is particle surface area, T is fluid temperature, $\mathrm{T}_{\mathrm{p}}$ is particle temperature. Heat transfer coefficient $h_{f p}$ can be derived from the relation of particle Nusselt number [57]:

$$
N u_{p}=\frac{h_{f p} D_{p}}{k}
$$

where $k$ is particle thermal conductivity and $D_{p}$ is particle diameter. Since $\operatorname{Re}_{p}<5000$, the RanzMarshall correlation [58] can be applied to determine the particle Nusselt number:

$$
N u_{p}=2\left(1+0.3 \operatorname{Re}_{p}^{1 / 2} \operatorname{Pr}^{1 / 3}\right)
$$

where Pr is from the fluid phase.

The particle participated radiative heat transfer $Q_{\text {rad }}$ is defined as:

$$
Q_{r a d}=\frac{A_{s}}{4} Q_{a, p}\left(G-4 \sigma T_{p}^{4}\right)
$$

where $\mathrm{Q}_{a, p}$ is absorption efficiency of particle, $\mathrm{G}$ is incident radiative heat flux, and $\sigma$ is the StefanBoltzmann constant. In this paper, $\mathrm{CeO}_{2}$ is assumed to have a $90 \%$ absorption efficiency. Reaction enthalpy of $\mathrm{CeO}_{2}$ reduction $\mathrm{Q}_{\text {reac }}$ in Eq. (4) is around $475 \mathrm{~kJ}$ per $1 / 2$ mole of $\mathrm{O}_{2}$ [59].

\subsubsection{Chemical Reaction}

In our study, the mixed chemical kinetic and equilibrium model was applied to simulate the $\mathrm{CeO} 2$ reduction of DEM particles. The reaction was set up based on the DEM particle phase and combined the mass transfer with the fluid phase via the former introduced source term $\left(S_{m, O_{2}}\right)$.

Based on the thermodynamic equilibrium model, which combines the crystal site model with the standard Gibbs free energy equation [42], the non-stoichiometric coefficient $\delta$ is a function of temperature and oxygen partial pressure [31]:

$$
\frac{\delta}{0.35-\delta}=\left(\frac{106,000 \mathrm{~Pa}}{P_{\mathrm{O}_{2}}}\right)^{0.217} \exp \left(\frac{-195.6 \mathrm{~kJ} / \mathrm{mol}}{R T}\right)
$$


The reaction rate in the DEM particle phase is expressed as $m_{p}$ in Eq. (25). To describe the reaction in the particle phase, the $\mathrm{CeO}_{2}$ reduction was regarded as the form of particle devolatilization. As the $\mathrm{CeO}_{2}$ reduction process is a non-stoichiometric reaction, the user reaction rate method (Eq. (31) is applied instead of the default first order rate method (Eq. (32)) [53].

$$
\begin{gathered}
\dot{m}_{p}=\frac{d m}{d t}=-r_{\text {user rate }} \\
\frac{d m}{d t}=-k m
\end{gathered}
$$

Based on the study of Ishida et al. [31], the $\mathrm{CeO}_{2}$ reduction rate can be expressed as:

$$
\frac{d x}{d t}=k_{r e d}(1-x)
$$

where $k_{\text {red }}$ is the reduction rate constant, $\mathrm{x}$ is the reaction fraction. The reaction fraction can be conveyed as:

$$
x=\frac{m-m_{i}}{m_{f}-m_{i}}
$$

where $m, m_{i}$, and $m_{f}$ are time-dependent particle mass, initial particle mass, and final particle mass, respectively.

Since $\mathrm{Ce}$ is conserved in the $\mathrm{CeO}_{2}$ reduction process, the concentration of vacant oxygen $\left(\left[\mathrm{O}_{\mathrm{vac}}\right]\right)$ and the concentration of cerium ([Ce]) have the following relation:

$$
\frac{\left[O_{v a c}\right]}{[\mathrm{Ce}]}=\delta
$$

Based on the above conservation between the concentration of oxygen and the concentration of cerium, the time-dependent particle mass $m$ in Eq. (31) can be shown as a function of $\delta$ :

$$
m=m_{i}-\frac{\delta n_{\mathrm{CeO}_{2}} M_{\mathrm{O}_{2}}}{2}
$$

where $n_{\mathrm{CeO}_{2}}$ is the moles of $\mathrm{CeO}_{2}$ and $\mathrm{M}_{\mathrm{O}_{2}}$ is the molecular weight of oxygen.

By substituting Eq. (30) and (33) into Eq. (31), the production generation rate is

$$
r_{\text {user rate }}=\frac{d m}{d t}=\left(m_{f}-m_{i}\right) \cdot k_{\text {red }} \cdot\left(1+\frac{\delta \frac{m_{i}}{M_{\mathrm{CeO}_{2}}} M_{O_{2}}}{2\left(m_{f}-m_{i}\right)}\right)
$$

The particle devolatilization rate based on Eq. (31) can be expressed as:

$$
\dot{m}_{p}=-\left(m_{f}-m_{i}\right) \cdot k_{\text {red }} \cdot\left(1+\frac{\delta \frac{m_{i}}{M_{C e O_{2}}} M_{O_{2}}}{2\left(m_{f}-m_{i}\right)}\right)
$$




\subsection{Boundary and Initial Conditions}

The boundary conditions are summarized in Table 1. A no-slip boundary condition is assumed on all fluid-solid interfaces. All the outer wall surfaces are adiabatic. The inlet surfaces are set as velocity inlet condition with the velocity taken from the volume flow rate. The outlets operate under atmospheric pressure. The boundary condition of the partition inner surface is taken as a diffuse radiation flux condition, which is determined by input radiation power. The radiative properties of partition and insulation boundaries depend on the material properties (listed in Table 1).

Table 1 Boundary conditions.

\begin{tabular}{ll}
\hline Surface & Boundary Conditions \\
\hline Inlets & $v_{\text {inlet }}=\frac{\dot{V}}{A_{\text {inlets }}} ; T_{\text {inlet }}=T_{\text {room }}$ \\
Outlets & $p_{\text {out }}=p_{\text {atm }}$ \\
Partition inner surface & $k_{\text {part }} \frac{\partial T}{\partial n}=S_{\text {rad }}+h_{\text {part }} \Delta T$ \\
Insulation inner surface & $k_{s} \frac{\partial T}{\partial n}=\Delta q_{r}+h_{s} \Delta T$ \\
All other surfaces & $\frac{\partial T}{\partial n}=0$ \\
\hline
\end{tabular}

The entire reduction process can be divided into two sections: 1) preheating and 2) reaction. The initial conditions are distinguished for each section. The preheating process is initiated from room temperature $(298 \mathrm{~K})$ and atmospheric pressure $(1 \mathrm{~atm})$ with pure Ar purge gas. The particle devolatilization is deactivated. After 20 mins radiation under $1.5 \mathrm{~kW}$ power, the preheating process is terminated. In the reaction stage, the initial conditions are taken from the preheating stage, except the inlet oxygen mole fraction is set as $10 \mathrm{ppm}$.

\subsection{Parameters}

The material properties of gas components, catalyst, and solid components are listed in Table 2. In this paper, four important parameters are studied to compare the performance via the control variable method. The four parameters can be classified into two groups. One is related to operating conditions, like radiant power and gas flow rate. The other is related to $\mathrm{CeO}_{2}$ particle packing, such as void fraction and particle size. To expand the study rationally, a base case is defined with a void fraction of 0.65 , a particle size of $5 \mathrm{~mm}$, under radiant power of $3.5 \mathrm{~kW}$ in the reaction stage, and the gas flow rate is set to $1.8 \mathrm{~L} / \mathrm{min}$ (specific parameters listed in Table 3 ). 
Table 2 Material properties.

\begin{tabular}{|c|c|c|c|}
\hline Material & Property & Expression & $T(K)$ \\
\hline \multirow[t]{6}{*}{$\mathrm{CeO}_{2}$} & Density $\left(\mathrm{kg} \mathrm{m}^{-3}\right)$ [54] & 7220 & 298 \\
\hline & Molecular weight $\left(\mathrm{g} \mathrm{mol}^{-1}\right)$ & 172.12 & \\
\hline & \multirow[t]{2}{*}{ Specific heat capacity $\left(\mathrm{J} \mathrm{kg}^{-1} \mathrm{~K}^{-1}\right)$ [54] } & $-0.0001271 \cdot T^{2}+0.2697656 \cdot T+299.8695684$ & $280-1100$ \\
\hline & & 444.27 & $>1100$ \\
\hline & Thermal conductivity $\left(\mathrm{W} \mathrm{m}^{-1} \mathrm{~K}^{-1}\right)[61]$ & $\begin{array}{l}-1.7234232 \cdot 10^{-9} \cdot T^{3}+1.1203174 \cdot 10^{-5} \cdot T^{2}- \\
0.024019964 \cdot T+17.800409\end{array}$ & $280-2000$ \\
\hline & Enthalpy of formation $\left(\mathrm{J} \mathrm{kmol}^{-1}\right)$ [62] & $-1.0887 \cdot 10^{9}$ & 298 \\
\hline \multirow[t]{6}{*}{$\begin{array}{l}\text { Quartz } \\
\text { Glass }\end{array}$} & Density $\left(\mathrm{kg} \mathrm{m}^{-3}\right)$ [63] & 2500 & 298 \\
\hline & \multirow[t]{2}{*}{ Specific heat capacity $\left(\mathrm{J} \mathrm{mol}^{-1} \mathrm{~K}^{-1}\right)[64]$} & $-0.0001 \cdot T^{2}+0.1791 \cdot T-0.173$ & $273-847$ \\
\hline & & $0.0072 \cdot T+61.717$ & $847-2000$ \\
\hline & Thermal Conductivity $\left(\mathrm{W} \mathrm{m}^{-1} \mathrm{~K}^{-1}\right)$ [65] & $1.18+3.14 \times 10^{-3} \cdot T+17966 / T^{2}$ & $273-2000$ \\
\hline & Emissivity [66] & 0.06 & $273-2000$ \\
\hline & Transmissivity [66] & 0.86 & $273-2000$ \\
\hline \multirow[t]{5}{*}{$\mathrm{Al}_{2} \mathrm{O}_{3}-\mathrm{SiO}_{2}$} & Density $\left(\mathrm{kg} \mathrm{m}^{-3}\right)$ [54] & 560.65 & 298 \\
\hline & \multirow[t]{2}{*}{ Specific heat capacity $\left(\mathrm{J} \mathrm{kg}^{-1} \mathrm{~K}^{-1}\right)[54]$} & $4 \cdot 10^{-7} \cdot T^{3}+1.3797 \cdot 10^{-3} \cdot T^{2}+1.5987289 \cdot T+447.6996$ & $\leq 1480$ \\
\hline & & 1118.44 & $>1480$ \\
\hline & Thermal Conductivity $\left(\mathrm{W} \mathrm{m}^{-1} \mathrm{~K}^{-1}\right)$ [54] & $0.00012926 \cdot T+0.019654$ & $280-2200$ \\
\hline & Emissivity [54] & 0.28 & \\
\hline
\end{tabular}


JEPT 2021; 3(1), doi:10.21926/jept.2101008

\begin{tabular}{llll}
\hline Argon & Density $\left(\mathrm{kg} \mathrm{m}^{-3}\right)[63]$ & 1.634 & 298 \\
& Viscosity $(\mathrm{Pa} \cdot \mathrm{s})[63]$ & $2.277 \cdot 10^{-5}$ & 298 \\
& $C_{p} / R[67]$ & 2.5 & $298-5000$ \\
& Thermal Conductivity $\left(\mathrm{W} \mathrm{m}^{-1} \mathrm{~K}^{-1}\right)[68]$ & $0.004834+4.83706 \cdot 10^{-5} \cdot \mathrm{T}-1.29 \cdot 10^{-8} \cdot \mathrm{T}^{2}+2.3533 \cdot 10^{-12} \cdot \mathrm{T}^{3}$ & $290-2400$ \\
Oxygen & Density $\left(\mathrm{kg} \mathrm{m}^{-3}\right)$ & 1.30878 & 298 \\
& Viscosity $(\mathrm{Pa} \cdot \mathrm{s})$ & $2.054 \cdot 10^{-5}$ & 298 \\
& $C_{p} / R[67]$ & $3.7825-2.9967 \cdot 10^{-3} \cdot \mathrm{T}+9.8473 \cdot 10^{-6} \cdot \mathrm{T}^{2}-9.6813 \cdot 10^{-9} \cdot \mathrm{T}^{3}$ & $200-1000$ \\
& & $+3.2437 \cdot 10^{-12} \cdot \mathrm{T}^{4}$ & $1000-5000$ \\
& & $3.6610+6.5637 \cdot 10^{-4} \cdot \mathrm{T}-1.4115 \cdot 10^{-7} \cdot \mathrm{T}^{2}+2.0580 \cdot 10^{-11} \cdot \mathrm{T}^{3}-$ & 1000 \\
& & $1.2991 \cdot 10^{-15} \cdot \mathrm{T}^{4}$ & $270-2500$ \\
\hline
\end{tabular}


Table 3 Studied reactor parameters.

\begin{tabular}{llll}
\hline Case & Parameter & \multicolumn{2}{l}{ Other Conditions } \\
\hline \multirow{2}{*}{ Gas Flow Rate $(\mathrm{L} / \mathrm{min})$} & $1.8,2.5,3.2$ & $\varepsilon$ & 0.65 \\
& & $\mathrm{~d}_{\mathrm{p}}$ & $5 \mathrm{~mm}$ \\
& & $\mathrm{P}$ & $3.5 \mathrm{~kW}$ \\
Solar Radiant Power $(\mathrm{kW})$ & $3.0,3.5,4.0,4.5$ & $\varepsilon$ & 0.65 \\
& & $\mathrm{~d}_{\mathrm{p}}$ & $5 \mathrm{~mm}$ \\
$\mathrm{CeO}_{2}$ Void Fractions & & $\dot{V}$ & $1.8 \mathrm{~L} / \mathrm{min}$ \\
& $0.50,0.60,0.65$ & $\mathrm{~d}_{\mathrm{p}}$ & $5 \mathrm{~mm}$ \\
& & $P$ & $3.5 \mathrm{Kw}$ \\
$\mathrm{CeO}_{2}$ Particle Size $(\mathrm{mm})$ & $3.0,5.0,6.0$ & $\dot{V}$ & $1.8 \mathrm{~L} / \mathrm{min}$ \\
& & $\varepsilon$ & 0.65 \\
& & $\dot{V}$ & $3.5 \mathrm{Kw}$ \\
& & & $1.8 \mathrm{~L} / \mathrm{min}$ \\
\hline
\end{tabular}

\subsection{Numerical Implementation}

The transport conservation equations are solved by the finite volume method with approximately 400,000 hexagonal cells in an unstructured mesh using STAR-CCM+ v. 12.02 [53]. A first-order implicit unsteady scheme is used for time integration with a time step of $2.0 \mathrm{~s}$. The second-order segregated flow/energy/species algorithms are applied in the computation of conservation equations. The DEM solver is introduced to solve the governing equations of discrete $\mathrm{CeO}_{2}$ particles [60]. The non-stoichiometric $\mathrm{CeO}_{2}$ reduction is defined via user-defined field functions as a source term. The transient simulations are performed on a high-performance cluster "FLARE" (6 x (1 Lenovo NeXtScale nx360m5 compute node, 64 GB RAM)).

\subsection{Validation}

To reduce the influences of mesh number and quality on the simulation results, the mesh independence analysis was applied to this study. Three different cell base sizes were selected to study the average temperature of the catalyst region for 10 minutes pre-heating process.

The relation between cell numbers and temperatures is shown in Figure 4 . With the mesh number increasing, the temperature differences between 400,000 case and 960,000 cases are almost negligible, especially for the gas phase. Therefore, the mesh number reaches a stable level. Considering the accuracy and calculation cost for the transient model, the mesh with 400,000 cells was selected in the following study. 


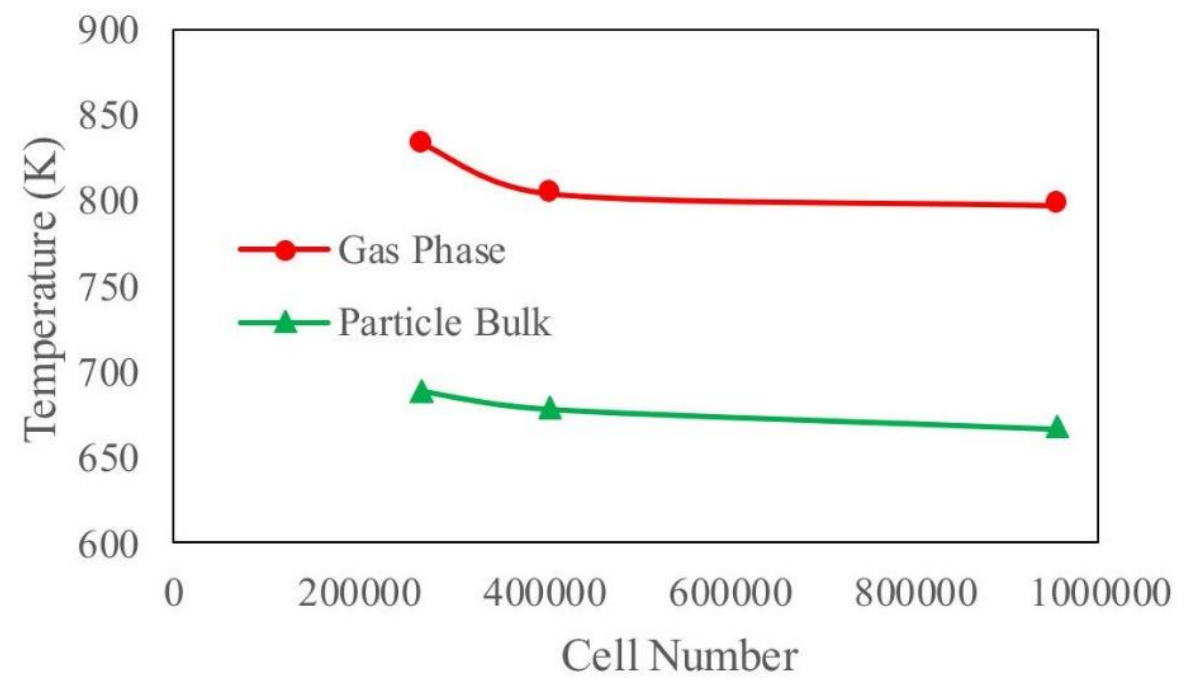

Figure 4 Mesh independence analysis.

The time-spatial analysis was applied to this model to ensure the time step is accurate enough to describe the processes in the reactor. Four different time steps $(0.5 s, 1 s, 2 s$, and $3 s)$ were used in the 20 minutes pre-heating process. The temperature was monitored by adding a vertical line probe in the center of the catalyst. Figure 5 shows the relation between temperature and position at the moment of 20 minutes under various time steps. The spatial-dependent temperature profiles have the same shape and the maximum difference is less than $4 \%$, which is in the tolerable range. Comprehensively considering the transient simulation target, the accuracy, and the calculation cost, 2 s time step was chosen in the following simulations.

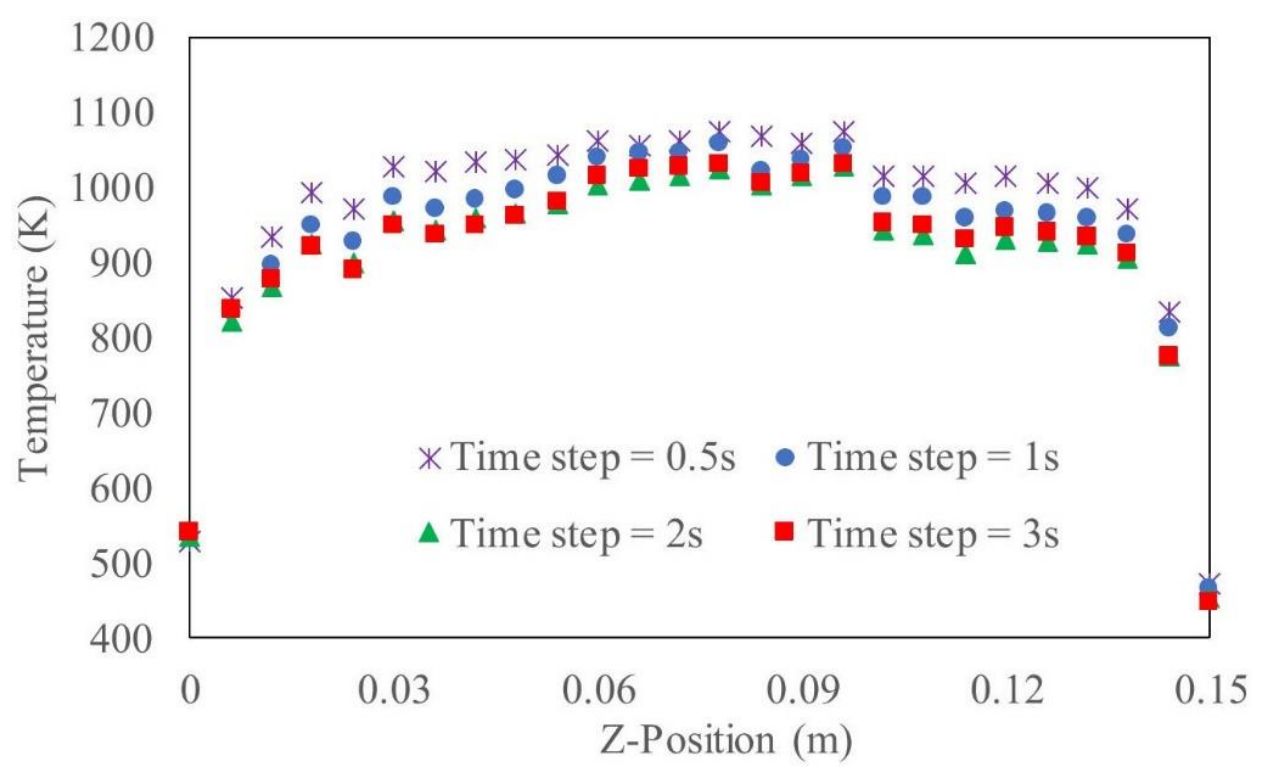

Figure 5 Time-spatial analysis.

The thermodynamic and $\mathrm{CeO}_{2}$ reduction models were validated against the experimental results obtained by Bulfin et al. [31], which used $100 \mathrm{~W}$ concentrated radiation power on a $\mathrm{CeO}_{2}$ pellet (5 $\mathrm{mm}$ diameter and $1 \mathrm{~mm}$ height) with $65 \%$ void fraction. It is visible from Figure 6 that the temperature and non-stoichiometric coefficient of $\mathrm{CeO}_{2}$ derived from the numerical model 
demonstrate comparable values to those derived from the experiment, with slight differences caused by influences of experimental setups and operating limitations [31].

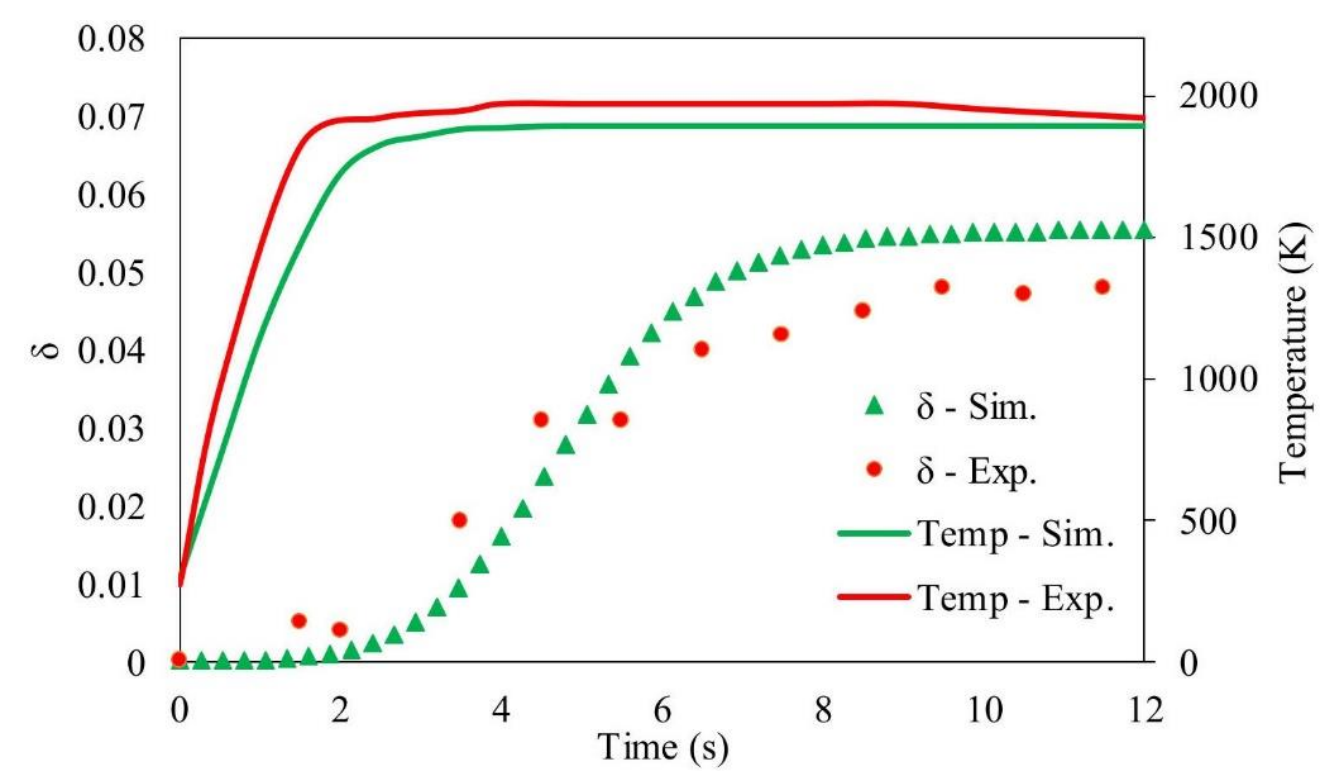

Figure 6 Validation of thermodynamic and chemical reaction models.

\section{Modeling Results and Discussions}

\subsection{Gas Flow Rates}

The purge gas is essential in $\mathrm{CeO}_{2}$ reduction to remove generated $\mathrm{O}_{2}$ from the particle surface to avoid the recombination with reduced $\mathrm{CeO}_{2}$. In this work, the influences of the inlet gas flow rate

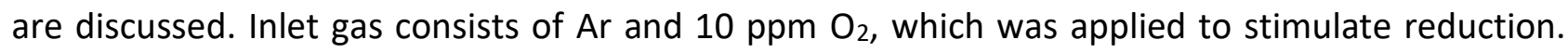
Three different flow rates were studied to compare the impacts on the temperatures and the $\mathrm{O}_{2}$ evolution rates during the reduction process. The transient temperature profiles of the gas and the particle phase are shown in Figure 7. The results reveal that the temperature differences are too small to distinguish under the given 15 mins reaction time. To clarify the results, temperature distributions between 10 mins and 15 mins are enlarged. From the enlarged temperature profiles, the $1.8 \mathrm{~L} / \mathrm{min}$ inlet gas flow rate shows relatively higher average temperatures in both phases. As slow gas flow rate takes less cold fluid to the reactive region, the convection losses are smaller than that of large gas flow rate. However, the temperature differences between $1.8 \mathrm{~L} / \mathrm{min}$ and $3.2 \mathrm{~L} / \mathrm{min}$ are $5 \mathrm{~K}$ and $0.5 \mathrm{~K}$ for gas phase and particle phase, respectively. This result reveals that various inlet gas flow rates have a negligible influence on temperature distribution under the current range. Since the input radiant power is large enough to control the temperature distribution, the influence on convection caused by gas flow changes small and may be ignored. The $\mathrm{O}_{2}$ evolution rate reveals a similar result (Figure 8). The peak reaction rates between $1.8 \mathrm{~L} / \mathrm{min}$ and $3.2 \mathrm{~L} / \mathrm{min}$ is less than 0.01 $\mathrm{mL}$ min-1 g-1 $\mathrm{CeO}_{2}$. Even though the gas flow rate may impact the $\mathrm{O}_{2}$ evolution rate, the result is not obvious under current operating conditions. 

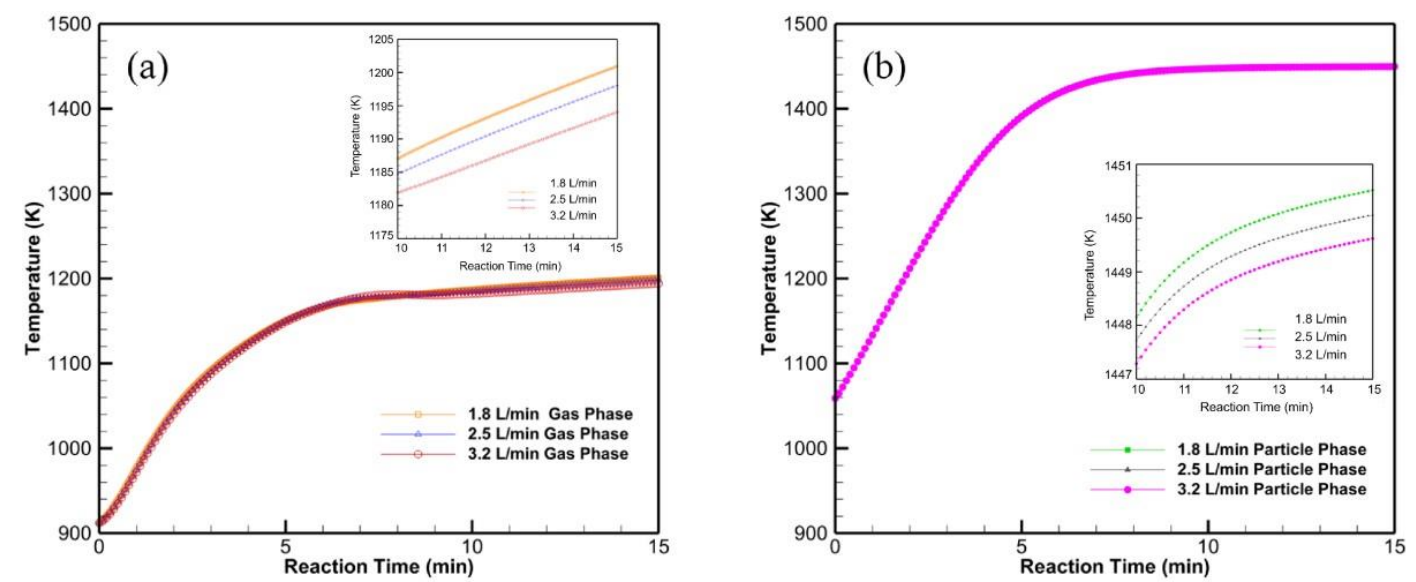

Figure 7 Temperature profiles of various inlet gas flow rate: (a) gas phase; (b) particle phase.
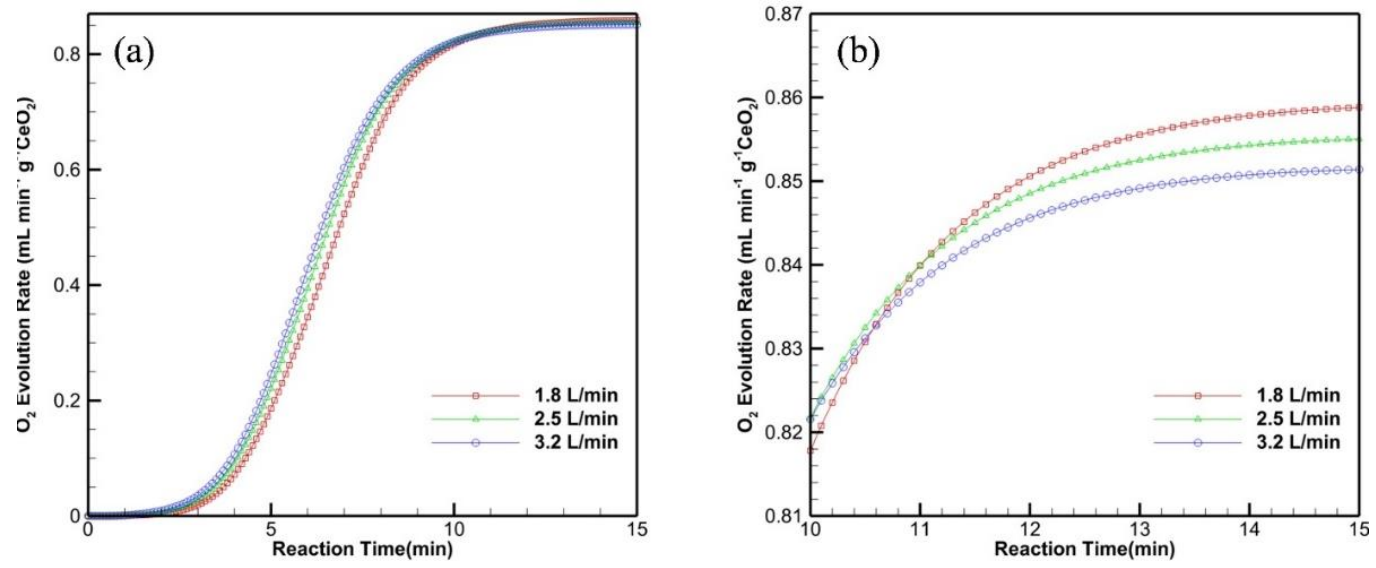

Figure 8 Oxygen evolution rates of various inlet gas flow rate.

\subsection{Solar Radiant Powers}

Solar radiant power is a significant factor in $\mathrm{CeO}_{2}$ reduction rate since it supplies the required thermal energy to support the endothermic reduction reaction. Four various radiant power levels between $3.0 \mathrm{~kW}$ and $4.5 \mathrm{~kW}$ were selected to investigate their influence on the reactive region, including incident radiation, temperature distribution, and reduction rate. Figure 9 (a) shows the scattering distribution of the radiant flux of the catalyst region's inner surface after 15 mins. The $Z$ positive direction is towards the solar receiver window. Higher input power results in higher radiant flux (as high as $4 \times 10^{6} \mathrm{~W} \mathrm{~m}^{-2}$ ), which also shows that the radiant flux is lower at the ceria top, even though the power is uniformly distributed on the surface. This phenomenon is caused by the diffuse radiation property. In this case, it is assumed that diffuse radiation works in the domains. Since the view factors are evenly distributed in all directions, less radiation accumulates at the top of the ceria domain, which results in a low radiant flux. Additionally, the radiant flux intervals for the different input power levels are nearly equal (consistent with Eq. (15)). The variations of radiant flux on radial direction can be reflected via temperature distribution, as shown in Figure 9 (b). Here, three different levels (top, middle, and bottom) were chosen to investigate the temperature profiles considering both radial and axial influences. At the top level, the temperature is the lowest with a relatively uniform distribution in the radial direction. This is due to the fluid not directly contacting 
the radiation absorption solid with a low-radiant flux. At the middle and bottom levels, the temperature profile distributions gradually decrease. Since incident radiation decreases due to absorption by solid particles which then radiate radiation, the radiation term in Eq. (18) decreases in the radial direction, which is consistent with a decrease temperature. Due to the high radiant flux to the middle level, the temperature is also higher.

The transient results under different input power are shown in Figure 9 (c) and (d). Figure 9 (c) shows the average temperature variations of the gas and particle phase in their respective domain during the 15 min reaction time. Since particles have higher specific heat and have better radiant properties compared with gases ( $\mathrm{Ar}$ and $\mathrm{O}_{2}$ are weak radiation absorbing species [56]), the particle phase temperatures are higher than the gas phases (difference between gas phase and particle phase is around $300 \mathrm{~K}$ ). Figure 9 (d) presents that reactive region under a $4.5 \mathrm{~kW}$ power input which produces the highest $\mathrm{O}_{2}$ formation rate due to its high temperature. Additionally, reaction rates sharply increase after 5 mins, due to temperature exceeds the minimum required reduction temperature (higher than $1000^{\circ} \mathrm{C}$, shown in Figure 9 (c)). After 10 mins, the average gas and particle phase temperatures of the reactive region stabilize, which provides a constant reaction rate. Under $3.5 \mathrm{~kW}$ power input, the peak $\mathrm{O}_{2}$ evolution rate reaches $0.8 \mathrm{~mL} \mathrm{~min}-1 \mathrm{~g}-1 \mathrm{CeO}_{2}$, which is comparable to that reported in the literature [69]. With $4.5 \mathrm{~kW}$ power input, the peak $\mathrm{O}_{2}$ evolution rate reaches $1.18 \mathrm{~mL}$ min-1 g- $1 \mathrm{CeO}_{2}$. The results show that higher power input provides a higher temperature in the reactive domain and correspondingly leads to higher $\mathrm{O}_{2}$ production. However, this observation does not mean the power input can continue to be increased without bound. An optimal power input should also consider solar-to-fuel efficiency. Eq. (7) shows the theoretical solar-to-fuel efficiency ignoring the energy of purge gas [22].
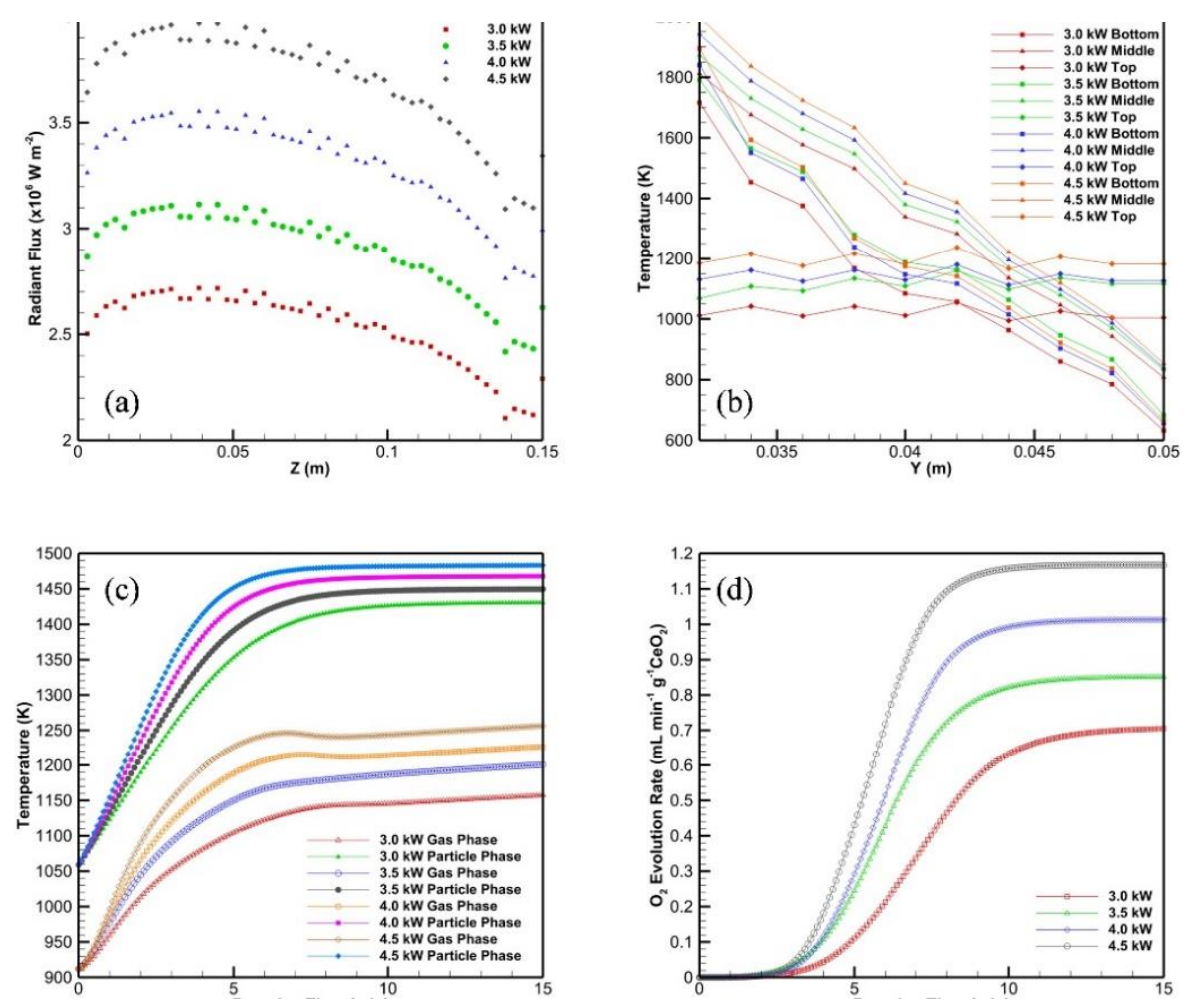

Figure 9 Profiles of various radiant powers: (a) radiant flux distributions along the axial direction; (b) temperature distributions along the radial direction; (c) transient temperature of fluid and particles; (d) oxygen evolution rates. 


\section{3 $\mathrm{CeO}_{2}$ Void Fractions}

Besides the influences caused by the operating condition, $\mathrm{CeO}_{2}$ morphology is also extremely important in the reduction process. In this work, DEM particles are used to approximate porous media via random injection, which provided similar gas paths among particles. Heat and mass transfer are considered macroscopically, which only occurs on the surface of particles by neglecting particles pores.

In this work, four different void fractions $(0.7,0.65,0.6$, and 0.5$)$ were considered to study temperature distributions and corresponding $\mathrm{O}_{2}$ evolution rates. Using the same particle size to fill the reactor's reactive region, a large void fraction implies that the packing is less dense, which provides less pressure drop in the packed section. Also, a larger void fraction allows incident radiation to penetrate deeper into the reactor radial direction, which results in higher reaction temperature. The resulting temperature distributions for the gas and particle phases are shown in Figure 10 which confirms that tighter (lower void fraction) packing results in low-temperature distributions for the gas and particle phases consistent with expectation, while higher void fraction (less tightly packed section) results in a higher temperature. Figure 11 shows the time-dependent temperature profiles and $\mathrm{O}_{2}$ evolution rates. As discussed above, the average temperatures of gas and particle phases are different due to the physical and material properties. In Figure 11 (a), particle temperature increases with an increased void fraction. However, the gas phase temperatures remain essentially constant for void fractions between $\varepsilon=0.7$ and $\varepsilon=0.65$ during the reduction process. From Eq. (18) and (21), the primary heat transfer mechanisms is convection from both the fluid-wall exchange and fluid-particle convection. When $\varepsilon=0.65$, the fluid-wall convection is greater but has a smaller energy source term for the particle phase. Under the current geometry, the fluid-wall convection and fluid-particle source term are balanced, which results in the close temperature distribution with $\varepsilon=0.70$. A similar temperature distribution leads to a similar $\mathrm{O}_{2}$ evolution rate in a section with increasing temperature. In Figure 11 (b), the $\mathrm{O}_{2}$ evolution rate is almost the same in the initial 7 mins for the void fractions between $\varepsilon=0.7$ and $\varepsilon=0.65$ due to a uniform temperature profile. After that, the temperatures are more or less stable, which reflects a stable oxygen generation rate with a slight difference. Since $\mathrm{O}_{2}$ evolution rate is based on per gram $\mathrm{CeO}_{2}, \varepsilon=0.70$ has a higher rate caused by the lower mass amount of $\mathrm{CeO}_{2}$ under a similar temperature condition. In order words, the catalyst with a void fraction of 0.7 can provide better heat transfer and achieve higher temperature distribution, which leads to a higher $\mathrm{O}_{2}$ evolution rate. 
(a)
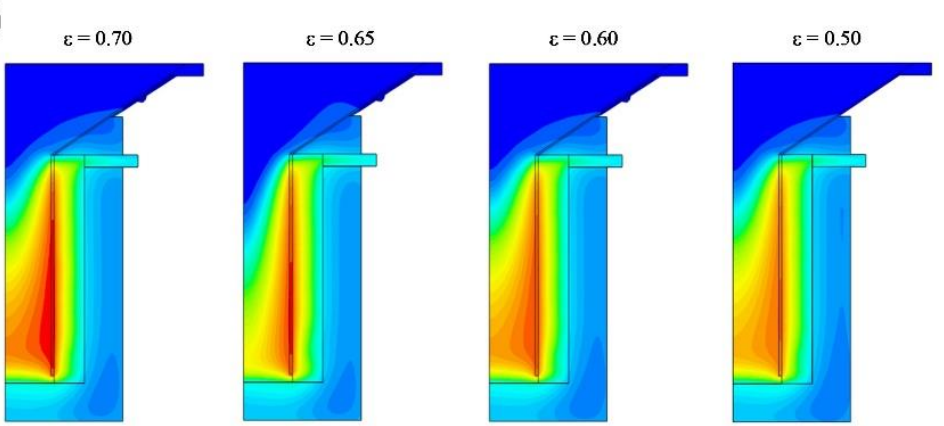

(b)

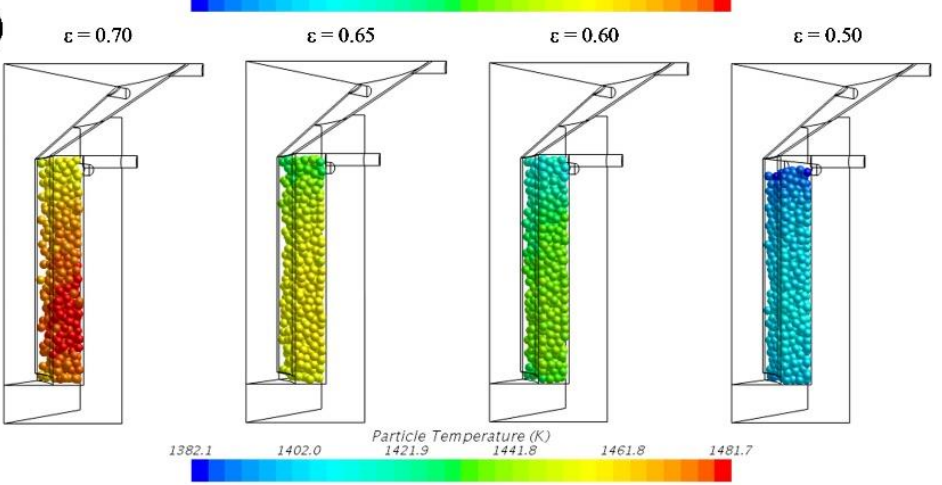

Figure 10 Simulated (a) gas phase and (b) particle phase temperature profiles under various $\mathrm{CeO}_{2}$ void fractions.
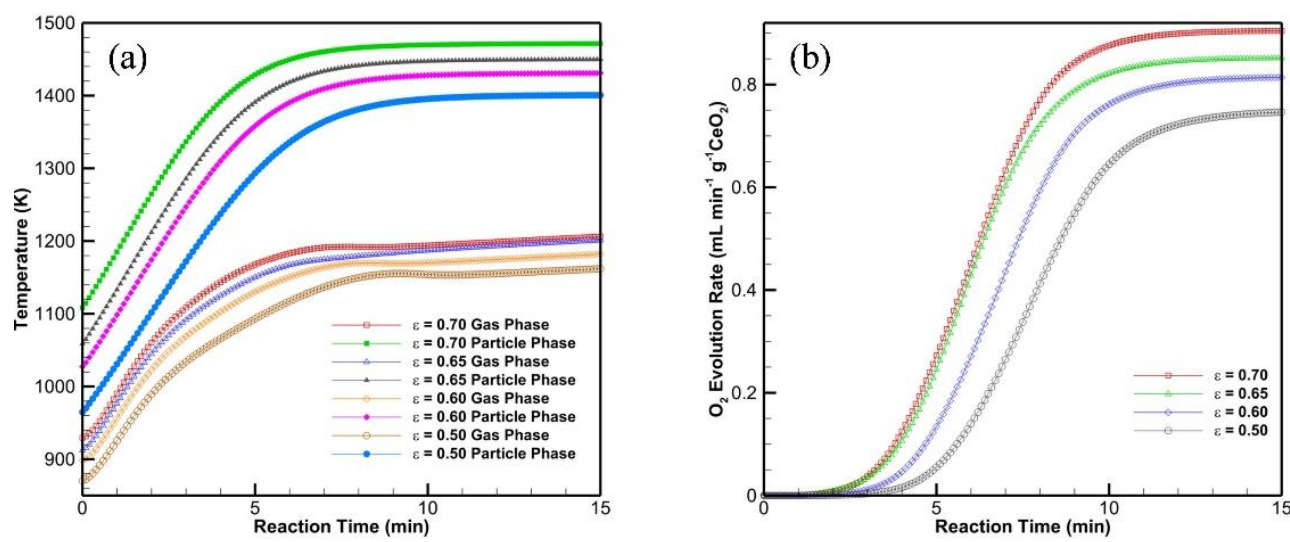

Figure 11 Time-dependent variable profiles under different void fractions: (a) average temperature; (b) $\mathrm{O}_{2}$ evolution rates.

\section{4 $\mathrm{CeO}_{2}$ Particle Sizes}

$\mathrm{CeO}_{2}$ particle size is another important factor that affects the structure of the reactive section. Under the same void fraction, smaller particles provide a more total specific surface area in the reactor. As $\mathrm{O}_{2}$ evolution reaction occurs on the surface of $\mathrm{CeO}_{2}$ particles, the larger specific surface area is beneficial to the reaction rate. However, smaller particles mean tighter packing, which is adverse to radiative heat transfer. Therefore, the influence of particle sizes may be dual directions. Figure 12 shows the temperature profiles of the gas phase and particle phase with $\varepsilon=0.65$ under various particle diameters. The result reveals that larger particles have advantages of deriving higher 
temperature since more incident radiation can reach particle surfaces due to the larger interspace between particles, which is shown in Figure 12 (b).

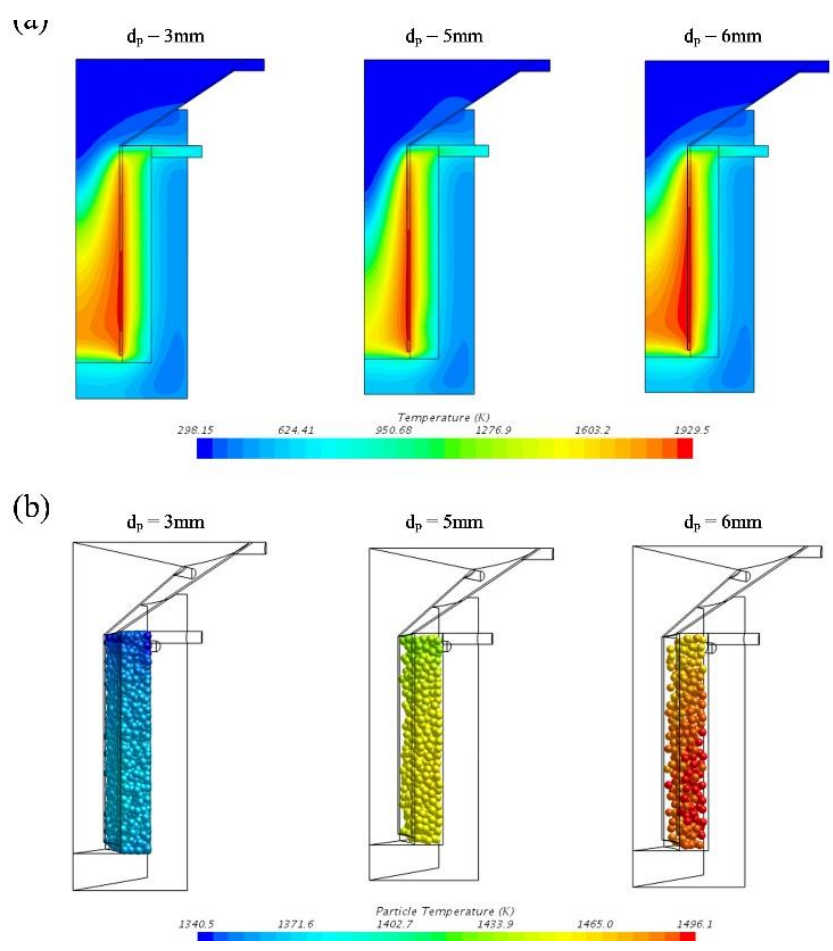

Figure 12 Simulated (a) gas phase and (b) particle phase temperature profiles under different particle sizes.

The transient variations of temperatures and $\mathrm{O}_{2}$ evolution rates are presented in Figure 13. From Figure $13(\mathrm{a})$, the particle temperature differences between $\mathrm{dp}=3 \mathrm{~mm}$ and $\mathrm{dp}=6 \mathrm{~mm}$ are distinct. The particle diameter $d p=3 \mathrm{~mm}$ gets a $150 \mathrm{~K}$ lower average particle temperature than that of $d p=6$ $\mathrm{mm}$. Compared with the temperature profile with other cases, $d p=3 \mathrm{~mm}$ derives the lowest temperature than other cases, which reveals that particle size has a stronger influence on temperature than other factors. Even though the temperature differences between different particle sizes are obvious, the gas phase temperatures seem discrepant. The gas phase temperature seems very close under different conditions, especially for $d p=5 \mathrm{~mm}$ and $d p=6 \mathrm{~mm}$. The result illustrates that the influence of particle-gas convection is weaker than that of gas-wall convection. In Figure $13(b), d p=3 \mathrm{~mm}$ shows the highest $\mathrm{O}_{2}$ evolution rate under these three cases, which is opposite to temperature results. As discussed above, specific surface area and temperature are both significant to the reaction rate. Under the $\mathrm{CeO}_{2}$ reduction process, the specific surface area is dominant. 

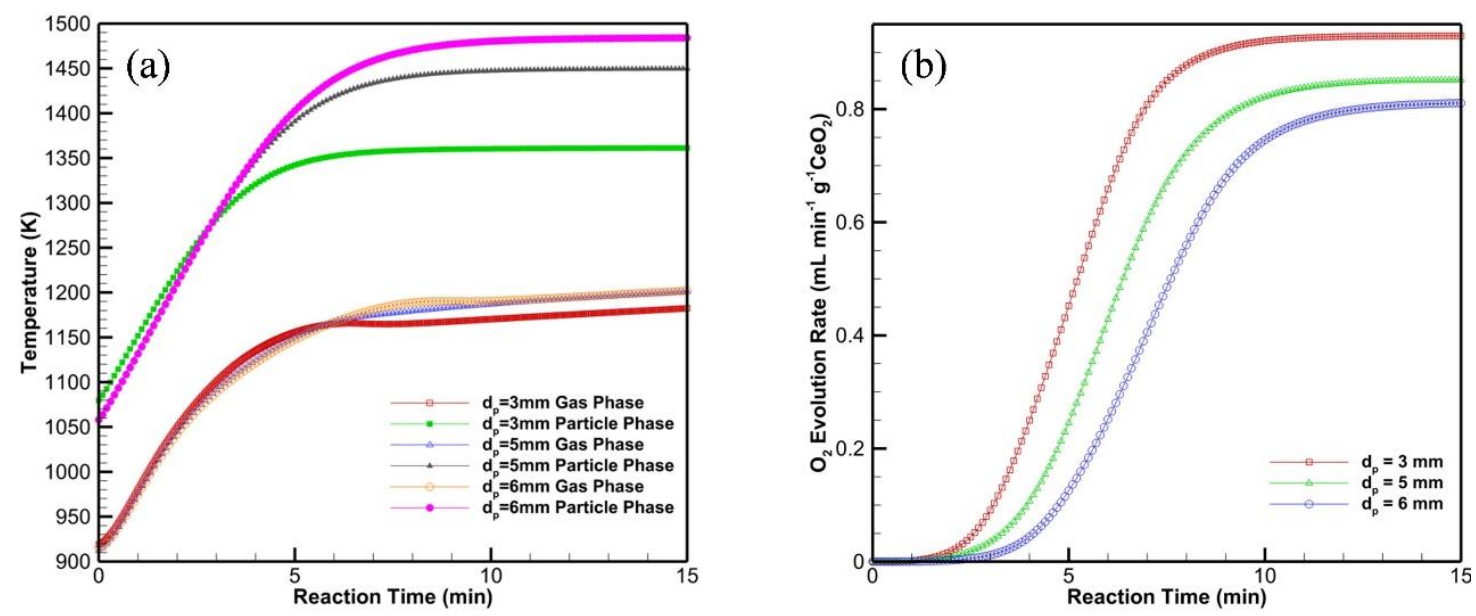

Figure 13 Time-dependent variable profiles under different particle sizes: (a) average temperature; (b) $\mathrm{O}_{2}$ evolution rates.

\section{Conclusions}

The DEM method was successfully employed to simulate the porous $\mathrm{CeO}_{2}$ structure in the transient CFD modeling of $\mathrm{CeO}_{2}$ reduction in a solar thermochemical reactor. Model validation was accomplished according to previously reported experimental data. The effects of the catalyst textures (particle size and void fraction) and process conditions (gas flow rate and radiative power input) on the temperature profiles and reaction rate were investigated. The gas flow rate showed slight influences on both the temperature distribution and reaction rates. The temperature is higher at a lower flow rate leading to a higher reaction rate. The higher solar radiant power input results in higher temperature and $\mathrm{O}_{2}$ evolution rate. A larger void fraction of the catalyst is advantageous to improve the thermal performances and $\mathrm{CeO}_{2}$ reduction rate. The reaction rate is remarkably enhanced by shrinking the particle size to derive more specific surface area of the catalyst. Further studies will focus on the study of $\mathrm{CO}_{2}$ splitting via reduced $\mathrm{CeO}_{2}$ and experimental validation of the thermal and reactive performances.

\section{Nomenclature}

\section{Symbols}

A

D

$f_{b}$

$F_{S}$

$F_{b}$

$\mathrm{H}$

I

I

$\mathrm{k}$

$k_{\text {red }}$

$\mathrm{m}_{\mathrm{f}}$

$m_{\mathrm{i}}$

surface area

diameter

body force (gravity)

particle surface force $(\mathrm{N})$

particle body force $(\mathrm{N})$

enthalpy $\left(\mathrm{J} \mathrm{kg}^{-1}\right)$

radiative intensity $\left(\mathrm{W} \mathrm{m}^{-2}\right)$

identity matrix

thermal conductivity $\left(\mathrm{W} \mathrm{m}^{-1} \mathrm{~K}^{-1}\right)$

reduction rate coefficient

final particle mass

initial particle mass 


\begin{tabular}{|c|c|}
\hline$\dot{m}_{p}$ & rate of mass transfer to particle \\
\hline $\mathrm{q}_{\mathrm{r}}$ & re-radiation $\left(\mathrm{W} \mathrm{m}^{-2}\right)$ \\
\hline $\mathrm{Q}$ & heat transfer (W) \\
\hline$Q_{a, p}$ & absorption efficiency of particle \\
\hline$Q_{t}$ & surface heat transfer (W) \\
\hline 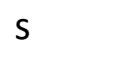 & distance in $\Omega$ direction \\
\hline$S_{E}$ & energy source ( $\mathrm{W} \mathrm{m}^{-2}$ or $\mathrm{W} \mathrm{m}^{-3}$ ) \\
\hline$S_{m}$ & mass source $\left(\mathrm{mol} \mathrm{m}^{-3} \mathrm{~s}^{-1}\right)$ \\
\hline $\mathrm{t}$ & time $(s)$ \\
\hline $\mathrm{T}$ & temperature $(\mathrm{K})$ \\
\hline$v$ & velocity $\left(\mathrm{m} \mathrm{s}^{-1}\right)$ \\
\hline$x$ & reaction fraction \\
\hline$Y_{i}$ & component concentration \\
\hline \multicolumn{2}{|c|}{ Greek Symbols } \\
\hline$\alpha$ & void fraction of fluid phase \\
\hline$\beta$ & extinction coefficient \\
\hline$\delta$ & non-stoichiometric coefficient \\
\hline$\kappa_{a}$ & absorption coefficient $\left(\mathrm{m}^{-1}\right)$ \\
\hline $\mathrm{K}_{\mathrm{pa}}$ & particle absorption coefficient $\left(\mathrm{m}^{-1}\right)$ \\
\hline$K_{p s}$ & particle scattering coefficient $\left(\mathrm{m}^{-1}\right)$ \\
\hline $\mathrm{K}_{\mathrm{s}}$ & scattering coefficient $\left(\mathrm{m}^{-1}\right)$ \\
\hline$\rho$ & density $\left(\mathrm{kg} \mathrm{m}^{-3}\right)$ \\
\hline$\sigma$ & Stefan-Boltzmann constant \\
\hline$\Omega$ & solid angle \\
\hline \multicolumn{2}{|c|}{ Subscripts } \\
\hline $\mathrm{b}$ & black body \\
\hline conv & convection \\
\hline$f$ & fluid \\
\hline fs & fluid-solid interphase \\
\hline $\mathrm{p}$ & particle \\
\hline part & partition \\
\hline $\mathrm{pb}$ & particle black body \\
\hline $\mathrm{rad}$ & radiation \\
\hline reac & reaction (reduction) \\
\hline s & insulation \\
\hline \multicolumn{2}{|c|}{ Abbreviation } \\
\hline DEM & Discrete Element Method \\
\hline DOM & Discrete Ordinates Model \\
\hline i.d. & inner diameter \\
\hline $\mathrm{Nu}$ & Nusselt number \\
\hline o.d. & outer diameter \\
\hline ppm & part per million \\
\hline $\operatorname{Pr}$ & Prandtl number \\
\hline $\operatorname{Re}$ & Reynold's number \\
\hline
\end{tabular}




\section{Acknowledgments}

We gratefully acknowledge the financial support from the Wayne and Gayle Laufer Foundation.

\section{Author Contributions}

Smith, Joseph: Conceptualization, Methodology, Supervision, Reviewing and Editing; Zhang, Han: Software, Data curation, Writing-Original draft preparation, Visualization, Investigation, Software, Validation, Writing.

\section{Competing Interests}

The authors have declared that no competing interests exist.

\section{References}

1. Energy related $\mathrm{CO}_{2}$ emissions [Internet]. Paris: IEA; 2020 [cited date 2020 February 11]. Available from: https://www.iea.org/data-and-statistics/charts/energy-related-CO 2 -emissions1990-2019.

2. Breyer C, Bogdanov D, Aghahosseini A, Gulagi A, Child M, Oyewo AS, et al. Solar photovoltaics demand for the global energy transition in the power sector. Prog Photovolt Res Appl. 2018; 26: 505-523

3. Zhu T, Swaminathan-Gopalan K, Stephani K, Ertekin E. Thermoelectric phonon-glass electroncrystal via ion beam patterning of silicon. Phys Rev B. 2018; 97: 174201.

4. Dincer I, Acar C. A review on clean energy solutions for better sustainability. Int J Energy Res. 2015; 39: 585-606.

5. Xu J, Wang F, Lv C, Xie H. Carbon emission reduction and reliable power supply equilibrium based daily scheduling towards hydro-thermal-wind generation system: A perspective from China. Energy Convers Manag. 2018; 164: 1-14.

6. Vaughan NE, Gough C, Mander S, Littleton EW, Welfle A, Gernaat DE, et al. Evaluating the use of biomass energy with carbon capture and storage in low emission scenarios. Environ Res Lett. 2018; 13: 044014.

7. Nguyen VN, Blum L. Syngas and synfuels from $\mathrm{H}_{2} \mathrm{O}$ and $\mathrm{CO}_{2}$ : Current status. Chemie Ing Tech. 2015; 87: 354-375.

8. Dayton DC, Turk B, Gupta R. Syngas cleanup, conditioning, and utilization. Thermochem Process Biomass Convers into Fuels Chem Power. 2019; 125-174. doi: 10.1002/9781119417637.ch5.

9. Bhosale RR. Thermodynamic efficiency analysis of zinc oxide based solar driven thermochemical $\mathrm{H}_{2} \mathrm{O}$ splitting cycle: Effect of partial pressure of $\mathrm{O}_{2}$, thermal reduction and $\mathrm{H}_{2} \mathrm{O}$ splitting temperatures. Int J Hydrog Energy. 2018; 43: 14915-14924.

10. Bachirou GL, Shuai $Y$, Zhang J, Huang $X$, Yuan $Y$, Tan $H$. Syngas production by simultaneous splitting of $\mathrm{H}_{2} \mathrm{O}$ and $\mathrm{CO}_{2}$ via iron oxide (Fe3O4) redox reactions under high-pressure. Int J Hydrog Energy. 2016; 41: 19936-19946.

11. Wu S, Zhou C, Doroodchi E, Nellore R, Moghtaderi B. A review on high-temperature thermochemical energy storage based on metal oxides redox cycle. Energy Convers Manag. 2018; 168: 421-453. 
12. Scheffe JR, Steinfeld A. Oxygen exchange materials for solar thermochemical splitting of $\mathrm{H}_{2} \mathrm{O}$ and $\mathrm{CO}_{2}$ : A review. Mater Today. 2014; 17: 341-348.

13. Chueh WC, Falter C, Abbott M, Scipio D, Furler P, Haile SM, et al. High-flux solar-driven thermochemical dissociation of $\mathrm{CO}_{2}$ and $\mathrm{H}_{2} \mathrm{O}$ using nonstoichiometric ceria. Science. 2010; 330: 1797-1801.

14. Romero $M$, Steinfeld $A$. Concentrating solar thermal power and thermochemical fuels. Energy Environ Sci. 2012; 5: 9234-9245.

15. Muhich CL, Ehrhart BD, Al-Shankiti I, Ward BJ, Musgrave CB, Weimer AW. A review and perspective of efficient hydrogen generation via solar thermal water splitting. Wiley Interdiscip Rev Energy Environ. 2016; 5: 261-287.

16. Charvin P, Abanades S, Flamant G, Lemort F. Two-step water splitting thermochemical cycle based on iron oxide redox pair for solar hydrogen production. Energy. 2007; 32: 1124-1133.

17. Dizaji HB, Hosseini H. A review of material screening in pure and mixed-metal oxide thermochemical energy storage (TCES) systems for concentrated solar power (CSP) applications. Renew Sust Energy Rev. 2018; 98: 9-26.

18. Bulfin B, Vieten J, Agrafiotis C, Roeb M, Sattler C. Applications and limitations of two step metal oxide thermochemical redox cycles: A review. J Mater Chem A. 2017; 5: 18951-18966.

19. Otsuka K, Hatano M, Morikawa A. Hydrogen from water by reduced cerium oxide. J Catal. 1983; 79: 493-496.

20. Abanades S, Flamant $G$. Thermochemical hydrogen production from a two-step solar-driven water-splitting cycle based on cerium oxides. Sol Energy. 2006; 80: 1611-1623.

21. Petrasch J, Klausner J. Integrated solar thermochemical cycles for energy storage and fuel production. Wiley Interdiscip Rev Energy Environ. 2012; 1: 347-361.

22. Bader R, Chandran RB, Venstrom LJ, Sedler SJ, Krenzke PT, De Smith RM. Design of a solar reactor to split $\mathrm{CO}_{2}$ via isothermal redox cycling of ceria. J Sol Energy Eng. 2015; 137.

23. Furler $P$, Scheffe JR, Steinfeld $A$. Syngas production by simultaneous splitting of $\mathrm{H}_{2} \mathrm{O}$ and $\mathrm{CO}_{2}$ via ceria redox reactions in a high-temperature solar reactor. Energy Environ Sci. 2012; 5: 60986103.

24. Furler $P$, Scheffe J, Gorbar M, Moes L, Vogt U, Steinfeld A. Solar thermochemical $\mathrm{CO}_{2}$ splitting utilizing a reticulated porous ceria redox system. Energy Fuels. 2012; 26: 7051-7059.

25. Venstrom LJ, Petkovich N, Rudisill S, Stein A, Davidson JH. The effects of morphology on the oxidation of ceria by water and carbon dioxide. J Sol Energy Eng. 2012; 134: 11005.

26. Zoller S, Koepf E, Roos P, Steinfeld A. Heat transfer model of a $50 \mathrm{~kW}$ solar receiver-reactor for thermochemical redox cycling using cerium dioxide. J Sol Energy Eng. 2019; 141: 21014.

27. Sedighi M, Padilla RV, Taylor RA, Lake M, Izadgoshasb I, Rose A. High-temperature, point-focus, pressurised gas-phase solar receivers: A comprehensive review. Energy Convers Manag. 2019; 185: 678-717.

28. de la Calle A, Bayon A. Annual performance of a thermochemical solar syngas production plant based on non-stoichiometric $\mathrm{CeO}_{2}$. Int J Hydrog Energy. 2019; 44: 1409-1424.

29. Groehn AJ, Lewandowski A, Yang R, Weimer AW. Hybrid radiation modeling for multi-phase solar-thermal reactor systems operated at high-temperature. Sol Energy. 2016; 140: 130-140.

30. Bulfin B, Call F, Lange M, Lubben O, Sattler C, Pitz-Paal R, et al. Thermodynamics of $\mathrm{CeO}_{2}$ thermochemical fuel production. Energy Fuels. 2015; 29: 1001-1009. 
31. Bulfin B, Lowe AJ, Keogh KA, Murphy BE., Lubben O, Krasnikov SA, et al. Analytical model of $\mathrm{CeO}_{2}$ oxidation and reduction. J Phys Chem C. 2013; 117: 24129-24137.

32. Zinkevich M, Djurovic D, Aldinger F. Thermodynamic modelling of the cerium-oxygen system. Solid State Ion. 2006; 177: 989-1001.

33. Ishida T, Gokon N, Hatamachi T, Kodama T. Kinetics of thermal reduction step of thermochemical two-step water splitting using $\mathrm{CeO}_{2}$ particles: Master-plot method for analyzing non-isothermal experiments. Energy Procedia. 2014; 49: 1970-1979.

34. Valades-Pelayo PJ, Villafán-Vidales $\mathrm{HI}$, Romero-Paredes $\mathrm{H}$, Arancibia-Bulnes CA. Modeling of a tubular solar reactor for continuous reduction of $\mathrm{CeO}_{2}$ : The effect of particle size and loading on radiative heat transfer and conversion. Chem Eng Sci. 2017; 162: 77-87.

35. Valle-Hernández J, Romero-Paredes $\mathrm{H}$, Pacheco-Reyes A. Modeling of the steam hydrolysis in a two-step process for hydrogen production by solar concentrated energy. AIP Conf Proc. 2017; 1850: 100016.

36. Valle-Hernández J, Romero-Paredes H, Arancibia-Bulnes CA, Villafan-Vidales HI, Espinosa-

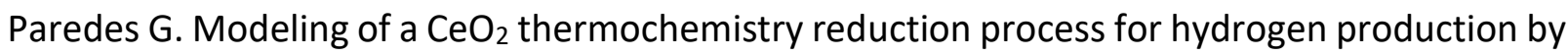
solar concentrated energy. AIP Conf Proc. 2016; 1734: 120008.

37. Arifin $\mathrm{D}$, Weimer AW. Kinetics and mechanism of solar-thermochemical $\mathrm{H}_{2}$ and $\mathrm{CO}$ production by oxidation of reduced $\mathrm{CeO}_{2}$. Sol Energy. 2018; 160: 178-185.

38. McDaniel AH, Ambrosini A, Coker EN, Miller JE, Chueh WC, O’Hayre R, et al. Nonstoichiometric perovskite oxides for solar thermochemical $\mathrm{H}_{2}$ and $\mathrm{CO}$ production. Energy Procedia. 2014; 49: 2009-2018.

39. Scheffe JR, McDaniel AH, Allendorf MD, Weimer AW. Kinetics and mechanism of solarthermochemical $\mathrm{H}_{2}$ production by oxidation of a cobalt ferrite-zirconia composite. Energy Environ Sci. 2013; 6: 963-973.

40. Kröger FA, Vink HJ. Relations between the concentrations of imperfections in crystalline solids. Solid State Phys. 1956; 3: 307-435.

41. Scheffe JR, Steinfeld A. Thermodynamic analysis of cerium-based oxides for solar thermochemical fuel production. Energy Fuels. 2012; 26: 1928-1936.

42. Bader R, Gampp L, Breuille T, Haussener S, Steinfeld A, Lipiński W. Unsteady Radiative heat transfer model of a ceria particle suspension undergoing solar thermochemical reduction. J Thermophys Heat Transf. 2019; 33: 63-77.

43. Haeussler A, Abanades S, Julbe A, Jouannaux J, Cartoixa B. Solar thermochemical fuel production from $\mathrm{H}_{2} \mathrm{O}$ and $\mathrm{CO}_{2}$ splitting via two-step redox cycling of reticulated porous ceria structures integrated in a monolithic cavity-type reactor. Energy. 2020; 117649.

44. Haeussler A, Abanades S, Julbe A, Jouannaux J, Drobek M, Ayral A, et al. Remarkable performance of microstructured ceria foams for thermochemical splitting of $\mathrm{H}_{2} \mathrm{O}$ and $\mathrm{CO}_{2}$ in a novel high-temperature solar reactor. Chem Eng Res Des. 2020; 156: 311-323.

45. Parthasarathy $P$, Le Clercq $P$. Heat transfer simulation in a high temperature solar reactor. Energy Procedia. 2015; 69: 1810-1818.

46. Kyrimis S, Le Clercq P, Brendelberger S. 3D modelling of a solar thermochemical reactor for MW scaling-up studies. AIP Conf Proc. 2019; 2126: 180013.

47. Ma H, Xu L, Zhao Y. CFD-DEM simulation of fluidization of rod-like particles in a fluidized bed. Powder Technol. 2017; 314: 355-366. 
48. Zhang G, Gutierrez M, Li M. A coupled CFD-DEM approach to model particle-fluid mixture transport between two parallel plates to improve understanding of proppant micromechanics in hydraulic fractures. Powder Technol. 2017; 308: 235-248.

49. Bellan S, Matsubara K, Cho HS, Gokon N, Kodama T. A CFD-DEM study of hydrodynamics with heat transfer in a gas-solid fluidized bed reactor for solar thermal applications. Int J Heat Mass Transf. 2018, 116: 377-392.

50. Bellan S, Kodama T, Matsubara K, Gokon N, Cho HS, Inoue K. Thermal performance of a 30kW fluidized bed reactor for solar gasification: A CFD-DEM study. Chem Eng J. 2019; 360: 1287-1300.

51. Morris $A B, M a Z$, Pannala S, Hrenya CM. Simulations of heat transfer to solid particles flowing through an array of heated tubes. Sol Energy. 2016, 130: 101-115.

52. STAR-CCM+ Theory Guide v. 12.06. Siemens AG. 2018.

53. STAR-CCM+ User Manual v. 12.06. 2018.

54. Furler $P$, Steinfeld $A$. Heat transfer and fluid flow analysis of a $4 \mathrm{~kW}$ solar thermochemical reactor for ceria redox cycling. Chem Eng Sci. 2015; 137: 373-383.

55. Khalsa AK, De Andrade D. Thermal reflectivity in solar collector prototype. Senior Design Project; 2008. Available from:

http://solar.sdsu.edu/Senior\%20Projects/Solar Trough/Solar Trough Design Khalsa DeAndr ade Final Report.pdf.

56. Brewster MQ. Thermal radiative transfer and properties. Hoboken: John Wiley Sons; 1992.

57. Hanley HJ, McCarty RD, Sengers JV. Viscosity and thermal conductivity coefficients of gaseous and liquid oxygen. 1974. Available from: https://core.ac.uk/download/pdf/42893032.pdf.

58. Ranz WE, Marshall WR. Evaporation from drops. Chem Eng Prog. 1952; 48: 141-146.

59. Marxer D, Furler P, Takacs M, Steinfeld A. Solar thermochemical splitting of CO2 into separate streams of $\mathrm{CO}$ and $\mathrm{O} 2$ with high selectivity, stability, conversion, and efficiency. Energy Environ Sci. 2017; 10: 1142-1149.

60. Cundall PA, Strack OD. A discrete numerical model for granular assemblies. Geotechnique. 1979; 29: 47-65.

61. Touloukian YS. Thermophysical properties of high temperature solid materials. Volume 4. Oxides and their solutions and mixtures. Part 1. Simple oxygen compounds and their mixtures, thermophysical and electronic properties information analysis center lafayette in. 1966.

62. Yaws CL. Thermophysical properties of chemicals and hydrocarbons. Norwich, NY: William Andrew; 2008.

63. STAR-CCM+ Material Databases. Siemens AG. 2017.

64. Richet $P$, Bottinga $Y$, Denielou L, Petitet JP, Tequi C. Thermodynamic properties of quartz, cristobalite and amorphous $\mathrm{SiO}_{2}$ : Drop calorimetry measurements between 1000 and $1800 \mathrm{~K}$ and a review from 0 to $2000 \mathrm{~K}$. Geochim Cosmochim Acta. 1982; 46: 2639-2658.

65. Andre S, Degiovanni A. A theoretical study of the transient coupled conduction and radiation heat transfer in glass: Phonic diffusivity measurements by the flash technique. Int J Heat Mass Transf. 1995; 38: 3401-3412.

66. Nicolau VP, Borges MT, Silva LM. Radiative properties of glass and coatings. 3rd Eur Conf Energy Perform Indoor Clim Build. 2002; 835-840.

67. McBride BJ. Coefficients for calculating thermodynamic and transport properties of individual species. Washington: NASA Langley Research Center; 1993.

68. Zimmermann D. Flow modeling of a solar thermogravimeter. Master Thesis ETH Zurich. 2012. 
69. Venstrom L, De Smith RM, Chandran RB, Boman DB, Krenzke PT, Davidson JH. Applicability of an equilibrium model to predict the conversion of $\mathrm{CO}_{2}$ to $\mathrm{CO}$ via the reduction and oxidation of a fixed bed of cerium dioxide. Energy Fuels. 2015; 29: 8168-8177.

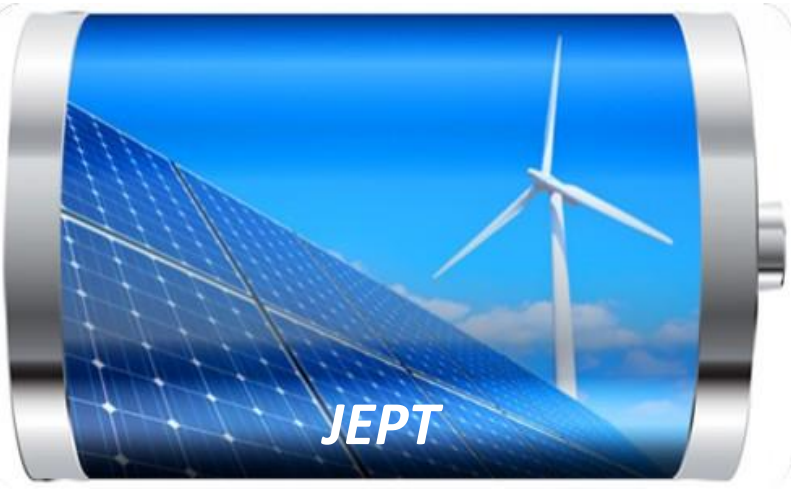

Enjoy JEPT by:

1. Submitting a manuscript

2. Joining in volunteer reviewer bank

3. Joining Editorial Board

4. Guest editing a special issue

For more details, please visit: http://www.lidsen.com/journal/jept 


\title{
Melting/Solidification Processes of PEG 1500 in Vertical and Horizontal Annular Enclosure
}

\author{
F. A. Hamad * , E. Egelle, Abbas Mohammed, S. Gooneratne, P. Russell
}

School of Science, Engineering and Design, Teesside University Middlesbrough, United Kingdom; EMails: $\quad$ F.hamad@tees.ac.uk; enenike e@yahoo.co.uk; P4195752@tees.ac.uk;

S.Gooneratne@tees.ac.uk; p.russell@tees.ac.uk

* Correspondence: F. A. Hamad; E-Mail: F.hamad@tees.ac.uk

Academic Editor: Joaquin Alonso-Montesinos

Special Issue: Photovoltaic Solar Systems and Solar Thermal Plants

Journal of Energy and Power Technology

2021, volume 3 , issue 1

doi:10.21926/jept.2101009
Received: December 01, 2020

Accepted: January 21, 2021

Published: January 31, 2021

\begin{abstract}
In this paper, the primary aim is to look at the fundamental melting/solidification processes of polyethylene glycol 1500 (PEG 1500) for energy storage - insulation to prolong the cooling time of pipelines in unexpected shut-down conditions, prevent/minimize the wax deposition, and hydrate formation. Polyethylene glycol 1500 was selected because its melting temperature is $>317 \mathrm{~K}$ making it a suitable candidate as lagging material to prevent wax deposition and hydrate formation in subsea oil pipelines. Experimental apparatus was designed with the Perspex to give an insight into the melting process. Vertical and horizontal annular geometries were used to consider the real-life cases. The vertical annular enclosure length is $950 \mathrm{~mm}$ and $34 \mathrm{~mm}$ width (Height/Width=27.94). The horizontal annular enclosure length is $300 \mathrm{~mm}$ and $15.9 \mathrm{~mm}$ width (Height/Width=18.87). The thermocouples and camera are used to collect the data for three cases of inner wall temperature of $333 \mathrm{~K}, 343 \mathrm{~K}$ and 353 $\mathrm{K}$ where is the heat added to the phase change material (PCM) for both cases. The main conclusions are: i) the horizontal annular case melt faster than the vertical case, in particular, at higher heating surface temperature of $353 \mathrm{~K}$, ii)The temperature of the inner region was
\end{abstract}

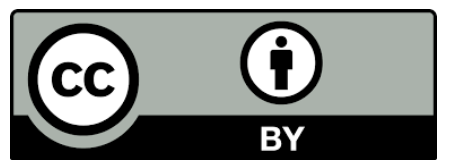

(C) 2021 by the author. This is an open access article distributed under the conditions of the Creative Commons by Attribution License, which permits unrestricted use, distribution, and reproduction in any medium or format, provided the original work is correctly cited. 
remained hot for long time which provide a good evidence that support the concept of using the PCM as heat storage-insulation material; iii) the melting percentage for horizontal case is $100 \%$ higher from the melting percentage of vertical case at $333 \mathrm{~K}$ which reduced to about $20 \%$ for $343 \mathrm{~K}$, iv) increasing the heating surface temperature substantially reduces the total melting time for both orientations.

\section{Keywords}

Annular geometry; melting/solidification; latent heat energy; storage - insulation application; polyethylene glycol 1500 (PEG 1500)

\section{Introduction}

The applications of the annular geometry are related to the shell and tube heat exchangers and thermal storage-reuse systems where the PCM exists in the annular space. Melting and solidification of the PCM occurs when there is heat exchange between the PCM and the hot fluid/ambient. This annular geometry could be horizontal, inclined or vertical based on the type of the application.

The new application of the present work is to prevent the wax deposits on the pipe wall and the hydrate formation at temperature less than $317 \mathrm{~K}$ The gas hydrates are an ice-like formation that can be formed under high pressure and low temperature conditions, when water become in contact with methane, ethane, hydrogen sulphide, carbon dioxide, etc. The gas molecules will be caged in the water molecules. The gas hydrates form solid ice plugs which will cause restricted flows and a full blockage. The wax deposition is another problem cause blockage of oil pipeline. As wax deposition and hydrate formation are mainly a result of cold temperatures, a prevention method in the form of thermal storage would make a perfect fitting towards attempting to solve the problem The PCM (Polyethylene glycol 1500 for synthesis. CAS 25322-68-3, 807489 Sigma-Aldrich, MERCK) selected for this study was polyethylene glycol 1500 (melting temperature $=337-341 \mathrm{~K}$ ) due to the ease of sourcing and low-cost benefits.

The main objectives of most of the publications in literature were to develop the knowledge in this area to improve the design and performance of the thermal storage systems by improving the rate of heat transfer. A number of ideas were on enhancing the heat transfer to accelerate the charging and discharging of energy such as fins [1-3] or changing the dimension of the system such as outer radius/inner radius ratio and length/diameter ratio [4, 5]. A summary of some recent publications on annular enclosure are given hereafter.

Ismail and Mello [5] developed a 2-dimensional model to study the effect of the outer shell to inner tube radius ratio (Ro/Ri) on the thermal performance of a PCM in a vertical cylinder. The results indicated that the solidification mass fraction decreased and the time necessary for the complete fusion increased by increasing Ro/Ri.

Longeon et al. [6] investigated experimentally and numerically, the heat exchange involved in a vertical annular latent storage unit filled with paraffin RT35. A testing loop with visualisation is built to analyse the influence of the heat transfer fluid (HTF) injection side on the system. The experimental test section is modelled with CFD simulations to explain the charge and discharge 
results. The combined study proves that an injection side coupled with free convection heat transfer mechanism influences the evolution of the PCM melting front.

Seddegh et al. [4] studied the effect of the geometrical and operational parameters on vertical cylindrical shell-and-tube latent heat thermal energy storage (LHTES) systems. Four different ratios of the shell-to-tube radius are considered with the PCM on the shell side and the heat transfer fluid (HTF) flowing through the tube. The results show that a shell to tube radius ratio of 5.4 gave better system performance in terms of the charging time and stored energy. The HTF temperature shows a reduction $68 \%$ in the charging time when the HTF temperature increases from 343 to $353 \mathrm{~K}$.

Kalapala and Devanuri [7] presented a numerical investigation on melting characteristics of a PCM placed in the annulus of a vertical shell and tube thermal storage unit of L/D ratio of 1-10. They found that increasing length/diameter (L/D) ratio increased the total melting time. By increasing L/D ratio from 1 to 4, total melting time increased by 1.75 times. They also found that mentioned that the shell to tube diameter ratio and L/D ratio are the design parameters, which need to be optimized.

Balikowski and Mollendort [8] investigated the melting process of two PCMs (Climsel 28 and Thermasorb 83) in a horizontal annulus unit. Bare- and spine fin-pipe latent heat thermal energy storage units were tested. Their results shown that the rate of charging (melting) and discharging (solidification) was improved with using fins.

Agyenim et al. [9] experimentally studied the erythritol melting/solidification processes in horizontal annulus heat thermal energy storage. They used three storage configurations including bare tube, circular finned tube and longitudinal finned tube. They observed that the longitudinal fins contributed in the best thermal performance of the LHTES unit amongst the other studied cases.

Azad et al. [10] investigated the melting of PCM in a cylindrical enclosure experimentally and numerically. The n-octadecane has been melted at HTF temperatures of 313, 323 and $333 \mathrm{~K}$. For 6 hours of heating, a linear increase in stored energy is observed at low wall temperature. At high wall temperatures, melting of the PCM slows down after a certain time. Once all melted, most of the energy still goes to the upper portion in the form of sensible heat to the liquid PCM, which slows down the melting of the PCM below the heating tube which is driven by conduction.

Jesumathy et al. [11] investigated the melting/solidification of Paraffin wax in horizontal doublepipe LHTES unit. They showed that heat transfer rate increased by $25 \%$ and $11 \%$ when the inlet temperature of HTF increased and decreased by $275 \mathrm{~K}$, respectively.

Pahamli et al. [12] also investigated the effect of inner pipe eccentricity on melting process of RT50 in the annular space of a horizontal double-pipe. They observed that the downward movement of the inner pipe contributed in the significant enhancement of the PCM melting process by shortening the total melting time by $64 \%$.

Tabassum [13] investigate the melting characteristics of impure PCM embedded between two concentric circular horizontal cylinders. The inner cylindrical tube was heated to a constant temperature by a heat transfer fluid while the outer tube was insulated. The results show that the melting rate increases rapidly up to the melting time of about $41.18 \mathrm{~min}$. Then, the melting rate increases but at much slower rate. The storage of thermal energy increases with the increase of the inner wall temperature and initial temperature of the solid PCM. The energy charged is greatly influenced by the change of the inner tube wall temperature compared to the change of the initial solid PCM temperature 
Gortychet al. [14] examine the process of heat accumulation and heat release for PCM in an annular space analytically and experimentally. The results showed that the thickness of the solidification layer depending on time and the distribution of the local heat transfer coefficient on the surface of the solidification front.

Mustafa et al. [15] compare the melting/solidification process between the PCM in the annulus (case A) and the inner tube (case B) numerically. The results showed that the melting time was significantly different in the two cases. it was shorter in case B than case A by almost $50 \%$. The results revealed that the solidification process in case $A$ was more rapid than case $B$ with the total solidification time of case A being lower by $43.4 \%$.

Saeid et al. [16] investigated the thermal behaviour in a vertical and horizontal shell-and-tube energy storage system using PCMs. The results indicate that the horizontal orientation has superior thermal performance during the charging and during part load energy charging. The results also show that increasing the hot HTF inlet temperature substantially reduces the total charging time for both orientations.

Al Siyabi I et al. [17] investigated the effect of inclination angle on melting of PCM in cylindrical thermal storage system. They found that the direction of buoyant force resulting from the melted liquid PCM has a major role in both melting rate and melting direction within the PCM storage.

From the above-mentioned works, the major influencing operating parameter is heating temperature, the inner and outer radius of the annular geometry and the length/width ratio of the annulus. Although, there are some experimental and numerical studies on annular geometry for thermal storage-reuse applications, but there is a need for more experimental data to fully understand the behaviour of melting and solidification process for thermal storage-insulation applications. In this study, experimental rig was built to study experimentally the melting/ solidification process of PEG 1500 in vertical annular enclosure of $950 \mathrm{~mm}$ length and $34 \mathrm{~mm}$ width and horizontal annular enclosure of $300 \mathrm{~mm}$ length and $15.9 \mathrm{~mm}$. The objectives of this paper are: i) To generate and report experimental data for the melting and solidification of the PCM from the inside to the outer side of the PCM annulus; ii) Use the recorded temperature to construct the melting front evolution during the melting process of vertical annulus; iii) Use the recorded temperature to construct the solidification contour. iv) Compare the melting percentage of the PCM between the vertical and horizontal annular geometry.

\section{Experimental Set-up and Procedure}

\subsection{Experimental Set-Up and PCM Compartment}

The experimental setup shown in Figure 1a for vertical and horizontal annular cases. The main components of the experimental set up comprise of, the heating water bath, thermocouples to measure temperature, temperature monitoring system (Pico data logger) and image recording system (digital camera). The thermocouples (TC) were placed in the PCM domain at different locations as shown in Figure $1 \mathrm{~b}$ for the vertical annular case and in Figure $1 \mathrm{c}$ for horizontal annular case. This apparatus has been designed with the Perspex to give an insight into the melting process. 
a

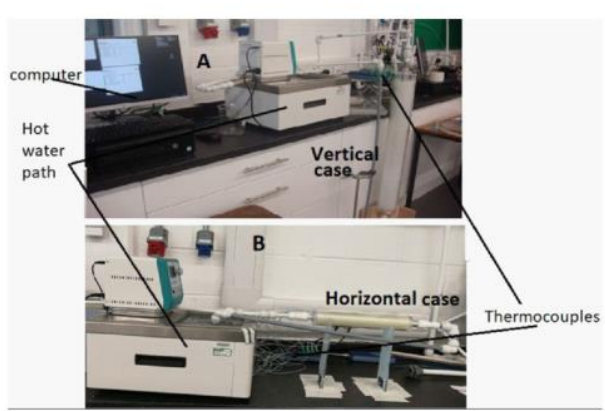

C

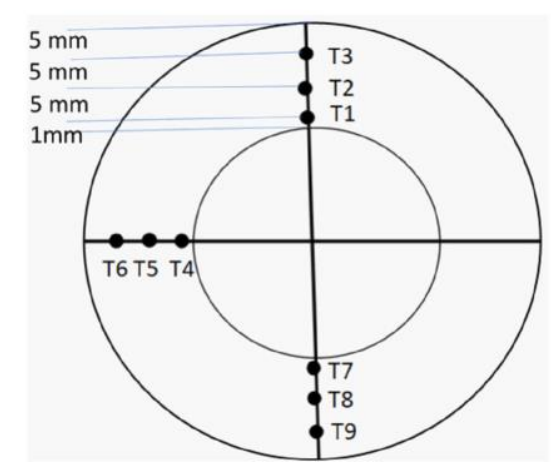

b

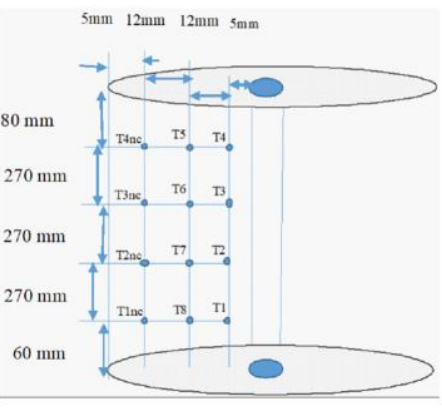

Figure 1 a. Photograph of the experimental set-up; b. Schematic diagram of the experimental set up showing the location of thermocouples in the vertical annular space dimensions; c. Schematic diagram of the experimental set up showing the location of thermocouples in the horizontal annular space dimensions.

The vertical annulus enclosure is formed from an outside cylinder made from a Perspex pipe 950 $\mathrm{mm}$ total length $(\mathrm{H}$ : height in rectangular enclose), outer diameter of $114 \mathrm{~mm}$ and inner diameter of $102 \mathrm{~mm}$. The inner aluminium pipe cylinder has $34 \mathrm{~mm}$ outer diameter, $28 \mathrm{~mm}$ inner diameter and $1,140 \mathrm{~mm}$ in length. The width of the annulus $(\mathrm{W}=$ inner diameter of Perspex pipe - outer diameter of aluminium pipe) is $34 \mathrm{~mm}$ and $950 \mathrm{~mm}$ length $(\mathrm{H} / \mathrm{W}=27.94)$. For larger volumes of PCM used in these experiments, the expansion upon melting becomes significant. This dictates that some free space $(50 \mathrm{~mm})$ filled with air is left above the PCM to allow room for the material to expand. The thermocouple attached in the space between the aluminium pipe and Perspex pipe by using ring shape thermocouples holder to stop them from being moved during the melting process.

The horizontal annulus enclosure was constructed from $3 \mathrm{~mm}$ thick Perspex material with dimensions of $300 \mathrm{~mm}$ long $\times 54 \mathrm{~mm}$ inner diameter, and $300 \mathrm{~mm}$ long $\times 22 \mathrm{~mm}$ outer diameter with $3 \mathrm{~mm}$ thick aluminium material. These dimensions produce annular space of $300 \mathrm{~m}$ length $(\mathrm{L})$ and 16 $\mathrm{mm}$ width (W) $(\mathrm{L} / \mathrm{W}=18.75)$. Free space of $15 \mathrm{~mm}$ from the annular length was left filled with air to allow space for PCM expansion.

The molten PCM was poured into the annular section of both enclosures whilst ensuring that no air bubbles were left in the PCM. The inner cylindrical aluminium pipe is connected to the hot water inlet from the heating water bath. The heating water bath is maintained at a constant temperature by the pump in a flowing circulation heater. The hot water flows through the aluminium tube. The desired temperature is maintained by heating the fluid with a controlled temperature heating water bath. The water bath continued to provide isothermal boundary condition of $353 \mathrm{~K}$ throughout the experimental progression. Tests were also performed at constant isothermal boundary conditions 
of 343 and $333 \mathrm{~K}$ respectively. All parts of the test sections were tightly joined to avoid any leakage. The maximum heat charging experiment duration is 375 mints.

The procedure for the experiment is therefore summarized below:

1. Turn on the power supply of the thermocouples control unit.

2. Turn on the heating water bath by adjusting the thermal control unit to certain desired heating temperature.

3. Open the valve and let the flow commence at the pump speed with an acceptable water volume flow rate

4. With the water circulation fully established at the required volume flow rate, record the temperature readings throughout the period of all the experiment.

\subsection{PCM Filling Procedure}

The main challenge while loading the PCM inside the enclosure is to maintain uniform PCM concentration in the entire annular cylindrical domain. In order not to have a segregation of the waxy particle inside the annular space, the waxy particles were first heated. The heating of the PCM to melting point was achieved using a heating plate maintained at a temperature above the melting point of the PCM $(333 \mathrm{~K})$. Once the melting is achieved, the content is immediately turned into the annular space to minimize the air bubble in the solidified PCM.

\subsection{Thermocouple for Temperature Data Acquisition}

The PCM temperatures inside the vertical annular cylindrical enclosure were measured by Type$K$ thermocouples. Twelve thermocouples were mounted at different locations in the same plane of the vertical annular section of the cylinder as shown in Figure $1 \mathrm{~b}$ to monitor the temperature during the phase change process. The temperature distributions is in angular direction was assumed homogenous as it was shown by Longeon et al [6]. For the horizontal annular case, the thermocouples attached in three planes to record the temperature variation between the top and the bottom of the horizontal annular space as the temperature distribution is not symmetrical as in the vertical case. Nine thermocouples are used and attached on ring shape thermocouples holder at three planes as shown in Figure 1c. On first thermocouples holder, T1 was placed near the Perspex pipe at the top of annulus, T2 attached to the middle point of the holder in horizontal plane. T3 attached near to the lower wall of the aluminium pipe. On the second thermocouples holder, T4 attached to the middle point at the top of the annulus, T5 attached at the top point on horizontal plane, T6 attached to near the lower wall of aluminium pipe. On the third thermocouple holder, T7 attached to the point near the top wall of the aluminium pipe, T8 attached to the middle point on horizontal plane, T9 attached near the lower Perspex wall. This thermocouple arrangement was used to record the temperatures in the vertical plane to show the temperature distribution during the melting process.

The data acquisition system for temperature reading was two 8 Channel Pico log data recorders connected to the computer system. A digital camera was used to capture the images of the PCM melting profile in the cylindrical Perspex pipe during the experiment at specified time intervals.

The experimental results are influenced by the inaccuracy in measurement of individual instruments. In present work, the uncertainty can be generated from temperature measurements 
using the thermocouple, the contours of melt front and the fraction of melted PCM which discussed in details by Hamad et al (2017) [18].

\section{Results and Discussion}

\subsection{Melting Evolution in the Vertical Annular Enclosure}

Different temperatures were initiated to establish the evolution of melting process of the PCM in the vertical annular enclosure. The lowest aluminium wall temperature used was the $333 \mathrm{~K}$. After the water flowing over a time of approximately 6 hours. The temperatures from thermocouples and the photos are recorded simultaneously to use both for analysis of melting evolution.

From the temperature records, as the melting front cannot be captured by the camera, before the melting front reach the Perspex pipe wall, the following procedure were used to construct the evolution of melting front using the temperature recoded by thermocouples:

1. The time for each thermocouple reaching the melting temperature $(318 \mathrm{k})$ is recoded.

2. The velocity for melting front is calculated from the distance between the first column of the thermocouples and the aluminium pipe and the time for melting of this distance as recorded in the first step. The same procedure is used for the second and third columns.

3. Then, the velocity and time are used to calculate the distance of the melting front from the aluminium pipe wall.

4. The average diameter of the melting fronts is used to calculate the melted volume. Then the melting percentage calculated by dividing the melted volume by the total annular volume.

The temperature evolution for melting at 333,343 and $353 \mathrm{~K}$ are given in Figures $2 \mathrm{a}, 2 \mathrm{~b}$ and $2 \mathrm{c}$. The images obtained for the cases when the melting front reach the Perspex wall were used to complete the explanation of melting process.

\subsubsection{Melting Evolution for the Case of $333 \mathrm{~K}$}

From the calculation of velocity using the temperature data from Figure 2a1 and 2a2, the melting evolution of the PCM at $333 \mathrm{~K}$ is given in Figure 3a. It can be observed that melting started and became steady all through the entire time. This lower temperature was unable to initiate significant melting of the PCM due to the melting temperature not fully reached by the low HTF temperature. The low temperature difference available was not able to create a buoyancy force and natural circulation to enhance heat transfer. The heat transfer by conduction can be considered as the dominated mode of heat transfer, which led to lower rate of heat transfer from the aluminium wall. There is a possibility of the steady state, which was reached, as the heat added to melted zone is approximately equal to overall heat losses from it. 

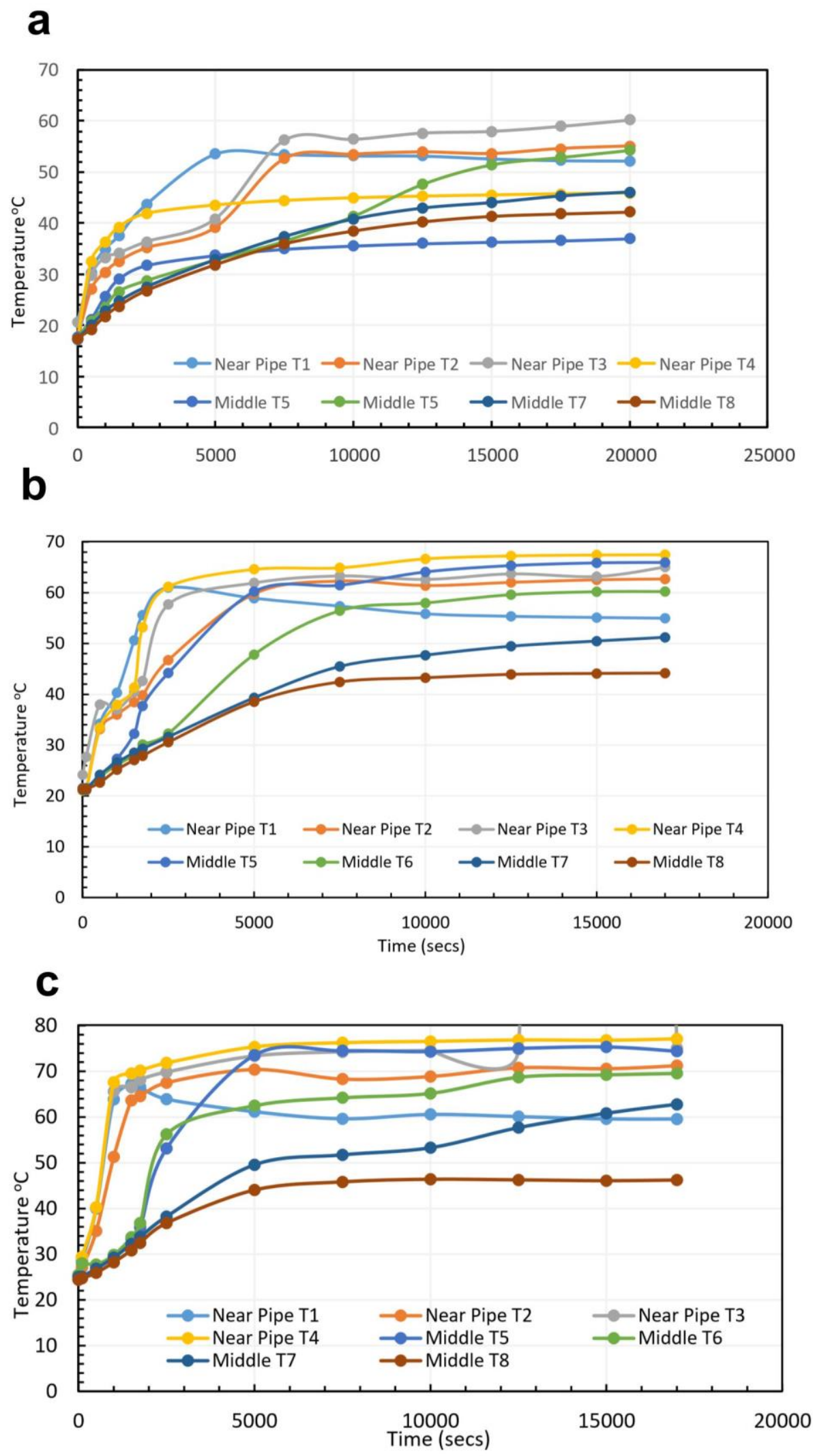

Figure 2 a. The temperature evolution for melting of $60^{\circ} \mathrm{C}$ (vertical case); b. The temperature evolution for melting of $70^{\circ} \mathrm{C}$ (vertical case); c. The temperature evolution for melting of $80^{\circ} \mathrm{C}$ (vertical case). 
a

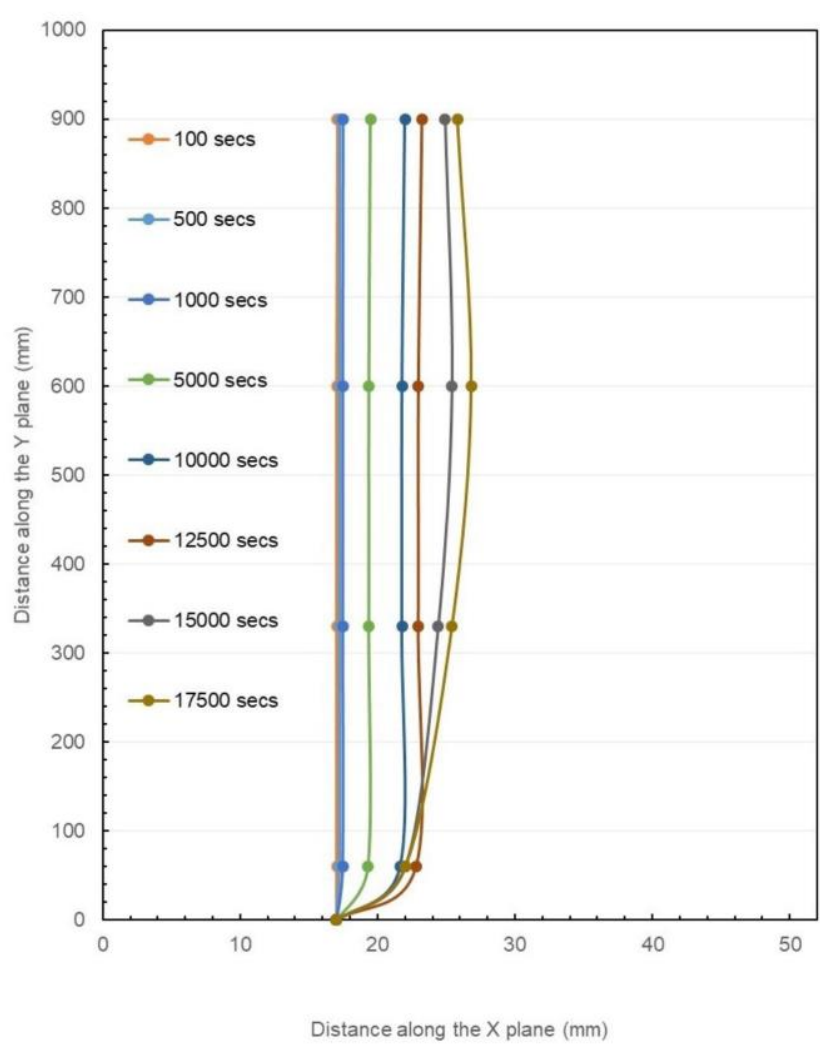

b

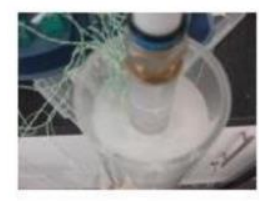

(a) $\mathrm{t}=100 \operatorname{secs}$

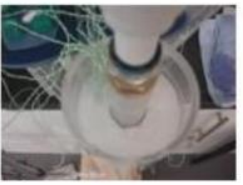

(d) $\mathrm{t}=2500 \mathrm{secs}$

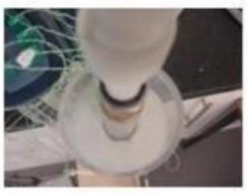

(g) $\mathrm{t}=10000$ secs

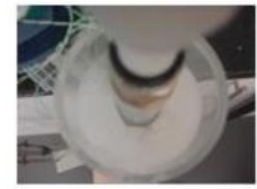

(j) $\mathrm{t}=17500 \mathrm{secs}$

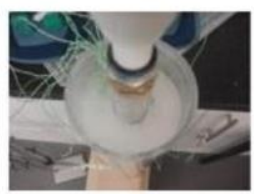

(b) $\mathrm{t}=500 \mathrm{secs}$

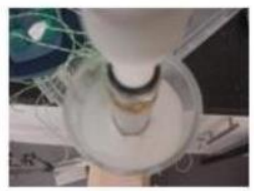

(e) $\mathrm{t}=5000$ secs

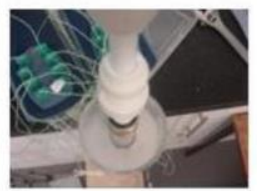

(h) $\mathrm{t}=12500 \mathrm{secs}$

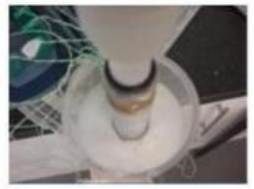

(k) $\mathrm{t}=20000$ secs

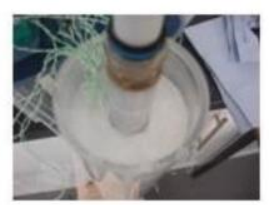

(c) $\mathrm{t}=1000 \mathrm{secs}$

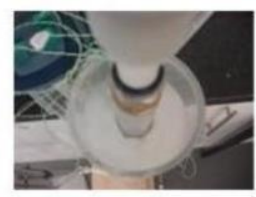

(f) $\mathrm{t}=7500$ secs

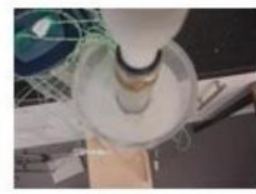

(1) $\mathrm{t}=15000 \mathrm{secs}$

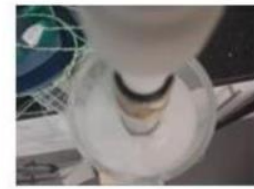

(1) $\mathrm{t}=22500 \mathrm{secs}$

Figure 3 a. The melting front profile of the PCM at the temperature of $60^{\circ} \mathrm{C}$ (vertical case); b. Photographs showing the melting progress for vertical case (heating temperatures $=60^{\circ} \mathrm{C}$ ).

The photos for this case are given in Figure 3b. It was recorded from the top of the annulus as the melting has progressed more than $10 \mathrm{~mm}$ from the aluminium pipe wall.

\subsubsection{Melting Evolution for the Case of $343 \mathrm{~K}$}

From the calculation of velocity using the temperatures data Figure $2 b 1$ and $2 b 2$, the melting evolution of the PCM at $343 \mathrm{~K}$ is given in Figure 4a. As the temperature higher than case a, the melting front moves faster as can be seen from the larger gaps between the graph reaching the Perspex pipe after around $10000 \mathrm{sec}$ from the start of heating. It can be seen from the images after the $10000 \mathrm{sec}$ that the melting start progressing downward reaching a distance of $150 \mathrm{~mm}$ from the upper level of PCM. This indicates that the higher temperature creates more circulation and increase the heat transfer by convection. The steady state is not reached in this case, as heat input to melted zone is more than the heat losses, which maintain the movement of melting front with the time. 
a

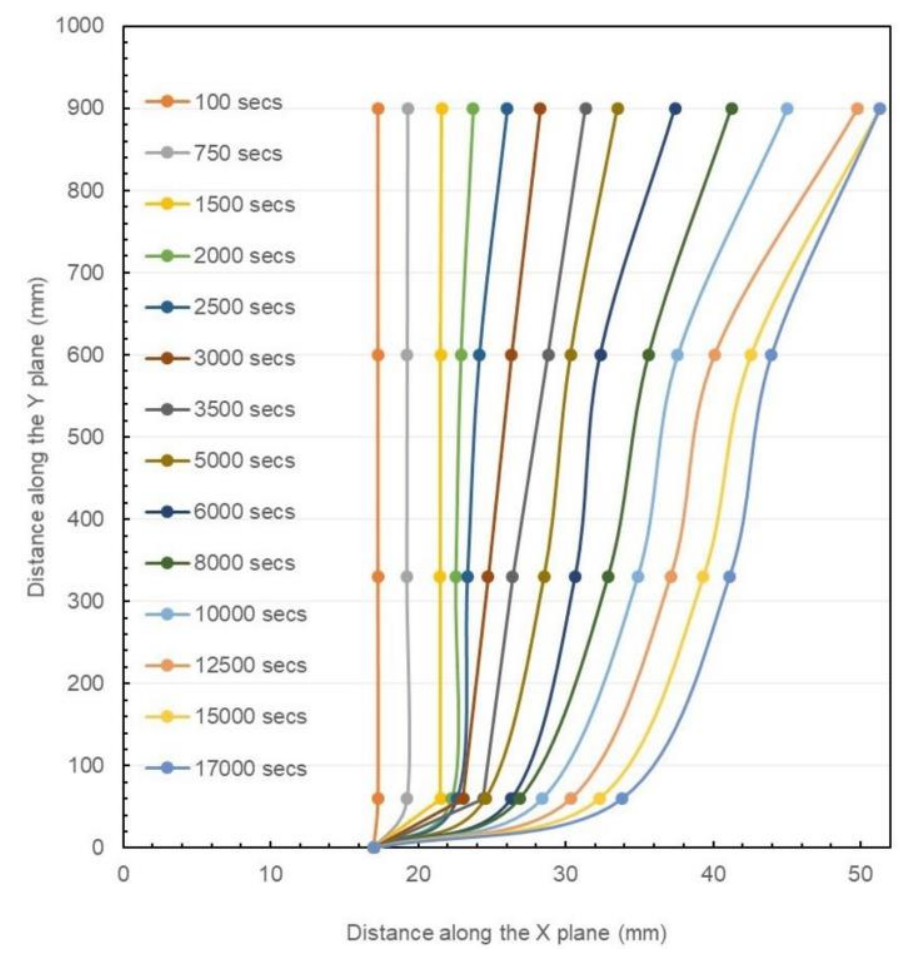

b

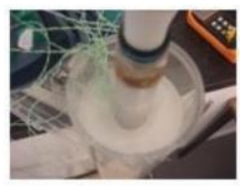

(a) $\mathrm{t}=100$ secs

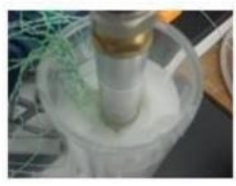

(d) $\mathrm{t}=2500$ secs

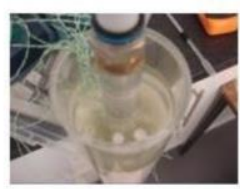

(g) $\mathrm{t}=10000$ secs

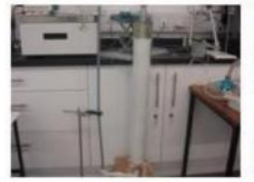

(j) $\mathrm{t}=12500$ secs

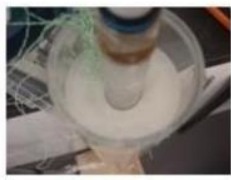

(b) $\mathrm{t}=500 \mathrm{secs}$

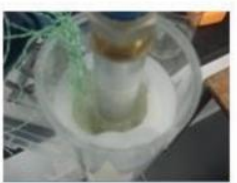

(e) $\mathrm{t}=5000$ secs

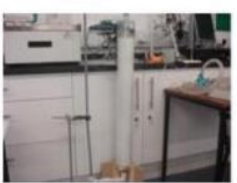

(h) $\mathrm{t}=10000$ secs

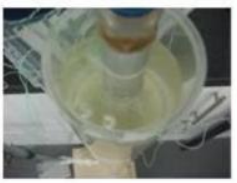

(k) $\mathrm{t}=15000 \mathrm{secs}$

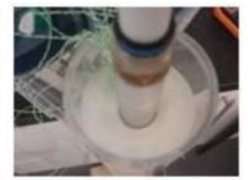

(c) $\mathrm{t}=1000$ secs

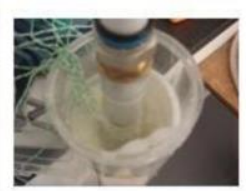

(f) $\mathrm{t}=7500$ secs

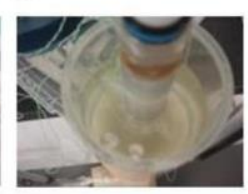

(1) $\mathrm{t}=12500 \mathrm{secs}$

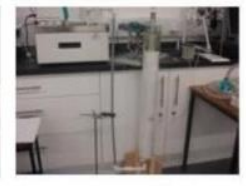

(1) $\mathrm{t}=17500$ secs

Figure 4 a. The melting profile front of the PCM at the temperature of $70^{\circ} \mathrm{C}$ (vertical case); b. Photographs showing the melting progress for vertical case (heating temperatures $=70^{\circ} \mathrm{C}$ ).

\subsubsection{Melting Evolution for the Case of $353 \mathrm{~K}$}

From the calculation of velocity using the temperatures data Figure $2 \mathrm{c} 1$ and $2 \mathrm{c} 2$, the melting evolution of the PCM at $353 \mathrm{~K}$ is given in Figure $5 \mathrm{a}$. As the temperature higher becomes higher than the cases $a$ and $b$, the melting front moves even faster and reached the Perspex pipe after around $7500 \mathrm{sec}$ from the start of heating. It can be seen from the images after the 7500 secs that the melting start progressing downward reaching a distance of $380 \mathrm{~mm}$ from the upper level of PCM at $15000 \mathrm{sec}$ and $520 \mathrm{~mm}$ at $20000 \mathrm{sec}$. This indicates that the heat transfer by convection becomes very strong compared to conduction which controlled the melting process in case a. The heat transfer is transient as the heat input to the melted zone is much more compared heat losses as the melting front moving much faster than other two cases. Figure 6 shows the variation of melting percentage with time. The melting percentage calculated by dividing the melted volume by the volume of the annular space. 


\section{a}

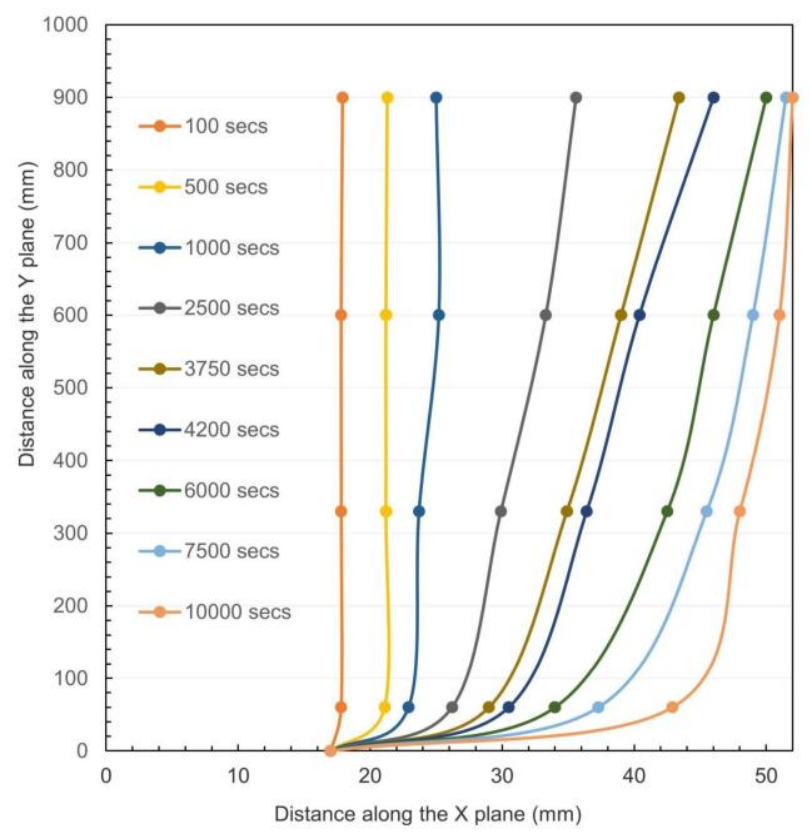

b

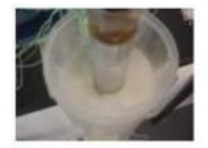

(a) $\mathrm{t}=100 \mathrm{secs}$

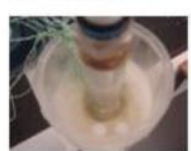

(d) $\mathrm{t}=2500$ secs

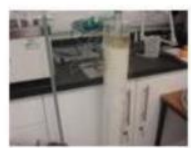

(g) $\mathrm{t}=7500 \mathrm{secs}$

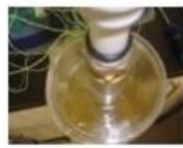

(j) $t=12500$ secs

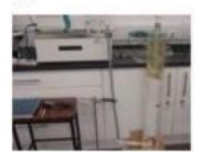

(m) $\mathrm{t}=15000 \mathrm{secs}$

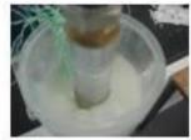

(b) $\mathrm{t}=500$ secs

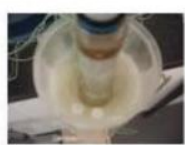

(e) $\mathrm{t}=5000$ secs

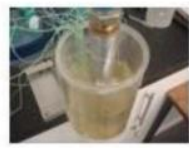

(h) $t=10000$ secs

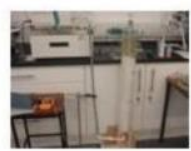

(k) $\mathrm{t}=12500$ secs

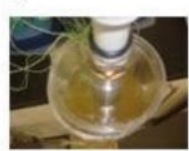

(n) $\mathrm{t}=17500$ secs

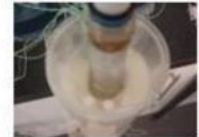

(c) $\mathrm{t}=1000 \mathrm{secs}$

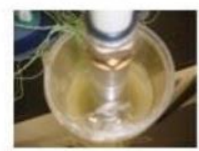

(f) $\mathrm{t}=7500$ secs

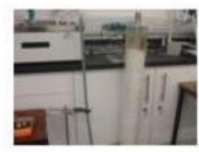

(i) $\mathrm{t}=10000$ secs

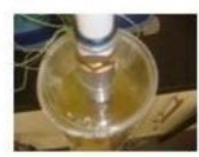

(1) $\mathrm{t}=15000 \mathrm{secs}$

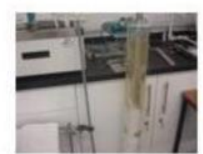

(o) $\mathrm{t}=17500 \mathrm{secs}$

Figure 5 a. The melting profile front of the PCM at the temperature of $80^{\circ} \mathrm{C}$ (vertical case); b. Photographs showing the melting progress for vertical case (heating temperatures $=80^{\circ} \mathrm{C}$ ).

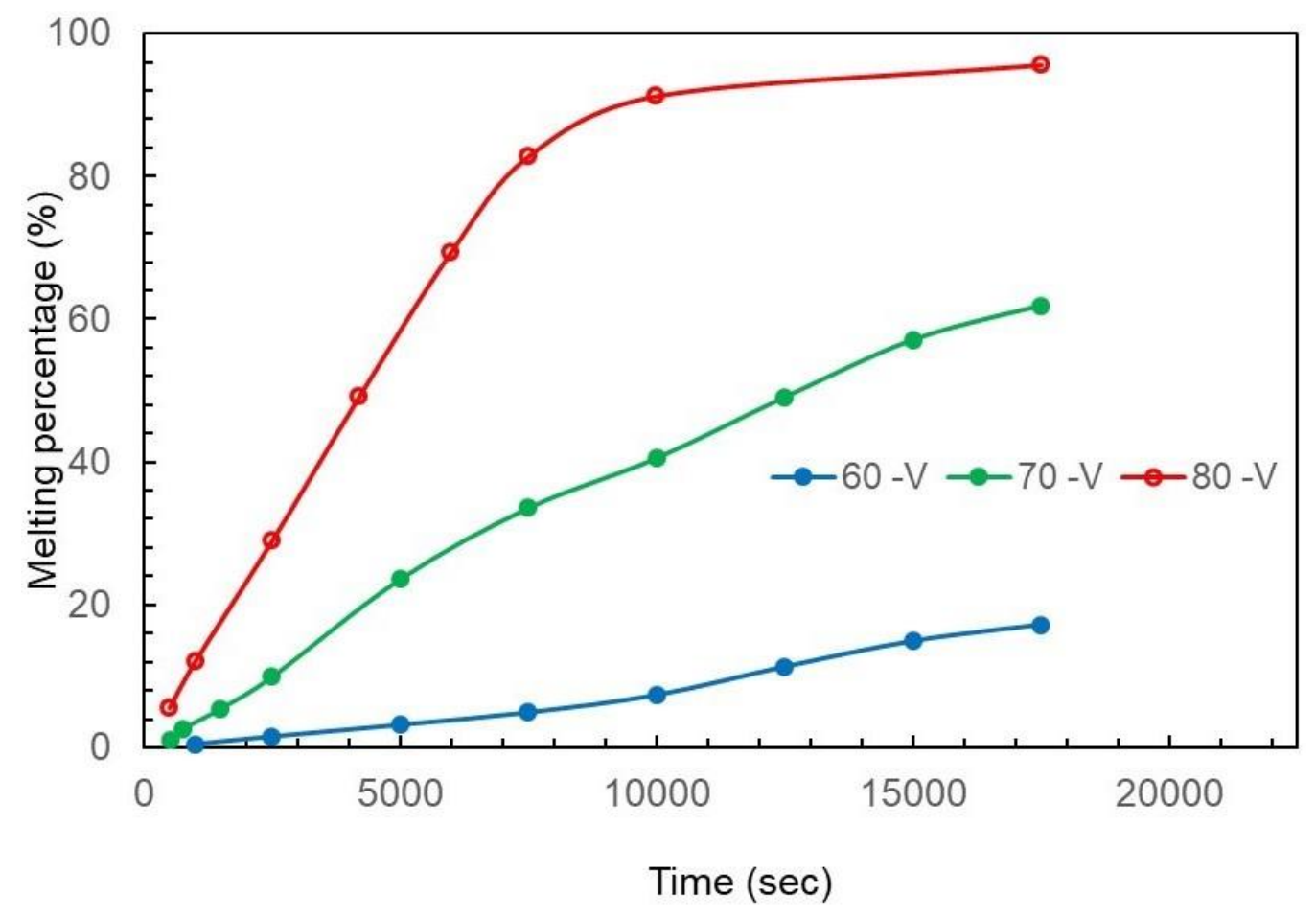

Figure 6 Comparison of melting percentage with time for $60 \circ \mathrm{C}, 70 \mathrm{oC}$ and $80 \mathrm{oC}$ heating wall (vertical case). 


\subsection{Melting Evolution in the Horizontal Annular Enclosure}

A camera was used to record the melt profile as the melt front progress with the time. Figure 7a, $7 \mathrm{~b}$ and $7 \mathrm{c}$ present a sequence of photos of the melting process of PEG 1500 at 333, 343, and $353 \mathrm{~K}$ respectively. Figure 8 shows a comparison of the calculated melting percentage for the three case.

a

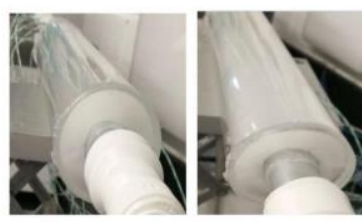

(a) $\mathrm{t}=100 \mathrm{sec}$

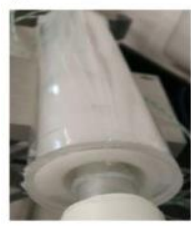

(e) $\mathrm{t}=5500 \mathrm{sec}$

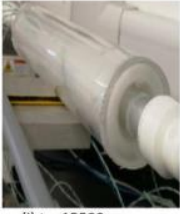

(i) $t=15500 \mathrm{sec}$

C

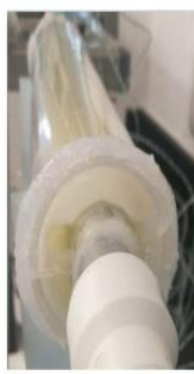

(a) $\mathrm{t}=500 \mathrm{sec}$

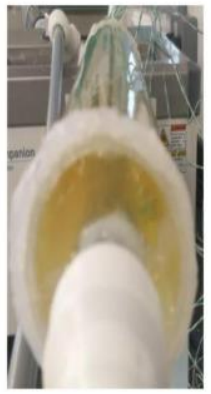

(a) $\mathrm{t}=5500 \mathrm{sec}$ (b) $\mathrm{t}=500 \mathrm{sec}$

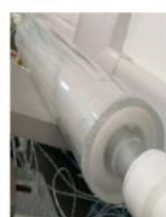

(f) $\mathrm{t}=7500 \mathrm{sec}$
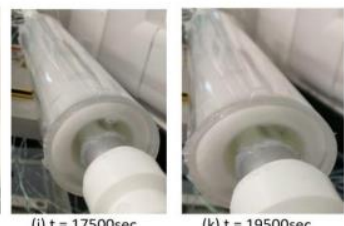

(c) $\mathrm{t}=1000 \mathrm{sec}$

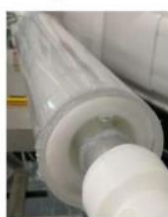

b

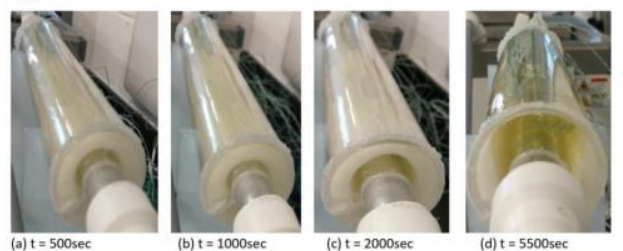

(d) $\mathrm{t}=2000 \mathrm{sec}$

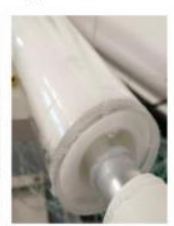

(h) $\mathrm{t}=12500 \mathrm{sec}$
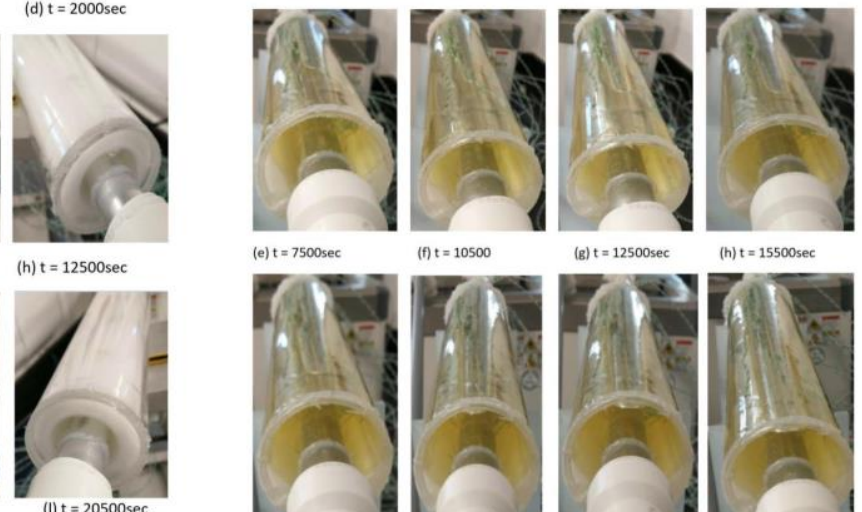

(e) $t=7500 \mathrm{sec}$

(f) $t=10500$
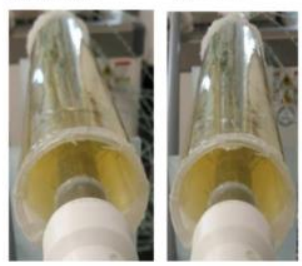

(g) $t=12500$ se

(h) $t=15500$ sec
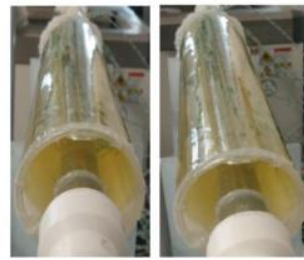

(j) $t=20500$ sec

$(k) t=22500$ sec

(L) $t=23500$ sec

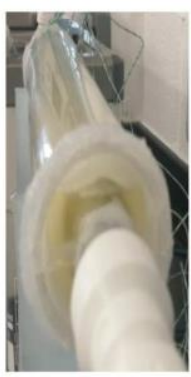

(a) $\mathrm{t}=1000 \mathrm{sec}$

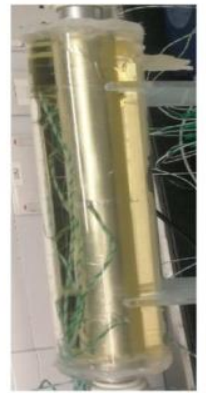

(a) $t=7500$ sec

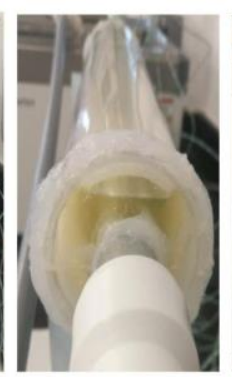

(a) $\mathrm{t}=2000 \mathrm{sec}$

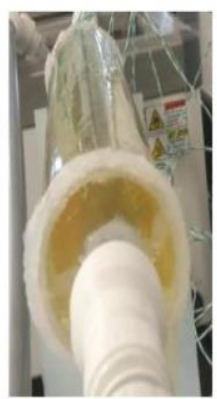

(a) $\mathrm{t}=10500 \mathrm{sec}$

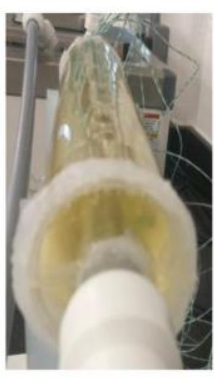

(a) $\mathrm{t}=4000 \mathrm{sec}$

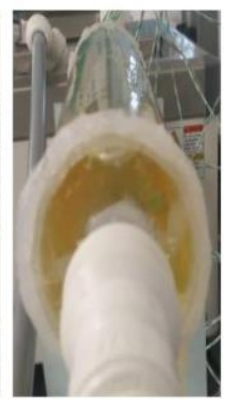

(a) $t=11500$ sec

Figure 7 a. Photographs showing the melting progress for horizontal case (heating temperatures $=60^{\circ} \mathrm{C}$ ); $\mathrm{b}$. Photographs showing the melting progress for horizontal case (heating temperatures $=70{ }^{\circ} \mathrm{C}$ ); c. Photographs showing the melting progress for horizontal case (heating temperatures $=80^{\circ} \mathrm{C}$ ). 


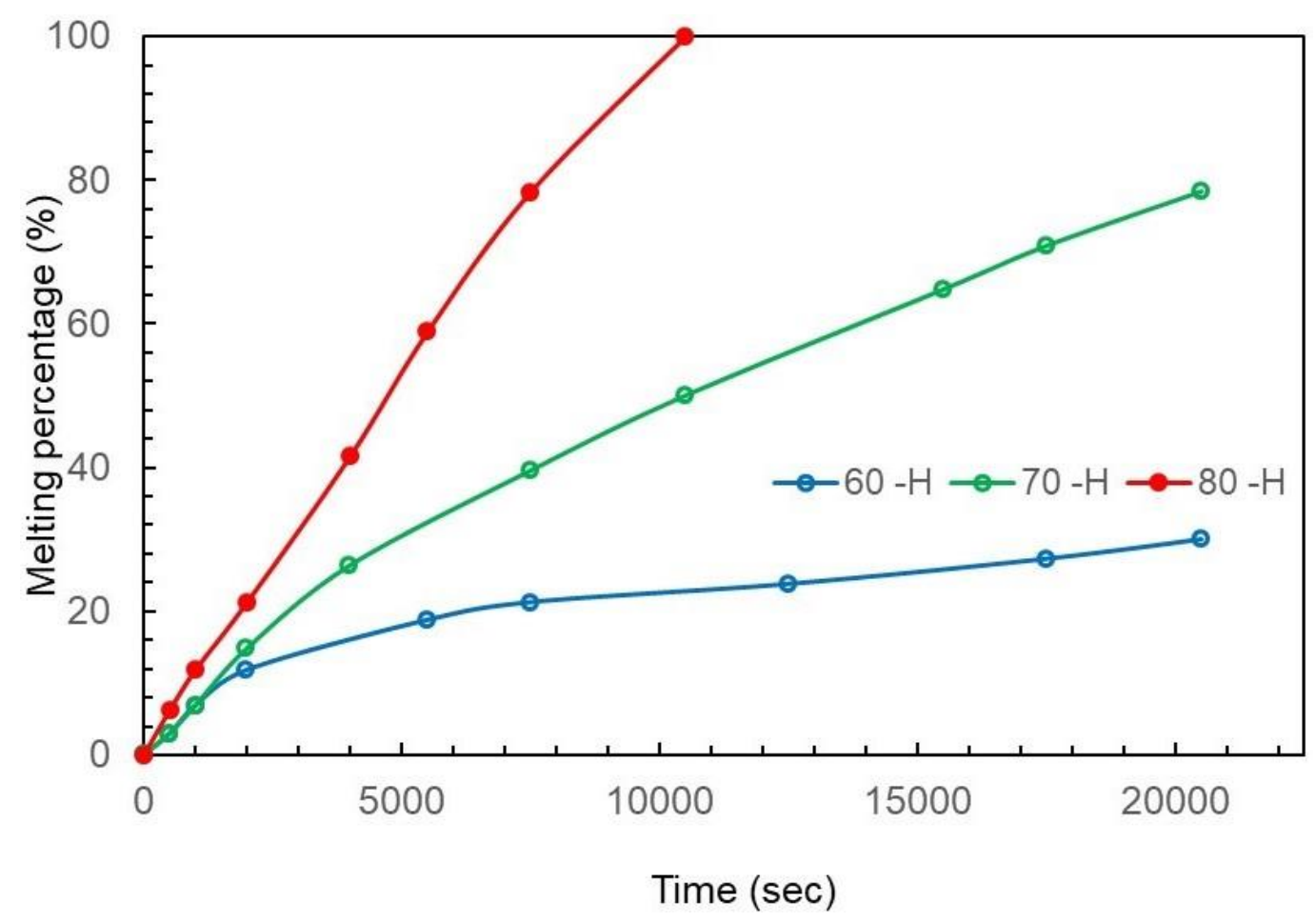

Figure 8 Comparison of melting percentage with time for $60 \circ \mathrm{C}, 70 \mathrm{oC}$ and $80 \mathrm{oC}$ heating wall ( $\mathrm{H}$ : horizontal case).

Due to the difficulty in calculating the melting percentage before the melting front reach the Perspex pipe wall, the temperature records from thermocouples were used to construct the evolution of melting front using the following procedure:

1. The time for each thermocouple reaching the melting temperature $(338 \mathrm{~K})$ is recoded.

2. The velocity for melting front is calculated from the distance between the first column of the thermocouples and the surface of the aluminium pipe. The same procedure is used for the second and third columns of thermocouples.

3. The velocity and time are used to calculate the distance of the melting front from the aluminium pipe wall.

4. The average diameter of the melting fronts is used to calculate the melted volume. Then the melting percentage calculated by dividing the melted volume by the total annular volume.

As the melting reach the outer surface, the photos from camera are used in combination with calculation of melting front from the temperature records to calculate the melting percentage.

For $333 \mathrm{~K}$ case, Figure $7 \mathrm{a}$ shows the photos of the melting progress with the time. From the graph in Figure 8, it can be observed that melted front is moving very fast for the first 2000 secs to reach $22 \%$ as the heat transfer take place by conduction with high temperature difference between the hot surface (inner aluminium cylinder) and the solid PCM. Then, the heat transfer rate reduced with time as the temperature difference between heating surface and melted PCM reduced which led to slower increase in the melting percentage for the rest of time. The melting graph highlighted that melting rate reduced with time due the reduction in heat transfer from the hot wall and the increase in heat losses from the test section as the surface temperature of the outer Perspex cylinder becomes a round $313 \mathrm{~K}$ after 10000 secs, which was higher than the surrounding air (295 K). The results indicated that the solid PCM layer adjacent to outer Perspex wall act as insulating material 
to reduce the heat losses. The low heat losses increase the temperature of the melted PCM and reduce the heat transfer from the heating wall which delay the PCM melting process.

For $343 \mathrm{~K}$ case, Figure $7 \mathrm{~b}$ shows the photos of the melting progress with the time. From the graph in figure 8 , it can be observed that the melting increased at high rate for the first 4000 secs to reach $40 \%$, then the melting rate reduced with the rest of the time The same explanation given for the case of $333 \mathrm{~K}$ heating temperature are applied to this case. However, melting percentage reached $90 \%$ after 22500 secs compared to $40 \%$ for the $333 \mathrm{~K}$ heating temperature due to the higher temperature of the heating surface. In this case, the higher heating surface temperature increased the overall temperature of the test section, which reduce the influence of solid PCM layer in controlling the heat transfer rate and lead to the high to the melting percentage.

For $353 \mathrm{~K}$ case, Figure $7 \mathrm{c}$ shows the photos of the melting progress with the time.

From the graph in Figure 8, it can be observed that the melting percentage is increased almost linearly and very fast compared to the cases of $333 \mathrm{~K}$ and $343 \mathrm{~K}$. The melting completed of the PCM was almost completed PCM in 10500 secs. The heat transfer and melting process evolution are very much similar to previous two cases of $333 \mathrm{~K}$ and $343 \mathrm{~K}$.

Figure 9 summarized the melting percentage for the vertical and horizontal cases and the three temperature 333,343 and $353 \mathrm{~K}$. It can be observed that there is melting percentage for horizontal case is twice the melting percentage for $333 \mathrm{~K}$ and reduced to about $20 \%$ for $343 \mathrm{~K}$. However, there is no significant differences for the high temperature of $353 \mathrm{~K}$. The reduction in discrepancy can be attributed to the increases of natural circulation of the melted PCM with highest temperature. It also be observed that the melting percentage reached $100 \%$ after 10500 secs while the melting percentage reached for vertical case reached $91 \%$ at 10000 secs and increased to $95.5 \%$ at 17500 secs. It may take another 10000 s to melt completely. This happen due to the difference in natural circulation mechanism between the two cases.

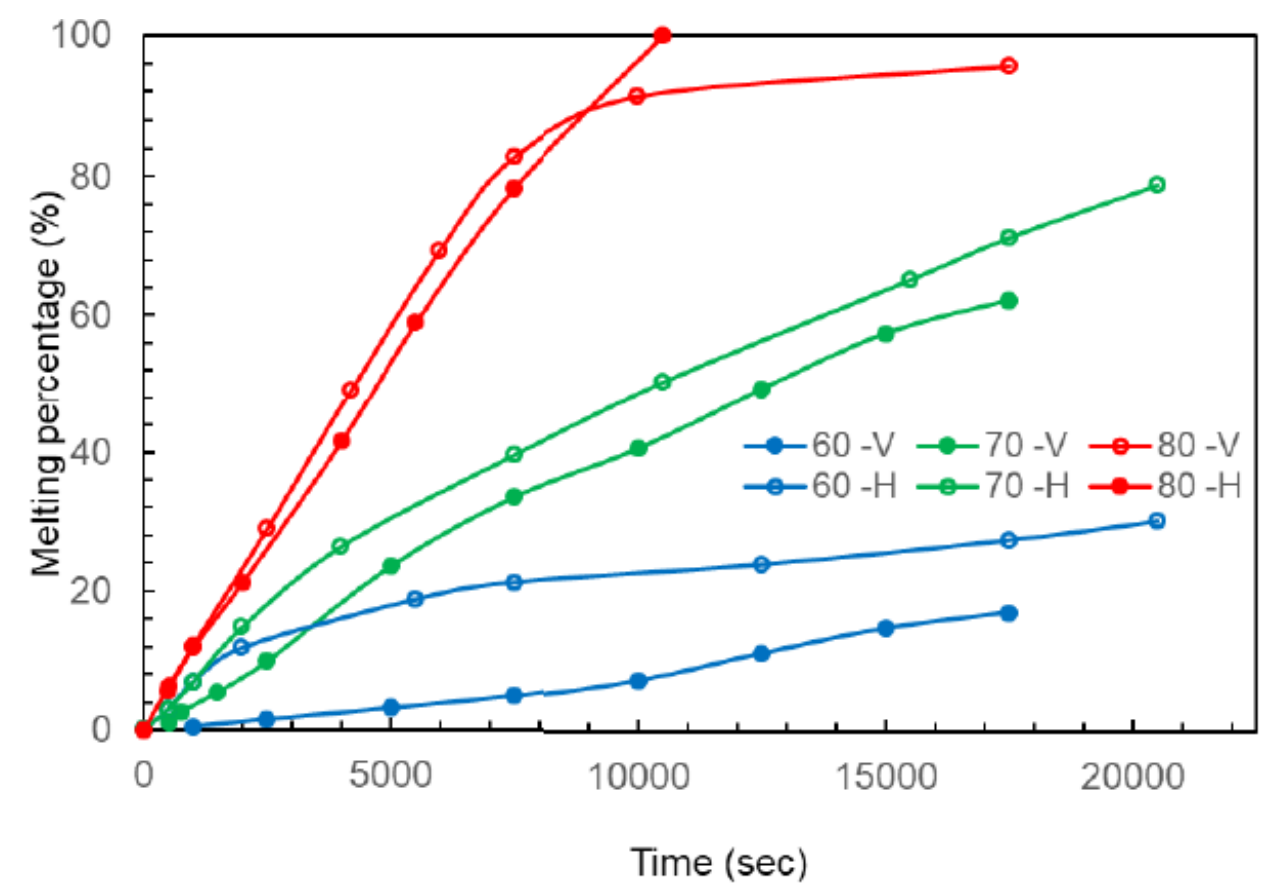

Figure 9 Comparison of melting percentage with time between vertical case $(\mathrm{V})$ and horizontal case $(\mathrm{H})$. 


\subsection{Solidification Evolution in the Vertical Annular Enclosure}

Solidification is a process controlled by conduction with minor convection as the temperature dropping continuously with time and the melted zone, which lead to continued reduction in natural circulation. While, melting process involves more natural convection than conduction as the temperature maintained higher than melting temperature by the added heat. When the temperature drops to freezing temperature, the natural convection cannot occur, and conduction across the growing solid layer controls the freezing rate. If the liquid is initially superheated, natural convection controls for minor and conduction for long times.

In present work, the temperatures were recorded from the thermocouples inside the PCM after the heating was switched off. The temperature started decreasing gradually with time as shown in Figures 10.

The time for the recoded temperatures in Figure 10 is used to construct the evolution contour of the solidification in similar procedure used for melting profile front. The procedure can be summarized as follows:

1. The time between the turn off time for the heating and the thermocouple reading recording the solidification temperature of $318 \mathrm{~K}$.

2. The velocity of the solidification front is calculated.

3. The location of the solidification front was calculated from the calculated velocity and time.

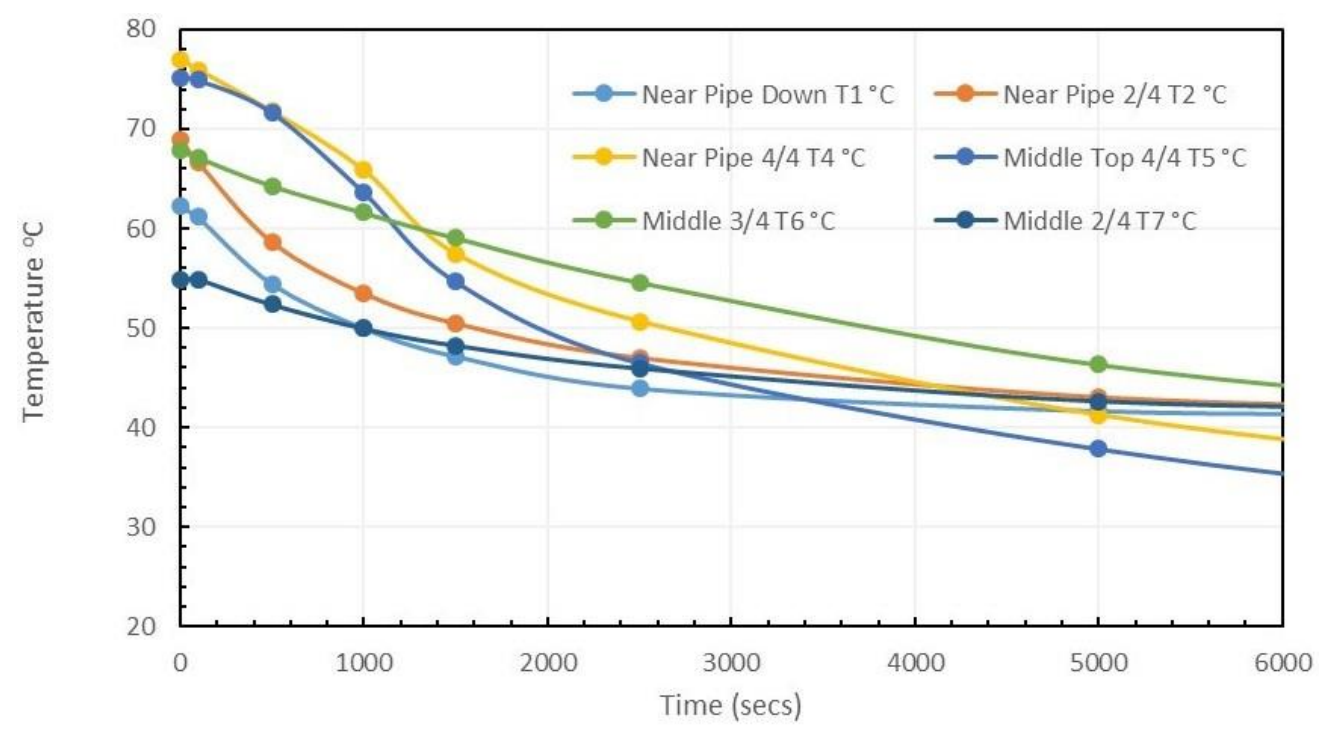

Figure 10 The Variation of temperature with time during solidification $\left(80^{\circ} \mathrm{C}\right)$ (vertical case).

The main difference between melting and solidification is in the movement of the interface. The melting front is moving in radial direction from the inner aluminium wall while the solidification is moving from all outer boundary toward the point of the highest temperature in the inner zone of the PCM. The movement of the solidification is presented in Figure 11. 


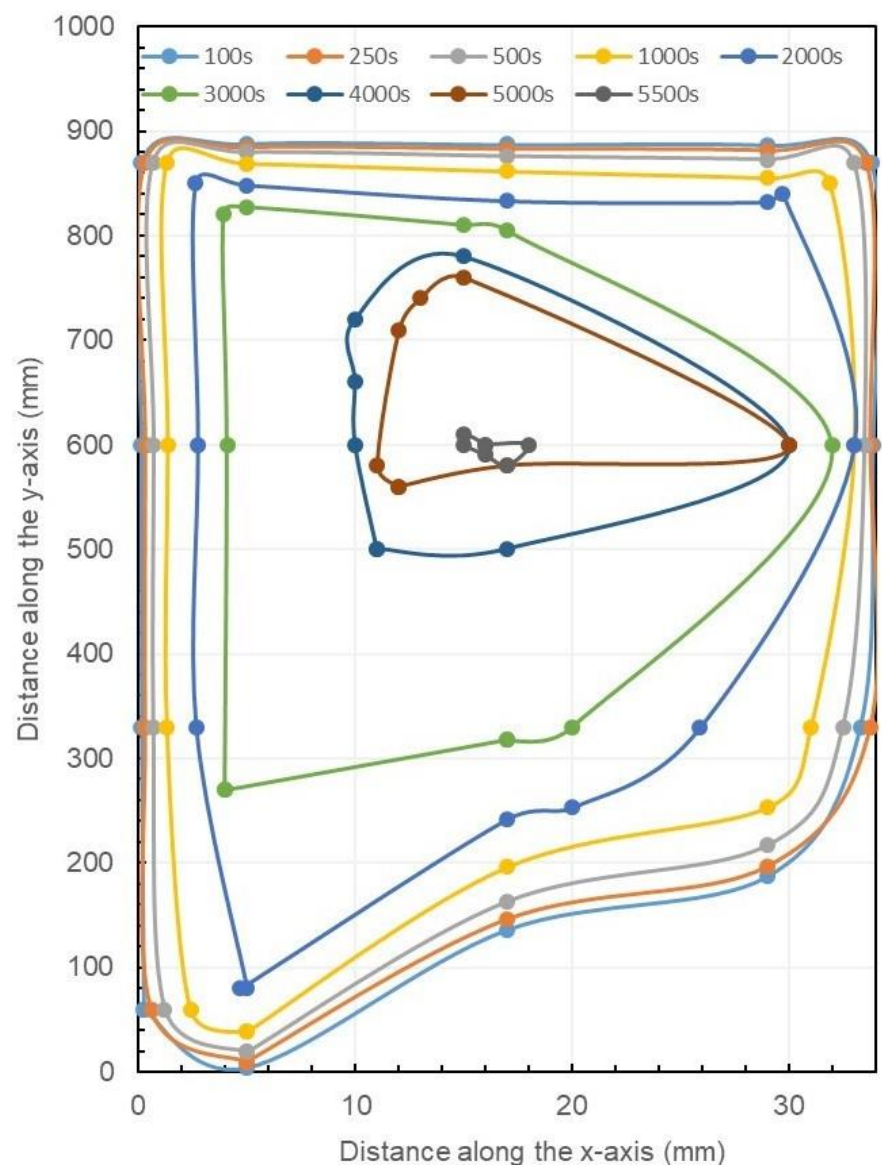

Figure 11 Solidification contour for the hot surface of $800 \mathrm{oc}$. (vertical case).

\section{Conclusions}

An experimental investigation has been conducted to investigate the melting behaviour of PCM in a vertical and horizontal annular enclosure between two concentric cylinders for heat storageinsulation applications. The study was performed for vertical and horizontal cases using PEG 1500 for heating surface temperatures of 333, 343 and $353 \mathrm{~K}$. It was clearly showed that inclination and heating surface temperature has a significant influence on melting/solidification characteristics. The following conclusions can be drawn:

1. The melting of the PCM in the annular enclosure started initially by conduction and then progressed to convection with the time when melted PCM appear near the heating wall. The results showed that the horizontal annular case melt faster than the vertical case, in particular, at higher heating surface temperature of $353 \mathrm{~K}$.

2. The increase in heating wall temperature showed a large influence on the melting rate of the PCM for vertical and horizontal cases.

3. In the case of solidification, the movement of interface was from the all the outer boundary toward the point of higher temperature at the inner melted PCM. The temperature of the inner region was remained hot for long time which provide a good evidence that support the concept of using the PCM as heat storage -insulation material.

4. The results show that the melting percentage for horizontal case is $100 \%$ higher from the melting percentage of vertical case at $333 \mathrm{~K}$ which reduced to about $20 \%$ for $343 \mathrm{~K}$. However, there 
is no significant differences for the high temperature of $353 \mathrm{~K}$. The reduction in discrepancy can be attributed to the increases of natural circulation of the melted PCM with highest temperature.

\section{Author Contributions}

F. A. Hamad, experimental design, conceptualization, writing, review; E. Egelle, Experimental measurements, literature review and draft preparation for vertical case; Abbas Mohammed, Experimental measurements literature review and draft preparation for horizontal case; S. Gooneratne, review and editing; P. Russell, experimental design, conceptualization, writing review; all authors have contributed in reviewing and editing the paper after submission to the journal.

\section{Competing Interests}

The authors have declared that no competing interests exist.

\section{References}

1. Chiu JN, Martin V. Submerged finned heat exchanger latent heat storage design and its experimental verification. Appl Energy. 2012; 93:507-516.

2. Kozak $Y$, Rozenfeld T, Ziskind G. Close-contact melting in vertical annular enclosures with a nonisothermal base: Theoretical modeling and application to thermal storage. Int J Heat Mass Transf. 2014; 72: 114-127.

3. Yagci OK, Avci M, Aydin O. Melting and solidification of PCM in a tube-in-shell unit: Effect of fin edge lengths' ratio. J Energy Storage. 2019; 24: 100802.

4. Seddegh S, Wang X, Joybari MM, Haghighat F. Investigation of the effect of geometric and operating parameters on thermal behavior of vertical shell-and-tube latent heat energy storage systems. Energy. 2017; 137: 69-82.

5. Ismail KA, Melo CA. Convection-based model for a PCM vertical storage unit. Int J Energy Res. 1998; 22: 1249-1265.

6. Longeon M, Soupart A, Fourmigué JF, Bruch A, Marty P. Experimental and numerical study of annular PCM storage in the presence of natural convection. Appl Energy. 2013; 112: 175-184.

7. Kalapala L, Devanuri JK. Parametric investigation to assess the melt fraction and melting time for a latent heat storage material based vertical shell and tube heat exchanger. Sol Energy. 2019; 193: 360-371.

8. Balikowski JR, Mollendorf JC., Performance of phase change materials in a horizontal annulus of a double-pipe heat exchanger in a water-circulating loop. J Heat Transfer. 2007; 129: 265272.

9. Agyenim F, Eames P, Smyth M. A comparison of heat transfer enhancement in a medium temperature thermal energy storage heat exchanger using fins. Sol Energy. 2009; 83: 15091520.

10. Azad M, Dineshan D, Groulx D, Donaldson A. Melting of Phase Change Materials in a cylindrical enclosure: Parameters influencing natural convection onset. Proceedings of the 4th International Forum on Heat Transfer; 2016 Nov 2-4; Sendai, Japan. Sendai, Japan: Sendai International Center. 
11. Jesumathy SP, Udayakumar M, Suresh S, Jegadheeswaran S. An experimental study on heat transfer characteristics of paraffin wax in horizontal double pipe heat latent heat storage unit. J Taiwan Inst Chem Eng. 2014; 45: 1298-1306.

12. Pahamli Y, Hosseini MJ, Ranjbar AA, Bahrampoury R., Inner pipe downward movement effect on melting of PCM in a double pipe heat exchanger. Appl Math Comput. 2018; 316: 30-42.

13. Tabassum T, Hasan M, Begum L., Thermal energy storage through melting of a commercial phase-change material in a horizontal cylindrical annulus. J Enhanc Heat Transf. 2018; 25: 211237.

14. Gortych M, Lipnicki Z, Weigand B., An experimental and theoretical study of the solidification process of phase change materials in a horizontal annular enclosure. Appl Therm Eng. 2019; 161: 114140.

15. Mahdi MS, Mahood HB, Hasan AF, Khadom AA, Campbell AN. Numerical study on the effect of the location of the phase change material in a concentric double pipe latent heat thermal energy storage unit. Therm Sci Eng Prog. 2019; 11: 40-49.

16. Seddegh S, Wang $X$, Joybari MM, Haghighat F. Investigation of the effect of geometric and operating parameters on thermal behavior of vertical shell-and-tube latent heat energy storage systems. Energy. 2017; 137: 69-82.

17. Al Siyabi I, Khanna S, Mallick T, Sundaram S. An experimental and numerical study on the effect of inclination angle of phase change materials thermal energy storage system. J Energy Storage. 2019; 23: 57-68.

18. Hamad FA, Egelle E, Cummings $K$, Russell $P$. Investigation of the melting process of polyethylene glycol 1500 (PEG 1500) in a rectagular enclosure. Int J Heat Mass Transf. 2017; 114: 1234-1247.

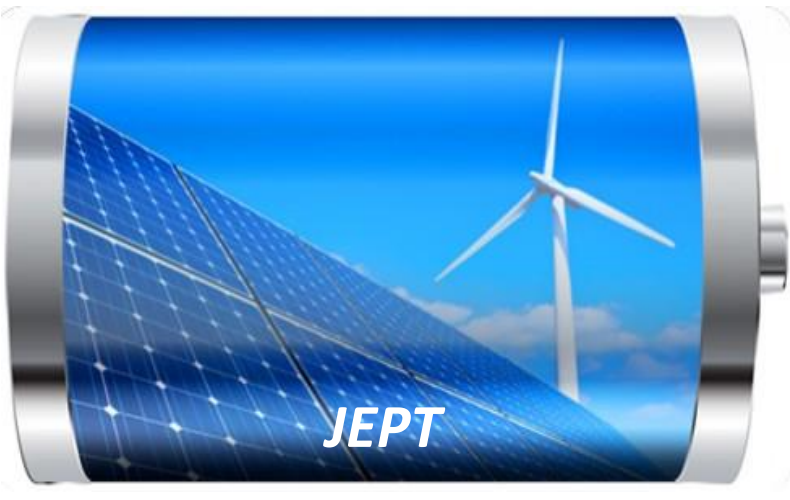

Enjoy JEPT by:

1. Submitting a manuscript

2. Joining in volunteer reviewer bank

3. Joining Editorial Board

4. Guest editing a special issue

For more details, please visit: http://www.lidsen.com/journal/jept 
Original Research

\title{
High Energy Density Rechargeable Aqueous Lithium Batteries with an Aqueous Hydroquinone Sulfonic Acid and Benzoquinone Sulfonic Acid Redox Couple Cathode
}

Hironori Takagi, Koichi Kakimoto, Daisuke Mori, Sou Taminato, Yasuo Takeda, Osamu Yamamoto *, Nobuyuki Imanishi

Graduate School of Engineering, Mie University, Tsu, Mie, 514-8507, Japan; E-Mails: 419m335@m.mie-u.ac.jp; 420m314@m.mie-u.ac,jp; daisuke.mori@chem.mie-u.ac.jp; taminato@chem.mie-u.ac.jp; takeda@chem.mie-u.ac.jp; yamamoto@chem.mie-u.ac.jp; imanishi@chem.mie-u.ac.jp

* Correspondence: Osamu Yamamoto; E-Mail: yamamoto@chem.mie-u.ac.jp

Academic Editor: Ahamed Irshad

Special Issue: Batteries: Past, Present and Future

Journal of Energy and Power Technology

2021, volume 3 , issue 1

doi:10.21926/jept.2101010
Received: January 12, 2021

Accepted: February 12, 2021

Published: February 24, 2021

\begin{abstract}
The demand for high energy density rechargeable batteries beyond lithium-ion batteries has increased for electric vehicles. In the present study, a novel high energy density rechargeable aqueous lithium battery was proposed. The battery was composed of a lithium metal anode, a lithium-stable non-aqueous electrolyte, a water-stable lithium-ion conducting solid electrolyte of $\mathrm{Li}_{1.4} \mathrm{Al}_{0.4} \mathrm{Ge}_{0.2} \mathrm{Ti}_{1.4}\left(\mathrm{PO}_{4}\right)_{3}$-epoxy- $\mathrm{TiO}_{2}$ separator, and a hydroquinone sulfonic acid (HQS)/benzoquinone sulfonic acid (BQS) redox couple in an aqueous acetic acid solution (HAC). An open-circuit voltage of $3.7 \mathrm{~V}$ at $25^{\circ} \mathrm{C}$ was recorded, and the theoretical energy density of the battery based on the reaction $2 \mathrm{Li}+\mathrm{BQS}+2 \mathrm{H}_{2} \mathrm{O}=2 \mathrm{LiOH}+\mathrm{HQS}$ was $833 \mathrm{Whkg}^{-1}$, about two times higher than that of the lithium-ion battery. The battery was successfully cycled at 0.5 $\mathrm{mA} \mathrm{cm}{ }^{-2}$ and $25^{\circ} \mathrm{C}$ with low polarization.
\end{abstract}

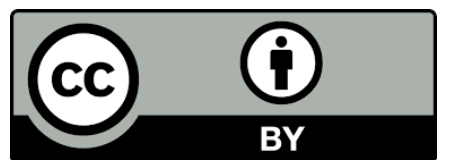

(c) 2021 by the author. This is an open access article distributed under the conditions of the Creative Commons by Attribution License, which permits unrestricted use, distribution, and reproduction in any medium or format, provided the original work is correctly cited. 


\section{Keywords}

High energy density battery; aqueous lithium battery; lithium metal electrode; benzoquinone sulfonic acid; solid electrolyte

\section{Introduction}

A lithium anode is the best candidate for a high energy density battery because of its high theoretical specific capacity of $3860 \mathrm{mAhg}^{-1}$ and highest electrochemical reduction potential of $3.04 \mathrm{~V}$ in comparison to a normal hydrogen electrode (NHE). Lithium reacts vigorously with water; therefore, an aqueous electrolyte is never used, and non-aqueous electrolytes were mainly developed for batteries with lithium anodes [1, 2]. In 2004, Visco et al. [3, 4] proposed a unique aqueous lithium-air battery that consisted of a lithium metal anode, a water-stable lithium-ion conducting solid electrolyte separator, an aqueous electrolyte, and an air electrode. The electrolyte separator of NASICON-type $\mathrm{Li}_{1+x} \mathrm{Al}_{x} \mathrm{Ti}_{2-\mathrm{x}}\left(\mathrm{PO}_{4}\right)_{3}$ (LATP) prevented direct contact between the lithium metal anode and the aqueous electrolyte solution. The aqueous lithium-air battery has been expected to give a high power density and to operate in an air atmosphere [5].

Mainly, two types of aqueous lithium batteries have been proposed in the literature; the one with an aqueous electrolyte and water-stable lithium intercalation electrodes [6], and the other with a water-stable solid electrolyte separator [3]. The former aqueous lithium battery exhibits a high power density; however, the specific energy density is lower than that of conventional lithiumion batteries [7]. At present, various aqueous lithium batteries with solid electrolyte separators have been suggested. Zhou and co-workers reported a prototype aqueous lithium battery with a solid electrolyte separator [8]. This cell was assembled using $1 \mathrm{M} \mathrm{LiClO}_{4}$ in ethylene carbonate and dimethyl carbonate as an electrolyte for the $\mathrm{Li}$ anode and an aqueous solution containing $1 \mathrm{M} \mathrm{LiOH}$ and $1 \mathrm{M} \mathrm{KOH}$ as the electrolytes for the $\mathrm{Ni}(\mathrm{OH})_{2}$ cathode. The two electrolytes were separated by the LATP. The energy density of this cell was calculated as $857 \mathrm{Whkg}^{-1}$ based on the weights of the electrode active materials. Similarly, a cell consisting of a lithium anode with a liquid electrolyte and water-soluble $\mathrm{Fe}(\mathrm{CN})_{6}{ }^{3-} / \mathrm{Fe}(\mathrm{CN})_{6}{ }^{4-}$ redox couple cathode separated by LATP was proposed by Goodenough et al. [9]. The theoretical specific energy density of this redox couple was recorded as $392 \mathrm{Whkg}^{-1}$, comparable to that of conventional lithium-ion batteries, although a higher power density was expected. Wu et al. reported a Li/gel-polymer electrolyte/LATP/aqueous electrolyte/ $\mathrm{LiMn}_{2} \mathrm{O}_{4}$ cell [10]. The specific energy density of the $\mathrm{Li} / \mathrm{LiMn}_{2} \mathrm{O}_{4}$ cell based on the mass of the electrodes was estimated to be $446 \mathrm{Whkg}^{-1}$. Imanishi and co-workers [11-13] proposed a higher specific energy density aqueous lithium battery that consisted of a lithium anode with a liquid electrolyte, an aqueous $\mathrm{MCl}_{2}\left(\mathrm{M}=\mathrm{Co}, \mathrm{Ni}\right.$, and $\mathrm{Sn}$ ) solution catholyte, and a $\mathrm{Li}_{1.4} \mathrm{Al}_{0.4} \mathrm{Ge}_{0.2} \mathrm{Ti}_{1.4}\left(\mathrm{PO}_{4}\right)_{3}$ (LAGTP) separator. The theoretical specific energy density of the $\mathrm{Li} / \mathrm{NiCl}_{2}$ cell was as high as 1047 Whkg ${ }^{-1}$ based on the weights of lithium and $\mathrm{NiCl}_{2}$ and was at least twice of the conventional lithiumion battery. The estimated specific energy density calculated using the mass of cell components with a $0.1 \mathrm{~mm}$ thick LAGTP separator (except packaging) was $670 \mathrm{Whkg}^{-1}$. Moreover, it has been shown in the literature that the battery with a liquid-type catholyte could be developed into a large scale battery without the degradation in cell performance due to the high diffusibility of the active material in the catholyte [13]. 
In this study, we have developed a novel aqueous lithium rechargeable battery with a watersoluble organic redox couple catholyte of 1,2-hydroquinone-3-sulfonic acid (HQS) and 1,2benzoquinone-3-sulfonic acid (BQS) and a lithium metal anode. Feng et al. [14] reported an organic cathode composed of 3,4,9,10-perylene-tetracarboxylic acid-dianhydride in an organic solvent, but the solubility of this compound in water was very low. Also, the catholyte and lithium metal anode were separated by LAGTP. The benzoquinone-hydroquinone couple is the prototypical example of an organic redox system, and their electrochemical behavior has been extensively studied for the past many years [15-17]. However, the solubility of benzoquinone in water is not very high. Narayanan et al. [18-20] reported an aqueous flow battery based on a water-soluble organic redox couple of 1,2-benzoquinone-3,5-disulfonic acid at the cathode and anthraquinone-2-sulfonic acid at the anode. A water-soluble aqueous solution of BQS/HQS was used as the catholyte. The cell reaction of the battery was the following;

$$
2 \mathrm{Li}+\mathrm{BQS}+2 \mathrm{H} 2 \mathrm{O}=2 \mathrm{LiOH}+\mathrm{HQS}
$$

The open-circuit voltage (OCV) of the cell was around $3.7 \mathrm{~V}$ at $25{ }^{\circ} \mathrm{C}$. The theoretical specific energy density based on the masses of the lithium anode, $\mathrm{H}_{2} \mathrm{O}$, and $\mathrm{BQS}$ with an OCV of $3.7 \mathrm{~V}$ was $833 \mathrm{Whkg}^{-1}$. The quinones are comparatively cheaper $\left(\sim \$ 5-10 \mathrm{~kg}^{-1}\right)$ [18]; hence are highly appropriate and attractive for large-size batteries for electric vehicles and various electrical energy storage applications.

\section{Experimental}

HQS was prepared by ion exchange of 1, 2-hydroquinone-3-sulfonic potassium salt (HQSK, Alfa Aesar) using an ion-exchange resin Amerlyst 15 DRY (Kurita Water, Japan). Since LAGTP had to be decomposed in a strongly acidic solution [21], the $\mathrm{pH}$ of the catholyte was adjusted to $\mathrm{pH} 2$. The aqueous solutions of $0.8 \mathrm{M} \mathrm{HQS}$ in $5.5 \mathrm{M} \mathrm{HAC}$ and $2 \mathrm{M} \mathrm{LiCl}, 0.015 \mathrm{M} \mathrm{H}_{2} \mathrm{SO}_{4}$, and $10 \mathrm{~m}$ 1-butyl-3methylimidazolium chloride (IBMmCl) with $1.0 \mathrm{M} \mathrm{HCl}$ (Sigma-Aldrich), all at pH 2.0, were examined as the catholyte. The $2 \mathrm{M} \mathrm{LiCl}$ was added to the $5.5 \mathrm{M} \mathrm{HAc}$ to increase the conductivity of the electrolyte. The electrochemical performance of the HQS/BQS redox reaction was studied using a beaker cell with a working electrode consisting of a mixture of a Pt-loaded carbon electrode (Pt-C) (Tanaka Kikinzoku, Japan) and polytetrafluoroethylene (PTFE) (Daikin Japan) (8:2 weight ratio), a Pt counter electrode, and $\mathrm{Ag} / \mathrm{AgCl}$ in saturated $\mathrm{KCl}$ reference electrode. The cathode mixture was pressed on a titanium mesh (Nikara, Japan).

The schematic diagram of an in-house-built Swagelok-type full test cell is shown in Figure 1. The cell consisted of a $200 \mu \mathrm{m}$ thick lithium metal anode (Honjometal, Japan), $\mathrm{Li}\left(\mathrm{CF}_{3} \mathrm{SO}_{2}\right)_{2} \mathrm{~N}$ (LiFSI) dissolved in tetra(ethylene)glycol dimethyl ether (G4) (2:1 mole ratio) and 1,3 dioxolane (DOL, 50 vol\%) as anode electrolyte, ca. $200 \mu \mathrm{m}$ thick LAGTP-epoxy-10 wt\% $\mathrm{TiO}_{2}$ as the solid electrolyte separator, an aqueous solution of $0.8 \mathrm{M} \mathrm{HQS}$ in $5.5 \mathrm{M} \mathrm{HAc}$ and $2 \mathrm{M} \mathrm{LiCl}$ as the catholyte, and a mixture of Pt-C and PTFE as the cathode. The anode electrolyte LiFSI-2G4-50 vol\% DOL was reported to suppress lithium dendrite formation at a high current density [22]. LAGTP-epoxy-TiO films were $^{2}$ prepared by a previously reported tape casting method [23]. Fine LAGTP powder was prepared by a sol-gel method using citric acid [24]. Briefly, stoichiometric amounts of $\mathrm{Ti}\left(\mathrm{OC}_{2} \mathrm{H}_{5}\right)_{4}$ and $\mathrm{Ge}\left(\mathrm{OC}_{2} \mathrm{O}_{5}\right)_{4}$ (Sigma Aldrich) were dissolved in ethylene glycol, added to a $0.2 \mathrm{M}$ aqueous solution of citric acid, and the solution was stirred well. After the gel formation, stoichiometric amounts of $\mathrm{LiNO}_{3}$, 
$\mathrm{Al}\left(\mathrm{NO}_{3}\right) 3.9 \mathrm{H} 2 \mathrm{O}$, and $\mathrm{NH}_{4} \mathrm{H}_{2} \mathrm{PO}_{4}$ (Nakalai Tesque, Japan) were added to the gel. The gel was kept at $220^{\circ} \mathrm{C}$ for several hours and was then heated at $580^{\circ} \mathrm{C}$ for $4 \mathrm{~h}$. The gel was then ground and heated again at $800{ }^{\circ} \mathrm{C}$ for $4 \mathrm{~h}$. A mixture of LAGTP, a fine $\mathrm{TiO}_{2}$ powder (Kojundo Chemicals Japan, $2 \mu \mathrm{m}$ particle size), Menhaden fish oil (Sigma Aldrich), Burver@B-98 (Sigma Aldrich), and benzyl-butylphthalate (Wako Chemicals, Japan) was ball-milled using a plenary mill (Fritsch, Mode 7). The obtained slurry was tape-casted onto a silicon-coated polyethylene substrate using a two doctor blade apparatus. The green tape-cast sheet was carefully dried at $4{ }^{\circ} \mathrm{C}$ followed by heating at $500{ }^{\circ} \mathrm{C}$ to remove organic additives, and finally sintered at $950{ }^{\circ} \mathrm{C}$. The LAGTP-epoxy- $\mathrm{TiO}_{2}$ films were prepared by dipping the $\mathrm{LAGTP}-\mathrm{TiO}_{2}$ film into a tetrahydrofuran solution containing $0.05 \mathrm{M} 1,3-$ phenylene diamine (Sigma-Aldrich) and 2,2-bis(4-glycidyloxy-phenyl)propane (Sigma-Aldrich), followed by a polymerization reaction at $170{ }^{\circ} \mathrm{C}$ for $24 \mathrm{~h}$ [25]. The amount of epoxy in the LAGTP$\mathrm{TiO}_{2}$ film estimated from the weight change was around ca $2 \mathrm{wt} \%$. Lastly, the Pt loaded porous carbon (Tanaka Kikinzoku, Japan) and polytetrafluoroethylene (PTFE) mixture were pressed onto a titanium mesh (Nikara, Japan).

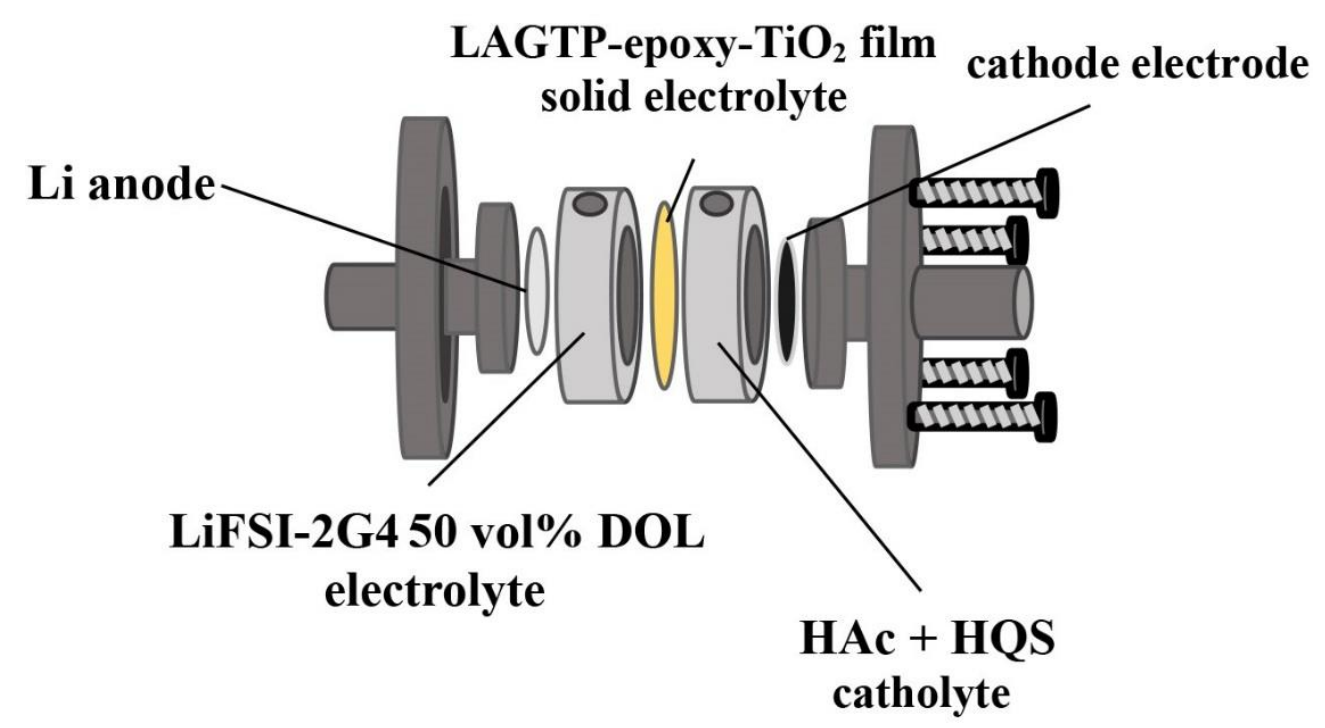

Figure 1 Schematic of the test cell.

Impedance measurements were conducted using a frequency analyzer (Solatron 1260) in the frequency range of $0.1 \mathrm{~Hz}$ to $1 \mathrm{M} \mathrm{Hz}$ at a bias voltage of $10 \mathrm{mV}$. Bulk and grain boundary conductivities of the samples were estimated from the complex impedance plots using Zview 2 software. Electrochemical measurements were conducted using a multichannel potentiogalvanostat (Bio-Logic Science Instrument VMPX).

\section{Results and Discussion}

The stability of the solid electrolyte in the aqueous catholyte is an important requirement for the separator in the aqueous lithium batteries. The stability of LATP in the aqueous electrolytes was examined by Imanishi and co-workers [21, 26-28]. They found that LATP was unstable in strong acid and alkaline aqueous solutions, and the lithium-ion conductivity of LATP was decreased considerably by its immersion in these solutions. We studied the impedance change of the tape cast LAGTP-epoxy- $-\mathrm{TiO}_{2}$ films in an aqueous solution of $0.8 \mathrm{M} \mathrm{HQS}$ in $5.5 \mathrm{M} \mathrm{HAC}$ and $2 \mathrm{M} \mathrm{LiCl}(\mathrm{pH}=2.0)$. 
The impedance profiles of Au/LAGTP-epoxy- $-\mathrm{TiO}_{2} / \mathrm{Au}$ as a function of the immersion period in Figure 2(a) showed that the initial total conductivity of the film was $3.7 \times 10^{-4} \mathrm{Scm}^{-1}$ at $25^{\circ} \mathrm{C}$. The bulk conductivity was not changed for two weeks, but the grain boundary conductivity was decreased. The total conductivity of the electrolyte after two weeks was $1.5 \times 10^{-4} \mathrm{Scm}^{-1}$, followed by no further significant change in total conductivity. The XRD patterns of the LAGTP-epoxy- $\mathrm{TiO}_{2}$ film before and after immersion in 0.8 M HQS in 5.5 M HAc and $2 \mathrm{M} \mathrm{LiCl}(\mathrm{pH}=2.0)$ for two weeks also showed no significant change. The other requirement of the solid electrolyte separator for the aqueous lithium cell is to be water impermeable. A water permeation test was conducted using an $\mathrm{H}$-type cell, where one chamber was filled with pure water, and the other chamber was filled with $1 \mathrm{M} \mathrm{LiCl}$ aqueous solution [25]. The water permeation rate was determined from the $\mathrm{Cl}^{-}$content in the chamber with water. The $\mathrm{LAGTP}-\mathrm{TiO}_{2}$ film without epoxy resin had a high lithium-ion conductivity of $8.9 \times 10^{-4} \mathrm{Scm}^{-}$ 1 , but exhibited water permeation through the film, while no water diffused through the LAGTPepoxy- $-\mathrm{TiO}_{2}$ film over a period of one week. The LAGTP-epoxy- $\mathrm{TiO}_{2}$ film was thus acceptable and recommended for the separator in the proposed aqueous lithium batteries.

(a)

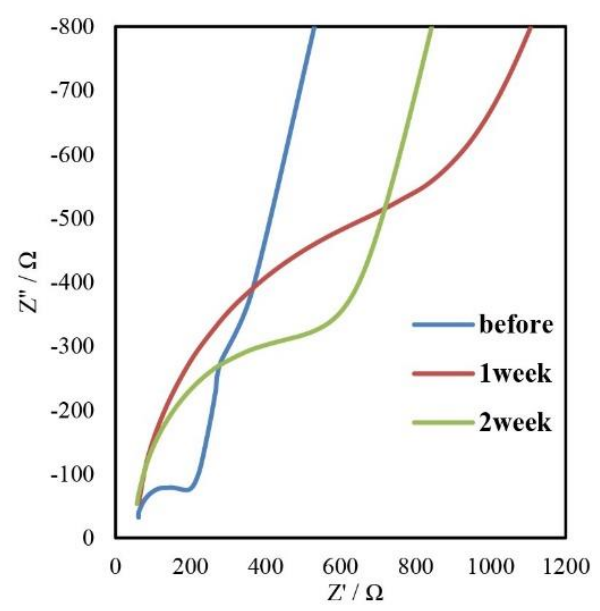

(b)

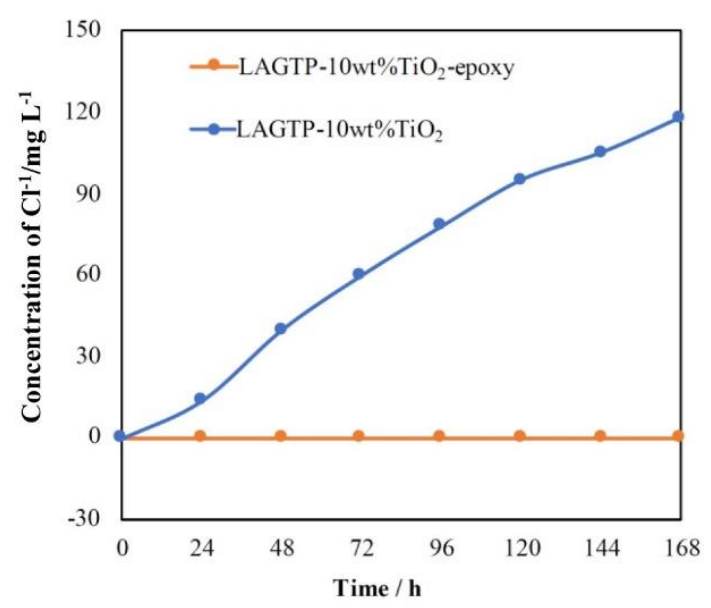

Figure 2 (a) Impedance profiles of the LAGTP epoxy- $-\mathrm{TiO}_{2}$ immersed in an aqueous solution of $0.8 \mathrm{M} \mathrm{HQS}$ in $5.5 \mathrm{M} \mathrm{HAC}$ and $2 \mathrm{M} \mathrm{LiCl}$ at $\mathrm{pH} 2.0$ and $25^{\circ} \mathrm{C}$ as a function of time

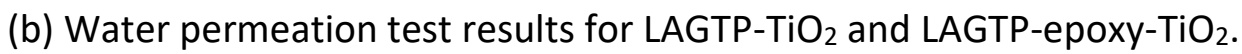

Figure 3 shows cyclic voltammograms (CV) for the Pt-C electrode of an aqueous solution of 0.8 $\mathrm{M} \mathrm{HQS}$ in $5.5 \mathrm{M} \mathrm{HAC}$ and $2 \mathrm{M} \mathrm{LiCl}$, an aqueous solution of $15 \mathrm{mM} \mathrm{H}_{2} \mathrm{SO}_{4}$, and $10 \mathrm{~m} \mathrm{IBMmCl}$ with 1.0 $\mathrm{M} \mathrm{HCl}\left(\mathrm{IBMmCl}-\mathrm{HCl}\right.$ ) aqueous solution at $25^{\circ} \mathrm{C}$, where HQS was oxidized to $\mathrm{BQS}$ at $0.5 \mathrm{~mA} \mathrm{~cm}^{-2}$ for $20 \mathrm{~h}$. The imidazole supporting electrolyte of IBM-1.0 M HCl was found to suppress the Michael addition reaction of benzoquinone with water [29]. The CV curve of $15 \mathrm{mM} \mathrm{H}_{2} \mathrm{SO}_{4}$ solution was similar to that of $1 \mathrm{mM} \mathrm{1,2}$ benzoquinone-3,5-disulfonic acid (BQDS) in $1 \mathrm{M} \mathrm{H}_{2} \mathrm{SO}_{4}$ solution [17]. The peak potential of $1.15 \mathrm{~V}$ vs. NHE was comparable to $1.13 \mathrm{~V}$ vs. NHE observed in the case of $1 \mathrm{mM}$ BQDS in $1 \mathrm{M} \mathrm{H}_{2} \mathrm{SO}_{4}$ solution [18] corresponding to the redox reaction of HQDS/BQDS. The hydrogen evolution reaction and oxygen evolution potentials at $\mathrm{pH} 2.0$ without overpotential were $-0.118 \mathrm{~V}$ vs. NHE and $1.111 \mathrm{~V}$ vs. NHE, respectively. The oxygen and hydrogen evolution reactions were observed at around $1.5 \mathrm{~V}$ vs. NHE and $0.0 \mathrm{~V}$ vs. NHE, respectively in $15 \mathrm{mM} \mathrm{H}_{2} \mathrm{SO}_{4}$. The $\mathrm{CV}$ for $5.5 \mathrm{M}$ $\mathrm{HAC}$ and $2 \mathrm{M} \mathrm{LiCl}$ and $10 \mathrm{~m} \mathrm{IBMmCl}$ with $1.0 \mathrm{M} \mathrm{HCl}$ indicated the initiation of the HQS oxidation 
reaction at around $0.7 \mathrm{~V}$ vs. $\mathrm{NHE}$, although no clear oxidation reaction peak was observed. The $\mathrm{CV}$ results for HQS in $15 \mathrm{mM} \mathrm{H}_{2} \mathrm{SO}_{4}$ confirmed that the HQS/BQS redox reaction proceeds by a twoelectron reaction of $\mathrm{BQS}+2 \mathrm{H}^{+}+2 \mathrm{e}=\mathrm{HQS}$ as reported previously for the hydroquinonebenzoquinone redox system in an aqueous solution [30]. Wu et al. observed the electrochemical oxidation reaction of $\mathrm{HAc}$ in an aqueous solution of $0.5 \mathrm{M} \mathrm{H}_{2} \mathrm{SO}_{4}$ at $1.14 \mathrm{~V}$ vs. $\mathrm{NHE}$ for a $\mathrm{Pt}_{60} \mathrm{Ru}_{10} \mathrm{Sn}_{30}$ electrode with a high Sn content [31], while Sine et al. suggested that the electrode with a low Sn content in $\mathrm{Pt}_{80} \mathrm{Ru}_{10} \mathrm{Sn}_{10}$ showed no oxidation of $\mathrm{HAc}$ in $1 \mathrm{M} \mathrm{HClO}_{3}-0.1 \mathrm{M} \mathrm{HAc}$ aqueous solution [32]. The results showed in the present study were in agreement with the above studies and exhibited no clear oxidation peak for HAc.

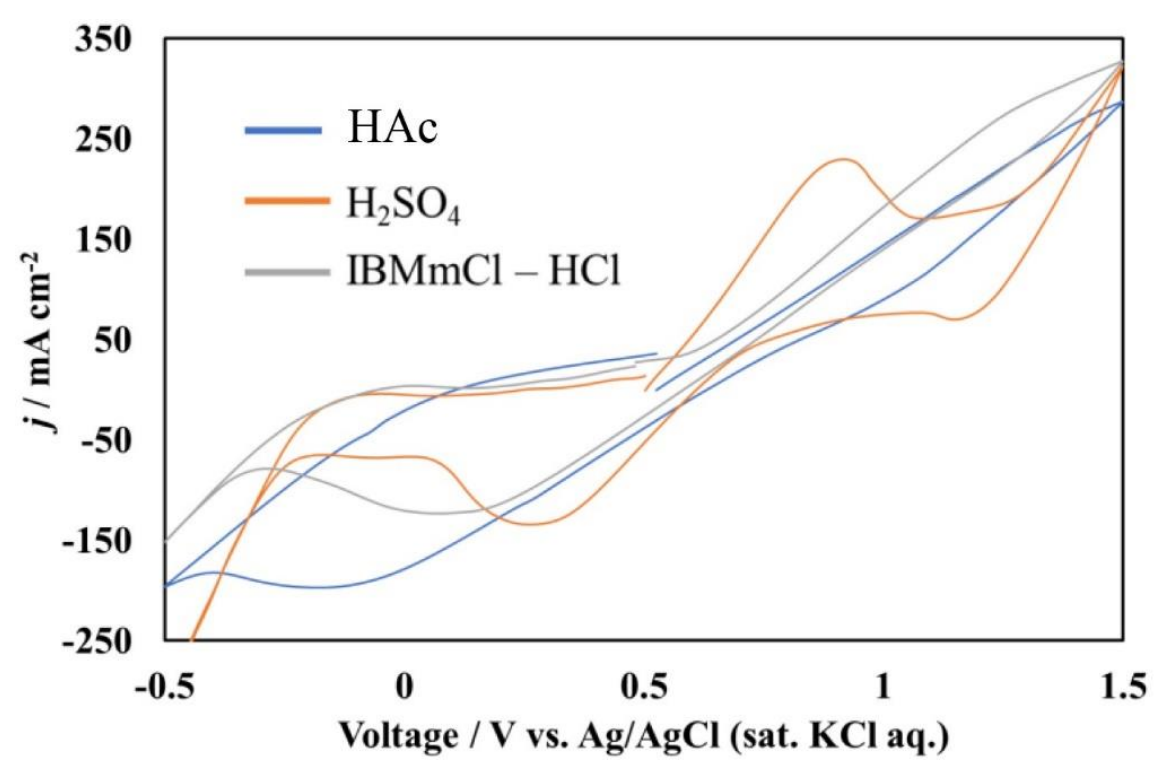

Figure 3 Cyclic voltammograms of the Pt-C electrode for $0.8 \mathrm{M} \mathrm{HQS}$ in $5.5 \mathrm{M} \mathrm{HAc}$ and 2 $\mathrm{M} \mathrm{LiCl}(\mathrm{HAC}), 15 \mathrm{mM} \mathrm{H}_{2} \mathrm{SO}_{4}\left(\mathrm{H}_{2} \mathrm{SO}_{4}\right)$, and $10 \mathrm{~m} \mathrm{IBMmCl}-1.0 \mathrm{M} \mathrm{HCl}(\mathrm{IBMmCl}-\mathrm{HCl}$ ) aqueous solutions at $25^{\circ} \mathrm{C}$. The scan rate was $5 \mathrm{mV} \mathrm{s}^{-1}$.

The redox reaction kinetics of HQS/BQS on Pt-C electrodes was examined in aqueous solutions with pH 2.0 at $25^{\circ} \mathrm{C}$. The overpotential (n) vs. current density (j) curves of $0.8 \mathrm{M}$ HQS in $5.5 \mathrm{M} \mathrm{HAc}$ $2 \mathrm{M} \mathrm{LiCl}(\mathrm{HAC}), 15 \mathrm{mM} \mathrm{H}_{2} \mathrm{SO}_{4}\left(\mathrm{H}_{2} \mathrm{SO}_{4}\right)$, and $10 \mathrm{~m} \mathrm{IBMmCl}-1.0 \mathrm{M} \mathrm{HCl}(\mathrm{IBMmCl}-\mathrm{HCl})$ aqueous solutions are shown in Figure 4, where the BQS reduction overpotentials were measured once around $20 \%$ of HQS was oxidized, and the overpotentials were recorded after the polarization for 10 min at each current density. The results showed that the oxidation overpotentials of HQS were low in the catholyte, whereas the reduction overpotentials of BQS were higher than the oxidation overpotential and depended on the catholyte. At a low current density, the lowest overpotential was observed in 0.8 M HQS taken in 5.5 M HAc-2 M LiCl (HAc), but at a high current density, the opposite was observed, and the overpotentials of the studied catholyte was higher than the other catholyte. The change in cell voltage with polarization period curves are shown in Figure 5 . The cells were charged initially at $0.5 \mathrm{mAcm}^{-2}$ for $10 \mathrm{mAh}$ and then discharged at $0.5 \mathrm{mAcm}^{-2}$. At this moment, around $80 \%$ of $\mathrm{BQS}$ was reduced to $\mathrm{HQS}$ with an overpotential of $0.5 \mathrm{~V}$. The flat discharge overpotentials of around $0.15 \mathrm{~V}$ at $0.5 \mathrm{mAcm}^{-2}$ were observed in $15 \mathrm{mM} \mathrm{H}_{2} \mathrm{SO}_{4}$ and $5.5 \mathrm{M} \mathrm{HAc}-2 \mathrm{M}$ $\mathrm{LiCl}$. 


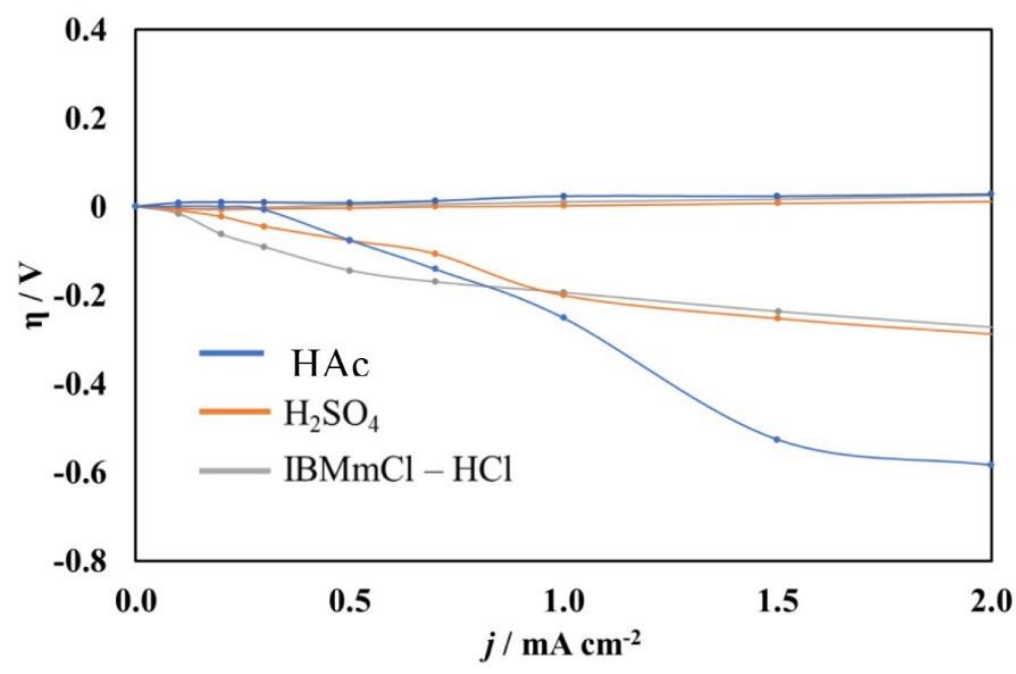

Figure 4 Overpotential ( $\eta$ ) vs. current density (j) curves for the oxidation of HQS and reduction of BQS on a Pt-C electrode in $0.8 \mathrm{M}$ HQS in $5.5 \mathrm{M} \mathrm{HAc}-2 \mathrm{M} \mathrm{LiCl}$ (HAc), $15 \mathrm{mM}$ $\mathrm{H}_{2} \mathrm{SO}_{4}\left(\mathrm{H}_{2} \mathrm{SO}_{4}\right)$, and $10 \mathrm{~m} \mathrm{IBMmCl}-1.0 \mathrm{M} \mathrm{HCl}(\mathrm{IBMmCl}-\mathrm{HCl})$ at $25^{\circ} \mathrm{C}$.

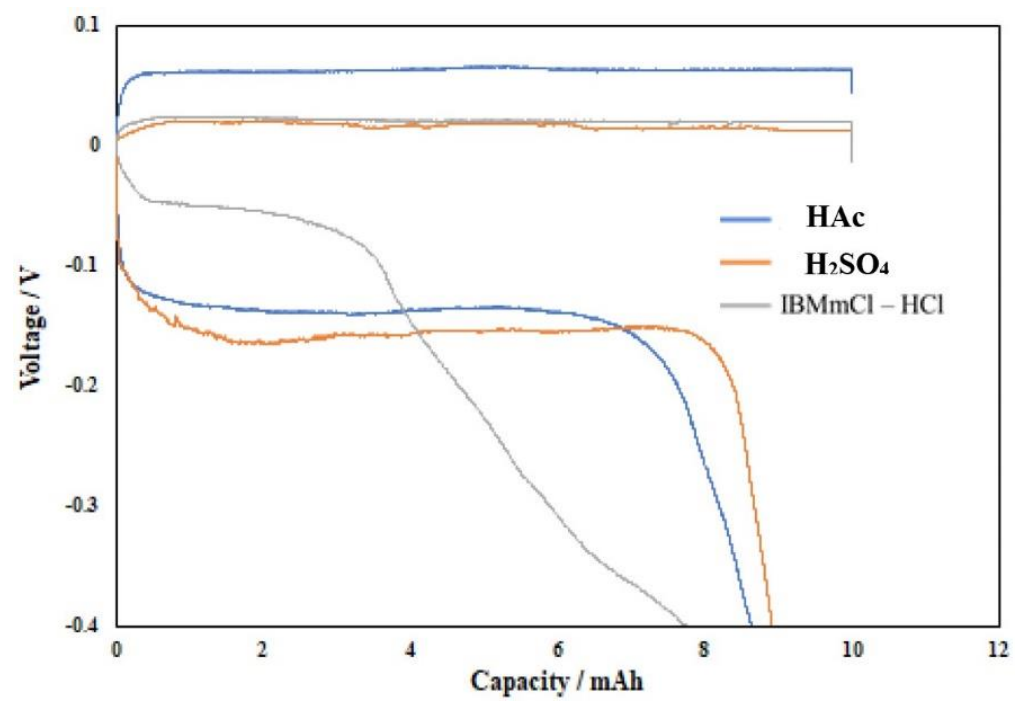

Figure 5 Electrode potential vs. capacity curves for HQS oxidation and BQS reduction on a Pt-C electrode in 0.8 M HQS in $5.5 \mathrm{M} \mathrm{HAc}-2 \mathrm{M} \mathrm{LiCl}(\mathrm{HAc}), 15 \mathrm{mM} \mathrm{H}_{2} \mathrm{SO}_{4}\left(\mathrm{H}_{2} \mathrm{SO}_{4}\right)$, and 10 $\mathrm{m}$ IBMmCl-1.0 M HCl (IBMmCl-HCl) at $25^{\circ} \mathrm{C}$.

The full cell performance of the lithium metal anode and the BQS/HQS redox couple cathode was also investigated. The Li/(LiFSI-2G)-50 vol\% DOL/LAGTP-epoxy-TiO $2 / 0.8 \mathrm{M} \mathrm{BQS} \mathrm{in} \mathrm{5.5} \mathrm{M} \mathrm{HAC-2} \mathrm{M} \mathrm{LiCl}$ aqueous solution/Pt-C cell was tested at $25{ }^{\circ} \mathrm{C}$. The OCV was $3.7 \mathrm{~V}$, which was slightly lower than that of $3.92 \mathrm{~V}$ estimated from the $\mathrm{BQS}$ reduction potential in $1 \mathrm{M} \mathrm{H}_{2} \mathrm{SO}_{4}$ reported in the literature [17]. The low OCV could be due to the high $\mathrm{pH}$ of the catholyte, the junction potential between LAGTP-epoxy- $\mathrm{TiO}_{2}$ and the aqueous catholyte and anode electrolyte, and low lithium-ion activity at the Li/anode electrolyte. The impedance profile of the full cell at $25{ }^{\circ} \mathrm{C}$ is shown in Figure 6 . The result showed two semicircles; one in the high-frequency range of $1 \mathrm{MHz}$ and $25 \mathrm{kHz}$ corresponds to the contribution of the grain boundary lithium-ion conduction in LAGTP-epoxy- $\mathrm{TiO}_{2}$ and the other in a low-frequency range of $25 \mathrm{kHz}$ and $0.1 \mathrm{kHz}$ attributed to the contribution of charge transfer 
resistance [33]. The intercept of the high-frequency semicircle with the real axis at high frequency is the sum of the bulk resistance of LAGTP-epoxy- $\mathrm{TiO}_{2}$ and the resistances of the buffer electrolyte and the catholyte. The ratio of these electrolyte resistances to the total cell resistance was around 0.5. In this study, a $1 \mathrm{~mm}$ thick anode electrolyte and $0.2 \mathrm{~mm}$ thick LAGTP-epoxy-TiO 2 separator were used. The electrolyte resistance could be reduced by reducing the thicknesses of these electrolytes. Figure 7 shows the charge and discharge overpotential vs. current density curve for the Li/(LiFSI-2G)-50 vol\% DOL/LAGTP-epoxy-TiO $2 / 0.8 \mathrm{M} \mathrm{HQS-5.5} \mathrm{M} \mathrm{HAc-2} \mathrm{M} \mathrm{LiCl} \mathrm{aqueous} \mathrm{cell,} \mathrm{where}$ the cell was precharged at $0.5 \mathrm{mAcm}^{-2}$ for $50 \mathrm{~h}$ (20\% of full charge). The charging overpotentials increased slightly with the increasing current density, while the discharging overpotential showed a significant increase. At $2 \mathrm{mAcm}^{-2}$, the round trip overpotential was $1.2 \mathrm{~V}$, corresponding to a 33\% energy loss.

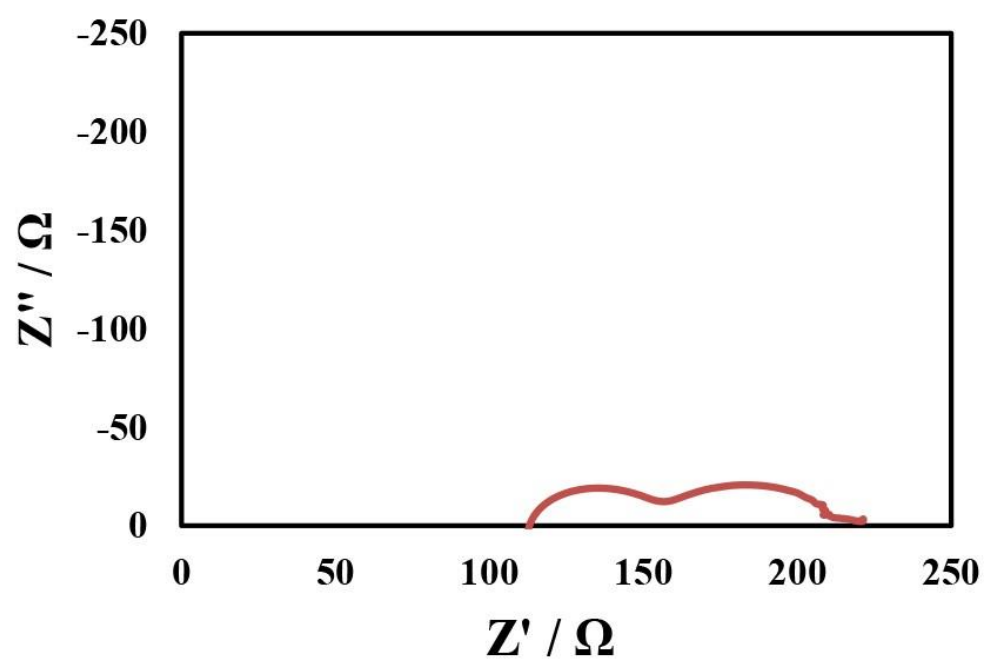

Figure 6 Impedance profile of Li/(LiFSI-2G)-50 vol\% DOL/LAGTP-epoxy-TiO $2 / 0.8 \mathrm{M} \mathrm{HQS}$ 5.5 M HAc-2 M LiCl aqueous solution/Pt-C cell at $25^{\circ} \mathrm{C}$.

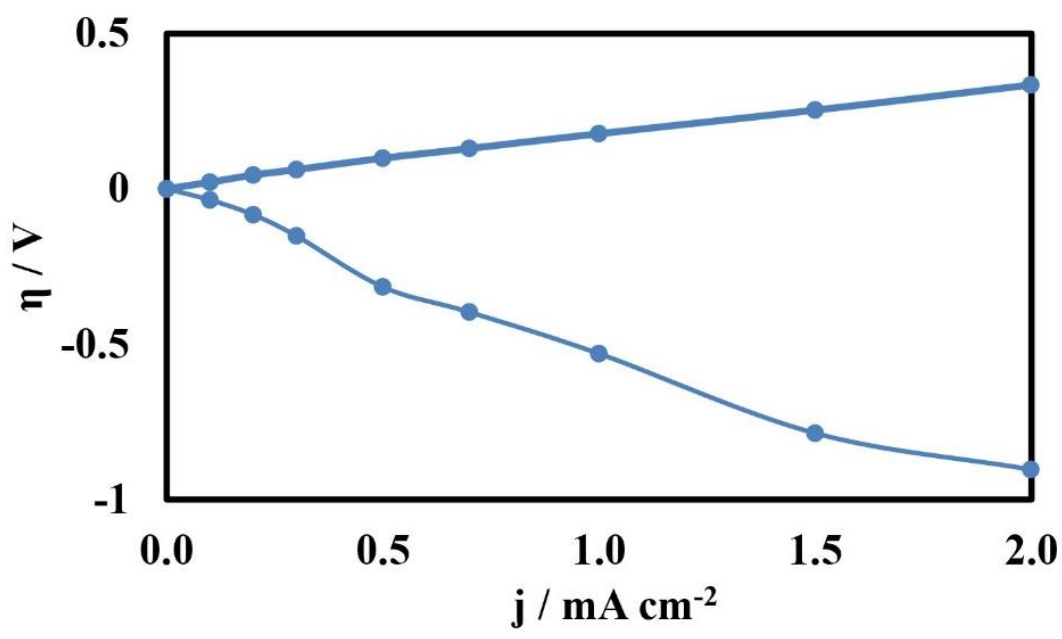

Figure 7 Overpotential ( $\eta$ ) vs. current density (j) curves for Li/(LiFSI-2G)-50 vol\% DOL/LAGTP-epoxy-TiO $2 / 0.8 \mathrm{M}$ HQS-5.5 M HAc-2 M LiCl aqueous solution/Pt-C cell at $25^{\circ} \mathrm{C}$. 
The cycling performance of the Li/(LiFSI-2G)-50 vol\% DOL/LAGTP-epoxy-TiO2/0.8 M HQS-5.5 M $\mathrm{HAc}-2 \mathrm{M} \mathrm{LiCl}$ aqueous solution/Pt-C cell at $0.5 \mathrm{~mA} \mathrm{~cm}^{-2}$ for $1 \mathrm{~h}$ discharge, $1 \mathrm{~h}$ rest, and then $1 \mathrm{~h}$ charge cycle at $25^{\circ} \mathrm{C}$ is shown in Figure 8 . The round-trip overpotential was $0.4 \mathrm{~V}$, which corresponds to a $12 \%$ energy loss. The discharge voltages were slightly decreased with each cycle. The deep charge and discharge profiles at $0.5 \mathrm{mAcm}^{-2}$ are shown in Figure 9. The cut-off voltages for charge and discharge were $4.5 \mathrm{~V}$ and $2.5 \mathrm{~V}$, respectively, and the mass of the charged HQS was $0.189 \mathrm{~g}(65 \mathrm{mAh})$. The observed charge and discharge capacities were 49.6 and $26.5 \mathrm{mAh}$, respectively, which corresponded to $76 \%$ and $41 \%$ utility of the active material in the catholyte, respectively. The potential plateau for the hydrogen evolution reaction was observed at around $2.2 \mathrm{~V}$. The calculated energy density of the proposed Li/HQSK cell was $358 \mathrm{Whkg}^{-1}$ based on the masses of $\mathrm{Li}, \mathrm{H}_{2} \mathrm{O}$, and $\mathrm{BQS}$ and $41 \%$ utilization of BQS with an operating voltage of $3.4 \mathrm{~V}$. The calculated energy density of the proposed new type aqueous lithium battery was comparable to that of the convention lithiumion battery. The calculated specific energy density of the cell generally depends on the specific area capacity [34], and the cell performance of a solid active material such as $\mathrm{LiCoO}_{2}$ in the lithium-ion batteries depends on the thickness of the electrode [35]. However, the cell performance of a cell with a liquid catholyte shows no significant dependence on the specific area capacity, as reported in the literature [11]. Hence, the proposed Li/BQS battery with a liquid catholyte could provide high energy density at a high area capacity.

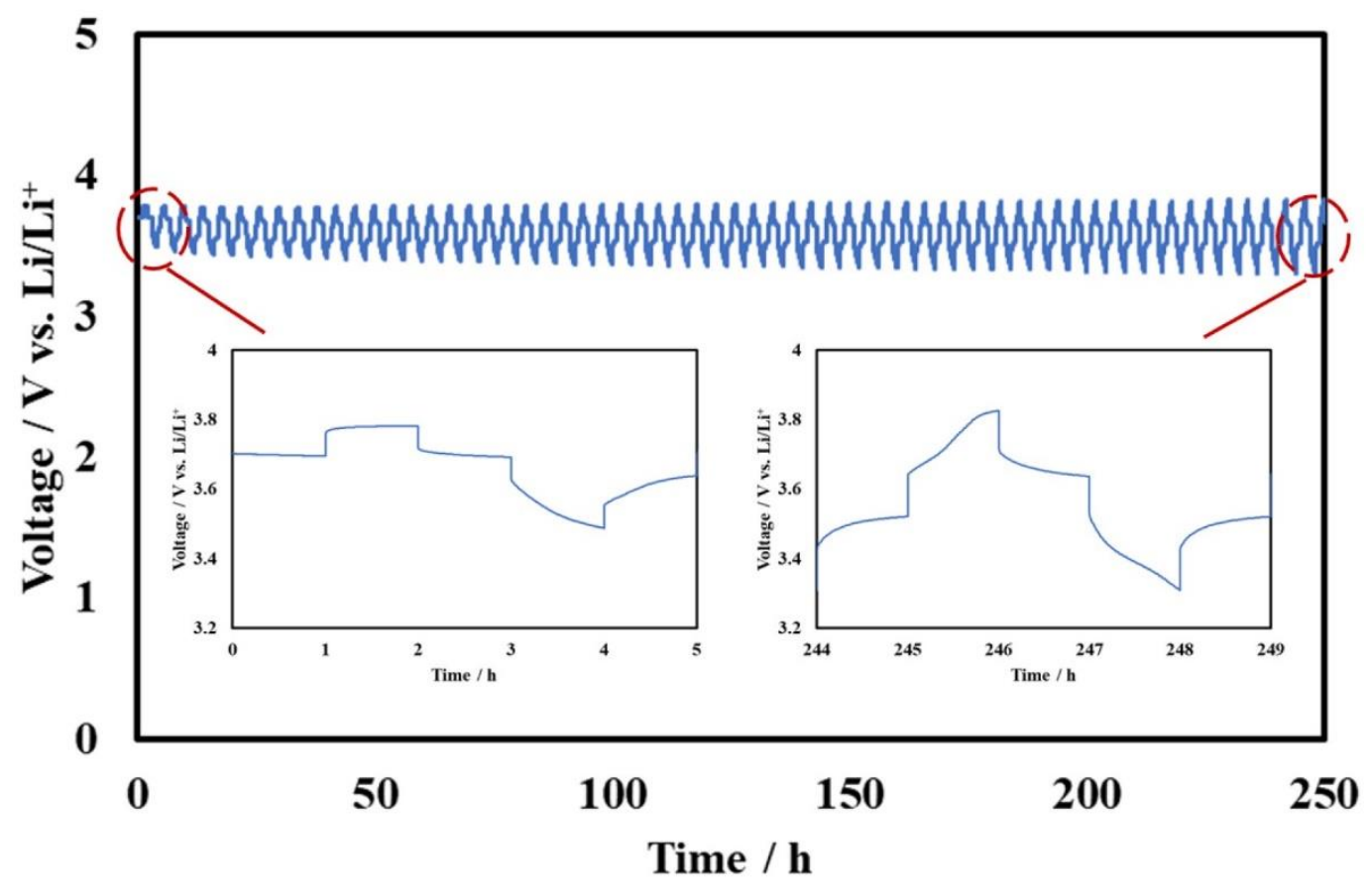

Figure 8 Cycling performance of the Li/(LiFSI-2G)-50 vol\%DOL/LAGTP-epoxy-TiO $2 / 0.8 \mathrm{M}$ HQS-5.5 M HAc-2 M LiCl aqueous solution/Pt-C cell at $0.5 \mathrm{mAcm}^{-2}$ and $25^{\circ} \mathrm{C}$. 


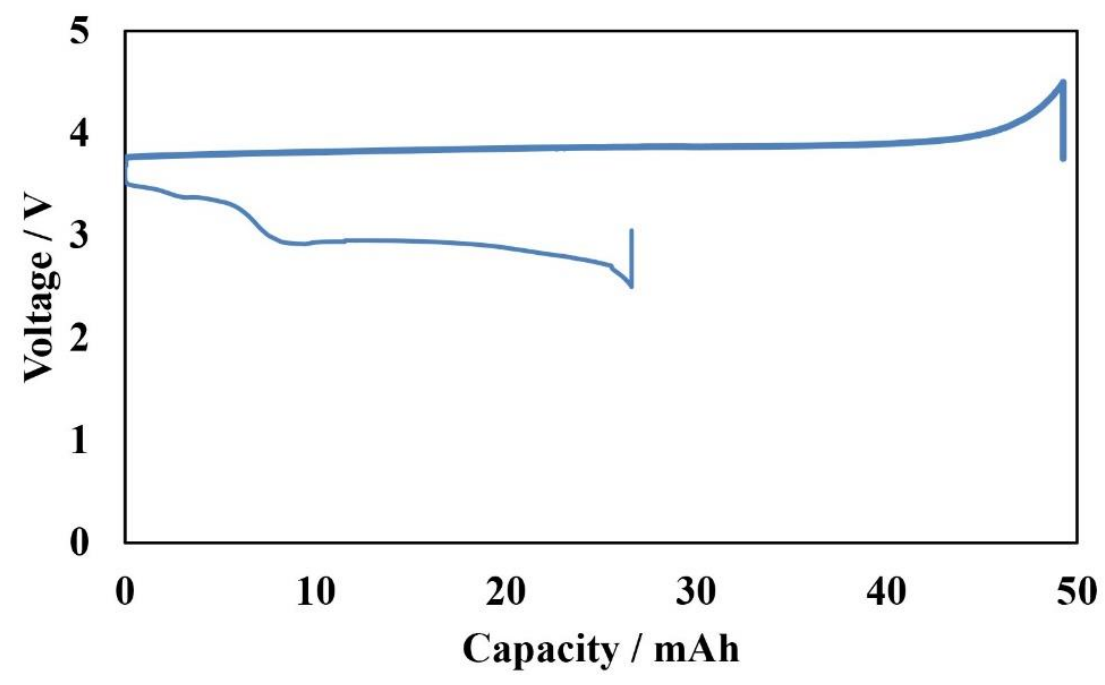

Figure 9 Charge and discharge curves of the Li/(LiFSI-2G)-50 vol\% DOL/LAGTP-epoxy$\mathrm{TiO}_{2} / 0.8 \mathrm{M} \mathrm{HQS}-5.5 \mathrm{M} \mathrm{HAc}-2 \mathrm{M} \mathrm{LiCl}$ aqueous solution/Pt-C cell at $0.5 \mathrm{mAcm}^{-2}$ and $25^{\circ} \mathrm{C}$.

\section{Conclusion}

The proposed novel aqueous lithium battery with a water-soluble and less expensive organic catholyte showed a higher theoretical specific energy density than conventional lithium-ion batteries. The cell performed well at $0.5 \mathrm{mAcm}^{-2}$ and $25^{\circ} \mathrm{C}$ and showed no significant degradation after 37 cycles. The battery with the aqueous catholyte could charge and discharge at a high specific area capacity, and the cost of the cathode active materials was lower than that of conventional lithium-ion batteries. Thus the proposed novel battery has a potential application for large-capacity batteries for electric vehicles and electric energy storage systems.

\section{Author Contributions}

HT prepared the solid electrolyte thin films, performed electrochemical measurements, and setup the full cell. KK prepared LAGTP fine powder. DM, S T, YT and OY supported and gave advice on each experimental work. $\mathrm{NI}$ managed the research direction and provided instructions and opportunities for discussions among all authors

\section{Funding}

This work was supported by JST Adaptable and Seamless Technology transfer Program through Target-driven R\&D (A-STEP) Grant Number JPMJTM120EP.

\section{Competing Interests}

The authors have declared that no competing interests exist.

\section{References}

1. Whittinham MS. Lithium batteries and cathode materials. Chem Rev. 2004; 104: 4271-4302.

2. Bruce PG, Freunberger SA, Hardwick $\mathrm{L}$, Tarascon JM. Li- $\mathrm{O}_{2}$ and Li-S batteries with high energy storage. Nat Mater. 2012; 11: 19-29. 
3. Visco SJ, Nimon E, Katz B, De Johne LC, Chu MY. Lithium metal aqueous batteries. Proceeding of 12th International Meeting on Lithium Batteries; 2004 June 27-July 2; Nara, Japan. Amsterdam: Elsevier.

4. Visco SJ, Nimon YS. Active metal/aqueous electrochemical cells and systems. U.S. Patent application; 2007; US7645543B2.

5. Imanishi N, Yamamoto O. Perspectives and challenges of rechargeable lithium-air batteries. Mater Today Adv. 2019; 4: 100031.

6. Li W, Dahn JR, Wainright DS. Rechargeable lithium batteries with aqueous electrolytes. Science. 1994; 264: 1115-1118.

7. Kim H, Hong J, Park KY, Kim H, Kim SW, Kang K. Aqueous rechargeable Li and Na ion batteries. Chem Rev. 2014; 114: 11788-11827.

8. Li H, Wang Y, Na H, Liu H, Zhou H. Rechargeable Ni-Li battery integrated aqueous/nonaqueous system. J Am Chem Soc. 2009; 131: 15098-15099.

9. Lu Y, Goodenough JB, Kim Y. Aqueous cathode for next-generation alkali-ion batteries. J Am Chem Soc. 2011; 133: 5756-5759.

10. Wang $X$, Hou Y, Zhu Y, Wu Y, Holze R. An aqueous rechargeable lithium battery using coated Li metal as anode. Sci Rep. 2013; 3: 1401.

11. Morita $Y$, Watanabe $S$, Zhang $P$, Wang $H$, Mori D, Matsuda $Y$, et al. High specific energy density aqueous lithium-metal chloride rechargeable batteries. J Electrochem Soc. 2017; 164: A1958.

12. Morita Y, Watanabe S, Mori D, Takeda Y, Yamamoto O, Imanishi N. High-energy-density rechargeable lithium-nickel chloride aqueous solution batteries. ACS Omega. 2018; 3: 55585562.

13. Watanabe S, Mori D, Taminato S, Matsuda Y, Yamamoto O, Takeda Y, et al. Aqueous lithium rechargeable battery with a Tin (II) chloride aqueous cathode and a water-stable lithium-ion conducting solid electrolyte. J Electrochem Soc. 2019; 166: A539.

14. Fei $H$, Liu Y, An Y, Xu X, Zeng G, Tian Y, et al. Stable all-solid-state potassium battery operating at room temperature with a composite polymer electrolyte and a sustainable organic cathode. J Power Sources. 2018; 399: 294-298.

15. Fieser LF. The tautomerism of hydroxy quinones. J Am Chem Soc. 1928; 50: 439-465.

16. Guin PS, Das S, Mandal PC. Electrochemical reduction of quinones in different media: A review. Int J Electrochem. 2011; 2011: 816202.

17. Bailey SI, Rritchie IM. A cyclic voltammetric study of the aqueous electrochemistry of some quinones. Electrochim Acta. 1985; 30: 3-12.

18. Yang B, Hoober-Burkhardt L, Wang F, Prakash GS, Narayanan SR. An inexpensive aqueous flow battery for large-scale electrical energy storage based on water-soluble organic redox couples. J Electrochem Soc. 2014: 161: A1371

19. Yang B, Hoober-Burkhardt L, Krishnamoorthy S, Murali A, Prakash GS, Narayanan SR. Highperformance aqueous organic flow battery with quinone-based redox couples at both electrodes. J Electrochem Soc. 2016; 163: A1442.

20. Hoober-Burkhardt L, Krishnamoorthy S, Yang B, Murali A, Nirmalchandar A, Prakash GS, et al. A New michael-reaction-resistant benzoquinone for aqueous organic redox flow batteries. J Electrochem Soc. 2017; 164: A600. 
21. Shimonishi $Y$, Zhang $T$, Johnson $P$, Imanishi N, Hirano A, Takeda $Y$, et al. A study on lithium/air secondary batteries - Stability of NASICON-type glass ceramic in acid solution. J Power Sources. 2010; 195: 6187-6191.

22. Wang $H$, Matsui $M$, Kuwata $H$, Sonoki $H$, Matsuda $Y$, Shang $X$, et al. A reversible dendrite-free high-areal-capacity lithium metal electrode. Nat Commun. 2017; 8: 15106.

23. Bai $F$, Shang $X$, Mori $D$, Taminato $S$, Matsumoto $M$, Watanabe $S$, et al. High lithium-ion conducting solid electrolyte thin film of $\mathrm{Li}_{1.4} \mathrm{Al}_{0.4} \mathrm{Ge}_{0.2} \mathrm{Ti}_{1.4}\left(\mathrm{PO}_{4}\right)_{3}-\mathrm{TiO}_{2}$ for aqueous lithium secondary batteries. Solid State Ion. 2019; 338: 127-133.

24. Kyono N, Bai F, Nemori H, Minami H, Mori D, Takeda $Y$, et al. Lithium-ion conducting solid electrolytes of $\mathrm{Li}_{1.4} \mathrm{Al}_{0.4} \mathrm{Ge}_{0.2} \mathrm{Ti}_{1.4}\left(\mathrm{PO}_{4}\right)_{3}$ and $\mathrm{MO}_{\mathbf{x}}(\mathrm{M}=\mathrm{Al}, \mathrm{Ti}$, and $\mathrm{Zr})$ composites. Solid State Ion. 2018; 324: 114-127.

25. Takahashi K, Johnson P, Imanishi N, Sammes N, Takeda Y, Yamamoto O. A water stable high lithium ion conducting $\mathrm{Li}_{1.4} \mathrm{Ti}_{1.6} \mathrm{Al}_{0.4}\left(\mathrm{PO}_{4}\right)_{3}$-epoxy resin hybrid sheet. J Electrochem Soc. 2012; 159: A1065.

26. Hasegawa S, Imanishi N, Zhang T, Xie J, Hirano A, Takeda Y, et al. Study on lithium/air secondary batteries-Stability of NASICON-type lithium ion conducting glass-ceramics with water. J Power Sources. 2009; 189: 371-377.

27. Shimonishi Y, Zhang T, Imanishi N, Im D, Lee DJ, Hirano A, et al. A study on lithium/air secondary batteries-Stability of the NASICON-type lithium ion conducting solid electrolyte in alkaline aqueous solutions. J Power Sources. 2011; 196: 5128-5132.

28. Zhang T, Imanishi N, Takeda Y, Yamamoto O. Aqueous lithium/air rechargeable batteries. Chem Lett. 2011; 40: 668-673.

29. Ye R, Henkensmeier D, Chen R. Imidazolium cation enabled reversibility of a hydroquinone derivative for designing aqueous redox electrolytes. Sustain Energy Fuel. 2020; 4: 2998-3005.

30. Quan M, Sanchez D, Wasylkiw MF, Smith DK. Voltammetry of quinones in unbuffered aqueous solution: Reassessing the roles of proton transfer and hydrogen bonding in the aqueous electrochemistry of quinones. J Am Chem Soc. 2007; 129: 12847-12856.

31. Wu G, Swaidan R, Cui G. Electrooxidations of ethanol, acetaldehyde and acetic acid using PtRuSn/C catalysts prepared by modified alcohol-reduction process. J Power Sources. 2007; 172: 180-188.

32. Siné $G$, Smida $D$, Limat $M$, Foti G, Comninellis C. Microemulsion synthesized Pt/Ru/Sn nanoparticles on BDD for alcohol electro-oxidation. J Electrochem Soc. 2007; 154: B170.

33. Bruce PG, West AR. Ionic conductivity of LISICON solid solutions, $\mathrm{Li}_{2+2 x} \mathrm{Zn}_{1-x} \mathrm{GeO}_{4}$. J Solid State Chem. 1982; 44: 354-365.

34. Park MS, Ma SB, Lee DJ, Im D, Doo SG, Yamamoto O. A highly reversible lithium metal anode. Sci Rep. 2014; 4: 3815-3822.

35. Zheng H, Li J, Song X, Liu G, Battaglia VS. A comprehensive understanding of electrode thickness effects on the electrochemical performances of Li-ion battery cathodes. Electrochim Acta. 2012; 71: 258-265. 
JEPT 2021; 3(1), doi:10.21926/jept.2101010

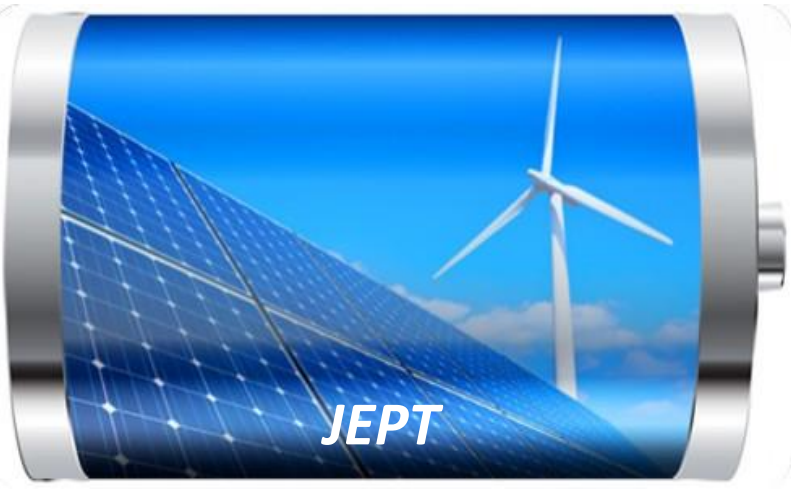

Enjoy JEPT by:

1. Submitting a manuscript

2. Joining in volunteer reviewer bank

3. Joining Editorial Board

4. Guest editing a special issue

For more details, please visit:

http://www.lidsen.com/journal/jept 
Review

\title{
Accelerated Controller Tuning for Wind Turbines Under Multiple Hazards
}

Aly Mousaad Aly ${ }^{*}$, Milad Rezaee

Windstorm Impact, Science and Engineering (WISE) Research Lab, Department of Civil and Environment Engineering, Louisiana State University, 3230H Patrick Taylor Hall, Baton Rouge, LA 70803, USA; E-Mails: aly@LSU.edu; mrezae2@lsu.edu

* Correspondence: Aly Mousaad Aly; E-Mail: aly@LSU.edu

Academic Editor: Aritra Ghosh

Special Issue: Progress of Wind Energy Technology and Its Maintenance

Journal of Energy and Power Technology

2021, volume 3 , issue 1

doi:10.21926/jept.2101011
Received: December 26, 2020

Accepted: February 19, 2021

Published: March 04, 2021

\begin{abstract}
During their lifecycle, wind turbines can be subjected to multiple hazard loads, such as highintensity wind, earthquake, wave, and mechanical unbalance. Excessive vibrations, due to these loads, can have detrimental effects on energy production, structural lifecycle, and the initial cost of wind turbines. Vibration control by various means, such as passive, active, and semi-active control systems provide crucial solutions to these issues. We developed a novel control theory that enables semi-active controller tuning under the complex structural behavior and inherent system nonlinearity. The proposed theory enables the evaluation of semi-active controllers' performance of multi-degrees-of-freedom systems, without the need for time-consuming simulations. A wide range of controllers can be tested in a fraction of a second, and their parameters can be tuned to achieve system-level performance for different optimization objectives.
\end{abstract}

\section{Keywords}

Control theory; semi-active control; vibration; wind turbines; multiple hazards; clean energy; smart structures; optimization; damper

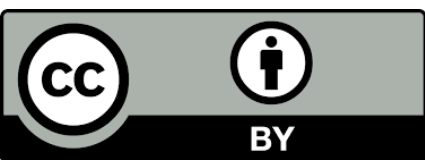

(C) 2021 by the author. This is an open access article distributed under the conditions of the Creative Commons by Attribution License, which permits unrestricted use, distribution, and reproduction in any medium or format, provided the original work is correctly cited. 


\section{Introduction}

The increasing energy consumption and the need for dependable, secure, and clean energy have led to explorations in non-conventional sources, particularly in renewable sources of energy. During the past decade, wind energy has been known as the fastest-growing source of electricity in the world, and it is anticipated to play a significant role in sustainably and powering the future. Today, wind energy has already become one of the main electricity sources in some countries such as Denmark, Portugal, Spain, and Germany, where the contribution of wind energy to the total power is $21 \%, 18 \%, 16 \%$, and $9 \%$, respectively [1]. In 2015, the contribution of wind energy to the nation's electricity in the United States was about $1 \%$, while potentially it could be increased up to $20 \%$, without major changes in the distribution system [2].

Wind turbines can be deployed in coastal zones, inland, and offshore areas, wherever the wind is powerful and uniform, and close enough to the market. However, installing wind turbines in areas with powerful winds and at a short distance to the market can lead to significant design challenges. Modern wind turbines likely face various natural hazards. The population concentration in coastal areas, and the availability of more powerful and uniform wind resources, accelerated the development of coastal and offshore wind farms. However, environmental loads, such as wind and waves are harsher in coastal and offshore areas, which can cause severe damages to wind turbines, or make the produced power more expensive and less dependable.

It has been less than 15 years since wind farms started to be installed in seismic prone areas. Therefore, due to the lack of experience, there is no guaranty that modern wind turbines can survive extreme earthquakes [3]. The effect of pulse-like near-fault ground motions on utility-scale landbased wind turbines was investigated [4]. Flexural testing was conducted on a full-scale 60-kW wind turbine's tower, telescopic steel pipe [5]. The concerns over the possibility of exposure of more modern wind turbines to multi-hazard loads make the understanding of the structural behavior more important than before. These concerns motivated a global endeavor to study and evaluate the performance of modern wind turbines, exposed to various loading scenarios. The efforts are continuing, to provide a comprehensive perspective of the performance and functionality at a system-level, for wind turbines to survive multi-hazard loads.

According to the findings of other researchers, an important trait of modern wind turbines that needs consideration is the flexibility of the structure and the challenge of damping deficit. Consequently, the exposure of a wind turbine to the intense environmental dynamic loads can cause excessive vibrations in the system. The undesirable motion can impose several aftereffects on the wind turbine, such as malfunctioning of mechanical parts in the nacelle or the rotor, power production disorders, reduced wind turbine's availability, crack deployment through the machine, reduced fatigue life of the system, increased likelihood of failure, and the need for more frequent maintenance. The direct and indirect consequences of these problems can lead to costly generated electricity.

Despite a limited number of studies on the evaluation and mitigation of wind turbines under multi-hazard loads, during the past decades, there were ongoing researches and advances in structural dynamic performance improvements of high-rise buildings and tall structures excited by wind and seismic loads [6-11]. It is shown in previous studies that employing external dampers is an 
effective method to control the vibration of lightly damped, tall, and flexible civil engineering structures [8, 12-16]. However, to design for multi-hazard loads, the aftereffects of applying a specific vibration mitigation method should be seen from a wider perspective. For instance, while vibration isolation is identified as an acceptable approach to increase the damping capacity of a structure under earthquakes, it might not be effective to mitigate wind-induced vibrations. The dynamic response of a base-isolated structure was numerically investigated and optimized for both wind and earthquake [17]. The results showed that base-isolation could not suppress excessive accelerations during high wind events. Similar results are observed from studies that investigated installing the vibration absorbers at the top of a turbine's tower, or the foundation, using semiactive MR dampers $[18,19]$. Implementing an isolation system is beneficial for reducing certain turbine responses, particularly for wind turbines in seismically active regions. In contrast, the hub displacement was increased. The other examples are tuned mass dampers (TMDs) and tuned liquid dampers (TLDs) that more or less have acceptable performance for wind-induced vibrations in wind turbines [10, 20-22]. There is no solid agreement, whether or not they are effective enough for reducing ground motion-induced vibrations. TMDs are shown to be effective in wind response control of wind turbines with soil-structure interaction [23]. Some researchers found them ineffective or not significantly effective to mitigate the seismic response [24-26]. Other researchers reported considerable response reduction using TMDs [27-32]. Moreover, having no control over tuning the controller during operation is another important problem of the passive dampers. To tune the passive TMD, comprehensive studies should be done [33], to investigate the performance of the controller under diverse load scenarios and tune the frequency for a specific case. However, the tuning frequency depends on the predefined load scenario and it is not adjustable, which is a drawback for the multi-hazard loading case, where different loading characteristics are expected. In contrast, smart dampers can produce variable control forces, which make them more effective, compared to passive TMDs as reported in various studies [34, 35].

The effectiveness of semi-active and active controllers in mitigating wind- and earthquakeinduced vibrations of tall structures was investigated extensively [10, 36-43]. While active dampers show an effective performance to reduce the responses since they inject significant non-dissipative energy to the system and they need external power, employing active dampers to control wind turbines' structural vibration is an imperfect solution due to: (1) the controller can cause instability [44], and (2) the power consumption can be a significant concern in this approach [45, 46]. However, semi-active dampers showed acceptable performance as they benefit from the positive characteristics of both passive and active controllers. Semi-active dampers do not suffer passive controllers' drawbacks, regarding the frequency tuning, and active controllers' problems, including the need for significant external power resources and instability challenges $[10,11]$. Wind turbines are already installed with power electronic devices, therefore it is convenient to control blade and tower vibrations by the use of power electronics-based controllers [47]. The literature has some detailed comparison between passive and semi-active TMDs, in terms of performance, dissipated energy, and TMD stroke [10, 48].

An important design task related to the application of controllers to suppress vibrations of a structure is tuning the controller's parameters according to the structure's properties, control objectives, and loading scenarios. In a semi-active damper, the performance of the controller and its success to suppress excessive vibrations depends on the desired control force, as well as the control algorithm that commands the damper. While there are a few recent studies on the 
advantages of employing semi-active dampers in wind turbines excited by multi-hazard loads [35, 49], the evaluation of the controller performance and structural behavior depends on the simulation of several cases with a family of controllers. Therefore, selecting a control approach is not generic and independent of the simulation.

Vibration mitigation through increasing damping can be an effective approach, to remedy these problems. However, vibration mitigation is not a straightforward approach that can be achieved following specific steps for all wind turbines, regardless of their environmental conditions. Since wind turbines may confront unequal natural hazards during their useful life, it should be taken into consideration that each type of natural hazard could have a specific impact on the structure, depending on the overall environmental conditions and structural characteristics. Therefore, it is of utmost importance to assess the structural performance and evaluate the mitigation approaches for various scenarios, to ascertain a proposed solution for one hazard does not make the structure more vulnerable to another hazard. Structural modeling and evaluating the performance of dynamic systems with multi-degree-of-freedom is generally a time-consuming process. Evaluating these systems with various control methods makes the models even more tedious and time-consuming. To overcome these issues, an innovative analytical approach is proposed to evaluate the performance of the controlled wind turbines that are modeled as multi-degree-of-freedom systems.

Considering the aforementioned points regarding the effects of multi-hazard loads on wind turbines, and structural control concerns, the current study aimed to address the context of the design of semi-active controllers for wind turbines under multi-hazard loads. This paper employs an energy-based analytical approach to design the controllers for a wind turbine in parked and operating conditions. An outer bracing MR damper system connected to the tower is presented. The control system is identified as an effective option for a wind turbine subjected to seismic and wind loads $[10,50]$. In the next sections, an introduction to wind turbine systems, dynamic modeling, and control will be presented, followed by an application example of a $5 \mathrm{MW}$ wind turbine subjected to wind and earthquake loads. The paper ends with a summary of the main findings.

\section{Wind Turbines}

Wind turbines are devices that harvest the power of the wind and convert it into a useful form of energy (electricity). Wind power makes the blades of a wind turbine turn around a rotor. The main shaft transfers this rotation to the generator via a gearbox unit and the generator converts the mechanical energy to electrical energy. A wind turbine consists of a hub, rotor, blades, nacelle, and tower. Figure 1 illustrates a detailed view of the inside of a wind turbine. The blades and the rotor are rotary parts, moving to bypass the wind through the rotor plane. The nacelle is a house for the mechanical parts and the tower is a foundation for the nacelle and the rotor to place them at an appropriate height. The materials that are used to build each component are continually changed and improved over the years, mainly because of significant changes in the height and size of the turbine, to harvest more wind energy by a single unit. 


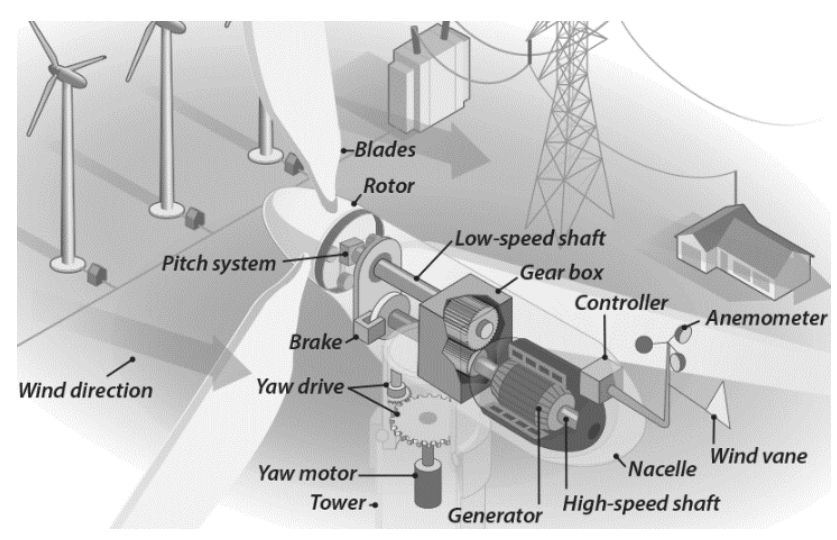

Figure 1 Anatomy of a wind turbine [51].

Developing technology yields increasing wind turbines' efficiency, rotor size, height, and more extracted energy. Developing technology also caused a continuous change in the material used in the construction of wind turbines. The trend is toward lighter weight and low-cost materials, especially for the blades and towers. The tower weight is typically $60 \%$ of the weight of the turbine above the foundation [52]. Therefore, the trend is toward using lighter steel and composite materials. An important issue that should be considered is the material fatigue properties. The majority of the components of a wind turbine, with a useful life of 30 years, are required to be able to sustain $4 \times 10^{8}$ fatigue stress cycles. This is even stricter than the fatigue stress cycles of other man-made structures, such as engines, aircraft, and bridges [52].

The tower can be made of various materials. Steel lattice is the material that was commonly used in early towers. However, because of the exposure of numbers of connections to corrosion, and the sensitivity of weak diagonals to wind excitation, the use of steel lattice towers is not safe to support the modern and higher capacity turbines [53]. Hence, steel lattice was replaced by cylindrical steel tubes in modern towers. Besides, for towers higher than $85 \mathrm{~m}$, concrete can have better performance to balance the vibration excitation, compared to steel tube towers [54]. However, concrete towers may suffer the problem of thermal constraints, leading to cracking, and consequently changing the natural frequency and structural characteristics. The main material used in wind turbines from 2001 to 2010 in the U.S. and worldwide was steel, particularly for large wind turbines [52]. Besides, in coastal areas, the part of fixed wind turbines that are placed in water is normally constructed from concrete.

According to the mentioned points, because the modern wind turbines are taller and lighter than the former generations, these structures are more flexible than before. This flexibility makes them sensitive to the environmental dynamic loads and causes excessive vibrations in the tower as well as the whole structure. Severe vibrations can cause functionality malfunctioning or permanent damages to acceleration-sensitive mechanical parts or may reduce the structure's fatigue life and increase the chance of systematic failure.

In a wind turbine, damage can occur everywhere from mechanical and electrical parts to structural parts. However, from a civil engineering point of view, the damages can occur through the foundation, the tower steel tube, bolts, or blades. Structural failures are reported in various countries, such as Scotland, Spain, Germany, Japan, France, Wales, Denmark, and New Zealand [55]. The investigation of wind turbine failures in Germany shows that all mechanical components have almost equal damage frequency as the primary structure [56]. 
Structural failure has been the third most common industrial accident in wind turbines, with 183 reported instances from the 1970s to 2017 [57]. Storm damage, tower collapse, poor control, lack of maintenance, and component failure were the main concerns in structural failures [57]. Figure 2 shows several structural and blade failures between the 1970s and 2017.

shows the collapse of two wind turbines, due to structural failure in Cape Breton, Canada, and Kansas, U.S. Failure of a wind turbine can have both societal and economic impacts. Modern wind energy harvesting is in a transient stage in most countries from just a potential source of energy to a dominant source of electricity. Condition monitoring and fault detection of wind turbines can improve the reliability of wind turbine power generation $[58,59]$. The failure of wind turbines can make doubt on the reliability of this energy and increase the power production costs. Considering the aforementioned points, and the consequences of failure, it is crucial to study wind turbines from a structural point of view, under dynamic loads, to advance their design.

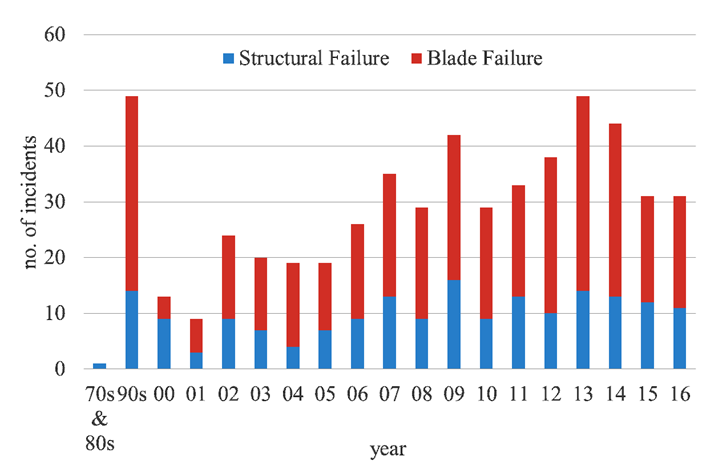

Figure 2 Structural and blade failures between 1970 and 2017.

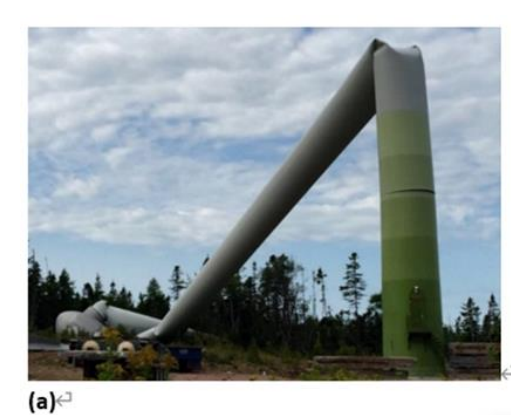

(a)

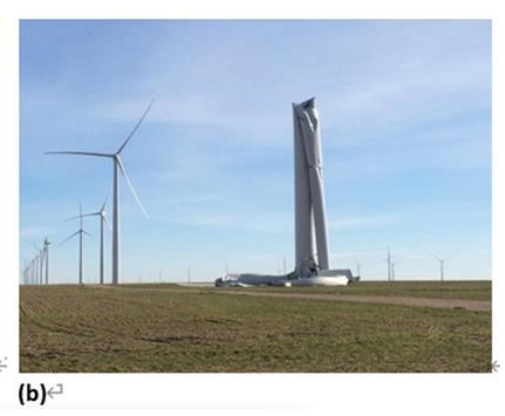

Figure 3 Wind turbine tower collapse: (a) Cape Breton, Canada [60], and (b) Kansas, USA [61].

\section{Structural Dynamics}

Under the action of environmental loads, a wind turbine, as a tall and flexible structure, responds according to the load characteristics and the structure's properties. Like other tall structures, there are several studies on the structural dynamic behavior of wind turbines, particularly by increasing the size of these structures, the static analysis becomes insufficient to design the support structures. As a very basic assumption, a wind turbine can be considered as a spring-dashpot single-degree-offreedom (SDOF) system excited with a harmonic or random force, or constrained mass at a top of a cantilevered beam [62, 63] (Figure 4). Here, the governing equation of motion for the system can be obtained from Newton's second law as follows. 


$$
M \ddot{X}+C \dot{X}+K X=F(t)
$$

Figure 4 Schematic representation of a wind turbine and its simplified equivalent SDOF structural and mechanical spring-dashpot system model.

Where $X$ is the displacement response, $M, C$, and $K$ are the mass, damping coefficient, and stiffness, respectively, and $F$ is the excitation force. The dots on the top of the displacement $(X)$ designate the $1^{\text {st }}$ and $2^{\text {nd }}$ derivatives, representing the velocity and acceleration of the system, respectively. This equation can be rewritten in another form that relates the responses to the damping ratio and the natural frequency of the system:

$$
\ddot{X}+2 \zeta \omega \dot{X}+\omega^{2} X=\frac{1}{M} F(t)
$$

here, $\omega$ and $\zeta$ are the natural frequency and the damping ratio of the system, respectively. As a very basic assumption, the natural frequency, $f_{n 1}$, can be approximated as follows [63].

$$
f_{n 1}^{2}=\frac{3.04}{4 \pi^{2}} \frac{E I}{(M+0.227 \mu L) L^{3}}
$$

$\mu$ is the tower mass per meter, $M$ is the top mass, $E l$ is the tower bending stiffness, and $L$ is the tower height. Considering the nature of the environmental loads on wind turbines, the structure should be designed to have the first natural frequency between the two limits called $1 \mathrm{P}$ and $3 \mathrm{P}$. Where $1 \mathrm{P}$ is a rotor loading frequency and $3 \mathrm{P}$ is the blade passing frequency for a three-bladed wind turbine. These frequency limits depend on the type of wind turbine, foundation, and the dominant environmental force. Designing the structure that its natural frequency places somewhere between $1 \mathrm{P}$ and $3 \mathrm{P}$ are called a soft-stiff design approach. Here, in this approach, the wind turbine is not that stiff to have a natural frequency higher than $3 \mathrm{P}$ (say $1 \mathrm{~Hz}$ ) and is not as soft as a system with a natural frequency of about $0.17 \mathrm{~Hz}$.

Simplifying a wind turbine as an SDOF system might cause the elimination of considering the real dynamic behavior of the system. Therefore, various studies are carried out on the dynamic of a wind turbine using a modal approach or finite element model that considers the structure as a multi-DOF (MDOF) system. 0 shows an MDOF model of a fixed offshore wind turbine with lumped mass at various heights of the tower in the parked position eliminating the blades-tower coupled interaction. An example of this model is one of the first studies carried out on the effect of wave loads on offshore fixed wind turbines [64]. In this study, a $750 \mathrm{~kW}$ two-bladed wind turbine was modeled as 
a six DOF system with concentrated masses in the nodes, and the masses were connected with a massless column having finite bending stiffness. Another method that is used in common simulation codes such as CAsT and FAST to find the response of parked or operating wind turbines is modeling based on the solution of the equation of motion for an MDOF beam element for each time step [48, 65-67].

In addition to the simplified models that mainly consider the wind turbine's tower or the wind turbine in the parked position, some models are more sophisticated which take the coupled effects of rotating blades, nacelle, and tower into account (Figure 5). Examples of these models are used in some studies [34, 68-71]. In most of these studies, the equations of motion of the system are developed based on the Euler-Lagrange formulation.

$$
\frac{d}{d t}\left(\frac{\partial T}{\partial \dot{q}_{i}}\right)-\frac{\partial T}{\partial q_{i}}+\frac{\partial V}{\partial q_{i}}=Q_{i}
$$

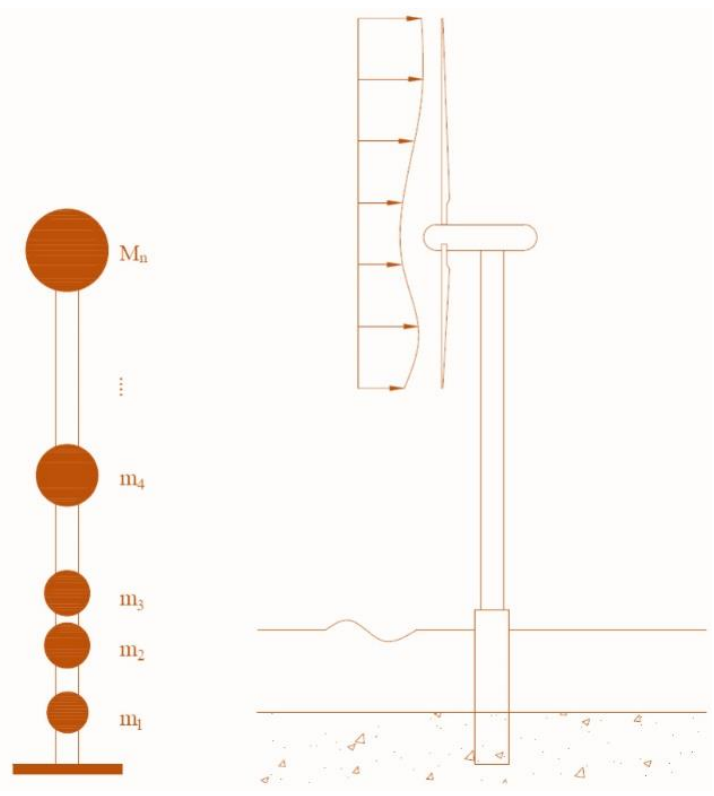

Figure 5 MDOF model of an offshore wind turbine on a mono-pile foundation.

Here, $T$ is the kinetic energy and $V$ is the potential energy of the system, respectively. $q_{i}$ is the displacement and $Q_{i}$ is the generalized loading corresponding to the $i$-th DOF. Therefore, the mass and stiffness matrices can be obtained by defining appropriate kinetic and potential energy equations. The potential energy term for a wind turbine, considering fore-aft and side-side direction of motion can be written as follows [69]:

$$
\begin{gathered}
\mathrm{V}_{\text {Total }}=\frac{1}{2} \sum_{j=0}^{3} \int_{0}^{L}\left[E I_{\text {in }}(x)\left(\frac{\partial \theta_{i, \text { in }}}{\partial x}\right)^{2} d x+E I_{\text {out }}(x)\left(\frac{\partial \theta_{i, \text { out }}}{\partial x}\right)^{2} d x\right. \\
+2 E I_{\text {inout }}(x)\left(\frac{\partial \theta_{i, \text { in }}}{\partial x}\right)\left(\frac{\partial \theta_{i, \text { out }}}{\partial x}\right) d x+N(x)\left(\frac{\partial u_{i, \text { in }}}{\partial x}\right)^{2} d x+N(x)\left(\frac{\partial u_{i, \text { out }}}{\partial x}\right)^{2} d x \\
\left.+G(x)\left(\frac{\partial u_{i, \text { in }}}{\partial x}\right)^{2} d x+G(x)\left(\frac{\partial u_{i, \text { out }}}{\partial x}\right)^{2} d x\right] \\
+\frac{1}{2} k_{n, \text { in }} \times q_{n, \text { in }}^{2}+\frac{1}{2} k_{n, \text { out }} \times q_{n, \text { out }}^{2}
\end{gathered}
$$


where $L$ and $E$ are the length and the modulus of elasticity of the blade, respectively. $\Omega$ is the rotor's rotational speed. The subscripts in and out are for in and out of rotor plane displacements and $k$ is the stiffness of the tower/nacelle. For an operating wind turbine, the rotation of the blade leads to an excessive centrifugal stiffening effect. The gravity causes additional stiffness in the blades. Therefore, $N(x)$ and $G(x)$ which denotes the centrifugal and gravitational forces can be defined as:

$$
\begin{gathered}
\mathrm{N}(x)=\Omega^{2} \int_{x}^{L} \mu(\xi) \xi d \xi \\
G(x)=-\frac{1}{2} g \cos \Psi_{i} \int_{x}^{L} \mu(\xi) d \xi
\end{gathered}
$$

where variable mass per unit length of the blade is denoted by $\mu(x)$, and $\psi$ is the azimuthal angle of the $i$ th blade because of the rotation. Blades have a $2 \pi / 3$ phase difference.

$$
\Psi_{i}=\Omega \mathrm{t}+\frac{2 \pi}{3}(i-1)
$$

Also, the rotations in in-plane and out-plane are:

$$
\begin{gathered}
\theta_{i, \text { in }}=\frac{\partial u_{i, \text { in }}}{\partial x} \\
\theta_{i, \text { out }}=\frac{\partial u_{i, \text { out }}}{\partial x}
\end{gathered}
$$

the parameters $I_{\text {in }}(x), I_{\text {out }}(x)$, and $l_{\text {inout }}(x)$ represent the in and out of plane second area moments of inertia and the second area products of inertia of the blade's cross-section, respectively. The crosssectional El can be found in NREL documents [72]. Kinetic energy also can be calculated from the following equation:

$$
T_{\text {Nacelle }}=\frac{1}{2} \sum_{i=1}^{3} \int_{0}^{L} \mu(x) v_{i}^{2} d x+\frac{1}{2} M_{n} \dot{q}_{n, \text { in }}^{2}+\frac{1}{2} M_{n} \dot{q}_{n, \text { out }}^{2}
$$

where, $v_{i}$, is the absolute velocity of the ith blade and $\dot{q}$ is absolute in- and out-plane velocity of the nacelle and $M_{n}$ is the mass of the tower [69]. Therefore, the wind turbine's mass, stiffness, and damping matrices can be derived.

Damping is assumed to be stiffness proportional for both the tower and the blades. Therefore, with the damping ratios of $\zeta_{n}$ and $\zeta_{b}$ and natural frequencies of $\omega_{n}$ and $\omega_{b}$ for the nacelle/tower and blades respectively, the damping can be obtained from the following relations:

$$
\begin{aligned}
& c_{n}=2 \frac{\zeta_{n}}{\omega_{n}} K_{n} \\
& c_{b}=2 \frac{\zeta_{b}}{\omega_{b}} K_{b}
\end{aligned}
$$


$K_{n}$ and $K_{b}$ are the stiffness matrices of the nacelle/tower and blades. For more details refer to [6871].

The presented models consider the structural properties of a wind turbine, regardless of the soilstructure interaction, controlled systems, and applied loads. More details on soil-structure interaction can be found in $[69,73-75]$. Having an appropriate wind turbine model provides a tool to investigate the system's responses and its problems. Since the focus of current research is to reduce the excessive vibrations in wind turbines, one should go through the methods to control the structure.

\section{Structural Control}

External dampers can be employed in structures to produce counter forces, reduce the responses, and therefore control structural vibrations. The first important step in the matureness of the application of structural control for civil engineering structures backs to 1994 that the first world conference on structural control was held in Los Angeles [44]. Since 1994, structural controllers are developed to a great extent and various types of them, including passive, semi-active, active, and hybrid controllers are investigated.

\subsection{Passive Control}

Passive control employs tuned devices and there will be no continuous adjustment during the operation. Therefore, there is no need for an external power source and the design concept is much simpler than other types of controllers. Passive dampers can improve the damping ratio of civil engineering structures, which normally are less than $2 \%$. Hence, passive dampers increase the dissipation capacity of the structures. Since the 1990s, impressive efforts have been done to implement the theory of applying external dampers into reality as a practical solution for civil engineering structure [76, 77].

Tuned mass dampers (TMDs), tuned liquid dampers (TLDs) and viscous fluid dampers are three examples of these controllers that have been used in full-scale civil engineering structures during the last decades. Tuned mass damper conceptually presented by [78]. It consists of a mass connected to the primary mass with a tuned spring and an oil dashpot damping system. The use of these systems is investigated widely for different types of civil engineering structures such as bridges, tall buildings, and wind turbines [20, 21, 31, 79]. Two of the tall buildings in the U.S. that are equipped with TMDs are John Hancock Tower in Boston and Citicorp Center in New York [44].

TLDs are similar to TMDs in concept where the motion of the fluid mass generates the counterforce to suppress vibrations of the system. Here, the dimension of the fluid tanker defines the damper's frequency that should be tuned to the natural frequency of the primary structure. Besides, the fluid viscosity and the opening of the barriers inside the tankers can adjust the damping ratio of the controller. Yokohama marine tower and Shin Yokohama are two examples of using TLDs in tall buildings $[44,80]$.

One commonly used class of dampers that employ fluids to dissipate energy is viscous damper (VD) that is widely used in mechanical systems and normally consists of a piston in the damper housing filled with a compound of silicone or oil [81]. Attractive parameters of this class of dampers that are interesting for civil engineering structures include the linear viscous response achieved over a broad frequency range, insensitivity to temperature, and compactness in comparison to stroke 
and output force [44]. 0 illustrates a typical damper of this type. The movement of the piston through the viscous fluid dissipates energy and if the fluid is purely viscous (e.g. Newtonian), then the damper force is directly proportional to the velocity of the piston [44]. Therefore, it can be modeled as follows:

$$
f_{d}=C_{0} \dot{x}
$$

Figure 6 shows the implementation of viscous fluid dampers in civil engineering applications, via a bracing system, to control the inter-story drift.

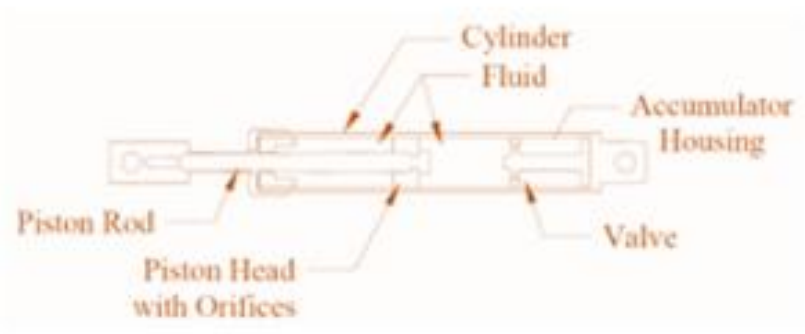

(a)

Figure 6 Fluid viscous dampers: (a) schematic representation from Tylor Devices [82], and (b) large viscous dampers in a building [83].

\subsection{Active Control}

In contrary to passive controller devices, active controllers inject energy into the system. These controllers apply force to the structure through actuators and in consequence, adjustments can be made. Due to the real-time changes that the active controllers make in the system, they may lead to instability with the consequences of structural/system failure [44]. Hence, the utmost endeavor should be done to design controllers to improve the dynamic responses of the system instead of making it worse. Considering this fact, different methods and algorithms are developed and various studies are carried out on active controllers. Some of these studies are: sliding mode control [84, 85], saturation control [86, 87], reliability-based control [88-91], fuzzy control [92-94] and neural control [95]. A schematic block diagram of the structural control problem is illustrated in Figure 7. As it is mentioned earlier, an important task is to specify an appropriate control strategy that receives the measured structural responses by sensors, calculate sufficient control signal, and send it to the actuator. So that the actuators improve the structure's performance by injecting enough forces to minimize the errors. 


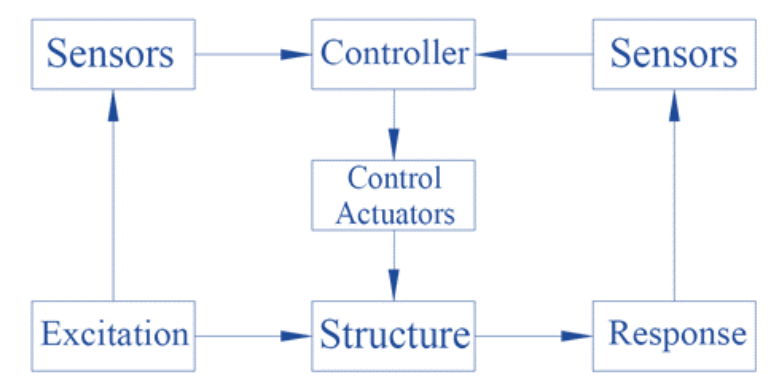

Figure 7 Block diagram of a generic structural control system.

Figure 8 depicts the installation of an active mass damper at the top of a tall building. Here, to control the system, a small mass (about $1 \%$ of the total mass of the system), is connected to the structure through an actuator. Sensors measure responses of the structure. A computer processes the data and according to the control algorithm, it sends appropriate signals to the amplifiers, which finally cause the reaction of the actuators to the excitation. The first full-scale installation of active control in a tall building was in the Kyobashi Center building in 1989. The installed control system consists of two AMDs, one to control transverse motion and the secondary AMD designed to reduce torsional motion [96]. For the list of civil engineering structures that employed external dampers refer to [96].

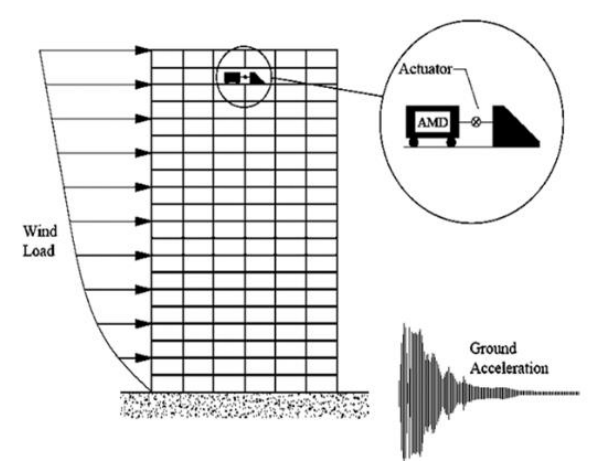

Figure 8 An active mass damper on top of a tall building [97].

\subsection{Hybrid Control}

Hybrid controllers are those that are a combination of active, semi-active, and passive dampers. An example of these systems is the structures that are equipped with various viscoelastic dampers and benefit from active base isolation, or active mass dampers [19]. In a hybrid control scheme, an active control may be used to enhance the performance of a passive control scheme. Alternatively, a passive control system can be employed in an active control scheme to reduce the required power [44].

\subsection{Semi-Active Control}

Semi-active dampers are bounded with active and passive dampers. These types of systems do not inject mechanical or non-dissipative energy into the system. Therefore, they can be considered as passive controllers that can be turned on to change the properties of the damper whenever it is desired. Control strategies based on semi-active devices benefit the best features of both passive 
and active control systems and is receiving the attention of the engineers and scientists as a practical, optimal solution to protect civil engineering structural systems against earthquake and wind loading [44]. Semi-active dampers can mimic the active controller without requiring the associated large power sources. For instance, magnetorheological dampers require only 20-50 watts of power and can operate just on battery power. This is super critical and beneficial during seismic or hurricane events when the main power source to the structure may fail. Besides, for a wind turbine, which is a power generator plant, it would not be beneficial if the control system itself consumes a large amount of power that the turbine generates.

Moreover, because a semi-active controller cannot inject mechanical power into the system, and conceptually is a passive damper with adjustable properties, it does not suffer the instability problems that an active damper can have. Some of the examples of semi-active dampers are variable-orifice fluid dampers and controllable fluid dampers.

\subsection{Variable-Orifice Dampers}

In these types of dampers, the electromechanical, variable-orifice valve controls the opening of the fluid passage and so the hydraulic head loss in the cylindrical piston. This simply translates to altering the fluid resistance or the property of the damper with a very low amount of power. Figure 9 shows a schematic representation of such a device. Some studies implemented such deva ice to control different types of civil engineering structures $[98,99]$.

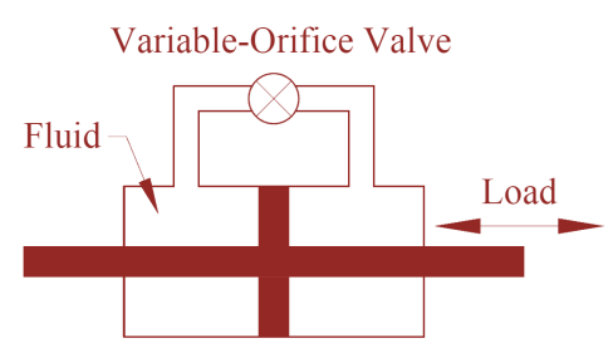

Figure 9 Variable-orifice damper [44].

\subsection{Controllable Fluid Damper}

Since controllable valve dampers have various moving mechanical parts, they potentially have malfunctioning probability and maintenance issues, which can reduce their reliability. Controllable fluid dampers are those that the property of the fluid can be changed by electrical (for electrorheological or ER fluids) or magnetic field (for magnetorheological or MR fluids). Hence, since there are no moving parts other than the piston, ER and MR dampers are very reliable. Moreover, they can respond to the changes in milliseconds, so that they decrease the delay considerably, which is significantly important in high-frequency events.

MR/ER free-flowing, linear viscous fluid properties can be changed with controllable yield strength, to a semisolid by exposing to an electric/magnetic field, almost immediately. Typically, MR fluid dampers that have received more interest rather than ER fluid dampers are consist of magnetically polarizable micron-sized particles dispersed in a carrier medium such as mineral or silicone oil. Applying a magnetic field, then forms particle chains in the fluid. Hence, the fluid 
becomes semisolid with viscoplastic behavior [44]. See [19, 36, 42, 100-102] for more information on the application of these types of dampers in civil engineering structures and wind turbines.

\section{MR Dampers}

To predict and simulate the behavior of an MR damper, an appropriate model should be developed and validated experimentally. In the literature, several conceptual mechanical models are presented by different mathematical expressions. Effective control models of MR dampers are those that precisely can reproduce the nonlinear behavior of the system altered by a magnetic field. According to Sahin et al. (2012) [103], there are two main categories of models: (i) non-parametric, and (ii) parametric models. In non-parametric models, the characteristics of the model are not necessarily physical parameters such as neural networks-based models [104, 105], neuro-fuzzybased models [106], and Chebyshev polynomials [107, 108]. Parametric models are those that consist of a set of mechanical elements such as springs, friction, and viscous dampers with various configurations. Therefore, the parameters of this category of models have the physical meaning which makes these types of models more desirable [103]. Brief information on some of the parametric models is presented as follows, including the formulations that are commonly used with these models.

\subsection{Bingham Model}

A simplified mechanical scheme of this model is shown in Figure 10-(a). A viscous damper is set parallel to a Coulomb friction element and the control force is given as follows:

$$
F_{M R}=f_{C} \operatorname{sgn}(\dot{x})+c_{0} \dot{x}+f_{0}
$$

(a)

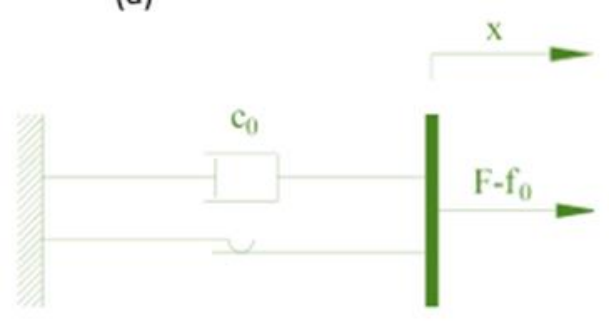

(b)

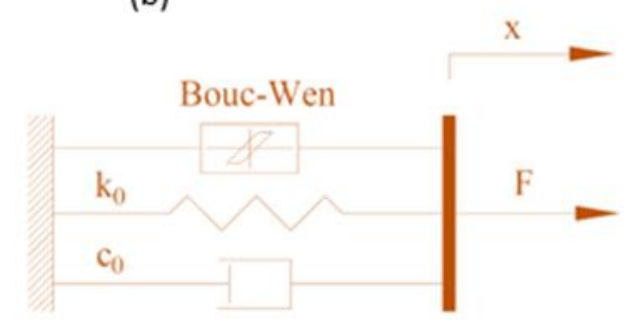

(c)

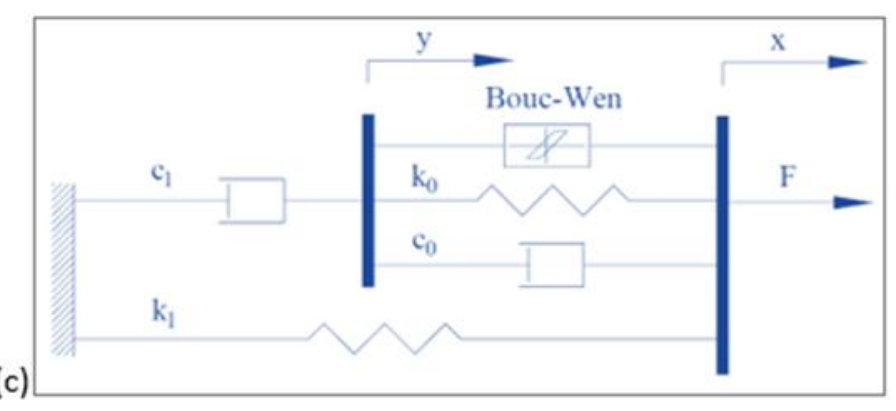

Figure 10 Mechanical modeling of the MR damper: (a) Bingham model, (b) Bouc-Wen model, and (c) modified Bouc-Wen model [109]. 
where, $c_{0}, f_{0}$, and $f_{c}$ are damping coefficient, force offset, and frictional force related to the fluid yield stress a detailed information can be found in $[109,110]$.

\subsection{Bouc-Wen Model}

As one of the earliest models, the Bouc-Wen model is used widely to model the dynamic behavior of MR dampers. A schematic simplified mechanical representation of this model is shown in Figure 10-(b). The control force of the MR damper according to this model can be calculated from the following equation:

$$
F_{M R}=c_{0} \dot{x}+k_{0}\left(x-x_{0}\right)+\alpha z
$$

here, $z$ is defined as:

$$
\dot{z}=-\gamma|\dot{x}| z|z|^{n-1}-\beta \dot{x}|z|^{n}+A \dot{x}
$$

To account for the unloading linearity and pre-yield to post-yield transmission, one can adjust the parameters $\gamma, 6$, and $A[8,109,111]$.

\subsection{Modified Bouc-Wen Model}

In both Bingham and Bouc-Wen models, the force-velocity response does not roll-off when the velocity has a small magnitude and the acceleration and velocity have opposite signs [109]. The modified version of the Bouc-Wen model can better predict the damper force in the region of small velocity with the opposite sign of acceleration and velocity. The mechanical scheme of the model is shown in Figure 10-(c), and the force can be calculated using the following formulations.

$$
\begin{gathered}
F_{M R}=c_{1} \dot{y}+k_{1}\left(x-x_{0}\right) \\
\dot{y}=\frac{1}{\left(c_{0}+c_{1}\right)}\left\{\alpha z+c_{0} \dot{x}+k_{0}(x-y)\right\} \\
\dot{z}=-\gamma|\dot{x}-y| z|z|^{n-1}-\beta(\dot{x}-\dot{y})|z|^{n}+A(\dot{x}-\dot{y})
\end{gathered}
$$

To find the parameters in this model for a 20 ton MR damper refer to [100] and for a 1.5 ton MR damper refer to [37]. There are some other models such as the Dahl model, Kwok model, and Gamota and Filisko (1991) that for more information you can refer to [103, 109].

\subsection{Control Algorithms}

During the past decade, significant development has been achieved in the field of active and semi-active controllers in civil engineering structures and various control algorithms are developed. Some of these control algorithms and strategies are based on applying direct velocity feedback (DVFB) control, $\mathrm{H}_{\infty}$ feedback control, wavelet function, fuzzy logic, genetic algorithms (GA), neural networks (NN), linear quadratic regulator (LQR), and linear Gaussian regulator (LGR) [94, 112-115].

One challenge in the use of semi-active technology is the nonlinear nature of these systems. The nonlinearity requires control algorithms that are appropriate for implementation in full-scale structures. Various control algorithms are presented for the semi-active systems, including the 
skyhook damper control algorithm [116], the bang-bang controller [98], Lyapunov stability theory [117], modulated homogeneous friction algorithm [118], and clipped-optimal controllers [36, 38, 39].

The clipped-optimal controller is known as one of the most common algorithms. According to this algorithm, the linear optimal controller gains, $\mathbf{K}_{c}(s)$, should be designed to calculate the desired control force, $f_{c}$ according to the measured structural responses, $\mathrm{y}$, and the measured force, $f$ applied to the system [119]:

$$
f_{c}=\mathcal{L}^{-1}\left\{-\boldsymbol{K}_{c}(s) \mathcal{L}\left\{\begin{array}{l}
\boldsymbol{y}_{f} \\
f
\end{array}\right\}\right\}
$$

and the control law is:

$$
v=V_{\max } H\left(\left\{f_{c}-f\right\} f\right)
$$

A comparison study on the performance of different control algorithms for a three-story building excited with the south-north direction of the El-Centro earthquake showed that the largest reduction in inter-story displacement and the third-floor displacement was achieved with the clipped-optimal algorithm [119]. However, the study shows that the maximum reduction in peak acceleration was obtained by applying the bang-bang approach [119]. The same comparison has been done in a three-story building excited with El-Centro earthquake and controlled by various controller strategies, including Passive-off and on, Lyapunov, Decentralized bang-bang, Quasi-bangbang, Maximum energy dissipation, Modulated homogenous friction, Clipped-optimal, and new quasi-bang-bang controller gives weight to the output in a way that is similar to a fuzzy logic controller [102]. The results revealed that the Lyapunov controller, quasi-bang-bang controller, and clipped-optimal control algorithm provided significant maximum displacement. Also, the clippedoptimal control algorithm gave a good reduction in the maximum absolute accelerations [102]. More information on the control algorithms is provided in the literature $[101,119,120]$.

\section{Controller Design}

In passive dampers, the system tuning includes setting the damping ratio and the frequency ratio once, without further adjustment during the operation. There are some studies on optimized passive damper parameters for wind and earthquake [21, 121-124]. The design procedure for semiactive controllers is more complicated. The performance of the controller depends on the selected algorithm that commands the damper. Moreover, the success of the controller to suppress excessive vibrations effectively depends on the desired control force as well as the control algorithm. Available methods to set the desired control force, and therefore design the optimal controller are based on the simulation. This makes existing methods to be very time-consuming and requires significant computational resources. An alternative to the common simulation-based methods is an energy-based probabilistic approach [125] for SDOF systems. In this approach, the probability of dissipativity of the controller is calculated by solving the Lyapunov function and substituting the results into a stochastic dissipativity index presented by [112]. Then the probability of dissipativity can be applied in an energy-based method to obtain the desired control force without the simulation [112]. 
Although there are several studies on the control of wind turbines for structural performance improvement, there are just limited studies on the evaluation of the performance of wind turbines for multihazard loads and mitigation of excessive vibrations. Looking into the responses of the structure for various loading scenarios and control approaches is an important task because various excitation sources (e.g. wind, wave, earthquake, rotor imbalance, etc.) affect the structure differently and the controllers might improve a specific response by scarifying the other. To prevent that we need to investigate the performance of the system for multihazard.

In the literature, various control methods, including passive, semi-active and active dampers installed in the nacelle, tower, or the foundation of the turbine are proposed and compared for wind and wave, or wind, and earthquake. For instance, the idea of installing vibration isolators for wind turbines is suggested in different studies. It is proposed to install vibration isolators at the top of the turbine's tower, or at the foundation using semi-active MR dampers to reduce the challenges regards the conventional base isolators for wind turbines $[18,19]$. Both wind and seismic loads are placed on the structure as disturbance forces and the responses are quantified. The results showed that implementing an isolation system was beneficial for reducing certain key parameters of the turbine's structural response, particularly for the wind turbines in seismically active regions.

Offshore and coastal wind turbines' vibration mitigation is also investigated through different numerical studies considering different types of floating and fixed foundations under the action of wind and wave. In a study that aimed to compare the effects of passive and active tuned mass dampers on a floating wind turbine, a reduction in fatigue loads of the tower base was compared [66]. However, the stroke of the active damper was much greater than the passive damper. This can be a limitation as the available space in the nacelle or at the top of the wind turbine is limited and it is mainly occupied by mechanical parts.

The impacts of pendulum-type passive and semi-active dampers at the top of both floating and fixed offshore wind turbines are also investigated for parked and operating wind turbines [48]. The TMD's oscillations absorbed the energy of tower fore-aft and side-to-side motion, simultaneously. However, since the semi-active TMD (SATMD) was activated around $32 \%$ of the time in the fore-aft direction and $31 \%$ in the side-to-side direction, the power source of the system was increased meaningfully [48]. The performance of TLCD for wind and wave loading on an offshore wind turbine was modeled [62]. The results showed that, while there was a significant reduction in displacement and acceleration at the top of the tower and base shear stress for parked and operating conditions, the reductions meaningfully depended on the wind-wave loads' properties, and in some of the cases, the TLCD even increased the responses.

In a recent study on the control of fixed offshore wind turbines, multihazard loading on wind turbines including wind, wave, and earthquake is evaluated [35]. A SATMD is installed in the nacelle of a wind turbine to control the along-wind displacement of the nacelle/tower and it is shown that the SATMD was more effective than a passive damper to reduce the resonance responses. However, it is not shown that the controller was designed effectively for multihazard.

\section{Advanced Control Theory to Accelerate the Optimal Tuning of Smart Structures}

The Windstorm Impact, Science and Engineering (WISE) research team at Louisiana State University (LSU) devoted efforts to developing advanced control theory to accelerate the optimal tuning of smart structures, to develop novel probabilistic analytical methods to address the complex 
behavior and inherent nonlinearity in semi-active control, for multiple hazards. The LSU WISE research group developed novel control methods that enabled semi-active controller tuning under complex behavior and inherent nonlinearity, without simulations [50]. The recently developed control theory enables the evaluation of semi-active controllers' performance of multi-degrees-offreedom systems, without significant computational effort [126]. With this analytical probabilistic control theory, a wide range of controllers can be evaluated in a fraction of a second while optimum control parameters can be tuned to achieve different control objectives. The potential applications include semi-active vibration control in flexible structures under multiple hazard loads brought by wind, wave, and earthquakes.

In the dynamics of structures under multiple hazards, vibration control offers attractive means for protecting structures and inhabitants, as well as balancing between resilience and sustainability. A fundamental task related to the design of semi-active controllers to attenuate structural vibrations is the tuning of the parameters based on the physical properties (mass, stiffness, and damping), mitigation objectives, nature of excitation, etc. Besides, in smart damping, the performance of the system to mitigate excessive vibrations is governed by the corresponding ideal active control force, and the algorithm alerting the damping characteristics of the device.

While there are several studies on the advantages of employing semi-active dampers to attenuate vibrations in flexible structures, the controller tuning process is mainly based on numerical simulations, hindering the consideration of different families of controllers, to achieve controller tuning-based performance under varying loading patterns, for instance, excitation due to multiple hazards. To address this challenge, the LSU WISE research team developed a novel control theory that enables the semi-active controller tuning, of multi-degrees-of-freedom systems, under complex behavior and inherent nonlinearity, without simulations [50]. According to the dissipative probability of the corresponding active control forces, a new concept of semi-active control gains was developed:

$$
\begin{gathered}
k_{s, i}=\frac{1}{2} G_{1, i}\left\{1+\sin ^{2}[\pi(P-0.5)]\right\} \quad \text { and } i=1,2, \ldots, m \\
\boldsymbol{k}_{s}=\operatorname{diag}\left(k_{s, i}\right) \\
\omega_{a}=\sqrt{\operatorname{eig}\left(M^{*-1}\left(\mathrm{~K}_{m, a}\right)\right)} ; \mathrm{K}_{m, a}=\mathrm{K}^{*}+\operatorname{diag}\left(\Phi G_{1}\right) \\
C_{s 1, i}=\frac{\left|G_{1, i}\right|}{\pi \omega_{s, i}}\left\{1+\sin ^{2}[\pi(P-0.5)]\right\} \text { and } i=1,2, \ldots, m \\
\omega_{s}=\sqrt{e i g\left(M^{*-1}\left(\mathrm{~K}^{*}+\mathrm{k}_{s}\right)\right)} \\
\left\{\frac{\pi}{2}+\left[\sin [\pi(P-0.5)] \times \sqrt{1-\sin ^{2}[\pi(P-0.5)]}+\tan ^{-1}\left(\frac{\sin [\pi(P-0.5)]}{\sqrt{1-\sin ^{2}[\pi(P-0.5)]}}\right)\right]\right\}
\end{gathered}
$$




$$
\mathbf{c}_{s}=\operatorname{diag}\left(c_{s 1, i}+c_{s 2, i}\right) \text { and } i=1,2, \ldots, m
$$

where $P$ is the dissipative probability of the corresponding optimal active control forces, $G_{1}$ and $G_{2}$ are the active gains, and $\mathrm{K}^{*}$ is the generalized stiffness of the primary structure [50].

With this new theory, a family of controllers can be studied, and their performance can be evaluated in a fraction of a second. This enables controller tuning to achieve optimum parameters for different design objectives. The potential applications of this control theory include vibration control in flexible structures under multiple hazard loads brought by, for example, wind, wave, and seismic loadings. To show the potentiality of the new theory, we considered semi-active controller design in both onshore and offshore wind turbines, in parked and operating positions, under wind, wave, and earthquake external forces, in addition to mechanical unbalance [50].

\section{Application Example}

A 5-MW NREL wind turbine is excited by multi-hazard loads (wind and earthquake). The Lagrangian approach was employed to model the wind turbine considering the blade/tower coupling. A control system was investigated: an outer bracing with magnetorheological (MR) dampers (Figure 11) $[50,10]$. The outer bracing system benefits a lever mechanism (Figure 11). The lever mechanism magnifies the control force transferred to the structure, which also compensates for the control force reduction due to its connection to the lower part of the tower, instead of the nacelle. The magnification factor is considered as 3.0, and the angle of attachment of the bracing member to the support is chosen as $\theta=45$ degrees [41].

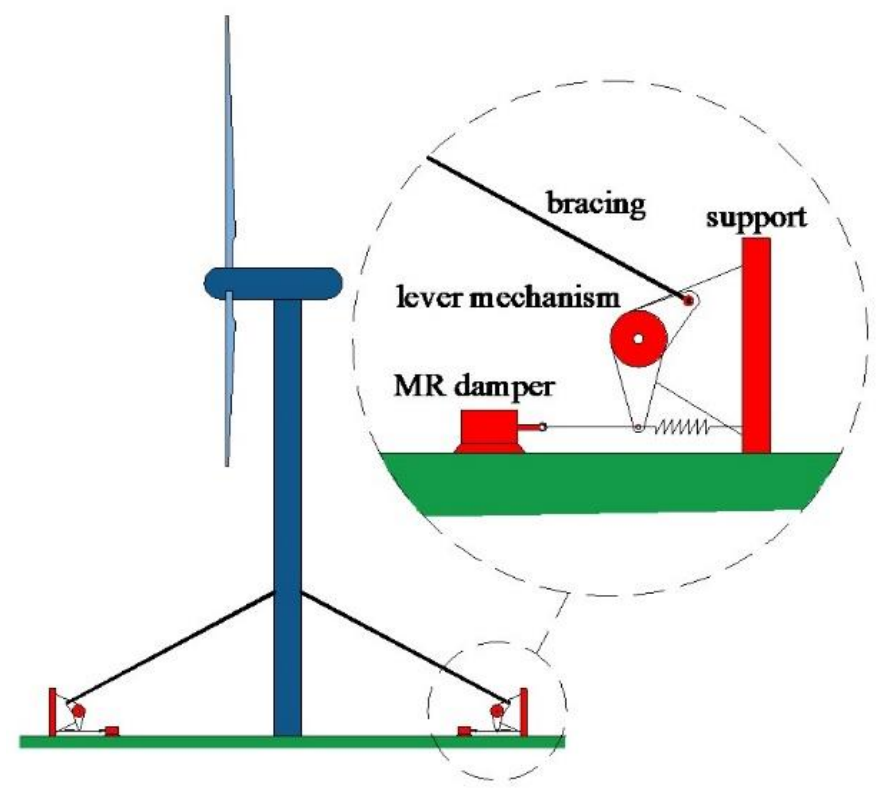

Figure 11 Modeling and control of a $5 \mathrm{MW}$ wind turbine: external bracing with magnetorheological (MR) dampers benefiting a lever mechanism to enhance the performance, and lumped mass model [127].

Since the probability of occurrence of both storm and earthquake events at the same time is too low, the occurrence of both was not considered. Instead, earthquake effects are evaluated for the structure equipped with the controller in operating conditions, which is more probable. Hence, the 
wind speed has a velocity of $25 \mathrm{~m} / \mathrm{s}$ and $12 \%$ turbulence intensity, the seismic excitation was simulated according to the El Centro 1940 earthquake. The outer bracing-damper system was designed and tuned based on the new analytical method (proposed control theory).

\subsection{Controller Tuning}

The new control theory was employed to accelerate the tuning of the semi-active controllers subjected to multiple hazards (Figure 12). A set of two large-scale MR dampers are employed and the force-time history, force-displacement, and force-velocity relationships for the MR damper in two extreme cases of passive-on and passive-off were obtained [50]. The simulated excitation to verify the model was a $1.0 \mathrm{~Hz}$ sinusoidal displacement with an amplitude of $1.27 \mathrm{~cm}$. Simulated control force-time, force-displacement, and force-velocity are similar to those obtained experimentally in [37].

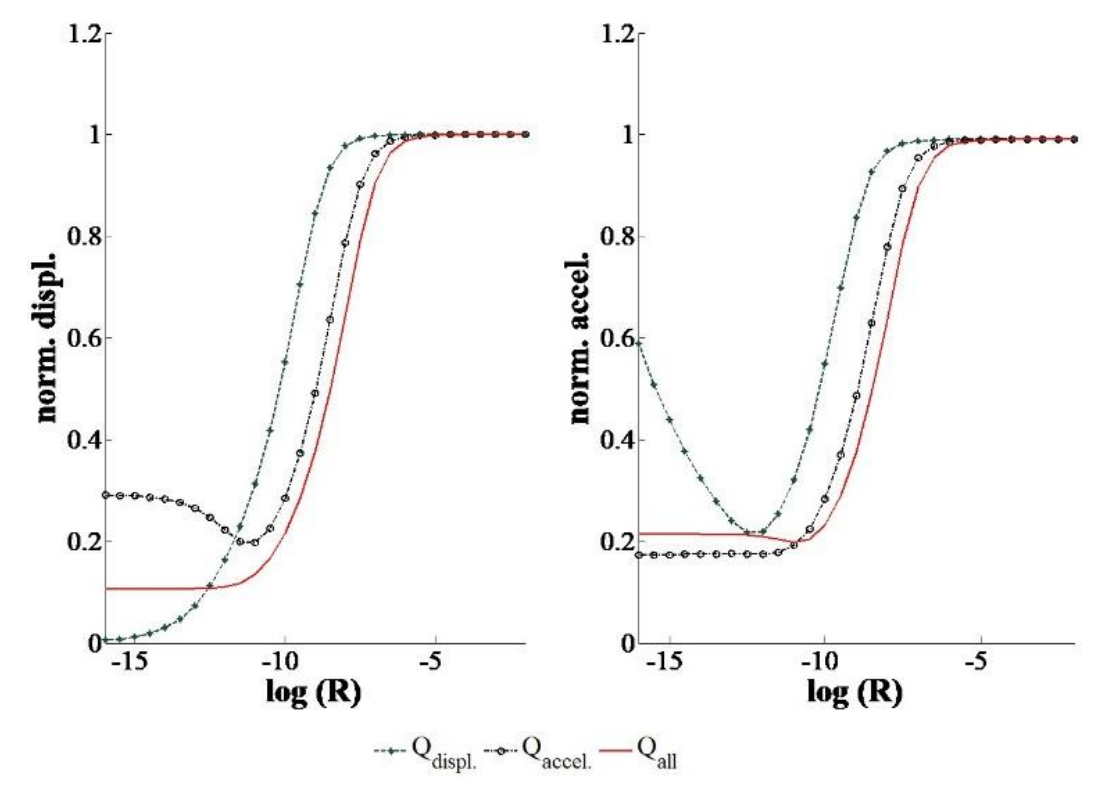

Figure 12 Results obtained by the proposed semi-active controller tuning theory: effect of weighting the $Q$ and $R$ matrices to achieve desired control forces for certain optimization objectives - minimizing normalized root-mean-square (RMS) displacement ( $\left.Q_{\text {displ. }}\right)$ and acceleration $\left(Q_{\text {accel. }}\right)$, as well as displacement and acceleration, simultaneously (Qall.).

Again, three control strategies of passive-on, passive-off, and clipped-optimal were considered. To achieve the optimum $Q$ and $R$ matrices in the LQR method, which yields the desired control force, the analytical method was applied for various controllers to control the first mode of the structure. Thus, four different approaches were defined for the weighting of the $Q$ matrix. The normalized RMS of the responses for all the controller scenarios plotted versus $R$ (Figure 12). The normalization for the displacement and acceleration is done by dividing the controlled responses by the corresponding uncontrolled responses.

The control force normalized over the weight of the lumped mass at the top of the tower. As was expected, the results obtained by the proposed semi-active controller tuning theory show that although weighting displacement, decreased the displacement, however, for more aggressive 
controllers the damper that was designed based on weighting displacement made the system stiffer, and therefore the acceleration responses increased.

The same issue was observed for weighting velocity. On the other hand, weighting acceleration provided appropriate responses for both acceleration and displacement for $\log (R)=-11$. Also, comparing the performance of $Q_{\text {all }}$ and $Q_{\text {acc }}$ shows that to achieve a specific reduction in the acceleration, both scenarios required almost the same control force.

\subsection{Parked Onshore Wind Turbine}

Passive-on and passive-off controllers also were examined and compared with the clippedoptimal strategy. The results are presented in Table 1. It is shown that the clipped-optimal controller exhibits the best performance in reducing both acceleration and displacement peaks. Passive-off is slightly better than clipped-optimal just in RMS of the acceleration. For the passive-on case, the MR damper's current is always at its maximum value, and for the passive-off case, it is always zero. The lever mechanism helps improve the performance of the dampers, leading to lower damping capacity or reduced number of devices. Nevertheless, the control force in the bracing system will be increased that yields the need for a stronger bracing link [42].

Table 4 Reduction in responses of the nacelle for a parked onshore wind turbine controlled with an outer bracing system.

percentage of reduction (controlled/uncontrolled)

\begin{tabular}{llllllll}
\hline \multicolumn{8}{c}{ percentage of reduction (controlled/uncontrolled) } \\
\hline & peak to peak & peak to peak & peak displ. & peak acc. & RMS displ. & RMS acc. \\
control strategy & displ. (\%) & accel. (\%) & $(\%)$ & $(\%)$ & $(\%)$ & $(\%)$ \\
passive-off & 92.14 & 93.05 & 82.43 & 80.54 & 62.68 & 89.56 \\
passive-on & 96.70 & 98.23 & 93.56 & 92.10 & 58.20 & 79.64 \\
clipped-optimal & 98.18 & 97.80 & 95.49 & 93.13 & 63.52 & 86.03 \\
\hline
\end{tabular}

\subsection{Operating Onshore Wind Turbine}

The performance of the MR damper to control the wind turbine undergoes operational and seismic loads were evaluated. The time history of the nacelle's responses is shown in Figure 13. It can be seen that the effect of the first shock of the ground acceleration was removed very fast (in less than $30 \mathrm{sec}$.). Also, the vibrations produced by the operational load were almost disappeared. A notable point is that the controller was available from the first seconds of the shock, which is an important aspect of the controller system particularly when the disturbance is the ground acceleration. Because, if there would be a delay in responding to the vibration, the structure may fail even before the controller start to react and reduce the responses. The comparison between applying controller with passive-on, passive-off, and clipped-optimal controller is presented in Table 2. It is shown that applying a clipped-optimal strategy reduced the peaks greater than other controllers did, but the RMS was slightly better for the passive-on controller. 


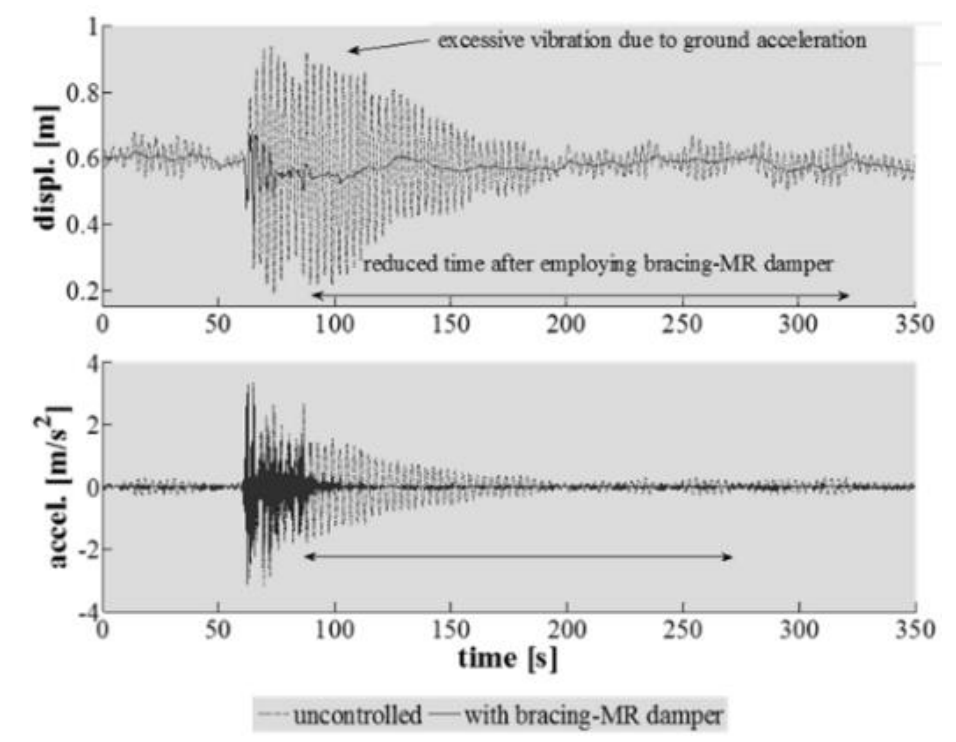

Figure 13 Displacement and acceleration time histories of the nacelle of an operating wind turbine coincident with seismic load: uncontrolled and controlled with the outer bracing system. Results obtained by the proposed semi-active controller tuning theory: time histories of uncontrolled and controlled (employing MR dampers with an outer bracing system) displacement and acceleration responses of the nacelle of an operating wind turbine under earthquake loading (El Centro horizontal component of ground acceleration).

Table 5 Reduction in responses of the nacelle for an operating onshore wind turbine controlled with the outer bracing system, coincident with an earthquake event.

\begin{tabular}{llllllll}
\hline \multicolumn{7}{c}{ percentage of reduction (controlled/uncontrolled) } \\
\hline control strategy & $\begin{array}{l}\text { peak to peak } \\
\text { displ. }(\%)\end{array}$ & peak to peak & peak displ. & peak acc. & RMS & displ. & RMS \\
passive-off & 81.69 & 81.02 & $(\%)$ & $(\%)$ & $(\%)$ & $(\%)$ \\
passive-on & 96.91 & 93.49 & 40.97 & 2.09 & 61.80 & 39.82 \\
clipped-optimal & 95.00 & 92.78 & 64.78 & 1.79 & 79.22 & 49.82 \\
\hline
\end{tabular}

The results show that for the operating wind turbine, earthquake excitations lead to excessive vibrations for a long period. However, the properly tuned semiactive damping system improved the performance of the wind turbine by reducing the magnitude and duration of excessive vibrations. For vibration control under multiple hazards, including wind and earthquake, the control system is immediately available, compared to, for instance, the classical slow responding tuned mass damper (Figure 13).

This new control theory reduced the design and performance evaluation time significantly. For instance, the CPU-time required for a single performance evaluation case considering specific weighting matrices, using a $2.5 \mathrm{GHz}$ Intel Core i5-2400S processor PC, was about $3 \mathrm{hrs}$. This amount of time increases significantly for aggressive primary active controllers, which means that several days/weeks are needed to evaluate the performance of a family of controllers under certain loading excisions. This amount of simulation time can increase exponentially when the objective is to design 
semi-active controllers for multiple hazards, for different optimization objectives - e.g. minimizing displacement, velocity, acceleration, or all responses. The new analytical control theory reduced the total time to a few seconds for a wide range of controllers and optimization objectives.

\section{Summary}

Structural design for multiple hazards is a challenge that requires the adoption and consideration of new control methods, theory, devices, and implementation techniques. Advanced control methods can yield more resilient and sustainable infrastructure, such as wind turbines, as well as other flexible structures, such as high-rise buildings, towers, and long-span bridges under the impact of wind, wave, and seismic excitations. The recently developed semi-active gain theory can be a benchmark for further investigations on structural control in wind turbines and other structures. A vibration mitigation technique with outer bracing using MR dampers is presented. To select the semi-active controller, the generic energy-based probabilistic approach was employed. This analytical approach reduced the time significantly. For an operating wind turbine, an earthquake event caused excessive vibrations for a period much longer than the event itself. The implementation of external dampers not only improved the performance of the system by reducing excessive vibrations but also reduced the duration of excessive vibrations. For vibration control under earthquakes, it is shown that the outer bracing system is immediately available to reduce the response. The time window required to eliminate the effect of the earthquake ground acceleration on the operating wind turbine for the outer bracing system was short. Besides, the analytical approach has potential applications in various mechanical systems. The goal is to build new structures and retrofit existing ones in ways that can balance resilience with sustainability, enhance safety, and reduce the huge cost of rebuilding due to multiple hazards. This will broadly impact the wind/structural engineering research field and facilitate effective investments in the infrastructure industry that will result in more resilient and sustainable communities, improve the quality of life, and contribute to economic growth.

\section{Author Contributions}

The authors contributed equally.

\section{Competing Interests}

The authors have declared that no competing interests exist.

\section{References}

1. WWEA. World wind energy report 2011. Bonn, Germany: World Wind Energy Association; 2011.

2. Laks JH, Pao LY, Wright AD. Control of wind turbines: Past, present, and future. Proceedings of 2009 American Control Conference; 2009 June 10-12; St Louis, MO, US. Piscataway: IEEE.

3. Katsanos El, Thöns S, Georgakis CT. Wind turbines and seismic hazard: A state-of-the-art review. Wind Energy. 2016; 19: 2113-2133.

4. Sigurðsson GÖ, Rupakhety R, Rahimi SE, Olafsson S. Effect of pulse-like near-fault ground motions on utility-scale land-based wind turbines. Bull Earthq Eng. 2020; 18: 953-968.

5. Campione G, Monaco A, Cannella F. Flexural test on a full-scale 60-kw wind turbine-tower 
telescopic steel pipe. Pract Period Struct Des Constr. 2019; 24: 04019012.

6. Aly AM, Gol Zaroudi H, Rezaee M. Hurricane hazard mitigation in traffic light support structures. Final Report. Baton Rouge: Louisiana Transportation Research Center; 2016; FHWA/LA.15/142TIRE. Available from: https://www.ltrc.Isu.edu/pdf/2016/14-2TIRE.pdf.

7. Kaloop MR, Hu JW, Bigdeli Y. Identification of the response of a controlled building structure subjected to seismic load by using nonlinear system models. Appl Sci. 2016; 6: 301.

8. Bigdeli Y, Kim D. Damping effects of the passive control devices on structural vibration control: TMD, TLC and TLCD for varying total masses. KSCE J Civ Eng. 2016; 20: 301-308.

9. Bigdeli Y, Kim D. Response control of irregular structures using structure-TLCD coupled system under seismic excitations. KSCE J Civ Eng. 2015; 19: 672-681.

10. Rezaee $M$, Aly AM. Vibration control in wind turbines for performance enhancement: $A$ comparative study. Wind Struct. 2016; 22: 107-131.

11. Aly AM, Abburu S. On the design of high-rise buildings for multihazard: Fundamental differences between wind and earthquake demand. Shock Vib. 2015; 2015: 148681.

12. Zheng M, Yang ZJ, Yang S, Still B. Modeling and mitigation of excessive dynamic responses of wind turbines founded in warm permafrost. Eng Struct. 2017; 148: 36-46.

13. Farghaly AA. Seismic analysis of adjacent buildings subjected to double pounding considering soil-structure interaction. Int J Adv Struct Eng. 2017; 9: 51-62.

14. Ramezani M, Bathaei A, Zahrai SM. Designing fuzzy systems for optimal parameters of TMDs to reduce seismic response of tall buildings. Smart Struct Syst. 2017; 20: 61-74.

15. AlHamaydeh M, Jaradat MA, Serry M, Sawaqed L, Hatamleh KS. Structural control of MRdampers with genetic algorithm-optimized Quasi-Bang-Bang controller. Proceedings of 2017 7th International Conference on Modeling, Simulation, and Applied Optimization (ICMSAO); 2017 April 4-6; Sharjah, UAE. Piscataway: IEEE.

16. Mohammadi RK, Haghighipour F. Implementation of uniform deformation theory in semi-active control of structures using fuzzy controller. Smart Struct Syst. 2017; 19: 351-360.

17. Vulcano A. Comparative study of the earthquake and wind dynamic responses of base-isolated buildings. J Wind Eng Ind Aerodyn. 1998; 74: 751-764.

18. Van der Woude C, Narasimhan S. A study on vibration isolation for wind turbine structures. Eng Struct. 2014; 60: 223-234.

19. Caterino N. Semi-active control of a wind turbine via magnetorheological dampers. J Sound Vib. 2015; 345: 1-17.

20. Murtagh PJ, Ghosh A, Basu B, Broderick BM. Passive control of wind turbine vibrations including blade/tower interaction and rotationally sampled turbulence. Wind Energy. 2008; 11: 305-317.

21. Si Y, Karimi HR, Gao H. Modelling and optimization of a passive structural control design for a spar-type floating wind turbine. Eng Struct. 2014; 69: 168-182.

22. Stewart GM, Lackner MA. The impact of passive tuned mass dampers and wind-wave misalignment on offshore wind turbine loads. Eng Struct. 2014; 73: 54-61.

23. Gaur S, Elias S, Höbbel T, Matsagar VA, Thiele K. Tuned mass dampers in wind response control of wind turbine with soil-structure interaction. Soil Dyn Earthq Eng. 2020; 132: 106071.

24. Gupta YP, Chandrasekaran AR. Absorber system for earthquake excitation. Proceedings of the Fourth World Conference on Earthquake Engineering; 1969 January 13th-18th; Santiago, Chile. Santiago: Universidad de Chile. pp.139-148.

25. Kaynia AM, Veneziano D, Biggs JM. Seismic effectiveness of tuned mass dampers. J Struct Div. 
1981; 107: 1465-1484.

26. Sladek JR, Klingner RE. Effect of tuned-mass dampers on seismic response. J Struct Eng. 1983; 109: 2004-2009.

27. Wirsching PH, Yao JT. Safety design concepts for seismic structures. Comput Struct. 1973; 3: 809826.

28. Wirsching $\mathrm{PH}$, Campbell GW. Minimal structural response under random excitation using the vibration absorber. Earthq Eng Struct Dyn. 1973; 2: 303-312.

29. Ohno S, Watari A, Sano I. Optimum tuning of the dynamic damper to control response of structures to earthquake ground motion. Proceedings of the Sixth World Conference on Earthquake Engineering; 1977 January 10th-14th; New Delhi, India. Pune: Sarita Prakashan. pp.157-161.

30. Villaverde R. Reduction seismic response with heavily-damped vibration absorbers. Earthq Eng Struct Dyn. 1985; 13: 33-42.

31. Villaverde R. Seismic control of structures with damped resonant appendages. Proc First World Conf Struct Control. 1994; 1: 113-122.

32. Villaverde R, Koyama LA. Damped resonant appendages to increase inherent damping in buildings. Earthq Eng Struct Dyn. 1993; 22: 491-507.

33. Dinh VN, Basu B. Passive control of floating offshore wind turbine nacelle and spar vibrations by multiple tuned mass dampers. Struct Control Health Monit. 2015; 22: 152-176.

34. Dinh VN, Basu B, Nagarajaiah S. Semi-active control of vibrations of spar type floating offshore wind turbines. Smart Struct Syst. 2016; 18: 683-705.

35. Sun C. Semi-active control of monopile offshore wind turbines under multi-hazards. Mech Syst Signal Process. 2018; 99: 285-305.

36. Dyke SJ, Spencer Jr BF, Sain MK, Carlson JD. Seismic response reduction using magnetorheological dampers. IFAC Proc Vol. 1996; 29: 5530-5535.

37. Dyke SJ, Spencer Jr BF, Sain MK, Carlson JD. Modeling and control of magnetorheological dampers for seismic response reduction. Smart Mater Struct. 1996; 5: 565-575.

38. Dyke SJ, Spencer Jr BF, Quast P, Kaspari Jr DC, Sain MK. Implementation of an active mass driver using acceleration feedback control. Comput Aided Civ Infrastruct Eng. 1996; 11: 305-323.

39. Dyke SJ, Spencer Jr BF, Quast P, Sain MK, Kaspari Jr DC, Soong TT. Acceleration feedback control of MDOF structures. J Eng Mech. 1996; 122: 907-918.

40. Occhiuzzi A. Additional viscous dampers for civil structures: Analysis of design methods based on effective evaluation of modal damping ratios. Eng Struct. 2009; 31: 1093-1101.

41. Aly AM, Zasso A, Resta F. On the dynamics of a very slender building under winds: Response reduction using MR dampers with lever mechanism. Struct Des Tall Spec Build. 2011; 20: 539551.

42. Zasso A, Aly AM, Resta F. MR dampers with lever mechanism for response reduction in high-rise buildings under wind loads. Proceedings of 5th European \& African Conference on Wind Engineering; 2009 July 19th-23rd; Florence, Italy. Firenze: Firenze University Press.

43. Sun C, Nagarajaiah S. Study on semi-active tuned mass damper with variable damping and stiffness under seismic excitations. Struct Control Health Monit. 2014; 21: 890-906.

44. Housner G, Bergman LA, Caughey TK, Chassiakos AG, Claus RO, Masri SF, et al. Structural control: Past, present, and future. J Eng Mech. 1997; 123: 897-971.

45. Choi SB, Hong SR, Sung KG, Sohn JW. Optimal control of structural vibrations using a mixed- 
mode magnetorheological fluid mount. Int J Mech Sci. 2008; 50: 559-568.

46. Weber F. Semi-active vibration absorber based on real-time controlled MR damper. Mech Syst Signal Process. 2014; 46: 272-288.

47. Basu B, Staino A, Basu M. Role of flexible alternating current transmission systems devices in mitigating grid fault-induced vibration of wind turbines. Wind Energy. 2014; 17: 1017-1033.

48. Tsouroukdissian AR, Lackner M, Cross-Whiter J, Park SM, Pourazarm P, Cava W La, et al. Smart novel semi-active tuned mass damper for fixed bottom and floating offshore wind. Proceeding of Offshore Technology Conference; 2016 May 2nd-5th; Houston, Texas, US. Houston: Offshore Technology Conference.

49. Sun C. Mitigation of offshore wind turbine responses under wind and wave loading: Considering soil effects and damage. Struct Control Health Monit. 2018; 25: e2117.

50. Rezaee M, Aly AM. Vibration control in wind turbines to achieve desired system-level performance under single and multiple hazard loadings. Struct Control Health Monit. 2018; 25: e2261.

51. Office of Energy Efficiency \& Renewable. The inside of a wind turbine [Internet]. Washington: Department of Energy. Available form: https://www.energy.gov/eere/wind/inside-wind-turbine.

52. Ancona D, McVeigh J. Wind turbine-materials and manufacturing fact sheet [Internet]. Washington: Office of Industrial Technologies, U.S. Department of Energy; 2001. Available from: http://www.perihq.com/documents/WindTurbine-MaterialsandManufacturing FactSheet.pdf.

53. Ciang CC, Lee JR, Bang HJ. Structural health monitoring for a wind turbine system: A review of damage detection methods. Meas Sci Technol. 2008; 19: 122001.

54. Harte R, Van Zijl GP. Structural stability of concrete wind turbines and solar chimney towers exposed to dynamic wind action. J Wind Eng Ind Aerodyn. 2007; 95: 1079-1096.

55. Rosenbloom E. A problem with wind power [Internet]. Available from: http://www.aweo.org/problemwithwind.html.

56. Hahn B, Durstewitz M, Rohrig K. Reliability of Wind Turbines: Experiences of 15 years with 1,500 WTs. Wind Energy. 2007: 329-332.

57. Caithness Windfarm Information Forum 2017. Summary of Wind Turbine Accident data to 31 December 2016. 2017.

58. Hameed Z, Hong YS, Cho YM, Ahn SH, Song CK. Condition monitoring and fault detection of wind turbines and related algorithms: A review. Renew Sustain Energ Rev. 2009; 13: 1-39.

59. Habibi H, Howard I, Simani S. Reliability improvement of wind turbine power generation using model-based fault detection and fault tolerant control: A review. Renew Energ. 2019; 135: 877896.

60. Brett Ruskin. Wind turbine collapse wreckage in Cape Breton combed for clues of cause [Internet]. Toronto: CBC News; 2016. Available from: https://www.cbc.ca/news/canada/novascotia/wind-turbine-collapse-point-tupper-cape-breton-enercon-renewable-1.3734562.

61. KWCH. Wind turbine collapse in Kansas. 2014.

62. Chen J, Liu Y, Bai X. Shaking table test and numerical analysis of offshore wind turbine tower systems controlled by TLCD. Earthq Eng Eng Vib. 2015; 14: 55-75.

63. van der Tempel J, Molenaar DP. Wind turbine structural dynamics-a review of the principles for modern power generation, onshore and offshore. Wind Eng. 2002; 26: 211-222.

64. Rogers N, Still D. Structural dynamics of offshore wind turbines subject to extreme wave loading. In: European Wind Energy Conference. London: James \& James; 1999. 
65. Xu N, Ishihara T. Analytical formulae for wind turbine tower loading in the parked condition by using quasi-steady analysis. Wind Eng. 2014; 38: 291-309.

66. Lackner MA, Rotea MA. Structural control of floating wind turbines. Mechatronics. 2011; 21 : 704-719.

67. Jonkman JM. Dynamics modeling and loads analysis of an offshore floating wind turbine. Golden, CO: National Renewable Energy Laboratory; 2007; NREL/TP-500-41958.

68. Quilligan A, O'Connor A, Pakrashi V. Fragility analysis of steel and concrete wind turbine towers. Eng Struct. 2012; 36: 270-282.

69. Fitzgerald B, Basu B. Structural control of wind turbines with soil structure interaction included. Eng Struct. 2016; 111: 131-151.

70. Hansen $\mathrm{MH}$. Improved modal dynamics of wind turbines to avoid stall-induced vibrations. Wind Energy. 2003; 6: 179-195.

71. Arrigan J, Pakrashi V, Basu B, Nagarajaiah S. Control of flapwise vibrations in wind turbine blades using semi-active tuned mass dampers. Struct Control Health Monit. 2011; 18: 840-851.

72. Jonkman J, Butterfield S, Musial W, Scott G. Definition of a 5-MW reference wind turbine for offshore system development. Golden, CO: National Renewable Energy Laboratory; 2019; NREL/TP-500-38060.

73. Abhinav KA, Saha N. Dynamic analysis of an offshore wind turbine including soil effects. Procedia Eng. 2015; 116: 32-39.

74. Harte M, Basu B, Nielsen SR. Dynamic analysis of wind turbines including soil-structure interaction. Eng Struct. 2012; 45: 509-518.

75. Lombardi D, Bhattacharya S, Wood DM. Dynamic soil-structure interaction of monopile supported wind turbines in cohesive soil. Soil Dyn Earthq Eng. 2013; 49: 165-180.

76. Soong TT, Constantinou MC. Passive and active structural vibration control. Cham: Springer International Publishing; 1994.

77. Soong TT, Dargush GF. Passive energy dissipation systems in structural engineering. Chichester: Wiley; 1997.

78. Den Hartog JP. Mechanical vibrations. 3rd ed. New York: McGraw-Hill Book Company; 1947.

79. Lin $\mathrm{YY}$, Cheng $\mathrm{CM}$, Lee $\mathrm{CH}$. A tuned mass damper for suppressing the coupled flexural and torsional buffeting response of long-span bridges. Eng Struct. 2000; 22: 1195-1204.

80. Tamura Y, Fujii K, Ohtsuki T, Wakahara T, Kohsaka R. Effectiveness of tuned liquid dampers under wind excitation. Eng Struct. 1995; 17: 609-621.

81. Makris N, Constantinou MC. Viscous dampers: Testing, modeling and application in vibration and seismic isolation. Buffalo: National Center for Research on Earthquake Engineering; 1990.

82. Constantinou MC, Symans MD, Tsopelas P, Taylor DP. Fluid viscous dampers in applications of seismic energy dissipation and seismic isolation. Proc ATC 17-1 Semin Seism Isol Passiv Energy Dissipation Act Control. 1993; 2: 581-592.

83. Vijayaraja. Passive energy dissipation devices in earthquake resistant buildings [Internet]. 2013. Available from: https://buildcivil.wordpress.com/2013/11/25/passive-energy-dissipationdevices/.

84. Nonami $\mathrm{K}$, Nishimura $\mathrm{H}$, Tian $\mathrm{H} . \mathrm{H}^{\infty} / \mu$ control-based frequency-shaped sliding mode control for flexible structures. Am Soc Mech Eng Publications PVP. 1994; 289: 19.

85. Yang JN, Wu JC, Agrawal AK. Sliding mode control for seismically excited linear structures. J Eng Mech. 1995; 121: 1386-1390. 
86. Chase JG, Smith HA. Robust $\mathrm{H}_{\infty}$ control considering actuator saturation. I: Theory. J Eng Mech. 1996; 122: 976-983.

87. Agrawal AK, Yang JN, Schmitendorf WE, Jabbari F. Stability of actively controlled structures with actuator saturation. J Struct Eng. 1997; 123: 505-512.

88. Spencer Jr BF, Sain MK, Kantor JC, Montemagno C. Probabilistic stability measures for controlled structures subject to real parameter uncertainties. Smart Mater Struct. 1992; 1: 294.

89. Spencer Jr BF, Sain MK, Won CH, Kaspari DC, Sain PM. Reliability-based measures of structural control robustness. Struct Saf. 1994; 15: 111-129.

90. Field Jr RV, Voulgaris PG, Bergman LA. Methods to compute probabilistic measures of robustness for structural systems. J Vib Control. 1996; 2: 447-463.

91. Field Jr RV, Voulgaris PG, Bergman LA. Probabilistic stability robustness of structural systems. J Eng Mech. 1996; 122: 1012-1021.

92. Nagarajaiah S. Fuzzy controller for structures with hybrid isolation system. In Structural Control: Proceedings of the 2nd World Conference on Structural Control. New York: Wiley; 1994.

93. Subramaniam RS, Reinhorn AM, Riley MA, Nagarajaiah S. Hybrid control of structures using fuzzy logic. Compu Aided Civ Infrastruct Eng. 1996; 11: 1-17.

94. Aly AM. Proposed robust tuned mass damper for response mitigation in buildings exposed to multidirectional wind. Struct Des Tall Spec Build. 2014; 23: 664-691.

95. Ghaboussi J, Joghataie A. Active control of structures using neural networks. J Eng Mech. 1995; 121: 555-567.

96. Spencer Jr BF, Nagarajaiah S. State of the art of structural control. J Struct Eng. 2003; 129: 845856.

97. Spencer BF, Sain MK. Controlling buildings: A new frontier in feedback. IEEE Control Syst. 1997; 17: 19-35.

98. Feng $Q$ and Shinozuka $M$. Use of a variable damper for hybrid control of bridge response under earthquake. Proceedings of the US national workshop on structural control research; 1990 October 25th-26th; Los Angeles, CA, US. Los Angeles: University of Southern California.

99. Kamagata S, Kobori T. Autonomous adaptive control of active variable stiffness system for seismic ground motion. Proceeding of First World Conference on Structural Control; 1994 August 3rd-5th; Los Angeles, CA, USA. Los Angeles: International Association for Structural Control.

100.Spencer BF, Carlson JD, Sain MK, Yang G. On the current status of magnetorheological dampers: Seismic protection of full-scale structures. Proceedings of the 1997 American Control Conference; 1997 June 6th; Albuquerque, NM, USA. Piscataway: Institute of Electrical and Electronics Engineer.

101.Spencer BF, Dyke SJ, Sain MK. Magnetorheological dampers: A new approach to seismic protection of structures. Proceedings of 35th IEEE Conference on Decision and Control; 1996 December 13th; Kobe, Japan. Piscataway: Institute of Electrical and Electronics Engineer.

102.Aly AM. Vibration control of buildings using magnetorheological damper: A new control algorithm. J Eng. 2013; 2013: 596078.

103.Şahin i, Engin T, Çeşmeci Ş. Comparison of some existing parametric models for magnetorheological fluid dampers. Smart Mater Struct. 2010; 19: 035012.

104.Chang CC, Roschke P. Neural network modeling of a magnetorheological damper. J Intell Mater Syst Struct. 1998; 9: 755-764. 
105.Chang CC, Zhou L. Neural network emulation of inverse dynamics for a magnetorheological damper. J Struct Eng. 2002; 128: 231-239.

106.Schurter K, Roschke P. Fuzzy modeling of a magnetorheological damper using ANFIS. Proceeding of Ninth IEEE International Conference on Fuzzy Systems; 2020 May 7th-10th; San Antonio, TX, USA. Piscataway: Institute of Electrical and Electronics Engineer.

107.Ehrgott RC, Masri SF. Modeling the oscillatory dynamic behaviour of electrorheological materials in shear. Smart Mater Struct. 1992; 1: 275.

108.Gavin HP, Hanson R, Filisko F. Electrorheological dampers, part II: Testing and modeling. J Appl Mech. 1996; 1050: 48109.

109.BF JS, Dyke SJ, Sain MK, Carlson JD. Phenomenological model of a magnetorheological damper. J Eng Mech. 1997; 123: 230-238.

110.Yang G, Spencer Jr BF, Carlson JD, Sain MK. Large-scale MR fluid dampers: Modeling and dynamic performance considerations. Eng Struct. 2002; 24: 309-323.

111. Aly AM, Zasso A, Resta F. On the dynamics of a very slender building under winds: Response reduction using MR dampers with lever mechanism. Struct Des Tall Spec Build. 2011; 20: 539551.

112.Inaudi JA. Performance of variable-damping systems: Theoretical analysis and simulation. Proceedings of the 3rd International Workshop on Structural Control; 2020 July 6th- 8th; Paris, France. London: World Scientific.

113. Fang JQ, Li QS, Jeary AP. Modified independent modal space control of m.d.o.f. systems. J Sound vib. 2003; 261: 421-441.

114.Yang JN, Li Z, Liu SC. Instantaneous optimal control with velocity and acceleration feedbacks. J Probabilistic Eng Mech. 1991; 16: 204-221.

115.Li QS, Liu DK, Fang JQ. Optimum design of actively controlled structures using genetic algorithms. Adv Struct Eng. 1999; 2: 109-118.

116. Karnopp D, Crosby MJ, Harwood RA. Vibration control using semi-active force generators. J Eng Ind. 1974; 96: 619-626.

117.Leitmann G. Semiactive control for vibration attenuation. J Intell Mater Syst Struct. 1994; 5: 841846.

118.Inaudi JA. Modulated homogeneous friction: A semi-active damping strategy. Earthq Eng Struct Dyn. 1997; 26: 361-376.

119.Dyke SJ, Spencer BF. A comparison of semi-active control strategies for the MR damper. Proceedings of the International Conference on Intelligent Information Systems (IIS 97); 1997 December 8th-10th; Grand Bahama Island, Bahamas. Piscataway: Institute of Electrical and Electronics Engineer.

120.Jansen LM, Dyke SJ. Semiactive control strategies for MR dampers: Comparative study. J Eng Mech. 2000; 126: 795-803.

121.Feng MQ, Mita A. Vibration control of tall buildings using mega subconfiguration. J Eng Mech. 1995; 121: 1082-1088.

122.Chang CC, Qu WL. Unified dynamic absorber design formulas for wind-induced vibration control of tall buildings. Struct Des Tall Build. 1998; 7: 147-166.

123. Min KW, Kim J, Lee HR. A design procedure of two-way liquid dampers for attenuation of windinduced responses of tall buildings. J Wind Eng Ind Aerodyn. 2014; 129: 22-30.

124.Pirner M, Urushadze S. Liquid damper for suppressing horizontal and vertical motions- 
parametric study. J Wind Eng Ind Aerodyn. 2007; 95: 1329-1349.

125.Aly AM, Christenson RE. On the evaluation of the efficacy of a smart damper: A new equivalent energy-based probabilistic approach. Smart Mater Struct. 2008; 17: 045008.

126. Rezaee M, Aly AM. Proposed theory of semiactive gains for smart dampers in MDOF systems. J Struct Eng. 2019; 145: 04019155.

127.Aly AM. Control of wind-induced motion in high-rise buildings with hybrid TM/MR dampers. Wind Struct. 2015; 21: 565-595.

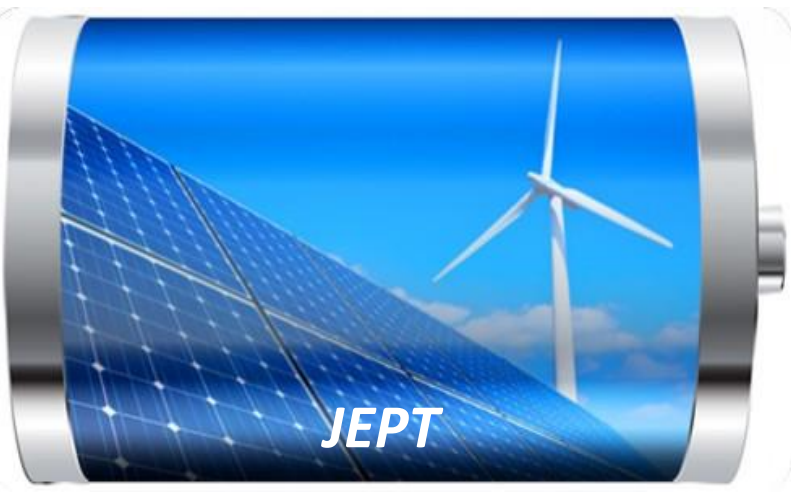

Enjoy JEPT by:

1. Submitting a manuscript

2. Joining in volunteer reviewer bank

3. Joining Editorial Board

4. Guest editing a special issue

For more details, please visit:

http://www.lidsen.com/journal/jept 
Original Research

\title{
Multi-Disciplinary and Multi-Scale Assessment of Marine Renewable Energy Structure in a Tidal System
}

\author{
Aurore Raoux * , Ilan Robin, Jean-Philippe Pezy, Anne-Claire Bennis, Jean-Claude Dauvin
}

Normandie Univ., UNICAEN, Laboratoire Morphodynamique Continentale et Côtière M2C, UMR 6143 M2C, 24 rue des Tilleuls, F-14000 Caen, France; E-Mails: raoux.aurore@gmail.com; ilan.robin@unicaen.fr; jean-philippe.pezy@unicaen.fr; anne-claire.bennis@unicaen.fr; jeanclaude.dauvin@unicaen.fr

* Correspondence: Aurore Raoux; E-Mail: raoux.aurore@gmail.com

Academic Editor: Andrés Elías Feijóo Lorenzo

Special Issue: Renewable Energy in Marine Environment

Journal of Energy and Power Technology

2021, volume 3, issue 1

doi:10.21926/jept.2101012
Received: October 08, 2020

Accepted: March 05, 2021

Published: March 17, 2021

\section{Abstract}

The French coast of the Atlantic and English Channel (EC) is promising for the development of Marine Renewable Energy (MRE), including wind, wave, and tidal stream, due to the high velocity of currents in some parts of the area. This paper, focusing on wind and tidal energy, discusses how the implementation of MRE converters influences biodiversity, and vice versa, through biofouling and reef effects. The understanding of these interactions requires the knowledge of the hydro-sedimentary conditions and the macrofauna. The research on these topics, performed at the Continental and Coastal Morphodynamic laboratory (M2C) (UNICAEN, France), is presented through a multi-disciplinary approach by i) studying the hydrodynamic conditions and the macrofauna in Alderney Race, ii) studying the biofouling effects on tidal turbines and their influence on the turbulent wake, iii) assessing the hydrosedimentary impacts induced by the offshore wind farm, like scouring, and iv) taking an ecosystem approach on MRE, such as the reef effect. From an ecological perspective, the reef effect can be responsible for changes in the structure and function of the ecosystem. Although

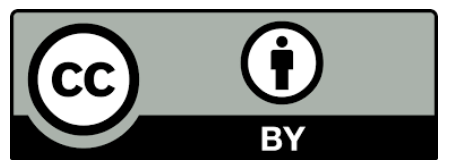

(C) 2021 by the author. This is an open access article distributed under the conditions of the Creative Commons by Attribution License, which permits unrestricted use, distribution, and reproduction in any medium or format, provided the original work is correctly cited. 
several studies have analyzed this effect at the species-or community-scale, the propagation of the reef effect at the ecosystem-scale remains unclear. Thus, understanding these ecosystem-scale effects is urgent for future research. From an engineering perspective, biofouling changes the structural characteristics (i.e., supplementary mass) of the converters and thus, affects their performance.

\section{Keywords}

English Channel; Alderney Race; energy characterization; environmental impacts; fluidstructure interaction; tidal turbine; offshore wind farm; biofouling effect; reef effect

\section{Introduction}

The European Union (EU) had set a target to derive $20 \%$ of its consumed energy from renewable energy sources by 2020. With more than 11 million $\mathrm{km}^{2}$ of water under its jurisdiction, France holds a huge potential for marine renewable energy. Currently, the construction of eight anchored offshore wind farms (OWFs) and four floating OWFs has been planned in metropolitan France (Figure 1). Among them, five anchored offshore wind farms will be built on the English Channel.

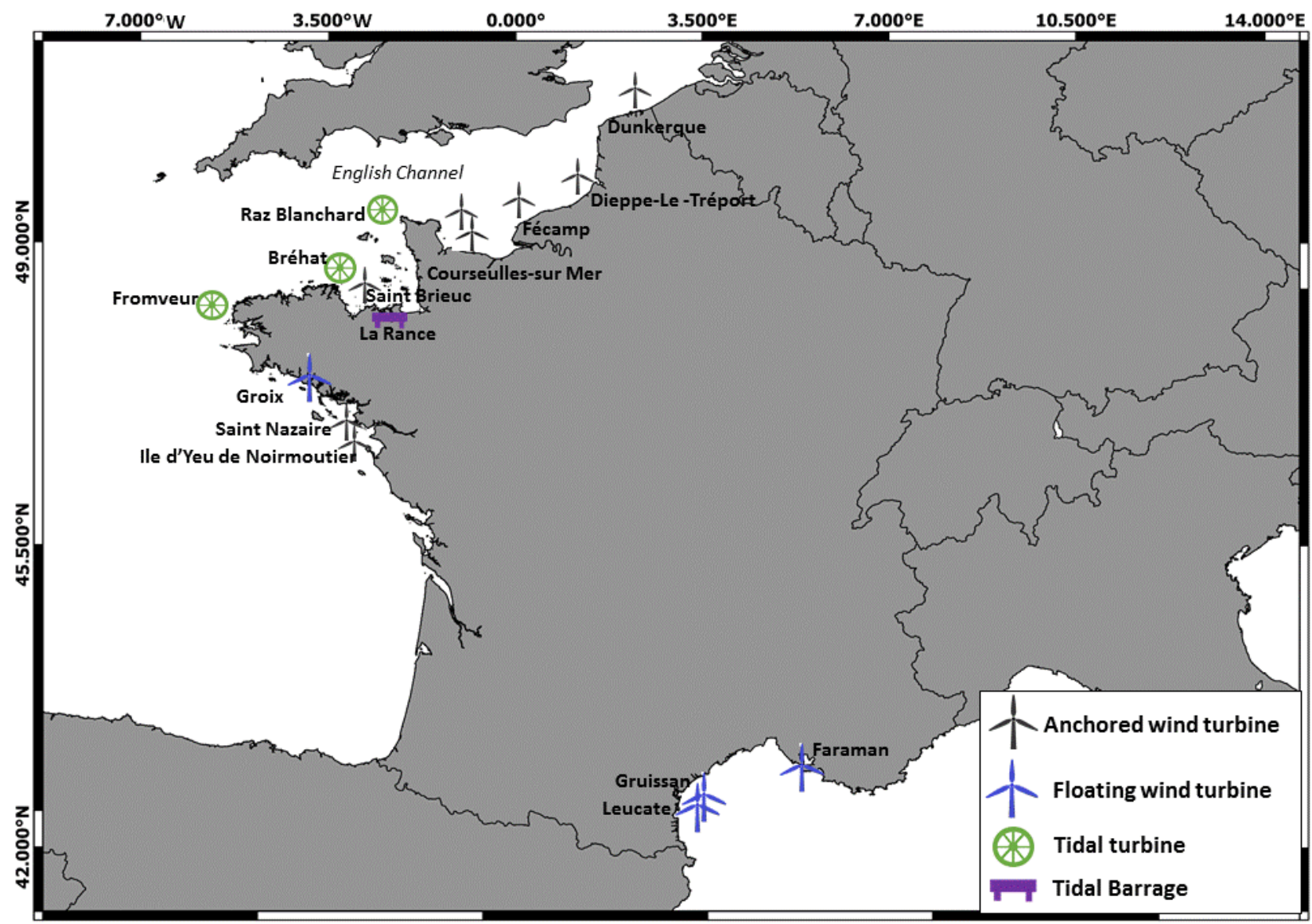

Figure 1 Marine renewable energy development along the French metropolitan coast. 
Till the end of 2020, no OWFs have been constructed due to the long administrative procedures and numerous appeals in courts at the French and the European levels [1]. Nevertheless, several studies have been performed to identify the environmental conditions and ecosystem functioning in selected sites before the implementation of OWFs. These surveys considered several biological groups, including macrobenthos, fish, birds, and mammals (including bats), while assessing the quality of seawater and hydro-sedimentary conditions.

Tidal energy is complementary to wind energy. The tidal power in Europe is estimated at 10 gigawatts (GW), with $4 \mathrm{GW}$ in France. French tidal energy sites are located in Brittany (Fromveur and Bréhat) and Normandy (Raz Blanchard) (Figure 1). The site with the highest tidal energy is the Alderney Race (Raz Blanchard) ( $3 \mathrm{GW}$ ). However, it is also the most complicated site due to complex hydrodynamics, with strong tidal currents (up to 12 knots), ocean waves, highly-energetic turbulent cells, and an uneven sea bed with different geological features (e.g., submarine faults, pebble dunes, etc.). Ten turbine-farms should be installed in Alderney Race to obtain a total power of $20 \mathrm{MW}$. The first French tidal turbines were connected to the electrical network in 2019 in the Ushant Race at Fromveur and at Bréhat (Figure 1).

In the last decade, research laboratories at the University of Caen Normandy had invested in renewable energy development in the marine environment, especially in the Normandy territory at the western part of the English Channel, in the north of Cotentin in the Raz Blanchard area, and the eastern part of the EC in the extended Bay of Seine.

The Continental and Coastal Morphodynamic laboratory (M2C) had developed multi-disciplinary approaches, such as in situ observations, high-frequency radar measurements, physical experimentations, trophic food models, and numerical modeling (Figure 2). The challenge was not only to accompany the development of MRE for each scientific discipline but also to share expertise and develop common projects on ecosystem functioning. This could be achieved by using physical and numerical modeling at different scales of observations, ranging from the consequences of biofouling of tidal turbines on energy production to the regional suspected impacts of offshore wind farming. Although previous studies have highlighted that the colonization of MRE structures by marine species (also called biofouling) can be responsible for both ecological and engineering consequences, its effects at the ecosystem-scale and on the performance of the offshore infrastructure remain poorly understood. Using a multi-disciplinary approach comprising benthos ecology, trophic web, and numerical modeling, along with expertise on the characteristics of the EC, the $\mathrm{M} 2 \mathrm{C}$ laboratory can make an important contribution to the understanding of key issues surrounding the MRE in the EC. The EC is a coastal sea with a strong tidal regime and dominant western winds, which ensures large-scale electricity production in the future. Notably, the M2C is also a member of the Working Group on Marine Benthal and Renewable Energy Developments (WGMBRED), a part of the International Council for the Exploration of the Sea (ICES), since 2013. Thus, this paper aims to present the progress of our research in the development of MRE in the EC. 


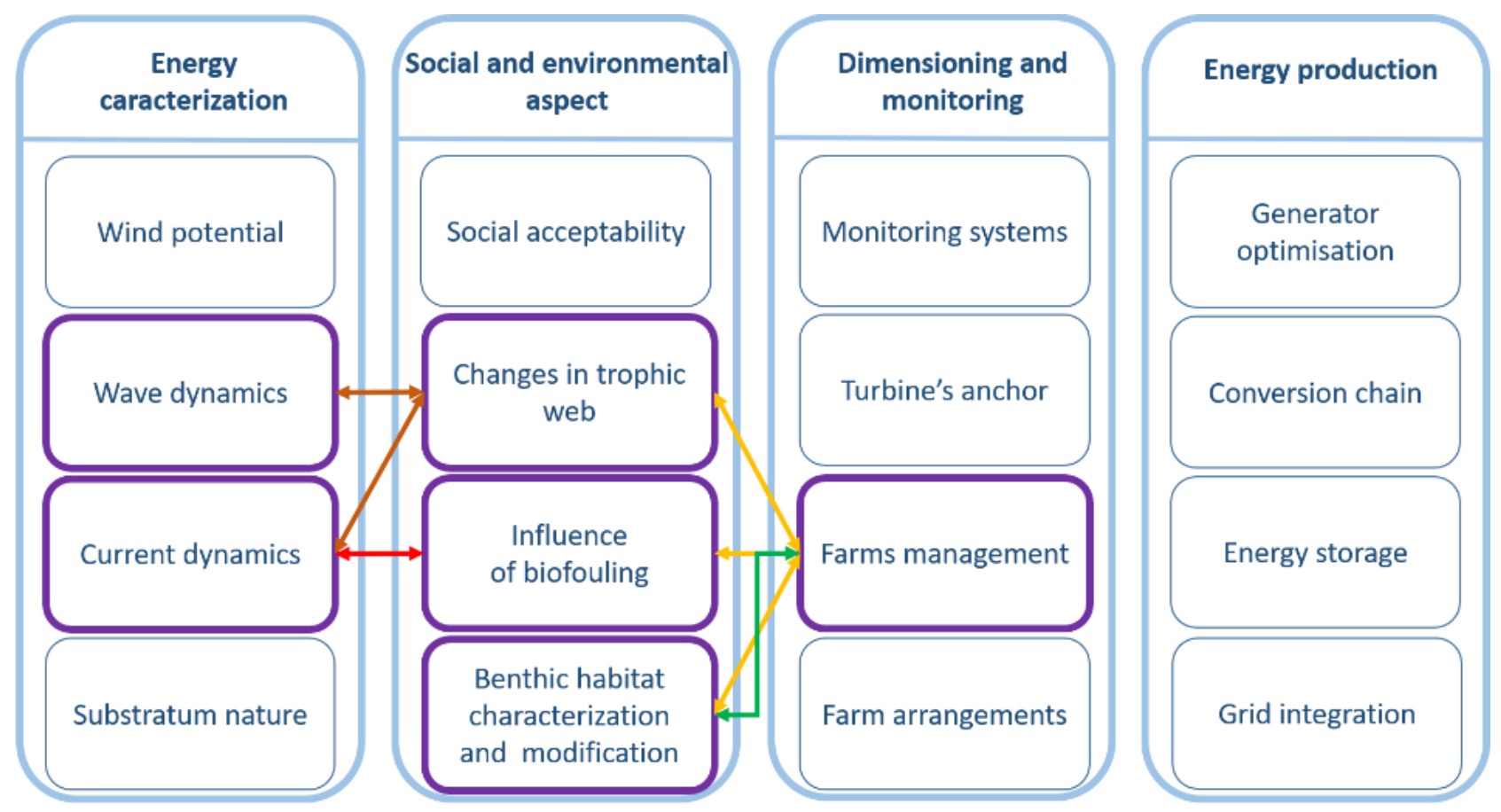

Study of the impact of biofouling on tidal turbine performances

Study of wave and current dynamics in order to study the impact on local ecosystems

Study of the impact of wind turbine farms on the local ecosystem

Study of local ecosystems before farm implantation

$\mathrm{M} 2 \mathrm{C}$ interest fields

Figure 2 Multi-disciplinary approaches developed by the Continental and Coastal Morphodynamic laboratory (M2C) on MRE.

\section{Context: The English Channel, a Megatidal Sea}

The English Channel (EC) is a shallow epi-continental sea shared between the United Kingdom in the north and France in the south, with a maximum depth of $174 \mathrm{~m}$ in the Hurd Deep (150 km in length and 1.5 to $5 \mathrm{~km}$ in width) (Figure 1) [2]. Its western basin is deeper than the Eastern basin and is influenced by the Atlantic waters. It shows the presence of a summer thermocline offshore Plymouth, while the eastern basin is mainly affected by the input of freshwater from the Seine River. The tidal regime is dominated by semidiurnal tides with a period of $12 \mathrm{~h}$ and $25 \mathrm{~min}$. The tidal ranges are greater along the French coast, mainly in the Mont-Saint-Michel Bay than along the British coast. The tidal residual propagates from the west to the east, with a water replacement time of approximately 500 days. Due to the Coriolis force, current velocities are higher along the French coast than the English coast, reaching greater than three knots in north Brittany, off the Cotentin Peninsula. In the Dover Strait, velocities exceed eight knots off the Cap de La Hague. Significant gyres are present around the Channel Islands and to the east of the capes of Fréhel, Barfleur, Antifer, and Gris Nez. Tidal currents dominate sedimentation processes; extensive pebbly sediments dominate areas of strong tidal currents located offshore and to the east of the capes, whereas fine sands and muddy fine sands appear in areas of weak tidal currents in bays and estuaries. Coarse sands and pebbles cover more than $80 \%$ of the EC seabed area; another particular feature of the sediments is their high proportions of calcareous biogenic material, reaching up to $80 \%$ in the bioclastic sediments in the central part of the western EC [2]. Located between $48^{\circ} 30^{\prime} \mathrm{N}$ and $51^{\circ}$ 
$10^{\prime} \mathrm{N}, \mathrm{EC}$ is a temperate sea and is a biogeographical transition zone between the Lusitanian and Boreal provinces, influenced by a balance between warm, temperate species during warm periods and boreal species during cool periods [2].

Moreover, the EC is among the most active coastal marine zones where cumulative human impacts are the greatest. The eastern basin is more impacted than the western basin due to widespread traditional activities such as maintenance of fisheries and the emergence of new activities such as granulate extraction and offshore wind farm installations [2, 3].

\section{Hydrodynamic conditions in Alderney Race}

To determine the shear of surface currents and sea states, high-frequency (HF) radars were implemented in Cap de la Hague in 2017. They monitored in real-time the surface hydrodynamic (currents and sea states). This innovative system, using two transmitting frequencies (24.5 MHz and $13.5 \mathrm{MHz}$ ), produced rare data of the spatialized hydrodynamic of the Alderney Race. First, these data have enabled validation of the numerical model (3D-fully wave-current model); for surface currents, a good agreement has a Root-Mean-Square-Error between 14 and $40 \mathrm{~cm} / \mathrm{s}$ for the neap tide and between 18 and $60 \mathrm{~cm} / \mathrm{s}$ for the spring tide [4, 5]. Maximum errors were found in shallow zones with consistently high current velocities. Second, some regions with a strong current shear were highlighted in the radar data; a new methodology for the radar data analysis in such regions is under development.

In addition to the HF radar measurements, some efforts in numerical modeling were performed during HY2DM. An innovative Large Eddy Simulation (LES) method, called LANS-alpha, was implemented in a coastal hydrodynamic numerical model, MARS3D [6], to improve the simulation of the marine turbulence $[7,8]$. This method differs from the classical dissipative LES in that this is a reformulation of the equations of fluid mechanics with alteration of the existing non-linear terms and the addition of extra non-linear terms. LANS-alpha does not use a sub-grid scale viscosity, unlike dissipative LES. After a validation step for a wind-driven motion over a seamount, this method was applied to the Alderney Race. A re-energization of the flow was observed with two types of turbulence energy decay in the inertial zone as expected for two-dimensional turbulence. This method mainly affects the turbulence induced by deformation; rotation-induced turbulence is slightly modified. The computing time is reduced by $30 \%$ by the use of LANS-alpha.

Furthermore, $\mathrm{M} 2 \mathrm{C}$ researchers studied the impact of the wave-current interactions on the tidal stream energy using numerical modeling. The 3D-fully wave-current simulations were performed with the coupled model, which combined the hydrodynamic numerical model, MARS 3D, and the spectral wave model, WAVEWATCH-III [9]. Data from this coupled model were tested and compared with in situ observed data and were found to have good scores [10]: i) the RMSE related to the sea level was around $0.13 \mathrm{~m}$ and $0.44 \mathrm{~m}$ at Cherbourg and Dièlette, respectively, ii) the PBIAS (percent bias) related to wind at ten-meters height was around $10 \%$ and lesser than $4 \%$ for magnitude and direction, respectively, iii) the significant wave height PBIAS was around $6 \%$ with an $\mathrm{R}^{2}$ (coefficient of determination) of 0.97 , iv) the NRMSE (Normalized RMSE) related to the magnitude of current was around 0.11 . The inclusion of wave effects has contributed to a decrease in the error in tidal energy estimate, which was lesser than $10 \%$ for the considered time-period (21-25 November 2017). Additionally, the impact of Eleanor's storm (on 2-3 January 2020) on tidal energy was simulated using the coupled model. This storm occurred during a spring tide. A strong reduction in tidal current 
magnitude $(0.4-0.5 \mathrm{~m} / \mathrm{s})$ was observed with PBIAS reduction by a factor of 1.89 and 1.76 for simulations with only tides and with both tides and wind, respectively [11].

\section{Macrofauna in the Alderney Race}

In the research project entitled "Pile \& Tide", financed by the ADEME (Agence de l'Environnement et de la Maîtrise de l'Energie), the M2C laboratory was in charge of the investigation of the benthic habitats (seabed composition and benthic communities) in the Alderney Race strait. The area is located in the western basin of the English Channel to the North of the Normand-Breton Gulf, with the strongest tidal currents in Europe. The water depth here varies between 20 and $80 \mathrm{~m}$. The sea bed is mainly composed of granite and calcareous rocks of the Cambrian, Silurian, and Cretaceous ages; some surficial sediments in small patches between rocky substrate are mainly composed of gravel and pebbles [12].

The data on the macrofauna of such a dynamic area are very rare and were obtained in the 1970s from the doctoral thesis of Retière (1979) [13], who described a mussel community, Musculus discors (L., 1767), in the area of the Alderney Race. Additionally, the horse mussel, Modiolus modiolus (Lamarck, 1799), was reported to form mussel beds. However, M. modiolus was not recorded recently in samplings in 2015-2016 [12], and its persistence in this area, which corresponds to the southern limit of its distribution in the north-eastern Atlantic, needs to be confirmed

During three sampling campaigns between 2015 and 2016, a total of 38 benthic stations were visited with a Rallier du Baty dredge. A single gear was used to sample the sea bed in hydrodynamic areas. Of the 38 operated dredges, 24 were unsuccessful, containing little to no sediments, and only 14 yielded sufficient volume of sediment ( 0.5 to $32 \mathrm{~L}$ ) composed of gravels and pebbles with or without sessile epifauna [12]. Despite a low number of effective stations, the collected fauna showed high diversity with 140 different taxa, dominated by crustaceans (69 taxa). The fauna was characterized by small species living either in interstitial positions, or as infauna, or protected (like erect hydrozoans). Most of the sampled species were adapted to live in high-energy hydrodynamic conditions and hard irregular sea beds. Following Michez et al. (2015) [14], three benthic habitats were identified [12]: (1) very mobile and coarse infralittoral sediments with scattered fauna; (2) circalittoral pebbles under strong hydrodynamic conditions with scattered fauna; and (3) mobile coastal circalittoral gravel and pebbles consisting of Spirobranchus triqueter (L., 1758), barnacles, and encrusted bryozoans. New locations of two crustacean species in the English Channel were reported for the amphipod, Elasmopus thalyae (Gouillieux and Sorbe, 2015) [15], found at the soft and hard bottoms of the Arcachon Bay [16] and the tanaid, Zeuxo holdichi (Bamber, 1990), described from the Arcachon Bay [17].

To provide additional information to standard benthic observations, Foveau et al. (2017) [18] had developed a new underwater imagery system to monitor the seafloor and benthic communities of the Alderney Race. Despite the difficulty in the acquisition, the underwater images highlighted the heterogeneous nature of the seabed in the Alderney Race. A total of 380 photos of the seafloor were obtained during a 2016 survey, among which 38\% were usable [18]. Two main sediment types were recognized: (1) gravel and blocks, mixed in a more or less cohesive way, and (2) bedrock. Sessile fauna was identified from the images, which included the barnacle, Balanus crenatus (Bruguière, 1789), hydrozoans belonging to the genus Sertularia, as well as poriferans and 
bryozoans. From the images, it was not possible to determine mobile species due to their small size and infaunal lifestyle.

In summary, the sea bed was mainly composed of a hard bottom with small enclaves of coarse sediments in the spaces between large granite blocks. This area remained difficult to explore due to its high heterogeneity and steep slopes (in some places, the vertical depth varied by $10 \mathrm{~m}$ over a horizontal distance of $5 \mathrm{~m} \mathrm{[18]).} \mathrm{Due} \mathrm{to} \mathrm{strong} \mathrm{currents,} \mathrm{the} \mathrm{hard} \mathrm{bottom} \mathrm{was} \mathrm{poorly} \mathrm{colonized} \mathrm{by}$ sessile fauna, except the mussel, Musculus discors, and the barnacle, Balanus crenatus, which dominated the epifauna. Nevertheless, the infauna was rich and consisted mainly of small species adapted to live in clean mobile sediments.

\section{Impact of Macrofauna on Tidal Turbine Performances}

After identifying the macrofauna of the Alderney Race, the $\mathrm{M} 2 \mathrm{C}$ researchers investigated its influence on the tidal turbine performance. In particular, the effects induced by biofouling on the turbulent wake were studied. Assessing these effects is a relevant issue for the tidal industry to minimize the maintenance of the turbines. Numerical modeling is challenging due to the need to solve the geometry of biofouling species in the mesh and to produce long time series for the diagnosis of turbine performance. Computational Fluid Dynamic (CFD) simulations were carried out to understand how species changed the flow near the blades using three main types of turbulence models (RANS, LES, DNS) available in OpenFoam [19]. The computational domain was composed of two meshes-one was fixed, while the other rotates and contained the rotor. The Arbitrary Mesh Interface (AMI) method was used to send the flow from one mesh to the other. As explained in Figure 3, the LES (Large Eddy Simulation) method with the Smagorinsky model was the best choice for this application; turbulent structures induced by biofouling were better represented than using RANS, and the computing time was less than using the DNS model. The simulated turbulent wake was found to be sensitive to the presence of organisms. When the colonization was located near the leading edge, the effects were much more significant. Moreover, a single organism or a small colony can disrupt the wake more than a large colony, leading to a sharkskin effect. Threedimensional effects were also investigated by Robin et al. (2019) [20] for the cases of forced and flow-induced rotations. Flow-induced rotation conforms to the energy chain better than forced rotation. In flow-induced rotation, the primary energy contained in the flow is transformed into mechanical energy before being converted into electricity. The wakes in both cases were compared, and, as expected, the signals were similar for similar speeds of rotation. It showed that flow-induced code does not interfere with the flow results. Moreover, biofouling modifies the rotor weight, which mainly affects the turbine's behavior when the flow strongly depends on time. The modification of the mass impacts the rotor's restart (transition mode) if it is heavily loaded with organisms. This problem was unlikely to occur in the Alderney Race but can appear at tidal turbines in shallower seas. The $\mathrm{M} 2 \mathrm{C}$ researchers are currently working on the coupling between OpenFoam (CFD) and Dorothy (vortex model) numerical codes to reduce the computing time. OpenFoam will handle the close-blade field while Dorothy will solve the wake in the far-field. Thus, we will have an efficient tool to study the impacts of different colonizations on the performance of the tidal turbines. 


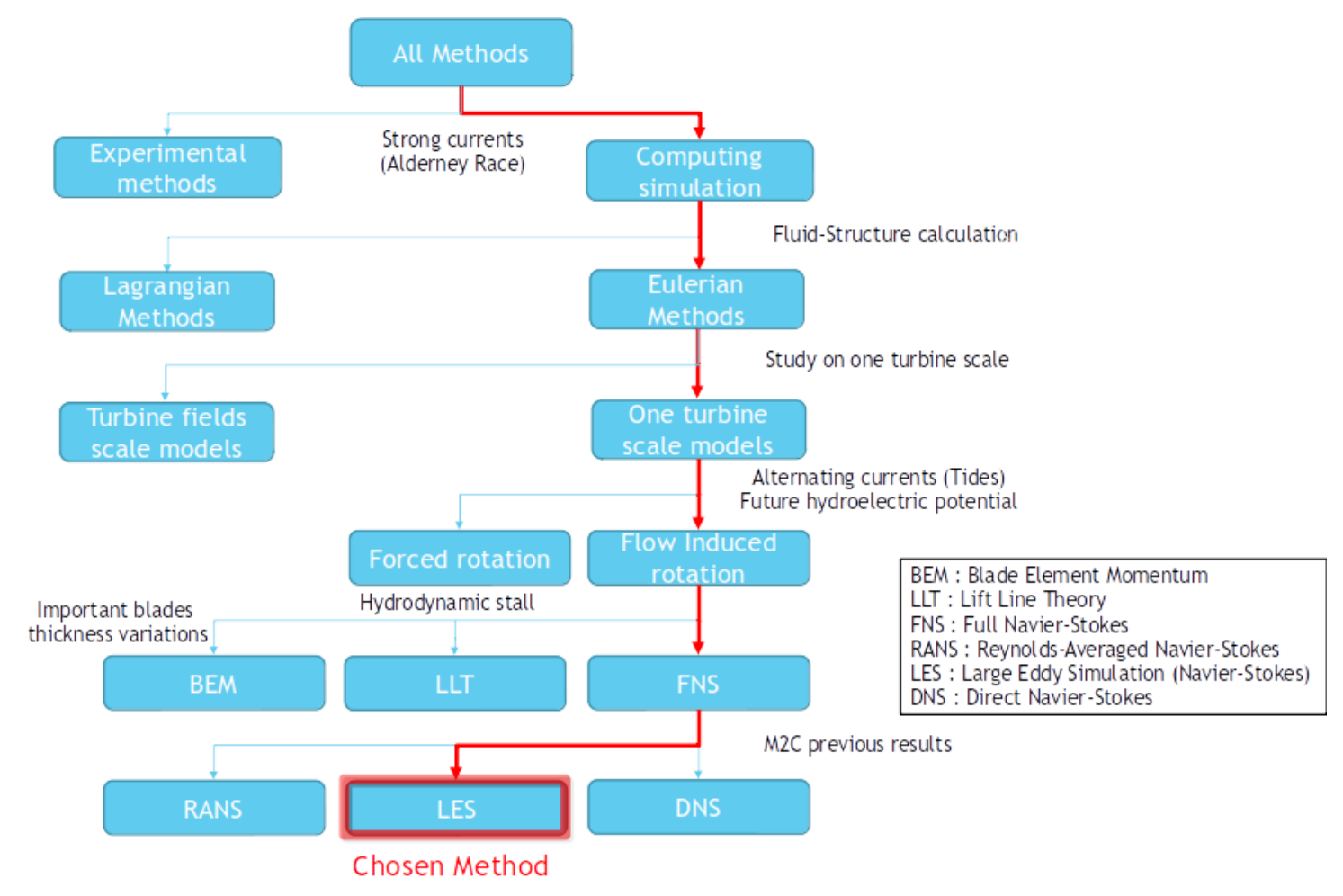

Figure 3 Decision tree for CTD method selection.

\section{Assessment of the Environmental Impacts of the Offshore Wind Farm Foundations}

In the Ofelia project (Interreg IVa France-England), the $\mathrm{M} 2 \mathrm{C}$ was in charge of the numerical modeling for the assessment of the hydro-sedimentary impacts of the monopiles on the future Courseulles s/mer wind farm [21, 22]. A subgrid-scale parameterization was developed and implemented in the three-dimensional hydrodynamical model, MARS 3D. This parameterization, based on Ganthy (2011) [23] and Kombiadou et al. (2014) [24] for the Zostera noltei meadows, was able to reproduce the changes in the flow due to the monopiles and the impacts on the seabed morphology by adding drag forces to the momentum and turbulence equations. Comparisons with simulations where the monopiles were explicitly solved in the mesh were done to evaluate the performance of the parameterization over a tidal cycle. We observed similar behaviors between the two types of simulations, where currents increased at the side of the monopile and decreased in front and downstream of the monopile. For a network of four monopiles, and according to the tidal cycle, wakes downstream of the monopile interacted with the other monopiles modifying the current velocity. For the effect of sediment transport, the increase in the flow around the monopile caused resuspension and erosion (scour), while the decrease in the flow generated sediment deposits around the monopile. These effects were in agreement with the ones observed experimentally (in-situ or laboratory) $[25,26]$. This new parameterization may be used to efficiently simulate the hydro-sedimentary changes induced by the monopiles. For other types of foundations, like gravity-based or tripod, results have to be carefully interpreted, and the expressions of the forces need to be adjusted.

\section{Toward an Ecosystem Approach of Marine Renewable Energy}


Noting that there was no integrated study of the effects of MRE on the ecosystem in the countries where they were implemented $[1,27]$, the $\mathrm{M} 2 \mathrm{C}$ laboratory promoted a sampling strategy to collect local data for future environmental monitoring programs of OWFs. The M2C developed an MRE ecosystem approach based on local biomass data (i.e., zooplankton, meiofauna, benthos, and demersal fish) and the link between demersal fish and the benthos, through stomach content studies $[1,27]$. Then, a trophic web model based on these data was built. In parallel, the $M 2 C$, in collaboration with the Biology of Aquatic Organisms and Ecosystems (BOREA) laboratory, explored a new way to understand the potential impacts of OWFs through food web models and flow analysis. They developed an ecosystem-integrated view of the future CSM OWF site to describe the marine ecosystems before OWF construction and to simulate the reef effect after OWF construction (Figure 4) $[27,28]$. Among the different trophic web modeling approaches currently available, the modeling tool Ecopath with Ecosim (EwE) was used [29-31] to describe the initial state of both future OWF sites. This was a mass-balance, zero-dimension modeling approach, which had previously been used to analyze the impact of a wide range of anthropogenic perturbations on marine ecosystems such as fishing, sea dumping operations, new species introductions, and infrastructure development [3237]. However, surprisingly few studies have combined trophic web modeling strength with OWF impact assessment [27, 38]. Thus, the M2C laboratory applied the EwE approach to model the trophic web of the area intended for the CSM OWF and the DLT OWF construction to obtain knowledge of both the systems and to characterize their ecological properties through Ecological Network Analysis (ENA) [1, 27, 28].

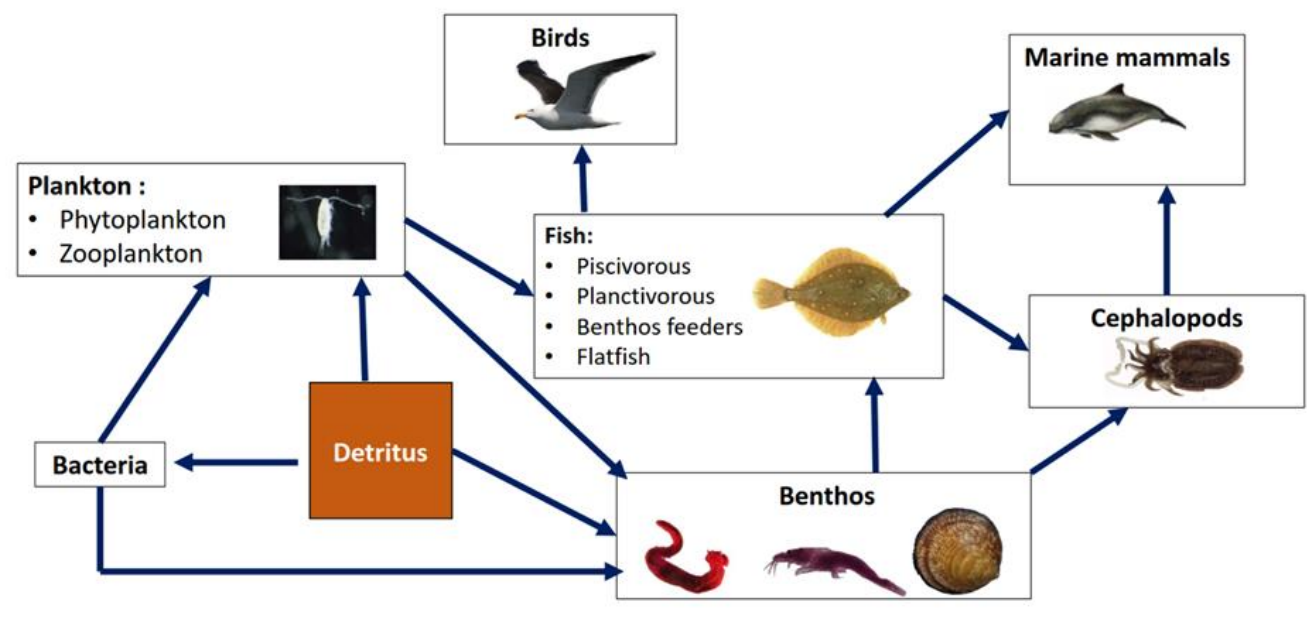

Figure 4 Trophic web of the Courseulles-sur-Mer ecosystem model (modified from Raoux et al., 2017 [27]).

\subsection{Ecopath Models of the Future CSM OWF and DLT OWF Sites}

An ecosystem model composed of 37 compartments (from phytoplankton to seabirds) was built to describe the situation "before" the CSM OWF construction and was called Before Offshore Wind Farm (BOWF) [27]. The compartment selection and aggregation were based on trophic interactions. Additionally, special attention was given to commercial species by placing them in monospecific compartments (for more details, see Raoux et al., 2017 [27]). This model was built by using the data collected during the Environmental Impact Assessment (EIA) of the CSM OWF site. The main results highlighted that the CSM trophic web is controlled by an intermediate trophic level dominated by 
the keystone species, the pouting (Trisopterus luscus). Similarly, an ecosystem model (from phytoplankton to seabirds) was built to describe the structure and function of the DLT OWF construction site. Ecosystem species were grouped into 28 compartments of similar trophic behavior (same prey and same predators). Additionally, benthic invertebrate macrofauna was subdivided into consumed and non-consumed species (more details in Pezy et al., 2018a). This model was built by using the data collected from sampling operations following the methods described by Pezy et al. (2018a) [1]. The main results highlighted that the trophic web is most likely detritus-based and that the ecosystem biomass is dominated by "non-consumed benthic invertebrates". This could be a trophic dead-end for fish due to the size of these filter feeders compared to the size of the fish in the DLT area. All information regarding the input and output parameters of the CSM and the DLT Ecopath models can be found in Raoux et al. (2017) [27] and Pezy et al. (2018a) [1], respectively.

The area intended for the construction of the CSM OWF and the DLT OWF had gravelly sand. The ecosystem biomass of both sites was dominated by benthic invertebrate filter feeders, with biomass of $22.5 \mathrm{gC} . \mathrm{m}^{-2}$ at the CSM site (dominated by the bivalve, Polititapes rhomboides) and $45 \mathrm{gC}^{-2} \mathrm{~m}^{-2}$ at the DLT site (dominated by the dog cockle, Glycymeris glycymeris, and the banded carpet clam, Polititapes rhomboides) [1, 39]. The comparison of the ENA between the gravelly sand of the OWF planned at CSM and at DLT highlighted that the total activity and ascendency values were higher for the CSM OWF. This was because the CSM OWF model had a greater number of compartments than the DLT OWF model and a higher intake of nutrients from the Seine River [40]. Additionally, between the two sites, the recycling value was higher at the area intended for the DLT OWF construction due to higher bivalve biomass at the DLT site [39]. This result highlighted the importance of considering an ecosystem perspective with physical and biological coupling in the OWF surveys. Thus, even if both these sites had a similar type of sediment, they had different emergent properties that could be explained partly by higher biomass and species richness at the DLT site.

\subsection{Simulation of the Reef Effect Due to the OWF Construction}

The reef effect has been described for epibenthic and demersal fauna as well as for benthopelagic fish (including commercial species) close to wind farm foundations [27, 28, 41-47]. Filter feeders, such as mussels and amphipods, tend to colonize and dominate the vertical structures of the turbines. Benthic predators such as crabs commonly inhabit the base of the foundations, and the scour protections [48]. In the North sea, several fish species (such as the pouting (Trisopterus luscus) and cod (Gadus morhua) have been shown to aggregate around the foundations, possibly because of the enhanced food supplies which could potentially improve their productivity [42-44]. Additionally, studies have also pointed out that the increase in the biomass of filter feeders, such as the blue mussel (Mytilus edulis), on turbines can alter the pelagic primary productivity by reducing the local concentration of phytoplankton $[46,47,49]$. This depletion of phytoplankton and primary production affect the benthic and fouling community and is responsible for changes in the functioning of the ecosystem, such as the local biogeochemical cycle $[46,49]$.

As mentioned previously, so far, there is no holistic study on the impact of the reef effect induced by OWF constructions on entire ecosystems. Hence, as a complementary approach to the traditional impact assessment, the objective of the $\mathrm{M} 2 \mathrm{C}$ laboratory, in collaboration with BOREA, was to develop an integrated ecosystem overview of the reef effect. Thus, an Ecosim model (the temporal 
dynamic module of EWE) was derived from projecting the evolution of the ecosystem over the next 30 years, after the simulated increase in the biomass of specific benthic and fish compartments associated with the OWF construction. Key findings from these analyses showed that the reef effect is predicted to lead to a detritivore-dominated trophic web [27], which is similar to the hypothesis of Norling and Kautsky (2008) [50]. An increase in the biomass of benthic invertebrates and benthosfeeding fish in response to the reef effect is predicted to attract apex predators, as hypothesized by Lindeboom et al. (2011) [45] and by Reubens et al. [44]), who demonstrated that poutings fed on the macrobenthos produced on the Thornton bank wind turbines in the Belgian part of the North Sea. Additionally, as mentioned previously, colonization of MRE structures by epibenthic communities (mussels and barnacles) can also affect the offshore infrastructure and its performance. Thus, one of the main challenges of the development of MRE is to understand how biofouling affects turbine performance.

\section{Important Gaps within the OWF Environmental Impact Assessment (EIA) Studies}

The EIA considers the sensitivity to potential disruptions of some groups of valuable species without accounting for the links between them [27]. Thus, the effects of the construction of OWFs on the structure and function of the ecosystem remain unclear. There is an urgent need for the OWF EIA to adopt a holistic and integrated view of the ecosystem in their analyses. Additionally, iconic or flagship species are particularly emphasized for their endangered status and popularity among the masses [51]. Thus, monitoring of biological entities other than top predators, particularly within the benthic community (including hard artificial structures), has not yet been sufficiently accounted for $[27,51,52]$. However, several studies have stressed the need to include the benthos within ecosystem monitoring of the MREs $[51,52]$. As mentioned below, the reef effect is considered to be the most important impact on the ecosystem with the arrival of new species. It is thus necessary to analyze the evolution of this new hard-substrate community. Additionally, a European review concluded that knowledge of changes in the functioning of soft-bottom benthic ecosystems due to MRE operations is limited and will require hypothesis-driven research combined with ecological modeling. This study also revealed that benthic sensitivity to MRE is higher than previously indicated [51]. Finally, Dannheim et al. (2019) [52] stressed the urgency to integrate benthic research on the MRE impact assessment with scientific approaches at the ecosystem level.

\section{Next steps}

The hydrodynamic of the Alderney Race is very complex, and further research is necessary to understand the physical mechanisms and their impacts on sediment transport and macrofauna. The $\mathrm{M} 2 \mathrm{C}$ researchers are planning to work on the characterization of the current shear and the interactions between waves, current, and turbulence near the bottom.

Laboratory experiments will be conducted to evaluate the impacts of species on the turbulent flow near the blade and in the wake. These results will be compared to the numerical results acquired by the $\mathrm{M} 2 \mathrm{C}$ researchers.

The reef effect creates important hard-bottom macrobenthic biomasses, but it also increases the availability of prey to higher trophic levels, especially demersal fishes. It will be essential to identify the part of the benthic biomass consumed by fish around the EMR by using stomach content or isotope analyses to construct a more realistic trophic model due to EMR deployments. This 
approach was proposed by the M2C laboratory for the CSM EMR site simultaneously with the evaluation of the changes in the soft-bottom and hard-bottom communities. The role of the EMR structures as a source for connecting populations of various species with a benthopelagic development began in October 2020 in the framework of a three-year Ph. D. program. This topic involved benthologists and physicians ensuring a multi-disciplinary approach by the $\mathrm{M} 2 \mathrm{C}$ laboratory to establish the English Channel as a model for the Megatidal Sea, where humans are highly dependent on the coastal ecosystem, which is under strong environmental forces.

\section{Acknowledgements}

These works were co-funded by the Normandy Region, the group 'Eoliennes Offshore du Calvados', the group 'Eoliennes en mer Dieppe-Le Tréport', the ANR HYD2M project (ANR-10-IEED0006-07), the ADEME Pile\&Tide project, the Interreg IVa France-Angleterre OFELIA project and the CPER Manche 2021 project. We also acknowledge Nathalie Niquil (BOREA, University of Caen), Guiomar Lopez (M2C, University of Caen), Laurent Benoit (M2C, University of Caen), Laurent Perez (M2C, University of Caen), Martial Boutet (M2C, University of Caen) and Feddy Adong (M2C, University of Caen). Results acquired with Ifremer MARS software. The authors acknowledge the Centre Régional Informatique et d'Applications Numériques de Normandie (CRIANN) for providing the computational resources and support.

\section{Author Contributions}

All authors have made a substantial, direct and intellectual contribution to the work, and approved it for publication.

\section{Competing Interests}

The authors have declared that no competing interests exist.

\section{References}

1. Pezy JP, Raoux A, Dauvin JC. An ecosystem approach for studying the impact of offshore wind farms: A French case study. ICES J Mar Sci. 2020; 77: 1238-1246.

2. Dauvin JC. The English Channel: La Manche. In World Seas: An Environmental Evaluation. 2nd ed. Volume I: Europe, the Americas and West Africa. Cambridge, MA: Academic Press Published; 2019. p. 153-188.

3. Halpern BS, Walbridge S, Selkoe KA, Kappel CV, Micheli F, D'Agrosa C, et al. A global map of human impact on marine ecosystems. Science. 2008; 319: 948-952.

4. Lopez G, Bennis AC, Barbin Y, Sentchev A, Benoît L, Marié L. Surface currents in the Alderney Race from high-frequency radar measurements and three-dimensional modelling. Philos Trans Royal Soc A. 2020; 378: 20190494.

5. Lopez G, Bennis AC, Barbin Y, Benoît L, Cambra R, Conley D, et al. Surface hydrodynamics of the Alderney Race from HF radar measurements. Proceedings of 13th European Wave and Tidal Energy Conference; 2019 September 1st; Naples, Italy. Paris: Centre pour la Communication Scientifique Directe.

6. Lazure P, Dumas F. An external-internal mode coupling for a 3D hydrodynamical model for 
applications at regional scale (MARS). Adv Water Resour. 2008; 31: 233-250.

7. Adong F, Bennis AC. Turbulence models for coastal simulation: Application to Alderney Race. Proceedings of 13th European Wave and Tidal Energy Conference; 2019 September 6th; Naples, Italy. Paris: Centre pour la Communication Scientifique Directe. p. 1-8.

8. Bennis AC, Adong F, Boutet M, Dumas F. LANS- $\alpha$ turbulence modeling for coastal sea: An application to Alderney Race. J Comput Phys. 2021; 432: 110155.

9. Tolman HL. User manual and system documentation of WAVEWATCH ${ }^{\circledR}{ }^{\circledR}$ version 4.18. NOAA/NWS/NCEP/MMAB; Technical Note 316; 2014.

10. Bennis AC, Furgerot L, Du Bois PB, Dumas F, Odaka T, Lathuiliere C, et al. Numerical modelling of three-dimensional wave-current interactions in complex environment: Application to alderney race. Appl Ocean Res. 2020; 95: 102021.

11. Bennis AC. Final report of the HYD2M project [Internet]. Plouzane: France energies marines; 2020. Available from: http://hyd2m.criann.fr/en/accueil/projet-2/.

12. Foveau A, Dauvin JC. Surprisingly diversified macrofauna in mobile gravels and pebbles from high-energy hydrodynamic environment of the 'Raz Blanchard' (English Channel). Reg Stud Mar Sci. 2017; 16: 188-197.

13. Retière C. Contribution à l'étude des peuplements benthiques du golfe normano-breton. Rennes, France: Université of Rennes; 1979.

14. Michez N, Bajjouk T, Aish A, Andersen AC, Ar Gall E, Baffreau A, et al. Typologie des habitats marins benthiques de la Manche, de la Mer du Nord et de l'Atlantique Version 2. Paris, Fance: Muséum national d'Histoire naturelle; SPN 2015-45; 2015.

15. Gouillieux B, Sorbe JC. Elasmopus thalyae sp. nov. (Crustacea: Amphipoda: Maeridae), a new benthic species from soft and hard bottoms of Arcachon Bay (SE Bay of Biscay). Zootaxa. 2015; 3905: 107-118.

16. Foveau A, Dauvin JC. Elasmopus thalyae (Crustacea: Amphipoda: Maeridae) in high energetic area off the North-west Cotentin peninsula, English Channel. Cah Biol Mar. 2017; 58: 363-366.

17. Foveau A, Pezy JP, Bauxi N, Baffreau A, Bachelet $Q$, Chouquet B, et al. Range extension of the tanaidid Zeuxo holdichi (Bamber, 1990) along the northern coasts of France? Cah Biol Mar. 2018; 59: 329-333.

18. Foveau A, Haquin S, Dauvin JC. Using underwater imagery as a complementary tool for benthos sampling in an area with high-energy hydrodynamic conditions. J Mar Biol Oceanogr. 2017; 6.

19. Rivier A, Bennis AC, Jean G, Dauvin JC. Hydrodynamic consequences of biofouling organisms on marine energy converters. Int J Mar Energy. 2018; 2: 101-109.

20. Robin I, Bennis AC, Dauvin JC, Gualous H. Impact of tidal turbine biofouling on turbulence and power generation. Proceedings of 24e Congrès Français de Mécanique; 2019 August 26th; Brest, France. Paris: Centre pour la Communication Scientifique Directe. pp. 1-12.

21. Rivier A, Bennis AC, Pinon G, Gross M, Magar V. Regional numerical modelling of offshore monopile wind turbine impacts on hydrodynamics and sediment transport. Boca Raton, FL: CRC Press; 2015. pp. 807-813.

22. Rivier A, Bennis AC, Pinon G, Magar V, Gross M. Parameterization of wind turbine impacts on hydrodynamics and sediment transport. Ocean Dyn. 2016; 66: 1285-1299.

23. Ganthy F. Rôle des herbiers de zostères (Zostera noltii) sur la dynamique sédimentaire du Bassin d'Arcachon. Bordeaux, Fance: Université de Bordeaux; 2011.

24. Kombiadou K, Ganthy F, Verney R, Plus M, Sottolichio A. Modelling the effects of Zostera noltei 
meadows on sediment dynamics: Application to the Arcachon lagoon. Ocean Dyn. 2014; 64: 1499-1516.

25. Rogan C, Miles J, Simmonds D, Iglesias G. The turbulent wake of a monopile foundation. Renew Energ. 2016; 93: 180-187.

26. Gunnoo H, Abcha N, Ezersky A. Frequency lock-in and phase synchronization of vortex shedding behind circular cylinder due to surface waves. Phy Lett A. 2016; 380: 863-868.

27. Raoux A, Tecchio S, Pezy JP, Lassalle G, Degraer S, Wilhelmsson D, et al. Benthic and fish aggregation inside an offshore wind farm: Which effects on the trophic web functioning? Ecol Indic. 2017; 72: 33-46.

28. Raoux A, Lassalle G, Pezy JP, Tecchio S, Safi G, Ernande B, et al. Measuring sensitivity of two OSPAR indicators for a coastal food web model under offshore wind farm construction. Ecol Indic. 2019; 96: 728-738.

29. Polovina JJ. Model of a coral reef ecosystem. I. The ECOPATH model and its application to French Frigate Shoals. Coral Reefs. 1984; 3: 1-11.

30. Christensen V, Walters CJ. Ecopath with Ecosim: Methods, capabilities and limitations. Ecol Modell. 2004; 172: 109-139.

31. Christensen V, Walters CJ, Pauly D, Forrest R. Ecopath with Ecosim version 6 user guide. Lenfest Ocean Futures Project. Vancouver, Canada: University of British Columbia, 2008. pp. 1-235.

32. Langseth BJ, Rogers $M$, Zhang $H$. Modeling species invasions in Ecopath with Ecosim: An evaluation using Laurentian Great Lakes models. Ecol Modell. 2012; 247: 251-261.

33. Heymans JJ, Tomczak MT. Regime shifts in the Northern Benguela ecosystem: Challenges for management. Ecol Modell. 2016; 331: 151-159.

34. Pezy JP, Raoux A, Marmin S, Balay P, Niquil N, Dauvin JC. Before-after analysis of the trophic network of an experimental dumping site in the eastern part of the Bay of Seine (English Channel). Mar Pollut Bull. 2017; 118: 101-111.

35. Pezy JP, Raoux A, Marmin S, Balay P, Dauvin JC. What are the most suitable indices to detect the structural and functional changes of benthic community after a local and short-term disturbance? Ecol Indic. 2018; 91 :232-240.

36. Raoux A, Baux N, Pezy JP, Balay P, Lesourd S, Dauvin JC. Evaluating ecosystem functioning of a long-term dumping site in the Bay of Seine (English Channel). Ecol Indic. 2020; 115: 106381.

37. Tecchio S, Rius AT, Dauvin JC, Lobry J, Lassalle G, Morin J, et al. The mosaic of habitats of the Seine estuary: Insights from food-web modelling and network analysis. Ecol Modell. 2015; 312: 91-101.

38. Raoux A, Pezy JP, Ernande B, Niquil N, Dauvin JC, Grangeré K. Isotopic analyses, a good tool to validate models in the context of marine renewable energy development and cumulative impacts. Estuar Coast Shelf Sci. 2020; 237: 106690.

39. Pezy JP, Raoux A, Dauvin JC. The environmental impact from an offshore windfarm: Challenge and evaluation methodology based on an ecosystem approach. Ecol Indic. 2020; 114: 106302.

40. Billen G, Garnier J, Lassaletta L. The nitrogen cascade from agricultural soils to the sea: Modelling nitrogen transfers at regional watershed and global scales. Philos Trans R Soc Lond B Biol Sci. 2013; 368: 20130123.

41. De Mesel I, Kerckhof F, Norro A, Rumes B, Degraer S. Succession and seasonal dynamics of the epifauna community on offshore wind farm foundations and their role as stepping stones for non-indigenous species. Hydrobiologia. 2015; 756: 37-50. 
42. Reubens JT, Degraer S, Vincx M. The ecology of benthopelagic fishes at offshore wind farms: A synthesis of 4 years of research. Hydrobiologia. 2014; 727: 121-136.

43. Reubens JT, Braeckman U, Vanaverbeke J, Van Colen C, Degraer S, Vincx M. Aggregation at windmill artificial reefs: CPUE of Atlantic cod (Gadus morhua) and pouting (Trisopterus luscus) at different habitats in the Belgian part of the North Sea. Fish Res. 2013; 139: 28-34.

44. Reubens JT, Degraer S, Vincx M. Aggregation and feeding behaviour of pouting (Trisopterus luscus) at wind turbines in the Belgian part of the North Sea. Fish Res. 2011; 108: 223-227.

45. Lindeboom HJ, Kouwenhoven HJ, Bergman MJ, Bouma S, Brasseur SM, Daan R, et al. Short-term ecological effects of an offshore wind farm in the Dutch coastal zone; a compilation. Environ Res Lett. 2011; 6: 035101.

46. Slavik K, Lemmen C, Zhang W, Kerimoglu O, Klingbeil K, Wirtz KW. The large-scale impact of offshore wind farm structures on pelagic primary productivity in the southern North Sea. Hydrobiologia. 2019; 845: 35-53.

47. Mavraki N, Degraer S, Vanaverbeke J, Braeckman U. Organic matter assimilation by hard substrate fauna in an offshore wind farm area: A pulse-chase study. ICES J Mar Sci. 2020; 77: 2681-2693.

48. Coates DA, Deschutter $Y$, Vincx M, Vanaverbeke J. Enrichment and shifts in macrobenthic assemblages in an offshore wind farm area in the Belgian part of the North Sea. Mar Environ Res. 2014; 95: 1-12.

49. Maar M, Bolding K, Petersen JK, Hansen JL, Timmermann K. Local effects of blue mussels around turbine foundations in an ecosystem model of Nysted off-shore wind farm, Denmark. J Sea Res. 2009; 62: 159-174.

50. Norling P, Kautsky N. Patches of the mussel Mytilus sp. are islands of high biodiversity in subtidal sediment habitats in the Baltic Sea. Aquat Biol. 2008; 4: 75-87.

51. Wilding TA, Gill AB, Boon A, Sheehan E, Dauvin JC, Pezy JP, et al. Turning off the DRIP ('Data-rich, information-poor')-rationalising monitoring with a focus on marine renewable energy developments and the benthos. Renew Sust Energ Rev. 2017; 74: 848-859.

52. Dannheim J, Degraer S, Jackson AC, Bergström L, Birchenough S, Brzana R, et al. Benthic effects of offshore renewables: Prioritizing the known unknowns. ICES J Mar Sci. 2019; 76: fsz018.

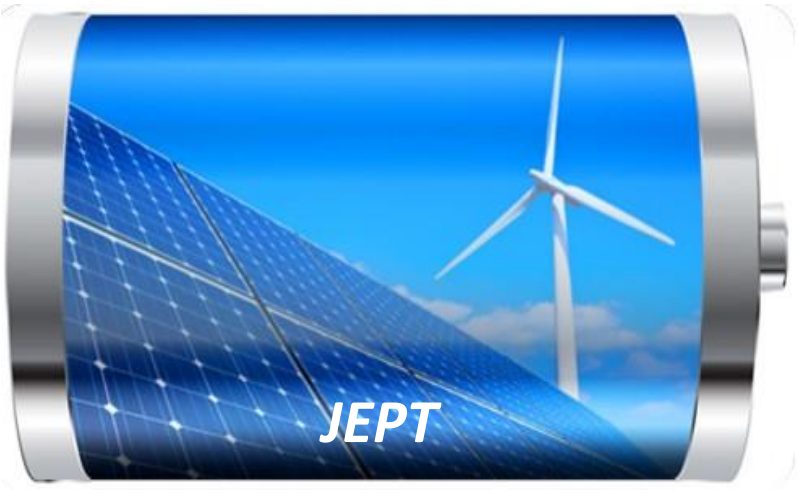

Enjoy JEPT by:

1. Submitting a manuscript

2. Joining in volunteer reviewer bank

3. Joining Editorial Board

4. Guest editing a special issue

For more details, please visit: http://www.lidsen.com/journal/jept 
Original Research

\title{
Crustal Reservoir Flow Simulation for Long-Range Spatially-Correlated Random Poroperm Media
}

Peter Leary ${ }^{1,2,+, *}$, Peter Malin ${ }^{2,+}$

1. GeoFlow Imaging, 43 High St, Auckland 1010, New Zealand; E-Mails:peter@geoflowimaging.com; pcl@asirseismic.com

2. Advanced Seismic Instrumentation \& Research, 317 E. Millbrook, Raleigh, NC 27609, USA; E-Mail: pem@asirseismic.com

† These authors contributed equally to this work.

* Correspondence: Peter Leary; E-Mail: peter@geoflowimaging.com

Academic Editor: Zhao Yang Dong

Special Issue: Modeling of Geothermal Systems

Journal of Energy and Power Technology

2021, volume 3 , issue 1

doi:10.21926/jept.2101013
Received: November 30, 2020

Accepted: March 15, 2021

Published: March 19, 2021

\begin{abstract}
In interest of accurately modelling wellbore production in convective geothermal systems, we draw attention to two approaches to numerical simulation of crustal fluid flow. The historical "strong" approach simulates the fluid mass conservation condition on a localised finite difference mesh approximation to poroelastic continua. The more flexible "weak" approach globally enforces fluid mass conservation by in effect integrating over all numerical mesh cells. Strong formulation crustal flow simulation emerged from numerical simulations of mathematically tractable conductive heat flow in media typified by normallydistributed/spatially-uncorrelated thermal property distributions. Strong formulation numerical stability problems quickly intrude, however, when confronted with the lognormally-distributed/spatially-correlated crustal permeability distributions that pervade the ambient crust. Weak flow conservation solver formulations accurately handle abrupt wide-ranging crustal permeability spatial fluctuations arising from these crustal permeability
\end{abstract}

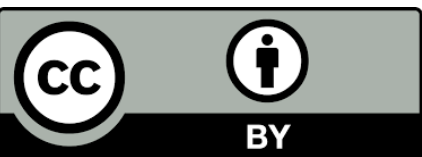

(C) 2021 by the author. This is an open access article distributed under the conditions of the Creative Commons by Attribution License, which permits unrestricted use, distribution, and reproduction in any medium or format, provided the original work is correctly cited. 
structures. We use two Matlab-based weak formulation flow simulation schemes to model wellbore-centric production fluid connectivity within large-scale spatially erratic convective geothermal flow structures described by the empirical poroperm relation $k(x, y, z) \sim$ $\exp (\alpha \phi(x, y, z))$ acting over cm-to-km scale lengths. Through weak formulation reservoir flow modelling of convective geothermal systems, the advancing ability to support large-scale crustal flow structure models with multi-channel surface seismic data acquisition/processing can guide geothermal field exploration and developmental drilling via feedback loops between crustal flow imaging data and accurate wellbore production modelling data.

\section{Keywords}

Convective geothermal flow; reservoir flow modelling; crustal permeability; finite element flow modelling

\section{Introduction}

The fundamental performance feature of geothermal energy extraction is production wellbore connectivity to spatially erratic convective cell flow structures. Wellbore power given by $Q \sim \rho C \times T$ $x \mathrm{~V}$ for fluids of volumetric heat capacity $\rho C$ scales with resource temperature $T$ and wellbore volumetric fluid flow rate $V=2 \pi r_{0} \phi v_{0} l$. For standard values of wellbore radius $r_{0}$ and open hole wellbore length $\ell$, geothermal well performance depends directly on formation bulk inflow rate $\phi v_{0}$. Assuming adequate reservoir porosities $\phi(x, y, z)$, Darcy's law gives wellbore power $Q$ in terms of formation permeability structure at the wellbore, $v_{0}=k\left(x_{0}, y_{0}, z_{0}\right) /\left.\mu \partial_{r} P(r)\right|_{r 0}$, with $\left.\partial_{r} P(r)\right|_{r 0}=$ $\mathrm{P}_{0} / \mathrm{r}_{0} / \log \left(\mathrm{R} / \mathrm{r}_{0}\right)$ for suitable flow system radius $\mathrm{R}$. Geothermal heat energy production rate $\mathrm{Q}$ is thus irreducibly controlled by permeability structures in the vicinity of the wellbore.

As we detail below, the empirical spatial variability of ambient crust permeability can be expressed by the mathematical form $k(x, y, z) \sim \exp (\alpha \phi(x, y, z))$ within a radius $R, v\left(\left(x-x_{0}\right)^{2}+\left(y-y_{0}\right)^{2}+\right.$ $\left.\left(z-z_{0}\right)^{2}\right)<R$, surrounding a wellbore site at location $\left(x_{0}, y_{0}, z_{0}\right)$. In this mathematical form, porosity $\phi(x, y, z)$ is normally distributed between $\phi_{m n}<\phi(x, y, z)<\phi_{m x}$ and is spatially correlated across cmto-km length scales. The normally distributed porosity field generates an associated lognormally distributed permeability field for large empirical values of poro-connectivity parameter $\alpha$. Small poroperm parameters $\alpha$ reduce spatial permeability spatial fluctuations to $k(x, y, z) \sim 1+\alpha \phi(x, y, z)$ that are consistent with thermal property fluctuations [1]. Such permeability structures are compatible with standard numerical flow simulation procedures developed for heat conduction in solids [2-5]. Empirical $\alpha$ values for the ambient crust are, however, of order 20 to 30 as set by the condition $3<\alpha \Phi<5$ for $\Phi \sim 0.2$ mean crustal reservoir porosities [6]. As such they are compatible with lognormal well-productivity data observed for ambient crustal geofluids worldwide. In consequence of empirical values $20<\alpha<30$, large-scale spatially-rapid variations in crustal permeability vastly exceed the magnitude and spatial complexity of rock thermal property distributions, thus destabilising standard crustal fluid flow modelling procedures.

To address numerical stability problems arising from large amplitude and rapidly varying ambient crustal permeability fluctuations, we here validate two non-traditional flow modelling procedures. These numerical procedures allow flow modelling to accurately connect geothermal production 
wellbore interval inflow velocity $v_{0}$ at wellbore sites $\left(x_{0}, y_{0}, z_{0}\right)$ to long-range spatially erratic poroconnectivity permeability structures exemplified by the empirical expression $k(x, y, z) \sim$ $\exp (\alpha \phi(x, y, z))$. Accurate wellbore inflow modelling of complex convective geothermal flow systems can interact efficiently with large-scale flow imaging data acquired by surface seismic sensor arrays to guide production well drilling [7]. The driver for accurate wellbore geothermal system flow modelling lies in the lognormal distribution of well productivity throughout the ambient crust [6]. It is proving uneconomic for convective geothermal energy production to drill large numbers of unproductive wells before locating productive and sustainable flow structures for targeted drilling [7]. Using advancing observational surface seismic sensor array data processing to remotely identify productive crustal flow systems will significantly reduce both exploration and exploitation drilling costs. An emerging use for precision flow modelling in ambient crustal volumes lies in tracking permeability enhancement (EGS) by systematic injection of wellbore fluids into deep hot crustal volumes [8-9].

Nomenclature

$Q \equiv$ Heat energy production rate in watts for a wellbore

$\rho C \equiv$ Volumetric heat capacity of wellbore fluid in joules per cubic meter of fluid

$\mathrm{T} \equiv$ Centigrade temperature of geothermal fluid entering a production wellbore

$\mathrm{V} \equiv$ Velocity of wellbore fluid in cubic meters per second

$r_{0} \equiv$ Radius of open production wellbore in meters

$\mathrm{v}_{0} \equiv$ Darcy fluid flow velocity at wellbore radius in meters per second

$\ell \equiv$ Axial length of open production wellbore in meters

$\left(x_{0}, y_{0}, z_{0}\right) \equiv$ Central location of a production well open interval in meters

$\phi(x, y, z) \equiv$ Spatially variable porosity of geothermal flow system near wellbore

$k(x, y, z) \equiv$ Spatially variable permeability of geothermal flow system near wellbore

$\mu \equiv$ Dynamic viscosity of wellbore fluid in Pascal-seconds

$P(r) \equiv$ Radial pressure of convective fluid near the wellbore in Pascal

$\mathrm{R} \equiv$ Effective radius in meters of open wellbore connectivity to convective geothermal system

$\alpha \equiv$ Empirical proportionality coefficient relating changes of logarithm of well-core permeability

to changes in well-core porosity, $\delta \log _{\mathrm{K}} \sim \alpha \delta \phi$

$\Phi \equiv$ Mean porosity of crustal volume of radius $\mathrm{R}$ hosting a geothermal flow structure

$\alpha \Phi \equiv$ Critical state product obeying empirical condition $3<\alpha \Phi<5$; for reservoir porosity range $0.1<\Phi<0.3,15-20<\alpha<25-30$

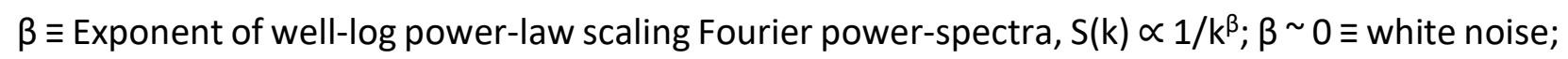
$\beta \sim 1$ = pink noise; $\beta \sim 2 \equiv$ red noise.

\section{Materials \& Methods-Computing Fluid Flow For Permeability $k(x, y, z) \sim \exp (\alpha \phi(x, y, z))$}

The fluid flow material properties of ambient crustal rock are set by a trio of widely attested empirical field relations (i)-(iii) derived respectively from crustal well-log, well-core, and wellproductivity data. Closely associated with these crustal poroperm statistical and spatial distribution empirics are microseismic event empirics (iv)-(vi) observed in crystalline basement, geothermal fields, and active deformation zones. Building on the appropriate flow simulation methodology, we can accurately model crustal reservoir flow systems derived from microseismic emission data 
embedded in numerical realisations of ambient crustal permeability structures $\mathrm{k}(\mathrm{x}, \mathrm{y}, \mathrm{z}) \sim$ $\exp (\alpha \phi(x, y, z))$ as mandated by the following ambient crustal flow structure empirics:

(i) Well-log spatial sequences of rock properties establish the existence of long-range powerlaw-scaling spatial correlations as a fundamental feature of the ambient brittle-fractured crust. Well-log Fourier power-spectra, $S(k) \propto 1 / k^{\beta}, \beta \sim 1$, span five decades of spatial frequency, $1 / \mathrm{cm}<k$ $<1 / \mathrm{km}$ [10-12]. Of particular interest are neutron porosity logs from hydrocarbon reservoirs, geothermal formations, and basement rock at up to $6 \mathrm{~km}$ depth. For contrast, the de facto standard assumption about crustal heterogeneity is lack of significant spatial correlation as denoted by "white noise" $(\beta=0)$ power-spectra, $S(k) \propto 1 / k^{0} \sim$ const.

(ii) Well-core data from hydrocarbon reservoirs, geothermal formations, and deep basement rock show close spatial correlation systematics, $\delta \operatorname{logk} \sim \alpha \delta \phi$, for spatially varying porosity $\phi$ and permeability $\mathrm{k}$ [6]. On well-core evidence, rock permeability is a physical feature of fractureconnectivity between spatially-correlated porosity structures at all scales. The constant $\alpha$ in the well-core poroperm spatial correlation is given by the empiric condition $\alpha \Phi \sim 3-5$ across two decades of mean porosity, $0.3 \%<\Phi<30 \%$ spanning the basement-to-reservoir formation spectrum [13-14]. The well-core empiric for porosity and permeability follows from considering that connectivity between $n$ pores scales as $n$-factorial, $n !=n(n-1)(n-2) \ldots .1$, so that changes in pore count $n \rightarrow n+\delta n$ lead to changes in the logarithm of permeability $\delta \log n ! \sim \log ((n+\delta n) !)-\log (n !) \sim \delta n \log (n)$, giving the observed well-log poroperm relation. The constant of proportionality $\alpha$ governs the degree to which pores are connected, with high degrees of pore connectivity leading to channelised flow structures.

(iii) Well-productivity lognormality follows directly from the integrated poroperm relation $\mathrm{K} \sim \mathrm{K}_{0}$ $\exp (\alpha \phi)$. The empirical condition $\alpha \Phi \sim 3-5$ guarantees the lognormal well-productivity distributions observed everywhere for groundwater aquifers, conventional and unconventional hydrocarbon reservoirs, convective geothermal systems, and mineral deposition abundances in fossil flow systems [15-16].

(iv) Ambient crust microseismicity event magnitude distributions in the magnitude range $-1<m$ $<2$ are lognormal rather than power-law/fractal, i.e., the standard Gutenberg-Richter relation [1718]. The observed lognormal magnitude distribution is congruent with crustal permeability lognormality (iii), indicating that ambient crustal microseismic slip events are closely associated with crustal permeability structures.

(v) Ambient crust permeability and microseismic event separations are spatially correlated according to two-point spatial correlation function $\Gamma(r) \sim 1 / r^{1 / 2}[19-20]$. The similar spatial correlation functions and scaling exponents indicate that microseismic slip events tend to occur on permeability structures rather than hypothetical fault structures.

(vi) Ambient crust basement rock microseismic event seismic waveform spectra are dominated by high frequencies, $200 \mathrm{~Hz}<\mathrm{f}<800 \mathrm{~Hz}$, characteristic of seismic slip events on spatially complex crustal permeability structures rather than on hypothetical planar fault-like dislocation structures [9].

Rates of wellbore heat energy extraction $Q$ from convective system volumetric flow $V=2 \pi r_{0} \phi v_{0} l$ thus depend explicitly on the details of production wellbore intersection flow $v_{0}$ given by Darcy's law, $v_{0}=k /\left.\mu \partial_{r} P(r)\right|_{r o}$, applied to the open hole length $\ell$ within the complex crustal flow structures $\mathrm{k}(\mathrm{x}, \mathrm{y}, \mathrm{z}) \sim \exp (\alpha \phi(\mathrm{x}, \mathrm{y}, \mathrm{z}))$ of radial dimension $\mathrm{R}$. For accurate wellbore flow modelling, computational methods for convective geothermal flow systems must be able to robustly handle the high- 
amplitude multi-scale spatial variations arising from the crustal flow empiric $k(x, y, z)$ $\exp (\alpha \phi(x, y, z))$.

Our crustal fluid flow modelling concern readily emerges from including the crustal poroperm, empiric $\mathrm{k}(\mathrm{x}, \mathrm{y}, \mathrm{z}) \sim \exp (\alpha \phi(\mathrm{x}, \mathrm{y}, \mathrm{z}))$ into Darcy's law (1) and the conservation of fluid mass in steady state flow (2). Darcy's law gives fluid flow velocity $\boldsymbol{v}$ in terms of the spatially variable formation permeability $\mathrm{k}$, fluid viscosity $\mu$ (here constant), and spatial gradient of fluid pressure $P$,

$$
\boldsymbol{v}(\mathrm{x}, \mathrm{y}, \mathrm{z})=\frac{\kappa(\mathrm{x}, \mathrm{y}, \mathrm{z})}{\mu} \nabla P(\mathrm{x}, \mathrm{y}, \mathrm{z})
$$

Steady-state reservoir flow models are mathematically derived from conservation of fluid mass constraint equation given by the spatial differential condition on fluid flow velocity requiring that zero net fluid moves into and out of any given crustal unit volume,

$$
\nabla v(\mathrm{x}, \mathrm{y}, \mathrm{z})=0
$$

Along with appropriate flow system boundary conditions, mass conservation condition (2) gives a closed mathematical system for numerical solution. For $k(x, y, z)=\exp (\alpha \phi(x, y, z))$ in $(1)-(2)$, the crustal flow system poro-connectivity parameter $\alpha$ defines the flow modelling computational task as finding pressure fields $P(x, y, z)$ that solve the equation

$$
\nabla^{2} P(\mathrm{x}, \mathrm{y}, \mathrm{z})+\alpha \nabla \varphi(\mathrm{x}, \mathrm{y}, \mathrm{z}) \cdot \nabla P(\mathrm{x}, \mathrm{y}, \mathrm{z})=0
$$

For small $\alpha$ and/or porosity spatial variations, the governing flow differential equation (3) is close to the mathematically and computationally well-known and highly tractable Laplace equation $\nabla^{2} P(\mathrm{x}, \mathrm{y}, \mathrm{z})=0$ and its generalisation the Poisson equation with known source term $\nabla^{2} P(\mathrm{x}, \mathrm{y}, \mathrm{z})=f(\mathrm{x}, \mathrm{y}, \mathrm{z})$ [2]. In consequence of the numerical stability offered by small values of $\alpha$ and/or small spatial variations in porosity, it quickly became routine to follow the lead of heat conduction numerical modelling in using the established "strong" methodology of finite-difference numerical approximation to poroelastic media to model crustal flow [4-5].

Of historical note in ambient crust flow computations are the introduction and persistence of the representative elementary volumes (REVs) [21] derived from effective media approximations such as dual porosity/permeability [22-24]. These numerical constructs of crustal flow simulation are based on pre-emptive smoothing of poroperm spatial variability, i.e., the artificial reduction or elimination of troublesome porosity structure spatial gradients [25-26]. Such pre-emptive smoothing is typically justified on the assumption that spatial variations in crustal flow properties are spatially uncorrelated and therefore subject to smoothing through spatial averaging. Where substantial spatial flow heterogeneity is required in a reservoir model, the heterogeneity is taken to define a set of distinct (and suitably uniform) formations that are sutured together in an overall flow structure. As illustrated by the widely used Tough2 flow simulation code, "strong" formulation method finite-difference numerical flow solvers based on pre-emptive generation of numerically tractable flow property distributions remain the mainstay of present reservoir fluid flow simulation [27-30].

Pre-emptive spatial averaging of reservoir flow property distributions for purposes of finite difference flow modelling numerical stability contradicts what we know about spatially correlated 
flow and wellbore fluid production. While spatially uncorrelated poroperm distributions have flat or constant spectra [12], the crustal empiric (i) shows that wellbore log spatial fluctuations, e.g., neutron porosity, have Fourier power-spectra that scale inversely with spatial frequency, $S(k) \propto 1 / k^{\beta}$, $\beta \sim 1$, over the five decades $1 / \mathrm{cm}<\mathrm{k}<1 / \mathrm{km}$. Further, as given by crustal empirics (ii)-(iii), spatially correlated porosity generates wellbore production distributions that are lognormal rather than normal.

The Figure 1 well production frequency distributions are computed for a 2D reservoir section with standard normal distribution of reservoir porosity ranging over $0.1<\phi<0.3$ with mean $\Phi^{\sim}$ 0.2 . The poro-connectivity parameter values $0<\alpha<30$ control the degree of flow connectivity and hence flow channelisation within the reservoir section. Low values of $\alpha$ correspond low degrees of flow channelisation, for which any given well has little chance of missing existing flow structures. The black fill histograms in the upper tier of Figure 1 show that for poro-connective parameter values $5<\alpha<15$, large percentages of wells produce at $>70 \%$ of normalised unit peak flow. Low degrees of crustal flow channelisation given by the $5<\alpha<15$ poro-connectivity parameter range correspond to the standard hypothetical concept of spatially uncorrelated reservoir flow heterogeneity and flow modelling. In such flow media, wells can be drilled indiscriminately (e.g., on a regular drill grid as is commonplace in hydrocarbon fields) with statistically assured well production levels. Cyan fills show, however, that high $\alpha$ values penalise indiscriminate drilling.
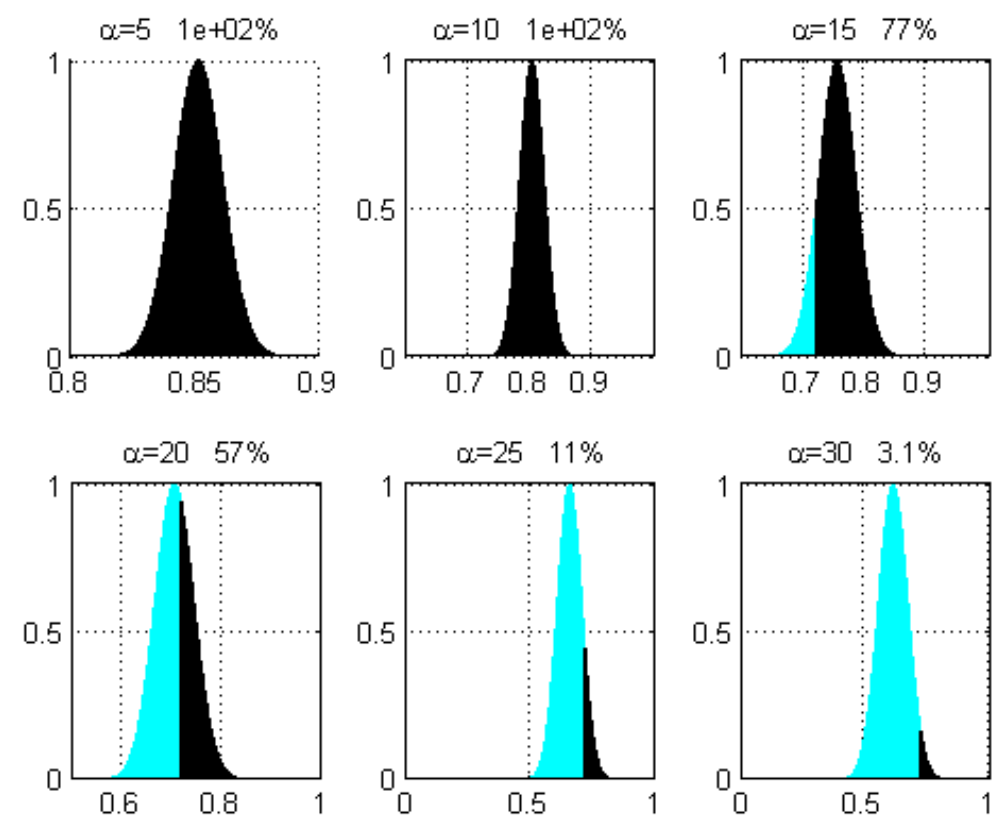

Figure 1 Six well productivity distributions for six values of empirical poro-connectivity parameter $\alpha$ in crustal reservoir permeability distributions $k(x, y, z) \sim \exp (\alpha \phi(x, y, z))$ for porosities $0.1<\phi<0.3$ with mean $\Phi \sim 0.2$. Well production for each $\alpha$ is normalised to unit peak value. Black/cyan histogram fills denote well production respectively above/below $70 \%$ peak productivity. For $5<\alpha<15$ most or all wells flow $>70 \%$ peak production. For $20<\alpha<30$-values observed in crustal reservoirs, however, $50 \%$ to $97 \%$ of wells flow $<70 \%$ peak production. While the impact of reduced well flow is moderate for hydrocarbon fluid production-both cyan and black wells pay-the impact on geothermal reservoir fluid production is critical-only the small number of lower tier black wells flow strongly enough to pay. 
Following ambient crustal empirics (i)-(iii), actual poro-connectivity parameters are in the range $20<\alpha<30$ for which reservoir fluid flow is increasingly channelised. With reservoir fluid flow restricted to ever higher degrees of poro-connectivity in ever narrower flow channels, the chance that wells fail to intersect existing flow channels rises substantially. Cyan fill portions of Figure 1 lower tier histograms show that for $\alpha \sim 20$ half of wells flow at productivity less than $70 \%$ of peak, with higher values $25<\alpha<30$ corresponding to spatially correlated flow channelling for which only $3 \%$ to $10 \%$ of wells produce greater than $70 \%$ unit peak production.

While the Figure 1 well productivity progression has only moderate impact on hydrocarbon production pay (low well productivity means that hydrocarbon pay is delayed not cancelled), the impact of crustal flow empirics on geothermal well production is critical. Only the most productive geothermal wells can turn power-producing turbines. All other such wells have little or no economic worth.

Figure 1 numerical simulations of reservoir flow structures depend on accurate flow modelling capability appropriate to flow channelisation encountered for the poro-connectivity parameter range $20<\alpha<30$ indicated by empirics (ii)-(iii). Solving the flow constraint equation (3) by the "strong" methods of finite difference expression evaluations on discrete numerical meshes faces numerical stability problems when the porosity gradient term $\nabla \phi(x, y, z)$ is ill-behaved and/or the poroperm empirical parameter $\alpha$ is large. Modelling channelised geothermal flow thus requires the use of the more mathematically-oriented "weak" solution methods developed to handle a wide class of constitutive functions such as $k(x, y, z) \sim \exp (\alpha \phi(x, y, z))$ for empirical values of poroconnectivity $3<\alpha \Phi<5$ (e.g., [31-35]).

As no mathematical expressions exist against which to test numerical flow simulations of $k(x, y, z)$ $\sim \exp (\alpha \phi(x, y, z))$ media, we base our wellbore flow validation on testing simple cases of flowing fluid through 2D numerical permeability sections $k(x, y) \sim \exp (\alpha \phi(x, y))$ for Figure 1 well productivity simulation statistics. We first test if the spatial distribution of fluid flow velocity computed by two weak-formulation flow solver algorithms agree, then establish that the weak-formulation flow solutions retain the spatial correlation scaling nature of the host permeability distribution established by crustal flow and microseismicity empirics (i)-(vi).

It is logical to expect that fluid flowing in the physical equivalent of our numerical simulation permeability distribution $\mathrm{k}(\mathrm{x}, \mathrm{y}, \mathrm{z}) \sim \exp (\alpha \phi(x, y, z))$ tracks the physical permeability structure. Flow simulations that preserve the host medium flow structure can be construed to give an "accurate" account of wellbore coupling to the flow medium. Similarly, numerical simulation flow structures that do not preserve these properties must be presumed to be "inaccurate".

\section{Results- Wellbore Interaction with $k(x, y) \sim \exp (\alpha \phi(x, y))$ Permeability Distributions}

The class of poroelastic flow materials under consideration is illustrated in Figure 2 crustal flow sections showing porosity spatial fluctuations and Figure 3 showing the associated permeability spatial fluctuations in accord with the crustal flow empirics (i)-(iii). From left to right, the degree of spatial correlation of porosity and permeability fluctuations increases from uncorrelated "white noise" (well-log spectral exponent $\beta=0$ ), to moderately correlated "pink noise" (exponent $\beta=1$ ), to strongly correlated "red noise" (exponent $\beta=2$ ). Applications of two weak-formulation flow simulation methodologies to the Figure 2 and Figure 3 poroperm sections are compared in Figure 4 and Figure 5. Figure 6, Figure 7, Figure 8 and Figure 9 validate that the two flow simulation methods 
generate velocity flow fields that preserve the spatial correlation properties of the empirical crustal permeability distributions $k(x, y) \sim \exp (\alpha \phi(x, y))$ for poro-connectivity parameter $\alpha=25$.
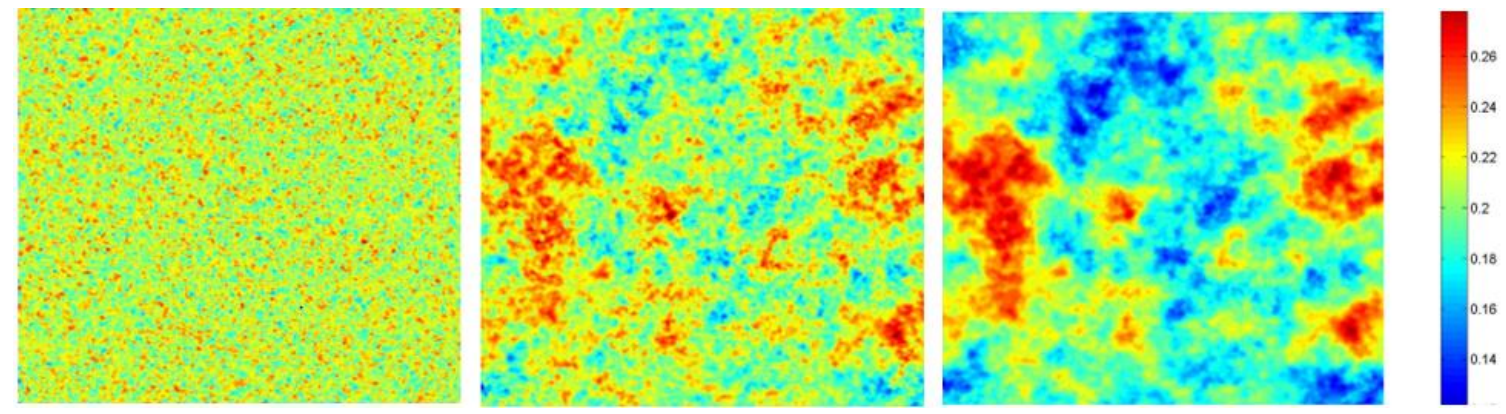

Figure 2 Numerical realisations of poroelastic crustal sections having (left to right) increasing degrees of power-law-scaling spatial correlation. (Left) $S(k) \propto 1 / k^{\beta}, \beta \sim 0$, uncorrelated "white noise". (Middle) $S(k) \propto 1 / k^{\beta}, \beta \sim 1$, moderately correlated " $1 / f^{\prime \prime}$ or "pink noise"). (Right) $S(k) \propto 1 / k^{\beta}, \beta \sim 2$, highly correlated Brownian or "red noise"). Numerical wellbores across each section return the designated power-law-scaling spectral exponent $\beta$.
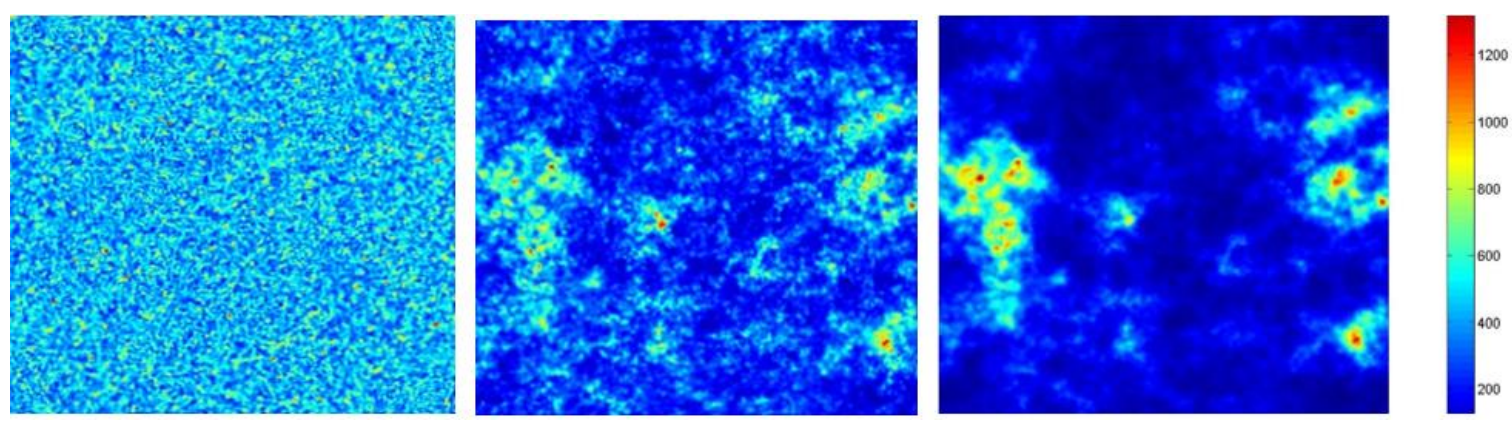

Figure 3 Numerical realisations of crustal permeability sections corresponding to Figure 2 porosity sections. Permeability is given by $\mathrm{k}(\mathrm{x}, \mathrm{y}) \sim \exp (\alpha \phi(\mathrm{x}, \mathrm{y}))$ for $\alpha=25$ and porosity $0.1<\phi<0.3$ with mean $\Phi \sim 0.2$. Permeability range shown at right is reduced overall by factors 4 and 2 for the left and middle permeability sections, respectively.
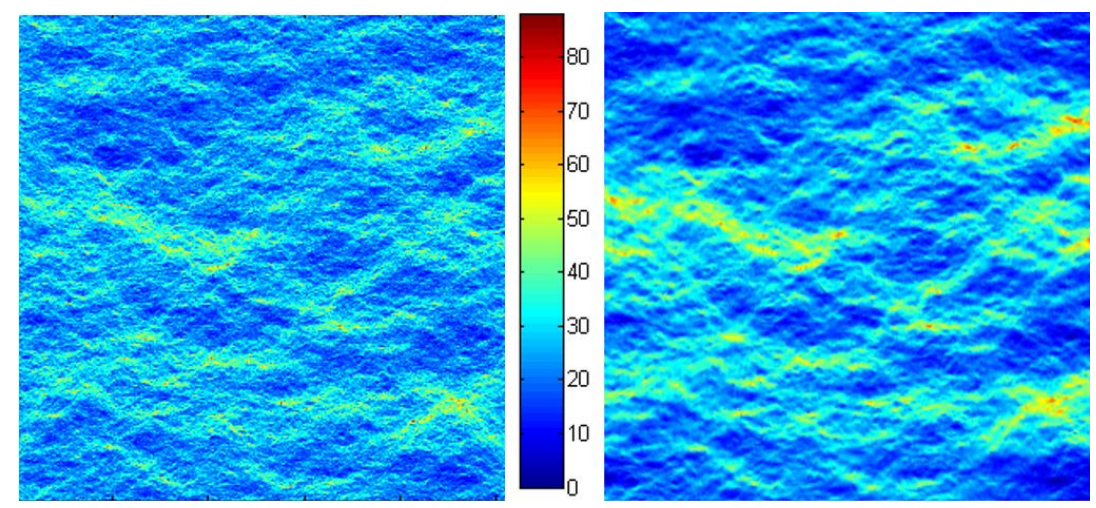

Figure 4 Channelised numerical flow amplitude distributions for Figure 3 middle permeability distribution with constant left/right pressure difference and zero-flow on upper/lower boundaries. (Left/right) Matlab-based finite-element [31-35] and MRST [36-37] flow solvers. 

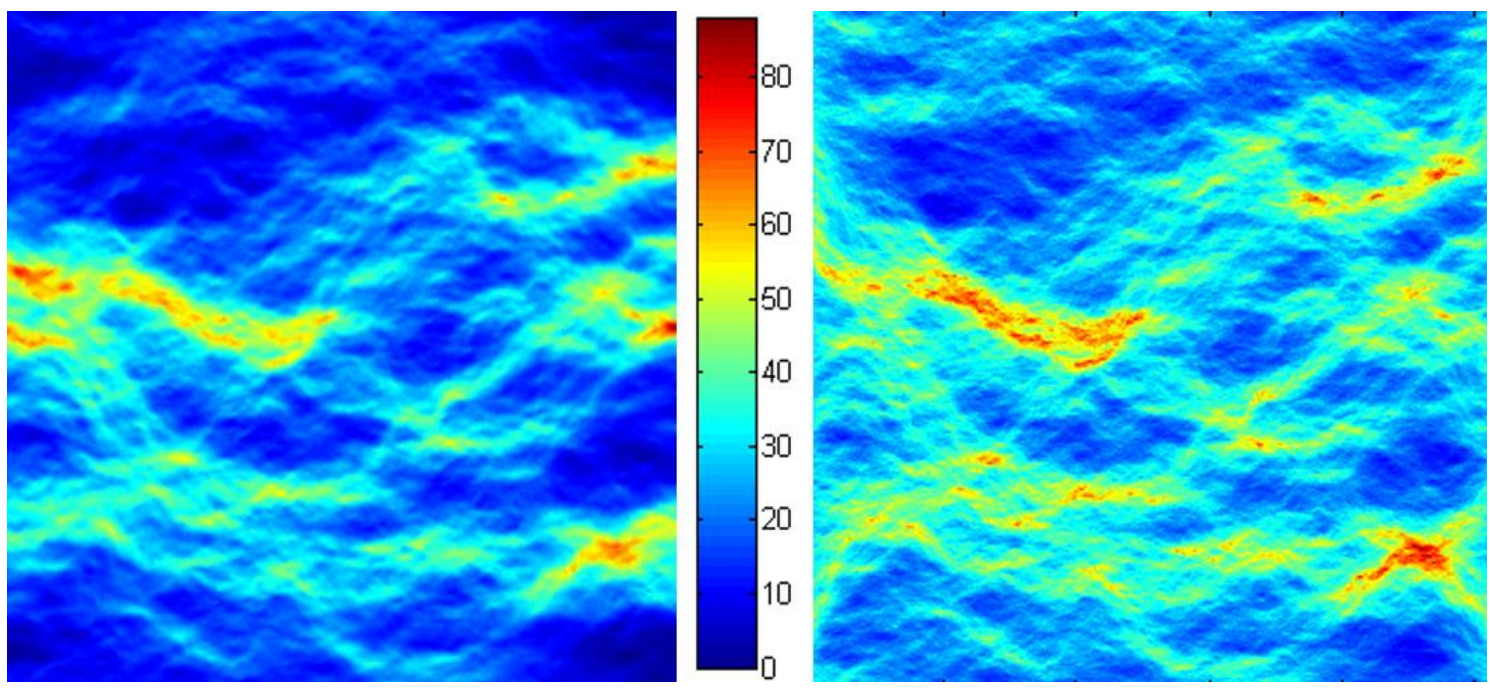

Figure 5 Channelised numerical flow amplitude spatial distributions for Figure 3 right permeability distribution with constant left/right pressure difference and zero-flow on upper/lower boundaries. (Left/right) Matlab-based finite- element [31-35] and MRST [36-37] flow solvers.

0

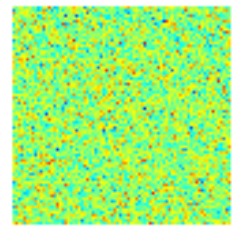

0.833333

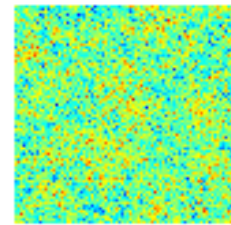

1.66667

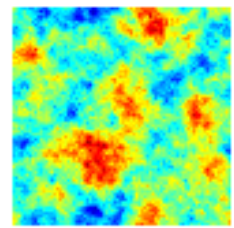

0.2775

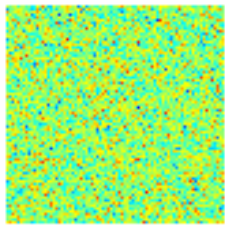

1.11083

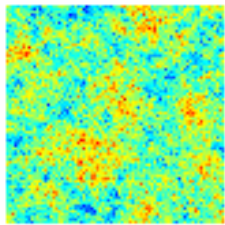

1.91667

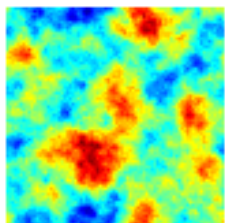

0.555

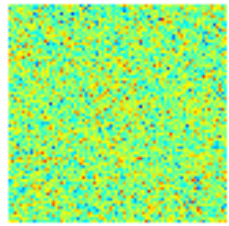

1.38833

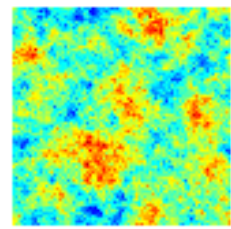

Figure 6 Sequence from upper tier left to lower tier right of 2D porosity sections according to degree of well-log power-law scaling correlation $S_{\phi}(k) \propto 1 / k^{\beta}$ with corresponding exponent $\beta$ given above each panel. 2D permeability sections for flow computation are given by $k(x, y, z) \sim \exp (\alpha \phi(x, y, z))$. 

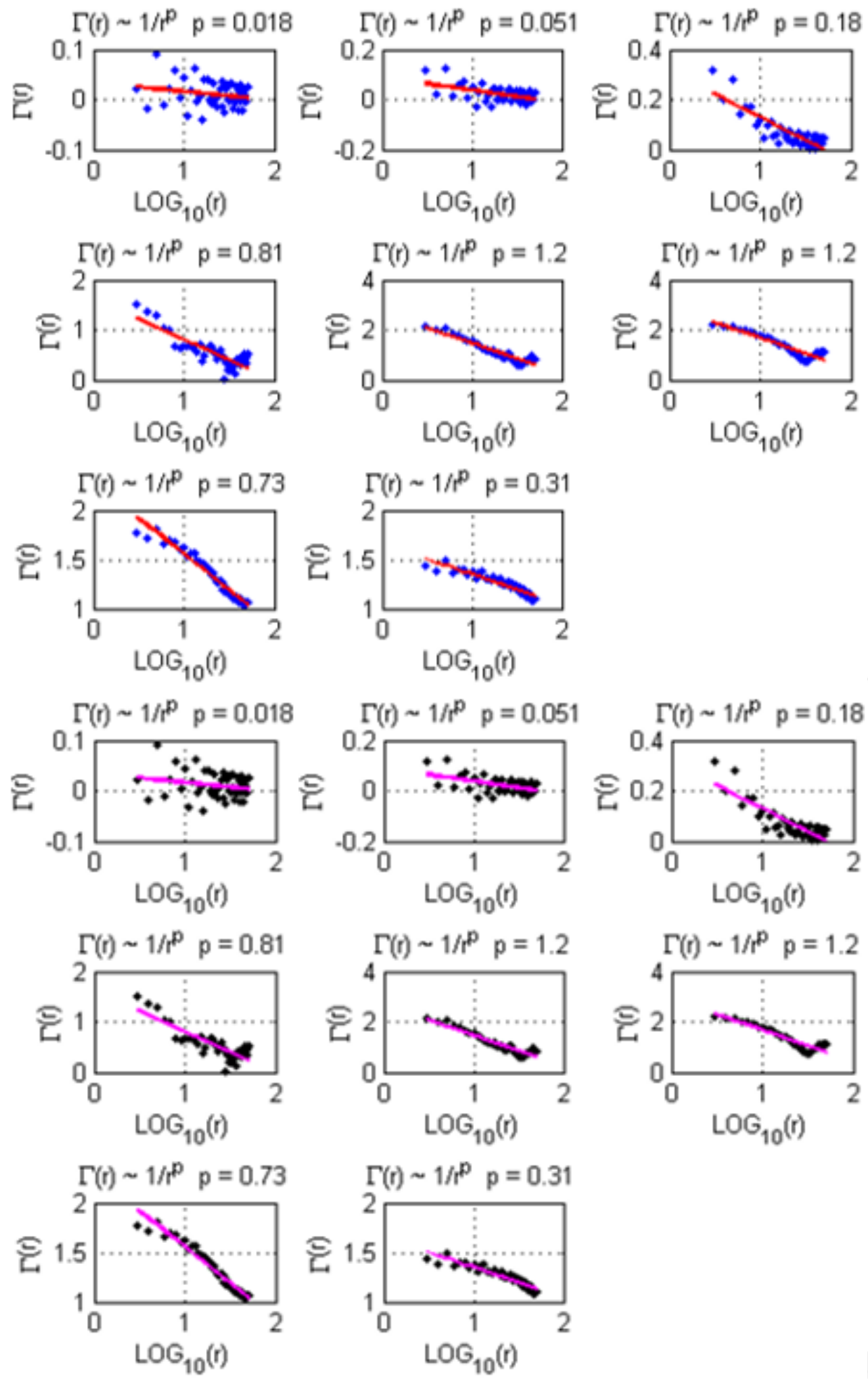

Figure 7 Plot groups for sequence of 2D permeability sections corresponding to Figure 6 2D porosity sections. Loglog plots within each group show the power-law trend of twopoint spatial correlation function fit to power-law exponent $p$. The upper plot group shows the spatial correlation of permeability input to the flow Matlab finite element flow modeller [31-35]. The modelled flow field reproduces the host permeability field to a high degree of fidelity. Virtually identical results proceed from the MRST flow solver shown in the lower plot group [36-37]. 

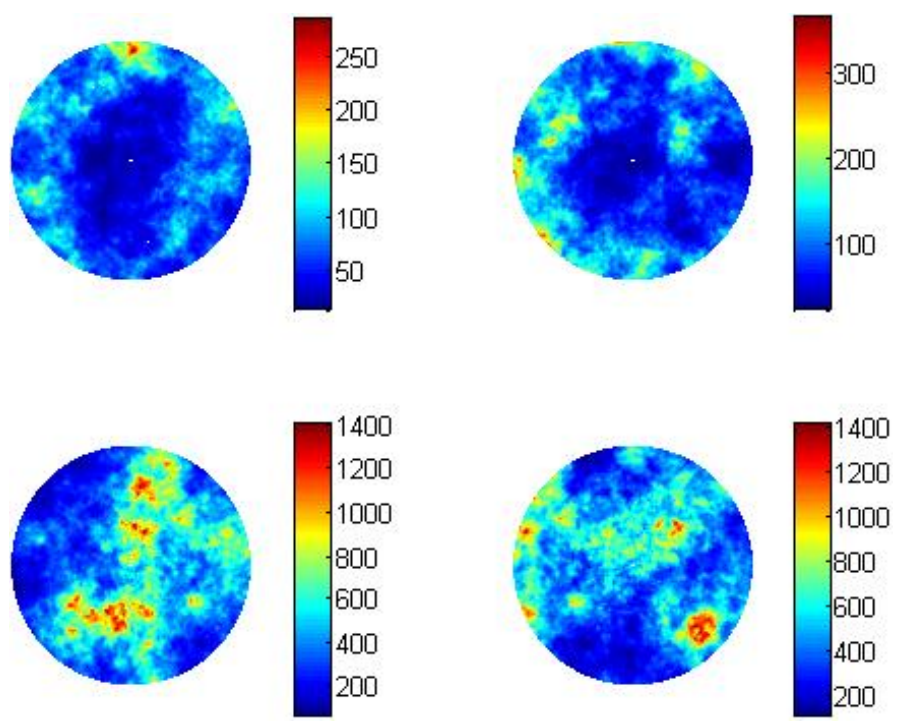

Figure 8 Selected wellbore-centric permeability distributions of $250 \mathrm{~m}$ diameter sections extracted from the central Figure $6 \mathrm{~km}$-scale permeability section with well-log fluctuation power spectral scaling exponent, $S_{\phi}(k) \propto 1 / k^{\beta}, \beta \sim 1.1$. The top pair, illustrating the low permeability sections, and the bottom pair, illustrating high permeability sections, summarise 169 random well-locations from the Figure 6 permeability section. Sidebars show nominal permeability scales. Figure 10 gives the lognormal wellbore production distribution for the 169 sections.
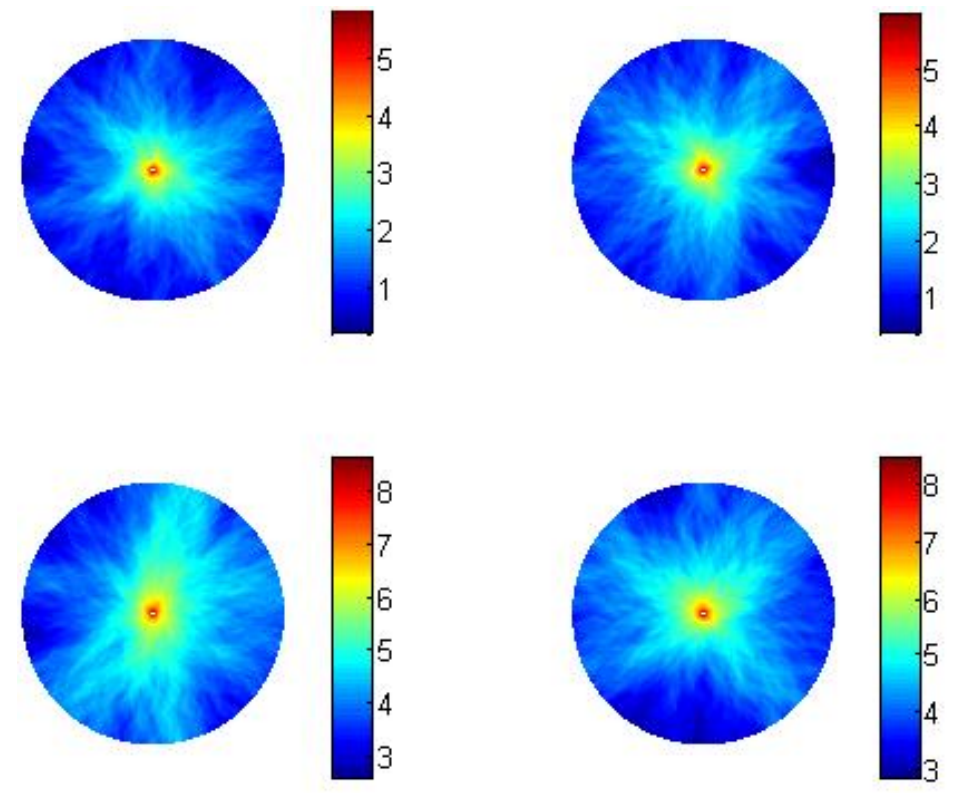

Figure 9 Production wellbore-centric inflow filamentary log(velocity) distributions for Figure 8 permeability sections. Logarithm of nominal inflow range shown in side-bars. As seen in Figure 10, the distribution of wellbore-centric inflow amplitudes is lognormal. 


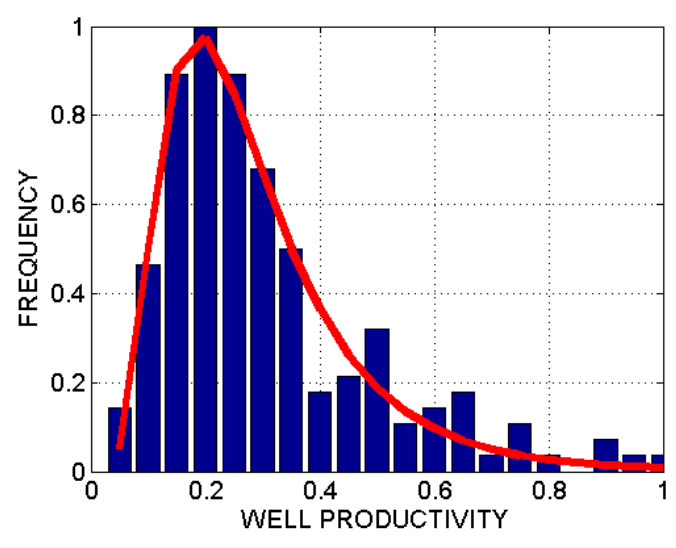

Figure 10 Distribution of normalised wellbore inflow amplitudes for a km-square permeability section sampled by 169 randomly located $250 \mathrm{~m}$-radius flow sections as illustrated in Figure 8. The central section of Figure 6 shows the km-square source permeability section with well-log spectral power scaling, $S_{\phi}(k) \propto 1 / k^{\beta}$, exponent $\beta \sim 1.1$. Red curve is the lognormal distribution fit to the histogram of wellbore flow amplitudes as illustrated in Figure 9.

Figure 2 and Figure 3 poroperm sections are 1024×1024 node numerical grids representing porosity in the range 0.1 (cool tints) to 0.3 (warm tints) and the corresponding permeability given by $k(x, y) \sim \exp (\alpha \phi(x, y))$ for $\alpha=25$. The middle panel $\beta=1$ "pink noise" poroperm sections represent most actual ambient crustal reservoir sections. Flow model computation applied to these sections should show that Darcy flow velocities increase/decrease in areas of high/low poroperm properties, respectively. The Figure 4 and Figure 5 flow velocity distributions do not, however, reproduce the poroperm distributions because fluid flow measures poroperm connectivity rather than poroperm values. The degree of poroperm connectivity given by fluid flow solvers is controlled by the parameter $\alpha$, thus giving the degree of flow channelisation. The flow continuity measure of spatially correlated poroperm properties given by the flow model solvers marks a fundamental departure from standard reservoir modelling that is based on uncorrelated poroperm properties. Standard modelling routinely assumes small values of $\alpha$, while actual crustal flow structures have high degrees of channelisation that systematically go unrecognised in standard reservoir modelling. Accurate flow continuity modelling involving channelled flow is paramount for incorporating microseismicity emission imaging data into structural profiles of convective geothermal flow systems [7].

Figure 4 and Figure 5 show the Darcy velocity amplitude fields generated from Eq. (1) evaluated for the weak-formulation pressure field solutions of constraint Eq. (3) using two Matlab-based solver implementations [31-32, 36-37]. These flow sections are applied respectively to the Figure 3 middle and right-hand spatially correlated permeability distributions. The flow model boundary conditions are constant differential pressure between the left/right vertical section boundaries for zero fluid flow condition across the upper/lower section boundaries.

Flow channelisation effects introduced by the spatially correlated permeability fields are the outstanding feature of Figure 4 and Figure 5, with the lesser/greater spatial correlation of Figure 3 middle/right creating the lesser/greater the channelisation of Figure 4 and Figure 5, respectively. Fluids entering at the left sides of the flow sections pass across to the right side of section via spatially erratic channelised poro-connectivity pathways. Flow velocity amplitudes denoted by the colour-bar vary by an order of magnitude. It is immediately evident from Figure 4 and Figure 5 that 
ideally positioned production wellbores will intersect high velocity flow structures (warm tints) and avoid low velocity flow structures (cool tints). The well productivity distributions associated with the high and low flow velocities of Figure 4 give the productivity frequency distributions appearing in Figure 1 for the $\alpha=25$ value of poro-connectivity parameter.

We note in Figure 4 and Figure 5 that the two flow solvers agree in their response to the gross scale distribution of permeability of Figure 3 sections. Where there are high permeabilities in the Figure 3 distributions, there are corresponding high flow velocities in Figure 4 and Figure 5, and equivalently for low permeabilities and flow velocities. The two solvers differ in the spatial discrimination of the flow response to the permeability distributions. The Matlab-based finiteelement solver [31-35] maintains a higher degree of contrast between high and low permeability domains than does the Matlab-based MRST solver [36-37]. The difference in velocity amplitude resolution is due to how the two solvers distribute the $k(x, y, z) \sim \exp (\alpha \phi(x, y, z))$ permeability heterogeneity between the computational mesh cells. The Matlab finite element solver evaluates the trial solutions globally between all mesh cells, while the MRST solver evaluates the trial solutions locally between nearest neighbour mesh cells. In principle, the finite element solver of Figure 4 and Figure 5 (left) is more accurate than the MRST solver of Figure 4 and Figure 5 (right), but the difference is likely to be less significant than other sources of modelling and observational error.

Figure 6 and Figure 7 assess the degree to which the two "weak" flow structure solver methodologies preserve the spatial correlation nature of ambient crust poroperm structures. From the crustal flow observational empiric (v), the two-point spatial correlation properties of crustal section permeability have the form $\Gamma(r) \sim 1 / r^{p}$, where the power-law exponent can in principle range from $0<p<1$. The observed two-point spatial correlation power-law exponent $p \sim 1 / 2$ of $(v)$ applies to volumetric distributions of permeability and microseismic events where the many-plane superposition of 2D distributions lowers the effective power-law exponent range. In the single-plane numerical simulation cases given in Figure 6 for 2D permeability sections, the exponent parameter range is $0<p<1$.

The full $0<p<1$ range of controlling numerical porosity spatial distributions in Figure 6 begins with the uncorrelated porosity $S_{\phi}(k) \propto 1 / k^{\beta}, \beta \sim 0$, in the upper left and proceeds to the maximally correlated porosity $S_{\phi}(k) \propto 1 / k^{\beta}, \beta \sim 2$ at lower right. The plot arrays of Figure 7 give the corresponding sequence of two-point $2 D$ section spatial correlation power-law fits $\Gamma(r) \sim 1 / r^{p}$ for permeability event-pair offsets $r$ for the two solver methods.

The upper set of Figure 7 plots shows that the two-point section power-law spatial correlation distributions for the originating permeability field of Figure 6 are matched in detail by permeability fields provided by the Matlab finite element solver through reverse expressing Darcy's law as $k(x, y)$ $=|\mathbf{v}(\mathrm{x}, \mathrm{y})| /|\nabla P(x, y)|$. As the input permeability field is essentially duplicated by the reverse permeability field, we are assured that the Matlab finite element solver is numerically robust for $\mathrm{k}(\mathrm{x}, \mathrm{y}, \mathrm{z}) \sim \exp (\alpha \phi(\mathrm{x}, \mathrm{y}, \mathrm{z}))$ crustal permeability fields for reasonable values of poro-connectivity parameter $\alpha$. Virtually identical results are provided by the MRST solver shown in the lower set of Figure 7 plots.

Two-point correlation power-law exponents $p \sim 0$ occur for spatially uncorrelated permeability derived from spatially uncorrelated porosity illustrated in Figure 6 top tier. There is no preferred pair-wise offset range for spatially uncorrelated distributions. As the controlling porosity spatial correlation increases towards the $\beta \sim 1$ porosity spatial correlation condition characteristic of crustal flow systems in the Figure 6 central tier, the pair-wise spatial correlation becomes optimally 
organised for fluid flow by favouring nearest-neighbour ranges before falling steeply for increasing pair-range with exponents $p \sim 1$. Intermediate values of two-point correlation exponent $p \sim 1 / 2$ occur for the high-correlation spectral distributions $S_{\phi}(k) \propto 1 / k^{\beta}, \beta \sim 2$ given in the Figure 6 lower tier.

Review of the Figure 7 sequence of two-point spatial correlation distributions for the Figure 6 sequence of porosity sections shows that well-log fluctuation power spectral scaling exponent $\beta \sim$ 1 observed in the ambient crust is the most efficient value for generating areas of high and low porosity given by warm/cool colours, respectively. As a general consequence of the reservoir flow structure empirical poroperm relation $\mathrm{k}(\mathrm{x}, \mathrm{y}, \mathrm{z}) \sim \exp (\alpha \phi(x, y, z))$, wellbores drilled into high porosity regions are systematically more permeable over wider crustal volumes and are therefore more productive than wellbores drilled into low porosity regions. This phenomenon is a mechanical picture of well productivity lognormal distribution of crustal flow empiric (iii) as seen in Figure 10.

From the ambient crust poroperm empirics (i)-(vi), we see how entrenched is the key drilling statistic observed world-wide in the form of lognormal well-productivity statistics for all crustal fluids. What we do not, however, at present know is how to proactively deal with the entrenched and inherent statistical uncertainty caused by flow channelling arising from universal spatially correlated poroperm randomness at all scales. As drilling outcome predictions based on the law of averages are poor in spatially correlated poroperm media, we can directly see from Figure 4 and Figure 5 channelised flow the need to acquire and quantify information about the large-scale reservoir flow structure if we are to reduce the overall cost of production well drilling in convective geothermal reservoirs [7].

Building on Figure 4 and Figure 5 channelised flow computations, we now return to the source of Figure 1 statistics arising from large-scale flow channelling structures in crustal reservoirs. Figure 8 and Figure 9 show 2D permeability section flow simulation data that visually quantify the drilling outcome problem posed by spatially correlated poroperm media. In composing Figure 8 , we start with a km-scale poroperm distribution such as illustrated in Figure 6 for porosity spatial correlation well-log spectral scaling exponents in range $1.1<\beta<1.3$ as given in flow empiric (i). Within the Figure $6 \mathrm{~km}$-scale permeability section, we can compute wellbore fluid inflow for a sequence of production wellbore locations that are taken to be at the centre of $250 \mathrm{~m}$ diameter drainage zones. Figure 8 compares two permeability distributions that are the least productive (above) with two of the most productive $250 \mathrm{~m}$ diameter flow sections (below). Figure 9 illustrates the wellbore-centric filamentary distributions of $\log ($ flow) amplitudes for the Figure 8 permeability sections. The accompanying side-bar nominal amplitude scales show the flow sections range over a factor of 6 in permeability and factor $20 \sim \mathrm{e}^{3}$ in wellbore flow. Figure 10 shows that the distribution of wellbore flows of the type illustrated in Figs 8-9 is lognormal, as observed for crustal reservoir flow systems worldwide as given in flow empiric (iii). In reference to Figure 1, Figure 10 shows that for a given permeability section (or volume) only the right-most handful of geothermal wells are productive in terms of spinning turbines. The need to acquire flow structure information in advance of drilling convective geothermal production wells is manifest.

\section{Discussion - Wellbore Flow Evaluation of 3D Convective Geothermal Flow Structures}

While Figures 1-10 are based on flow model computations for 2D sections embodying the spatial correlation properties of crustal flow systems, applying these spatial-correlation outcomes to actual reservoirs requires $3 \mathrm{D}$ computation. Via the essential universality of ambient crustal fluid flow 
empirics (i)-(vi), we can expand from 2D generic reservoir section flow simulations of Figs 1-10 intended for geothermal flow systems to 3D applications using recent field-scale fluid flow data acquired in crystalline basement rock [14] and hydrocarbon-bearing shale formations [7].

In Figure 11 we illustrate the Matlab-implemented finite-element flow solver applied to 3D wellbore-centric flow modelling of heat advective flow transport at $1.5 \mathrm{~km}$ depth in basement rock [14]. To match the steady-state temperature profile observed in the wellbore, inflow to the well was conjectured to occur at the observed temperature anomaly depths. The inflow structure was modelled as a planar zone of enhanced permeability as might be attributed to a standard ambient fracture/fault structure. Flow boundary conditions were adjusted until the model wellbore temperature profile matched the observed wellbore flow profile. The 3D finite-element flow modelling procedure provided good model matches for multiple wellbore temperature profiles, indicating that 3D fluid flow modelling in spatially complex media is feasible.

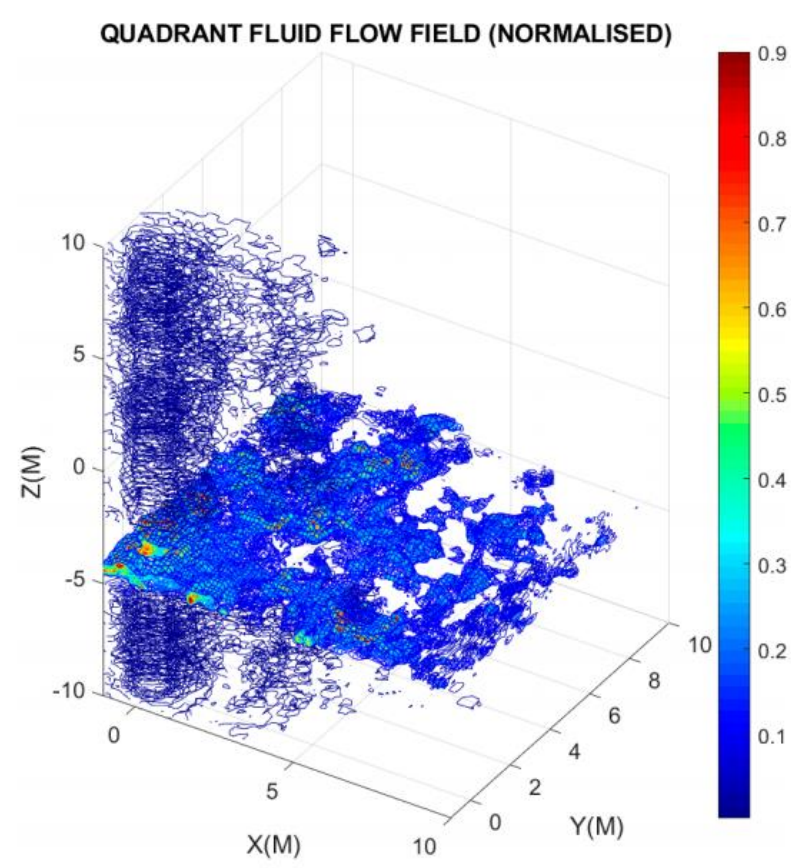

Figure 11 Quarter-section of 3D version of 2D wellbore-centric inflow modelling of crustal flow systems $k(x, y) \sim \exp (\alpha \phi(x, y))$ illustrated in Figures 1-10. The pictured volumetric flow distribution is dominated by a horizontal planar flow structure which transports heat to the wellbore fluid [14].

In a second 3D field-scale flow image, Figure 12 displays surface seismic emission acquisition/processing image data obtained during shale formation stimulation and production of a $1 \mathrm{~km}$-scale crustal volume [7]. The three-panel time sequence of flow stimulation of the shale formation shows tracking of seismic emission energy associated with fluid flow (white/black smudges). Fluid from a lateral wellbore (black line) enters the formation via the imaged fracture system (red lines) at the point of wellbore intersection (left panel), then spreads (mid/right panels) through the fracture-connectivity pathways to flow-stimulate seismic emissions elsewhere in the fracture structure of the shale formation. Crustal fluid flow empirics (i)-(vi) show that such seismic emission flow imaging data acquired for convective geothermal flow systems can provide flowmodel structures within which to embed precision flow images $V$ to give production well output $Q$ 
$\sim \rho C \times T \times V$. Production well drilling guided by combining enhanced-flow seismic emission data and precision wellbore-centric flow modelling generated from wellbore flow data can greatly increase the efficiency of convective geothermal system drilling.

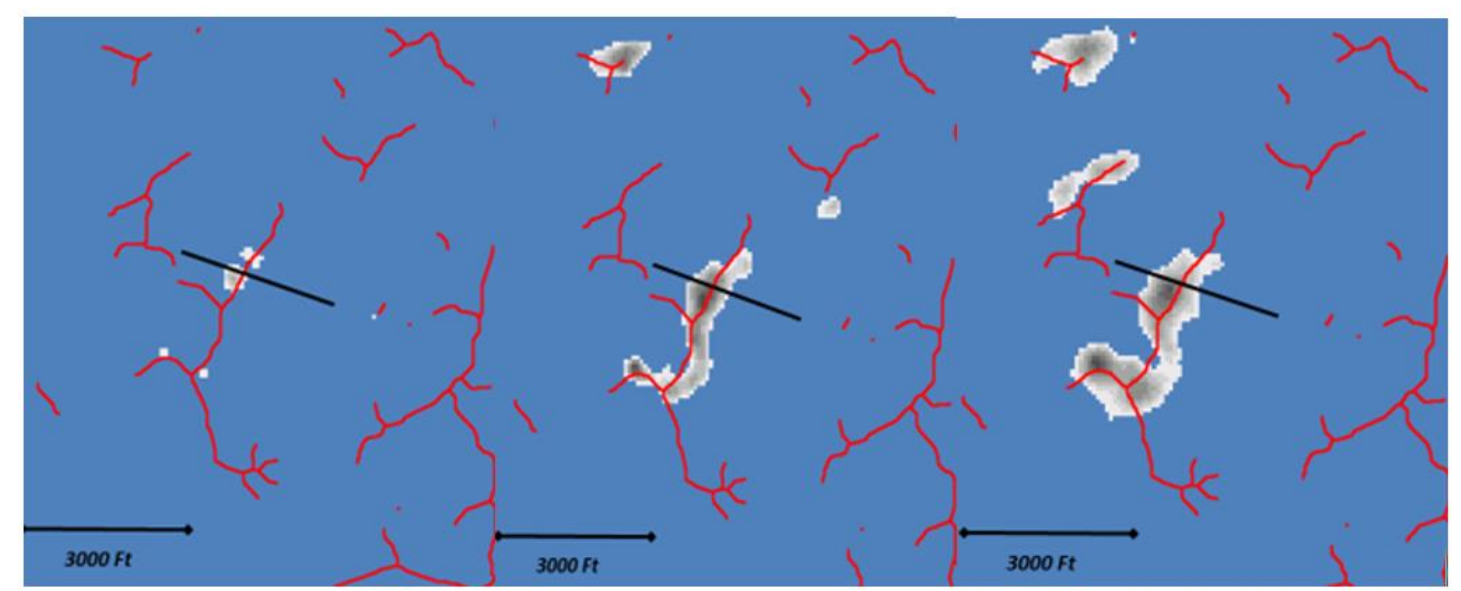

Figure 12 Time sequence of fracture-system enhanced flow structure from hydrocarbon-bearing shale formation as depicted by surface seismic sensor array data acquisition/processing [7]. Red lines represent fracture-flow system. Black/white smudges represent seismic emissions generated by stimulation fluids injected into the crust from a horizontal well (black line). Seismic emissions progress as shown in the minute-by-minute sequence following the time of initial fluid injection at left. The fact that seismic emissions are confined to the red-line domain connected to the injection wellbore indicate that fluid is detected by seismic emissions associated with fluid flow along the connected red-line fracture system. This "channelled" field-scale flow image is equivalent to the Figure 4 and Figure 5 model flow images.

Figure 11 and Figure 12 stand as guide to using the Matlab-based finite element flow solver (or equivalent) to model flow into a convective geothermal production wellbore drilled into a km-scale crustal volume. A km-scale reservoir model volume with 256 nodes to a side can represent an actual geothermal field flow system at $4 \mathrm{~m}$ spatial resolution through seismic emission data acquired by a multi-channel surface sensor array as outlined in [7]. The reservoir model comprises a generic 3D crustal empiric permeability number field $k(x, y, z) \sim \exp (\alpha \phi(x, y, z))$ that is embedded with a 3D enhanced flow structure image derived from field seismic emission data.

\section{Conclusions}

In convective geothermal reservoirs, channelised flow arising from highly attested spatially correlated crustal poroperm properties leads to lognormal distributions of well productivity. While hydrocarbon production can extract pay from both low and high productivity wells, the persistent high risk of low productivity non-pay geothermal wells greatly hinders investment in convective geothermal power projects [7]. Geothermal drilling risk can be addressed by the growing ability of multi-channel seismic emission data acquisition/processing to locate high-flow geothermal drilling targets and avoid low-flow areas. 
Crustal flow channelisation empirics at scales from $\mathrm{cm}$ to $\mathrm{km}$ is given in terms of the spatial correlation properties of crustal permeability distribution $k(x, y, z) \sim \exp (\alpha \phi(x, y, z))$ for large values of the poro-connectivity parameter $\alpha$. Flow modelling for geothermal production wells must handle the spatially-erratic high-amplitude spatial fluctuations arising from the $k(x, y, z) \sim \exp (\alpha \phi(x, y, z))$ empiric. Two "weak formulation" flow solver algorithms implemented in Matlab demonstrate that these numerical methods give stable accurate reservoir flow modelling capability for the realistic range of poro-connectivity parameter $\alpha$. We project that large scale seismic emission flow imaging data embedded in the crustal empiric permeability structure $k(x, y, z) \sim \exp (\alpha \phi(x, y, z))$ will allow precision wellbore flow modelling to refine reservoir flow structures and quantitatively model production wellbore flow for sustainable and progressive resource operation of convective flow systems.

\section{Author Contributions}

PCL performed the computations; PCL and PEM jointly prepared the manuscript.

\section{Competing Interests}

The authors have declared that no competing interests exist.

\section{References}

1. Clauser $\mathrm{C}$, Huenges $\mathrm{E}$. Thermal conductivity of rocks and minerals. Rock physics and phase relations: A handbook of physical constants. Washington: American Geophysical Union; 1995.

2. Carslaw HS, Jaeger JC. Conduction of heat in solids. Oxford: Clarendon Press; 1959. p. 510.

3. Theis CV. The relation between the lowering of the piezometric surface and the rate and duration of discharge of a well using ground-water storage. Eos Trans Am Geophys Union. 1959; 16: 519-524.

4. Muskat M. The flow of fluids through porous media. J Appl Phys. 1937; 8: 274-282.

5. Kruger P, Ramey HJ. Workshop on geothermal reservoir engineering. Stanford: Stanford University Workshop; 1975.

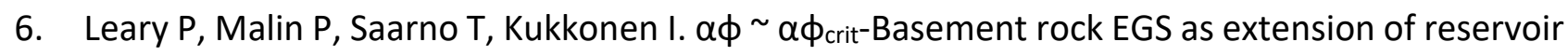
rock flow processes. Proceedings of 43th Workshop on Geothermal Reservoir Engineering; 2018 February 12-14; Stanford, CA, USA. Stanford: Stanford University.

7. Leary $P$, Malin $P$, Saunders $G$, Sicking $C$. Seismic imaging of convective geothermal flow systems to increase well productivity. J Energ Power Technol. 2020; 2: 012.

8. Leary P, Malin P, Saarno T, Heikkinen P. St1 deep heat egs project-computation of wellbore-towellbore permeability stimulation. Proceedings of World Geothermal Congress; 2021 May 2126; Reykjavik, Iceland. Bonn: International Geothermal Association.

9. Leary P, Malin P. MEQ permeability-modelling high frequency emissions from stimulationinduced seismic activity in the ambient crust. Proceedings of World Geothermal Congress; 2021 May 21-26; Reykjavik, Iceland. Bonn: International Geothermal Association.

10. Leary PC. Rock as a critical-point system and the inherent implausibility of reliable earthquake prediction. Geophys J Int. 1997; 131: 451-466. 
11. Leary PC. Fractures and physical heterogeneity in crustal rock, in heterogeneity in the crust and upper mantle: Nature, scaling, and seismic properties. New York: Kluwer Academic/Plenum Publishers; 2002. p. 155-p. 186.

12. Leary $P$, Malin P, Niemi R. Fluid flow \& heat transport computation for power-law scaling poroperm media. Geofluids. 2017; 2017: 9687325.

13. Leary P, Malin P, Pogacnik J, Rugis J, Valles B, Geiser P. Lognormality, $d k \sim k d \phi$, EGS, and all that. Proceedings of 39th Stanford Geothermal Workshop, Stanford University; 2014 February 24-26; Stanford, CA, USA. Stanford: Stanford University.

14. Leary $P$, Malin P, Saarno T, Kukkonen I. Prospects for assessing enhanced geothermal system (EGS) basement rock flow stimulation by wellbore temperature data. Energies. 2017; 10: 1979.

15. Success of geothermal wells: A global study, international finance corporation [Internet]. Washington: International Finance Corporation; 2013. Available from: https://www.ifc.org/wps/wcm/connect/topics ext content/ifc external corporate site/sust ainability-at-ifc/publications/publications gpn geothermal-wells.

16. Malin P, Leary P, Shalev E, Rugis J, Valles B, Boese C, et al. Flow lognormality and spatial correlation in crustal reservoirs: II-Where-to-drill guidance via acoustic/seismic imaging. Proceedings of the World Geothermal Congress; 2015 April 19-24; Melbourne, Australia. Bonn: International Geothermal Association.

17. Leary P, Malin P, Saarno T. A physical basis for the Gutenberg-Richter fractal scaling. Proceedings of 45th Workshop on Geothermal Reservoir Engineering; 2020 February 10-12; Stanford, CA, USA. Stanford: Stanford University.

18. Zaky DA, Nugraha AD, Sule R, Jousset P. Spatial analysis of earthquake frequency-magnitude distribution at geothermal region in the south of Bandung, West Java, Indonesia. Potsdam: 9th International Workshop on Statistical Seismology; 2015.

19. Leary P, Malin P, Saarno T, Heikkinen P, Diningrat W. Coupling crustal seismicity to crustal permeability - Power-law spatial correlation for EGS-induced and hydrothermal seismicity. Proceedings of the 44th Workshop on Geothermal Reservoir Engineering; 2019 February 11-13; Stanford, CA, USA. Stanford: Stanford University.

20. Leary $P$, Malin $P$. Correlation function $\Gamma_{\text {meq }}(r) \sim 1 / r^{1 / 2}$ coupling of microseismicity to permeability - The basis for fluid flow seismic image targeting for geothermal production wells. Reykjavik: Proceedings World Geothermal Congress; 2020.

21. Bear J. Dynamics of fluids in porous media, New York: American Elsevier; 1972.

22. Warren JE, Price HS. Flow in heterogeneous porous media. Soc Pet Eng J. 1961; 1: 153-169.

23. Warren JE, Skiba FF. Macroscopic dispersion. Soc Pet Eng J. 1964; 4: 215-230.

24. Warren JE, Root PJ. The behavior of naturally fractured reservoirs. Soc Pet Eng J. 1963; 3: 245255.

25. Hubbert MK. Motion of ground water. Trans N Y Acad Sci. 1941; 3: 39-55.

26. Hubbert MK. Darcy's law and the field equations of the flow of underground fluids. Int Assoc Sci Hydrol. Bull. 1957; 2: 23-59.

27. Pruess K, Oldenburg C, Moridis G. TOUGH2 User's Guide, Version 2.1 [Internet]. Berkeley: Lawrence Berkeley National Laboratory; 1999. Avilable from: https://www.osti.gov/biblio/751729-tough2-user-guide-version.

28. Pruess $\mathrm{K}$. The TOUGH codes-A Family of simulation tools for multiphase flow and transport processes in permeable media. Vadose Zone J. 2004; 3: 738-746. 
29. Peaceman DW. Interpretation of well-block pressures in numerical reservoir simulation. Soc Pet Eng J. 1978; 18: 183-194.

30. Thomas K, Dixon TN, Pierson RG. Fractured reservoir simulation. Soc Pet Eng J. 1983; 23: 42-54.

31. Partial differential equation toolbox user's guide [Internet]. Natick: The MathWorks Inc. Available from: http://www.math.chalmers.se/ mohammad/teaching/PDE1 TM/pde1 lab/lab2/toolboxguid e.pdf.

32. Hughes TJ. The finite element method: Linear static and dynamic finite element analysis. New York: Dover Publications; 2020.

33. Leary $P$, Malin $P$, Niemi R. Finite-element modelling of wellbore-observed fracture-borne heat advection - application to EGS stimulation in basement rock. Proceedings of 41st Workshop Geothermal Reservoir Engineering; 2016 February 22-24; Stanford, CA, USA. Stanford: Stanford University.

34. Brehme $M$, Regenspurg S, Leary $P$, Bulut $F$, Milsch $H$, Petrauskas $S$, et al. Injection-triggered occlusion of flow pathways in geothermal operations. Geofluids. 2018; 2018: 4694829.

35. Brehme $M$, Leary $P$, Milsch $H$, Petrauskas $S$, Valickas $R$, Kamah $Y$, et al. Natural and altered physical flow structures in the earth's crust with applications for geothermal energy. Proceedings of 43rd Workshop on Geothermal Reservoir Engineering; 2018 February 12-14; Stanford, CA, USA. Stanford: Stanford University.

36. Aarnes J, Gimse T, Lie KA. An introduction to the numerics of flow in porous media using Matlab. In Geometric Modelling, Numerical Simulation, and Optimization. Berlin: Springer; 2006.

37. Lie KA. User guide for the MATLAB Reservoir Simulation Toolbox (MRST). Cambridge: Cambridge University Press; 2019.

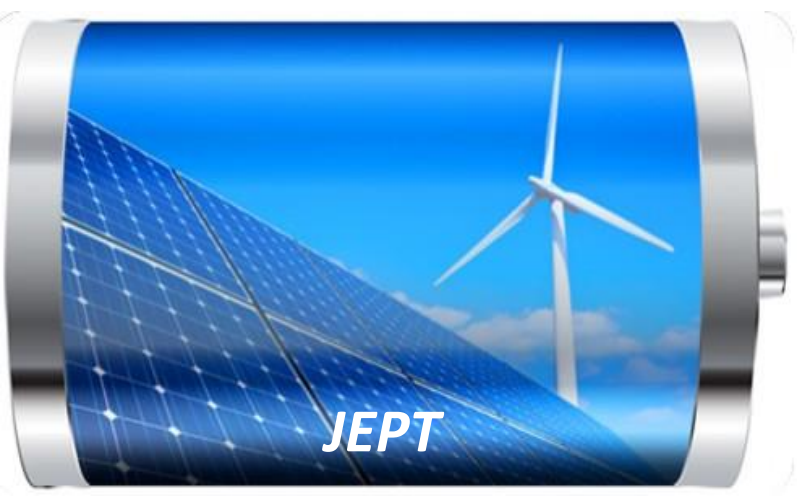

Enjoy JEPT by:

1. Submitting a manuscript

2. Joining in volunteer reviewer bank

3. Joining Editorial Board

4. Guest editing a special issue

For more details, please visit: http://www.lidsen.com/journal/jept 
Original Research

\title{
Nowcasting Wind, Wave, Current and Turbulence Intensity for Offshore Wind Power Operation and Maintenance
}

\author{
Shih-Ang Hsu * \\ Coastal Studies Institute, Louisiana State University, Baton Rouge, LA 70803, USA; E-Mail: \\ sahsu@lsu.edu
}

* Correspondence: Shih-Ang Hsu; E-Mail: sahsu@lsu.edu

Academic Editor: Aritra Ghosh

\section{Special Issue:}

Journal of Energy and Power Technology

2021, volume 3 , issue 1

doi:10.21926/jept.2101014
Received: December 21, 2020

Accepted: March 21, 2021

Published: March 24, 2021

\begin{abstract}
In order to improve offshore wind power operation and maintenance (O\&M), particularly during tropical and non-tropical cyclones, short-term forecasts or nowcasts up to 6 hours of meteorological and oceanographic (met-ocean) parameters including wind, waves, currents and turbulence intensity are needed. On the basis of numerous air-sea and wind-wave interaction experiments, datasets are analyzed including those from simultaneous measurements of wind and waves during Hurricane Wilma. Formulas are presented for nowcasts of met-ocean parameters. For quality assurance, these proposed formulas are further verified by independent datasets as provided in the literature. This manual-like guide should be useful for offshore wind-power O\&M technicians and operators.
\end{abstract}

\section{Keywords}

Offshore wind power; wind-wave interaction; tropical and non-tropical cyclones; turbulence intensity; nowcast met-ocean parameters

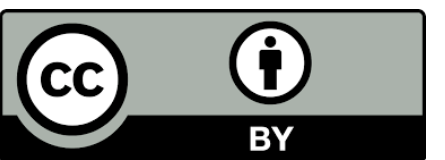

(C) 2021 by the author. This is an open access article distributed under the conditions of the Creative Commons by Attribution License, which permits unrestricted use, distribution, and reproduction in any medium or format, provided the original work is correctly cited. 


\section{Introduction}

According to the Glossary of the U. S. National Weather Service (https://w1.weather.gov/glossary/index.php?letter=n), nowcast is a short-term weather forecast, generally out to six hours or less. While global or regional weather forecasts are routinely available, e.g. from the European Centre for Medium-Range Weather Forecasts (ECWMF) or the U.S. National Center for Environmental Prediction (NCEP), it is the purpose of this study to supplement or valueadded nowcast for a smaller area within the forecasted domain such as an offshore wind farm. Using the wind or wave forecasts by the ECWMF or NCEP, we further add the nowcast for wind-driven current and overwater turbulence intensity, which are needed for offshore wind power operation and maintenance (O\&M).

According to [1-3], for aerodynamically rough flow over the ocean, approximately, the wind speed at $10 \mathrm{~m}$ (meters), $U_{10} \geq 9 \mathrm{~m} / \mathrm{s}$ and for a wind sea, the wave steepness, $H_{s} / L_{p} \geq 0.020$. Here $H_{s}$ is the significant wave height $(m), L_{p}\left(=1.56 T_{p}{ }^{2}\right)$ is the dominant wavelength $(m)$ and $T_{p}$ is the peak wave period (in seconds). Note that the wave parameter, $H_{s} / L_{p}$, is called wave steepness. Therefore, we define here that when $U_{10} \leq 9 \mathrm{~m} / \mathrm{s}$ and $H_{s} / L_{p} \leq 0.020$, fair weather and swell dominant conditions exist and they are not of major meteorological and oceanographic (met-ocean) concerns from the viewpoints of offshore wind power O\&M. In other words, when a wind farm is under gale or tropical cyclone conditions, nowcasts of wind, wave, current and turbulence intensity are more important. Following topics are our proposed manual-like guide for such an endeavor.

\section{Nowcasting Wind and Waves}

According to [3], during extra-tropical or tropical cyclone conditions, approximately,

$$
\mathrm{U}_{10}=\frac{35 \mathrm{H}_{\mathrm{s}}}{\mathrm{T}_{\mathrm{p}}}
$$

Here $T_{p}$ is the peak or dominant wave period (in seconds).

Further verification of Eq. (1) during a tropical cyclone is presented in Figure 1. Since the slope is unity and the correlation coefficient $R=0.85$, we can say that Eq. (1) is useful for operational use. In order to validate Eq. (1) under non-tropical cyclone conditions, Table 1 is provided and the result is shown in Figure 2. If one accepts the statistics given in Figure 3 and Figure 4, Eq. (1) is also applicable in Lake Ontario [4] and the Southern Ocean [5].

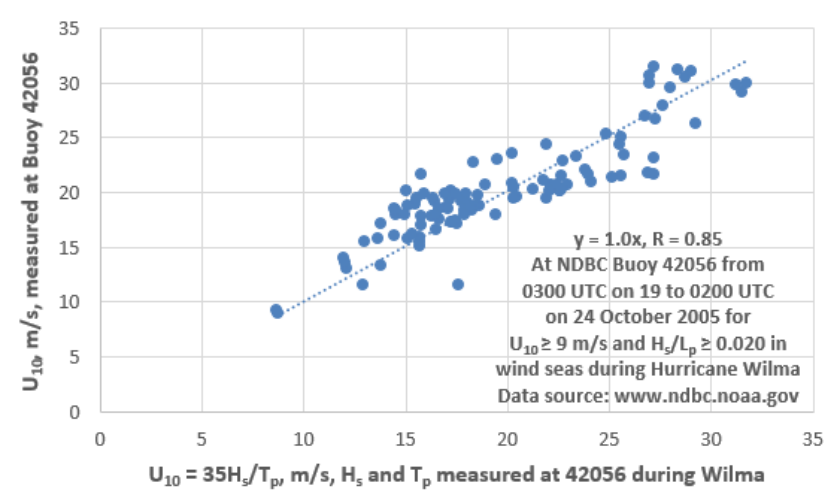

Figure 1 Verifying Eq. (1) at Buoy 42056 during Hurricane Wilma in 2005. 
Table 1 Datasets for the four non-tropical cyclones used in this study (Data source: www.ndbc.noaa.gov). Note that the wind speed measured at Buoys 45008 and 42360 were adjusted to $U_{10}$ according to the wind-gust method as presented in [3].

\begin{tabular}{lllll}
\hline Year & Month & Day & Buoy & Location \\
\hline 1993 & 3 & $12-14$ & 42003 & Gulf of Mexico \\
1996 & 11 & $2-5$ & 46035 & Bering Sea \\
2003 & 11 & $13-14$ & 45008 & Lake Huron \\
2018 & 12 & $26-27$ & 42360 & Gulf of Mexico \\
\hline
\end{tabular}

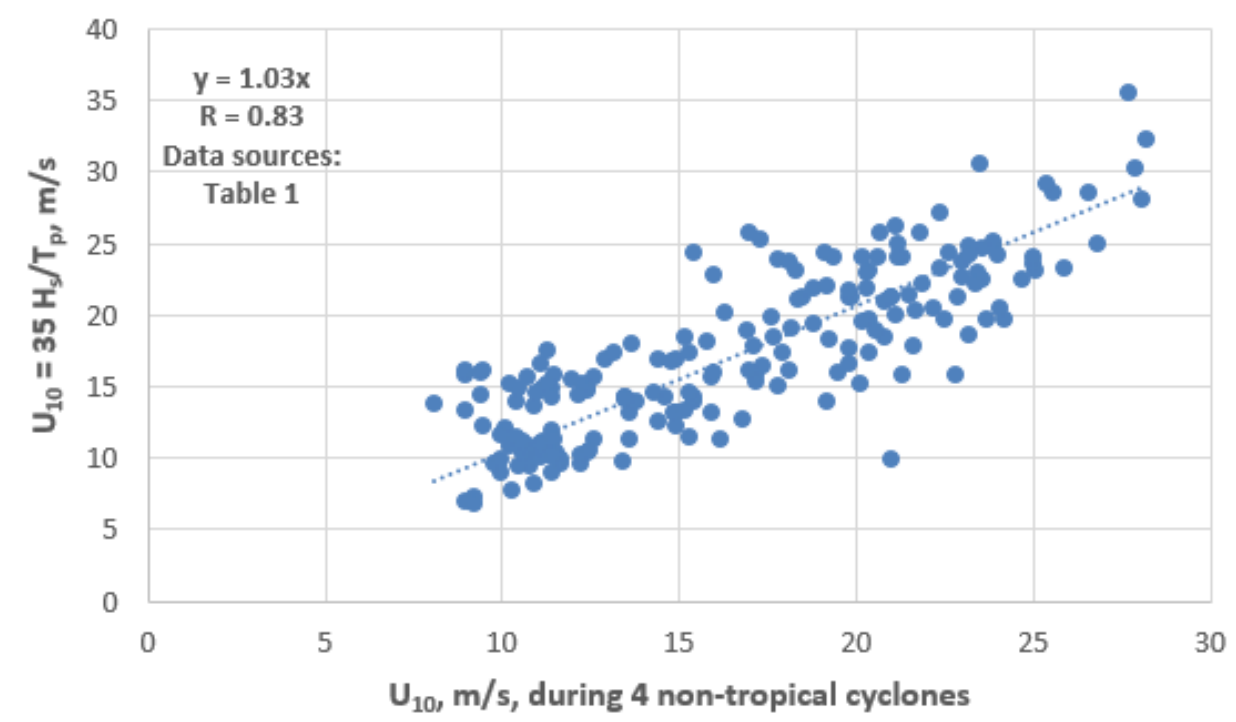

Figure 2 A verification of Eq. (1) under non-tropical cyclone conditions.

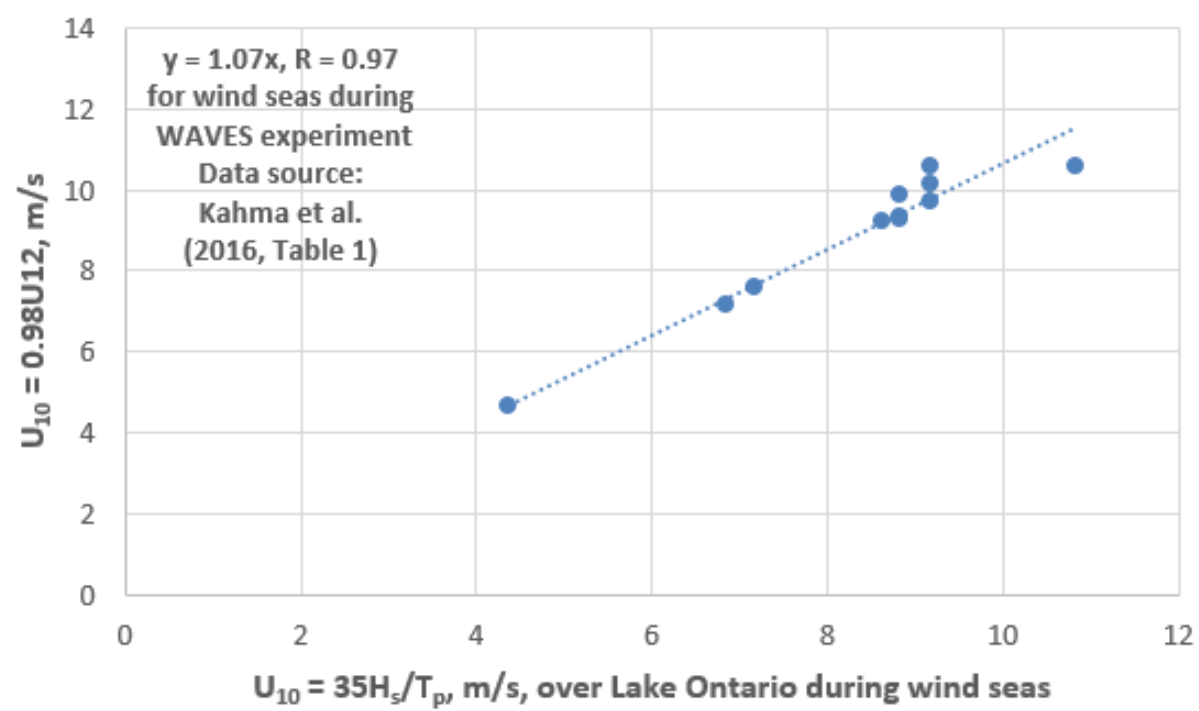

Figure 3 Validating Eq. (1) in Lake Ontario. 


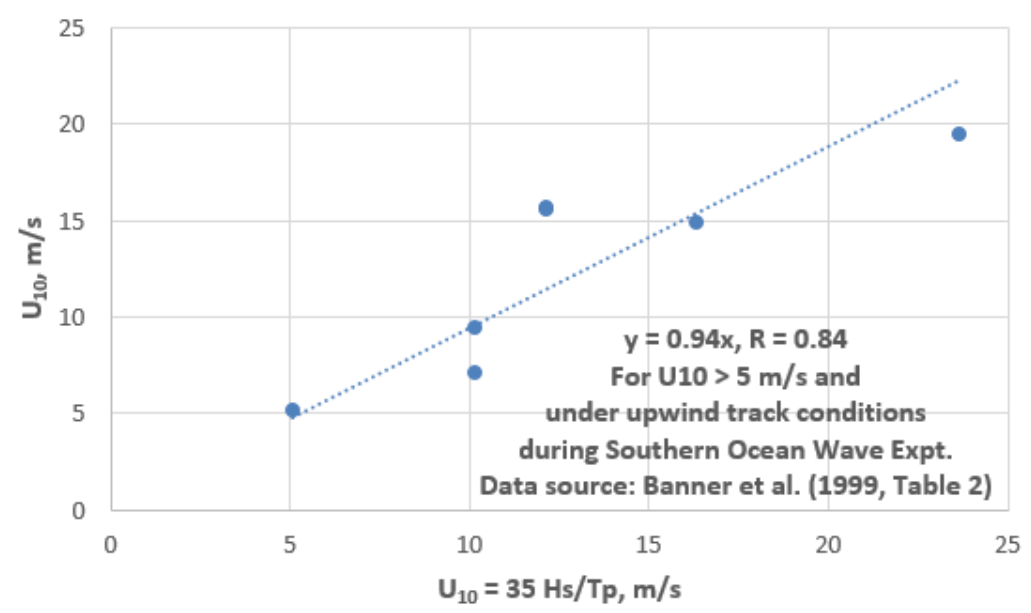

Figure 4 Validating Eq. (1) in the Southern Ocean.

Table 2 Examples of estimating winds and currents using the extreme wave measurements during Hurricane Ivan in 2004 based on Teague et al. [14].

\begin{tabular}{llllll}
\hline Parameter & M3 & M4 & M5 & M6 & Mean \\
\hline $\mathrm{H}_{\mathrm{s}}, \mathrm{m}$ measured & 18.0 & 16.1 & 17.7 & 14.8 & 16.7 \\
$\mathrm{U}_{10}, \mathrm{~m} / \mathrm{s}$ estimated & 47.5 & 43.1 & 46.8 & 40.1 & 44.4 \\
$\mathrm{U}_{\text {sea, }} \mathrm{m} / \mathrm{s}$ measured & 1.73 & 1.96 & 1.91 & 1.82 & 1.86 \\
$\mathrm{U}_{\text {sea, }} \mathrm{m} / \mathrm{s}$ estimated & 2.09 & 1.77 & 2.04 & 1.57 & 1.87 \\
\hline
\end{tabular}

If $T_{p}$ is not available, on the basis of Figure 5 during Hurricane Wilma near the National Data Buoy Center (NDBC) Buoy 42056 (see https://www.ndbc.noaa.gov/hurricanes/2005/wilma/), it may be estimated as

$$
\frac{\mathrm{H}_{\mathrm{s}}}{\mathrm{T}_{\mathrm{p}}}=0.0064 \mathrm{H}_{\mathrm{s}}+0.16
$$

With $\mathrm{R}=0.96$.

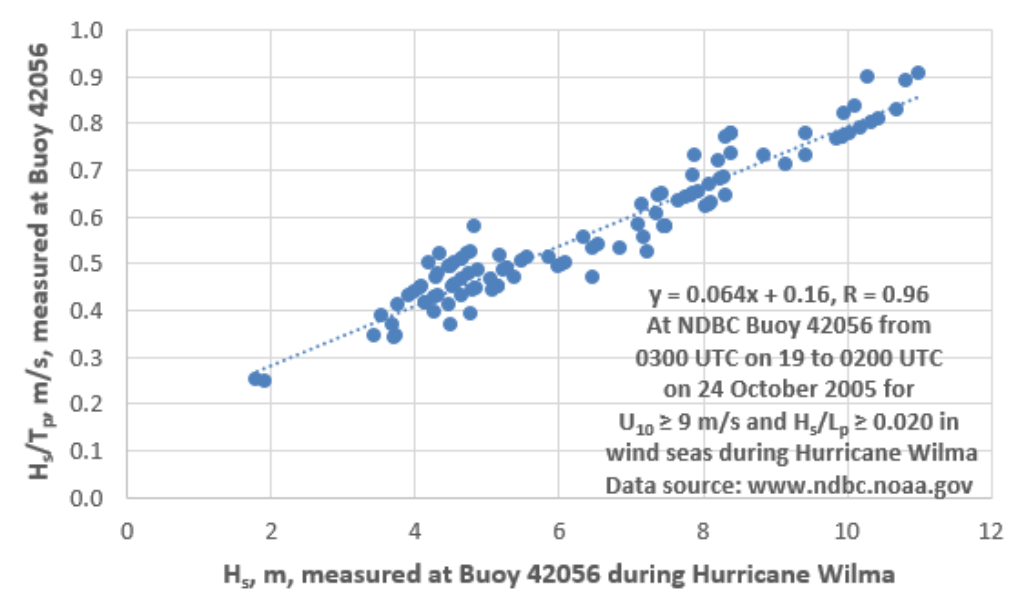

Figure 5 Relation between $\mathrm{H}_{s} / \mathrm{T}_{\mathrm{p}}$ and $\mathrm{H}_{\mathrm{s}}$. 
So that $\mathrm{U}_{10}$ and $\mathrm{H}_{\mathrm{s}}$ are linearly related and according to Figure 6 , we have

$$
\mathrm{H}_{\mathrm{s}}=0.43 \mathrm{U}_{10}-2.4
$$

With $R=0.91$, Eq. (3) may be used to nowcast $H_{s}$ if $U_{10}$ is available and vice versa.

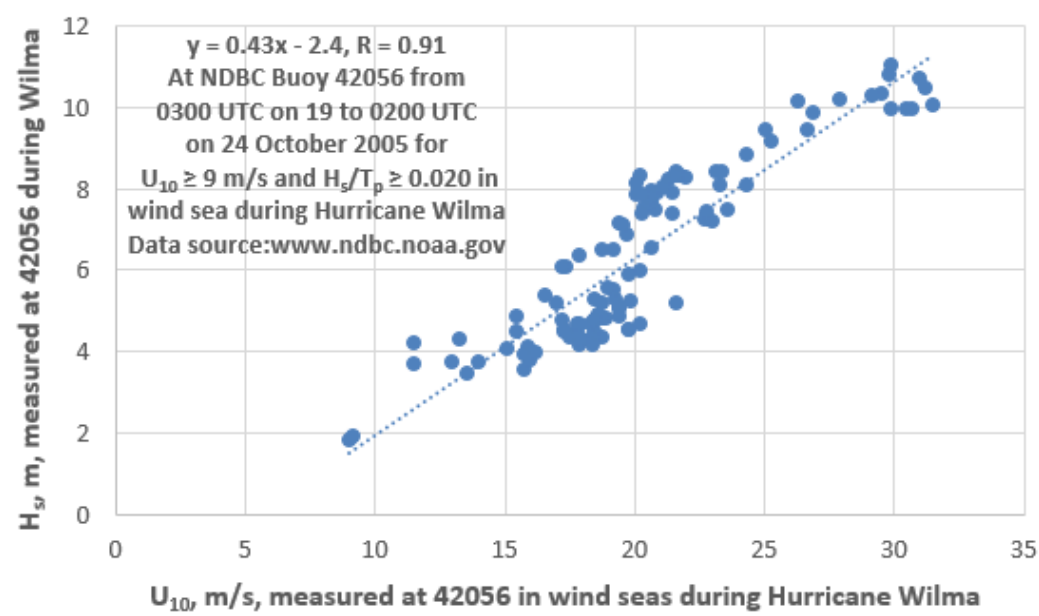

Figure 6 Relation between $\mathrm{H}_{\mathrm{s}}$ and $\mathrm{U}_{10}$.

\section{Nowcasting Overwater Turbulence Intensity}

Overwater turbulence intensity (TI) is an important parameter for offshore wind power $\mathrm{O} \& \mathrm{M}$ [6]. According to [7-9], when the atmospheric stability is near neutral [10], we have

$$
\mathrm{TI}=\frac{2.5 \mathrm{U}_{*}}{\mathrm{U}_{10}}=\frac{1}{\operatorname{Ln}\left(\frac{10}{\mathrm{Z}_{\mathrm{o}}}\right)}
$$

And

$$
\frac{\mathrm{Z}_{\mathrm{o}}}{\mathrm{H}_{\mathrm{s}}}=1200\left(\frac{\mathrm{H}_{\mathrm{s}}}{\mathrm{L}_{\mathrm{p}}}\right)^{4.5}
$$

Here $U *$ is the friction velocity in $\mathrm{m} / \mathrm{s}$ and $\mathrm{Z}_{\mathrm{o}}$ is the aerodynamic roughness parameter in meters.

Simultaneous measurements of met-ocean parameters including $U_{10}, H_{s}$ and $T_{p}$ are available at Buoy 42056 during Hurricane Wilma. These data can be analyzed using Eqs. (4) and (5). Figure 7 and Figure 8 are the results for the relations between $\mathrm{TI}$ at $10-\mathrm{m}$ with $\mathrm{U}_{10}$ and $\mathrm{H}_{\mathrm{s}}$, respectively, so that

$$
\mathrm{TI}=0.0028 \mathrm{U}_{10}+0.0581
$$

With $\mathrm{R}=0.79$ and,

$$
\mathrm{TI}=0.006 \mathrm{H}_{\mathrm{s}}+0.0753
$$

With $\mathrm{R}=0.83$. 


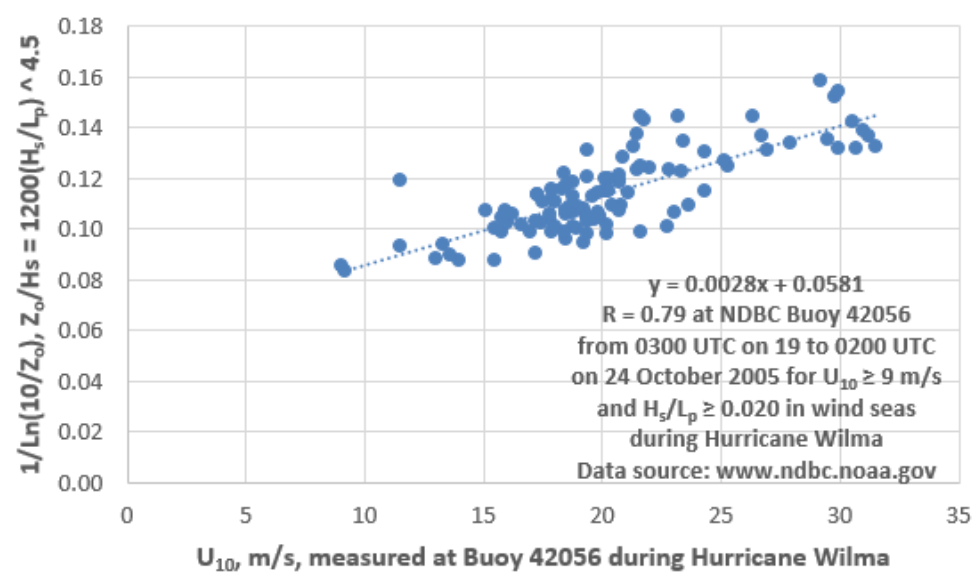

Figure 7 Variation of $\mathrm{Tl}$ at $10 \mathrm{~m}$ with $\mathrm{U}_{10}$.

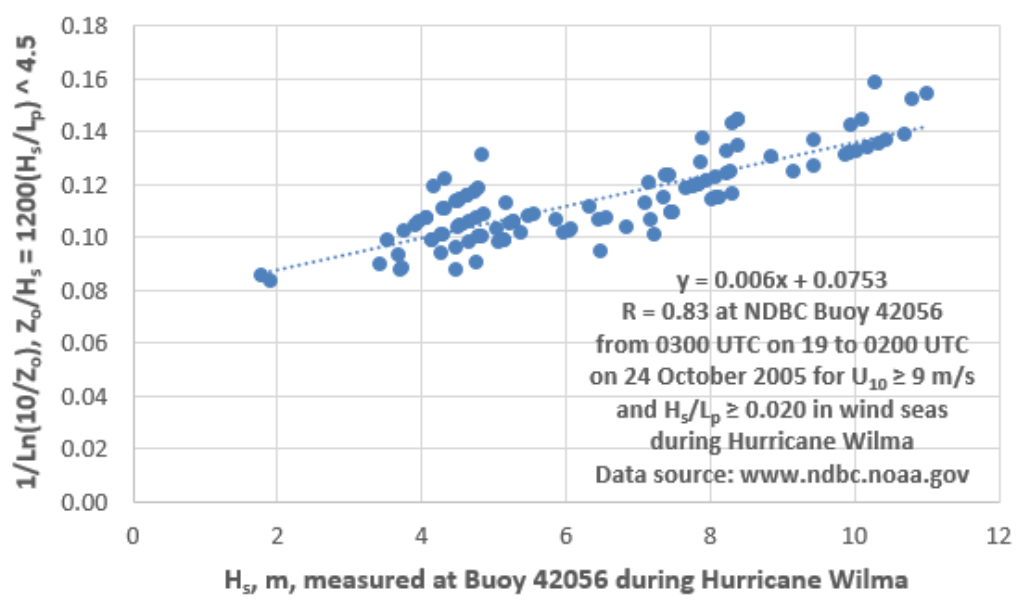

Figure 8 Variation of $\mathrm{Tl}$ at $10 \mathrm{~m}$ with $\mathrm{H}_{\mathrm{s}}$.

For quality assurance, Eq. (6) is compared with the TI formula by Smith [11]. It is a surprise that the result is nearly identical, indicating Eq. (6) is very useful for offshore wind power $O \& M$, since it extends from gale force wind to hurricane condition.

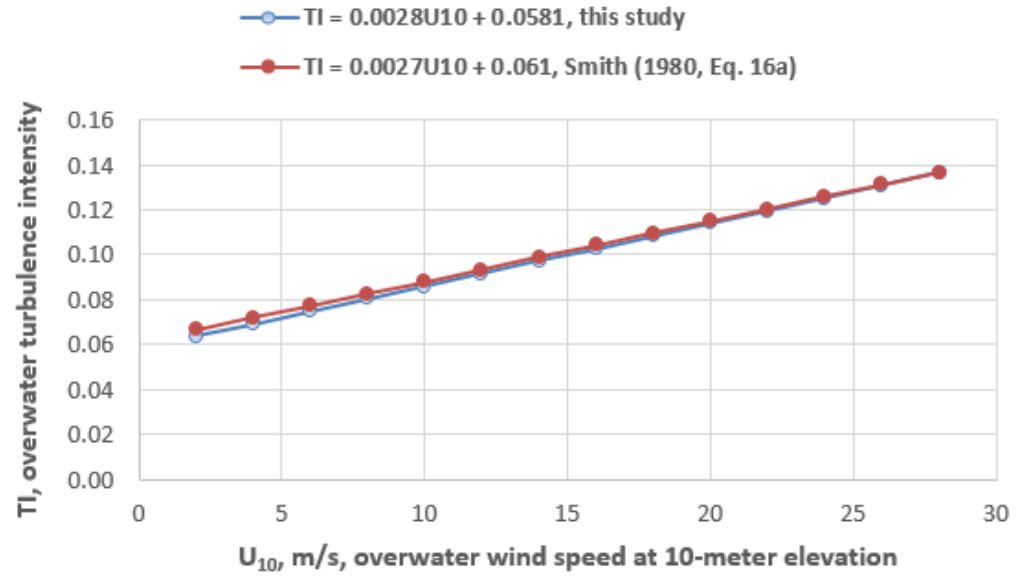

Figure 9 Comparison of Variation of $\mathrm{TI}$ at $10 \mathrm{~m}$ between Eq. (6) and the TI formula by Smith [11]. 


\section{Nowcasting Wind-Driven Currents}

Wind-driven currents are integral parts of total currents which also include those generated by the astronomical tides. For wind-driven currents, $U_{\text {sea, }}$ in $\mathrm{m} / \mathrm{s}$, according to [12] Eq. 4,

$$
\mathrm{U}_{\text {sea }}=0.57 \mathrm{U}_{*}
$$

From Eqs. (4) and (6), we have

$$
\mathrm{U}_{*}=0.4 \mathrm{U}_{10}\left(0.0028 \mathrm{U}_{10}+0.0581\right)
$$

On the basis of the datasets provided in [11] and [13], a verification of Eq. (9) is presented in Figure 10. Since the slope is unity and $R=0.97$, Eq. (9) is recommended for operational use.

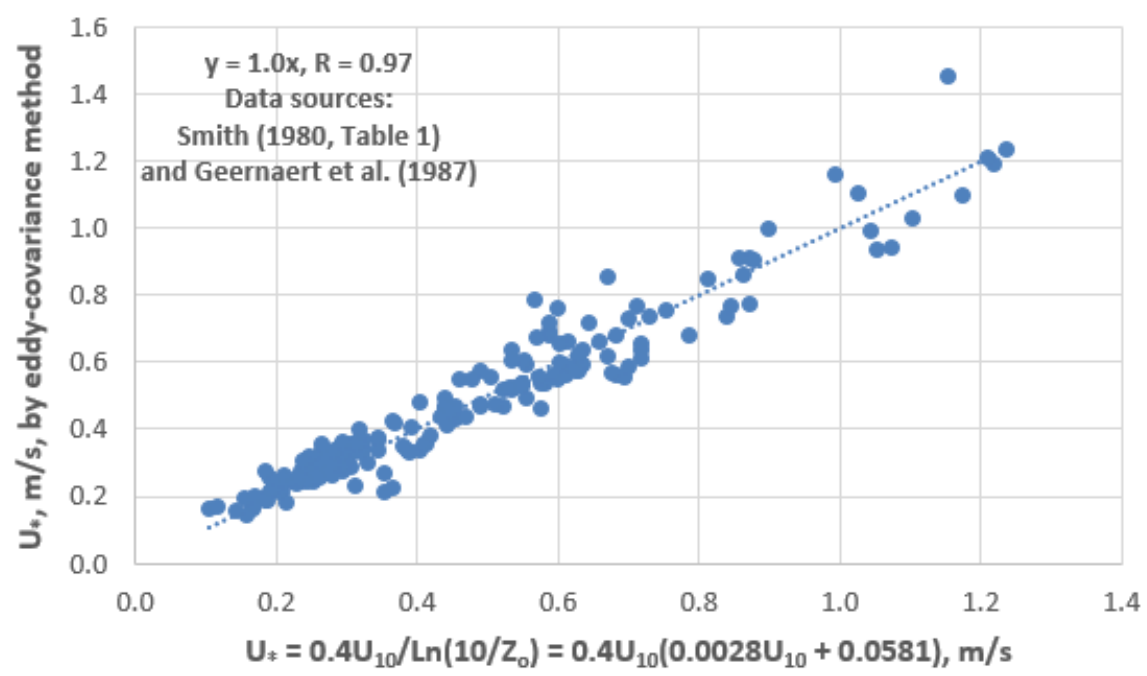

Figure 10 A verification of Eq. (9).

Now, by substituting Eq. (9) into Eq. (8), one gets

$$
\mathrm{U}_{\text {sea }}=0.23 \mathrm{U}_{10}\left(0.0028 \mathrm{U}_{10}+0.0581\right)
$$

Using Eq. (3) to convert $\mathrm{H}_{\mathrm{s}}$ to $\mathrm{U}_{10}$ for the datasets presented in [14] during Hurricane Ivan, Eq. (10) is validated in Table 2. Since the mean near-surface current is almost identical (in red) between the estimated and the measured, Eq. (10) is recommended for use in nowcasting.

\section{Conclusions}

On the basis of aforementioned analyses and discussions, it is concluded that

(1) Wind or waves can be nowcasted using Eq. (3) if either the wind or wave forecast is available;

(2) Overwater turbulence intensity at 10-m elevation can be nowcasted using Eq. (6) if the wind speed at $10-\mathrm{m}$ is available or Eq. (7) if the significant wave height is available;

(3) Overwater friction velocity can be nowcasted using Eq. (9); and

(4) Wind-driven currents can be nowcasted using Eq. (10). 


\section{Author Contributions}

Shih-Ang Hsu did all work.

\section{Competing Interests}

The author has declared that no competing interests exist.

\section{References}

1. Andreas EL, Mahrt L, Vickers D. A new drag relation for aerodynamically rough flow over the ocean. J Atmos Sci. 2012; 69: 2520-2537.

2. Drennan WM, Taylor PK, Yelland MJ. Parameterizing the sea surface roughness. J Phys Oceanogr. 2005; 35: 835-848.

3. Hsu SA, He Y, Shen H. Buoy measurements of wind-wave relations during Hurricane Matthew in 2016. J Phys Oceanogr. 2017; 47: 2603-2609.

4. Kahma KK, Donelan MA, Drennan WM, Terray EA. Evidence of energy and momentum flux from swell to wind. J Phys Oceanogr. 2016; 46: 2143-2156.

5. Banner ML, Chen W, Walsh EJ, Jensen JB, Lee S, Fandry C. The Southern Ocean waves experiment. Part I: Overview and mean results. J Phys Oceanogr. 1999; 29: 2130-2145.

6. Argyle $\mathrm{P}$, Watson $\mathrm{S}$, Montavon $\mathrm{C}$, Jones $\mathrm{I}$, Smith $\mathrm{M}$. Modelling turbulence intensity within a large offshore wind farm. Wind Energy. 2018; 21: 1329-1343.

7. Hsu SA, Blanchard BW. Estimating overwater turbulence intensity from routine gust-factor measurements. J Appl Meteorol. 2004; 43: 1911-1916.

8. Hsu SA. Estimating overwater friction velocity and exponent of power-law wind profile from gust factor during storms. J Waterw Port Coast Ocean Eng. 2003; 129: 174-177.

9. Taylor PK, Yelland MJ. The dependence of sea surface roughness on the height and steepness of the waves. J Phys Oceanogr. 2001; 31: 572-590.

10. Hsu SA. An overwater stability criterion for the offshore and coastal dispersion model. Boundary Layer Meteorol. 1992; 60: 397-402.

11. Smith SD. Wind stress and heat flux over the ocean in gale force winds. J Phys Oceanogr. 1980; 10: 709-726.

12. Wu J. Sea-surface drift currents induced by wind and waves. J Phys Oceanogr. 1983; 13: 14411451.

13. Geernaert GL, Larsen SE, Hansen F. Measurements of the wind stress, heat flux, and turbulence intensity during storm conditions over the North Sea. J Geophys Res Oceans. 1987; 92: 1312713139.

14. Teague WJ, Jarosz E, Wang DW, Mitchell DA. Observed oceanic response over the upper continental slope and outer shelf during Hurricane Ivan. J Phys Oceanogr. 2007; 37: 2181-2206. 
JEPT 2021; 3(1), doi:10.21926/jept.2101014

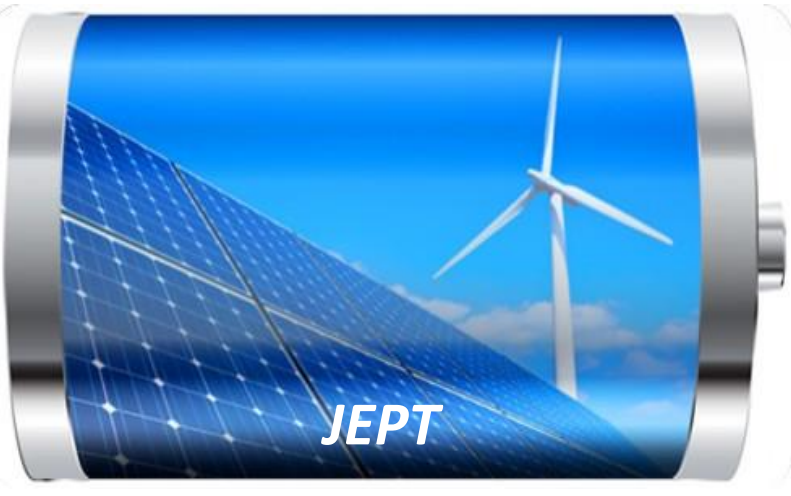

Enjoy JEPT by:

1. Submitting a manuscript

2. Joining in volunteer reviewer bank

3. Joining Editorial Board

4. Guest editing a special issue

For more details, please visit:

http://www.lidsen.com/journal/jept 


\author{
LIDSEN Publishing Inc. \\ 2000 Auburn Drive, One Chagrin \\ Highlands, Suite 200, Beachwood, OH 44122 \\ USA \\ Tel.: +1-216-370-7293 \\ Fax: +1-216-378-7505 \\ http://www.lidsen.com/
}

Journal of Energy and Power Technology Editorial Office,

E-mail: jept@lidsen.com

http://www.lidsen.com/journals/jept 
LIDSEN Publishing Inc.

2000 Auburn Drive, One Chagrin

Highlands, Suite 200, Beachwood, OH 44122

USA

Tel.: +1-216-370-7293

Fax: +1-216-378-7505

http://www.lidsen.com/

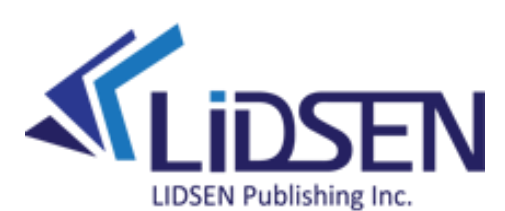

ISSN 2690-1692 